This document was prepared in conjunction with work accomplished under Contract No. DE-AC09-96SR18500 with the U.S. Department of Energy.

This work was prepared under an agreement with and funded by the U.S. Government. Neither the U. S. Government or its employees, nor any of its contractors, subcontractors or their employees, makes any express or implied: 1 . warranty or assumes any legal liability for the accuracy, completeness, or for the use or results of such use of any information, product, or process disclosed; or 2 . representation that such use or results of such use would not infringe privately owned rights; or 3 . endorsement or recommendation of any specifically identified commercial product, process, or service. Any views and opinions of authors expressed in this work do not necessarily state or reflect those of the United States Government, or its contractors, or subcontractors. 
June 12, 2007

From: T. B. Edwards

Statistical Consulting Section

M. D. Joner, Technical Reviewer

Date

R. C. Tuckfield, Manager

Date

Statistical Consulting Section

\section{A Statistical Review of the Chemical Composition Measurements and PCT Results for the Glasses Fabricated as Part of the US Test Matrix}




\subsection{EXECUTIVE SUMMARY}

The Savannah River National Laboratory (SRNL) is part of a consortium that is looking to improve the retention of aluminum, chromium, and sulfate in high level radioactive waste (HLW) glass. Such glass has been produced by the Defense Waste Processing Facility (DWPF) at the Savannah River Site (SRS) in South Carolina since it began operating in 1996 and is planned to be produced by the River Protection Project - Waste Treatment Plant (WTP) at the Hanford Site in Washington. The consortium conducting this study, which is designated as Task \#6 by the Department of Energy (DOE) Environmental Management (EM) program sponsoring this effort, is made up of personnel from SRNL, the Pacific Northwest National Laboratory (PNNL), and the V.G. Khlopin Radium Institute (KRI). Coordinated glass experimental work will be performed by each member of the consortium. The glasses that are being studied were selected to further the understanding of composition-property relationships within the glass regions of interest to both DWPF and WTP.

Forty-five (45) glasses, making up the US test matrix, were batched and fabricated to support the study. The chemical compositions of these glasses were measured by SRNL's Process Science Analytical Laboratory (PSAL) under the auspices of an analytical plan. In addition, two heat treatments (quenched and centerline canister cooled, $\mathrm{ccc}$ ) of each glass were subjected to the 7day Product Consistency Test (PCT) to assess their durabilities. More specifically, the Method A of the PCT (ASTM C-1285-2002) was used for these tests. Measurements of the resulting leachate solutions were conducted by PSAL under the auspices of three analytical plans.

A statistical review of the PSAL measurements of the chemical compositions and of the PCT results for the glasses making up the US test matrix is provided in this memorandum. Target, measured, and measured bias-corrected compositional views were determined for these glasses. The durability results for the US study glasses are compared to those of the Environmental Assessment (EA) glass. All of the US glasses yielded PCTs that are lower than those of the EA glass. The largest PCT values are those measured for the ccc versions of US-27 and US-18 whose boron normalized leachate (NL[B]) values in grams per liter $(\mathrm{g} / \mathrm{L})$ were $16.4 \mathrm{~g} / \mathrm{L}$ (based on the targeted composition) and $10.7 \mathrm{~g} / \mathrm{L}$ (based on the targeted composition), respectively. The $16.4 \mathrm{~g} / \mathrm{L}$ is just below the value of $16.695 \mathrm{~g} / \mathrm{L}$ for EA's NL[B] that was reported by Jantzen et al. in WSRC-TR-92-0346, Revision 1. For the quenched version of the glasses, the largest NL[B] value is $0.67 \mathrm{~g} / \mathrm{L}$ (based on the targeted composition). Thus, some statistically significant differences were seen between the quenched and ccc versions for some of the glasses. It should be noted that the thirty (30) glasses making up the KRI test matrix were not included in these analyses. 


\subsection{INTRODUCTION}

The Savannah River National Laboratory (SRNL) is part of a consortium that is looking to improve the retention of aluminum, chromium, and sulfate in high level radioactive waste (HLW) glass. Such glass has been produced by the Defense Waste Processing Facility (DWPF) at the Savannah River Site (SRS) in South Carolina since it began operating in 1996 and is planned to be produced by the River Protection Project - Waste Treatment Plant (WTP) at the Hanford Site in Washington. The consortium conducting this study, which is designated as Task \#6 by the Department of Energy (DOE) Environmental Management (EM) program sponsoring this effort, is made up of personnel from SRNL, the Pacific Northwest National Laboratory (PNNL), and the V. R. Khlopin Radium Institute (KRI). Coordinated glass experimental work is being performed by each member of the consortium. The glasses that are being studied were selected to further the understanding of composition-property relationships within the glass regions of interest to both DWPF and WTP [1].

Forty-five (45) glasses, making up the US portion of the test matrix, were batched and fabricated to support the study. The chemical compositions of these glasses were measured by SRNL's Process Science Analytical Laboratory (PSAL) under the auspices of an analytical plan [2]. The first objective of this memorandum is to provide a statistical analysis of the chemical composition measurements that were generated by PSAL for the US glasses. More specifically, assessments of measured compositions against their targeted values are made. The results from this analysis are presented in Section 3.

The durability of these 45 US glasses was measured using the Product Consistency Test (PCT) as defined in ASTM C-1285-2002 [3]. Two heat treatments were utilized during the fabrication of each of these glasses. Specifically, each of the glasses was quenched (i.e., rapidly cooled) and a sample of each glass was cooled in accordance with the centerline canister cooling (ccc) regime. Both heat treatments of each glass were subjected to the PCT. SRNL's PSAL measured the elemental concentrations of the resulting leachate solutions from the PCTs.

Due to the large number of PCTs needed to complete the testing for the two heat treatments of the 45 glasses, the PCTs were submitted to PSAL in three sets. PSAL completed these measurements under the auspices of three analytical plans ([4], [5], and [6]). The second objective of this memorandum is to provide a review of the PCT measurements and a comparison of these durability results to the acceptance criteria for durability that relies on PCT measurements of the Environmental Assessment (EA) standard glass. The results from these efforts are presented in Section 4.

Section 5 provides a summary of the overall conclusions from these investigations and analyses, which were conducted using JMP Version 6.0.2 [7].

\subsection{A STATISTICAL REVIEW OF THE CHEMICAL COMPOSITION MEASUREMENTS FOR THE US GLASSES}

In this section, the measured and targeted compositions of the US glasses are presented and compared. The targeted compositions for these glasses are provided in Table A1 of Appendix A.

Chemical composition measurements for these glasses were conducted by PSAL following an analytical plan [2]. Two dissolution methods were utilized in measuring these chemical compositions: samples prepared by lithium metaborate (LM) dissolution were measured for calcium $(\mathrm{Ca})$, chromium $(\mathrm{Cr})$, potassium $(\mathrm{K})$, magnesium $(\mathrm{Mg})$, manganese $(\mathrm{Mn})$, sodium $(\mathrm{Na})$, lead $(\mathrm{Pb})$, sulfur $(\mathrm{S})$, strontium $(\mathrm{Sr})$, titanium $(\mathrm{Ti})$, zinc $(\mathrm{Zn})$, and zirconium $(\mathrm{Zr})$ concentrations. Samples prepared by the sodium peroxide fusion (PF) method were measured for aluminum (Al), 
boron (B), iron (Fe), lithium (Li), nickel (Ni), and silicon (Si) concentrations. All of the prepared samples were analyzed (twice for each element of interest) by Inductively Coupled Plasma Emission Spectroscopy (ICP-ES) (with the instrumentation being re-calibrated between the duplicate analyses).

Table A2 in Appendix A provides the elemental concentration measurements derived from the samples prepared using LM, and Table A3 in Appendix A provides the measurements derived from the samples prepared using PF. Measurements for samples of the Batch 1 and Low-Activity Reference Material (LRM) standards that were included in the PSAL analytical plan along with the study glasses are also provided in these two tables.

The elemental concentrations were converted to oxide concentrations by multiplying the values for each element by the gravimetric factor for the corresponding oxide. During this process, each elemental concentration that was determined to be below the detection limit of the analytical procedures used by the PSAL was reduced to half of that detection limit as the oxide concentration was determined.

In the sections that follow, the analytical sequences of the measurements are explored, the measurements of the standards are investigated and used for bias correction, the measurements for each glass are reviewed including an analysis of their components of variation, the average chemical compositions (measured and bias-corrected) for each glass are determined, and comparisons are made between the measurements and the targeted composition for the glasses of this study.

\subsection{Measurements in Analytical Sequence}

Exhibit A1 in Appendix A provides plots of the measurements generated by the PSAL for samples prepared using the LM method. The plots are in analytical sequence with different symbols and colors being used to represent each of the study and standard glasses. Similar plots for the samples prepared using the PF method are provided in Exhibit A2 in Appendix A. These plots include the oxide values derived from all of the measurement data from Tables A2 and A3. While no problems are readily apparent in these preliminary plots of the results, additional reviews of these results are presented in the sections that follow.

\subsection{Batch 1 and LRM Standard Results}

In this section, the PSAL measurements of the chemical compositions of the Batch 1 and LRM glasses are reviewed. These measurements are investigated across the ICP analytical blocks, and the results are used to bias correct the measurements for the US study glasses.

Exhibit A3 in Appendix A provides statistical analyses of the Batch 1 and LRM results generated by the LM prep method by block for each oxide of interest. The results include analysis of variance (ANOVA) investigations looking for statistically significant differences between the block means for each of the oxides for each of the standards. The results from the statistical tests for the Batch 1 standard may be summarized as follows: $\mathrm{CaO}, \mathrm{Cr}_{2} \mathrm{O}_{3}, \mathrm{~K}_{2} \mathrm{O}, \mathrm{MgO}$, and $\mathrm{NiO}$ have measurements that indicate a significant ICP calibration effect on the block averages at the 5\% significance level. The results from the statistical tests for the LRM standard may be summarized as follows: $\mathrm{CaO}, \mathrm{K}_{2} \mathrm{O}, \mathrm{MgO}, \mathrm{NiO}, \mathrm{TiO}_{2}$, and $\mathrm{ZrO}_{2}$ have measurements that indicate a significant ICP calibration effect on the block averages at the 5\% significance level. The reference values for the oxide concentrations of the standards are given in the header for each set of measurements in the exhibit.

Exhibit A4 in Appendix A provides a similar set of analyses for the measurements derived from samples prepared via the PF method. The results from the statistical tests for the Batch 1 standard 
may be summarized as follows: $\mathrm{Li}_{2} \mathrm{O}, \mathrm{MnO}$, and $\mathrm{SiO}_{2}$ have measurements that indicate a significant ICP calibration effect on the block averages at the 5\% significance level. The results from the statistical tests for the LRM standard may be summarized as follows: $\mathrm{B}_{2} \mathrm{O}_{3}, \mathrm{Li}_{2} \mathrm{O}$, and $\mathrm{SiO}_{2}$ have measurements that indicate a significant ICP calibration effect on the block averages at the 5\% significance level. The reference values for the oxide concentrations of the standards are given in the headers for each set of measurements in the exhibit.

Thus, some of these results provide incentive for adjusting the measurements by the effect of the ICP calibration as revealed through the Batch 1 measurements. Therefore, the oxide measurements of the study glasses are to be bias corrected for the effect of the ICP calibration on each of the analytical blocks. The basis for this bias correction is presented as part of Exhibits A3 and $\mathrm{A} 4$ - the average measurement for Batch 1 for each ICP block for $\mathrm{Al}_{2} \mathrm{O}_{3}, \mathrm{~B}_{2} \mathrm{O}_{3}, \mathrm{CaO}, \mathrm{Cr}_{2} \mathrm{O}_{3}$, $\mathrm{Fe}_{2} \mathrm{O}_{3}, \mathrm{~K}_{2} \mathrm{O}, \mathrm{Li}_{2} \mathrm{O}, \mathrm{MgO}, \mathrm{MnO}, \mathrm{Na}_{2} \mathrm{O}, \mathrm{NiO}, \mathrm{SiO}_{2}$, and $\mathrm{TiO}_{2}$. The Batch 1 results were used to conduct the bias correction as long as the reference value for the oxide concentration in the Batch 1 glass was greater than or equal to $0.1 \mathrm{wt} \%$. Thus, applying this approach and based upon the information in the exhibits, the Batch 1 results were used to bias correct the $\mathrm{Al}_{2} \mathrm{O}_{3}, \mathrm{~B}_{2} \mathrm{O}_{3}, \mathrm{CaO}$, $\mathrm{Cr}_{2} \mathrm{O}_{3}, \mathrm{Fe}_{2} \mathrm{O}_{3}, \mathrm{~K}_{2} \mathrm{O}, \mathrm{Li}_{2} \mathrm{O}, \mathrm{MgO}, \mathrm{MnO}, \mathrm{Na}_{2} \mathrm{O}, \mathrm{NiO}, \mathrm{SiO}_{2}$, and $\mathrm{TiO}_{2}$ measurements. No bias correction was conducted for $\mathrm{PbO}, \mathrm{SO}_{3}, \mathrm{SrO}, \mathrm{ZnO}$, or $\mathrm{ZrO}_{2}$ given that little if any of these oxides are present in the Batch 1 standard.

The bias correction was conducted as follows. For each oxide, let $\overline{\mathrm{a}}_{\mathrm{ij}}$ be the average measurement for the $\mathrm{i}^{\text {th }}$ oxide at analytical block $\mathrm{j}$ for Batch 1 , and let $\mathrm{t}_{\mathrm{i}}$ be the reference value for the $\mathrm{i}^{\text {th }}$ oxide for Batch 1. (The averages and reference values are provided in Exhibits A3 and A4.) Let $\bar{c}_{i j k}$ be the average measurement for the $i^{\text {th }}$ oxide at analytical block $\mathrm{j}$ for the $\mathrm{k}^{\text {th }}$ glass. The bias adjustment was conducted as follows

$$
\overline{\mathrm{c}}_{\mathrm{ijk}} \bullet\left(1-\frac{\overline{\mathrm{a}}_{\mathrm{ij}}-\mathrm{t}_{\mathrm{i}}}{\overline{\mathrm{a}}_{\mathrm{ij}}}\right)=\overline{\mathrm{c}}_{\mathrm{ijk}} \bullet \frac{\mathrm{t}_{\mathrm{i}}}{\overline{\mathrm{a}}_{\mathrm{ij}}}
$$

Bias-corrected measurements are indicated by a "bc" suffix, and such adjustments were performed for all of the oxides of this study except for $\mathrm{PbO}, \mathrm{SO}_{3}, \mathrm{SrO}, \mathrm{ZnO}$, and $\mathrm{ZrO}_{2}$. Both measured and measured "bc" values are included in the discussion that follows. In these discussions bias-corrected values for $\mathrm{PbO}, \mathrm{SO}_{3}, \mathrm{SrO}, \mathrm{ZnO}$, and $\mathrm{ZrO}_{2}$ are included for completeness (e.g., to allow a sum of oxides to be computed for the bias-corrected results). These bias-corrected values are the same as the original $\mathrm{PbO}, \mathrm{SO}_{3}, \mathrm{SrO}, \mathrm{ZnO}$, and $\mathrm{ZrO}_{2}$ values (i.e., once again, no bias correction was performed for this group of oxides).

\subsection{Composition Measurements by Glass Number}

Exhibits A5 and A6 in Appendix A provide plots of the oxide concentration measurements by Glass ID in groups of 15 (with the measurements for the glass standards, Batch 1 and LRM, being provided in the fourth group) for the measured and bias-corrected (bc) values for the LM and PF preparation methods, respectively. The results by Lab ID, which indicates the preparation number and the ICP block number, are shown for each of the Glass ID's along with the targeted concentrations. Different symbols and colors are used to represent the different glasses. These plots show the individual measurements across each preparation method for the two ICP calibrations. A review of the plots presented in these exhibits reveals the repeatability of the individual, oxide values for each glass. From such a review, the following observations are offered. For the LM results, there is a great deal of scatter in the $\mathrm{Na}_{2} \mathrm{O}$ values for US-01, US-06, US-09, US-11, US-14, US-18, and US-38; in the $\mathrm{SrO}, \mathrm{ZnO}$, and $\mathrm{ZrO}_{2}$ values for US-08 and US27; and in the $\mathrm{CaO}$ for US-36. For the PF results, there is a great deal of scatter in the $\mathrm{Fe}_{2} \mathrm{O}_{3}$ 
values for US-14 and US-17, in the $\mathrm{SiO}_{2}$ values for US-01, US-04, US-05, US-15, US-16, US-17, US-19, and US-23. For some of the other study glasses, bias-correcting the $\mathrm{SiO}_{2}$ measurements resulted in increasing the scatter of the values. In the next section, there is an attempt to average out the scatter that was noted for some of these measurements, and the resulting average compositions are then compared to their targeted compositions.

\subsection{Measured versus Targeted Compositions}

The four measurements for each oxide for each glass (over both preparation methods) were averaged to determine a representative chemical composition for each glass. These determinations were conducted both for the measured and for the bias-corrected data. A sum of oxides was also computed for each glass based upon both the measured and bias-corrected values. Exhibit A7 in Appendix A provides plots showing results for each glass for each oxide to help highlight the comparisons among the measured, bias-corrected, and target value for each oxide.

Some observations from the plots of Exhibit A7 are offered: The most significant observation from this exhibit is the strong indication that two glass samples were switched as they were introduced into the PSAL analytical process. These two glasses are US-28 and US-37.

Another observation of interest relates to the behavior of the $\mathrm{Cr}_{2} \mathrm{O}_{3}$ measurements. The $\mathrm{Cr}_{2} \mathrm{O}_{3}$ measurements are consistently below the targeted values for study glasses whose targeted $\mathrm{Cr}_{2} \mathrm{O}_{3}$ concentrations are $0.4 \mathrm{wt} \%$ or greater.

Although not the primary objective of this task, the compositional data do provide some insight into $\mathrm{SO}_{3}$ solubility in high-level waste glasses. Given the $\mathrm{SO}_{3}$ measurements on the LRM glass suggest that the analytical techniques provide a "true" measurement of the $\mathrm{SO}_{3}$ retained in the glass, the results of the US test matrix glasses suggest that there is a dependence of $\mathrm{SO}_{3}$ retention with composition. For select US test matrix glasses, full retention of $\mathrm{SO}_{3}$ is shown (i.e., target equal measured) while other glasses show a significant reduction in the $\mathrm{SO}_{3}$ retained in glass (i.e., measured values significantly lower than targeted values).

Table A4 in Appendix A provides a summary of the average compositions as well as the targeted composition and some associated differences and relative differences. Notice that the targeted sums of oxides for the Batch 1 and LRM standards do not sum to $100 \%$ due to an incomplete coverage of the oxides in the standard glasses. All of the sums of oxides (both measured and bias-corrected) for the study glasses fall within the interval of 95 to $105 \mathrm{wt} \%$. Entries in Table A4 show the absolute differences and percent relative differences (when the targeted concentration for the oxide is at least $0.5 \mathrm{wt} \%$ ) between the measured or bias-corrected values and the targeted values. These differences are shaded when the percent relative differences are greater than or equal to 5\%. Also note that in addition to the entries in this table for US-28 and US-37 (showing the measured and measured-bias corrected values as originally reported), there are entries labeled US-28* and US-37* (showing the measured and measured-biased corrected values arranged properly). Thus, the US-28* and US-37* compositions should be used to represent these glasses along with the information for the other US glasses in Table A4 in providing the three views of the composition of these study glasses.

Display 1 provides a comparison between the measurements and bias-corrected measurements for US-44 and US-45. These glasses were duplicates; that is, they had the same targeted compositions. The comparisons indicate a measure of the repeatability of the batching process which appears to be acceptable (the percent differences for most of the oxides are less than 5\% and percent differences for all of the oxides are less than $10 \mathrm{wt} \%$ ). The largest percent difference between the two sets of measurements is the $8.1 \%$ difference for $\mathrm{Li}_{2} \mathrm{O}$ with US-44's value of $4.3919 \mathrm{wt} \%$ and US-45's value of $4.0636 \mathrm{wt} \%$. Considering the targeted value of $4.0488 \mathrm{wt} \%$ 
for this oxide, it appears that the $\mathrm{Li}_{2} \mathrm{O}$ value for US-44 would be the more unusual of the two glasses.

Display 1. Comparison Between the Chemical Composition Measurements for Replicates US-44 and US-45

\begin{tabular}{|c|c|c|c|c|c|c|c|c|c|}
\hline & & & & & & US-44 & US-45 & Difference & \\
\hline & US-44 & & & & & Measured & Measured & Measured & $\%$ \\
\hline & US-45 & US-44 & US-45 & Difference & $\%$ & Bias- & Bias- & Bias- & Difference \\
\hline & Targeted & Measured & Measured & Measured & Difference & Corrected & Corrected & Corrected (BC) & Measured \\
\hline Oxide & $(\mathrm{wt} \%)$ & $(w t \%)$ & $(\mathrm{wt} \%)$ & $(w t \%)$ & Measured & $(w t \%)$ & $(w t \%)$ & $(w t \%)$ & $\mathrm{BC}$ \\
\hline $\mathrm{A} 12 \mathrm{O} 3$ & 12.8029 & 13.6327 & 13.2123 & 0.4204 & $3.2 \%$ & 13.8261 & 13.4881 & 0.3380 & $2.5 \%$ \\
\hline $\mathrm{B} 2 \mathrm{O} 3$ & 12.5864 & 12.4610 & 12.8635 & -0.4025 & $-3.2 \%$ & 12.3775 & 12.7786 & -0.4011 & $-3.2 \%$ \\
\hline $\mathrm{CaO}$ & 0.5553 & 0.5439 & 0.5422 & 0.0017 & $0.3 \%$ & 0.6064 & 0.5837 & 0.0227 & $3.9 \%$ \\
\hline $\mathrm{Cr} 2 \mathrm{O} 3$ & 0.3051 & 0.2616 & 0.2558 & 0.0058 & $2.3 \%$ & 0.2548 & 0.2502 & 0.0046 & $1.8 \%$ \\
\hline $\mathrm{Fe} 2 \mathrm{O} 3$ & 8.8598 & 8.7426 & 8.9321 & -0.1894 & $-2.2 \%$ & 8.7753 & 8.9240 & -0.1486 & $-1.7 \%$ \\
\hline $\mathrm{K} 2 \mathrm{O}$ & 0.6397 & 0.6445 & 0.6640 & -0.0196 & $-3.0 \%$ & 0.5672 & 0.5892 & -0.0220 & $-3.9 \%$ \\
\hline $\mathrm{Li} 2 \mathrm{O}$ & 4.0488 & 4.3919 & 4.0636 & 0.3283 & $8.1 \%$ & 4.4618 & 4.1216 & 0.3402 & $8.3 \%$ \\
\hline $\mathrm{MgO}$ & 0.2525 & 0.2550 & 0.2467 & 0.0083 & $3.4 \%$ & 0.2748 & 0.2661 & 0.0087 & $3.3 \%$ \\
\hline $\mathrm{MnO}$ & 1.8339 & 1.7560 & 1.7915 & -0.0355 & $-2.0 \%$ & 1.8655 & 1.8984 & -0.0330 & $-1.8 \%$ \\
\hline $\mathrm{Na} 2 \mathrm{O}$ & 12.7286 & 13.9181 & 13.4396 & 0.4785 & $3.6 \%$ & 13.3541 & 12.9576 & 0.3966 & $3.1 \%$ \\
\hline $\mathrm{NiO}$ & 0.3587 & 0.3394 & 0.3321 & 0.0073 & $2.2 \%$ & 0.3736 & 0.3664 & 0.0073 & $2.0 \%$ \\
\hline $\mathrm{PbO}$ & 0.6665 & 0.6070 & 0.5911 & 0.0159 & $2.7 \%$ & 0.6070 & 0.5911 & 0.0159 & $2.7 \%$ \\
\hline $\mathrm{SiO} 2$ & 39.5480 & 37.4378 & 39.6305 & -2.1928 & $-5.9 \%$ & 38.2395 & 40.3297 & -2.0902 & $-5.5 \%$ \\
\hline $\mathrm{SO} 3$ & 0.2222 & 0.2278 & 0.2222 & 0.0056 & $2.5 \%$ & 0.2278 & 0.2222 & 0.0056 & $2.5 \%$ \\
\hline $\mathrm{SrO}$ & 1.6195 & 1.4073 & 1.4339 & -0.0266 & $-1.9 \%$ & 1.4073 & 1.4339 & -0.0266 & $-1.9 \%$ \\
\hline $\mathrm{TiO} 2$ & 0.4650 & 0.4487 & 0.4529 & -0.0042 & $-0.9 \%$ & 0.4880 & 0.4876 & 0.0005 & $0.1 \%$ \\
\hline $\mathrm{ZnO}$ & 1.0237 & 0.9653 & 0.9607 & 0.0047 & $0.5 \%$ & 0.9653 & 0.9607 & 0.0047 & $0.5 \%$ \\
\hline $\mathrm{ZrO} 2$ & 1.4636 & 1.3076 & 1.3130 & -0.0054 & $-0.4 \%$ & 1.3076 & 1.3130 & -0.0054 & $-0.4 \%$ \\
\hline Sum & 99.9800 & 99.3483 & 100.9477 & -1.5993 & $-1.6 \%$ & 99.9798 & 101.5621 & -1.5823 & $-1.6 \%$ \\
\hline
\end{tabular}

\subsection{A Statistical REVieW OF THE PCT Results}

The study glasses, after being batched and fabricated, were subjected to the 7-day PCT to assess their durabilities. More specifically, Method A of the PCT (ASTM C-1285-2002 [3]) was used for these measurements. Durability is the critical product quality metric for DWPF glass studies. Two heat treatments (quenching and a ccc regime) were used during the fabrication of each of the study glasses. Both heat treatments for each study glass were subjected to the PCT (in triplicate). PCTs were also conducted in triplicate for samples of the EA glass and for samples of the Approved Reference Material (ARM) glass. Blanks (samples consisting only of ASTM Type I water) were also submitted for the PCT.

Analytical plans (see [4], [5], and [6]) were provided to the PSAL to support the measurement of the compositions of the solutions resulting from the PCTs which were conducted in three sets. Samples of a multi-element, standard solution were also included in the analytical plans (as a check on the accuracy of the ICP-ES used for these measurements). In this and the following sections, the measurements generated by the PSAL for these PCTs are presented and reviewed. 
Table B1 in Appendix B provides the elemental leachate concentration measurements determined by the PSAL for the solution samples generated by the PCTs. One of the quality control checkpoints for the PCT procedure is solution-weight loss over the course of the 7-day test. None of these PCT results indicated a solution-weight loss problem. Any measurement in Table B1 below the detection limit of the analytical procedure (indicated by a "<") was replaced by $1 / 2$ of the detection limit in subsequent analyses. In addition to adjustments for detection limits, the values were adjusted for the dilution factors: the values for the study glasses, the blanks, and the ARM glass in Table B1 were multiplied by 1.6667 to determine the values in parts per million (ppm) and the values for EA were multiplied by 16.6667. Table B2 in Appendix B provides the resulting measurements. ${ }^{1}$

In the sections that follow, the analytical sequence of the measurements is explored, the measurements of the standards are investigated and used to assess the overall accuracy of the ICP measurement process, the measurements for each glass are reviewed, plots are provided that explore the effects of heat treatment on the PCTs for these glasses, the PCTs are normalized using the compositions (targeted, measured, and bias-corrected) presented in Table A4, and the normalized PCTs are compared to those of the EA glass.

\subsection{Measurements in Analytical Sequence}

Exhibits B1 and B2 in Appendix B provide plots of the leachate (ppm) concentrations in analytical sequence as generated by the PSAL for all of the data and for the data from only the study glasses, respectively. A different color and symbol are used for each study glass or standard. No problems are seen in these plots.

\subsection{Results for the Samples of the Multi-Element Solution Standard}

Exhibit B3 in Appendix B provides analyses of the PSAL measurements of the samples of the multi-element solution standard by ICP analytical (or calibration) block by analytical set. An analysis of variance (ANOVA) investigating for statistically significant differences among the set/block averages for these samples for each element of interest is included in these exhibits. These results indicate a statistically significant (at a 5\% significance level) difference among the average measurements over these sets/blocks for all of the elements of interest. However, no bias correction of the PCT results for the study glasses was conducted. This approach was taken since the triplicate PCTs for a single study glass were placed in different ICP blocks. Averaging the ppm's for each set of triplicates helps to minimize the impact of the ICP effects.

Display 2 summarizes the average measurements and the reference values for the 4 primary elements of interest. By in large, the results indicate consistent and accurate measurements from the PSAL processes used to conduct these analyses. The exceptions to this are the $17.60 \mathrm{ppm} \mathrm{Si}$ measurement and the $77.27 \mathrm{ppm}$ Na measurement for the standard in Set/Block 3/1. While each of these measurements is low compared to its reference value for the standard, this is not seen to be a significant problem, and no other issues are evident in these results.

1 Note that the switching of glasses US-28 and US-37 that was identified for the chemical compositions is not thought to have affected the PCT measurements for this pair of glasses. Thus, no switching of the PCT measurements was deemed necessary. 
Display 2: Results from Samples of the Multi-Element Solution Standard

\begin{tabular}{|c|c|c|c|c|}
\hline Analytical & B & $\mathbf{L i}$ & $\mathrm{Na}$ & Si \\
\hline Set/Block & (ppm) & (ppm) & (ppm) & (ppm) \\
\hline $1 / 1$ & 20.13 & 9.83 & 82.80 & 49.37 \\
\hline $1 / 2$ & 19.93 & 9.66 & 82.30 & 48.67 \\
\hline $1 / 3$ & 20.33 & 9.81 & 82.27 & 49.80 \\
\hline $1 / 4$ & 20.57 & 9.71 & 82.30 & 50.07 \\
\hline $1 / 5$ & 20.63 & 9.78 & 82.87 & 49.77 \\
\hline $1 / 6$ & 20.80 & 9.90 & 81.10 & 50.30 \\
\hline $2 / 1$ & 20.77 & 9.98 & 81.07 & 49.93 \\
\hline $2 / 2$ & 20.30 & 9.82 & 81.17 & 49.47 \\
\hline $2 / 3$ & 20.73 & 9.92 & 80.87 & 51.07 \\
\hline $2 / 4$ & 21.23 & 10.03 & 82.07 & 51.13 \\
\hline $2 / 5$ & 20.70 & 10.03 & 81.97 & 50.70 \\
\hline $2 / 6$ & 20.23 & 9.74 & 80.77 & 50.93 \\
\hline $3 / 1$ & 17.60 & 9.85 & 77.27 & 49.30 \\
\hline $3 / 2$ & 20.13 & 9.84 & 81.50 & 49.43 \\
\hline $3 / 3$ & 20.23 & 9.90 & 80.63 & 49.13 \\
\hline $3 / 4$ & 20.20 & 9.82 & 81.93 & 49.57 \\
\hline $3 / 5$ & 20.47 & 9.84 & 80.17 & 49.67 \\
\hline $3 / 6$ & 20.10 & 9.92 & 80.87 & 49.27 \\
\hline Grand Average & 20.28 & 9.86 & 81.33 & 49.86 \\
\hline Reference Value & 20 & 10 & 81 & 50 \\
\hline$\%$ difference & $1.42 \%$ & $-1.44 \%$ & $0.40 \%$ & $-0.27 \%$ \\
\hline
\end{tabular}

\subsection{Measurements by Glass Number}

Exhibit B4 in Appendix B provides plots of the leachate concentrations for each type of submitted sample: the study glasses and the standards (EA (101), ARM (102), the multi-element solution standard (100), and blanks (103)). Exhibit B5 in Appendix B provides plots of the leachate concentrations for the PCT results of the study glasses (in the three sets of 15 glasses each) by heat treatment. These plots allow for the assessment of the repeatability of the measurements, which suggests some scatter in the triplicate values for some analytes for some of the glasses. Also, note that some differences between the values for the two heat treatments for some glasses are evident. Specifically, glass \#18, glass \#27, and glass \#42 show the biggest differences between their quenched and ccc counterparts for B, Li, Na, and $\mathrm{Si}$.

\subsection{Normalized PCT Results}

PCT leachate concentrations are typically normalized using the cation composition (expressed as a weight percent) in the glass to obtain a grams-per-liter $(\mathrm{g} / \mathrm{L})$ leachate concentration. The normalization of the PCTs is usually conducted using the measured compositions of the glasses. This is the preferred normalization process for the PCTs. For completeness, the targeted cation and the bias-corrected cation compositions were also used to conduct this normalization.

As is the usual convention, the common logarithm of the normalized PCT (normalized leachate, NL) for each element of interest was determined and used for comparison. To accomplish this computation, one must 
1.Determine the common logarithm of the elemental parts per million (ppm) leachate concentration for each of the triplicates and each of the elements of interest (these values are provided in Table B2 of Appendix B),

2.Average the common logarithms over the triplicates for each element of interest, and then

Normalizing Using Measured Composition (preferred method)

3. Subtract a quantity equal to 1 plus the common logarithm of the average cation measured concentration (expressed as a weight percent of the glass) from the average computed in step 2 .

Or Normalizing Using Target Composition

3. Subtract a quantity equal to 1 plus the common logarithm of the target cation concentration (expressed as a weight percent of the glass) from the average computed in step 2 .

Or Normalizing Using Measured Bias-Corrected Composition

3. Subtract a quantity equal to 1 plus the common logarithm of the measured bias-corrected cation concentration (expressed as a weight percent of the glass) from the average computed in step 2.

Exhibit B6 in Appendix B provides scatter plots for these results for each analytical set of PCTs and offers an opportunity to investigate the consistency in the leaching across the elements for the glasses of this study. All combinations of the normalizations of the PCTs (i.e., those generated using the targeted, measured, and bias-corrected compositional views) and both heat treatments are represented in the series of scatter plots. Consistency in the leaching across the elements is typically demonstrated by a high degree of linear correlation among the values for pairs of these elements. For each set of PCTs, the Si results appear to yield the smallest correlations were paired with each of the other elements. The smallest is the 0.3747 correlation between Si and B for the Set \#3 results. The reason for some of these correlations being this low is not known.

Display 3 summarizes the normalized PCTs for the glasses of this study. The results are listed by glass identifier and show the PCTs for both heat treatments normalized using measured, biascorrected, and targeted compositions. Note that the PCT results for ARM and EA are also provided. In comparing the results for the US study glasses to the results for EA, note that all of the US glasses yielded PCTs that are lower than those of the EA glass. The largest PCT values are for the ccc versions of US-27 and US-18 with NL[B] values of $16.4 \mathrm{~g} / \mathrm{L}$ (based on the targeted composition) and $10.7 \mathrm{~g} / \mathrm{L}$ (based on the targeted composition), respectively. The 16.4 $\mathrm{g} / \mathrm{L}$ is just below the value of $16.695 \mathrm{~g} / \mathrm{L}$ for EA's NL[B] that was reported in [8]. For the quenched version of the glasses, the largest $\mathrm{NL}[\mathrm{B}]$ value is $0.67 \mathrm{~g} / \mathrm{L}$ (based on the targeted composition). Also note the similarity of PCT results for US-44 and US-45. These two glasses had the same targeted composition, and their PCT results differ by less than $0.1 \mathrm{~g} / \mathrm{L}$ for any of the four elements over all of the compositional views and heat treatments. A closer comparison between the quenched and ccc versions of the study glasses is presented in the next section. 
Display 3: Normalized PCTs by Glass ID, Heat Treatment, and Compositional View

\begin{tabular}{|c|c|c|c|c|c|c|c|c|c|c|}
\hline $\begin{array}{c}\text { Glass } \\
\text { ID }\end{array}$ & $\begin{array}{c}\text { Heat } \\
\text { Treatment }\end{array}$ & Comp View & $\begin{array}{c}\log N L[B \\
(g / L)]\end{array}$ & $\begin{array}{c}\log \mathrm{NL} \\
{[\mathrm{Li}(\mathrm{g} / \mathrm{L})]}\end{array}$ & $\begin{array}{c}\log \mathrm{NL}[\mathrm{Na} \\
(\mathrm{g} / \mathrm{L})]\end{array}$ & $\begin{array}{c}\log \mathrm{NL} \\
{[\mathrm{Si}(\mathrm{g} / \mathrm{L})]}\end{array}$ & $\begin{array}{l}\text { NL B } \\
(\mathrm{g} / \mathrm{L})\end{array}$ & $\begin{array}{c}\begin{array}{c}\text { NL Li } \\
(\mathrm{g} / \mathrm{L})\end{array} \\
\end{array}$ & $\begin{array}{c}\begin{array}{c}\text { NL Na } \\
(\mathrm{g} / \mathrm{L})\end{array} \\
\end{array}$ & $\begin{array}{l}\mathrm{NL} \mathrm{Si} \\
(\mathrm{g} / \mathrm{L}) \\
\end{array}$ \\
\hline ARM & & reference & -0.2928 & -0.2463 & -0.2935 & -0.5555 & 0.510 & 0.567 & 0.509 & 0.278 \\
\hline ARM & & reference & -0.2956 & -0.2500 & -0.2982 & -0.5556 & 0.506 & 0.562 & 0.503 & 0.278 \\
\hline ARM & & reference & -0.2738 & -0.2349 & -0.3096 & -0.5531 & 0.532 & 0.582 & 0.490 & 0.280 \\
\hline EA & & reference & 1.2551 & 0.9846 & 1.1272 & 0.5952 & 17.995 & 9.652 & 13.403 & 3.937 \\
\hline EA & & reference & 1.2529 & 0.9886 & 1.1348 & 0.6010 & 17.903 & 9.741 & 13.640 & 3.990 \\
\hline EA & & reference & 1.2615 & 1.0010 & 1.1345 & 0.6081 & 18.260 & 10.023 & 13.630 & 4.056 \\
\hline US-01 & $\mathrm{ccc}$ & measured & -0.3594 & -0.2682 & -0.3536 & -0.3779 & 0.437 & 0.539 & 0.443 & 0.419 \\
\hline US-01 & $\mathrm{ccc}$ & measured bc & -0.3511 & -0.2737 & -0.3432 & -0.3899 & 0.446 & 0.533 & 0.454 & 0.407 \\
\hline US-01 & $\mathrm{ccc}$ & target & -0.3349 & -0.2663 & -0.2958 & -0.4043 & 0.462 & 0.542 & 0.506 & 0.394 \\
\hline US-01 & quenched & measured & -0.3517 & -0.2584 & -0.3306 & -0.3666 & 0.445 & 0.552 & 0.467 & 0.430 \\
\hline US-01 & quenched & measured bc & -0.3434 & -0.2639 & -0.3202 & -0.3787 & 0.454 & 0.545 & 0.478 & 0.418 \\
\hline US-01 & quenched & target & -0.3272 & -0.2565 & -0.2728 & -0.3931 & 0.471 & 0.554 & 0.534 & 0.405 \\
\hline US-02 & $\mathrm{ccc}$ & measured & 0.1447 & 0.1046 & -0.1454 & -0.4496 & 1.395 & 1.272 & 0.716 & 0.355 \\
\hline US-02 & $\mathrm{ccc}$ & measured bc & 0.1668 & 0.1017 & -0.1296 & -0.4504 & 1.468 & 1.264 & 0.742 & 0.354 \\
\hline US-02 & $\mathrm{ccc}$ & target & 0.1453 & 0.0888 & -0.1182 & -0.4633 & 1.397 & 1.227 & 0.762 & 0.344 \\
\hline US-02 & quenched & measured & 0.2383 & 0.1966 & -0.0757 & -0.4505 & 1.731 & 1.573 & 0.840 & 0.354 \\
\hline US-02 & quenched & measured bc & 0.2604 & 0.1937 & -0.0599 & -0.4513 & 1.821 & 1.562 & 0.871 & 0.354 \\
\hline US-02 & quenched & target & 0.2389 & 0.1808 & -0.0485 & -0.4643 & 1.734 & 1.516 & 0.894 & 0.343 \\
\hline US-03 & $\mathrm{ccc}$ & measured & -0.5032 & -0.3178 & -0.8219 & -0.5739 & 0.314 & 0.481 & 0.151 & 0.267 \\
\hline US-03 & $\mathrm{ccc}$ & measured bc & -0.4864 & -0.3232 & -0.8039 & -0.5910 & 0.326 & 0.475 & 0.157 & 0.256 \\
\hline US-03 & $\mathrm{ccc}$ & target & -0.4888 & -0.3231 & -0.7831 & -0.5919 & 0.325 & 0.475 & 0.165 & 0.256 \\
\hline US-03 & quenched & measured & -0.4625 & -0.3639 & -0.6523 & -0.5500 & 0.345 & 0.433 & 0.223 & 0.282 \\
\hline US-03 & quenched & measured bc & -0.4456 & -0.3693 & -0.6344 & -0.5672 & 0.358 & 0.427 & 0.232 & 0.271 \\
\hline US-03 & quenched & target & -0.4480 & -0.3692 & -0.6136 & -0.5680 & 0.356 & 0.427 & 0.243 & 0.270 \\
\hline US-04 & $\mathrm{ccc}$ & measured & -0.4919 & -0.3401 & -0.5970 & -0.5875 & 0.322 & 0.457 & 0.253 & 0.259 \\
\hline US-04 & $\mathrm{ccc}$ & measured bc & -0.4750 & -0.3456 & -0.5791 & -0.6048 & 0.335 & 0.451 & 0.264 & 0.248 \\
\hline US-04 & $\mathrm{ccc}$ & target & -0.4800 & -0.3403 & -0.5476 & -0.6129 & 0.331 & 0.457 & 0.283 & 0.244 \\
\hline US-04 & quenched & measured & -0.4689 & -0.3012 & -0.5928 & -0.5730 & 0.340 & 0.500 & 0.255 & 0.267 \\
\hline US-04 & quenched & measured bc & -0.4520 & -0.3066 & -0.5749 & -0.5903 & 0.353 & 0.494 & 0.266 & 0.257 \\
\hline US-04 & quenched & target & -0.4570 & -0.3013 & -0.5434 & -0.5984 & 0.349 & 0.500 & 0.286 & 0.252 \\
\hline US-05 & $\mathrm{ccc}$ & measured & -0.3233 & -0.1697 & -0.4726 & -0.5479 & 0.475 & 0.677 & 0.337 & 0.283 \\
\hline US-05 & $\mathrm{ccc}$ & measured bc & -0.3012 & -0.1727 & -0.4709 & -0.5488 & 0.500 & 0.672 & 0.338 & 0.283 \\
\hline US-05 & $\mathrm{ccc}$ & target & -0.3098 & -0.1685 & -0.4454 & -0.5636 & 0.490 & 0.679 & 0.359 & 0.273 \\
\hline US-05 & quenched & measured & -0.4812 & -0.1597 & -0.7310 & -0.6033 & 0.330 & 0.692 & 0.186 & 0.249 \\
\hline US-05 & quenched & measured bc & -0.4591 & -0.1626 & -0.7294 & -0.6042 & 0.347 & 0.688 & 0.186 & 0.249 \\
\hline US-05 & quenched & target & -0.4678 & -0.1584 & -0.7038 & -0.6189 & 0.341 & 0.694 & 0.198 & 0.240 \\
\hline US-06 & $\mathrm{ccc}$ & measured & -0.5276 & -0.3501 & -0.5554 & -0.6405 & 0.297 & 0.447 & 0.278 & 0.229 \\
\hline US-06 & $\mathrm{ccc}$ & measured bc & -0.5108 & -0.3556 & -0.5541 & -0.6577 & 0.308 & 0.441 & 0.279 & 0.220 \\
\hline US-06 & $\mathrm{ccc}$ & target & -0.5132 & -0.3487 & -0.5628 & -0.6504 & 0.307 & 0.448 & 0.274 & 0.224 \\
\hline US-06 & quenched & measured & -0.4873 & -0.2951 & -0.5297 & -0.6309 & 0.326 & 0.507 & 0.295 & 0.234 \\
\hline US-06 & quenched & measured bc & -0.4705 & -0.3005 & -0.5284 & -0.6481 & 0.338 & 0.501 & 0.296 & 0.225 \\
\hline US-06 & quenched & target & -0.4730 & -0.2936 & -0.5371 & -0.6408 & 0.337 & 0.509 & 0.290 & 0.229 \\
\hline US-07 & $\mathrm{ccc}$ & measured & -0.4830 & -0.3290 & -0.5976 & -0.6550 & 0.329 & 0.469 & 0.253 & 0.221 \\
\hline US-07 & $\mathrm{ccc}$ & measured bc & -0.4801 & -0.3350 & -0.5872 & -0.6627 & 0.331 & 0.462 & 0.259 & 0.217 \\
\hline US-07 & $\mathrm{ccc}$ & target & -0.4923 & -0.3453 & -0.5707 & -0.6468 & 0.322 & 0.452 & 0.269 & 0.226 \\
\hline US-07 & quenched & measured & -0.4423 & -0.2719 & -0.5590 & -0.6377 & 0.361 & 0.535 & 0.276 & 0.230 \\
\hline US-07 & quenched & measured bc & -0.4394 & -0.2780 & -0.5486 & -0.6453 & 0.364 & 0.527 & 0.283 & 0.226 \\
\hline US-07 & quenched & target & -0.4516 & -0.2883 & -0.5321 & -0.6294 & 0.354 & 0.515 & 0.294 & 0.235 \\
\hline US-08 & $\mathrm{ccc}$ & measured & -0.4121 & -0.2754 & -0.5052 & -0.4309 & 0.387 & 0.530 & 0.312 & 0.371 \\
\hline US-08 & $\mathrm{ccc}$ & measured bc & -0.3899 & -0.2783 & -0.5036 & -0.4317 & 0.407 & 0.527 & 0.314 & 0.370 \\
\hline US-08 & $\mathrm{ccc}$ & target & -0.3890 & -0.2728 & -0.4893 & -0.4484 & 0.408 & 0.534 & 0.324 & 0.356 \\
\hline US-08 & quenched & measured & -0.3861 & -0.2192 & -0.5041 & -0.4021 & 0.411 & 0.604 & 0.313 & 0.396 \\
\hline US-08 & quenched & measured bc & -0.3639 & -0.2221 & -0.5025 & -0.4030 & 0.433 & 0.600 & 0.314 & 0.395 \\
\hline US-08 & quenched & target & -0.3629 & -0.2166 & -0.4882 & -0.4196 & 0.434 & 0.607 & 0.325 & 0.381 \\
\hline US-09 & $\mathrm{ccc}$ & measured & -0.3588 & -0.2858 & -0.5453 & -0.6097 & 0.438 & 0.518 & 0.285 & 0.246 \\
\hline US-09 & $\mathrm{ccc}$ & measured bc & -0.3560 & -0.2927 & -0.5436 & -0.6189 & 0.441 & 0.510 & 0.286 & 0.240 \\
\hline US-09 & $\mathrm{ccc}$ & target & -0.3545 & -0.2742 & -0.5823 & -0.6113 & 0.442 & 0.532 & 0.262 & 0.245 \\
\hline US-09 & quenched & measured & -0.3682 & -0.2653 & -0.3588 & -0.5521 & 0.428 & 0.543 & 0.438 & 0.280 \\
\hline US-09 & quenched & measured bc & -0.3653 & -0.2721 & -0.3571 & -0.5613 & 0.431 & 0.534 & 0.439 & 0.275 \\
\hline US-09 & quenched & target & -0.3639 & -0.2536 & -0.3958 & -0.5537 & 0.433 & 0.558 & 0.402 & 0.279 \\
\hline US-10 & $\mathrm{ccc}$ & measured & -0.4703 & -0.3323 & -0.4482 & -0.5979 & 0.339 & 0.465 & 0.356 & 0.252 \\
\hline US-10 & $\mathrm{ccc}$ & measured bc & -0.4620 & -0.3377 & -0.4324 & -0.6100 & 0.345 & 0.460 & 0.369 & 0.245 \\
\hline US-10 & $\mathrm{ccc}$ & target & -0.4576 & -0.3198 & -0.4217 & -0.6229 & 0.349 & 0.479 & 0.379 & 0.238 \\
\hline US-10 & quenched & measured & -0.4620 & -0.3168 & -0.4349 & -0.5875 & 0.345 & 0.482 & 0.367 & 0.259 \\
\hline US-10 & quenched & measured bc & -0.4538 & -0.3223 & -0.4191 & -0.5996 & 0.352 & 0.476 & 0.381 & 0.251 \\
\hline US-10 & quenched & target & -0.4494 & -0.3044 & -0.4083 & -0.6125 & 0.355 & 0.496 & 0.391 & 0.244 \\
\hline US-11 & $\mathrm{ccc}$ & measured & -0.1158 & -0.0460 & -0.3124 & -0.6397 & 0.766 & 0.900 & 0.487 & 0.229 \\
\hline US-11 & $\mathrm{ccc}$ & measured bc & -0.0989 & -0.0515 & -0.3059 & -0.6568 & 0.796 & 0.888 & 0.494 & 0.220 \\
\hline US-11 & $\mathrm{ccc}$ & target & -0.0982 & -0.0393 & -0.2913 & -0.6377 & 0.798 & 0.914 & 0.511 & 0.230 \\
\hline
\end{tabular}


Display 3: Normalized PCTs by Glass ID, Heat Treatment, and Compositional View

\begin{tabular}{|c|c|c|c|c|c|c|c|c|c|c|}
\hline $\begin{array}{c}\text { Glass } \\
\text { ID }\end{array}$ & $\begin{array}{c}\text { Heat } \\
\text { Treatment }\end{array}$ & Comp View & $\begin{array}{c}\log N L[B \\
(g / L)]\end{array}$ & $\begin{array}{c}\log \mathrm{NL} \\
{[\mathrm{Li}(\mathrm{g} / \mathrm{L})]}\end{array}$ & $\begin{array}{c}\log \mathrm{NL}[\mathrm{Na} \\
(\mathrm{g} / \mathrm{L})]\end{array}$ & $\begin{array}{c}\log \mathrm{NL} \\
{[\mathrm{Si}(\mathrm{g} / \mathrm{L})]}\end{array}$ & $\begin{array}{l}\text { NL B } \\
(\mathrm{g} / \mathrm{L})\end{array}$ & $\begin{array}{l}\text { NL Li } \\
(\mathrm{g} / \mathrm{L})\end{array}$ & $\begin{array}{c}\begin{array}{c}\text { NL Na } \\
(\mathrm{g} / \mathrm{L})\end{array} \\
\end{array}$ & $\begin{array}{l}\mathrm{NL} \mathrm{Si} \\
(\mathrm{g} / \mathrm{L}) \\
\end{array}$ \\
\hline US-11 & quenched & measured & -0.5008 & -0.2459 & -0.6159 & -0.7140 & 0.316 & 0.568 & 0.242 & 0.193 \\
\hline US-11 & quenched & measured bc & -0.4839 & -0.2514 & -0.6094 & -0.7311 & 0.328 & 0.561 & 0.246 & 0.186 \\
\hline US-11 & quenched & target & -0.4832 & -0.2392 & -0.5948 & -0.7120 & 0.329 & 0.577 & 0.254 & 0.194 \\
\hline US-12 & $\mathrm{ccc}$ & measured & 0.2300 & 0.1752 & -0.0158 & -0.4142 & 1.698 & 1.497 & 0.964 & 0.385 \\
\hline US-12 & $\mathrm{ccc}$ & measured bc & 0.2468 & 0.1698 & -0.0092 & -0.4314 & 1.765 & 1.478 & 0.979 & 0.370 \\
\hline US-12 & $\mathrm{ccc}$ & target & 0.2415 & 0.1746 & 0.0395 & -0.4158 & 1.744 & 1.495 & 1.095 & 0.384 \\
\hline US-12 & quenched & measured & 0.0923 & 0.0490 & -0.1036 & -0.4484 & 1.237 & 1.120 & 0.788 & 0.356 \\
\hline US-12 & quenched & measured bc & 0.1091 & 0.0436 & -0.0971 & -0.4656 & 1.286 & 1.106 & 0.800 & 0.342 \\
\hline US-12 & quenched & target & 0.1038 & 0.0484 & -0.0483 & -0.4500 & 1.270 & 1.118 & 0.895 & 0.355 \\
\hline US-13 & $\mathrm{ccc}$ & measured & -0.5268 & -0.3686 & -0.5594 & -0.6560 & 0.297 & 0.428 & 0.276 & 0.221 \\
\hline US-13 & $\mathrm{ccc}$ & measured bc & -0.5239 & -0.3754 & -0.5530 & -0.6652 & 0.299 & 0.421 & 0.280 & 0.216 \\
\hline US-13 & $\mathrm{ccc}$ & target & -0.5244 & -0.3625 & -0.5353 & -0.6503 & 0.299 & 0.434 & 0.292 & 0.224 \\
\hline US-13 & quenched & measured & -0.4561 & -0.3071 & -0.5058 & -0.6231 & 0.350 & 0.493 & 0.312 & 0.238 \\
\hline US-13 & quenched & measured bc & -0.4532 & -0.3140 & -0.4993 & -0.6323 & 0.352 & 0.485 & 0.317 & 0.233 \\
\hline US-13 & quenched & target & -0.4537 & -0.3010 & -0.4817 & -0.6174 & 0.352 & 0.500 & 0.330 & 0.241 \\
\hline US-14 & $\mathrm{ccc}$ & measured & 0.4150 & 0.3479 & 0.1962 & -0.4660 & 2.600 & 2.228 & 1.571 & 0.342 \\
\hline US-14 & $\mathrm{ccc}$ & measured bc & 0.4232 & 0.3424 & 0.1977 & -0.4780 & 2.650 & 2.200 & 1.577 & 0.333 \\
\hline US-14 & $\mathrm{ccc}$ & target & 0.4180 & 0.3478 & 0.1953 & -0.4710 & 2.618 & 2.227 & 1.568 & 0.338 \\
\hline US-14 & quenched & measured & 0.4917 & 0.4233 & 0.2648 & -0.4640 & 3.103 & 2.651 & 1.840 & 0.344 \\
\hline US-14 & quenched & measured bc & 0.5000 & 0.4179 & 0.2663 & -0.4760 & 3.162 & 2.618 & 1.846 & 0.334 \\
\hline US-14 & quenched & target & 0.4948 & 0.4232 & 0.2638 & -0.4690 & 3.124 & 2.650 & 1.836 & 0.340 \\
\hline US-15 & $\mathrm{ccc}$ & measured & 0.5566 & 0.4905 & 0.2961 & -0.6260 & 3.603 & 3.094 & 1.977 & 0.237 \\
\hline US-15 & $\mathrm{ccc}$ & measured bc & 0.5595 & 0.4845 & 0.3024 & -0.6337 & 3.627 & 3.051 & 2.007 & 0.232 \\
\hline US-15 & $\mathrm{ccc}$ & target & 0.5619 & 0.4909 & 0.3216 & -0.6337 & 3.647 & 3.097 & 2.097 & 0.232 \\
\hline US-15 & quenched & measured & 0.6620 & 0.5934 & 0.3936 & -0.6550 & 4.592 & 3.921 & 2.475 & 0.221 \\
\hline US-15 & quenched & measured bc & 0.6649 & 0.5874 & 0.4000 & -0.6626 & 4.623 & 3.867 & 2.512 & 0.217 \\
\hline US-15 & quenched & target & 0.6673 & 0.5938 & 0.4192 & -0.6626 & 4.649 & 3.925 & 2.625 & 0.217 \\
\hline US-16 & $\mathrm{ccc}$ & measured & -0.0663 & -0.0277 & -0.3805 & -0.5622 & 0.858 & 0.938 & 0.416 & 0.274 \\
\hline US-16 & $\mathrm{ccc}$ & measured bc & -0.0634 & -0.0337 & -0.3702 & -0.5697 & 0.864 & 0.925 & 0.426 & 0.269 \\
\hline US-16 & $\mathrm{ccc}$ & target & -0.0632 & -0.0265 & -0.3440 & -0.5751 & 0.865 & 0.941 & 0.453 & 0.266 \\
\hline US-16 & quenched & measured & -0.1970 & -0.0974 & -0.5446 & -0.5871 & 0.635 & 0.799 & 0.285 & 0.259 \\
\hline US-16 & quenched & measured bc & -0.1941 & -0.1034 & -0.5343 & -0.5946 & 0.640 & 0.788 & 0.292 & 0.254 \\
\hline US-16 & quenched & target & -0.1939 & -0.0962 & -0.5081 & -0.6000 & 0.640 & 0.801 & 0.310 & 0.251 \\
\hline US-17 & $\mathrm{ccc}$ & measured & -0.3066 & -0.2322 & -0.5261 & -0.3865 & 0.494 & 0.586 & 0.298 & 0.411 \\
\hline US-17 & $\mathrm{ccc}$ & measured bc & -0.2984 & -0.2376 & -0.5194 & -0.3986 & 0.503 & 0.579 & 0.302 & 0.399 \\
\hline US-17 & $\mathrm{ccc}$ & target & -0.3168 & -0.2488 & -0.5052 & -0.4242 & 0.482 & 0.564 & 0.312 & 0.377 \\
\hline US-17 & quenched & measured & -0.2969 & -0.1875 & -0.5616 & -0.3696 & 0.505 & 0.649 & 0.274 & 0.427 \\
\hline US-17 & quenched & measured bc & -0.2887 & -0.1929 & -0.5549 & -0.3817 & 0.514 & 0.641 & 0.279 & 0.415 \\
\hline US-17 & quenched & target & -0.3071 & -0.2042 & -0.5407 & -0.4073 & 0.493 & 0.625 & 0.288 & 0.391 \\
\hline US-18 & $\mathrm{ccc}$ & measured & 1.0042 & 0.9158 & 0.5477 & -0.0638 & 10.097 & 8.238 & 3.530 & 0.863 \\
\hline US-18 & $\mathrm{ccc}$ & measured bc & 1.0211 & 0.9104 & 0.5490 & -0.0810 & 10.498 & 8.136 & 3.540 & 0.830 \\
\hline US-18 & $\mathrm{ccc}$ & target & 1.0277 & 0.9145 & 0.5223 & -0.0644 & 10.659 & 8.213 & 3.329 & 0.862 \\
\hline US-18 & quenched & measured & -0.3042 & -0.2235 & -0.2092 & -0.3932 & 0.496 & 0.598 & 0.618 & 0.404 \\
\hline US-18 & quenched & measured bc & -0.2873 & -0.2289 & -0.2079 & -0.4103 & 0.516 & 0.590 & 0.620 & 0.389 \\
\hline US-18 & quenched & target & -0.2807 & -0.2248 & -0.2346 & -0.3937 & 0.524 & 0.596 & 0.583 & 0.404 \\
\hline US-19 & $\mathrm{ccc}$ & measured & -0.3920 & -0.2707 & -0.4997 & -0.5049 & 0.405 & 0.536 & 0.316 & 0.313 \\
\hline US-19 & $\mathrm{ccc}$ & measured bc & -0.3699 & -0.2736 & -0.4839 & -0.5058 & 0.427 & 0.533 & 0.328 & 0.312 \\
\hline US-19 & $\mathrm{ccc}$ & target & -0.3834 & -0.2725 & -0.4664 & -0.5251 & 0.414 & 0.534 & 0.342 & 0.298 \\
\hline US-19 & quenched & measured & -0.3608 & -0.2345 & -0.4979 & -0.4741 & 0.436 & 0.583 & 0.318 & 0.336 \\
\hline US-19 & quenched & measured bc & -0.3387 & -0.2374 & -0.4820 & -0.4751 & 0.458 & 0.579 & 0.330 & 0.335 \\
\hline US-19 & quenched & target & -0.3521 & -0.2363 & -0.4645 & -0.4943 & 0.444 & 0.580 & 0.343 & 0.320 \\
\hline US-20 & $\mathrm{ccc}$ & measured & -0.3892 & -0.2552 & -0.4923 & -0.5054 & 0.408 & 0.556 & 0.322 & 0.312 \\
\hline US-20 & $\mathrm{ccc}$ & measured bc & -0.3671 & -0.2582 & -0.4764 & -0.5063 & 0.429 & 0.552 & 0.334 & 0.312 \\
\hline US-20 & $\mathrm{ccc}$ & target & -0.3789 & -0.2597 & -0.4629 & -0.5239 & 0.418 & 0.550 & 0.344 & 0.299 \\
\hline US-20 & quenched & measured & -0.3616 & -0.2225 & -0.4796 & -0.4869 & 0.435 & 0.599 & 0.331 & 0.326 \\
\hline US-20 & quenched & measured bc & -0.3394 & -0.2254 & -0.4637 & -0.4879 & 0.458 & 0.595 & 0.344 & 0.325 \\
\hline US-20 & quenched & target & -0.3512 & -0.2269 & -0.4502 & -0.5055 & 0.445 & 0.593 & 0.355 & 0.312 \\
\hline US-21 & $\mathrm{ccc}$ & measured & -0.4031 & -0.2581 & -0.5525 & -0.5238 & 0.395 & 0.552 & 0.280 & 0.299 \\
\hline US-21 & $\mathrm{ccc}$ & measured bc & -0.4002 & -0.2650 & -0.5345 & -0.5330 & 0.398 & 0.543 & 0.292 & 0.293 \\
\hline US-21 & $\mathrm{ccc}$ & target & -0.4020 & -0.2515 & -0.5112 & -0.5220 & 0.396 & 0.560 & 0.308 & 0.301 \\
\hline US-21 & quenched & measured & -0.3811 & -0.2244 & -0.5392 & -0.4468 & 0.416 & 0.596 & 0.289 & 0.357 \\
\hline US-21 & quenched & measured bc & -0.3781 & -0.2313 & -0.5212 & -0.4560 & 0.419 & 0.587 & 0.301 & 0.350 \\
\hline US-21 & quenched & target & -0.3800 & -0.2178 & -0.4979 & -0.4450 & 0.417 & 0.606 & 0.318 & 0.359 \\
\hline US-22 & $\mathrm{ccc}$ & measured & -0.2793 & -0.2584 & -0.2932 & -0.3712 & 0.526 & 0.552 & 0.509 & 0.425 \\
\hline US-22 & $\mathrm{ccc}$ & measured bc & -0.2710 & -0.2638 & -0.2869 & -0.3832 & 0.536 & 0.545 & 0.517 & 0.414 \\
\hline US-22 & $\mathrm{ccc}$ & target & -0.2742 & -0.2615 & -0.2434 & -0.3805 & 0.532 & 0.548 & 0.571 & 0.416 \\
\hline US-22 & quenched & measured & -0.2521 & -0.2397 & -0.3379 & -0.4349 & 0.560 & 0.576 & 0.459 & 0.367 \\
\hline US-22 & quenched & measured bc & -0.2439 & -0.2451 & -0.3316 & -0.4470 & 0.570 & 0.569 & 0.466 & 0.357 \\
\hline US-22 & quenched & target & $\begin{array}{l}-0.2471 \\
\end{array}$ & -0.2428 & -0.2881 & -0.4442 & 0.566 & 0.572 & 0.515 & 0.360 \\
\hline
\end{tabular}


Display 3: Normalized PCTs by Glass ID, Heat Treatment, and Compositional View

\begin{tabular}{|c|c|c|c|c|c|c|c|c|c|c|}
\hline $\begin{array}{c}\text { Glass } \\
\text { ID }\end{array}$ & $\begin{array}{c}\text { Heat } \\
\text { Treatment }\end{array}$ & Comp View & $\begin{array}{c}\log N L[B \\
(g / L)]\end{array}$ & $\begin{array}{c}\log \mathrm{NL} \\
{[\mathrm{Li}(\mathrm{g} / \mathrm{L})]}\end{array}$ & $\begin{array}{c}\log \mathrm{NL}[\mathrm{Na} \\
(\mathrm{g} / \mathrm{L})]\end{array}$ & $\begin{array}{c}\log \mathrm{NL} \\
{[\mathrm{Si}(\mathrm{g} / \mathrm{L})]}\end{array}$ & $\begin{array}{l}\text { NL B } \\
(\mathrm{g} / \mathrm{L})\end{array}$ & $\begin{array}{l}\text { NL Li } \\
(\mathrm{g} / \mathrm{L})\end{array}$ & $\begin{array}{c}\begin{array}{c}\text { NL Na } \\
(\mathrm{g} / \mathrm{L})\end{array} \\
\end{array}$ & $\begin{array}{l}\mathrm{NL} \mathrm{Si} \\
(\mathrm{g} / \mathrm{L}) \\
\end{array}$ \\
\hline US-23 & $\mathrm{ccc}$ & measured & -0.3596 & -0.2418 & -0.4930 & -0.4790 & 0.437 & 0.573 & 0.321 & 0.332 \\
\hline US-23 & $\mathrm{ccc}$ & measured bc & -0.3513 & -0.2473 & -0.4751 & -0.4911 & 0.445 & 0.566 & 0.335 & 0.323 \\
\hline US-23 & $\mathrm{ccc}$ & target & -0.3582 & -0.2486 & -0.4473 & -0.4925 & 0.438 & 0.564 & 0.357 & 0.322 \\
\hline US-23 & quenched & measured & -0.3486 & -0.2089 & -0.4885 & -0.4404 & 0.448 & 0.618 & 0.325 & 0.363 \\
\hline US-23 & quenched & measured bc & -0.3403 & -0.2143 & -0.4706 & -0.4525 & 0.457 & 0.610 & 0.338 & 0.353 \\
\hline US-23 & quenched & target & -0.3472 & -0.2157 & -0.4428 & -0.4539 & 0.450 & 0.609 & 0.361 & 0.352 \\
\hline US-24 & $\mathrm{ccc}$ & measured & -0.4068 & -0.2773 & -0.4907 & -0.4981 & 0.392 & 0.528 & 0.323 & 0.318 \\
\hline US-24 & $\mathrm{ccc}$ & measured bc & -0.3899 & -0.2827 & -0.4748 & -0.5154 & 0.407 & 0.522 & 0.335 & 0.305 \\
\hline US-24 & $\mathrm{ccc}$ & target & -0.3897 & -0.2623 & -0.4519 & -0.5278 & 0.408 & 0.547 & 0.353 & 0.297 \\
\hline US-24 & quenched & measured & -0.4200 & -0.2690 & -0.5102 & -0.5073 & 0.380 & 0.538 & 0.309 & 0.311 \\
\hline US-24 & quenched & measured bc & -0.4031 & -0.2744 & -0.4944 & -0.5245 & 0.395 & 0.532 & 0.320 & 0.299 \\
\hline US-24 & quenched & target & -0.4028 & -0.2540 & -0.4715 & -0.5369 & 0.396 & 0.557 & 0.338 & 0.290 \\
\hline US-25 & $\mathrm{ccc}$ & measured & 0.1503 & 0.0960 & -0.0735 & -0.3551 & 1.413 & 1.247 & 0.844 & 0.441 \\
\hline US-25 & $\mathrm{ccc}$ & measured bc & 0.1532 & 0.0891 & -0.0631 & -0.3643 & 1.423 & 1.228 & 0.865 & 0.432 \\
\hline US-25 & $\mathrm{ccc}$ & target & 0.1500 & 0.0923 & -0.0346 & -0.3723 & 1.413 & 1.237 & 0.924 & 0.424 \\
\hline US-25 & quenched & measured & 0.0595 & 0.0179 & -0.1467 & -0.4490 & 1.147 & 1.042 & 0.713 & 0.356 \\
\hline US-25 & quenched & measured bc & 0.0624 & 0.0110 & -0.1363 & -0.4582 & 1.155 & 1.026 & 0.731 & 0.348 \\
\hline US-25 & quenched & target & 0.0592 & 0.0142 & -0.1078 & -0.4662 & 1.146 & 1.033 & 0.780 & 0.342 \\
\hline US-26 & $\mathrm{ccc}$ & measured & 0.5451 & 0.4143 & 0.0667 & -0.4871 & 3.509 & 2.596 & 1.166 & 0.326 \\
\hline US-26 & $\mathrm{ccc}$ & measured bc & 0.5672 & 0.4114 & 0.0732 & -0.4880 & 3.691 & 2.579 & 1.184 & 0.325 \\
\hline US-26 & $\mathrm{ccc}$ & target & 0.5566 & 0.4058 & 0.0836 & -0.4814 & 3.603 & 2.546 & 1.212 & 0.330 \\
\hline US-26 & quenched & measured & -0.3891 & -0.2753 & -0.3950 & -0.5817 & 0.408 & 0.531 & 0.403 & 0.262 \\
\hline US-26 & quenched & measured bc & -0.3670 & -0.2782 & -0.3885 & -0.5827 & 0.430 & 0.527 & 0.409 & 0.261 \\
\hline US-26 & quenched & target & -0.3776 & -0.2838 & -0.3781 & -0.5760 & 0.419 & 0.520 & 0.419 & 0.265 \\
\hline US-27 & $\mathrm{ccc}$ & measured & 1.1808 & 0.8651 & 0.8372 & 0.3655 & 15.164 & 7.330 & 6.874 & 2.320 \\
\hline US-27 & $\mathrm{ccc}$ & measured bc & 1.2031 & 0.8622 & 0.8475 & 0.3648 & 15.962 & 7.281 & 7.039 & 2.316 \\
\hline US-27 & $\mathrm{ccc}$ & target & 1.2158 & 0.8534 & 0.8725 & 0.3622 & 16.435 & 7.136 & 7.457 & 2.302 \\
\hline US-27 & quenched & measured & 0.0616 & 0.0240 & 0.1585 & -0.0775 & 1.152 & 1.057 & 1.441 & 0.836 \\
\hline US-27 & quenched & measured bc & 0.0839 & 0.0210 & 0.1689 & -0.0783 & 1.213 & 1.050 & 1.475 & 0.835 \\
\hline US-27 & quenched & target & 0.0966 & 0.0123 & 0.1939 & -0.0809 & 1.249 & 1.029 & 1.563 & 0.830 \\
\hline US-28 & $\mathrm{ccc}$ & target & 0.1388 & 0.0994 & -0.0420 & -0.5552 & 1.376 & 1.257 & 0.908 & 0.278 \\
\hline US-28 & quenched & target & 0.2322 & 0.1773 & 0.0211 & -0.5743 & 1.707 & 1.504 & 1.050 & 0.267 \\
\hline US-28* & $\mathrm{ccc}$ & measured & 0.1384 & 0.0938 & -0.0637 & -0.5541 & 1.375 & 1.241 & 0.864 & 0.279 \\
\hline US-28* & $\mathrm{ccc}$ & measured bc & 0.1466 & 0.0884 & -0.0534 & -0.5662 & 1.402 & 1.226 & 0.884 & 0.272 \\
\hline US-28* & quenched & measured & 0.2317 & 0.1718 & -0.0006 & -0.5732 & 1.705 & 1.485 & 0.999 & 0.267 \\
\hline US-28* & quenched & measured bc & 0.2400 & 0.1663 & 0.0098 & -0.5853 & 1.738 & 1.467 & 1.023 & 0.260 \\
\hline US-29 & $\mathrm{ccc}$ & measured & 0.1732 & 0.1456 & 0.2236 & -0.1183 & 1.490 & 1.398 & 1.673 & 0.762 \\
\hline US-29 & $\mathrm{ccc}$ & measured bc & 0.1954 & 0.1427 & 0.2394 & -0.1189 & 1.568 & 1.389 & 1.736 & 0.760 \\
\hline US-29 & $\mathrm{ccc}$ & target & 0.1862 & 0.1379 & 0.2424 & -0.1299 & 1.535 & 1.374 & 1.748 & 0.742 \\
\hline US-29 & quenched & measured & 0.2491 & 0.1748 & 0.3010 & -0.1012 & 1.774 & 1.496 & 2.000 & 0.792 \\
\hline US-29 & quenched & measured bc & 0.2713 & 0.1719 & 0.3168 & -0.1019 & 1.868 & 1.486 & 2.074 & 0.791 \\
\hline US-29 & quenched & target & 0.2621 & 0.1672 & 0.3198 & -0.1129 & 1.828 & 1.469 & 2.088 & 0.771 \\
\hline US-30 & $\mathrm{ccc}$ & measured & -0.2011 & -0.2033 & -0.4079 & -0.6040 & 0.629 & 0.626 & 0.391 & 0.249 \\
\hline US-30 & $\mathrm{ccc}$ & measured bc & -0.1982 & -0.2101 & -0.3921 & -0.6132 & 0.634 & 0.616 & 0.405 & 0.244 \\
\hline US-30 & $\mathrm{ccc}$ & target & -0.1938 & -0.2061 & -0.3544 & -0.5958 & 0.640 & 0.622 & 0.442 & 0.254 \\
\hline US-30 & quenched & measured & -0.2476 & -0.2399 & -0.4624 & -0.6508 & 0.565 & 0.576 & 0.345 & 0.223 \\
\hline US-30 & quenched & measured bc & -0.2447 & -0.2467 & -0.4466 & -0.6600 & 0.569 & 0.567 & 0.358 & 0.219 \\
\hline US-30 & quenched & target & -0.2403 & -0.2427 & -0.4088 & -0.6426 & 0.575 & 0.572 & 0.390 & 0.228 \\
\hline US-31 & $\mathrm{ccc}$ & measured & -0.5119 & -0.3082 & -0.7651 & -0.6059 & 0.308 & 0.492 & 0.172 & 0.248 \\
\hline US-31 & $\mathrm{ccc}$ & measured bc & -0.5036 & -0.3136 & -0.7492 & -0.6179 & 0.314 & 0.486 & 0.178 & 0.241 \\
\hline US-31 & $\mathrm{ccc}$ & target & -0.5126 & -0.3062 & -0.6960 & -0.6021 & 0.307 & 0.494 & 0.201 & 0.250 \\
\hline US-31 & quenched & measured & -0.3022 & -0.1986 & -0.5976 & -0.5487 & 0.499 & 0.633 & 0.253 & 0.283 \\
\hline US-31 & quenched & measured bc & -0.2939 & -0.2041 & -0.5817 & -0.5608 & 0.508 & 0.625 & 0.262 & 0.275 \\
\hline US-31 & quenched & target & -0.3029 & -0.1967 & -0.5285 & -0.5450 & 0.498 & 0.636 & 0.296 & 0.285 \\
\hline US-32 & $\mathrm{ccc}$ & measured & 0.4486 & 0.3600 & 0.1532 & -0.4691 & 2.810 & 2.291 & 1.423 & 0.340 \\
\hline US-32 & $\mathrm{ccc}$ & measured bc & 0.4515 & 0.3538 & 0.1596 & -0.4768 & 2.828 & 2.258 & 1.444 & 0.334 \\
\hline US-32 & $\mathrm{ccc}$ & target & 0.4498 & 0.3728 & 0.1781 & -0.4713 & 2.817 & 2.360 & 1.507 & 0.338 \\
\hline US-32 & quenched & measured & 0.5509 & 0.4608 & 0.2458 & -0.4642 & 3.556 & 2.889 & 1.761 & 0.343 \\
\hline US-32 & quenched & measured bc & 0.5537 & 0.4546 & 0.2522 & -0.4720 & 3.579 & 2.849 & 1.787 & 0.337 \\
\hline US-32 & quenched & target & 0.5520 & 0.4737 & 0.2707 & -0.4664 & 3.565 & 2.976 & 1.865 & 0.342 \\
\hline US-33 & $\mathrm{ccc}$ & measured & -0.5102 & -0.2586 & -0.0699 & -0.4316 & 0.309 & 0.551 & 0.851 & 0.370 \\
\hline US-33 & $\mathrm{ccc}$ & measured bc & -0.4931 & -0.2641 & -0.0635 & -0.4488 & 0.321 & 0.544 & 0.864 & 0.356 \\
\hline US-33 & $\mathrm{ccc}$ & target & -0.4632 & -0.2450 & -0.0702 & -0.4314 & 0.344 & 0.569 & 0.851 & 0.370 \\
\hline US-33 & quenched & measured & -0.4883 & -0.2973 & -0.0467 & -0.4494 & 0.325 & 0.504 & 0.898 & 0.355 \\
\hline US-33 & quenched & measured bc & -0.4712 & -0.3028 & -0.0403 & -0.4665 & 0.338 & 0.498 & 0.911 & 0.342 \\
\hline US-33 & quenched & target & -0.4413 & -0.2838 & -0.0470 & -0.4491 & 0.362 & 0.520 & 0.897 & 0.356 \\
\hline US-34 & $\mathrm{ccc}$ & measured & 0.5703 & 0.5064 & 0.4473 & -0.3492 & 3.718 & 3.209 & 2.801 & 0.448 \\
\hline US-34 & $\mathrm{ccc}$ & measured bc & 0.5871 & 0.5009 & 0.4489 & -0.3664 & 3.865 & 3.169 & 2.811 & 0.430 \\
\hline US-34 & $\mathrm{ccc}$ & target & 0.5849 & 0.5049 & 0.4344 & $\begin{array}{l}-0.3481 \\
\end{array}$ & 3.845 & 3.198 & 2.719 & 0.449 \\
\hline
\end{tabular}


Display 3: Normalized PCTs by Glass ID, Heat Treatment, and Compositional View

\begin{tabular}{|c|c|c|c|c|c|c|c|c|c|c|}
\hline $\begin{array}{c}\text { Glass } \\
\text { ID }\end{array}$ & $\begin{array}{c}\text { Heat } \\
\text { Treatment }\end{array}$ & Comp View & $\begin{array}{c}\log N L[B \\
(g / L)]\end{array}$ & $\begin{array}{c}\log \mathrm{NL} \\
{[\mathrm{Li}(\mathrm{g} / \mathrm{L})]}\end{array}$ & $\begin{array}{c}\log \mathrm{NL}[\mathrm{Na} \\
(\mathrm{g} / \mathrm{L})]\end{array}$ & $\begin{array}{c}\log \mathrm{NL} \\
{[\mathrm{Si}(\mathrm{g} / \mathrm{L})]}\end{array}$ & $\begin{array}{l}\text { NL B } \\
(\mathrm{g} / \mathrm{L})\end{array}$ & $\begin{array}{c}\text { NL Li } \\
(\mathrm{g} / \mathrm{L})\end{array}$ & $\begin{array}{c}\text { NL Na } \\
(\mathrm{g} / \mathrm{L})\end{array}$ & $\begin{array}{l}\mathrm{NL} \mathrm{Si} \\
(\mathrm{g} / \mathrm{L})\end{array}$ \\
\hline US-34 & quenched & measured & 0.6039 & 0.5356 & 0.4851 & -0.3293 & 4.017 & 3.432 & 3.056 & 0.469 \\
\hline US-34 & quenched & measured bc & 0.6208 & 0.5301 & 0.4867 & -0.3465 & 4.176 & 3.389 & 3.067 & 0.450 \\
\hline US-34 & quenched & target & 0.6186 & 0.5341 & 0.4722 & -0.3282 & 4.155 & 3.420 & 2.966 & 0.470 \\
\hline US-35 & $\mathrm{ccc}$ & measured & 0.6005 & 0.5435 & 0.3564 & -0.6589 & 3.985 & 3.495 & 2.272 & 0.219 \\
\hline US-35 & $\mathrm{ccc}$ & measured bc & 0.6034 & 0.5366 & 0.3743 & -0.6681 & 4.012 & 3.440 & 2.367 & 0.215 \\
\hline US-35 & $\mathrm{ccc}$ & target & 0.5976 & 0.5497 & 0.4011 & -0.6592 & 3.959 & 3.546 & 2.518 & 0.219 \\
\hline US-35 & quenched & measured & -0.0931 & -0.0470 & -0.2424 & -0.6837 & 0.807 & 0.897 & 0.572 & 0.207 \\
\hline US-35 & quenched & measured bc & -0.0902 & -0.0538 & -0.2245 & -0.6929 & 0.812 & 0.883 & 0.596 & 0.203 \\
\hline US-35 & quenched & target & -0.0960 & -0.0407 & -0.1977 & -0.6840 & 0.802 & 0.910 & 0.634 & 0.207 \\
\hline US-36 & $\mathrm{ccc}$ & measured & -0.0935 & -0.0994 & -0.3528 & -0.6137 & 0.806 & 0.796 & 0.444 & 0.243 \\
\hline US-36 & $\mathrm{ccc}$ & measured bc & -0.0906 & -0.1062 & -0.3349 & -0.6229 & 0.812 & 0.783 & 0.463 & 0.238 \\
\hline US-36 & $\mathrm{ccc}$ & target & -0.0956 & -0.1033 & -0.3068 & -0.6223 & 0.803 & 0.788 & 0.493 & 0.239 \\
\hline US-36 & quenched & measured & -0.1850 & -0.1553 & -0.4211 & -0.6371 & 0.653 & 0.699 & 0.379 & 0.231 \\
\hline US-36 & quenched & measured bc & -0.1821 & -0.1622 & -0.4032 & -0.6463 & 0.658 & 0.688 & 0.395 & 0.226 \\
\hline US-36 & quenched & target & -0.1870 & -0.1592 & -0.3751 & -0.6457 & 0.650 & 0.693 & 0.422 & 0.226 \\
\hline US-37 & $\mathrm{ccc}$ & target & 0.3280 & 0.2203 & 0.1507 & -0.2831 & 2.128 & 1.661 & 1.415 & 0.521 \\
\hline US-37 & quenched & target & -0.1392 & -0.1799 & -0.0308 & -0.3998 & 0.726 & 0.661 & 0.932 & 0.398 \\
\hline US-37* & $\mathrm{ccc}$ & measured & 0.2941 & 0.2158 & 0.1343 & -0.2911 & 1.968 & 1.644 & 1.362 & 0.512 \\
\hline US-37* & $\mathrm{ccc}$ & measured bc & 0.2971 & 0.2098 & 0.1446 & -0.2988 & 1.982 & 1.621 & 1.395 & 0.503 \\
\hline US-37* & quenched & measured & -0.1731 & -0.1844 & -0.0472 & -0.4079 & 0.671 & 0.654 & 0.897 & 0.391 \\
\hline US-37* & quenched & measured bc & -0.1702 & -0.1904 & -0.0368 & -0.4155 & 0.676 & 0.645 & 0.919 & 0.384 \\
\hline US-38 & $\mathrm{ccc}$ & measured & -0.1084 & -0.1017 & 0.0802 & -0.3000 & 0.779 & 0.791 & 1.203 & 0.501 \\
\hline US-38 & $\mathrm{ccc}$ & measured bc & -0.1001 & -0.1071 & 0.0816 & -0.3121 & 0.794 & 0.781 & 1.207 & 0.487 \\
\hline US-38 & $\mathrm{ccc}$ & target & -0.0974 & -0.0894 & 0.0500 & -0.3073 & 0.799 & 0.814 & 1.122 & 0.493 \\
\hline US-38 & quenched & measured & -0.1135 & -0.1567 & 0.0646 & -0.3555 & 0.770 & 0.697 & 1.160 & 0.441 \\
\hline US-38 & quenched & measured bc & -0.1052 & -0.1622 & 0.0659 & -0.3675 & 0.785 & 0.688 & 1.164 & 0.429 \\
\hline US-38 & quenched & target & -0.1025 & -0.1444 & 0.0344 & -0.3627 & 0.790 & 0.717 & 1.082 & 0.434 \\
\hline US-39 & $\mathrm{ccc}$ & measured & -0.3680 & -0.2824 & -0.7405 & -0.5409 & 0.429 & 0.522 & 0.182 & 0.288 \\
\hline US-39 & $\mathrm{ccc}$ & measured bc & -0.3459 & -0.2853 & -0.7391 & -0.5418 & 0.451 & 0.518 & 0.182 & 0.287 \\
\hline US-39 & $\mathrm{ccc}$ & target & -0.3593 & -0.2856 & -0.7385 & -0.5587 & 0.437 & 0.518 & 0.183 & 0.276 \\
\hline US-39 & quenched & measured & -0.3134 & -0.2553 & -0.6593 & -0.5444 & 0.486 & 0.556 & 0.219 & 0.285 \\
\hline US-39 & quenched & measured bc & -0.2913 & -0.2582 & -0.6579 & -0.5453 & 0.511 & 0.552 & 0.220 & 0.285 \\
\hline US-39 & quenched & target & -0.3047 & -0.2586 & -0.6573 & -0.5623 & 0.496 & 0.551 & 0.220 & 0.274 \\
\hline US-40 & ccc & measured & 0.1856 & 0.1414 & -0.1182 & -0.5407 & 1.533 & 1.385 & 0.762 & 0.288 \\
\hline US-40 & $\mathrm{ccc}$ & measured bc & 0.1885 & 0.1353 & -0.1003 & -0.5484 & 1.543 & 1.366 & 0.794 & 0.283 \\
\hline US-40 & $\mathrm{ccc}$ & target & 0.1876 & 0.1426 & -0.0781 & -0.5347 & 1.540 & 1.389 & 0.835 & 0.292 \\
\hline US-40 & quenched & measured & 0.0369 & 0.0302 & -0.2343 & -0.5296 & 1.089 & 1.072 & 0.583 & 0.295 \\
\hline US-40 & quenched & measured bc & 0.0398 & 0.0242 & -0.2164 & -0.5372 & 1.096 & 1.057 & 0.608 & 0.290 \\
\hline US-40 & quenched & target & 0.0389 & 0.0314 & -0.1943 & -0.5236 & 1.094 & 1.075 & 0.639 & 0.299 \\
\hline US-41 & $\mathrm{ccc}$ & measured & -0.5450 & -0.3003 & -0.7809 & -0.6671 & 0.285 & 0.501 & 0.166 & 0.215 \\
\hline US-41 & $\mathrm{ccc}$ & measured bc & -0.5421 & -0.3072 & -0.7705 & -0.6763 & 0.287 & 0.493 & 0.170 & 0.211 \\
\hline US-41 & $\mathrm{ccc}$ & target & -0.5514 & -0.3092 & -0.7469 & -0.6864 & 0.281 & 0.491 & 0.179 & 0.206 \\
\hline US-41 & quenched & measured & -0.4808 & -0.3000 & -0.7143 & -0.6551 & 0.331 & 0.501 & 0.193 & 0.221 \\
\hline US-41 & quenched & measured bc & -0.4778 & -0.3069 & -0.7039 & -0.6643 & 0.333 & 0.493 & 0.198 & 0.217 \\
\hline US-41 & quenched & target & -0.4872 & -0.3089 & -0.6803 & -0.6743 & 0.326 & 0.491 & 0.209 & 0.212 \\
\hline US-42 & $\mathrm{ccc}$ & measured & -0.0501 & 0.3044 & 0.5460 & -0.7134 & 0.891 & 2.015 & 3.516 & 0.193 \\
\hline US-42 & $\mathrm{ccc}$ & measured bc & -0.0472 & 0.2982 & 0.5640 & -0.7211 & 0.897 & 1.987 & 3.664 & 0.190 \\
\hline US-42 & $\mathrm{ccc}$ & target & -0.0338 & 0.3014 & 0.5617 & -0.7080 & 0.925 & 2.002 & 3.645 & 0.196 \\
\hline US-42 & quenched & measured & 0.0970 & 0.0430 & 0.2031 & -0.1532 & 1.250 & 1.104 & 1.596 & 0.703 \\
\hline US-42 & quenched & measured bc & 0.0999 & 0.0368 & 0.2211 & -0.1609 & 1.259 & 1.088 & 1.664 & 0.690 \\
\hline US-42 & quenched & target & 0.1133 & 0.0400 & 0.2188 & -0.1478 & 1.298 & 1.097 & 1.655 & 0.712 \\
\hline US-43 & $\mathrm{ccc}$ & measured & 0.2388 & 0.2504 & -0.3189 & -0.4463 & 1.733 & 1.780 & 0.480 & 0.358 \\
\hline US-43 & $\mathrm{ccc}$ & measured bc & 0.2417 & 0.2443 & -0.3086 & -0.4540 & 1.745 & 1.755 & 0.491 & 0.352 \\
\hline US-43 & $\mathrm{ccc}$ & target & 0.2441 & 0.2498 & -0.2793 & -0.4632 & 1.754 & 1.778 & 0.526 & 0.344 \\
\hline US-43 & quenched & measured & -0.3981 & -0.2252 & -0.4843 & -0.5352 & 0.400 & 0.595 & 0.328 & 0.292 \\
\hline US-43 & quenched & measured bc & -0.3951 & -0.2314 & -0.4739 & -0.5429 & 0.403 & 0.587 & 0.336 & 0.287 \\
\hline US-43 & quenched & target & -0.3928 & -0.2259 & -0.4446 & -0.5521 & 0.405 & 0.594 & 0.359 & 0.280 \\
\hline US-44 & $\mathrm{ccc}$ & measured & -0.2867 & -0.2805 & -0.3445 & -0.5120 & 0.517 & 0.524 & 0.452 & 0.308 \\
\hline US-44 & ccc & measured bc & -0.2838 & -0.2874 & -0.3266 & -0.5212 & 0.520 & 0.516 & 0.471 & 0.301 \\
\hline US-44 & $\mathrm{ccc}$ & target & -0.2911 & -0.2452 & -0.3058 & -0.5358 & 0.512 & 0.569 & 0.495 & 0.291 \\
\hline US-44 & quenched & measured & -0.2772 & -0.2826 & -0.3398 & -0.5257 & 0.528 & 0.522 & 0.457 & 0.298 \\
\hline US-44 & quenched & measured bc & -0.2743 & -0.2895 & -0.3218 & -0.5349 & 0.532 & 0.514 & 0.477 & 0.292 \\
\hline US-44 & quenched & target & -0.2815 & -0.2473 & -0.3010 & -0.5496 & 0.523 & 0.566 & 0.500 & 0.282 \\
\hline US-45 & $\mathrm{ccc}$ & measured & -0.3537 & -0.2633 & -0.3606 & -0.5490 & 0.443 & 0.545 & 0.436 & 0.282 \\
\hline US-45 & $\mathrm{ccc}$ & measured bc & -0.3509 & -0.2695 & -0.3447 & -0.5566 & 0.446 & 0.538 & 0.452 & 0.278 \\
\hline US-45 & $\mathrm{ccc}$ & target & -0.3443 & -0.2617 & -0.3370 & -0.5481 & 0.453 & 0.547 & 0.460 & 0.283 \\
\hline US-45 & quenched & measured & -0.3340 & -0.2465 & -0.3305 & -0.5445 & 0.463 & 0.567 & 0.467 & 0.285 \\
\hline US-45 & quenched & measured bc & -0.3311 & -0.2527 & -0.3147 & -0.5521 & 0.467 & 0.559 & 0.485 & 0.280 \\
\hline US-45 & quenched & target & -0.3245 & -0.2450 & -0.3069 & -0.5436 & 0.474 & 0.569 & 0.493 & 0.286 \\
\hline
\end{tabular}




\subsection{Effects of Heat Treatment on PCTs}

Exhibit B7 in Appendix B provides a series of plots and statistical comparisons that show the effects of heat treatment on the common logarithm ppm-responses of interest of the triplicate PCTs for each element for each study glass. The ccc version of a given glass yielded measurements indicating a significantly (at the 5\% significance level) different mean $\log (\mathrm{ppm})$ response than the quenched version of the glass for a given element if the Prob $>|\mathbf{t}|$ value in the exhibit is 0.05 or smaller. For example, the first glass that shows a statistically significant PCT difference between its quenched and ccc versions is US-03, and for this glass, all four of the elements of interest (i.e., $\mathrm{B}, \mathrm{Li}, \mathrm{Na}$, and $\mathrm{Si}$ ), show a statistically significant difference between their average quenched PCT result versus their average ccc PCT result at the $5 \%$ significance level.

Exhibit B8 in Appendix B provides a series of plots that show the effects of heat treatment on the PCT response based on the three different compositional views: measured, measured biascorrected, and targeted. These plots allow for an assessment of the differences in PCT responses from a practical perspective. Note the differences in the quenched and ccc versions of US-18, US-27, and US-42 that were highlighted earlier. Also, note similar differences for US-26 in some of the plots. Figure 1 provides a summary plot of the PCT results (based upon the targeted compositional view) for these glasses. From the information in Exhibit B7, the ccc version of the US-18 glass is statistically less durable than its quenched counterpart for all four elements of interest. For US-26, none of the differences are statistically significant (at the 5\% level). For US27 , the ccc version of the glass is statistically less durable than its quenched counterpart for all four elements of interest. For US-42, there is a statistically significant difference between the durability of the quenched and ccc versions of this glass for each element of interest: the quenched version is more durable for boron and silicon, but less durable for lithium and sodium.

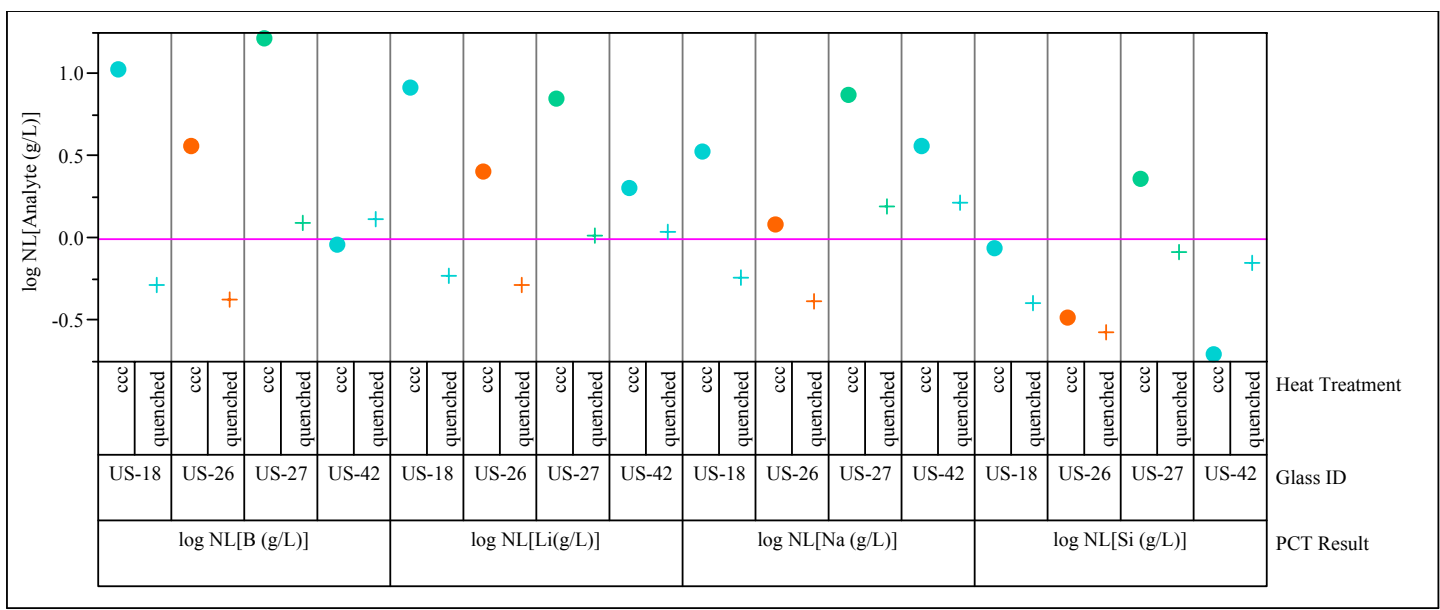

Figure 1. Normalized PCT Results for US-18, US-26, US-27, and US-42 Based Upon Targeted Compositions 


\subsection{CONCLUSIONS}

A statistical review of the PSAL measurements of the chemical compositions and of the PCT results for the glasses making up the US test matrix is provided in this memorandum. Target, measured, and measured bias-corrected compositional views were determined for these glasses. While some statistically significant differences were seen between the quenched and ccc versions for some of the glasses, all of the PCTs for the study glasses were less than the PCT measured for the EA standard glass.

\subsection{REFERENCES}

[1] Fox, K. M. et al., "Glass Selection Strategy: Development of US and KRI Test Matrices," WSRC-STI-2006-00205, Revision 0, September, 2006.

[2] Edwards, T. B., "An Analytical Plan for Measuring the Chemical Compositions of Glasses from the Task \#6 Study," SRNL-SCS-2006-00036, October 10, 2006.

[3] ASTM C-1285-2002, "Standard Test Methods for Determining Chemical Durability of Nuclear Waste Glasses: The Product Consistency Test (PCT)," ASTM, 2002.

[4] Edwards, T. B., "An Analytical Plan for Measuring the PCT Solutions for the First Set of Glasses from the Task \#6 Study," SRNL-SCS-2006-00035, September 20, 2006.

[5] Edwards, T. B., "An Analytical Plan for Measuring the PCT Solutions for the Second Set of Glasses from the Task \#6 Study,” SRNL-SCS-2006-00037, October 16, 2006.

[6] Edwards, T. B., "An Analytical Plan for Measuring the PCT Solutions for the Third Set of Glasses from the Task \#6 Study," SRNL-SCS-2006-00041, November 6, 2006.

[7] SAS Institute, Inc., JMP®: Statistics and Graphics Guide, Version 6.0.2, SAS Institute, Inc., Cary, NC, 2005.

[8] Jantzen, C. M., N. E. Bibler, D. C. Beam, C. L. Crawford, and M. A. Pickett, "Characterization of the Defense Waste Processing Facility (DWPF) Environmental Assessment (EA) Glass Standard Reference Material (U)”, WSRC-TR-92-346, Rev. 1, 1993. 


\section{Appendix A:}

\section{Tables and Exhibits Supporting the Analysis of the Chemical Composition Measurements for the US Study Glasses}


Table A1. Targeted Oxide Concentrations (in mass fractions) for the US Study Glass

\begin{tabular}{|c|c|c|c|c|c|c|c|c|c|c|c|c|c|c|c|c|c|c|c|c|}
\hline Glass ID & $\mathrm{Al}_{2} \mathrm{O}_{3}$ & $\mathbf{B}_{2} \mathrm{O}_{3}$ & $\mathrm{CaO}$ & $\mathrm{Cr}_{2} \mathrm{O}_{3}$ & $\mathrm{Fe}_{2} \mathrm{O}_{3}$ & $\mathrm{~K}_{2} \mathrm{O}$ & $\mathrm{Li}_{2} \mathrm{O}$ & MgO & MnO & $\mathrm{Na}_{2} \mathrm{O}$ & NiO & PbO & $\mathrm{RuO}_{2}$ & $\mathrm{SO}_{3}$ & $\mathrm{SiO}_{2}$ & $\begin{array}{l}\text { SrO } \\
\end{array}$ & $\overline{T i O}_{2}$ & $\begin{array}{l}\mathrm{ZnO} \\
\end{array}$ & $\mathrm{ZrO}_{2}$ & Sum \\
\hline US-01 & $\begin{array}{l}0.14929 \\
\end{array}$ & $\begin{array}{l}0.07891 \\
\end{array}$ & $\begin{array}{l}0.00000 \\
\end{array}$ & 0.00300 & 0.08589 & 0.01000 & 0.05616 & $\begin{array}{l}0.00500 \\
\end{array}$ & $\begin{array}{l}0.02498 \\
\end{array}$ & 0.12372 & 0.00057 & 0.00100 & $\begin{array}{l}0.00020 \\
\end{array}$ & $\begin{array}{l}0.00000 \\
\end{array}$ & 0.45530 & $\begin{array}{l}0.00100 \\
\end{array}$ & 0.00000 & 0.00000 & 0.00500 & 1.00000 \\
\hline US-02 & 0.15997 & 0.16855 & 0.00000 & 0.00000 & 0.06589 & 0.00000 & 0.05291 & 0.00000 & 0.00634 & 0.12334 & 0.00000 & 0.00000 & 0.00020 & 0.00000 & 0.41679 & 0.00000 & 0.00000 & 0.00100 & 0.00500 & 1.00000 \\
\hline US-03 & 0.15997 & 0.17309 & 0.01000 & 0.00000 & 0.06526 & 0.01000 & 0.04070 & 0.00499 & 0.00000 & 0.07884 & 0.00293 & 0.00099 & 0.00020 & 0.00000 & 0.44206 & 0.00100 & 0.00998 & 0.00000 & 0.00000 & 1.00000 \\
\hline $\begin{array}{l}\text { US-04 } \\
\end{array}$ & $\begin{array}{l}0.15996 \\
\end{array}$ & 0.11286 & $\begin{array}{l}0.00999 \\
\end{array}$ & 0.00001 & 0.08265 & 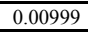 & 0.03742 & $\begin{array}{l}0.00499 \\
\end{array}$ & 0.02189 & $\begin{array}{l}0.10333 \\
\end{array}$ & 0.00001 & 0.00099 & 0.00020 & 0.00499 & 0.43378 & 0.00099 & $\begin{array}{l}0.00999 \\
\end{array}$ & 0.00099 & 0.00499 & 1.00000 \\
\hline US-05 & 0.15994 & 0.16700 & 0.00702 & 0.00001 & 0.08156 & 0.00999 & 0.02001 & 0.00499 & 0.02408 & 0.09581 & 0.00001 & 0.00100 & 0.00020 & 0.00498 & 0.40642 & 0.00100 & 0.00998 & 0.00100 & 0.00500 & 1.00000 \\
\hline $\begin{array}{l}\text { US- } 06 \\
\end{array}$ & 0.15997 & 0.14065 & 0.00999 & 0.00000 & 0.08154 & 0.00999 & 0.02473 & 0.00499 & 0.02998 & 0.10778 & 0.00001 & 0.00000 & 0.00020 & 0.00500 & 0.41420 & 0.00099 & 0.00999 & 0.00000 & 0.00000 & 1.00000 \\
\hline US-07 & 0.15996 & 0.13488 & $\begin{array}{l}0.00999 \\
\end{array}$ & 0.00001 & 0.07300 & $\begin{array}{l}0.00999 \\
\end{array}$ & 0.02660 & $\begin{array}{l}0.00499 \\
\end{array}$ & 0.02998 & 0.10668 & 0.00202 & 0.00099 & 0.00020 & 0.00499 & 0.41878 & 0.00099 & $\begin{array}{l}0.00999 \\
\end{array}$ & 0.00099 & 0.00499 & 1.00000 \\
\hline US-08 & 0.15193 & 0.09555 & 0.00000 & 0.00000 & 0.09747 & 0.01000 & 0.04864 & 0.00000 & $\begin{array}{l}0.02437 \\
\end{array}$ & $\begin{array}{l}0.09814 \\
\end{array}$ & $\begin{array}{l}0.00001 \\
\end{array}$ & $\begin{array}{l}0.00099 \\
\end{array}$ & 0.00020 & $\begin{array}{l}0.00499 \\
\end{array}$ & $\begin{array}{l}0.45772 \\
\end{array}$ & 0.00000 & 0.01000 & 0.00000 & 0.00000 & 1.00000 \\
\hline US-09 & 0.13366 & 0.15048 & 0.01000 & 0.00300 & 0.10880 & 0.00000 & 0.02000 & 0.00500 & 0.00000 & 0.14115 & 0.00000 & 0.00100 & 0.00020 & 0.00500 & 0.41172 & 0.00000 & 0.01000 & 0.00000 & 0.00000 & 1.00000 \\
\hline US-10 & $\begin{array}{l}0.14123 \\
\end{array}$ & 0.10992 & 0.00999 & $\begin{array}{l}0.00001 \\
\end{array}$ & 0.10685 & 0.00999 & 0.02001 & $\begin{array}{l}0.00499 \\
\end{array}$ & \begin{tabular}{|l}
0.02737 \\
\end{tabular} & 0.12674 & 0.00001 & 0.00099 & $\begin{array}{l}0.00020 \\
\end{array}$ & $\begin{array}{l}0.00499 \\
\end{array}$ & 0.41976 & 0.00099 & 0.00999 & 0.00099 & 0.00499 & 1.00000 \\
\hline US-11 & 0.13703 & 0.19976 & 0.01000 & 0.00000 & 0.07761 & 0.01000 & 0.02000 & 0.00500 & 0.02999 & 0.06686 & 0.00000 & 0.00100 & 0.00020 & 0.00500 & 0.43657 & 0.00000 & 0.00000 & 0.00100 & 0.00000 & 1.00000 \\
\hline US-12 & 0.13239 & 0.16081 & 0.01000 & 0.00145 & 0.08743 & 0.01000 & $\begin{array}{l}0.05999 \\
\end{array}$ & 0.00000 & 0.00000 & 0.11158 & 0.01837 & 0.00000 & 0.00020 & 0.00500 & $\begin{array}{l}0.39129 \\
\end{array}$ & 0.00100 & 0.00498 & 0.00100 & 0.00452 & 1.00000 \\
\hline US-13 & 0.15997 & $\begin{array}{l}0.14569 \\
\end{array}$ & \begin{tabular}{|l|l}
0.00999 \\
\end{tabular} & 0.00000 & 0.07300 & \begin{tabular}{|l|l}
0.00999 \\
\end{tabular} & $\begin{array}{l}0.02155 \\
\end{array}$ & \begin{tabular}{|l|l|}
0.00499 \\
\end{tabular} & $\begin{array}{l}0.02800 \\
\end{array}$ & $\begin{array}{l}0.11528 \\
\end{array}$ & 0.00282 & 0.00099 & $\begin{array}{l}0.00020 \\
\end{array}$ & $\begin{array}{l}0.00000 \\
\end{array}$ & 0.41654 & 0.00000 & $\begin{array}{l}0.01000 \\
\end{array}$ & $\begin{array}{l}0.00100 \\
\end{array}$ & 0.00000 & 1.00000 \\
\hline US-14 & 0.13016 & 0.17898 & 0.00397 & 0.00000 & 0.09415 & 0.01000 & 0.05739 & 0.00499 & 0.02599 & 0.11224 & 0.00000 & 0.00071 & 0.00020 & 0.00000 & 0.36522 & 0.00000 & 0.01000 & 0.00100 & 0.00500 & 1.00000 \\
\hline US-15 & 0.09998 & 0.17796 & 0.00500 & 0.00300 & 0.04999 & 0.01000 & 0.02000 & 0.00000 & 0.00000 & 0.14997 & 0.00000 & 0.00100 & 0.00020 & 0.00500 & 0.46591 & 0.00100 & 0.01000 & 0.00100 & 0.00000 & 1.00000 \\
\hline US-16 & 0.13316 & 0.18807 & 0.01000 & 0.00080 & 0.09026 & 0.01000 & 0.02555 & 0.00000 & 0.02483 & 0.06298 & 0.00003 & 0.00000 & 0.00020 & 0.00000 & 0.43911 & 0.00000 & 0.01000 & 0.00000 & 0.00500 & 1.00000 \\
\hline $\begin{array}{l}\text { US-17 } \\
\end{array}$ & 0.11656 & 0.11775 & 0.00000 & 0.00107 & 0.06223 & 0.00000 & 0.05363 & 0.00500 & \begin{tabular}{|c|}
0.02999 \\
\end{tabular} & 0.08582 & $\begin{array}{l}0.01085 \\
\end{array}$ & 0.00000 & 0.00020 & 0.00000 & $\begin{array}{l}0.49990 \\
\end{array}$ & $\begin{array}{l}0.00100 \\
\end{array}$ & 0.01000 & 0.00100 & 0.00500 & 1.00000 \\
\hline US-18 & 0.15997 & 0.09128 & 0.01000 & 0.00000 & 0.09746 & 0.00000 & 0.04951 & 0.00000 & 0.02999 & 0.13407 & 0.00000 & 0.00100 & 0.00020 & 0.00000 & 0.41452 & 0.00100 & 0.01000 & 0.00100 & 0.00000 & 1.00000 \\
\hline $\begin{array}{l}\text { US-19 } \\
\end{array}$ & 0.14473 & 0.14401 & 0.00700 & 0.00069 & 0.08228 & 0.00777 & 0.03638 & 0.00333 & 0.01988 & 0.10802 & 0.00209 & 0.00070 & 0.00020 & 0.00277 & 0.42809 & 0.00061 & 0.00805 & 0.00066 & 0.00275 & 1.00000 \\
\hline $\begin{array}{l}\text { US-20 } \\
\end{array}$ & $\begin{array}{l}0.14473 \\
\end{array}$ & $\begin{array}{l}0.14401 \\
\end{array}$ & $\begin{array}{l}0.00700 \\
\end{array}$ & 0.00069 & 0.08228 & $\begin{array}{l}0.00777 \\
\end{array}$ & 0.03638 & 0.00333 & $\begin{array}{l}0.01988 \\
\end{array}$ & 0.10802 & 0.00209 & 0.00070 & 0.00020 & $\begin{array}{l}0.00277 \\
\end{array}$ & 0.42809 & 0.00061 & 0.00805 & 0.00066 & 0.00275 & 1.00000 \\
\hline US-21 & 0.14219 & 0.13755 & 0.00376 & 0.00080 & 0.08452 & 0.00749 & 0.03000 & 0.00374 & 0.01779 & 0.10753 & 0.00501 & 0.00079 & 0.00020 & 0.00125 & $\begin{array}{l}0.44955 \\
\end{array}$ & 0.00080 & 0.00543 & 0.00030 & 0.00130 & 1.00000 \\
\hline US-22 & 0.13340 & 0.11687 & 0.00749 & 0.00080 & 0.09467 & $\begin{array}{l}0.00749 \\
\end{array}$ & 0.04998 & \begin{tabular}{|l|}
0.00374 \\
\end{tabular} & 0.02249 & 0.11777 & 0.00918 & 0.00030 & 0.00020 & 0.00125 & \begin{tabular}{|l|l|}
0.42401 \\
\end{tabular} & 0.00079 & $\begin{array}{l}0.00749 \\
\end{array}$ & 0.00079 & 0.00130 & 1.00000 \\
\hline US-23 & 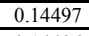 & $\begin{array}{ll}0.13321 \\
\end{array}$ & $\begin{array}{l}0.00750 \\
\end{array}$ & 0.00080 & 0.07623 & 0.00250 & 0.03499 & 0.00125 & 0.02250 & 0.11681 & $\begin{array}{l}0.00500 \\
\end{array}$ & 0.00080 & $\begin{array}{l}0.00020 \\
\end{array}$ & $\begin{array}{l}0.00374 \\
\end{array}$ & 0.44460 & 0.00030 & 0.00250 & 0.00080 & 0.00130 & 1.00000 \\
\hline US-24 & 0.14496 & 0.12250 & 0.00749 & 0.00081 & 0.07787 & 0.00749 & 0.03000 & 0.00374 & 0.02249 & 0.11573 & 0.00501 & 0.00079 & 0.00020 & 0.00374 & 0.44432 & 0.00079 & 0.00749 & 0.00079 & 0.00379 & 1.00000 \\
\hline US-25 & 0.11585 & 0.16247 & 0.00750 & 0.00218 & 0.08540 & 0.00287 & 0.04999 & 0.00375 & 0.02165 & 0.10883 & 0.01449 & 0.00030 & 0.00020 & 0.00375 & 0.40788 & 0.00080 & $\begin{array}{l}0.00750 \\
\end{array}$ & 0.00080 & 0.00380 & 1.00000 \\
\hline $\begin{array}{l}\text { US-26 } \\
\end{array}$ & $\begin{array}{l}0.18829 \\
\end{array}$ & 0.10685 & 0.01000 & 0.00548 & 0.04999 & 0.00000 & $\begin{array}{l}0.05999 \\
\end{array}$ & 0.00500 & 0.00000 & 0.11017 & 0.00000 & 0.00000 & 0.00020 & 0.00000 & 0.38054 & $\begin{array}{l}0.02999 \\
\end{array}$ & 0.01000 & 0.02000 & $\begin{array}{l}0.02349 \\
\end{array}$ & 1.00000 \\
\hline US-27 & 0.10403 & 0.06484 & 0.00000 & 0.01000 & 0.15183 & 0.01000 & 0.05999 & 0.00500 & 0.00000 & 0.16868 & 0.00000 & 0.00000 & 0.00020 & 0.00500 & 0.40044 & 0.00000 & 0.00000 & 0.02000 & 0.00000 & 1.00000 \\
\hline US-28 & 0.17472 & 0.17958 & 0.00000 & 0.00000 & 0.05722 & 0.01000 & 0.02000 & 0.00000 & 0.00000 & 0.13355 & 0.00000 & 0.00000 & 0.00020 & 0.00500 & 0.35974 & 0.02999 & 0.01000 & 0.02000 & 0.00000 & 1.00000 \\
\hline US-29 & 0.09998 & 0.08726 & 0.01000 & 0.00671 & 0.04999 & 0.01000 & 0.05999 & 0.00500 & 0.00000 & 0.18684 & 0.00000 & 0.01000 & 0.00020 & 0.00000 & 0.45263 & 0.01176 & 0.00964 & 0.00000 & 0.00000 & 1.00000 \\
\hline $\begin{array}{l}\text { US-30 } \\
\end{array}$ & 0.12646 & $\begin{array}{l}0.18190 \\
\end{array}$ & 0.01000 & 0.00818 & 0.05328 & 0.01000 & $\begin{array}{l}0.05998 \\
\end{array}$ & 0.00000 & 0.03497 & $\begin{array}{l}0.04999 \\
\end{array}$ & 0.00000 & 0.00000 & 0.00020 & 0.00500 & $\begin{array}{l}0.37524 \\
\end{array}$ & \begin{tabular}{|c|}
0.02999 \\
\end{tabular} & $\begin{array}{l}0.01000 \\
\end{array}$ & 0.00481 & $\begin{array}{l}0.03999 \\
\end{array}$ & 1.00000 \\
\hline US-31 & $\begin{array}{l}0.12668 \\
\end{array}$ & 0.14521 & 0.00000 & 0.00000 & 0.15448 & $\begin{array}{l}0.00000 \\
\end{array}$ & 0.04908 & 0.00500 & 0.00741 & 0.07097 & 0.00000 & 0.01000 & 0.00020 & 0.00500 & 0.38598 & $\begin{array}{c}0.02999 \\
\end{array}$ & $\begin{array}{l}0.01000 \\
\end{array}$ & 0.00000 & 0.00000 & 1.00000 \\
\hline $\begin{array}{l}\text { US-32 } \\
\end{array}$ & 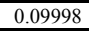 & 0.19012 & $\begin{array}{c}0.01000 \\
\end{array}$ & 0.00000 & 0.04999 & 0.00000 & 0.05999 & 0.00000 & 0.03999 & 0.07503 & 0.00000 & 0.01000 & 0.00020 & $\begin{array}{l}0.00500 \\
\end{array}$ & 0.43971 & 0.00000 & 0.00000 & 0.02000 & 0.00000 & 1.00000 \\
\hline US-33 & 0.11345 & 0.04999 & 0.00000 & 0.01000 & 0.04999 & 0.00000 & 0.02000 & 0.00500 & 0.03999 & $\begin{array}{ll}0.19761 \\
\end{array}$ & 0.00965 & 0.01000 & 0.00020 & 0.00500 & 0.45914 & 0.02999 & 0.00000 & 0.00000 & 0.00000 & 1.00000 \\
\hline US-34 & 0.09998 & $\begin{array}{l}0.13691 \\
\end{array}$ & 0.00000 & 0.00000 & 0.06385 & 0.01000 & 0.02420 & 0.00500 & $\begin{array}{l}0.03999 \\
\end{array}$ & 0.19996 & 0.00000 & 0.00000 & 0.00020 & 0.00000 & $\begin{array}{l}0.34993 \\
\end{array}$ & $\begin{array}{l}0.02999 \\
\end{array}$ & 0.00000 & 0.00000 & $\begin{array}{l}0.03999 \\
\end{array}$ & 1.00000 \\
\hline US-35 & 0.10016 & $\begin{array}{l}0.19958 \\
\end{array}$ & $\begin{array}{l}0.01000 \\
\end{array}$ & 0.00518 & 0.11352 & $\begin{array}{l}0.00000 \\
\end{array}$ & $\begin{array}{l}0.02000 \\
\end{array}$ & $\begin{array}{l}0.00500 \\
\end{array}$ & \begin{tabular}{|l|l}
0.03999 \\
\end{tabular} & $\begin{array}{l}0.07941 \\
\end{array}$ & 0.00000 & 0.00000 & $\begin{array}{l}0.00020 \\
\end{array}$ & $\begin{array}{l}0.00000 \\
\end{array}$ & 0.37995 & \begin{tabular}{|l|}
0.02703 \\
\end{tabular} & 0.00000 & $\begin{array}{l}0.02000 \\
\end{array}$ & 0.00000 & 1.00000 \\
\hline $\begin{array}{l}\text { US-36 } \\
\end{array}$ & 0.12858 & 0.15512 & $\begin{array}{l}0.00207 \\
\end{array}$ & $\begin{array}{l}0.00511 \\
\end{array}$ & 0.09761 & 0.00518 & 0.02422 & 0.00045 & 0.01206 & 0.12519 & 0.00558 & 0.01000 & 0.00020 & 0.00000 & \begin{tabular}{|l|l|}
0.38841 \\
\end{tabular} & 0.00007 & 0.00007 & 0.00007 & 0.03999 & 1.00000 \\
\hline US-37 & \begin{tabular}{|l}
0.09998 \\
\end{tabular} & 0.05770 & 0.01000 & 0.00000 & 0.16997 & 0.00000 & 0.02000 & 0.00000 & 0.00000 & 0.18886 & 0.00000 & 0.01000 & $\begin{array}{l}0.00020 \\
\end{array}$ & 0.00000 & $\begin{array}{l}0.37331 \\
\end{array}$ & 0.00000 & 0.01000 & 0.02000 & 0.03999 & 1.00000 \\
\hline US-38 & 0.09998 & 0.07314 & 0.00789 & 0.00000 & 0.04999 & 0.01000 & 0.02000 & 0.00000 & 0.03999 & $\begin{array}{l}0.19725 \\
\end{array}$ & 0.02905 & 0.01000 & 0.00020 & 0.00500 & 0.39753 & $\begin{array}{l}0.02999 \\
\end{array}$ & 0.01000 & 0.02000 & 0.00000 & 1.00000 \\
\hline $\begin{array}{l}\text { US-39 } \\
\end{array}$ & 0.11545 & $\begin{array}{l}0.16348 \\
\end{array}$ & 0.00000 & 0.00000 & 0.07502 & 0.01000 & 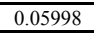 & 0.00500 & 0.00000 & $\begin{array}{l}0.04999 \\
\end{array}$ & 0.01828 & 0.01000 & 0.00020 & 0.00000 & 0.42465 & 0.00797 & 0.00000 & 0.02000 & \begin{tabular}{|c|}
0.03999 \\
\end{tabular} & 1.00000 \\
\hline US-40 & 0.15518 & 0.17000 & 0.00000 & 0.00425 & 0.11416 & 0.01000 & 0.02512 & 0.00000 & 0.02759 & 0.10131 & 0.00001 & 0.00999 & 0.00020 & 0.00000 & 0.37297 & 0.00000 & $\begin{array}{l}0.00922 \\
\end{array}$ & 0.00000 & 0.00000 & 1.00000 \\
\hline US-41 & $\begin{array}{l}0.09998 \\
\end{array}$ & 0.15554 & 0.01000 & 0.00000 & 0.15532 & 0.00999 & 0.03724 & 0.00000 & 0.00000 & 0.06519 & 0.00000 & 0.01000 & 0.00020 & 0.00000 & 0.42654 & 0.02999 & 0.00000 & 0.00000 & 0.00000 & 1.00000 \\
\hline US-42 & 0.19996 & 0.06187 & 0.01000 & 0.00000 & 0.04999 & 0.01000 & 0.05999 & 0.00000 & 0.03999 & 0.18820 & 0.00000 & 0.01000 & 0.00020 & 0.00000 & 0.35492 & 0.00000 & 0.00000 & 0.01489 & 0.00000 & 1.00000 \\
\hline US-43 & 0.17167 & 0.08645 & 0.01000 & 0.00000 & 0.08857 & $\begin{array}{l}0.00999 \\
\end{array}$ & 0.04905 & 0.00500 & 0.00812 & 0.10295 & 0.00198 & 0.01000 & 0.00020 & 0.00500 & 0.39701 & 0.00472 & 0.00477 & 0.00453 & $\begin{array}{l}0.03999 \\
\end{array}$ & 1.00000 \\
\hline $\begin{array}{l}\text { US-44 } \\
\end{array}$ & 0.12803 & $\begin{array}{l}0.12586 \\
\end{array}$ & 0.00555 & 0.00305 & 0.08860 & $\begin{array}{l}0.00640 \\
\end{array}$ & 0.04049 & 0.00252 & 0.01834 & 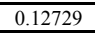 & 0.00359 & 0.00666 & 0.00020 & 0.00222 & $\begin{array}{l}0.39548 \\
\end{array}$ & $\begin{array}{c}0.01619 \\
\end{array}$ & 0.00465 & $\begin{array}{l}0.01024 \\
\end{array}$ & 0.01464 & 1.00000 \\
\hline US-45 & $\begin{array}{l}0.12803 \\
\end{array}$ & 0.12586 & 0.00555 & 0.00305 & 0.08860 & $\begin{array}{l}0.00640 \\
\end{array}$ & 0.04049 & 0.00252 & 0.01834 & $\begin{array}{l}0.12729 \\
\end{array}$ & 0.00359 & 0.00666 & $\begin{array}{l}0.00020 \\
\end{array}$ & 0.00222 & $\begin{array}{l}0.39548 \\
\end{array}$ & 0.01619 & 0.00465 & $\begin{array}{ll}0.01024 \\
\end{array}$ & 0.01464 & 1.00000 \\
\hline
\end{tabular}


Table A2. Measured Elemental Concentrations (wt\%) for Samples Prepared Using Lithium Metaborate

\begin{tabular}{|c|c|c|c|c|c|c|c|c|c|c|c|c|c|c|c|}
\hline Glass ID & 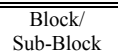 & Sequence & Lab ID & $\mathrm{Ca}(\mathrm{wt} \%)$ & $\mathrm{Cr}(\mathrm{wt} \%)$ & $\mathrm{K}(\mathrm{wt} \%)$ & $\operatorname{Mg}(\mathrm{wt} \%)$ & $\mathrm{Na}(\mathrm{wt} \%)$ & $\mathrm{Ni}(\mathrm{wt} \%)$ & $\mathrm{Pb}(\mathrm{wt} \%)$ & S (wt\%) & $\operatorname{Sr}(w t \%)$ & $\mathrm{Ti}(\mathrm{wt} \%)$ & $\mathrm{Zn}(\mathrm{wt} \%)$ & $\mathrm{Zr}(\mathrm{wt} \%)$ \\
\hline Batch 1 & $1-1$ & 1 & BCHLM111 & 0.854 & 0.075 & 3.24 & 0.79 & 6.69 & 0.533 & 0.001 & $<0.050$ & $<0.010$ & 0.383 & $<0.010$ & 0.062 \\
\hline LRM & $1-1$ & 2 & LRMLM111 & 0.384 & 0.135 & 1.26 & 0.059 & 14.9 & 0.135 & 0.082 & 0.084 & $<0.010$ & 0.058 & $<0.010$ & 0.656 \\
\hline US-38 & $1-1$ & $\frac{2}{3}$ & Z07LM21 & 0.536 & 0.011 & 0.816 & $<0.010$ & 14.6 & 2.2 & 0.806 & 0.19 & 2.19 & 0.555 & 1.391 & 0.011 \\
\hline US-39 & $1-1$ & 4 & Z31LM11 & 0.019 & 0.01 & 0.87 & 0.296 & 4.07 & 1.34 & 0.839 & $<0.050$ & 0.604 & $<0.010$ & 1.42 & 2.58 \\
\hline US-18 & $1-1$ & 5 & Z12LM11 & 0.641 & 0.011 & $<0.100$ & $<0.010$ & 10.3 & $<0.010$ & 0.083 & $<0.050$ & 0.083 & 0.568 & 0.096 & $<0.010$ \\
\hline US-09 & $1-1$ & 6 & $\begin{array}{l}\text { Z14LM21 } \\
\end{array}$ & 0.656 & 0.15 & $<0.100$ & 0.296 & 10.6 & $<0.010$ & 0.081 & 0.155 & 0.011 & 0.557 & $<0.010$ & $<0.010$ \\
\hline US-38 & $1-1$ & 7 & Z07LM11 & 0.557 & 0.011 & 0.853 & $<0.010$ & 15.2 & 2.18 & 0.827 & 0.193 & 2.29 & 0.57 & 1.433 & 0.011 \\
\hline US-14 & $1-1$ & 8 & Z24LM21 & 0.293 & 0.009 & 0.839 & 0.302 & 8.78 & $<0.010$ & 0.059 & $<0.050$ & $<0.010$ & 0.582 & 0.093 & 0.345 \\
\hline US- 06 & $1-1$ & 9 & Z39LM21 & 0.69 & 0.013 & 0.839 & 0.299 & 8.71 & $<0.010$ & $<0.010$ & 0.14 & 0.092 & 0.55 & 0.019 & 0.02 \\
\hline US-18 & $1-1$ & 10 & Z12LM21 & 0.65 & 0.01 & $<0.100$ & $<0.010$ & 10.6 & $<0.010$ & 0.084 & $<0.050$ & 0.082 & 0.583 & 0.084 & 0.002 \\
\hline US-05 & $1-1$ & 11 & $\begin{array}{l}\text { Z30LM11 } \\
\end{array}$ & 0.49 & 0.013 & 0.841 & 0.297 & 7.62 & $<0.010$ & 0.084 & 0.111 & 0.094 & 0.586 & 0.078 & 0.361 \\
\hline Batch 1 & $1-1$ & $\frac{11}{12}$ & BCHLM112 & 0.822 & 0.073 & 3.11 & 0.769 & 6.82 & 0.514 & $<0.010$ & $<0.050$ & $\begin{array}{l}<0.010 \\
\end{array}$ & 0.369 & $<0.010$ & 0.065 \\
\hline LRM & $1-1$ & 13 & LRMLM112 & 0.399 & 0.134 & 1.2 & 0.058 & 15.2 & 0.134 & 0.081 & 0.085 & $<0.010$ & 0.058 & $<0.010$ & 0.664 \\
\hline US-14 & $1-1$ & 14 & Z24LM11 & 0.354 & $<0.010$ & 0.827 & 0.294 & 8.64 & $<0.010$ & 0.059 & $<0.050$ & $<0.010$ & 0.569 & 0.079 & 0.333 \\
\hline US-08 & $1-1$ & 15 & Z34LM11 & 0.068 & 0.023 & 0.792 & 0.013 & 7.6 & $<0.010$ & 0.078 & 0.152 & 0.108 & 0.522 & 0.08 & 0.085 \\
\hline US-06 & $1-1$ & $\frac{16}{16}$ & Z39LM11 & 0.68 & 0.013 & 0.855 & 0.299 & 8.68 & $<0.010$ & $<0.010$ & 0.143 & 0.092 & 0.554 & 0.012 & 0.018 \\
\hline US-34 & $1-1$ & 17 & Z27LM11 & 0.016 & $<0.010$ & 0.911 & 0.297 & 14.4 & $<0.010$ & $<0.010$ & $<0.050$ & 2.34 & $<0.010$ & $<0.010$ & 2.62 \\
\hline US-34 & $1-1$ & 18 & Z27LM21 & 0.033 & 0.011 & 0.899 & 0.296 & 14.8 & $<0.010$ & $<0.010$ & $<0.050$ & 2.29 & $<0.010$ & $<0.010$ & 2.62 \\
\hline US- 05 & $1-1$ & 19 & Z30LM21 & 0.485 & 0.012 & 0.867 & 0.303 & 7.59 & $<0.010$ & 0.085 & 0.112 & 0.1 & 0.609 & 0.079 & 0.383 \\
\hline US- 08 & $1-1$ & 20 & Z34LM21 & 0.117 & 0.033 & 0.806 & 0.046 & 7.71 & $<0.010$ & 0.075 & 0.147 & 0.197 & 0.529 & 0.135 & 0.149 \\
\hline US-39 & $1-1$ & 21 & Z31LM21 & 0.014 & 0.01 & 0.912 & 0.295 & 4.02 & 1.3 & 0.833 & $<0.050$ & 0.629 & $<0.010$ & 1.415 & 2.63 \\
\hline US- 09 & $1-1$ & 22 & Z14LM11 & 0.69 & 0.159 & $<0.100$ & 0.303 & 8.51 & $<0.010$ & 0.085 & 0.159 & 0.018 & 0.581 & $<0.010$ & $<0.010$ \\
\hline Batch 1 & $1-1$ & 23 & BCHLM113 & 0.837 & 0.073 & 3.15 & 0.769 & 6.44 & 0.514 & $<0.010$ & $<0.050$ & $<0.010$ & 0.372 & $<0.010$ & 0.067 \\
\hline LRM & $1-1$ & 24 & LRMLM113 & 0.403 & 0.135 & 1.22 & 0.058 & 14.7 & 0.134 & 0.081 & 0.084 & $<0.010$ & 0.058 & $<0.010$ & 0.672 \\
\hline Batch 1 & $1-2$ & 1 & BCHLM121 & 0.835 & 0.074 & 3.15 & 0.794 & 6.72 & 0.532 & $<0.010$ & $<0.050$ & $<0.010$ & 0.376 & $<0.010$ & 0.06 \\
\hline LRM & $1-2$ & $\frac{1}{2}$ & LRMLM121 & 0.383 & 0.133 & 1.22 & 0.056 & 15.2 & 0.133 & 0.079 & 0.083 & $<0.010$ & 0.056 & $<0.010$ & 0.651 \\
\hline US-39 & $1-2$ & 3 & Z31LM12 & 0.017 & $<0.010$ & 0.871 & 0.307 & 3.45 & 1.41 & 0.868 & $<0.050$ & 0.622 & $<0.010$ & 1.473 & 2.65 \\
\hline US-14 & $1-2$ & 4 & Z24LM22 & 0.283 & $<0.010$ & 0.795 & 0.303 & 8.9 & $<0.010$ & 0.059 & $<0.050$ & $<0.010$ & 0.575 & 0.093 & 0.34 \\
\hline US-18 & $1-2$ & $\frac{7}{5}$ & Z12LM12 & 0.623 & $<0.010$ & $<0.100$ & $\begin{array}{l}0.0010 \\
<0.010\end{array}$ & 8.28 & $<0.010$ & 0.082 & $<0.050$ & 0.08 & 0.568 & 0.096 & $<0.010$ \\
\hline US-39 & $1-2$ & 6 & Z31LM22 & 0.012 & $<0.010$ & 0.853 & 0.298 & 3.36 & 1.34 & 0.843 & $<0.050$ & 0.609 & $<0.010$ & 1.429 & 2.58 \\
\hline US- 09 & $1-2$ & 7 & Z14LM22 & 0.642 & 0.151 & $<0.100$ & 0.299 & 8.35 & $<0.010$ & 0.081 & 0.151 & 0.01 & 0.554 & $<0.010$ & $<0.010$ \\
\hline US- 05 & $1-2$ & 8 & Z30LM12 & 0.47 & 0.011 & 0.798 & 0.297 & 7.46 & $<0.010$ & 0.083 & 0.114 & 0.09 & 0.574 & 0.077 & 0.356 \\
\hline US- 09 & $1-2$ & 9 & Z14LM12 & 0.647 & 0.157 & $<0.100$ & 0.305 & 11 & $<0.010$ & 0.085 & 0.16 & 0.016 & 0.565 & $<0.010$ & $<0.010$ \\
\hline US-34 & $1-2$ & 10 & Z27LM22 & 0.03 & $<0.010$ & 0.843 & 0.301 & 14.2 & $<0.010$ & $<0.010$ & $<0.050$ & 2.21 & $<0.010$ & $<0.010$ & 2.57 \\
\hline US-38 & $1-2$ & 11 & Z07LM12 & 0.558 & 0.01 & 0.844 & $<0.010$ & 12.2 & 2.23 & 0.826 & 0.196 & 2.24 & 0.565 & 1.429 & 0.021 \\
\hline Batch 1 & $1-2$ & 12 & BCHLM122 & 0.84 & 0.074 & 3.1 & 0.802 & 6.86 & 0.537 & $<0.010$ & $<0.050$ & $<0.010$ & 0.379 & $<0.010$ & 0.063 \\
\hline LRM & $1-2$ & 13 & LRMLM122 & 0.384 & 0.134 & 1.17 & 0.057 & 15.3 & 0.134 & 0.081 & 0.084 & $<0.010$ & 0.057 & $<0.010$ & 0.659 \\
\hline US- 38 & $1-2$ & 14 & Z07LM22 & 0.55 & $<0.010$ & 0.838 & $<0.010$ & 12.6 & 2.3 & 0.818 & 0.194 & 2.24 & 0.562 & 1.416 & 0.014 \\
\hline US- 05 & $1-2$ & $\frac{77}{15}$ & Z30LM22 & 0.473 & 0.01 & 0.042 & 0.302 & 7.6 & $<0.010$ & 0.084 & 0.118 & 0.098 & 0.596 & 0.077 & 0.365 \\
\hline US-18 & $1-2$ & 16 & Z12LM22 & 0.637 & $<0.010$ & $<0.100$ & $<0.010$ & 8.34 & $<0.010$ & 0.084 & $<0.050$ & 0.081 & 0.576 & 0.083 & $<0.010$ \\
\hline US-06 & $1-2$ & 17 & Z39LM12 & 0.662 & 0.012 & 0.814 & 0.297 & 7.13 & $<0.010$ & $<0.010$ & 0.14 & 0.089 & 0.543 & 0.01 & 0.016 \\
\hline US-06 & $\frac{1-2}{1-2}$ & $\frac{17}{18}$ & $\begin{array}{l}\text { ZSPLMN22 } \\
\text { Z39L }\end{array}$ & 0.002 & 0.011 & 0.821 & 0.298 & 6.13 & $<0.010$ & $<0.010$ & 0.14 & $\begin{array}{l}0.00 \% \\
0.09\end{array}$ & $\begin{array}{ll}0.3454 \\
0.54\end{array}$ & 0.017 & 0.016 \\
\hline US- 08 & $1-2$ & 19 & Z34LM12 & 0.065 & 0.022 & 0.767 & 0.011 & 7.37 & $<0.010$ & 0.077 & 0.157 & 0.107 & 0.52 & 0.079 & 0.081 \\
\hline US- 08 & $1-2$ & 20 & Z34LM22 & 0.113 & 0.032 & 0.781 & 0.043 & 7.53 & $<0.010$ & 0.074 & 0.152 & 0.195 & 0.52 & 0.134 & 0.141 \\
\hline US-14 & $1-2$ & 21 & Z24LM12 & 0.351 & $<0.010$ & 0.81 & 0.298 & 6.91 & $<0.010$ & 0.058 & $<0.050$ & $<0.010$ & 0.57 & 0.079 & 0.341 \\
\hline US-34 & $1-2$ & 22 & Z27LM12 & 0.014 & $<0.010$ & 0.86 & 0.299 & 14.2 & $<0.010$ & $<0.010$ & $<0.050$ & 2.24 & $<0.010$ & $<0.010$ & 2.57 \\
\hline Batch 1 & $1-2$ & $\frac{22}{23}$ & BCHLM123 & 0.857 & 0.075 & 3.12 & 0.809 & 6.7 & 0.541 & $<0.010$ & $<0.050$ & $<<0.010$ & 0.384 & $<0.010$ & 0.074 \\
\hline LRM & $1-2$ & 24 & LRMLM123 & 0.397 & 0.135 & 1.19 & 0.057 & 14.8 & 0.135 & 0.081 & 0.091 & $<0.010$ & 0.058 & $<0.010$ & 0.671 \\
\hline Batch 1 & $2-1$ & 1 & BCHLM211 & 0.815 & 0.076 & 3.25 & 0.792 & 6.78 & 0.532 & $<0.010$ & $<0.050$ & $<0.010$ & 0.373 & 0.01 & 0.064 \\
\hline
\end{tabular}


Table A2. Measured Elemental Concentrations (wt\%) for Samples Prepared Using Lithium Metaborate

\begin{tabular}{|c|c|c|c|c|c|c|c|c|c|c|c|c|c|c|c|}
\hline Glass ID & $\begin{array}{c}\text { Block/ } \\
\text { Sub-Block }\end{array}$ & Sequence & Lab ID & $\mathrm{Ca}(\mathrm{wt} \%)$ & $\mathrm{Cr}(\mathrm{wt} \%)$ & $\mathrm{K}(\mathrm{wt} \%)$ & $\mathrm{Mg}(\mathrm{wt} \%)$ & $\mathrm{Na}(\mathrm{wt} \%)$ & $\mathrm{Ni}(\mathrm{wt} \%)$ & $\mathrm{Pb}(\mathrm{wt} \%)$ & $\mathrm{S}(\mathrm{wt} \%)$ & $\mathrm{Sr}(\mathrm{wt} \%)$ & $\mathrm{Ti}(\mathrm{wt} \%)$ & $\mathrm{Zn}(\mathrm{wt} \%)$ & $\mathrm{Zr}(\mathrm{wt} \%)$ \\
\hline LRM & $2-1$ & 2 & LRMLM211 & 0.378 & 0.135 & 1.31 & 0.058 & 15.2 & 0.136 & 0.083 & 0.088 & $<0.010$ & 0.058 & $<0.010$ & 0.644 \\
\hline US-33 & $2-1$ & 3 & $\begin{array}{l}\text { Z09LM21 } \\
\end{array}$ & 0.016 & 0.488 & $<0.100$ & 0.298 & 14.7 & 0.694 & 0.834 & 0.193 & 2.19 & $<0.010$ & 0.097 & $<0.010$ \\
\hline US-11 & $2-1$ & 4 & Z20LM11 & 0.7 & 0.011 & 0.816 & 0.309 & 4.53 & $<0.010$ & 0.087 & 0.107 & $<0.010$ & $<0.010$ & 0.084 & 0.011 \\
\hline $\begin{array}{l}\text { US-22 } \\
\end{array}$ & $2-1$ & 5 & $\begin{array}{l}\text { Z17LM11 } \\
\end{array}$ & 0.498 & 0.055 & 0.611 & 0.229 & 9.67 & 0.671 & 0.029 & 0.06 & 0.06 & 0.435 & 0.069 & 0.089 \\
\hline US-15 & $2-1$ & 6 & Z42LM11 & 0.438 & 0.162 & 0.814 & $<0.010$ & 12 & $<0.010$ & 0.085 & 0.168 & 0.078 & 0.561 & 0.082 & 0.013 \\
\hline US-26 & $2-1$ & 7 & Z37LM11 & 0.664 & 0.258 & $<0.100$ & 0.29 & 8.66 & $<0.010$ & $<0.010$ & $<<0.050$ & 2.16 & 0.555 & 1.35 & 1.52 \\
\hline US-13 & $2-1$ & 8 & Z04LM21 & 0.66 & 0.015 & 0.807 & 0.302 & 9.05 & 0.208 & 0.083 & $<0.050$ & $<0.010$ & 0.568 & 0.082 & 0.053 \\
\hline US-26 & $2-1$ & 9 & $\begin{array}{l}\text { Z37LM21 } \\
\end{array}$ & 0.674 & 0.312 & $<0.100$ & 0.295 & 8.75 & $<0.010$ & $<0.010$ & $<0.050$ & 2.18 & 0.565 & 1.401 & 1.54 \\
\hline US-11 & $2-1$ & 10 & Z20LM21 & 0.659 & 0.011 & 0.828 & 0.306 & 6.14 & $<0.010$ & 0.087 & 0.097 & $<0.010$ & $<0.010$ & 0.08 & 0.015 \\
\hline US-12 & $2-1$ & 11 & $\begin{array}{l}\text { Z01LM11 } \\
\end{array}$ & 0.698 & 0.086 & 0.876 & $<0.010$ & 9.59 & 1.4 & 0.003 & 0.187 & 0.081 & 0.305 & 0.09 & 0.333 \\
\hline Batch 1 & $2-1$ & 12 & BCHLM212 & 0.795 & 0.075 & 3.17 & 0.788 & 6.87 & 0.529 & $<0.010$ & $<0.050$ & $<0.010$ & 0.374 & $<0.010$ & 0.072 \\
\hline LRM & $2-1$ & 13 & LRMLM212 & 0.384 & 0.136 & 1.24 & 0.059 & 15.3 & 0.135 & 0.083 & 0.086 & $<0.010$ & 0.058 & 0.018 & 0.655 \\
\hline US-17 & $2-1$ & 14 & Z02LM11 & 0.057 & 0.062 & $<0.100$ & 0.295 & 7.41 & 0.774 & $<0.010$ & $<0.050$ & 0.074 & 0.562 & 0.085 & 0.32 \\
\hline US-15 & $2-1$ & 15 & Z42LM21 & 0.356 & 0.152 & 0.778 & $<0.010$ & 11.8 & $<0.010$ & 0.079 & 0.158 & 0.073 & 0.524 & 0.074 & 0.021 \\
\hline US-17 & $2-1$ & 16 & $\begin{array}{l}\text { Z02LM21 } \\
\end{array}$ & 0.056 & 0.062 & $<0.100$ & 0.295 & 6.74 & 0.773 & $<0.010$ & $<0.050$ & 0.073 & 0.561 & 0.084 & 0.304 \\
\hline US-22 & $2-1$ & 17 & $\begin{array}{l}\text { Z17LM21 } \\
\end{array}$ & 0.526 & 0.055 & 0.64 & 0.233 & 9.94 & 0.665 & 0.029 & 0.059 & 0.063 & 0.449 & 0.072 & 0.095 \\
\hline $\begin{array}{l}\text { US-12 } \\
\end{array}$ & $2-1$ & 18 & $\begin{array}{l}\text { Z01LM21 } \\
\end{array}$ & 0.698 & 0.078 & 0.867 & $<0.010$ & 9.76 & 1.36 & $<0.010$ & 0.183 & 0.081 & 0.299 & 0.088 & 0.32 \\
\hline US-13 & $2-1$ & 19 & Z04LM11 & 0.66 & 0.014 & 0.821 & 0.299 & 9.4 & 0.207 & 0.081 & $<0.050$ & $<0.010$ & 0.563 & 0.082 & $<0.010$ \\
\hline US-32 & $2-1$ & 20 & $\begin{array}{l}\text { Z06LM21 } \\
\end{array}$ & 0.671 & 0.011 & $<0.100$ & $<0.010$ & 6.06 & $<0.010$ & 0.825 & 0.167 & $<0.010$ & $<0.010$ & 1.411 & 0.034 \\
\hline US-33 & $2-1$ & 21 & Z09LM11 & 0.035 & 0.5 & $<0.100$ & 0.34 & 15 & 0.702 & 0.832 & 0.216 & 2.24 & $<0.010$ & 0.096 & $<0.010$ \\
\hline $\begin{array}{l}\text { US-32 } \\
\end{array}$ & $2-1$ & 22 & $\begin{array}{l}\text { Z06LM11 } \\
\end{array}$ & 0.671 & 0.013 & $<0.100$ & $<0.010$ & 5.93 & $<0.010$ & 0.839 & 0.166 & $<0.010$ & $<0.010$ & 1.438 & 0.033 \\
\hline Batch 1 & $2-1$ & 23 & BCHLM213 & 0.811 & 0.076 & 3.18 & 0.792 & 6.97 & 0.532 & $<0.010$ & $<0.050$ & $<0.010$ & 0.379 & $<0.010$ & 0.07 \\
\hline LRM & $2-1$ & 24 & LRMLM213 & 0.382 & 0.135 & 1.28 & 0.058 & 15.5 & 0.134 & 0.082 & 0.082 & $<0.010$ & 0.058 & $<0.010$ & 0.648 \\
\hline Batch 1 & $2-2$ & 1 & BCHLM221 & 0.789 & 0.073 & 3.08 & 0.78 & 6.56 & 0.525 & $<0.010$ & $<0.050$ & $<0.010$ & 0.371 & $<0.010$ & 0.065 \\
\hline LRM & $2-2$ & 2 & LRMLM221 & 0.373 & 0.134 & 1.17 & 0.058 & 14.7 & 0.134 & 0.082 & 0.084 & $<0.010$ & 0.057 & $<0.010$ & 0.639 \\
\hline $\begin{array}{l}\text { US-17 } \\
\end{array}$ & $2-2$ & 3 & $\begin{array}{l}\text { Z02LM22 } \\
\end{array}$ & 0.053 & 0.06 & $<0.100$ & 0.293 & 6.18 & 0.77 & $<0.010$ & $<0.050$ & 0.069 & 0.556 & 0.083 & 0.296 \\
\hline US- 26 & $2-2$ & 4 & Z37LM22 & 0.639 & 0.297 & $<0.100$ & 0.282 & 8.28 & $<0.010$ & $<0.010$ & $<0.050$ & 2.07 & 0.541 & 1.341 & 1.47 \\
\hline US-15 & $2-2$ & 5 & Z42LM22 & 0.371 & 0.161 & 0.791 & $<0.010$ & 11.7 & $<0.010$ & 0.082 & 0.162 & 0.075 & 0.555 & 0.079 & 0.017 \\
\hline US-33 & $2-2$ & 6 & $\begin{array}{l}\text { Z09LM12 } \\
\end{array}$ & 0.034 & 0.501 & $<<0.100$ & 0.341 & 14.6 & 0.705 & 0.84 & 0.214 & 2.2 & $<0.010$ & 0.096 & 0.004 \\
\hline $\begin{array}{l}\text { US-12 } \\
\end{array}$ & $2-2$ & 7 & $\begin{array}{l}\text { Z01LM12 } \\
\end{array}$ & 0.692 & 0.085 & 0.847 & $<0.010$ & 9.19 & 1.36 & $<0.010$ & 0.179 & 0.079 & 0.307 & 0.09 & 0.328 \\
\hline US-13 & $2-2$ & 8 & Z04LM22 & 0.648 & 0.014 & 0.777 & 0.302 & 8.84 & 0.208 & 0.083 & $<0.050$ & $<0.010$ & 0.567 & 0.081 & 0.044 \\
\hline US-22 & $2-2$ & 9 & $\begin{array}{l}\text { Z17LM12 } \\
\end{array}$ & 0.49 & 0.054 & 0.594 & 0.227 & 9.62 & 0.667 & 0.026 & 0.05 & 0.058 & 0.434 & 0.068 & 0.087 \\
\hline US-12 & $2-2$ & 10 & $\begin{array}{l}\text { Z01LM22 } \\
\end{array}$ & 0.683 & 0.078 & 0.821 & $<0.010$ & 9.06 & 1.36 & $<0.010$ & 0.181 & 0.078 & 0.3 & 0.088 & 0.319 \\
\hline $\begin{array}{l}\text { US-22 } \\
\end{array}$ & $2-2$ & 11 & $\begin{array}{l}\text { Z17LM22 } \\
\end{array}$ & 0.508 & 0.054 & 0.606 & 0.233 & 9.96 & 0.667 & 0.028 & 0.055 & 0.06 & 0.446 & 0.071 & 0.092 \\
\hline Batch 1 & $2-2$ & 12 & BCHLM222 & 0.794 & 0.075 & 3.06 & 0.792 & 6.82 & 0.533 & $<0.010$ & $<<0.050$ & $<0.010$ & 0.376 & $<0.010$ & 0.068 \\
\hline LRM & $2-2$ & 13 & LRMLM222 & 0.377 & 0.135 & 1.18 & 0.058 & 15.3 & 0.135 & 0.082 & 0.086 & $<0.010$ & 0.057 & 0.017 & 0.645 \\
\hline US-17 & $2-2$ & 14 & Z02LM12 & 0.025 & 0.059 & $<<0.100$ & 0.3 & 6.39 & 0.777 & $<0.010$ & $<0.050$ & 0.07 & 0.569 & 0.083 & 0.292 \\
\hline US-33 & $2-2$ & 15 & Z09LM22 & 0.015 & 0.49 & $<0.100$ & 0.3 & 14.3 & 0.696 & 0.834 & 0.187 & 2.19 & $<<0.010$ & 0.096 & $<0.010$ \\
\hline $\begin{array}{l}\text { US-32 } \\
\end{array}$ & $2-2$ & 16 & $\begin{array}{l}\text { Z06LM22 } \\
\end{array}$ & 0.657 & 0.01 & $<0.100$ & $<0.010$ & 5.78 & $<0.010$ & 0.83 & 0.163 & $<0.010$ & $<0.010$ & 1.424 & 0.031 \\
\hline US-11 & $2-2$ & 17 & $\begin{array}{l}\text { Z20LM12 } \\
\end{array}$ & 0.694 & 0.01 & 0.793 & 0.308 & 4.19 & $<0.010$ & 0.087 & 0.098 & $<0.010$ & $<0.010$ & 0.083 & $<0.010$ \\
\hline US-13 & $2-2$ & 18 & $\begin{array}{l}\text { Z04LM12 } \\
\end{array}$ & 0.651 & 0.012 & 0.789 & 0.3 & 8.87 & 0.207 & 0.081 & $<0.050$ & $<0.010$ & 0.563 & 0.081 & $<0.010$ \\
\hline US-26 & $2-2$ & 19 & $\begin{array}{l}\text { Z37LM12 } \\
\end{array}$ & 0.662 & 0.258 & $<<0.100$ & 0.292 & 8.3 & $<0.010$ & $<0.010$ & $<0.050$ & 2.14 & 0.557 & 1.359 & 1.51 \\
\hline US-15 & $2-2$ & 20 & $\begin{array}{l}\text { Z42LM12 } \\
\end{array}$ & 0.438 & 0.161 & 0.802 & $<0.010$ & 11.7 & $<0.010$ & 0.084 & 0.165 & 0.077 & 0.565 & 0.081 & 0.019 \\
\hline US-11 & $2-2$ & 21 & Z20LM22 & 0.673 & 0.01 & 0.832 & 0.308 & 5.97 & $<0.010$ & 0.086 & 0.097 & $<0.010$ & $<0.010$ & 0.08 & $<0.010$ \\
\hline US-32 & $2-2$ & 22 & Z06LM12 & 0.669 & 0.011 & $<0.100$ & $<0.010$ & 5.81 & $<0.010$ & 0.827 & 0.163 & $<0.010$ & $<0.010$ & 1.425 & 0.032 \\
\hline Batch 1 & $2-2$ & 23 & BCHLM223 & 0.812 & 0.074 & 3.08 & 0.778 & 6.67 & 0.525 & $<0.010$ & $<0.050$ & $<0.010$ & 0.375 & $<0.010$ & 0.067 \\
\hline LRM & $2-2$ & 24 & $\begin{array}{l}\text { LRMLM223 } \\
\end{array}$ & 0.384 & 0.133 & 1.19 & 0.057 & 15.2 & 0.133 & 0.081 & 0.086 & $<0.010$ & 0.057 & $<0.010$ & 0.651 \\
\hline Batch 1 & $3-1$ & 1 & BCHLM311 & 0.816 & 0.075 & 3.11 & 0.787 & 6.75 & 0.534 & $<0.010$ & $<0.050$ & $<0.010$ & 0.374 & 0.01 & 0.062 \\
\hline LRM & $3-1$ & 2 & LRMLM311 & 0.367 & 0.135 & 1.2 & 0.058 & 15.4 & 0.135 & 0.083 & 0.088 & $<0.010$ & 0.058 & $<0.010$ & 0.64 \\
\hline US-37 & $3-1$ & 3 & Z32LM11 & 0.017 & $<0.010$ & 0.782 & $<0.010$ & 10.7 & $<0.010$ & $<0.010$ & 0.152 & 2.09 & 0.557 & 1.406 & 0.012 \\
\hline
\end{tabular}


Table A2. Measured Elemental Concentrations (wt\%) for Samples Prepared Using Lithium Metaborate

\begin{tabular}{|c|c|c|c|c|c|c|c|c|c|c|c|c|c|c|c|}
\hline Glass ID & $\begin{array}{c}\text { Block/ } \\
\text { Sub-Block }\end{array}$ & Sequence & Lab ID & $\mathrm{Ca}(\mathrm{wt} \%)$ & $\mathrm{Cr}(\mathrm{w} t \%)$ & $\mathrm{K}(\mathrm{wt} \%)$ & $\mathrm{Mg}(\mathrm{w} t \%)$ & $\mathrm{Na}(\mathrm{w} t \%)$ & $\mathrm{Ni}(\mathrm{wt} \%)$ & $\mathrm{Pb}(\mathrm{wt} \%)$ & $\mathrm{S}(\mathrm{wt} \%)$ & $\mathrm{Sr}(\mathrm{wt} \%)$ & $\mathrm{Ti}(\mathrm{wt} \%)$ & $\mathrm{Zn}(\mathrm{wt} \%)$ & $\mathrm{Zr}(\mathrm{wt} \%)$ \\
\hline US-16 & $3-1$ & 4 & Z44LM21 & 0.624 & 0.056 & 0.807 & $<0.010$ & 4.99 & 0.016 & $<0.010$ & $<0.050$ & $<0.010$ & 0.552 & $<0.010$ & 0.346 \\
\hline US-25 & $3-1$ & 5 & Z08ILM11 & 0.504 & 0.102 & 0.246 & 0.224 & 8.77 & 1.01 & 0.031 & 0.132 & 0.067 & 0.435 & 0.087 & 0.263 \\
\hline US-07 & $3-1$ & 6 & $\begin{array}{l}\text { Z19LM21 } \\
\end{array}$ & 0.634 & 0.011 & 0.781 & 0.298 & 8.01 & 0.148 & 0.083 & 0.144 & 0.072 & 0.554 & 0.079 & 0.315 \\
\hline US-27 & $3-1$ & 7 & Z36LM21 & 0.014 & 0.57 & 0.835 & 0.288 & 13.6 & $\begin{array}{l}<0.010 \\
\end{array}$ & 0.031 & $\begin{array}{ll}0.188 \\
\end{array}$ & $\begin{array}{l}<0.010 \\
\end{array}$ & 0.01 & 1.374 & 0.518 \\
\hline US-27 & $3-1$ & 8 & Z36LM11 & 0.025 & 0.58 & 0.833 & 0.299 & 13.4 & $<0.010$ & $<0.010$ & 0.198 & $<0.010$ & $<0.010$ & 1.408 & 0.092 \\
\hline US-07 & $3-1$ & 9 & Z19LM11 & 0.636 & 0.011 & 0.789 & 0.297 & 8.61 & 0.147 & 0.083 & 0.142 & 0.073 & 0.553 & 0.078 & 0.308 \\
\hline US-43 & $3-1$ & 10 & $\begin{array}{l}\text { Z21LM11 } \\
\end{array}$ & 0.629 & $<0.010$ & 0.791 & 0.288 & 8.32 & 0.142 & 0.815 & 0.161 & 0.342 & 0.268 & 0.348 & 2.45 \\
\hline US-25 & $3-1$ & 11 & $\begin{array}{l}\text { Z08LM21 } \\
\end{array}$ & 0.501 & 0.101 & 0.249 & 0.225 & 8.74 & 1 & 0.028 & 0.136 & 0.067 & 0.434 & 0.081 & 0.273 \\
\hline Batch 1 & $3-1$ & 12 & BCHLM312 & 0.791 & 0.075 & 3.06 & 0.786 & 6.8 & 0.532 & $<0.010$ & $<0.050$ & $<0.010$ & 0.374 & $<0.010$ & 0.07 \\
\hline LRM & $3-1$ & 13 & LRMLM312 & 0.369 & 0.135 & 1.2 & 0.058 & 15.2 & 0.135 & 0.083 & 0.084 & $<0.010$ & 0.057 & 0.01 & 0.649 \\
\hline US-41 & $3-1$ & 14 & Z29LM11 & 0.665 & 0.011 & 0.823 & $<0.010$ & 5.21 & $<0.010$ & 0.834 & $<0.050$ & 2.18 & $<0.010$ & $<0.010$ & $<0.010$ \\
\hline US-37 & $3-1$ & 15 & $\begin{array}{l}\text { Z32LM21 } \\
\end{array}$ & 0.033 & $<0.010$ & 0.782 & $<0.010$ & 10.3 & $<0.010$ & 0.003 & 0.155 & 2.1 & 0.559 & 1.404 & $<0.010$ \\
\hline US-16 & $3-1$ & 16 & Z44LM11 & 0.651 & 0.056 & 0.821 & $<0.010$ & 5.08 & $<0.010$ & $<0.010$ & $<0.050$ & $<0.010$ & 0.567 & $<0.010$ & 0.365 \\
\hline US-28 & $3-1$ & 17 & Z33LM21 & 0.664 & 0.01 & $<0.100$ & $<0.010$ & 14.7 & $<0.010$ & 0.815 & $<0.050$ & 0.041 & 0.567 & 1.415 & 2.53 \\
\hline US-28 & $3-1$ & 18 & Z33LM11 & 0.657 & $<0.010$ & $<<0.100$ & $<0.010$ & 14.3 & 0.014 & 0.811 & $<0.050$ & 0.027 & 0.549 & 1.374 & 2.53 \\
\hline US-41 & $3-1$ & 19 & Z29LM21 & 0.65 & 0.011 & 0.808 & $<0.010$ & 5.07 & 0.014 & 0.815 & $<0.050$ & 2.17 & $<0.010$ & $<0.010$ & 0.013 \\
\hline $\begin{array}{l}\text { US-01 } \\
\end{array}$ & $3-1$ & 20 & Z43LM21 & 0.015 & 0.177 & 0.785 & 0.297 & 9.94 & 0.043 & 0.052 & $<0.050$ & 0.071 & 0.01 & $<0.010$ & 0.321 \\
\hline US-01 & $3-1$ & 21 & Z43LM11 & 0.149 & 0.186 & 0.804 & 0.307 & 10.4 & 0.044 & 0.056 & $<0.050$ & 0.075 & $<0.010$ & $<0.010$ & 0.336 \\
\hline US-43 & $3-1$ & 22 & Z21LM21 & 0.636 & $<0.010$ & 0.795 & 0.294 & 8.33 & 0.144 & 0.828 & 0.167 & 0.347 & 0.271 & 0.356 & 2.49 \\
\hline Batch 1 & $3-1$ & 23 & BCHLM313 & 0.758 & 0.075 & 3.12 & 0.776 & 6.87 & 0.528 & $<0.010$ & $<0.050$ & $<0.010$ & 0.366 & $<0.010$ & 0.067 \\
\hline LRM & $3-1$ & 24 & LRMLM313 & 0.362 & 0.134 & 1.18 & 0.057 & 15.4 & 0.134 & 0.082 & 0.085 & $<0.010$ & 0.057 & $<0.010$ & 0.645 \\
\hline Batch 1 & $3-2$ & 1 & BCHLM321 & 0.81 & 0.075 & 3.11 & 0.793 & 6.79 & 0.536 & $<0.010$ & $<0.050$ & $<0.010$ & 0.375 & $<0.010$ & 0.06 \\
\hline LRM & $3-2$ & 2 & LRMLM321 & 0.372 & 0.135 & 1.2 & 0.057 & 14.5 & 0.136 & 0.082 & 0.087 & $<0.010$ & 0.057 & $<0.010$ & 0.647 \\
\hline US-37 & $3-2$ & 3 & Z32LM22 & 0.032 & $<0.010$ & 0.777 & $<0.010$ & 9.86 & $<0.010$ & $<0.010$ & 0.152 & 2.06 & 0.563 & 1.418 & $<0.010$ \\
\hline US-43 & $3-2$ & 4 & $\begin{array}{l}\text { Z21LM22 } \\
\end{array}$ & 0.662 & $<0.010$ & 0.828 & 0.3 & 8.57 & 0.147 & 0.841 & 0.166 & 0.357 & 0.279 & 0.362 & 2.54 \\
\hline US-01 & $3-2$ & 5 & $\begin{array}{l}\text { Z43LM22 } \\
\end{array}$ & 0.016 & 0.18 & 0.822 & 0.304 & 10.2 & 0.043 & 0.053 & $<0.050$ & 0.074 & 0.01 & $\begin{array}{l}<0.010 \\
\end{array}$ & 0.338 \\
\hline US-37 & $3-2$ & 6 & Z32LM12 & 0.017 & $<0.010$ & 0.81 & $<0.010$ & 10.8 & $<0.010$ & $<0.010$ & 0.152 & 2.14 & 0.567 & 1.422 & 0.016 \\
\hline US-07 & $3-2$ & 7 & $\begin{array}{l}\text { Z19LM22 } \\
\end{array}$ & 0.651 & 0.01 & 0.813 & 0.301 & 8.54 & 0.148 & 0.083 & 0.142 & 0.075 & 0.56 & 0.078 & 0.319 \\
\hline US-28 & $3-2$ & 8 & $\begin{array}{l}\text { Z33LM22 } \\
\end{array}$ & 0.662 & $<0.010$ & $<0.100$ & $<0.010$ & 14.7 & $<0.010$ & 0.823 & $<0.050$ & 0.042 & 0.571 & 1.424 & 2.54 \\
\hline US-41 & $3-2$ & 9 & Z29LM22 & 0.665 & 0.01 & 0.823 & $<0.010$ & 5.21 & 0.014 & 0.829 & $<0.050$ & 2.16 & $<<0.010$ & $<0.010$ & 0.014 \\
\hline US-28 & $3-2$ & 10 & Z33LM12 & 0.667 & $<0.010$ & $<0.100$ & $<0.010$ & 14.5 & 0.014 & 0.824 & $<0.050$ & 0.029 & 0.562 & 1.398 & 2.56 \\
\hline US-27 & $3-2$ & 11 & Z36LM22 & 0.014 & 0.571 & 0.869 & 0.289 & 13.4 & $<0.010$ & 0.031 & 0.19 & $<0.010$ & $<0.010$ & 1.379 & 0.543 \\
\hline Batch 1 & $3-2$ & 12 & BCHLM322 & 0.812 & 0.075 & 3.16 & 0.79 & 6.91 & 0.534 & $<0.010$ & $<0.050$ & $<0.010$ & 0.377 & $<0.010$ & 0.07 \\
\hline LRM & $3-2$ & 13 & LRMLM322 & 0.387 & 0.135 & 1.24 & 0.057 & 15.4 & 0.135 & 0.083 & 0.087 & $<0.010$ & 0.058 & $<0.010$ & 0.667 \\
\hline US-07 & $3-2$ & 14 & $\begin{array}{l}\text { Z19LM12 } \\
\end{array}$ & 0.647 & $<0.010$ & 0.8 & 0.3 & 8.52 & 0.147 & 0.084 & 0.148 & 0.075 & 0.561 & 0.078 & 0.31 \\
\hline US-41 & $3-2$ & 15 & $\begin{array}{l}\text { Z29LM12 } \\
\end{array}$ & 0.687 & 0.01 & 0.853 & $<0.010$ & 5.43 & $<0.010$ & 0.842 & $<0.050$ & 2.26 & $<0.010$ & $<0.010$ & $<0.010$ \\
\hline US-25 & $3-2$ & 16 & $\begin{array}{l}\text { Z08LM12 } \\
\end{array}$ & 0.514 & 0.102 & 0.256 & 0.225 & 9.01 & 0.995 & 0.03 & 0.135 & 0.07 & 0.438 & 0.087 & 0.264 \\
\hline US-27 & $3-2$ & 17 & Z36LM12 & 0.025 & 0.573 & 0.854 & 0.298 & 13.9 & $<0.010$ & $<0.010$ & 0.198 & $<0.010$ & $<<0.010$ & 1.406 & 0.092 \\
\hline US-16 & $3-2$ & 18 & $\begin{array}{l}\text { Z44LM12 } \\
\end{array}$ & 0.678 & 0.056 & 0.868 & $<0.010$ & 5.29 & $<0.010$ & $<0.010$ & $<0.050$ & $<0.010$ & 0.583 & $<0.010$ & 0.373 \\
\hline US-25 & $3-2$ & 19 & Z08LM22 & 0.526 & 0.1 & 0.26 & 0.225 & 8.8 & 0.995 & 0.027 & 0.137 & 0.072 & 0.441 & $\begin{array}{l}0.081 \\
\end{array}$ & 0.268 \\
\hline US-16 & $3-2$ & 20 & $\begin{array}{l}\text { Z44LM22 } \\
\end{array}$ & 0.656 & 0.055 & 0.854 & $<0.010$ & 4.97 & 0.015 & $<0.010$ & $<0.050$ & $<0.010$ & 0.566 & $<0.010$ & 0.356 \\
\hline US-43 & $3-2$ & 21 & $\begin{array}{l}\text { Z21LM12 } \\
\end{array}$ & 0.656 & $<0.010$ & 0.829 & 0.29 & 8.25 & 0.141 & 0.814 & 0.161 & 0.36 & 0.272 & 0.35 & 2.49 \\
\hline US-01 & $3-2$ & 22 & $\begin{array}{l}\text { Z43LM12 } \\
\end{array}$ & 0.164 & 0.198 & 0.901 & 0.329 & 11.4 & 0.046 & 0.06 & $<0.050$ & 0.083 & $<0.010$ & $<0.010$ & 0.377 \\
\hline Batch 1 & $3-2$ & 23 & BCHLM323 & 0.802 & 0.074 & 3.13 & 0.781 & 6.92 & 0.528 & $<0.010$ & $<0.050$ & $<0.010$ & 0.373 & $<0.010$ & 0.07 \\
\hline LRM & $3-2$ & 24 & LRMLM323 & 0.38 & 0.134 & 1.21 & 0.057 & 15.8 & 0.135 & 0.082 & 0.086 & $<0.010$ & 0.057 & $<0.010$ & 0.654 \\
\hline Batch 1 & $4-1$ & 1 & BCHLM411 & 0.782 & 0.075 & 3.08 & 0.787 & 6.55 & 0.532 & $<0.010$ & $<0.050$ & $<0.010$ & 0.372 & $<0.010$ & 0.068 \\
\hline LRM & $4-1$ & 2 & LRMLM411 & 0.363 & 0.134 & 1.24 & 0.058 & 15.5 & 0.135 & 0.082 & 0.083 & $<0.010$ & 0.057 & $<0.010$ & 0.636 \\
\hline US-42 & $4-1$ & 3 & $\begin{array}{l}\text { Z15LM11 } \\
\end{array}$ & 0.616 & $<0.010$ & 0.808 & $<0.010$ & 14.7 & $<0.010$ & 0.812 & 0.02 & $<0.010$ & $<<0.010$ & $\begin{array}{l}1.096 \\
\end{array}$ & $<0.010$ \\
\hline US-03 & $4-1$ & 4 & Z26LM21 & 0.644 & 0.011 & 0.782 & 0.298 & 6.41 & 0.215 & 0.083 & $<0.050$ & 0.072 & 0.556 & $<0.010$ & $<0.010$ \\
\hline US-40 & $4-1$ & 5 & Z22LM21 & 0.018 & 0.183 & 0.837 & $<0.010$ & 8.33 & $<0.010$ & 0.853 & $<0.050$ & $<0.010$ & 0.514 & $<0.010$ & $<0.010$ \\
\hline
\end{tabular}


Table A2. Measured Elemental Concentrations (wt\%) for Samples Prepared Using Lithium Metaborate

\begin{tabular}{|c|c|c|c|c|c|c|c|c|c|c|c|c|c|c|c|}
\hline Glass ID & $\begin{array}{c}\text { Block/ } \\
\text { Sub-Block }\end{array}$ & Sequence & Lab ID & $\mathrm{Ca}(\mathrm{wt} \%)$ & $\mathrm{Cr}(\mathrm{wt} \%)$ & $\mathrm{K}(\mathrm{wt} \%)$ & $\mathrm{Mg}(\mathrm{wt} \%)$ & $\mathrm{Na}(\mathrm{wt} \%)$ & $\mathrm{Ni}(\mathrm{wt} \%)$ & $\mathrm{Pb}(\mathrm{wt} \%)$ & $\mathrm{S}(\mathrm{wt} \%)$ & $\mathrm{Sr}(\mathrm{wt} \%)$ & $\mathrm{Ti}(\mathrm{wt} \%)$ & $\mathrm{Zn}(\mathrm{wt} \%)$ & $\mathrm{Zr}(\mathrm{wt} \%)$ \\
\hline US-35 & $4-1$ & 6 & Z11LM21 & 0.65 & 0.302 & $<0.100$ & 0.302 & 6.63 & $<0.010$ & $<0.010$ & $<0.050$ & 1.96 & $<0.010$ & 1.42 & $<0.010$ \\
\hline US-04 & $4-1$ & 7 & Z10LM11 & 0.62 & 0.011 & 0.766 & 0.295 & 8.57 & $<0.010$ & 0.082 & 0.152 & 0.072 & 0.545 & 0.075 & 0.312 \\
\hline US-44 & $4-1$ & 8 & Z25LM21 & 0.4 & 0.178 & 0.533 & 0.155 & 10.6 & 0.268 & 0.561 & 0.093 & 1.19 & 0.267 & 0.774 & 0.965 \\
\hline $\begin{array}{l}\text { US-35 } \\
\end{array}$ & $4-1$ & 9 & $\begin{array}{l}\text { Z11LM11 } \\
\end{array}$ & 0.655 & 0.295 & $<0.100$ & 0.297 & 6.8 & 0.012 & $<0.010$ & $<0.050$ & 1.98 & $\begin{array}{l}<0.010 \\
\end{array}$ & 1.384 & 0.012 \\
\hline US- 36 & $4-1$ & 10 & Z41LM11 & 0.159 & 0.264 & 0.461 & 0.027 & 10.4 & 0.385 & 0.84 & $<0.050$ & $<0.010$ & 0.013 & 0.012 & 2.55 \\
\hline $\begin{array}{ll}\text { US-21 } \\
\end{array}$ & $4-1$ & 11 & Z16LM11 & 0.254 & 0.044 & 0.605 & 0.225 & 9.01 & 0.369 & 0.068 & 0.052 & 0.062 & 0.308 & 0.028 & 0.085 \\
\hline Batch 1 & $4-1$ & 12 & BCHLM412 & 0.789 & 0.075 & 3.13 & 0.793 & 6.8 & 0.535 & $<0.010$ & $<0.050$ & $<0.010$ & 0.374 & $<0.010$ & 0.07 \\
\hline LRM & $4-1$ & 13 & $\begin{array}{l}\text { LRMLM412 } \\
\end{array}$ & 0.371 & 0.135 & 1.23 & 0.058 & 15.8 & 0.136 & 0.083 & 0.088 & $<0.010$ & 0.057 & $<0.010$ & 0.643 \\
\hline US-44 & $4-1$ & 14 & Z25LM11 & 0.376 & 0.178 & 0.534 & 0.152 & 10.2 & 0.263 & 0.56 & 0.089 & 1.18 & 0.269 & 0.771 & 0.955 \\
\hline US- 03 & $4-1$ & 15 & $\begin{array}{l}\text { Z26LM11 } \\
\end{array}$ & 0.656 & 0.011 & 0.804 & 0.307 & 6.65 & 0.221 & 0.087 & $<0.050$ & 0.075 & 0.571 & $<0.010$ & $<0.010$ \\
\hline US-21 & $4-1$ & 16 & Z16LM21 & 0.266 & 0.037 & 0.6 & 0.225 & 8.76 & 0.327 & 0.069 & 0.051 & 0.063 & 0.306 & 0.03 & 0.064 \\
\hline $\begin{array}{l}\text { US-36 } \\
\end{array}$ & $4-1$ & 17 & Z41LM21 & 0.334 & 0.268 & 0.472 & 0.027 & 10.5 & 0.397 & 0.851 & $<0.050$ & $<0.010$ & 0.013 & 0.01 & 2.56 \\
\hline US-42 & $4-1$ & 18 & Z15LM21 & 0.633 & $<0.010$ & 0.817 & $<0.010$ & 14.2 & $<0.010$ & 0.823 & $<0.050$ & $<0.010$ & $<0.010$ & 1.09 & 0.021 \\
\hline US-40 & $4-1$ & 19 & Z22LM11 & 0.046 & 0.246 & 0.823 & $<0.010$ & 8.34 & $<0.010$ & 0.843 & $<0.050$ & $<0.010$ & 0.519 & 0.014 & $<0.010$ \\
\hline US-23 & $4-1$ & 20 & $\begin{array}{l}\text { Z28LM11 } \\
\end{array}$ & 0.476 & 0.047 & 0.211 & 0.077 & 9.91 & 0.371 & 0.072 & 0.134 & 0.025 & 0.149 & 0.071 & 0.1 \\
\hline US-04 & $4-1$ & 21 & $\begin{array}{l}\text { Z10LM21 } \\
\end{array}$ & 0.637 & 0.013 & 0.798 & 0.3 & 8.96 & $<0.010$ & 0.084 & 0.163 & 0.075 & 0.556 & 0.076 & 0.317 \\
\hline $\begin{array}{l}\text { US-23 } \\
\end{array}$ & $4-1$ & 22 & $\begin{array}{l}\text { Z28LM21 } \\
\end{array}$ & 0.482 & 0.043 & 0.211 & 0.075 & 9.87 & 0.346 & 0.073 & 0.132 & 0.024 & 0.15 & 0.074 & 0.103 \\
\hline Batch 1 & $4-1$ & 23 & BCHLM413 & 0.766 & 0.074 & 3.14 & 0.792 & 7.37 & 0.534 & $<0.010$ & $<0.050$ & $<0.010$ & 0.37 & $<0.010$ & 0.067 \\
\hline LRM & $4-1$ & 24 & LRMLM413 & 0.358 & 0.136 & 1.23 & 0.058 & 16.4 & 0.136 & 0.084 & 0.088 & $<0.010$ & 0.056 & $<0.010$ & 0.642 \\
\hline Batch 1 & $4-2$ & 1 & BCHLM421 & 0.784 & 0.075 & 3.12 & 0.794 & 7.23 & 0.534 & $<0.010$ & $<0.050$ & $<0.010$ & 0.37 & $<0.010$ & 0.06 \\
\hline LRM & $4-2$ & 2 & LRMLM421 & 0.362 & 0.136 & 1.21 & 0.058 & 16.3 & 0.137 & 0.084 & 0.083 & $<0.010$ & 0.058 & $<0.010$ & 0.642 \\
\hline US-23 & $4-2$ & 3 & $\begin{array}{l}\text { Z28LM22 } \\
\end{array}$ & 0.476 & 0.044 & 0.208 & 0.076 & 9.51 & 0.349 & 0.074 & 0.132 & 0.025 & 0.152 & 0.075 & 0.103 \\
\hline US-44 & $4-2$ & 4 & Z25LM22 & 0.399 & 0.178 & 0.532 & 0.155 & 10.3 & 0.269 & 0.562 & 0.095 & 1.19 & 0.268 & 0.776 & 0.964 \\
\hline US-36 & $4-2$ & 5 & $\begin{array}{l}\text { Z41LM22 } \\
\end{array}$ & 0.331 & 0.271 & 0.463 & 0.027 & 10 & 0.4 & 0.855 & $<0.050$ & $<0.010$ & 0.014 & 0.01 & 2.56 \\
\hline $\begin{array}{ll}\text { US-21 } \\
\end{array}$ & $4-2$ & 6 & Z16LM12 & 0.253 & 0.045 & 0.603 & 0.226 & 8.64 & 0.371 & 0.07 & 0.049 & 0.063 & 0.309 & 0.029 & 0.086 \\
\hline $\begin{array}{l}\text { US-36 } \\
\end{array}$ & $4-2$ & 7 & $\begin{array}{l}\text { Z41LM12 } \\
\end{array}$ & 0.163 & 0.272 & 0.468 & 0.028 & 10.4 & 0.396 & 0.864 & $<0.050$ & $<0.010$ & 0.014 & 0.012 & 2.61 \\
\hline US-44 & $4-2$ & 8 & Z25LM12 & 0.38 & 0.182 & 0.541 & 0.153 & 10.2 & 0.267 & 0.571 & 0.088 & 1.2 & 0.272 & 0.781 & 0.988 \\
\hline US-21 & $4-2$ & 9 & Z16LM22 & 0.266 & 0.038 & 0.601 & 0.227 & 8.68 & 0.33 & 0.07 & 0.052 & 0.064 & 0.308 & 0.031 & 0.07 \\
\hline US-04 & $4-2$ & 10 & $\begin{array}{l}\text { Z10LM22 } \\
\end{array}$ & 0.639 & 0.013 & 0.799 & 0.304 & 8.69 & $<0.010$ & 0.086 & 0.16 & 0.076 & 0.565 & 0.078 & 0.323 \\
\hline $\begin{array}{l}\text { US-04 } \\
\end{array}$ & $4-2$ & 11 & $\begin{array}{l}\text { Z10LM12 } \\
\end{array}$ & 0.634 & 0.012 & 0.795 & 0.298 & 8.13 & $<0.010$ & 0.083 & 0.159 & 0.077 & 0.555 & 0.077 & 0.322 \\
\hline Batch 1 & $4-2$ & 12 & BCHLM422 & 0.782 & 0.076 & 3.18 & 0.8 & 6.75 & 0.542 & $<0.010$ & $<0.050$ & $<0.010$ & 0.376 & $<0.010$ & 0.07 \\
\hline LRM & $4-2$ & 13 & LRMLM422 & 0.361 & 0.136 & 1.21 & 0.058 & 14.9 & 0.136 & 0.084 & 0.086 & $<0.010$ & 0.057 & $<0.010$ & 0.643 \\
\hline US-03 & $4-2$ & 14 & Z26LM22 & 0.636 & 0.011 & 0.773 & 0.301 & 6.05 & 0.219 & 0.086 & $<0.050$ & 0.073 & 0.56 & $<0.010$ & $<0.010$ \\
\hline $\begin{array}{l}\text { US-42 } \\
\end{array}$ & $4-2$ & 15 & $\begin{array}{l}\text { Z15LM22 } \\
\end{array}$ & 0.628 & $<0.010$ & 0.804 & $<0.010$ & 14.1 & $<0.010$ & 0.831 & $<0.050$ & $<0.010$ & $<0.010$ & 1.1 & $<0.010$ \\
\hline US-23 & $4-2$ & 16 & $\begin{array}{l}\text { Z28LM12 } \\
\end{array}$ & 0.479 & 0.048 & 0.212 & 0.078 & 9.22 & 0.377 & 0.072 & 0.132 & 0.026 & 0.151 & 0.073 & 0.101 \\
\hline US- 03 & $4-2$ & 17 & Z26LM12 & 0.655 & 0.011 & 0.805 & 0.308 & 6.47 & 0.222 & 0.088 & $<0.050$ & 0.076 & 0.572 & $<0.010$ & $<0.010$ \\
\hline US-35 & $4-2$ & 18 & Z11LM22 & 0.659 & 0.305 & $<<0.100$ & 0.303 & 6.3 & $<0.010$ & 0.006 & $<0.050$ & 1.97 & $<0.010$ & 1.435 & $<0.010$ \\
\hline US-35 & $4-2$ & 19 & Z11LM12 & 0.658 & 0.301 & $<0.100$ & 0.299 & 6.39 & 0.012 & 0.002 & $<0.050$ & 2 & $<<0.010$ & 1.405 & $<0.010$ \\
\hline US-40 & $4-2$ & 20 & $\begin{array}{l}\text { Z22LM12 } \\
\end{array}$ & 0.047 & 0.249 & 0.827 & $<0.010$ & 8.02 & $<0.010$ & 0.855 & $<0.050$ & $<0.010$ & 0.525 & 0.014 & $<0.010$ \\
\hline US-40 & $4-2$ & 21 & $\begin{array}{l}\text { Z22LM22 } \\
\end{array}$ & 0.019 & 0.187 & 0.859 & $<0.010$ & 8.28 & $<0.010$ & 0.87 & $<0.050$ & $<0.010$ & 0.525 & $<0.010$ & $<0.010$ \\
\hline US-42 & $4-2$ & 22 & Z15LM12 & 0.622 & $<0.010$ & 0.818 & $<0.010$ & 14.9 & $<0.010$ & 0.828 & $<0.050$ & $<0.010$ & $<0.010$ & 1.114 & $<0.010$ \\
\hline Batch 1 & $4-2$ & 23 & BCHLM423 & 0.79 & 0.076 & 3.18 & 0.797 & 7.07 & 0.54 & $<0.010$ & $<0.050$ & $<0.010$ & 0.377 & $<0.010$ & 0.067 \\
\hline LRM & $4-2$ & 24 & LRMLM423 & 0.331 & 0.132 & 1.21 & 0.059 & 15.8 & 0.136 & 0.08 & 0.079 & $<0.010$ & 0.058 & 0.012 & 0.651 \\
\hline Batch 1 & $5-1$ & 1 & BCHLM511 & 0.795 & 0.074 & 3.15 & 0.787 & 6.97 & 0.531 & $<0.010$ & $<0.050$ & $<0.010$ & 0.372 & $<0.010$ & 0.065 \\
\hline LRM & $5-1$ & 2 & LRMLM511 & 0.374 & 0.134 & 1.23 & 0.057 & 15.4 & 0.135 & 0.082 & 0.083 & $<0.010$ & 0.056 & $<0.010$ & 0.64 \\
\hline US-10 & $5-1$ & 3 & Z05LM21 & 0.658 & 0.011 & 0.817 & 0.301 & 9.71 & $<0.010$ & 0.081 & 0.16 & 0.076 & 0.567 & 0.08 & 0.319 \\
\hline US-20 & $5-1$ & 4 & Z40LM21 & 0.484 & 0.046 & 0.642 & 0.204 & 8.73 & 0.155 & 0.062 & 0.105 & 0.049 & 0.471 & 0.058 & 0.202 \\
\hline $\begin{array}{l}\text { US-24 } \\
\end{array}$ & $5-1$ & 5 & Z45LM11 & 0.503 & 0.041 & 0.622 & 0.223 & 9.51 & 0.363 & 0.075 & 0.13 & 0.059 & 0.419 & 0.068 & 0.349 \\
\hline US- 20 & $5-1$ & 6 & Z40LM11 & 0.584 & 0.043 & 0.605 & 0.2 & 8.54 & 0.149 & 0.058 & 0.099 & 0.046 & 0.453 & 0.056 & 0.197 \\
\hline US-24 & $5-1$ & 7 & Z45LM21 & 0.493 & 0.038 & 0.603 & 0.222 & 9.05 & 0.35 & 0.067 & 0.126 & 0.059 & 0.417 & 0.063 & 0.21 \\
\hline
\end{tabular}


Table A2. Measured Elemental Concentrations (wt\%) for Samples Prepared Using Lithium Metaborate

\begin{tabular}{|c|c|c|c|c|c|c|c|c|c|c|c|c|c|c|c|}
\hline Glass ID & $\begin{array}{c}\text { Block } / \\
\text { Sub-Block }\end{array}$ & Sequence & Lab ID & $\mathrm{Ca}(\mathrm{wt} \%)$ & $\mathrm{Cr}(\mathrm{wt} \%)$ & $\mathrm{K}(\mathrm{wt} \%)$ & $\mathrm{Mg}(\mathrm{wt} \%)$ & $\mathrm{Na}(\mathrm{wt} \%)$ & $\mathrm{Ni}(\mathrm{wt} \%)$ & $\mathrm{Pb}(\mathrm{wt} \%)$ & $\mathrm{S}(\mathrm{wt} \%)$ & $\mathrm{Sr}(\mathrm{wt} \%)$ & $\mathrm{Ti}(\mathrm{wt} \%)$ & $\mathrm{Zn}(\mathrm{wt} \%)$ & $\mathrm{Zr}(\mathrm{wt} \%)$ \\
\hline US-30 & $5-1$ & 8 & Z03LM11 & 0.73 & 0.447 & 0.858 & $<0.010$ & 4.32 & $<0.010$ & $<0.010$ & 0.193 & 2.37 & 0.616 & 0.388 & 2.8 \\
\hline US-31 & $5-1$ & 9 & Z13LM21 & 0.033 & 0.008 & $<0.100$ & 0.29 & 6.22 & $<0.010$ & 0.817 & 0.145 & 2.14 & 0.571 & $<0.010$ & 0.018 \\
\hline $\begin{array}{l}\text { US-19 } \\
\end{array}$ & $5-1$ & 10 & Z18LM11 & 0.479 & 0.035 & 0.634 & 0.203 & 8.64 & 0.138 & 0.061 & 0.105 & 0.049 & 0.461 & 0.054 & 0.164 \\
\hline US-02 & $5-1$ & 11 & $\begin{array}{l}\text { Z35LM11 } \\
\end{array}$ & 0.044 & 0.02 & $<0.100$ & $<0.010$ & 9.68 & 0.019 & $<0.010$ & $<0.050$ & 0.031 & 0.038 & 0.1 & 0.346 \\
\hline Batch 1 & $5-1$ & 12 & BCHLM512 & 0.792 & 0.074 & 3.1 & 0.792 & 6.85 & 0.533 & $<0.010$ & $<0.050$ & $<0.010$ & 0.373 & $<0.010$ & 0.07 \\
\hline LRM & $5-1$ & 13 & $\begin{array}{l}\text { LRMLM512 } \\
\end{array}$ & 0.368 & 0.136 & 1.24 & 0.058 & 15.4 & 0.137 & 0.083 & 0.083 & $<0.010$ & 0.057 & $<0.010$ & 0.649 \\
\hline $\begin{array}{l}\text { US-30 } \\
\end{array}$ & $5-1$ & 14 & $\begin{array}{l}\text { Z03LM21 } \\
\end{array}$ & 0.65 & 0.348 & 0.763 & $<0.010$ & 3.95 & $<0.010$ & $<0.010$ & 0.17 & 2.14 & 0.546 & 0.34 & 2.46 \\
\hline US-45 & $5-1$ & 15 & Z38LM21 & 0.373 & 0.174 & 0.542 & 0.148 & 9.92 & 0.263 & 0.547 & 0.087 & 1.19 & 0.268 & 0.763 & 0.968 \\
\hline US-45 & $5-1$ & 16 & Z38LM11 & 0.388 & 0.176 & 0.537 & 0.15 & 9.89 & 0.26 & 0.555 & 0.089 & 1.2 & 0.272 & 0.784 & 0.981 \\
\hline US-02 & $5-1$ & 17 & Z35LM21 & 0.045 & 0.013 & $<0.100$ & $<0.010$ & 9.46 & 0.011 & $<0.010$ & $<0.050$ & 0.028 & 0.037 & 0.098 & 0.334 \\
\hline US-29 & $5-1$ & 18 & Z23LM11 & 0.663 & 0.376 & 0.8 & 0.303 & 14.1 & $<0.010$ & 1.23 & $<0.050$ & 0.9 & 0.543 & $<0.010$ & $<0.010$ \\
\hline US-10 & $5-1$ & 19 & Z05LM11 & 0.651 & 0.012 & 0.809 & 0.304 & 9.87 & $<0.010$ & 0.083 & 0.157 & 0.075 & 0.567 & 0.079 & 0.319 \\
\hline US-19 & $5-1$ & 20 & Z18LM21 & 0.475 & 0.041 & 0.635 & 0.204 & 8.6 & 0.16 & 0.061 & 0.107 & 0.049 & 0.464 & 0.053 & 0.172 \\
\hline US-29 & $5-1$ & 21 & Z23LM21 & 0.66 & 0.384 & 0.804 & 0.299 & 14.5 & $<0.010$ & 1.2 & $<0.050$ & 0.902 & 0.542 & $<0.010$ & $<0.010$ \\
\hline US-31 & $5-1$ & 22 & $\begin{array}{l}\text { Z13LM11 } \\
\end{array}$ & 0.047 & 0.011 & $<0.100$ & 0.29 & 6.12 & 0.03 & 0.82 & 0.146 & 2.12 & 0.57 & $<0.010$ & $<0.010$ \\
\hline Batch 1 & $5-1$ & 23 & BCHLM513 & 0.795 & 0.075 & 3.16 & 0.797 & 7.17 & 0.537 & $<0.010$ & $<0.050$ & $<0.010$ & 0.376 & $<0.010$ & 0.067 \\
\hline LRM & $5-1$ & 24 & $\begin{array}{l}\text { LRMLM513 } \\
\end{array}$ & 0.328 & 0.13 & 1.26 & 0.058 & 16 & 0.135 & 0.079 & 0.083 & $<0.010$ & 0.056 & 0.011 & 0.653 \\
\hline Batch 1 & $5-2$ & 1 & BCHLM521 & 0.81 & 0.075 & 3.07 & 0.799 & 6.65 & 0.537 & $<0.010$ & $<0.050$ & $<0.010$ & 0.382 & $<0.010$ & 0.067 \\
\hline LRM & $5-2$ & 2 & LRMLM521 & 0.341 & 0.132 & 1.18 & 0.058 & 15.2 & 0.135 & 0.079 & 0.081 & $<0.010$ & 0.058 & 0.012 & 0.657 \\
\hline US-30 & $5-2$ & 3 & Z03LM22 & 0.675 & 0.35 & 0.791 & $\angle 0.010$ & 4.16 & $<0.010$ & $<0.010$ & 0.174 & 2.19 & 0.559 & 0.344 & 2.53 \\
\hline US- 20 & $5-2$ & 4 & Z40LM12 & 0.606 & 0.045 & 0.626 & 0.201 & 8.38 & 0.151 & 0.059 & 0.101 & 0.05 & 0.464 & 0.057 & 0.222 \\
\hline US- 20 & $5-2$ & 5 & Z40LM22 & 0.493 & 0.047 & 0.642 & 0.204 & 8.65 & 0.156 & 0.062 & 0.102 & 0.05 & 0.471 & 0.059 & 0.203 \\
\hline US-31 & $5-2$ & 6 & Z13LM12 & 0.049 & 0.012 & $<0.100$ & 0.29 & 6.17 & 0.03 & 0.812 & 0.152 & 2.15 & 0.57 & $<0.010$ & $<0.010$ \\
\hline US-24 & $5-2$ & 7 & $\begin{array}{l}\text { Z45LM22 } \\
\end{array}$ & 0.51 & 0.039 & 0.622 & 0.222 & 9.23 & 0.351 & 0.068 & 0.13 & 0.062 & 0.424 & 0.064 & 0.213 \\
\hline US-02 & $5-2$ & 8 & Z35LM22 & 0.049 & 0.014 & $<0.100$ & $<0.010$ & 9.72 & 0.012 & $<0.010$ & $<0.050$ & 0.032 & 0.038 & 0.099 & 0.334 \\
\hline US-29 & $5-2$ & 9 & Z23LM12 & 0.688 & 0.371 & 0.838 & 0.299 & 14.6 & $<0.010$ & 1.2 & $<0.050$ & 0.922 & 0.547 & $<0.010$ & $<0.010$ \\
\hline US-10 & $5-2$ & 10 & Z05LM12 & 0.676 & 0.013 & 0.834 & 0.301 & 10.1 & $<0.010$ & 0.082 & 0.158 & 0.077 & 0.573 & 0.079 & 0.322 \\
\hline US-02 & $5-2$ & 11 & $\begin{array}{l}\text { Z35LM12 } \\
\end{array}$ & 0.045 & 0.021 & $<0.100$ & $<0.010$ & 10.1 & 0.019 & $<0.010$ & $<0.050$ & 0.031 & 0.039 & 0.099 & 0.343 \\
\hline Batch 1 & $5-2$ & 12 & BCHLM522 & 0.816 & 0.075 & 3.12 & 0.789 & 6.98 & 0.534 & $<0.010$ & $<0.050$ & $<0.010$ & 0.378 & $<0.010$ & 0.07 \\
\hline LRM & $5-2$ & 13 & $\begin{array}{l}\text { LRMLM522 } \\
\end{array}$ & 0.343 & 0.131 & 1.2 & 0.058 & 15.9 & 0.135 & 0.079 & 0.086 & $<0.010$ & 0.058 & 0.012 & 0.661 \\
\hline US-19 & $5-2$ & 14 & Z18LM12 & 0.496 & 0.036 & 0.657 & 0.202 & 8.63 & 0.138 & 0.062 & 0.105 & 0.052 & 0.468 & 0.055 & 0.164 \\
\hline US-45 & $5-2$ & 15 & $\begin{array}{l}\text { Z38LM22 } \\
\end{array}$ & 0.389 & 0.175 & 0.566 & 0.148 & 9.87 & 0.263 & 0.543 & 0.089 & 1.23 & 0.273 & 0.762 & 0.958 \\
\hline US-31 & $5-2$ & 16 & $\begin{array}{l}\text { Z13LM22 } \\
\end{array}$ & 0.035 & $<0.010$ & $<0.100$ & 0.289 & 6.18 & $<0.010$ & 0.809 & 0.147 & 2.17 & 0.577 & $<0.010$ & $<0.010$ \\
\hline US-29 & $5-2$ & 17 & Z23LM22 & 0.676 & 0.384 & 0.827 & 0.3 & 14.7 & $<0.010$ & 1.2 & $<0.050$ & 0.916 & 0.548 & 0.01 & $<0.010$ \\
\hline US-19 & $5-2$ & 18 & Z18LM22 & 0.488 & 0.042 & 0.654 & 0.204 & 8.74 & 0.16 & 0.061 & 0.105 & 0.052 & 0.47 & 0.054 & 0.171 \\
\hline US-30 & $5-2$ & 19 & Z03LM12 & 0.742 & 0.446 & 0.879 & $<0.010$ & 4.35 & $<0.010$ & $<0.010$ & 0.197 & 2.4 & 0.618 & 0.388 & 2.8 \\
\hline US-10 & $5-2$ & 20 & $\begin{array}{l}\text { Z05LM22 } \\
\end{array}$ & 0.666 & 0.012 & 0.831 & 0.302 & 10.3 & $<0.010$ & 0.082 & 0.163 & 0.076 & 0.57 & 0.081 & 0.334 \\
\hline US-45 & $5-2$ & 21 & $\begin{array}{l}\text { Z38LM12 } \\
\end{array}$ & 0.4 & 0.175 & 0.56 & 0.149 & 10.2 & 0.258 & 0.55 & 0.091 & 1.23 & 0.273 & 0.778 & 0.981 \\
\hline US-24 & $5-2$ & 22 & Z45LM12 & 0.534 & 0.042 & 0.658 & 0.224 & 9.76 & 0.365 & 0.075 & 0.135 & 0.064 & 0.43 & 0.069 & 0.364 \\
\hline Batch 1 & $5-2$ & 23 & BCHLM523 & 0.852 & 0.076 & 3.08 & 0.795 & 6.95 & 0.538 & $<0.010$ & $<0.050$ & $<0.010$ & 0.381 & 0.01 & 0.067 \\
\hline LRM & $5-2$ & 24 & LRMLM523 & 0.388 & 0.137 & 1.19 & 0.058 & 16.1 & 0.136 & 0.082 & 0.084 & $<0.010$ & 0.058 & $<0.010$ & 0.659 \\
\hline
\end{tabular}




\section{Table A3. Measured Elemental Concentrations (wt\%)} for Samples Prepared Using Peroxide Fusion

\begin{tabular}{|c|c|c|c|c|c|c|c|c|c|}
\hline Glass ID & $\begin{array}{c}\text { Block/ } \\
\text { Sub-Block }\end{array}$ & Sequence & Lab ID & $\mathrm{Al}(\mathrm{wt} \%)$ & B $(w t \%)$ & $\mathrm{Fe}(w \mathrm{w} \%)$ & $\mathrm{Li}(\mathrm{wt} \%)$ & $\begin{array}{c}\text { Mn } \\
(w t \%)\end{array}$ & $\mathrm{Si}(\mathrm{wt} \%)$ \\
\hline Batch 1 & $1 / 1$ & 1 & BCHPF111 & 2.59 & 2.64 & 9.12 & 2.08 & 1.3 & 23 \\
\hline LRM & $1 / 1$ & 2 & LRMPF111 & 5.42 & 2.61 & 1.14 & 0.09 & $<0.100$ & 25.3 \\
\hline US-27 & $1 / 1$ & 4 & Z36PF11 & 5.59 & 2.08 & 10.3 & 2.71 & $<0.100$ & 18.2 \\
\hline US-29 & $1 / 1$ & 5 & Z23PF11 & 5.35 & 2.69 & 3.44 & 2.7 & $<0.100$ & 19.8 \\
\hline US-20 & $1 / 1$ & 6 & Z40PF21 & 7.61 & 4.45 & 5.65 & 1.65 & 1.47 & 19.2 \\
\hline US-29 & $1 / 1$ & 9 & Z23PF21 & 5.43 & 2.72 & 3.25 & 2.75 & $<0.100$ & 20.1 \\
\hline US-39 & $1 / 1$ & 10 & Z31PF21 & 6.14 & 5.03 & 5.01 & 2.7 & $<0.100$ & 19.1 \\
\hline US-08 & $1 / 1$ & 11 & Z34PF11 & 8.2 & 3.06 & 6.26 & 2.26 & 1.71 & 20.4 \\
\hline Batch 1 & $1 / 1$ & 12 & BCHPF112 & 2.5 & 2.39 & 8.77 & 2.01 & 1.24 & 22.9 \\
\hline LRM & $1 / 1$ & 13 & LRMPF112 & 5.17 & 2.42 & 0.912 & 0.069 & $<0.100$ & 24.6 \\
\hline US-05 & $1 / 1$ & 18 & Z30PF21 & 8.45 & 5.21 & 5.39 & 0.919 & 1.81 & 18.5 \\
\hline US-39 & $1 / 1$ & 19 & Z31PF11 & 6.32 & 5.18 & 5.03 & 2.79 & $<0.100$ & 19 \\
\hline US-19 & $1 / 1$ & 20 & Z18PF11 & 7.68 & 4.48 & 5.36 & 1.66 & 1.43 & 19 \\
\hline US-19 & $1 / 1$ & 21 & Z18PF21 & 7.68 & 4.49 & 5.48 & 1.68 & 1.45 & 19.4 \\
\hline US-02 & $1 / 1$ & 22 & Z35PF21 & 8.38 & 5.17 & 4.45 & 2.34 & 0.424 & 18.6 \\
\hline Batch 1 & $1 / 1$ & 23 & BCHPF113 & 2.5 & 2.44 & 8.72 & 2.03 & 1.22 & 23.1 \\
\hline LRM & $1 / 1$ & 24 & LRMPF113 & 5.17 & 2.45 & 0.907 & 0.07 & $<0.100$ & 25.4 \\
\hline Batch 1 & $1 / 2$ & 1 & BCHPF121 & 2.52 & 2.65 & 8.99 & 2.04 & 1.28 & 24 \\
\hline LRM & $1 / 2$ & 2 & LRMPF121 & 5.21 & 2.61 & 0.973 & 0.072 & $<0.100$ & 25.3 \\
\hline US-19 & $1 / 2$ & 3 & Z18PF22 & 7.75 & 4.6 & 5.65 & 1.69 & 1.52 & 19.3 \\
\hline US-29 & $1 / 2$ & 4 & Z23PF12 & 5.39 & 2.86 & 3.54 & 2.73 & $<0.100$ & 20.8 \\
\hline US-39 & $1 / 2$ & 5 & Z31PF22 & 6.17 & 5.13 & 5.11 & 2.72 & $<0.100$ & 18.8 \\
\hline LRM & $1 / 2$ & 13 & LRMPF122 & 5.21 & 2.54 & 1 & 0.073 & $<0.100$ & 25.5 \\
\hline US-08 & $1 / 2$ & 14 & Z34PF12 & 8.18 & 3.17 & 6.39 & 2.26 & 1.76 & 20.9 \\
\hline US-39 & $1 / 2$ & 15 & Z31PF12 & 6.45 & 5.38 & 5.39 & 2.85 & $<0.100$ & 19.3 \\
\hline US-19 & $1 / 2$ & 16 & Z18PF12 & 7.78 & 4.68 & 5.73 & 1.7 & 1.55 & 18.7 \\
\hline US-05 & $1 / 2$ & 17 & Z30PF22 & 8.5 & 5.39 & 5.77 & 0.93 & 1.94 & 18.5 \\
\hline US-20 & $1 / 2$ & 18 & Z40PF12 & 7.83 & 4.76 & 6.12 & 1.7 & 1.6 & 19.1 \\
\hline US-05 & $1 / 2$ & 19 & Z30PF12 & 8.66 & 5.52 & 5.7 & 0.945 & 1.95 & 18.1 \\
\hline US-29 & $1 / 2$ & 20 & Z23PF22 & 5.51 & 2.9 & 3.44 & 2.77 & $<0.100$ & 21.7 \\
\hline US-08 & $1 / 2$ & 21 & Z34PF22 & 8.34 & 3.24 & 6.58 & 2.3 & 1.83 & 20.5 \\
\hline US-02 & $1 / 2$ & 22 & Z35PF12 & 8.65 & 5.37 & 4.8 & 2.41 & 0.501 & 19.4 \\
\hline Batch 1 & $1 / 2$ & 23 & BCHPF123 & 2.55 & 2.61 & 9.25 & 2.06 & 1.32 & 23.9 \\
\hline LRM & $1 / 2$ & 24 & LRMPF123 & 5.29 & 2.64 & 1.03 & 0.074 & $<0.100$ & 26.5 \\
\hline Batch 1 & $2 / 1$ & 1 & BCHPF211 & 2.57 & 2.58 & 9.18 & 2.09 & 1.26 & 22.4 \\
\hline LRM & $2 / 1$ & 2 & LRMPF211 & 5.42 & 2.58 & 1.15 & 0.098 & $<0.100$ & 25.1 \\
\hline US-11 & $2 / 1$ & 3 & Z20PF11 & 7.75 & 6.45 & 4.95 & 0.945 & 2.42 & 20.6 \\
\hline US-34 & $2 / 1$ & 4 & Z27PF21 & 5.53 & 4.5 & 4.57 & 1.14 & 3.22 & 16.7 \\
\hline US-06 & $2 / 1$ & 5 & Z39PF21 & 8.63 & 4.52 & 5.48 & 1.16 & 2.34 & 19 \\
\hline US-18 & $2 / 1$ & 6 & Z12PF11 & 8.9 & 2.99 & 6.73 & 2.34 & 2.4 & 19.5 \\
\hline US-12 & $2 / 1$ & 7 & Z01PF21 & 7.25 & 5.1 & 6.3 & 2.8 & $<0.100$ & 18.1 \\
\hline US-34 & $2 / 1$ & 8 & Z27PF11 & 5.38 & 4.27 & 4.34 & 1.12 & 3.09 & 16.2 \\
\hline US-06 & $2 / 1$ & 9 & Z39PF11 & 8.56 & 4.5 & 5.63 & 1.16 & 2.38 & 18.6 \\
\hline US-18 & $2 / 1$ & 10 & Z12PF21 & 8.67 & 2.92 & 6.81 & 2.3 & 2.38 & 19 \\
\hline US-04 & $2 / 1$ & 11 & Z10PF11 & 8.69 & 3.6 & 5.75 & 1.76 & 1.72 & 19.4 \\
\hline
\end{tabular}




\section{Table A3. Measured Elemental Concentrations (wt\%)} for Samples Prepared Using Peroxide Fusion

\begin{tabular}{|c|c|c|c|c|c|c|c|c|c|}
\hline Glass ID & $\begin{array}{c}\text { Block/ } \\
\text { Sub-Block }\end{array}$ & Sequence & Lab ID & $\mathrm{Al}(\mathrm{wt} \%)$ & B $(w t \%)$ & $\mathrm{Fe}(w \mathrm{w} \%)$ & $\mathrm{Li}(w \mathrm{w} \%)$ & $\begin{array}{c}\text { Mn } \\
(w t \%)\end{array}$ & $\mathrm{Si}(\mathrm{wt} \%)$ \\
\hline US-24 & $2 / 1$ & 20 & Z45PF21 & 7.97 & 3.89 & 5.11 & 1.41 & 1.72 & 19.6 \\
\hline US-24 & $2 / 1$ & 22 & Z45PF11 & 7.97 & 3.98 & 5.65 & 1.51 & 1.88 & 19.5 \\
\hline Batch 1 & $2 / 1$ & 23 & BCHPF213 & 2.53 & 2.41 & 9.17 & 2.06 & 1.27 & 22.5 \\
\hline LRM & $2 / 1$ & 24 & LRMPF213 & 5.27 & 2.42 & 0.89 & 0.074 & $<0.100$ & 24.4 \\
\hline US-12 & $2 / 2$ & 3 & Z01PF12 & 7.17 & 5.12 & 6.19 & 2.75 & $<0.100$ & 18.3 \\
\hline US-03 & $2 / 2$ & 4 & Z26PF12 & 8.45 & 5.6 & 4.8 & 1.85 & $<0.100$ & 20 \\
\hline US-06 & $2 / 2$ & 5 & Z39PF22 & 8.6 & 4.54 & 5.59 & 1.15 & 2.39 & 19.6 \\
\hline US-33 & $2 / 2$ & 6 & Z09PF12 & 6.26 & 1.78 & 3.54 & 0.939 & 3.26 & 21.5 \\
\hline US-11 & $2 / 2$ & 7 & Z20PF12 & 7.75 & 6.5 & 4.95 & 0.945 & 2.47 & 21.2 \\
\hline Batch 1 & $2 / 2$ & 12 & BCHPF222 & 2.53 & 2.58 & 9.13 & 2.01 & 1.3 & 23.2 \\
\hline LRM & $2 / 2$ & 13 & LRMPF222 & 5.21 & 2.58 & 1 & 0.07 & $<0.100$ & 25.4 \\
\hline US-11 & $2 / 2$ & 14 & Z20PF22 & 7.6 & 6.51 & 4.96 & 0.938 & 2.42 & 20.5 \\
\hline US-18 & $2 / 2$ & 15 & Z12PF12 & 8.88 & 3.08 & 6.67 & 2.29 & 2.42 & 19.9 \\
\hline US-24 & $2 / 2$ & 16 & Z45PF22 & 7.95 & 3.98 & 5.11 & 1.38 & 1.75 & 19.5 \\
\hline US-18 & $2 / 2$ & 17 & Z12PF22 & 8.59 & 2.98 & 6.72 & 2.24 & 2.36 & 19 \\
\hline US-06 & $2 / 2$ & 18 & Z39PF12 & 8.54 & 4.5 & 5.66 & 1.14 & 2.4 & 18.5 \\
\hline US-33 & $2 / 2$ & 19 & Z09PF22 & 6.39 & 1.79 & 3.65 & 0.955 & 3.37 & 21.7 \\
\hline US-12 & $2 / 2$ & 20 & Z01PF22 & 7.27 & 5.1 & 6.37 & 2.75 & $<0.100$ & 18.2 \\
\hline US-04 & $2 / 2$ & 21 & Z10PF22 & 8.67 & 3.64 & 5.62 & 1.72 & 1.71 & 18.9 \\
\hline US-04 & $2 / 2$ & 22 & Z10PF12 & 8.58 & 3.62 & 5.55 & 1.71 & 1.68 & 18.9 \\
\hline Batch 1 & $2 / 2$ & 23 & BCHPF223 & 2.51 & 2.48 & 8.84 & 1.99 & 1.27 & 22.6 \\
\hline US-28 & $3 / 1$ & 7 & Z33PF11 & 5.57 & 1.97 & 12.8 & 0.908 & $<0.100$ & 18.3 \\
\hline US-28 & $3 / 1$ & 8 & Z33PF21 & 5.51 & 1.83 & 12.1 & 0.889 & $<0.100$ & 17.6 \\
\hline US-43 & $3 / 1$ & 9 & Z21PF11 & 9.35 & 2.65 & 6.39 & 2.26 & 0.518 & 18.5 \\
\hline US-16 & $3 / 1$ & 10 & Z44PF21 & 7.25 & 5.89 & 6.42 & 1.16 & 1.96 & 20.5 \\
\hline US-15 & $3 / 1$ & 11 & Z42PF11 & 5.5 & 5.63 & 3.68 & 0.897 & $<0.100$ & 22.1 \\
\hline Batch 1 & $3 / 1$ & 12 & BCHPF312 & 2.51 & 2.34 & 9.25 & 2 & 1.26 & 23.6 \\
\hline LRM & $3 / 1$ & 13 & LRMPF312 & 5.24 & 2.44 & 0.95 & 0.035 & $<0.100$ & 26.1 \\
\hline US-45 & $3 / 1$ & 14 & Z38PF21 & 6.85 & 3.92 & 6.05 & 1.84 & 1.3 & 18.5 \\
\hline US-16 & $3 / 1$ & 15 & Z44PF11 & 7.19 & 5.86 & 6.14 & 1.15 & 1.88 & 20.6 \\
\hline US-15 & $3 / 1$ & 16 & Z42PF21 & 5.41 & 5.54 & 3.53 & 0.887 & $<0.100$ & 21.3 \\
\hline US-07 & $3 / 1$ & 17 & Z19PF11 & 8.54 & 4.16 & 4.8 & 1.18 & 2.27 & 20.1 \\
\hline US-32 & $3 / 1$ & 18 & Z06PF21 & 5.56 & 5.91 & 3.35 & 2.76 & 3.14 & 20.9 \\
\hline US-43 & $3 / 1$ & 19 & Z21PF21 & 9.3 & 2.74 & 6.47 & 2.24 & 0.525 & 17.5 \\
\hline US-40 & $3 / 1$ & 20 & Z22PF21 & 8.41 & 5.32 & 7.99 & 1.15 & 2.16 & 18 \\
\hline US-42 & $3 / 1$ & 21 & Z15PF11 & 10.7 & 1.95 & 3.55 & 2.72 & 3.12 & 16.8 \\
\hline US-40 & $3 / 1$ & 22 & Z22PF11 & 8.23 & 5.2 & 7.66 & 1.11 & 2.08 & 17.5 \\
\hline Batch 1 & $3 / 1$ & 23 & BCHPF313 & 2.53 & 2.36 & 8.95 & 2 & 1.22 & 23.4 \\
\hline LRM & $3 / 1$ & 24 & LRMPF313 & 5.22 & 2.36 & 0.925 & 0.034 & $<0.100$ & 25.3 \\
\hline Batch 1 & $3 / 2$ & 1 & BCHPF321 & 2.52 & 2.55 & 8.77 & 2.03 & 1.27 & 22.2 \\
\hline LRM & $3 / 2$ & 2 & LRMPF321 & 5.14 & 2.51 & 0.989 & 0.134 & $<0.100$ & 24.3 \\
\hline US-16 & $3 / 2$ & 3 & Z44PF22 & 7.22 & 5.94 & 6.2 & 1.24 & 1.94 & 18.8 \\
\hline US-32 & $3 / 2$ & 4 & Z06PF12 & 5.52 & 5.9 & 3.55 & 2.96 & 3.41 & 20.8 \\
\hline US-28 & $3 / 2$ & 5 & Z33PF12 & 5.53 & 2.01 & 12.2 & 0.986 & $<0.100$ & 18 \\
\hline
\end{tabular}




\section{Table A3. Measured Elemental Concentrations (wt\%)} for Samples Prepared Using Peroxide Fusion

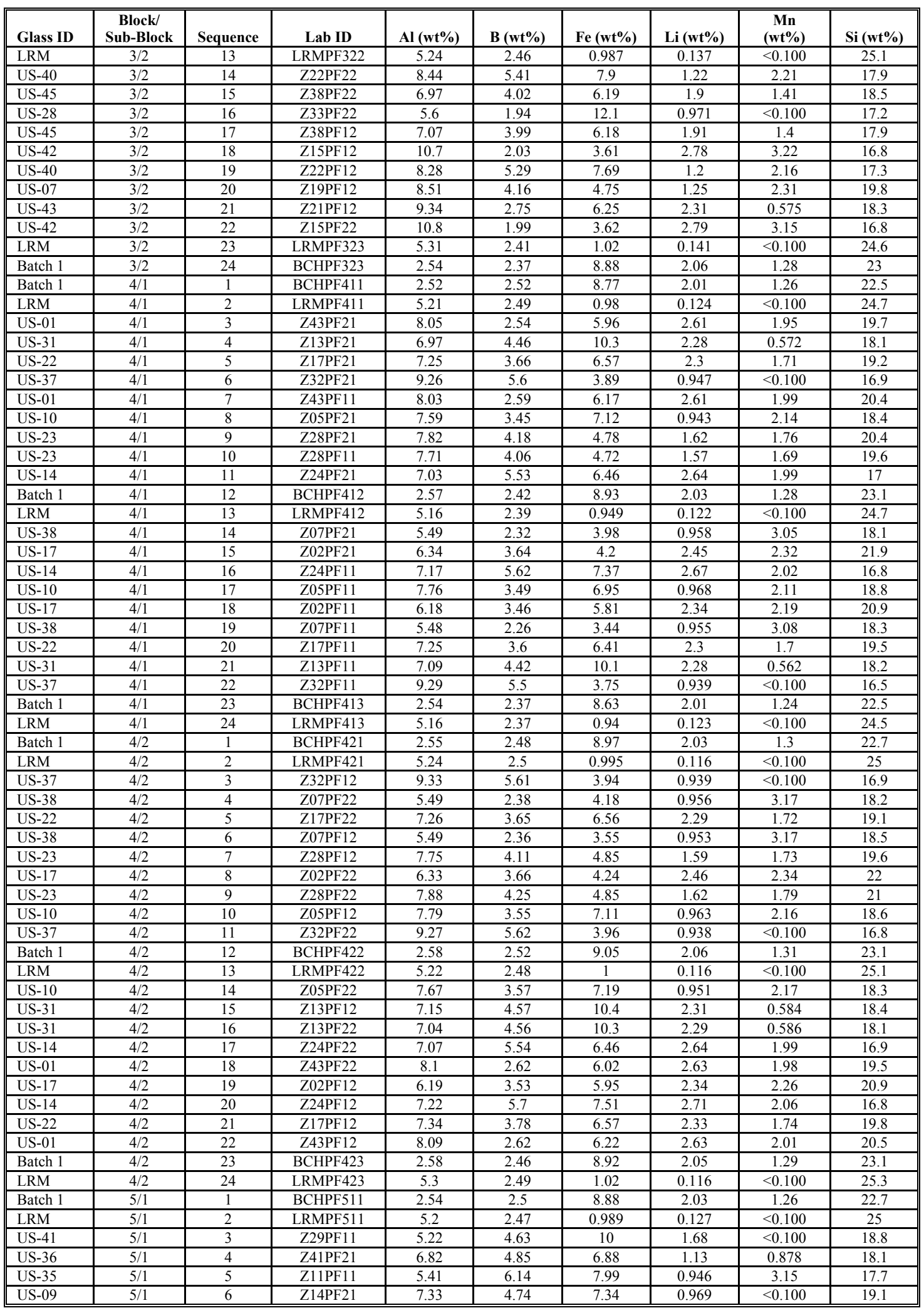




\section{Table A3. Measured Elemental Concentrations (wt\%)} for Samples Prepared Using Peroxide Fusion

\begin{tabular}{|c|c|c|c|c|c|c|c|c|c|}
\hline Glass ID & $\begin{array}{c}\text { Block/ } \\
\text { Sub-Block }\end{array}$ & Sequence & Lab ID & $\mathrm{Al}(w \mathrm{t} \%)$ & B (wt\%) & $\mathrm{Fe}(w \mathrm{w} \%)$ & Li (wt\%) & $\begin{array}{c}\text { Mn } \\
(w t \%)\end{array}$ & $\mathrm{Si}(\mathrm{wt} \%)$ \\
\hline US-09 & $5 / 1$ & 8 & Z14PF11 & 7.31 & 4.66 & 7.41 & 0.96 & $<0.100$ & 19.1 \\
\hline US-36 & $5 / 1$ & 10 & Z41PF11 & 6.86 & 4.67 & 6.64 & 1.12 & 0.853 & 17.4 \\
\hline US-30 & $5 / 1$ & 11 & Z03PF21 & 7 & 5.66 & 3.75 & 2.77 & 2.73 & 17.9 \\
\hline Batch 1 & $5 / 1$ & 12 & BCHPF512 & 2.54 & 2.32 & 8.96 & 2.02 & 1.27 & 22.9 \\
\hline US-13 & $5 / 1$ & 15 & Z04PF21 & 8.68 & 4.52 & 4.87 & 1.03 & 2.18 & 19.7 \\
\hline US-30 & $5 / 1$ & 16 & Z03PF11 & 6.83 & 5.69 & 3.71 & 2.78 & 2.71 & 17.7 \\
\hline US-41 & $5 / 1$ & 17 & Z29PF21 & 5.47 & 4.8 & 10.7 & 1.71 & $<0.100$ & 19.2 \\
\hline US-44 & $5 / 1$ & 18 & Z25PF21 & 7.41 & 3.76 & 6.14 & 2.21 & 1.38 & 17.5 \\
\hline US-13 & $5 / 1$ & 19 & Z04PF11 & 8.69 & 4.5 & 4.88 & 1.02 & 2.15 & 19.6 \\
\hline LRM & $5 / 1$ & 24 & LRMPF513 & 5.24 & 2.34 & 0.918 & 0.126 & $<0.100$ & 24.7 \\
\hline Batch 1 & $5 / 2$ & 1 & BCHPF521 & 2.52 & 2.51 & 9 & 2.01 & 1.26 & 22.8 \\
\hline LRM & $5 / 2$ & 2 & LRMPF521 & 5.15 & 2.46 & 0.956 & 0.1 & $<0.100$ & 25 \\
\hline US-13 & $5 / 2$ & 3 & Z04PF22 & 8.54 & 4.59 & 4.97 & 1.01 & 2.21 & 19.9 \\
\hline US-36 & $5 / 2$ & 4 & Z41PF12 & 6.87 & 4.81 & 6.82 & 1.1 & 0.853 & 17.7 \\
\hline US-41 & $5 / 2$ & 5 & Z29PF12 & 5.26 & 4.72 & 10.1 & 1.69 & $<0.100$ & 19 \\
\hline US-21 & $5 / 2$ & 6 & Z16PF22 & 7.86 & 4.39 & 5.64 & 1.41 & 1.34 & 21 \\
\hline US-35 & $5 / 2$ & 7 & Z11PF12 & 5.45 & 6.21 & 8.01 & 0.92 & 3.15 & 17.9 \\
\hline US-09 & $5 / 2$ & 8 & Z14PF12 & 7.32 & 4.74 & 7.45 & 0.94 & $<0.100$ & 19.2 \\
\hline US-44 & $5 / 2$ & 9 & Z25PF22 & 7.39 & 3.91 & 6.26 & 2.19 & 1.38 & 17.6 \\
\hline US-35 & $5 / 2$ & 10 & Z11PF22 & 5.49 & 6.22 & 7.92 & 0.94 & 3.17 & 17.8 \\
\hline US-30 & $5 / 2$ & 11 & Z03PF22 & 6.88 & 5.77 & 3.8 & 2.75 & 2.75 & 18 \\
\hline US-44 & $5 / 2$ & 19 & Z25PF12 & 6.98 & 3.91 & 6.08 & 1.87 & 1.34 & 17.5 \\
\hline US-36 & $5 / 2$ & 20 & Z41PF22 & 6.83 & 4.85 & 6.79 & 1.11 & 0.84 & 18 \\
\hline US-25 & $5 / 2$ & 21 & Z08PF12 & 6.38 & 5.09 & 5.98 & 2.29 & 1.63 & 18.2 \\
\hline US-09 & $5 / 2$ & 22 & Z14PF22 & 7.34 & 4.74 & 7.35 & 0.947 & $<0.100$ & 19.3 \\
\hline Batch 1 & $5 / 2$ & 23 & BCHPF523 & 2.55 & 2.43 & 9.01 & 2.03 & 1.26 & 23.3 \\
\hline LRM & $5 / 2$ & 24 & LRMPF523 & 5.21 & 2.4 & 0.96 & 0.101 & $<0.100$ & 25.4 \\
\hline
\end{tabular}


Table A4. Measured, Measured Bias-Corrected, and Targeted Compositions by Glass Number

\begin{tabular}{|c|c|c|c|c|c|c|c|c|c|}
\hline & & & & Measured & & & & & \\
\hline Glass & & & Measured & $\begin{array}{c}\text { Bias- } \\
\text { Corrected }\end{array}$ & Targeted & Diff of & Diff of & $\%$ Diff of & $\%$ Diff of \\
\hline$\#$ & GlassID & Oxide & $\left(w^{\prime} \%\right)$ & $(w t \%)$ & $(\mathrm{wt} \%)$ & Measured & Meas BC & Measured & Meas BC \\
\hline 100 & Batch 1 & Al2O3 & 4.7974 & 4.8770 & 4.8770 & -0.0796 & 0.0000 & $-1.6 \%$ & $0.0 \%$ \\
\hline 100 & Batch 1 & $\mathrm{~B} 2 \mathrm{O} 3$ & 7.9714 & 7.7770 & 7.7770 & 0.1944 & 0.0000 & $2.5 \%$ & $0.0 \%$ \\
\hline 100 & Batch 1 & $\mathrm{CaO}$ & 1.1288 & 1.2200 & 1.2200 & -0.0912 & 0.0000 & $-7.5 \%$ & $0.0 \%$ \\
\hline 100 & Batch 1 & $\mathrm{Cr} 2 \mathrm{O} 3$ & 0.1092 & 0.1070 & 0.1070 & 0.0022 & 0.0000 & & \\
\hline 100 & Batch 1 & $\mathrm{Fe} 2 \mathrm{O} 3$ & 12.8301 & 12.8390 & 12.8390 & -0.0089 & 0.0000 & $-0.1 \%$ & $0.0 \%$ \\
\hline 100 & Batch 1 & $\mathrm{~K} 2 \mathrm{O}$ & 3.7700 & 3.3270 & 3.3270 & 0.4430 & 0.0000 & $13.3 \%$ & $0.0 \%$ \\
\hline 100 & Batch 1 & $\mathrm{Li} 2 \mathrm{O}$ & 4.3747 & 4.4290 & 4.4290 & -0.0543 & 0.0000 & $-1.2 \%$ & $0.0 \%$ \\
\hline 100 & Batch 1 & $\mathrm{MgO}$ & 1.3095 & 1.4190 & 1.4190 & -0.1095 & 0.0000 & $-7.7 \%$ & $0.0 \%$ \\
\hline 100 & Batch 1 & $\mathrm{MnO}$ & 1.6403 & 1.7260 & 1.7260 & -0.0857 & 0.0000 & $-5.0 \%$ & $0.0 \%$ \\
\hline 100 & Batch 1 & $\mathrm{Na} 2 \mathrm{O}$ & 9.2239 & 9.0030 & 9.0030 & 0.2209 & 0.0000 & $2.5 \%$ & $0.0 \%$ \\
\hline 100 & Batch 1 & $\mathrm{NiO}$ & 0.6772 & 0.7510 & 0.7510 & -0.0738 & 0.0000 & $-9.8 \%$ & $0.0 \%$ \\
\hline 100 & Batch 1 & $\mathrm{PbO}$ & 0.0052 & 0.0052 & 0.0000 & 0.0052 & 0.0052 & & \\
\hline 100 & Batch 1 & $\mathrm{SiO} 2$ & 49.1540 & 50.2200 & 50.2200 & -1.0660 & 0.0000 & $-2.1 \%$ & $0.0 \%$ \\
\hline 100 & Batch 1 & $\mathrm{SO} 3$ & 0.0624 & 0.0624 & 0.0000 & 0.0624 & 0.0624 & & \\
\hline 100 & Batch 1 & $\mathrm{SrO}$ & 0.0059 & 0.0059 & 0.0000 & 0.0059 & 0.0059 & & \\
\hline 100 & Batch 1 & $\mathrm{TiO} 2$ & 0.6256 & 0.6770 & 0.6770 & -0.0514 & 0.0000 & $-7.6 \%$ & $0.0 \%$ \\
\hline 100 & Batch 1 & $\mathrm{ZnO}$ & 0.0068 & 0.0068 & 0.0000 & 0.0068 & 0.0068 & & \\
\hline 100 & Batch 1 & $\mathrm{ZrO} 2$ & 0.0902 & 0.0902 & 0.0980 & -0.0078 & -0.0078 & & \\
\hline 100 & Batch 1 & Sum & 97.7828 & 98.5427 & 98.4700 & -0.6872 & 0.0727 & $-0.7 \%$ & $0.1 \%$ \\
\hline 200 & LRM & $\mathrm{A} 12 \mathrm{O} 3$ & 9.8789 & 10.0430 & 10.0000 & -0.1211 & 0.0430 & $-1.2 \%$ & $0.4 \%$ \\
\hline 200 & LRM & B2O3 & 7.9617 & 7.7675 & 8.0000 & -0.0383 & -0.2325 & $-0.5 \%$ & $-2.9 \%$ \\
\hline 200 & LRM & $\mathrm{CaO}$ & 0.5202 & 0.5622 & 0.5000 & 0.0202 & 0.0622 & $4.0 \%$ & $12.4 \%$ \\
\hline 200 & LRM & $\mathrm{Cr} 2 \mathrm{O} 3$ & 0.1964 & 0.1924 & 0.2000 & -0.0036 & -0.0076 & & \\
\hline 200 & LRM & $\mathrm{Fe} 2 \mathrm{O} 3$ & 1.3994 & 1.4005 & 1.0000 & 0.3994 & 0.4005 & $39.9 \%$ & $40.1 \%$ \\
\hline 200 & LRM & $\mathrm{K} 2 \mathrm{O}$ & 1.4652 & 1.2929 & 1.5000 & -0.0348 & -0.2071 & $-2.3 \%$ & $-13.8 \%$ \\
\hline 200 & LRM & $\mathrm{Li} 2 \mathrm{O}$ & 0.2023 & 0.2046 & 0.1000 & 0.1023 & 0.1046 & & \\
\hline 200 & LRM & $\mathrm{MgO}$ & 0.0958 & 0.1038 & 0.1000 & -0.0042 & 0.0038 & & \\
\hline 200 & LRM & $\mathrm{MnO}$ & 0.0646 & 0.0680 & 0.1000 & -0.0354 & -0.0320 & & \\
\hline 200 & LRM & $\mathrm{Na} 2 \mathrm{O}$ & 20.7457 & 20.2492 & 20.0000 & 0.7457 & 0.2492 & $3.7 \%$ & $1.2 \%$ \\
\hline 200 & LRM & $\mathrm{NiO}$ & 0.1718 & 0.1906 & 0.1000 & 0.0718 & 0.0906 & & \\
\hline 200 & LRM & $\mathrm{PbO}$ & 0.0881 & 0.0881 & 0.1000 & -0.0119 & -0.0119 & & \\
\hline 200 & LRM & $\mathrm{SiO} 2$ & 53.6964 & 54.8662 & 54.3700 & -0.6736 & 0.4962 & $-1.2 \%$ & $0.9 \%$ \\
\hline 200 & LRM & SO3 & 0.2120 & 0.2120 & 0.2000 & 0.0120 & 0.0120 & & \\
\hline 200 & LRM & $\mathrm{SrO}$ & 0.0059 & 0.0059 & 0.0000 & 0.0059 & 0.0059 & & \\
\hline 200 & LRM & $\mathrm{TiO} 2$ & 0.0956 & 0.1035 & 0.1000 & -0.0044 & 0.0035 & & \\
\hline 200 & LRM & $\mathrm{ZnO}$ & 0.0086 & 0.0086 & 0.0000 & 0.0086 & 0.0086 & & \\
\hline 200 & LRM & $\mathrm{ZrO} 2$ & 0.8795 & 0.8795 & 1.0000 & -0.1205 & -0.1205 & $-12.0 \%$ & $-12.0 \%$ \\
\hline 200 & LRM & Sum & 97.6883 & 98.2385 & 97.3700 & 0.3183 & 0.8685 & $0.3 \%$ & $0.9 \%$ \\
\hline 1 & US-01 & $\mathrm{Al} 2 \mathrm{O} 3$ & 15.2435 & 15.3894 & 14.9289 & 0.3146 & 0.4605 & $2.1 \%$ & $3.1 \%$ \\
\hline 1 & US-01 & $\mathrm{B} 2 \mathrm{O} 3$ & 8.3476 & 8.1903 & 7.8908 & 0.4568 & 0.2995 & $5.8 \%$ & $3.8 \%$ \\
\hline 1 & US-01 & $\mathrm{CaO}$ & 0.1203 & 0.1314 & 0.0000 & 0.1203 & 0.1314 & & \\
\hline 1 & US-01 & $\mathrm{Cr} 2 \mathrm{O} 3$ & 0.2708 & 0.2649 & 0.2999 & -0.0292 & -0.0350 & & \\
\hline 1 & US-01 & $\mathrm{Fe} 2 \mathrm{O} 3$ & 8.7104 & 8.8111 & 8.5893 & 0.1212 & 0.2218 & $1.4 \%$ & $2.6 \%$ \\
\hline 1 & US-01 & $\mathrm{K} 2 \mathrm{O}$ & 0.9974 & 0.8842 & 0.9998 & -0.0024 & -0.1156 & $-0.2 \%$ & $-11.6 \%$ \\
\hline 1 & US-01 & Li2O & 5.6406 & 5.7117 & 5.6159 & 0.0247 & 0.0958 & $0.4 \%$ & $1.7 \%$ \\
\hline 1 & US-01 & $\mathrm{MgO}$ & 0.5128 & 0.5586 & 0.4999 & 0.0129 & 0.0587 & & \\
\hline 1 & US-01 & $\mathrm{MnO}$ & 2.5598 & 2.6737 & 2.4975 & 0.0623 & 0.1762 & $2.5 \%$ & $7.1 \%$ \\
\hline 1 & US-01 & $\mathrm{Na} 2 \mathrm{O}$ & 14.1338 & 13.7990 & 12.3719 & 1.7619 & 1.4271 & $14.2 \%$ & $11.5 \%$ \\
\hline 1 & US-01 & $\mathrm{NiO}$ & 0.0560 & 0.0621 & 0.0568 & -0.0008 & 0.0054 & & \\
\hline 1 & US-01 & $\mathrm{PbO}$ & 0.0595 & 0.0595 & 0.1000 & -0.0405 & -0.0405 & & \\
\hline 1 & US-01 & $\mathrm{SiO} 2$ & 42.8395 & 44.0451 & 45.5296 & -2.6901 & -1.4844 & $-5.9 \%$ & $-3.3 \%$ \\
\hline 1 & US-01 & $\mathrm{SO} 3$ & 0.0624 & 0.0624 & 0.0000 & 0.0624 & 0.0624 & & \\
\hline 1 & US-01 & $\mathrm{SrO}$ & 0.0896 & 0.0896 & 0.1000 & -0.0104 & -0.0104 & & \\
\hline 1 & US-01 & $\mathrm{TiO} 2$ & 0.0125 & 0.0136 & 0.0000 & 0.0125 & 0.0136 & & \\
\hline 1 & US-01 & $\mathrm{ZnO}$ & 0.0062 & 0.0062 & 0.0000 & 0.0062 & 0.0062 & & \\
\hline 1 & US-01 & $\mathrm{ZrO} 2$ & 0.4633 & 0.4633 & 0.4999 & -0.0366 & -0.0366 & & \\
\hline 1 & US-01 & Sum & 100.1261 & 101.2161 & 99.9800 & 0.1461 & 1.2361 & $0.1 \%$ & $1.2 \%$ \\
\hline 2 & US-02 & $\mathrm{Al} 2 \mathrm{O} 3$ & 16.0419 & 16.3551 & 15.9968 & 0.0451 & 0.3583 & $0.3 \%$ & $2.2 \%$ \\
\hline 2 & US-02 & $\mathrm{B} 2 \mathrm{O} 3$ & 16.8803 & 16.0425 & 16.8552 & 0.0251 & -0.8127 & $0.1 \%$ & $-4.8 \%$ \\
\hline 2 & US-02 & $\mathrm{CaO}$ & 0.0640 & 0.0689 & 0.0000 & 0.0640 & 0.0689 & & \\
\hline 2 & US-02 & $\mathrm{Cr} 2 \mathrm{O} 3$ & 0.0248 & 0.0243 & 0.0000 & 0.0248 & 0.0243 & & \\
\hline 2 & US-02 & $\mathrm{Fe} 2 \mathrm{O} 3$ & 6.5766 & 6.5608 & 6.5893 & -0.0127 & -0.0285 & $-0.2 \%$ & $-0.4 \%$ \\
\hline 2 & US-02 & $\mathrm{K} 2 \mathrm{O}$ & 0.0602 & 0.0534 & 0.0000 & 0.0602 & 0.0534 & & \\
\hline 2 & US-02 & $\mathrm{Li} 2 \mathrm{O}$ & 5.1024 & 5.1370 & 5.2913 & -0.1889 & -0.1543 & $-3.6 \%$ & $-2.9 \%$ \\
\hline 2 & US-02 & $\mathrm{MgO}$ & 0.0083 & 0.0089 & 0.0000 & 0.0083 & 0.0089 & & \\
\hline 2 & US-02 & $\mathrm{MnO}$ & 0.5907 & 0.6173 & 0.6342 & -0.0435 & -0.0169 & $-6.9 \%$ & $-2.7 \%$ \\
\hline 2 & US-02 & $\mathrm{Na} 2 \mathrm{O}$ & 13.1295 & 12.6600 & 12.3340 & 0.7955 & 0.3260 & $6.4 \%$ & $2.6 \%$ \\
\hline 2 & US-02 & $\mathrm{NiO}$ & 0.0194 & 0.0214 & 0.0000 & 0.0194 & 0.0214 & & \\
\hline
\end{tabular}


Table A4. Measured, Measured Bias-Corrected, and Targeted Compositions by Glass Number

\begin{tabular}{|c|c|c|c|c|c|c|c|c|c|}
\hline & & & & Measured & & & & & \\
\hline Glass & & & Measured & $\begin{array}{c}\text { Bias- } \\
\text { Corrected }\end{array}$ & Targeted & Diff of & Diff of & $\%$ Diff of & $\%$ Diff of \\
\hline$\#$ & GlassID & Oxide & $(\mathrm{wt} \%)$ & $(w t \%)$ & $(\mathrm{wt} \%)$ & Measured & Meas BC & Measured & Meas BC \\
\hline 2 & US-02 & $\mathrm{PbO}$ & 0.0054 & 0.0054 & 0.0000 & 0.0054 & 0.0054 & & \\
\hline 2 & US-02 & $\mathrm{SiO} 2$ & 40.3793 & 40.4559 & 41.6792 & -1.3000 & -1.2233 & $-3.1 \%$ & $-2.9 \%$ \\
\hline 2 & US-02 & SO3 & 0.0624 & 0.0624 & 0.0000 & 0.0624 & 0.0624 & & \\
\hline 2 & US-02 & $\mathrm{SrO}$ & 0.0361 & 0.0361 & 0.0000 & 0.0361 & 0.0361 & & \\
\hline 2 & US-02 & TiO2 & 0.0634 & 0.0682 & 0.0000 & 0.0634 & 0.0682 & & \\
\hline 2 & US-02 & $\mathrm{ZnO}$ & 0.1232 & 0.1232 & 0.1000 & 0.0233 & 0.0233 & & \\
\hline 2 & US-02 & $\mathrm{ZrO} 2$ & 0.4583 & 0.4583 & 0.4999 & -0.0416 & -0.0416 & & \\
\hline 2 & US-02 & Sum & 99.6262 & 98.7593 & 99.9800 & -0.3538 & -1.2207 & $-0.4 \%$ & $-1.2 \%$ \\
\hline 3 & US-03 & $\mathrm{A} 12 \mathrm{O} 3$ & 16.0560 & 16.3588 & 15.9968 & 0.0592 & 0.3620 & $0.4 \%$ & $2.3 \%$ \\
\hline 3 & US-03 & $\mathrm{B} 2 \mathrm{O} 3$ & 17.8946 & 17.2129 & 17.3085 & 0.5861 & -0.0957 & $3.4 \%$ & $-0.6 \%$ \\
\hline 3 & US-03 & $\mathrm{CaO}$ & 0.9063 & 1.0104 & 0.9998 & -0.0935 & 0.0106 & $-9.3 \%$ & $1.1 \%$ \\
\hline 3 & US-03 & $\mathrm{Cr} 2 \mathrm{O} 3$ & 0.0161 & 0.0157 & 0.0000 & 0.0161 & 0.0157 & & \\
\hline 3 & US-03 & $\mathrm{Fe} 2 \mathrm{O} 3$ & 6.7053 & 6.6494 & 6.5259 & 0.1794 & 0.1236 & $2.7 \%$ & $1.9 \%$ \\
\hline 3 & US-03 & $\mathrm{K} 2 \mathrm{O}$ & 0.9528 & 0.8386 & 0.9998 & -0.0470 & -0.1612 & $-4.7 \%$ & $-16.1 \%$ \\
\hline 3 & US-03 & $\mathrm{Li2O}$ & 4.0205 & 4.0714 & 4.0701 & -0.0496 & 0.0013 & $-1.2 \%$ & $0.0 \%$ \\
\hline 3 & US-03 & $\mathrm{MgO}$ & 0.5033 & 0.5425 & 0.4989 & 0.0044 & 0.0436 & & \\
\hline 3 & US-03 & $\mathrm{MnO}$ & 0.0646 & 0.0678 & 0.0000 & 0.0646 & 0.0678 & & \\
\hline 3 & US-03 & $\mathrm{Na} 2 \mathrm{O}$ & 8.6205 & 8.2721 & 7.8841 & 0.7363 & 0.3879 & $9.3 \%$ & $4.9 \%$ \\
\hline 3 & US-03 & $\mathrm{NiO}$ & 0.2790 & 0.3071 & 0.2929 & -0.0139 & 0.0142 & & \\
\hline 3 & US-03 & $\mathrm{PbO}$ & 0.0926 & 0.0926 & 0.0990 & -0.0063 & -0.0063 & & \\
\hline 3 & US-03 & $\mathrm{SiO} 2$ & 42.4116 & 44.1198 & 44.2064 & -1.7948 & -0.0866 & $-4.1 \%$ & $-0.2 \%$ \\
\hline 3 & US-03 & $\mathrm{SO} 3$ & 0.0624 & 0.0624 & 0.0000 & 0.0624 & 0.0624 & & \\
\hline 3 & US-03 & $\mathrm{SrO}$ & 0.0875 & 0.0875 & 0.1000 & -0.0125 & -0.0125 & & \\
\hline 3 & US-03 & $\mathrm{TiO} 2$ & 0.9420 & 1.0246 & 0.9978 & -0.0558 & 0.0268 & $-5.6 \%$ & $2.7 \%$ \\
\hline 3 & US-03 & $\mathrm{ZnO}$ & 0.0062 & 0.0062 & 0.0000 & 0.0062 & 0.0062 & & \\
\hline 3 & US-03 & $\mathrm{ZrO} 2$ & 0.0068 & 0.0068 & 0.0000 & 0.0068 & 0.0068 & & \\
\hline 3 & US-03 & Sum & 99.6282 & 100.7465 & 99.9800 & -0.3518 & 0.7665 & $-0.4 \%$ & $0.8 \%$ \\
\hline 4 & US-04 & $\mathrm{Al} 2 \mathrm{O} 3$ & 16.3678 & 16.6764 & 15.9958 & 0.3720 & 0.6806 & $2.3 \%$ & $4.3 \%$ \\
\hline 4 & US-04 & $\mathrm{B} 2 \mathrm{O} 3$ & 11.5997 & 11.1576 & 11.2862 & 0.3135 & -0.1286 & $2.8 \%$ & $-1.1 \%$ \\
\hline 4 & US-04 & $\mathrm{CaO}$ & 0.8850 & 0.9865 & 0.9988 & -0.1138 & -0.0123 & $-11.4 \%$ & $-1.2 \%$ \\
\hline 4 & US-04 & $\mathrm{Cr} 2 \mathrm{O} 3$ & 0.0179 & 0.0174 & 0.0010 & 0.0169 & 0.0164 & & \\
\hline 4 & US-04 & $\mathrm{Fe} 2 \mathrm{O} 3$ & 8.0456 & 7.9777 & 8.2655 & -0.2199 & -0.2878 & $-2.7 \%$ & $-3.5 \%$ \\
\hline 4 & US-04 & $\mathrm{K} 2 \mathrm{O}$ & 0.9510 & 0.8369 & 0.9988 & -0.0478 & -0.1619 & $-4.8 \%$ & $-16.2 \%$ \\
\hline 4 & US-04 & Li2O & 3.7407 & 3.7878 & 3.7417 & -0.0010 & 0.0461 & $0.0 \%$ & $1.2 \%$ \\
\hline 4 & US-04 & $\mathrm{MgO}$ & 0.4962 & 0.5349 & 0.4989 & -0.0027 & 0.0360 & & \\
\hline 4 & US-04 & $\mathrm{MnO}$ & 2.1886 & 2.2980 & 2.1886 & 0.0000 & 0.1094 & $0.0 \%$ & $5.0 \%$ \\
\hline 4 & US-04 & $\mathrm{Na} 2 \mathrm{O}$ & 11.5760 & 11.1081 & 10.3325 & 1.2434 & 0.7756 & $12.0 \%$ & $7.5 \%$ \\
\hline 4 & US-04 & $\mathrm{NiO}$ & 0.0064 & 0.0070 & 0.0010 & 0.0054 & 0.0060 & & \\
\hline 4 & US-04 & $\mathrm{PbO}$ & 0.0902 & 0.0902 & 0.0990 & -0.0088 & -0.0088 & & \\
\hline 4 & US-04 & $\mathrm{SiO} 2$ & 40.9141 & 42.5750 & 43.3777 & -2.4636 & -0.8027 & $-5.7 \%$ & $-1.9 \%$ \\
\hline 4 & US-04 & $\mathrm{SO} 3$ & 0.3958 & 0.3958 & 0.4989 & -0.1031 & -0.1031 & & \\
\hline 4 & US-04 & $\mathrm{SrO}$ & 0.0887 & 0.0887 & 0.0990 & -0.0103 & -0.0103 & & \\
\hline 4 & US-04 & $\mathrm{TiO} 2$ & 0.9262 & 1.0073 & 0.9988 & -0.0726 & 0.0085 & $-7.3 \%$ & $0.9 \%$ \\
\hline 4 & US-04 & $\mathrm{ZnO}$ & 0.0952 & 0.0952 & 0.0990 & -0.0038 & -0.0038 & & \\
\hline 4 & US-04 & $\mathrm{ZrO} 2$ & 0.4302 & 0.4302 & 0.4989 & -0.0687 & -0.0687 & & \\
\hline 4 & US-04 & Sum & 98.8153 & 100.0708 & 99.9800 & -1.1648 & 0.0908 & $-1.2 \%$ & $0.1 \%$ \\
\hline 5 & US-05 & $\mathrm{A} 12 \mathrm{O} 3$ & 16.1411 & 16.4562 & 15.9938 & 0.1473 & 0.4624 & $0.9 \%$ & $2.9 \%$ \\
\hline 5 & US-05 & $\mathrm{B} 2 \mathrm{O} 3$ & 17.2265 & 16.3702 & 16.6999 & 0.5266 & -0.3297 & $3.2 \%$ & $-2.0 \%$ \\
\hline 5 & US-05 & $\mathrm{CaO}$ & 0.6709 & 0.6958 & 0.7025 & -0.0316 & -0.0067 & $-4.5 \%$ & $-1.0 \%$ \\
\hline 5 & US-05 & Cr2O3 & 0.0168 & 0.0166 & 0.0011 & 0.0157 & 0.0155 & & \\
\hline 5 & US-05 & $\mathrm{Fe} 2 \mathrm{O} 3$ & 7.9670 & 7.9478 & 8.1562 & -0.1892 & -0.2084 & $-2.3 \%$ & $-2.6 \%$ \\
\hline 5 & US-05 & $\mathrm{K} 2 \mathrm{O}$ & 1.0083 & 0.8854 & 0.9988 & 0.0094 & -0.1134 & $0.9 \%$ & $-11.4 \%$ \\
\hline 5 & US-05 & $\mathrm{Li} 2 \mathrm{O}$ & 2.0065 & 2.0201 & 2.0006 & 0.0059 & 0.0195 & $0.3 \%$ & $1.0 \%$ \\
\hline 5 & US-05 & $\mathrm{MgO}$ & 0.4971 & 0.5394 & 0.4989 & -0.0018 & 0.0405 & & \\
\hline 5 & US-05 & $\mathrm{MnO}$ & 2.4339 & 2.5445 & 2.4080 & 0.0259 & 0.1365 & $1.1 \%$ & $5.7 \%$ \\
\hline 5 & US-05 & $\mathrm{Na} 2 \mathrm{O}$ & 10.2010 & 10.1622 & 9.5815 & 0.6195 & 0.5807 & $6.5 \%$ & $6.1 \%$ \\
\hline 5 & US-05 & $\mathrm{NiO}$ & 0.0064 & 0.0071 & 0.0010 & 0.0054 & 0.0061 & & \\
\hline 5 & US-05 & $\mathrm{PbO}$ & 0.0905 & 0.0905 & 0.1000 & -0.0095 & -0.0095 & & \\
\hline 5 & US-05 & $\mathrm{SiO} 2$ & 39.2027 & 39.2867 & 40.6422 & -1.4396 & -1.3555 & $-3.5 \%$ & $-3.3 \%$ \\
\hline 5 & US-05 & SO3 & 0.2840 & 0.2840 & 0.4979 & -0.2139 & -0.2139 & & \\
\hline 5 & US-05 & $\mathrm{SrO}$ & 0.1129 & 0.1129 & 0.1000 & 0.0130 & 0.0130 & & \\
\hline 5 & US-05 & TiO2 & 0.9862 & 1.0614 & 0.9978 & -0.0116 & 0.0636 & $-1.2 \%$ & $6.4 \%$ \\
\hline 5 & US-05 & $\mathrm{ZnO}$ & 0.0968 & 0.0968 & 0.1000 & -0.0032 & -0.0032 & & \\
\hline 5 & US-05 & $\mathrm{ZrO} 2$ & 0.4947 & 0.4947 & 0.4999 & -0.0052 & -0.0052 & & \\
\hline 5 & US- 05 & Sum & 99.4432 & 99.0724 & 99.9800 & -0.5368 & -0.9076 & $-0.5 \%$ & $-0.9 \%$ \\
\hline 6 & US-06 & $\mathrm{Al} 2 \mathrm{O} 3$ & 16.2166 & 16.5226 & 15.9968 & 0.2198 & 0.5258 & $1.4 \%$ & $3.3 \%$ \\
\hline 6 & US-06 & $\mathrm{B} 2 \mathrm{O} 3$ & 14.5378 & 13.9856 & 14.0647 & 0.4732 & -0.0790 & $3.4 \%$ & $-0.6 \%$ \\
\hline 6 & US-06 & $\mathrm{CaO}$ & 0.9487 & 0.9838 & 0.9988 & -0.0501 & -0.0150 & $-5.0 \%$ & $-1.5 \%$ \\
\hline
\end{tabular}


Table A4. Measured, Measured Bias-Corrected, and Targeted Compositions by Glass Number

\begin{tabular}{|c|c|c|c|c|c|c|c|c|c|}
\hline & & & & Measured & & & & & \\
\hline Glass & & & Measured & $\begin{array}{c}\text { Bias- } \\
\text { Corrected }\end{array}$ & Targeted & Diff of & Diff of & $\%$ Diff of & $\%$ Diff of \\
\hline$\#$ & GlassID & Oxide & $(\mathrm{wt} \%)$ & $(w t \%)$ & $(\mathrm{wt} \%)$ & Measured & Meas BC & Measured & Meas BC \\
\hline 6 & US-06 & $\mathrm{Cr} 2 \mathrm{O} 3$ & 0.0179 & 0.0177 & 0.0000 & 0.0179 & 0.0177 & & \\
\hline 6 & US-06 & $\mathrm{Fe} 2 \mathrm{O} 3$ & 7.9920 & 7.9252 & 8.1540 & -0.1620 & -0.2288 & $-2.0 \%$ & $-2.8 \%$ \\
\hline 6 & US-06 & $\mathrm{K} 2 \mathrm{O}$ & 1.0025 & 0.8803 & 0.9988 & 0.0037 & -0.1185 & $0.4 \%$ & $-11.9 \%$ \\
\hline 6 & US-06 & $\mathrm{Li2O}$ & 2.4812 & 2.5127 & 2.4731 & 0.0081 & 0.0396 & $0.3 \%$ & $1.6 \%$ \\
\hline 6 & US-06 & $\mathrm{MgO}$ & 0.4946 & 0.5367 & 0.4989 & -0.0043 & 0.0378 & & \\
\hline 6 & US-06 & $\mathrm{MnO}$ & 3.0698 & 3.2229 & 2.9984 & 0.0714 & 0.2245 & $2.4 \%$ & $7.5 \%$ \\
\hline 6 & US-06 & $\mathrm{Na} 2 \mathrm{O}$ & 10.5953 & 10.5638 & 10.7776 & -0.1823 & -0.2138 & $-1.7 \%$ & $-2.0 \%$ \\
\hline 6 & US-06 & $\mathrm{NiO}$ & 0.0064 & 0.0071 & 0.0010 & 0.0054 & 0.0061 & & \\
\hline 6 & US-06 & $\mathrm{PbO}$ & 0.0054 & 0.0054 & 0.0000 & 0.0054 & 0.0054 & & \\
\hline 6 & US-06 & $\mathrm{SiO} 2$ & 40.4863 & 42.1195 & 41.4202 & -0.9340 & 0.6993 & $-2.3 \%$ & $1.7 \%$ \\
\hline 6 & US-06 & SO3 & 0.3514 & 0.3514 & 0.4999 & -0.1485 & -0.1485 & & \\
\hline 6 & US-06 & $\mathrm{SrO}$ & 0.1073 & 0.1073 & 0.0990 & 0.0083 & 0.0083 & & \\
\hline 6 & US-06 & $\mathrm{TiO} 2$ & 0.9120 & 0.9815 & 0.9988 & -0.0868 & -0.0173 & $-8.7 \%$ & $-1.7 \%$ \\
\hline 6 & US-06 & $\mathrm{ZnO}$ & 0.0180 & 0.0180 & 0.0000 & 0.0180 & 0.0180 & & \\
\hline 6 & US-06 & $\mathrm{ZrO} 2$ & 0.0236 & 0.0236 & 0.0000 & 0.0236 & 0.0236 & & \\
\hline 6 & US-06 & Sum & 99.2669 & 100.7653 & 99.9800 & -0.7131 & 0.7853 & $-0.7 \%$ & $0.8 \%$ \\
\hline 7 & US-07 & $\mathrm{A} 12 \mathrm{O} 3$ & 15.9710 & 16.3047 & 15.9958 & -0.0248 & 0.3089 & $-0.2 \%$ & $1.9 \%$ \\
\hline 7 & US-07 & $\mathrm{B} 2 \mathrm{O} 3$ & 13.2016 & 13.1143 & 13.4875 & -0.2859 & -0.3732 & $-2.1 \%$ & $-2.8 \%$ \\
\hline 7 & US-07 & $\mathrm{CaO}$ & 0.8983 & 0.9813 & 0.9988 & -0.1005 & -0.0175 & $-10.1 \%$ & $-1.8 \%$ \\
\hline 7 & US-07 & $\mathrm{Cr} 2 \mathrm{O} 3$ & 0.0135 & 0.0132 & 0.0010 & 0.0125 & 0.0122 & & \\
\hline 7 & US-07 & $\mathrm{Fe} 2 \mathrm{O} 3$ & 6.7732 & 6.7674 & 7.3001 & -0.5269 & -0.5327 & $-7.2 \%$ & $-7.3 \%$ \\
\hline 7 & US-07 & $\mathrm{K} 2 \mathrm{O}$ & 0.9586 & 0.8499 & 0.9988 & -0.0402 & -0.1489 & $-4.0 \%$ & $-14.9 \%$ \\
\hline 7 & US-07 & $\mathrm{Li} 2 \mathrm{O}$ & 2.5620 & 2.5979 & 2.6601 & -0.0981 & -0.0622 & $-3.7 \%$ & $-2.3 \%$ \\
\hline 7 & US-07 & $\mathrm{MgO}$ & 0.4958 & 0.5401 & 0.4989 & -0.0031 & 0.0412 & & \\
\hline 7 & US-07 & $\mathrm{MnO}$ & 2.9117 & 3.0856 & 2.9984 & -0.0867 & 0.0872 & $-2.9 \%$ & $2.9 \%$ \\
\hline 7 & US-07 & $\mathrm{Na} 2 \mathrm{O}$ & 11.3502 & 11.0822 & 10.6678 & 0.6823 & 0.4144 & $6.4 \%$ & $3.9 \%$ \\
\hline 7 & US-07 & $\mathrm{NiO}$ & 0.1877 & 0.2082 & 0.2017 & -0.0140 & 0.0065 & & \\
\hline 7 & US-07 & $\mathrm{PbO}$ & 0.0897 & 0.0897 & 0.0990 & -0.0093 & -0.0093 & & \\
\hline 7 & US-07 & $\mathrm{SiO} 2$ & 42.6790 & 43.4419 & 41.8776 & 0.8015 & 1.5643 & $1.9 \%$ & $3.7 \%$ \\
\hline 7 & US-07 & SO3 & 0.3596 & 0.3596 & 0.4989 & -0.1393 & -0.1393 & & \\
\hline 7 & US-07 & $\mathrm{SrO}$ & 0.0872 & 0.0872 & 0.0990 & -0.0118 & -0.0118 & & \\
\hline 7 & US-07 & $\mathrm{TiO} 2$ & 0.9291 & 1.0105 & 0.9988 & -0.0697 & 0.0117 & $-7.0 \%$ & $1.2 \%$ \\
\hline 7 & US-07 & $\mathrm{ZnO}$ & 0.0974 & 0.0974 & 0.0990 & -0.0016 & -0.0016 & & \\
\hline 7 & US-07 & $\mathrm{ZrO} 2$ & 0.4228 & 0.4228 & 0.4989 & -0.0761 & -0.0761 & & \\
\hline 7 & US-07 & Sum & 99.9882 & 101.0538 & 99.9800 & 0.0082 & 1.0738 & $0.0 \%$ & $1.1 \%$ \\
\hline 8 & US-08 & $\mathrm{A} 12 \mathrm{O} 3$ & 15.5222 & 15.8253 & 15.1931 & 0.3292 & 0.6322 & $2.2 \%$ & $4.2 \%$ \\
\hline 8 & US-08 & $\mathrm{B} 2 \mathrm{O} 3$ & 10.0783 & 9.5765 & 9.5552 & 0.5231 & 0.0213 & $5.5 \%$ & $0.2 \%$ \\
\hline 8 & US-08 & $\mathrm{CaO}$ & 0.1270 & 0.1317 & 0.0000 & 0.1270 & 0.1317 & & \\
\hline 8 & US-08 & $\mathrm{Cr} 2 \mathrm{O} 3$ & 0.0402 & 0.0398 & 0.0000 & 0.0402 & 0.0398 & & \\
\hline 8 & US-08 & $\mathrm{Fe} 2 \mathrm{O} 3$ & 9.1036 & 9.0831 & 9.7466 & -0.6430 & -0.6636 & $-6.6 \%$ & $-6.8 \%$ \\
\hline 8 & US-08 & $\mathrm{K} 2 \mathrm{O}$ & 0.9474 & 0.8320 & 0.9998 & -0.0524 & -0.1678 & $-5.2 \%$ & $-16.8 \%$ \\
\hline 8 & US-08 & $\mathrm{Li} 2 \mathrm{O}$ & 4.8925 & 4.9257 & 4.8638 & 0.0287 & 0.0619 & $0.6 \%$ & $1.3 \%$ \\
\hline 8 & US-08 & $\mathrm{MgO}$ & 0.0468 & 0.0509 & 0.0000 & 0.0468 & 0.0509 & & \\
\hline 8 & US-08 & $\mathrm{MnO}$ & 2.2596 & 2.3626 & 2.4374 & -0.1778 & -0.0749 & $-7.3 \%$ & $-3.1 \%$ \\
\hline 8 & US-08 & $\mathrm{Na} 2 \mathrm{O}$ & 10.1808 & 10.1428 & 9.8137 & 0.3671 & 0.3291 & $3.7 \%$ & $3.4 \%$ \\
\hline 8 & US-08 & $\mathrm{NiO}$ & 0.0064 & 0.0071 & 0.0010 & 0.0054 & 0.0061 & & \\
\hline 8 & US-08 & $\mathrm{PbO}$ & 0.0819 & 0.0819 & 0.0990 & -0.0171 & -0.0171 & & \\
\hline 8 & US-08 & $\mathrm{SiO} 2$ & 43.9626 & 44.0498 & 45.7717 & -1.8091 & -1.7219 & $-4.0 \%$ & $-3.8 \%$ \\
\hline 8 & US-08 & SO3 & 0.3795 & 0.3795 & 0.4989 & -0.1194 & -0.1194 & & \\
\hline 8 & US-08 & $\mathrm{SrO}$ & 0.1795 & 0.1795 & 0.0000 & 0.1795 & 0.1795 & & \\
\hline 8 & US-08 & $\mathrm{TiO} 2$ & 0.8719 & 0.9384 & 0.9998 & -0.1279 & -0.0614 & $-12.8 \%$ & $-6.1 \%$ \\
\hline 8 & US-08 & $\mathrm{ZnO}$ & 0.1332 & 0.1332 & 0.0000 & 0.1332 & 0.1332 & & \\
\hline 8 & US-08 & $\mathrm{ZrO} 2$ & 0.1540 & 0.1540 & 0.0000 & 0.1540 & 0.1540 & & \\
\hline 8 & US-08 & Sum & 98.9674 & 98.8936 & 99.9800 & -1.0126 & -1.0864 & $-1.0 \%$ & $-1.1 \%$ \\
\hline 9 & US-09 & $\mathrm{A} 12 \mathrm{O} 3$ & 13.8406 & 14.0371 & 13.3663 & 0.4743 & 0.6709 & $3.5 \%$ & $5.0 \%$ \\
\hline 9 & US-09 & B2O3 & 15.1979 & 15.0973 & 15.0479 & 0.1500 & 0.0494 & $1.0 \%$ & $0.3 \%$ \\
\hline 9 & US-09 & $\mathrm{CaO}$ & 0.9217 & 0.9559 & 0.9998 & -0.0781 & -0.0439 & $-7.8 \%$ & $-4.4 \%$ \\
\hline 9 & US-09 & $\mathrm{Cr} 2 \mathrm{O} 3$ & 0.2255 & 0.2230 & 0.2999 & -0.0745 & -0.0769 & & \\
\hline 9 & US-09 & $\mathrm{Fe} 2 \mathrm{O} 3$ & 10.5619 & 10.6021 & 10.8800 & -0.3181 & -0.2779 & $-2.9 \%$ & $-2.6 \%$ \\
\hline 9 & US-09 & $\mathrm{K} 2 \mathrm{O}$ & 0.0602 & 0.0529 & 0.0000 & 0.0602 & 0.0529 & & \\
\hline 9 & US-09 & $\mathrm{Li} 2 \mathrm{O}$ & 2.0539 & 2.0865 & 1.9996 & 0.0543 & 0.0869 & $2.7 \%$ & $4.3 \%$ \\
\hline 9 & US-09 & $\mathrm{MgO}$ & 0.4987 & 0.5411 & 0.4999 & -0.0012 & 0.0412 & & \\
\hline 9 & US-09 & $\mathrm{MnO}$ & 0.0646 & 0.0686 & 0.0000 & 0.0646 & 0.0686 & & \\
\hline 9 & US-09 & $\mathrm{Na} 2 \mathrm{O}$ & 12.9610 & 12.9106 & 14.1149 & -1.1539 & -1.2044 & $-8.2 \%$ & $-8.5 \%$ \\
\hline 9 & US-09 & $\mathrm{NiO}$ & 0.0064 & 0.0071 & 0.0000 & 0.0064 & 0.0071 & & \\
\hline 9 & US-09 & $\mathrm{PbO}$ & 0.0894 & 0.0894 & 0.1000 & -0.0106 & -0.0106 & & \\
\hline 9 & US-09 & $\mathrm{SiO} 2$ & 41.0211 & 41.8993 & 41.1719 & -0.1509 & 0.7273 & $-0.4 \%$ & $1.8 \%$ \\
\hline 9 & US-09 & SO3 & 0.3901 & 0.3901 & 0.4999 & -0.1098 & -0.1098 & & \\
\hline
\end{tabular}


Table A4. Measured, Measured Bias-Corrected, and Targeted Compositions by Glass Number

\begin{tabular}{|c|c|c|c|c|c|c|c|c|c|}
\hline & & & & Measured & & & & & \\
\hline Glass & & & Measured & $\begin{array}{c}\text { Bias- } \\
\text { Corrected }\end{array}$ & Targeted & Diff of & Diff of & $\%$ Diff of & $\%$ Diff of \\
\hline$\#$ & GlassID & Oxide & $(w t \%)$ & $(w t \%)$ & $(\mathrm{wt} \%)$ & Measured & Meas BC & Measured & Meas BC \\
\hline 9 & US-09 & $\mathrm{SrO}$ & 0.0163 & 0.0163 & 0.0000 & 0.0163 & 0.0163 & & \\
\hline 9 & US-09 & TiO2 & 0.9412 & 1.0129 & 0.9998 & -0.0586 & 0.0131 & $-5.9 \%$ & $1.3 \%$ \\
\hline 9 & US-09 & $\mathrm{ZnO}$ & 0.0062 & 0.0062 & 0.0000 & 0.0062 & 0.0062 & & \\
\hline 9 & US-09 & $\mathrm{ZrO} 2$ & 0.0068 & 0.0068 & 0.0000 & 0.0068 & 0.0068 & & \\
\hline 9 & US-09 & Sum & 98.8634 & 100.0032 & 99.9800 & -1.1166 & 0.0232 & $-1.1 \%$ & $0.0 \%$ \\
\hline 10 & US-10 & $\mathrm{A} 12 \mathrm{O} 3$ & 14.5539 & 14.6931 & 14.1233 & 0.4306 & 0.5698 & $3.0 \%$ & $4.0 \%$ \\
\hline 10 & US-10 & $\mathrm{B} 2 \mathrm{O} 3$ & 11.3179 & 11.1044 & 10.9925 & 0.3255 & 0.1119 & $3.0 \%$ & $1.0 \%$ \\
\hline 10 & US-10 & $\mathrm{CaO}$ & 0.9273 & 0.9984 & 0.9988 & -0.0715 & -0.0004 & $-7.2 \%$ & $0.0 \%$ \\
\hline 10 & US-10 & $\mathrm{Cr} 2 \mathrm{O} 3$ & 0.0175 & 0.0172 & 0.0010 & 0.0165 & 0.0162 & & \\
\hline 10 & US-10 & $\mathrm{Fe} 2 \mathrm{O} 3$ & 10.1401 & 10.2569 & 10.6855 & -0.5453 & -0.4286 & $-5.1 \%$ & $-4.0 \%$ \\
\hline 10 & US-10 & $\mathrm{K} 2 \mathrm{O}$ & 0.9911 & 0.8793 & 0.9988 & -0.0077 & -0.1195 & $-0.8 \%$ & $-12.0 \%$ \\
\hline 10 & US-10 & $\mathrm{Li} 2 \mathrm{O}$ & 2.0587 & 2.0847 & 2.0006 & 0.0581 & 0.0841 & $2.9 \%$ & $4.2 \%$ \\
\hline 10 & US-10 & $\mathrm{MgO}$ & 0.5008 & 0.5403 & 0.4989 & 0.0019 & 0.0414 & & \\
\hline 10 & US-10 & $\mathrm{MnO}$ & 2.7696 & 2.8927 & 2.7369 & 0.0327 & 0.1558 & $1.2 \%$ & $5.7 \%$ \\
\hline 10 & US-10 & $\mathrm{Na} 2 \mathrm{O}$ & 13.4733 & 12.9919 & 12.6736 & 0.7997 & 0.3183 & $6.3 \%$ & $2.5 \%$ \\
\hline 10 & US-10 & $\mathrm{NiO}$ & 0.0064 & 0.0070 & 0.0010 & 0.0054 & 0.0060 & & \\
\hline 10 & US-10 & $\mathrm{PbO}$ & 0.0883 & 0.0883 & 0.0990 & -0.0106 & -0.0106 & & \\
\hline 10 & US-10 & $\mathrm{SiO} 2$ & 39.6305 & 40.7465 & 41.9756 & -2.3451 & -1.2291 & $-5.6 \%$ & $-2.9 \%$ \\
\hline 10 & US-10 & $\mathrm{SO} 3$ & 0.3983 & 0.3983 & 0.4989 & -0.1006 & -0.1006 & & \\
\hline 10 & US-10 & $\mathrm{SrO}$ & 0.0899 & 0.0899 & 0.0990 & -0.0091 & -0.0091 & & \\
\hline 10 & US-10 & $\mathrm{TiO} 2$ & 0.9495 & 1.0223 & 0.9988 & -0.0493 & 0.0235 & $-4.9 \%$ & $2.4 \%$ \\
\hline 10 & US-10 & $\mathrm{ZnO}$ & 0.0993 & 0.0993 & 0.0990 & 0.0003 & 0.0003 & & \\
\hline 10 & US-10 & $\mathrm{ZrO} 2$ & 0.4370 & 0.4370 & 0.4989 & -0.0619 & -0.0619 & & \\
\hline 10 & US-10 & Sum & 98.4494 & 99.3474 & 99.9800 & -1.5306 & -0.6326 & $-1.5 \%$ & $-0.6 \%$ \\
\hline 11 & US-11 & $\mathrm{Al} 2 \mathrm{O} 3$ & 14.4405 & 14.7133 & 13.7027 & 0.7378 & 1.0106 & $5.4 \%$ & $7.4 \%$ \\
\hline 11 & US-11 & $\mathrm{B} 2 \mathrm{O} 3$ & 20.8006 & 20.0080 & 19.9755 & 0.8250 & 0.0325 & $4.1 \%$ & $0.2 \%$ \\
\hline 11 & US-11 & $\mathrm{CaO}$ & 0.9536 & 1.0359 & 0.9998 & -0.0462 & 0.0361 & $-4.6 \%$ & $3.6 \%$ \\
\hline 11 & US-11 & $\mathrm{Cr} 2 \mathrm{O} 3$ & 0.0153 & 0.0150 & 0.0000 & 0.0153 & 0.0150 & & \\
\hline 11 & US-11 & $\mathrm{Fe} 2 \mathrm{O} 3$ & 7.0734 & 7.0141 & 7.7607 & -0.6873 & -0.7467 & $-8.9 \%$ & $-9.6 \%$ \\
\hline 11 & US-11 & $\mathrm{K} 2 \mathrm{O}$ & 0.9845 & 0.8671 & 0.9998 & -0.0153 & -0.1327 & $-1.5 \%$ & $-13.3 \%$ \\
\hline 11 & US-11 & $\mathrm{Li} 2 \mathrm{O}$ & 2.0307 & 2.0566 & 1.9996 & 0.0311 & 0.0570 & $1.6 \%$ & $2.9 \%$ \\
\hline 11 & US-11 & $\mathrm{MgO}$ & 0.5103 & 0.5549 & 0.4999 & 0.0104 & 0.0550 & & \\
\hline 11 & US-11 & $\mathrm{MnO}$ & 3.1279 & 3.2839 & 2.9994 & 0.1285 & 0.2845 & $4.3 \%$ & $9.5 \%$ \\
\hline 11 & US-11 & $\mathrm{Na} 2 \mathrm{O}$ & 7.0197 & 6.9156 & 6.6861 & 0.3336 & 0.2295 & $5.0 \%$ & $3.4 \%$ \\
\hline 11 & US-11 & $\mathrm{NiO}$ & 0.0064 & 0.0071 & 0.0000 & 0.0064 & 0.0071 & & \\
\hline 11 & US-11 & $\mathrm{PbO}$ & 0.0934 & 0.0934 & 0.1000 & -0.0065 & -0.0065 & & \\
\hline 11 & US-11 & $\mathrm{SiO} 2$ & 43.8557 & 45.6185 & 43.6565 & 0.1991 & 1.9620 & $0.5 \%$ & $4.5 \%$ \\
\hline 11 & US-11 & SO3 & 0.2491 & 0.2491 & 0.4999 & -0.2508 & -0.2508 & & \\
\hline 11 & US-11 & $\mathrm{SrO}$ & 0.0059 & 0.0059 & 0.0000 & 0.0059 & 0.0059 & & \\
\hline 11 & US-11 & $\mathrm{TiO} 2$ & 0.0083 & 0.0090 & 0.0000 & 0.0083 & 0.0090 & & \\
\hline 11 & US-11 & $\mathrm{ZnO}$ & 0.1018 & 0.1018 & 0.1000 & 0.0018 & 0.0018 & & \\
\hline 11 & US-11 & $\mathrm{ZrO} 2$ & 0.0122 & 0.0122 & 0.0000 & 0.0122 & 0.0122 & & \\
\hline 11 & US-11 & Sum & 101.2893 & 102.5613 & 99.9800 & 1.3093 & 2.5813 & $1.3 \%$ & $2.6 \%$ \\
\hline 12 & US-12 & $\mathrm{A} 12 \mathrm{O} 3$ & 13.6800 & 13.9380 & 13.2389 & 0.4411 & 0.6991 & $3.3 \%$ & $5.3 \%$ \\
\hline 12 & US-12 & $\mathrm{B} 2 \mathrm{O} 3$ & 16.5100 & 15.8844 & 16.0809 & 0.4291 & -0.1965 & $2.7 \%$ & $-1.2 \%$ \\
\hline 12 & US-12 & $\mathrm{CaO}$ & 0.9693 & 1.0529 & 0.9998 & -0.0305 & 0.0531 & $-3.1 \%$ & $5.3 \%$ \\
\hline 12 & US-12 & Cr2O3 & 0.1195 & 0.1169 & 0.1446 & -0.0252 & -0.0277 & & \\
\hline 12 & US-12 & $\mathrm{Fe} 2 \mathrm{O} 3$ & 9.0107 & 8.9348 & 8.7434 & 0.2673 & 0.1914 & $3.1 \%$ & $2.2 \%$ \\
\hline 12 & US-12 & $\mathrm{K} 2 \mathrm{O}$ & 1.0272 & 0.9045 & 0.9998 & 0.0274 & -0.0953 & $2.7 \%$ & $-9.5 \%$ \\
\hline 12 & US-12 & $\mathrm{Li} 2 \mathrm{O}$ & 5.9904 & 6.0660 & 5.9988 & -0.0084 & 0.0672 & $-0.1 \%$ & $1.1 \%$ \\
\hline 12 & US-12 & $\mathrm{MgO}$ & 0.0083 & 0.0090 & 0.0000 & 0.0083 & 0.0090 & & \\
\hline 12 & US-12 & $\mathrm{MnO}$ & 0.0646 & 0.0678 & 0.0000 & 0.0646 & 0.0678 & & \\
\hline 12 & US-12 & $\mathrm{Na} 2 \mathrm{O}$ & 12.6712 & 12.4824 & 11.1579 & 1.5133 & 1.3245 & $13.6 \%$ & $11.9 \%$ \\
\hline 12 & US-12 & $\mathrm{NiO}$ & 1.7433 & 1.9437 & 1.8369 & -0.0936 & 0.1068 & $-5.1 \%$ & $5.8 \%$ \\
\hline 12 & US-12 & $\mathrm{PbO}$ & 0.0048 & 0.0048 & 0.0000 & 0.0048 & 0.0048 & & \\
\hline 12 & US-12 & $\mathrm{SiO} 2$ & 38.9887 & 40.5644 & 39.1293 & -0.1406 & 1.4351 & $-0.4 \%$ & $3.7 \%$ \\
\hline 12 & US-12 & $\mathrm{SO} 3$ & 0.4557 & 0.4557 & 0.4999 & -0.0442 & -0.0442 & & \\
\hline 12 & US-12 & $\mathrm{SrO}$ & 0.0943 & 0.0943 & 0.1000 & -0.0057 & -0.0057 & & \\
\hline 12 & US-12 & $\mathrm{TiO} 2$ & 0.5050 & 0.5471 & 0.4979 & 0.0071 & 0.0492 & & \\
\hline 12 & US-12 & $\mathrm{ZnO}$ & 0.1108 & 0.1108 & 0.1000 & 0.0108 & 0.0108 & & \\
\hline 12 & US-12 & $\mathrm{ZrO} 2$ & 0.4390 & 0.4390 & 0.4519 & -0.0129 & -0.0129 & & \\
\hline 12 & US-12 & Sum & 102.3929 & 103.6165 & 99.9800 & 2.4129 & 3.6365 & $2.4 \%$ & $3.6 \%$ \\
\hline 13 & US-13 & $\mathrm{A} 12 \mathrm{O} 3$ & 16.3158 & 16.5472 & 15.9968 & 0.3190 & 0.5504 & $2.0 \%$ & $3.4 \%$ \\
\hline 13 & US-13 & $\mathrm{B} 2 \mathrm{O} 3$ & 14.6505 & 14.5527 & 14.5690 & 0.0816 & -0.0163 & $0.6 \%$ & $-0.1 \%$ \\
\hline 13 & US-13 & $\mathrm{CaO}$ & 0.9161 & 0.9952 & 0.9988 & -0.0827 & -0.0036 & $-8.3 \%$ & $-0.4 \%$ \\
\hline 13 & US-13 & $\mathrm{Cr} 2 \mathrm{O} 3$ & 0.0201 & 0.0197 & 0.0000 & 0.0201 & 0.0197 & & \\
\hline 13 & US-13 & $\mathrm{Fe} 2 \mathrm{O} 3$ & 7.0234 & 7.0498 & 7.3003 & -0.2769 & -0.2505 & $-3.8 \%$ & $-3.4 \%$ \\
\hline 13 & US-13 & $\mathrm{K} 2 \mathrm{O}$ & 0.9619 & 0.8470 & 0.9988 & -0.0369 & -0.1518 & $-3.7 \%$ & $-15.2 \%$ \\
\hline
\end{tabular}


Table A4. Measured, Measured Bias-Corrected, and Targeted Compositions by Glass Number

\begin{tabular}{|c|c|c|c|c|c|c|c|c|c|}
\hline & & & & Measured & & & & & \\
\hline Glass & & & Measured & $\begin{array}{c}\text { Bias- } \\
\text { Corrected }\end{array}$ & Targeted & Diff of & Diff of & $\%$ Diff of & $\%$ Diff of \\
\hline$\#$ & GlassID & Oxide & $(w t \%)$ & $(\mathrm{wt} \%)$ & $(\mathrm{wt} \%)$ & Measured & Meas BC & Measured & Meas BC \\
\hline 13 & US-13 & $\mathrm{Li} 2 \mathrm{O}$ & 2.1852 & 2.2200 & 2.1547 & 0.0305 & 0.0653 & $1.4 \%$ & $3.0 \%$ \\
\hline 13 & US-13 & $\mathrm{MgO}$ & 0.4987 & 0.5423 & 0.4989 & -0.0002 & 0.0434 & & \\
\hline 13 & US-13 & $\mathrm{MnO}$ & 2.8116 & 2.9868 & 2.8000 & 0.0116 & 0.1868 & $0.4 \%$ & $6.7 \%$ \\
\hline 13 & US-13 & $\mathrm{Na} 2 \mathrm{O}$ & 12.1859 & 12.0059 & 11.5281 & 0.6578 & 0.4778 & $5.7 \%$ & $4.1 \%$ \\
\hline 13 & US-13 & $\mathrm{NiO}$ & 0.2640 & 0.2944 & 0.2816 & -0.0176 & 0.0128 & & \\
\hline 13 & US-13 & $\mathrm{PbO}$ & 0.0883 & 0.0883 & 0.0990 & -0.0106 & -0.0106 & & \\
\hline 13 & US-13 & $\mathrm{SiO} 2$ & 42.1977 & 43.1011 & 41.6543 & 0.5434 & 1.4468 & $1.3 \%$ & $3.5 \%$ \\
\hline 13 & US-13 & SO3 & 0.0624 & 0.0624 & 0.0000 & 0.0624 & 0.0624 & & \\
\hline 13 & US-13 & $\mathrm{SrO}$ & 0.0059 & 0.0059 & 0.0000 & 0.0059 & 0.0059 & & \\
\hline 13 & US-13 & $\mathrm{TiO} 2$ & 0.9428 & 1.0214 & 0.9998 & -0.0570 & 0.0216 & $-5.7 \%$ & $2.2 \%$ \\
\hline 13 & US-13 & $\mathrm{ZnO}$ & 0.1015 & 0.1015 & 0.1000 & 0.0015 & 0.0015 & & \\
\hline 13 & US-13 & $\mathrm{ZrO} 2$ & 0.0361 & 0.0361 & 0.0000 & 0.0361 & 0.0361 & & \\
\hline 13 & US-13 & Sum & 101.2681 & 102.4774 & 99.9800 & 1.2881 & 2.4974 & $1.3 \%$ & $2.5 \%$ \\
\hline 14 & US-14 & $\mathrm{A} 12 \mathrm{O} 3$ & 13.4580 & 13.5867 & 13.0164 & 0.4416 & 0.5704 & $3.4 \%$ & $4.4 \%$ \\
\hline 14 & US-14 & $\mathrm{B} 2 \mathrm{O} 3$ & 18.0234 & 17.6850 & 17.8983 & 0.1250 & -0.2134 & $0.7 \%$ & $-1.2 \%$ \\
\hline 14 & US-14 & $\mathrm{CaO}$ & 0.4481 & 0.4647 & 0.3970 & 0.0511 & 0.0676 & & \\
\hline 14 & US-14 & $\mathrm{Cr} 2 \mathrm{O} 3$ & 0.0088 & 0.0087 & 0.0000 & 0.0088 & 0.0087 & & \\
\hline 14 & US-14 & $\mathrm{Fe} 2 \mathrm{O} 3$ & 9.9364 & 10.0512 & 9.4148 & 0.5216 & 0.6363 & $5.5 \%$ & $6.8 \%$ \\
\hline 14 & US-14 & $\mathrm{K} 2 \mathrm{O}$ & 0.9851 & 0.8650 & 0.9998 & -0.0147 & -0.1348 & $-1.5 \%$ & $-13.5 \%$ \\
\hline 14 & US-14 & $\mathrm{Li} 2 \mathrm{O}$ & 5.7375 & 5.8098 & 5.7387 & -0.0013 & 0.0711 & $0.0 \%$ & $1.2 \%$ \\
\hline 14 & US-14 & $\mathrm{MgO}$ & 0.4962 & 0.5384 & 0.4989 & -0.0027 & 0.0395 & & \\
\hline 14 & US-14 & $\mathrm{MnO}$ & 2.6018 & 2.7176 & 2.5993 & 0.0025 & 0.1183 & $0.1 \%$ & $4.6 \%$ \\
\hline 14 & US-14 & $\mathrm{Na} 2 \mathrm{O}$ & 11.1985 & 11.1599 & 11.2237 & -0.0252 & -0.0638 & $-0.2 \%$ & $-0.6 \%$ \\
\hline 14 & US-14 & $\mathrm{NiO}$ & 0.0064 & 0.0071 & 0.0000 & 0.0064 & 0.0071 & & \\
\hline 14 & US-14 & $\mathrm{PbO}$ & 0.0633 & 0.0633 & 0.0710 & -0.0077 & -0.0077 & & \\
\hline 14 & US-14 & $\mathrm{SiO} 2$ & 36.1007 & 37.1167 & 36.5224 & -0.4217 & 0.5943 & $-1.2 \%$ & $1.6 \%$ \\
\hline 14 & US-14 & $\mathrm{SO} 3$ & 0.0624 & 0.0624 & 0.0000 & 0.0624 & 0.0624 & & \\
\hline 14 & US-14 & $\mathrm{SrO}$ & 0.0059 & 0.0059 & 0.0000 & 0.0059 & 0.0059 & & \\
\hline 14 & US-14 & TiO2 & 0.9574 & 1.0304 & 0.9998 & -0.0424 & 0.0306 & $-4.2 \%$ & $3.1 \%$ \\
\hline 14 & US-14 & $\mathrm{ZnO}$ & 0.1071 & 0.1071 & 0.1000 & 0.0071 & 0.0071 & & \\
\hline 14 & US-14 & $\mathrm{ZrO} 2$ & 0.4589 & 0.4589 & 0.4999 & -0.0410 & -0.0410 & & \\
\hline 14 & US-14 & Sum & 100.6558 & 101.7388 & 99.9800 & 0.6758 & 1.7588 & $0.7 \%$ & $1.8 \%$ \\
\hline 15 & US-15 & $\mathrm{A} 12 \mathrm{O} 3$ & 10.3214 & 10.5370 & 9.9980 & 0.3234 & 0.5390 & $3.2 \%$ & $5.4 \%$ \\
\hline 15 & US-15 & $\mathrm{B} 2 \mathrm{O} 3$ & 18.0153 & 17.8966 & 17.7964 & 0.2189 & 0.1002 & $1.2 \%$ & $0.6 \%$ \\
\hline 15 & US-15 & $\mathrm{CaO}$ & 0.5607 & 0.6092 & 0.4999 & 0.0608 & 0.1093 & & \\
\hline 15 & US-15 & $\mathrm{Cr} 2 \mathrm{O} 3$ & 0.2324 & 0.2274 & 0.2999 & -0.0675 & -0.0725 & & \\
\hline 15 & US-15 & $\mathrm{Fe} 2 \mathrm{O} 3$ & 5.1362 & 5.1319 & 4.9990 & 0.1372 & 0.1329 & $2.7 \%$ & $2.7 \%$ \\
\hline 15 & US-15 & $\mathrm{K} 2 \mathrm{O}$ & 0.9592 & 0.8449 & 0.9998 & -0.0406 & -0.1549 & $-4.1 \%$ & $-15.5 \%$ \\
\hline 15 & US-15 & $\mathrm{Li} 2 \mathrm{O}$ & 2.0017 & 2.0295 & 1.9996 & 0.0021 & 0.0299 & $0.1 \%$ & $1.5 \%$ \\
\hline 15 & US-15 & $\mathrm{MgO}$ & 0.0083 & 0.0090 & 0.0000 & 0.0083 & 0.0090 & & \\
\hline 15 & US-15 & $\mathrm{MnO}$ & 0.0646 & 0.0684 & 0.0000 & 0.0646 & 0.0684 & & \\
\hline 15 & US-15 & $\mathrm{Na} 2 \mathrm{O}$ & 15.9064 & 15.6740 & 14.9970 & 0.9094 & 0.6770 & $6.1 \%$ & $4.5 \%$ \\
\hline 15 & US-15 & $\mathrm{NiO}$ & 0.0064 & 0.0071 & 0.0000 & 0.0064 & 0.0071 & & \\
\hline 15 & US-15 & $\mathrm{PbO}$ & 0.0889 & 0.0889 & 0.1000 & -0.0111 & -0.0111 & & \\
\hline 15 & US-15 & $\mathrm{SiO} 2$ & 45.7810 & 46.5908 & 46.5907 & -0.8097 & 0.0001 & $-1.7 \%$ & $0.0 \%$ \\
\hline 15 & US-15 & $\mathrm{SO} 3$ & 0.4076 & 0.4076 & 0.4999 & -0.0923 & -0.0923 & & \\
\hline 15 & US-15 & $\mathrm{SrO}$ & 0.0896 & 0.0896 & 0.1000 & -0.0104 & -0.0104 & & \\
\hline 15 & US-15 & $\mathrm{TiO} 2$ & 0.9195 & 0.9961 & 0.9998 & -0.0803 & -0.0037 & $-8.0 \%$ & $-0.4 \%$ \\
\hline 15 & US-15 & $\mathrm{ZnO}$ & 0.0983 & 0.0983 & 0.1000 & -0.0016 & -0.0016 & & \\
\hline 15 & US-15 & $\mathrm{ZrO} 2$ & 0.0236 & 0.0236 & 0.0000 & 0.0236 & 0.0236 & & \\
\hline 15 & US-15 & Sum & 100.6210 & 101.3300 & 99.9800 & 0.6410 & 1.3500 & $0.6 \%$ & $1.4 \%$ \\
\hline 16 & US-16 & $\mathrm{A} 12 \mathrm{O} 3$ & 13.6044 & 13.8888 & 13.3159 & 0.2885 & 0.5729 & $2.2 \%$ & $4.3 \%$ \\
\hline 16 & US-16 & $\mathrm{B} 2 \mathrm{O} 3$ & 18.9411 & 18.8164 & 18.8074 & 0.1336 & 0.0090 & $0.7 \%$ & $0.0 \%$ \\
\hline 16 & US-16 & $\mathrm{CaO}$ & 0.9126 & 0.9968 & 0.9998 & -0.0872 & -0.0030 & $-8.7 \%$ & $-0.3 \%$ \\
\hline 16 & US-16 & $\mathrm{Cr} 2 \mathrm{O} 3$ & 0.0815 & 0.0797 & 0.0798 & 0.0017 & -0.0001 & & \\
\hline 16 & US-16 & $\mathrm{Fe} 2 \mathrm{O} 3$ & 8.8641 & 8.8559 & 9.0262 & -0.1620 & -0.1703 & $-1.8 \%$ & $-1.9 \%$ \\
\hline 16 & US-16 & $\mathrm{K} 2 \mathrm{O}$ & 1.0089 & 0.8944 & 0.9998 & 0.0091 & -0.1054 & $0.9 \%$ & $-10.5 \%$ \\
\hline 16 & US-16 & $\mathrm{Li} 2 \mathrm{O}$ & 2.5620 & 2.5979 & 2.5552 & 0.0068 & 0.0427 & $0.3 \%$ & $1.7 \%$ \\
\hline 16 & US-16 & $\mathrm{MgO}$ & 0.0083 & 0.0090 & 0.0000 & 0.0083 & 0.0090 & & \\
\hline 16 & US-16 & $\mathrm{MnO}$ & 2.4856 & 2.6344 & 2.4833 & 0.0023 & 0.1511 & $0.1 \%$ & $6.1 \%$ \\
\hline 16 & US-16 & $\mathrm{Na} 2 \mathrm{O}$ & 6.8512 & 6.6896 & 6.2985 & 0.5527 & 0.3911 & $8.8 \%$ & $6.2 \%$ \\
\hline 16 & US-16 & $\mathrm{NiO}$ & 0.0130 & 0.0145 & 0.0035 & 0.0096 & 0.0110 & & \\
\hline 16 & US-16 & $\mathrm{PbO}$ & 0.0054 & 0.0054 & 0.0000 & 0.0054 & 0.0054 & & \\
\hline 16 & US-16 & $\mathrm{SiO} 2$ & 42.6256 & 43.3697 & 43.9110 & -1.2855 & -0.5413 & $-2.9 \%$ & $-1.2 \%$ \\
\hline 16 & US-16 & $\mathrm{SO} 3$ & 0.0624 & 0.0624 & 0.0000 & 0.0624 & 0.0624 & & \\
\hline 16 & US-16 & $\mathrm{SrO}$ & 0.0059 & 0.0059 & 0.0000 & 0.0059 & 0.0059 & & \\
\hline 16 & US-16 & $\mathrm{TiO} 2$ & 0.9458 & 1.0286 & 0.9998 & -0.0540 & 0.0288 & $-5.4 \%$ & $2.9 \%$ \\
\hline 16 & US-16 & $\mathrm{ZnO}$ & 0.0062 & 0.0062 & 0.0000 & 0.0062 & 0.0062 & & \\
\hline
\end{tabular}


Table A4. Measured, Measured Bias-Corrected, and Targeted Compositions by Glass Number

\begin{tabular}{|c|c|c|c|c|c|c|c|c|c|}
\hline & & & & Measured & & & & & \\
\hline Glass & & & Measured & $\begin{array}{c}\text { Bias- } \\
\text { Corrected }\end{array}$ & Targeted & Diff of & Diff of & $\%$ Diff of & $\%$ Diff of \\
\hline$\#$ & GlassID & Oxide & $(w t \%)$ & $(w t \%)$ & $(\mathrm{wt} \%)$ & Measured & Meas BC & Measured & Meas BC \\
\hline 16 & US-16 & $\mathrm{ZrO} 2$ & 0.4863 & 0.4863 & 0.4999 & -0.0136 & -0.0136 & & \\
\hline 16 & US-16 & Sum & 99.4702 & 100.4419 & 99.9800 & -0.5098 & 0.4619 & $-0.5 \%$ & $0.5 \%$ \\
\hline 17 & US-17 & $\mathrm{A} 12 \mathrm{O} 3$ & 11.8283 & 11.9417 & 11.6559 & 0.1723 & 0.2857 & $1.5 \%$ & $2.5 \%$ \\
\hline 17 & US-17 & $\mathrm{B} 2 \mathrm{O} 3$ & 11.5031 & 11.2868 & 11.7751 & -0.2720 & -0.4882 & $-2.3 \%$ & $-4.1 \%$ \\
\hline 17 & US-17 & $\mathrm{CaO}$ & 0.0668 & 0.0725 & 0.0000 & 0.0668 & 0.0725 & & \\
\hline 17 & US-17 & $\mathrm{Cr} 2 \mathrm{O} 3$ & 0.0888 & 0.0869 & 0.1071 & -0.0184 & -0.0203 & & \\
\hline 17 & US-17 & $\mathrm{Fe} 2 \mathrm{O} 3$ & 7.2200 & 7.3030 & 6.2230 & 0.9970 & 1.0800 & $16.0 \%$ & $17.4 \%$ \\
\hline 17 & US-17 & $\mathrm{K} 2 \mathrm{O}$ & 0.0602 & 0.0531 & 0.0000 & 0.0602 & 0.0531 & & \\
\hline 17 & US-17 & $\mathrm{Li} 2 \mathrm{O}$ & 5.1616 & 5.2268 & 5.3635 & -0.2019 & -0.1367 & $-3.8 \%$ & $-2.5 \%$ \\
\hline 17 & US-17 & $\mathrm{MgO}$ & 0.4904 & 0.5333 & 0.4999 & -0.0095 & 0.0334 & & \\
\hline 17 & US-17 & $\mathrm{MnO}$ & 2.9407 & 3.0713 & 2.9994 & -0.0587 & 0.0719 & $-2.0 \%$ & $2.4 \%$ \\
\hline 17 & US-17 & $\mathrm{Na} 2 \mathrm{O}$ & 9.0046 & 8.8668 & 8.5818 & 0.4228 & 0.2849 & $4.9 \%$ & $3.3 \%$ \\
\hline 17 & US-17 & $\mathrm{NiO}$ & 0.9843 & 1.0974 & 1.0845 & -0.1002 & 0.0129 & $-9.2 \%$ & $1.2 \%$ \\
\hline 17 & US-17 & $\mathrm{PbO}$ & 0.0054 & 0.0054 & 0.0000 & 0.0054 & 0.0054 & & \\
\hline 17 & US-17 & $\mathrm{SiO} 2$ & 45.8345 & 47.1238 & 49.9900 & -4.1555 & -2.8662 & $-8.3 \%$ & $-5.7 \%$ \\
\hline 17 & US-17 & SO3 & 0.0624 & 0.0624 & 0.0000 & 0.0624 & 0.0624 & & \\
\hline 17 & US-17 & $\mathrm{SrO}$ & 0.0846 & 0.0846 & 0.1000 & -0.0154 & -0.0154 & & \\
\hline 17 & US-17 & $\mathrm{TiO} 2$ & 0.9374 & 1.0155 & 0.9998 & -0.0624 & 0.0157 & $-6.2 \%$ & $1.6 \%$ \\
\hline 17 & US-17 & $\mathrm{ZnO}$ & 0.1043 & 0.1043 & 0.1000 & 0.0043 & 0.0043 & & \\
\hline 17 & US-17 & $\mathrm{ZrO} 2$ & 0.4093 & 0.4093 & 0.4999 & -0.0906 & -0.0906 & & \\
\hline 17 & US-17 & Sum & 96.7867 & 98.3447 & 99.9800 & -3.1933 & -1.6353 & $-3.2 \%$ & $-1.6 \%$ \\
\hline 18 & US-18 & $\mathrm{A} 12 \mathrm{O} 3$ & 16.5520 & 16.8642 & 15.9968 & 0.5552 & 0.8674 & $3.5 \%$ & $5.4 \%$ \\
\hline 18 & US-18 & $\mathrm{B} 2 \mathrm{O} 3$ & 9.6356 & 9.2674 & 9.1275 & 0.5080 & 0.1398 & $5.6 \%$ & $1.5 \%$ \\
\hline 18 & US-18 & $\mathrm{CaO}$ & 0.8923 & 0.9254 & 0.9998 & -0.1075 & -0.0744 & $-10.7 \%$ & $-7.4 \%$ \\
\hline 18 & US-18 & Cr2O3 & 0.0113 & 0.0112 & 0.0000 & 0.0113 & 0.0112 & & \\
\hline 18 & US-18 & $\mathrm{Fe} 2 \mathrm{O} 3$ & 9.6255 & 9.5442 & 9.7464 & -0.1210 & -0.2022 & $-1.2 \%$ & $-2.1 \%$ \\
\hline 18 & US-18 & $\mathrm{K} 2 \mathrm{O}$ & 0.0602 & 0.0529 & 0.0000 & 0.0602 & 0.0529 & & \\
\hline 18 & US-18 & $\mathrm{Li} 2 \mathrm{O}$ & 4.9355 & 4.9977 & 4.9510 & -0.0154 & 0.0468 & $-0.3 \%$ & $0.9 \%$ \\
\hline 18 & US-18 & $\mathrm{MgO}$ & 0.0083 & 0.0090 & 0.0000 & 0.0083 & 0.0090 & & \\
\hline 18 & US-18 & $\mathrm{MnO}$ & 3.0860 & 3.2402 & 2.9994 & 0.0866 & 0.2408 & $2.9 \%$ & $8.0 \%$ \\
\hline 18 & US-18 & $\mathrm{Na} 2 \mathrm{O}$ & 12.6442 & 12.6074 & 13.4069 & -0.7627 & -0.7995 & $-5.7 \%$ & $-6.0 \%$ \\
\hline 18 & US-18 & $\mathrm{NiO}$ & 0.0064 & 0.0071 & 0.0000 & 0.0064 & 0.0071 & & \\
\hline 18 & US-18 & $\mathrm{PbO}$ & 0.0897 & 0.0897 & 0.1000 & -0.0103 & -0.0103 & & \\
\hline 18 & US-18 & $\mathrm{SiO} 2$ & 41.3955 & 43.0662 & 41.4524 & -0.0569 & 1.6138 & $-0.1 \%$ & $3.9 \%$ \\
\hline 18 & US-18 & SO3 & 0.0624 & 0.0624 & 0.0000 & 0.0624 & 0.0624 & & \\
\hline 18 & US-18 & $\mathrm{SrO}$ & 0.0964 & 0.0964 & 0.1000 & -0.0036 & -0.0036 & & \\
\hline 18 & US-18 & $\mathrm{TiO} 2$ & 0.9570 & 1.0299 & 0.9998 & -0.0428 & 0.0301 & $-4.3 \%$ & $3.0 \%$ \\
\hline 18 & US-18 & $\mathrm{ZnO}$ & 0.1117 & 0.1117 & 0.1000 & 0.0117 & 0.0117 & & \\
\hline 18 & US-18 & $\mathrm{ZrO} 2$ & 0.0057 & 0.0057 & 0.0000 & 0.0057 & 0.0057 & & \\
\hline 18 & US-18 & Sum & 100.1757 & 101.9889 & 99.9800 & 0.1957 & 2.0089 & $0.2 \%$ & $2.0 \%$ \\
\hline 19 & US-19 & $\mathrm{A} 12 \mathrm{O} 3$ & 14.5917 & 14.8766 & 14.4727 & 0.1190 & 0.4039 & $0.8 \%$ & $2.8 \%$ \\
\hline 19 & US-19 & $\mathrm{B} 2 \mathrm{O} 3$ & 14.6908 & 13.9613 & 14.4010 & 0.2898 & -0.4397 & $2.0 \%$ & $-3.1 \%$ \\
\hline 19 & US-19 & $\mathrm{CaO}$ & 0.6779 & 0.7298 & 0.6996 & -0.0217 & 0.0302 & $-3.1 \%$ & $4.3 \%$ \\
\hline 19 & US-19 & $\mathrm{Cr} 2 \mathrm{O} 3$ & 0.0563 & 0.0550 & 0.0686 & -0.0124 & -0.0136 & & \\
\hline 19 & US-19 & $\mathrm{Fe} 2 \mathrm{O} 3$ & 7.9420 & 7.9234 & 8.2281 & -0.2861 & -0.3047 & $-3.5 \%$ & $-3.7 \%$ \\
\hline 19 & US-19 & $\mathrm{K} 2 \mathrm{O}$ & 0.7770 & 0.6894 & 0.7773 & -0.0003 & -0.0879 & $0.0 \%$ & $-11.3 \%$ \\
\hline 19 & US-19 & $\mathrm{Li} 2 \mathrm{O}$ & 3.6223 & 3.6468 & 3.6377 & -0.0154 & 0.0092 & $-0.4 \%$ & $0.3 \%$ \\
\hline 19 & US-19 & $\mathrm{MgO}$ & 0.3370 & 0.3636 & 0.3328 & 0.0042 & 0.0308 & & \\
\hline 19 & US-19 & $\mathrm{MnO}$ & 1.9207 & 2.0079 & 1.9878 & -0.0671 & 0.0201 & $-3.4 \%$ & $1.0 \%$ \\
\hline 19 & US-19 & $\mathrm{Na} 2 \mathrm{O}$ & 11.6636 & 11.2450 & 10.8018 & 0.8618 & 0.4432 & $8.0 \%$ & $4.1 \%$ \\
\hline 19 & US-19 & $\mathrm{NiO}$ & 0.1896 & 0.2092 & 0.2090 & -0.0194 & 0.0001 & & \\
\hline 19 & US-19 & $\mathrm{PbO}$ & 0.0660 & 0.0660 & 0.0703 & -0.0043 & -0.0043 & & \\
\hline 19 & US-19 & $\mathrm{SiO} 2$ & 40.8606 & 40.9512 & 42.8088 & -1.9482 & -1.8576 & $-4.6 \%$ & $-4.3 \%$ \\
\hline 19 & US-19 & $\mathrm{SO} 3$ & 0.2634 & 0.2634 & 0.2774 & -0.0140 & -0.0140 & & \\
\hline 19 & US-19 & $\mathrm{SrO}$ & 0.0597 & 0.0597 & 0.0609 & -0.0012 & -0.0012 & & \\
\hline 19 & US-19 & $\mathrm{TiO} 2$ & 0.7769 & 0.8364 & 0.8048 & -0.0280 & 0.0315 & $-3.5 \%$ & $3.9 \%$ \\
\hline 19 & US-19 & $\mathrm{ZnO}$ & 0.0672 & 0.0672 & 0.0665 & 0.0007 & 0.0007 & & \\
\hline 19 & US-19 & $\mathrm{ZrO} 2$ & 0.2266 & 0.2266 & 0.2749 & -0.0483 & -0.0483 & & \\
\hline 19 & US-19 & Sum & 98.7892 & 98.1785 & 99.9800 & -1.1908 & -1.8015 & $-1.2 \%$ & $-1.8 \%$ \\
\hline 20 & US-20 & $\mathrm{A} 12 \mathrm{O} 3$ & 14.5633 & 14.8477 & 14.4727 & 0.0906 & 0.3750 & $0.6 \%$ & $2.6 \%$ \\
\hline 20 & US-20 & $\mathrm{B} 2 \mathrm{O} 3$ & 14.7471 & 14.0135 & 14.4010 & 0.3461 & -0.3876 & $2.4 \%$ & $-2.7 \%$ \\
\hline 20 & US-20 & $\mathrm{CaO}$ & 0.7580 & 0.8161 & 0.6996 & 0.0584 & 0.1165 & $8.4 \%$ & $16.7 \%$ \\
\hline 20 & US-20 & $\mathrm{Cr} 2 \mathrm{O} 3$ & 0.0661 & 0.0647 & 0.0686 & -0.0025 & -0.0039 & & \\
\hline 20 & US-20 & $\mathrm{Fe} 2 \mathrm{O} 3$ & 8.3459 & 8.3266 & 8.2281 & 0.1178 & 0.0985 & $1.4 \%$ & $1.2 \%$ \\
\hline 20 & US-20 & $\mathrm{K} 2 \mathrm{O}$ & 0.7574 & 0.6720 & 0.7773 & -0.0199 & -0.1053 & $-2.6 \%$ & $-13.5 \%$ \\
\hline 20 & US-20 & $\mathrm{Li} 2 \mathrm{O}$ & 3.6007 & 3.6252 & 3.6377 & -0.0369 & -0.0125 & $-1.0 \%$ & $-0.3 \%$ \\
\hline 20 & US-20 & $\mathrm{MgO}$ & 0.3354 & 0.3618 & 0.3328 & 0.0026 & 0.0290 & & \\
\hline 20 & US-20 & $\mathrm{MnO}$ & 1.9691 & 2.0587 & 1.9878 & -0.0187 & 0.0709 & $-0.9 \%$ & $3.6 \%$ \\
\hline
\end{tabular}


Table A4. Measured, Measured Bias-Corrected, and Targeted Compositions by Glass Number

\begin{tabular}{|c|c|c|c|c|c|c|c|c|c|}
\hline & & & & Measured & & & & & \\
\hline Glass & & & Measured & $\begin{array}{c}\text { Bias- } \\
\text { Corrected }\end{array}$ & Targeted & Diff of & Diff of & $\%$ Diff of & $\%$ Diff of \\
\hline$\#$ & GlassID & Oxide & $(w t \%)$ & $(\mathrm{wt} \%)$ & $(\mathrm{wt} \%)$ & Measured & Meas BC & Measured & Meas BC \\
\hline 20 & US-20 & $\mathrm{Na} 2 \mathrm{O}$ & 11.5591 & 11.1431 & 10.8018 & 0.7573 & 0.3413 & $7.0 \%$ & $3.2 \%$ \\
\hline 20 & US-20 & $\mathrm{NiO}$ & 0.1944 & 0.2144 & 0.2090 & -0.0147 & 0.0054 & & \\
\hline 20 & US-20 & $\mathrm{PbO}$ & 0.0649 & 0.0649 & 0.0703 & -0.0054 & -0.0054 & & \\
\hline 20 & US-20 & $\mathrm{SiO} 2$ & 41.0211 & 41.1090 & 42.8088 & -1.7877 & -1.6998 & $-4.2 \%$ & $-4.0 \%$ \\
\hline 20 & US-20 & SO3 & 0.2541 & 0.2541 & 0.2774 & -0.0233 & -0.0233 & & \\
\hline 20 & US-20 & $\mathrm{SrO}$ & 0.0577 & 0.0577 & 0.0609 & -0.0032 & -0.0032 & & \\
\hline 20 & US-20 & $\mathrm{TiO} 2$ & 0.7752 & 0.8346 & 0.8048 & -0.0296 & 0.0298 & $-3.7 \%$ & $3.7 \%$ \\
\hline 20 & US-20 & $\mathrm{ZnO}$ & 0.0716 & 0.0716 & 0.0665 & 0.0051 & 0.0051 & & \\
\hline 20 & US-20 & $\mathrm{ZrO} 2$ & 0.2783 & 0.2783 & 0.2749 & 0.0034 & 0.0034 & & \\
\hline 20 & US-20 & Sum & 99.4193 & 98.8137 & 99.9800 & -0.5607 & -1.1663 & $-0.6 \%$ & $-1.2 \%$ \\
\hline 21 & US-21 & $\mathrm{A} 12 \mathrm{O} 3$ & 14.9459 & 15.1578 & 14.2189 & 0.7270 & 0.9389 & $5.1 \%$ & $6.6 \%$ \\
\hline 21 & US-21 & $\mathrm{B} 2 \mathrm{O} 3$ & 13.7892 & 13.6965 & 13.7549 & 0.0343 & -0.0584 & $0.2 \%$ & $-0.4 \%$ \\
\hline 21 & US-21 & $\mathrm{CaO}$ & 0.3634 & 0.4052 & 0.3761 & -0.0126 & 0.0291 & & \\
\hline 21 & US-21 & $\mathrm{Cr} 2 \mathrm{O} 3$ & 0.0599 & 0.0584 & 0.0803 & -0.0203 & -0.0219 & & \\
\hline 21 & US-21 & $\mathrm{Fe} 2 \mathrm{O} 3$ & 7.8848 & 7.9142 & 8.4520 & -0.5672 & -0.5378 & $-6.7 \%$ & $-6.4 \%$ \\
\hline 21 & US-21 & $\mathrm{K} 2 \mathrm{O}$ & 0.7255 & 0.6385 & 0.7489 & -0.0234 & -0.1104 & $-3.1 \%$ & $-14.7 \%$ \\
\hline 21 & US-21 & $\mathrm{Li} 2 \mathrm{O}$ & 3.0464 & 3.0948 & 3.0004 & 0.0460 & 0.0944 & $1.5 \%$ & $3.1 \%$ \\
\hline 21 & US-21 & $\mathrm{MgO}$ & 0.3744 & 0.4035 & 0.3739 & 0.0004 & 0.0296 & & \\
\hline 21 & US-21 & $\mathrm{MnO}$ & 1.7399 & 1.8483 & 1.7789 & -0.0390 & 0.0694 & $-2.2 \%$ & $3.9 \%$ \\
\hline 21 & US-21 & $\mathrm{Na} 2 \mathrm{O}$ & 11.8253 & 11.3467 & 10.7534 & 1.0720 & 0.5933 & $10.0 \%$ & $5.5 \%$ \\
\hline 21 & US-21 & $\mathrm{NiO}$ & 0.4444 & 0.4892 & 0.5009 & -0.0565 & -0.0117 & $-11.3 \%$ & $-2.3 \%$ \\
\hline 21 & US-21 & $\mathrm{PbO}$ & 0.0746 & 0.0746 & 0.0790 & -0.0044 & -0.0044 & & \\
\hline 21 & US-21 & $\mathrm{SiO} 2$ & 45.1392 & 46.1060 & 44.9546 & 0.1846 & 1.1514 & $0.4 \%$ & $2.6 \%$ \\
\hline 21 & US-21 & $\mathrm{SO} 3$ & 0.1273 & 0.1273 & 0.1250 & 0.0024 & 0.0024 & & \\
\hline 21 & US-21 & $\mathrm{SrO}$ & 0.0745 & 0.0745 & 0.0800 & -0.0055 & -0.0055 & & \\
\hline 21 & US-21 & $\mathrm{TiO} 2$ & 0.5133 & 0.5583 & 0.5430 & -0.0297 & 0.0153 & $-5.5 \%$ & $2.8 \%$ \\
\hline 21 & US-21 & $\mathrm{ZnO}$ & 0.0367 & 0.0367 & 0.0300 & 0.0067 & 0.0067 & & \\
\hline 21 & US-21 & $\mathrm{ZrO} 2$ & 0.1030 & 0.1030 & 0.1300 & -0.0270 & -0.0270 & & \\
\hline 21 & US-21 & Sum & 101.2679 & 102.1336 & 99.9800 & 1.2879 & 2.1536 & $1.3 \%$ & $2.2 \%$ \\
\hline 22 & US-22 & $\mathrm{A} 12 \mathrm{O} 3$ & 13.7461 & 13.8776 & 13.3397 & 0.4064 & 0.5379 & $3.0 \%$ & $4.0 \%$ \\
\hline 22 & US-22 & $\mathrm{B} 2 \mathrm{O} 3$ & 11.8251 & 11.6021 & 11.6875 & 0.1376 & -0.0853 & $1.2 \%$ & $-0.7 \%$ \\
\hline 22 & US-22 & $\mathrm{CaO}$ & 0.7073 & 0.7683 & 0.7489 & -0.0416 & 0.0194 & $-5.5 \%$ & $2.6 \%$ \\
\hline 22 & US-22 & $\mathrm{Cr} 2 \mathrm{O} 3$ & 0.0797 & 0.0779 & 0.0800 & -0.0003 & -0.0021 & & \\
\hline 22 & US-22 & $\mathrm{Fe} 2 \mathrm{O} 3$ & 9.3324 & 9.4401 & 9.4674 & -0.1350 & -0.0273 & $-1.4 \%$ & $-0.3 \%$ \\
\hline 22 & US-22 & $\mathrm{K} 2 \mathrm{O}$ & 0.7381 & 0.6499 & 0.7489 & -0.0107 & -0.0989 & $-1.4 \%$ & $-13.2 \%$ \\
\hline 22 & US-22 & $\mathrm{Li} 2 \mathrm{O}$ & 4.9624 & 5.0251 & 4.9980 & -0.0356 & 0.0271 & $-0.7 \%$ & $0.5 \%$ \\
\hline 22 & US-22 & $\mathrm{MgO}$ & 0.3822 & 0.4156 & 0.3739 & 0.0083 & 0.0417 & & \\
\hline 22 & US-22 & $\mathrm{MnO}$ & 2.2176 & 2.3162 & 2.2486 & -0.0309 & 0.0677 & $-1.4 \%$ & $3.0 \%$ \\
\hline 22 & US-22 & $\mathrm{Na} 2 \mathrm{O}$ & 13.2070 & 13.0155 & 11.7767 & 1.4303 & 1.2388 & $12.1 \%$ & $10.5 \%$ \\
\hline 22 & US-22 & $\mathrm{NiO}$ & 0.8494 & 0.9470 & 0.9181 & -0.0688 & 0.0289 & $-7.5 \%$ & $3.1 \%$ \\
\hline 22 & US-22 & $\mathrm{PbO}$ & 0.0302 & 0.0302 & 0.0300 & 0.0002 & 0.0002 & & \\
\hline 22 & US-22 & $\mathrm{SiO} 2$ & 41.5024 & 42.6695 & 42.4006 & -0.8982 & 0.2689 & $-2.1 \%$ & $0.6 \%$ \\
\hline 22 & US-22 & SO3 & 0.1398 & 0.1398 & 0.1250 & 0.0149 & 0.0149 & & \\
\hline 22 & US-22 & $\mathrm{SrO}$ & 0.0713 & 0.0713 & 0.0790 & -0.0077 & -0.0077 & & \\
\hline 22 & US-22 & $\mathrm{TiO} 2$ & 0.7356 & 0.7969 & 0.7489 & -0.0133 & 0.0480 & $-1.8 \%$ & $6.4 \%$ \\
\hline 22 & US-22 & $\mathrm{ZnO}$ & 0.0871 & 0.0871 & 0.0790 & 0.0082 & 0.0082 & & \\
\hline 22 & US-22 & $\mathrm{ZrO} 2$ & 0.1226 & 0.1226 & 0.1300 & -0.0074 & -0.0074 & & \\
\hline 22 & US-22 & Sum & 100.7363 & 102.0527 & 99.9800 & 0.7563 & 2.0727 & $0.8 \%$ & $2.1 \%$ \\
\hline 23 & US-23 & $\mathrm{A} 12 \mathrm{O} 3$ & 14.7192 & 14.8601 & 14.4971 & 0.2221 & 0.3630 & $1.5 \%$ & $2.5 \%$ \\
\hline 23 & US-23 & $\mathrm{B} 2 \mathrm{O} 3$ & 13.3626 & 13.1112 & 13.3214 & 0.0412 & -0.2102 & $0.3 \%$ & $-1.6 \%$ \\
\hline 23 & US-23 & $\mathrm{CaO}$ & 0.6692 & 0.7460 & 0.7499 & -0.0807 & -0.0039 & $-10.8 \%$ & $-0.5 \%$ \\
\hline 23 & US-23 & $\mathrm{Cr} 2 \mathrm{O} 3$ & 0.0665 & 0.0648 & 0.0800 & -0.0135 & -0.0152 & & \\
\hline 23 & US-23 & $\mathrm{Fe} 2 \mathrm{O} 3$ & 6.8626 & 6.9414 & 7.6227 & -0.7602 & -0.6814 & $-10.0 \%$ & $-8.9 \%$ \\
\hline 23 & US-23 & $\mathrm{K} 2 \mathrm{O}$ & 0.2536 & 0.2232 & 0.2500 & 0.0036 & -0.0268 & & \\
\hline 23 & US-23 & $\mathrm{Li} 2 \mathrm{O}$ & 3.4446 & 3.4881 & 3.4993 & -0.0547 & -0.0112 & $-1.6 \%$ & $-0.3 \%$ \\
\hline 23 & US-23 & $\mathrm{MgO}$ & 0.1269 & 0.1367 & 0.1250 & 0.0019 & 0.0118 & & \\
\hline 23 & US-23 & $\mathrm{MnO}$ & 2.2499 & 2.3499 & 2.2496 & 0.0004 & 0.1003 & $0.0 \%$ & $4.5 \%$ \\
\hline 23 & US-23 & $\mathrm{Na} 2 \mathrm{O}$ & 12.9779 & 12.4540 & 11.6811 & 1.2968 & 0.7729 & $11.1 \%$ & $6.6 \%$ \\
\hline 23 & US-23 & $\mathrm{NiO}$ & 0.4591 & 0.5053 & 0.4999 & -0.0408 & 0.0054 & & \\
\hline 23 & US-23 & $\mathrm{PbO}$ & 0.0784 & 0.0784 & 0.0800 & -0.0016 & -0.0016 & & \\
\hline 23 & US-23 & $\mathrm{SiO} 2$ & 43.1069 & 44.3178 & 44.4603 & -1.3534 & -0.1425 & $-3.0 \%$ & $-0.3 \%$ \\
\hline 23 & US-23 & $\mathrm{SO} 3$ & 0.3308 & 0.3308 & 0.3739 & -0.0431 & -0.0431 & & \\
\hline 23 & US-23 & $\mathrm{SrO}$ & 0.0296 & 0.0296 & 0.0300 & -0.0004 & -0.0004 & & \\
\hline 23 & US-23 & $\mathrm{TiO} 2$ & 0.2510 & 0.2730 & 0.2500 & 0.0011 & 0.0231 & & \\
\hline 23 & US-23 & $\mathrm{ZnO}$ & 0.0912 & 0.0912 & 0.0800 & 0.0112 & 0.0112 & & \\
\hline 23 & US-23 & $\mathrm{ZrO} 2$ & 0.1374 & 0.1374 & 0.1300 & 0.0075 & 0.0075 & & \\
\hline 23 & US-23 & Sum & 99.2173 & 100.1388 & 99.9800 & -0.7628 & 0.1588 & $-0.8 \%$ & $0.2 \%$ \\
\hline 24 & US-24 & $\mathrm{A} 12 \mathrm{O} 3$ & 14.9696 & 15.2517 & 14.4961 & 0.4735 & 0.7556 & $3.3 \%$ & $5.2 \%$ \\
\hline
\end{tabular}


Table A4. Measured, Measured Bias-Corrected, and Targeted Compositions by Glass Number

\begin{tabular}{|c|c|c|c|c|c|c|c|c|c|}
\hline & & & & Measured & & & & & \\
\hline Glass & & & Measured & $\begin{array}{c}\text { Bias- } \\
\text { Corrected }\end{array}$ & Targeted & Diff of & Diff of & $\%$ Diff of & $\%$ Diff of \\
\hline$\#$ & GlassID & Oxide & $(w t \%)$ & $(\mathrm{wt} \%)$ & $(\mathrm{wt} \%)$ & Measured & Meas BC & Measured & Meas BC \\
\hline 24 & US-24 & $\mathrm{B} 2 \mathrm{O} 3$ & 12.7428 & 12.2576 & 12.2496 & 0.4932 & 0.0080 & $4.0 \%$ & $0.1 \%$ \\
\hline 24 & US-24 & $\mathrm{CaO}$ & 0.7136 & 0.7681 & 0.7489 & -0.0353 & 0.0192 & $-4.7 \%$ & $2.6 \%$ \\
\hline 24 & US-24 & $\mathrm{Cr} 2 \mathrm{O} 3$ & 0.0585 & 0.0572 & 0.0810 & -0.0225 & -0.0238 & & \\
\hline 24 & US-24 & $\mathrm{Fe} 2 \mathrm{O} 3$ & 7.6668 & 7.6022 & 7.7875 & -0.1207 & -0.1853 & $-1.6 \%$ & $-2.4 \%$ \\
\hline 24 & US-24 & $\mathrm{K} 2 \mathrm{O}$ & 0.7544 & 0.6694 & 0.7489 & 0.0055 & -0.0795 & $0.7 \%$ & $-10.6 \%$ \\
\hline 24 & US-24 & $\mathrm{Li} 2 \mathrm{O}$ & 3.1056 & 3.1447 & 3.0004 & 0.1052 & 0.1443 & $3.5 \%$ & $4.8 \%$ \\
\hline 24 & US-24 & $\mathrm{MgO}$ & 0.3694 & 0.3985 & 0.3739 & -0.0045 & 0.0246 & & \\
\hline 24 & US-24 & $\mathrm{MnO}$ & 2.3371 & 2.4537 & 2.2486 & 0.0885 & 0.2051 & $3.9 \%$ & $9.1 \%$ \\
\hline 24 & US-24 & $\mathrm{Na} 2 \mathrm{O}$ & 12.6544 & 12.2011 & 11.5734 & 1.0810 & 0.6278 & $9.3 \%$ & $5.4 \%$ \\
\hline 24 & US-24 & $\mathrm{NiO}$ & 0.4546 & 0.5015 & 0.5009 & -0.0463 & 0.0006 & $-9.2 \%$ & $0.1 \%$ \\
\hline 24 & US-24 & $\mathrm{PbO}$ & 0.0768 & 0.0768 & 0.0790 & -0.0022 & -0.0022 & & \\
\hline 24 & US-24 & $\mathrm{SiO} 2$ & 41.5024 & 43.1849 & 44.4324 & -2.9299 & -1.2474 & $-6.6 \%$ & $-2.8 \%$ \\
\hline 24 & US-24 & $\mathrm{SO} 3$ & 0.3252 & 0.3252 & 0.3739 & -0.0487 & -0.0487 & & \\
\hline 24 & US-24 & $\mathrm{SrO}$ & 0.0721 & 0.0721 & 0.0790 & -0.0068 & -0.0068 & & \\
\hline 24 & US-24 & $\mathrm{TiO} 2$ & 0.7047 & 0.7587 & 0.7489 & -0.0441 & 0.0098 & $-5.9 \%$ & $1.3 \%$ \\
\hline 24 & US-24 & $\mathrm{ZnO}$ & 0.0822 & 0.0822 & 0.0790 & 0.0032 & 0.0032 & & \\
\hline 24 & US-24 & $\mathrm{ZrO} 2$ & 0.3836 & 0.3836 & 0.3789 & 0.0047 & 0.0047 & & \\
\hline 24 & US-24 & Sum & 98.9735 & 100.1892 & 99.9800 & -1.0065 & 0.2092 & $-1.0 \%$ & $0.2 \%$ \\
\hline 25 & US-25 & $\mathrm{Al} 2 \mathrm{O} 3$ & 12.1070 & 12.2788 & 11.5849 & 0.5221 & 0.6939 & $4.5 \%$ & $6.0 \%$ \\
\hline 25 & US-25 & $\mathrm{B} 2 \mathrm{O} 3$ & 16.2363 & 16.1278 & 16.2468 & -0.0104 & -0.1190 & $-0.1 \%$ & $-0.7 \%$ \\
\hline 25 & US-25 & $\mathrm{CaO}$ & 0.7153 & 0.7814 & 0.7499 & -0.0345 & 0.0316 & $-4.6 \%$ & $4.2 \%$ \\
\hline 25 & US-25 & $\mathrm{Cr} 2 \mathrm{O} 3$ & 0.1480 & 0.1448 & 0.2176 & -0.0697 & -0.0729 & & \\
\hline 25 & US-25 & $\mathrm{Fe} 2 \mathrm{O} 3$ & 8.4031 & 8.4343 & 8.5402 & -0.1371 & -0.1058 & $-1.6 \%$ & $-1.2 \%$ \\
\hline 25 & US-25 & $\mathrm{K} 2 \mathrm{O}$ & 0.3045 & 0.2699 & 0.2871 & 0.0174 & -0.0172 & & \\
\hline 25 & US-25 & $\mathrm{Li} 2 \mathrm{O}$ & 4.9571 & 5.0359 & 4.9990 & -0.0419 & 0.0369 & $-0.8 \%$ & $0.7 \%$ \\
\hline 25 & US-25 & $\mathrm{MgO}$ & 0.3727 & 0.4060 & 0.3749 & -0.0022 & 0.0311 & & \\
\hline 25 & US-25 & $\mathrm{MnO}$ & 2.0885 & 2.2186 & 2.1645 & -0.0760 & 0.0541 & $-3.5 \%$ & $2.5 \%$ \\
\hline 25 & US-25 & $\mathrm{Na} 2 \mathrm{O}$ & 11.9028 & 11.6221 & 10.8831 & 1.0197 & 0.7390 & $9.4 \%$ & $6.8 \%$ \\
\hline 25 & US-25 & $\mathrm{NiO}$ & 1.2725 & 1.4117 & 1.4494 & -0.1769 & -0.0377 & $-12.2 \%$ & $-2.6 \%$ \\
\hline 25 & US-25 & $\mathrm{PbO}$ & 0.0312 & 0.0312 & 0.0300 & 0.0012 & 0.0012 & & \\
\hline 25 & US-25 & $\mathrm{SiO} 2$ & 39.2027 & 40.0433 & 40.7880 & -1.5853 & -0.7447 & $-3.9 \%$ & $-1.8 \%$ \\
\hline 25 & US-25 & $\mathrm{SO} 3$ & 0.3371 & 0.3371 & 0.3749 & -0.0378 & -0.0378 & & \\
\hline 25 & US-25 & $\mathrm{SrO}$ & 0.0816 & 0.0816 & 0.0800 & 0.0016 & 0.0016 & & \\
\hline 25 & US-25 & $\mathrm{TiO} 2$ & 0.7289 & 0.7928 & 0.7499 & -0.0209 & 0.0430 & $-2.8 \%$ & $5.7 \%$ \\
\hline 25 & US-25 & $\mathrm{ZnO}$ & 0.1046 & 0.1046 & 0.0800 & 0.0246 & 0.0246 & & \\
\hline 25 & US-25 & $\mathrm{ZrO} 2$ & 0.3607 & 0.3607 & 0.3799 & -0.0193 & -0.0193 & & \\
\hline 25 & US-25 & Sum & 99.3545 & 100.4826 & 99.9800 & -0.6255 & 0.5026 & $-0.6 \%$ & $0.5 \%$ \\
\hline 26 & US-26 & $\mathrm{A} 12 \mathrm{O} 3$ & 18.8336 & 19.2014 & 18.8291 & 0.0045 & 0.3724 & $0.0 \%$ & $2.0 \%$ \\
\hline 26 & US-26 & $\mathrm{B} 2 \mathrm{O} 3$ & 10.9718 & 10.4286 & 10.6853 & 0.2865 & -0.2567 & $2.7 \%$ & $-2.4 \%$ \\
\hline 26 & US-26 & $\mathrm{CaO}$ & 0.9231 & 1.0027 & 0.9998 & -0.0767 & 0.0029 & $-7.7 \%$ & $0.3 \%$ \\
\hline 26 & US-26 & $\mathrm{Cr} 2 \mathrm{O} 3$ & 0.4111 & 0.4021 & 0.5484 & -0.1373 & -0.1463 & $-25.0 \%$ & $-26.7 \%$ \\
\hline 26 & US-26 & $\mathrm{Fe} 2 \mathrm{O} 3$ & 5.1255 & 5.1144 & 4.9990 & 0.1265 & 0.1154 & $2.5 \%$ & $2.3 \%$ \\
\hline 26 & US-26 & $\mathrm{K} 2 \mathrm{O}$ & 0.0602 & 0.0531 & 0.0000 & 0.0602 & 0.0531 & & \\
\hline 26 & US-26 & $\mathrm{Li} 2 \mathrm{O}$ & 5.8828 & 5.9228 & 5.9988 & -0.1160 & -0.0760 & $-1.9 \%$ & $-1.3 \%$ \\
\hline 26 & US-26 & $\mathrm{MgO}$ & 0.4805 & 0.5224 & 0.4999 & -0.0194 & 0.0225 & & \\
\hline 26 & US-26 & $\mathrm{MnO}$ & 0.0646 & 0.0675 & 0.0000 & 0.0646 & 0.0675 & & \\
\hline 26 & US-26 & $\mathrm{Na} 2 \mathrm{O}$ & 11.4546 & 11.2848 & 11.0175 & 0.4371 & 0.2673 & $4.0 \%$ & $2.4 \%$ \\
\hline 26 & US-26 & $\mathrm{NiO}$ & 0.0064 & 0.0071 & 0.0000 & 0.0064 & 0.0071 & & \\
\hline 26 & US-26 & $\mathrm{PbO}$ & 0.0054 & 0.0054 & 0.0000 & 0.0054 & 0.0054 & & \\
\hline 26 & US-26 & $\mathrm{SiO} 2$ & 38.5609 & 38.6416 & 38.0544 & 0.5065 & 0.5872 & $1.3 \%$ & $1.5 \%$ \\
\hline 26 & US-26 & SO3 & 0.0624 & 0.0624 & 0.0000 & 0.0624 & 0.0624 & & \\
\hline 26 & US-26 & $\mathrm{SrO}$ & 2.5278 & 2.5278 & 2.9994 & -0.4716 & -0.4716 & $-15.7 \%$ & $-15.7 \%$ \\
\hline 26 & US-26 & TiO2 & 0.9249 & 1.0019 & 0.9998 & -0.0749 & 0.0021 & $-7.5 \%$ & $0.2 \%$ \\
\hline 26 & US-26 & $\mathrm{ZnO}$ & 1.6964 & 1.6964 & 1.9996 & -0.3032 & -0.3032 & $-15.2 \%$ & $-15.2 \%$ \\
\hline 26 & US-26 & $\mathrm{ZrO} 2$ & 2.0397 & 2.0397 & 2.3491 & -0.3094 & -0.3094 & $-13.2 \%$ & $-13.2 \%$ \\
\hline 26 & US-26 & Sum & 100.0316 & 99.9822 & 99.9800 & 0.0516 & 0.0022 & $0.1 \%$ & $0.0 \%$ \\
\hline 27 & US-27 & $\mathrm{A} 12 \mathrm{O} 3$ & 10.6048 & 10.8119 & 10.4035 & 0.2014 & 0.4084 & $1.9 \%$ & $3.9 \%$ \\
\hline 27 & US-27 & $\mathrm{B} 2 \mathrm{O} 3$ & 7.0274 & 6.6760 & 6.4837 & 0.5438 & 0.1923 & $8.4 \%$ & $3.0 \%$ \\
\hline 27 & US-27 & $\mathrm{CaO}$ & 0.0273 & 0.0298 & 0.0000 & 0.0273 & 0.0298 & & \\
\hline 27 & US-27 & Cr2O3 & 0.8382 & 0.8200 & 0.9998 & -0.1616 & -0.1798 & $-16.2 \%$ & $-18.0 \%$ \\
\hline 27 & US-27 & $\mathrm{Fe} 2 \mathrm{O} 3$ & 15.1798 & 15.1462 & 15.1827 & -0.0028 & -0.0365 & $0.0 \%$ & $-0.2 \%$ \\
\hline 27 & US-27 & $\mathrm{K} 2 \mathrm{O}$ & 1.0212 & 0.9054 & 0.9998 & 0.0214 & -0.0944 & $2.1 \%$ & $-9.4 \%$ \\
\hline 27 & US-27 & $\mathrm{Li} 2 \mathrm{O}$ & 5.8397 & 5.8794 & 5.9988 & -0.1591 & -0.1194 & $-2.7 \%$ & $-2.0 \%$ \\
\hline 27 & US-27 & $\mathrm{MgO}$ & 0.4867 & 0.5302 & 0.4999 & -0.0132 & 0.0303 & & \\
\hline 27 & US-27 & $\mathrm{MnO}$ & 0.0646 & 0.0675 & 0.0000 & 0.0646 & 0.0675 & & \\
\hline 27 & US-27 & $\mathrm{Na} 2 \mathrm{O}$ & 18.2991 & 17.8677 & 16.8681 & 1.4310 & 0.9996 & $8.5 \%$ & $5.9 \%$ \\
\hline 27 & US-27 & $\mathrm{NiO}$ & 0.0064 & 0.0071 & 0.0000 & 0.0064 & 0.0071 & & \\
\hline 27 & US-27 & $\mathrm{PbO}$ & 0.0194 & 0.0194 & 0.0000 & 0.0194 & 0.0194 & & \\
\hline
\end{tabular}


Table A4. Measured, Measured Bias-Corrected, and Targeted Compositions by Glass Number

\begin{tabular}{|c|c|c|c|c|c|c|c|c|c|}
\hline & & & & Measured & & & & & \\
\hline Glass & & & Measured & $\begin{array}{c}\text { Bias- } \\
\text { Corrected }\end{array}$ & Targeted & Diff of & Diff of & $\%$ Diff of & $\%$ Diff of \\
\hline$\#$ & GlassID & Oxide & $(\mathrm{wt} \%)$ & $(w t \%)$ & $(\mathrm{wt} \%)$ & Measured & Meas BC & Measured & Meas BC \\
\hline 27 & US-27 & $\mathrm{SiO} 2$ & 39.7375 & 39.8029 & 40.0442 & -0.3068 & -0.2414 & $-0.8 \%$ & $-0.6 \%$ \\
\hline 27 & US-27 & SO3 & 0.4832 & 0.4832 & 0.4999 & -0.0167 & -0.0167 & & \\
\hline 27 & US-27 & $\mathrm{SrO}$ & 0.0059 & 0.0059 & 0.0000 & 0.0059 & 0.0059 & & \\
\hline 27 & US-27 & TiO2 & 0.0104 & 0.0114 & 0.0000 & 0.0104 & 0.0114 & & \\
\hline 27 & US-27 & $\mathrm{ZnO}$ & 1.7325 & 1.7325 & 1.9996 & -0.2671 & -0.2671 & $-13.4 \%$ & $-13.4 \%$ \\
\hline 27 & US-27 & $\mathrm{ZrO} 2$ & 0.4204 & 0.4204 & 0.0000 & 0.4204 & 0.4204 & & \\
\hline 27 & US-27 & Sum & 101.8045 & 101.2167 & 99.9800 & 1.8245 & 1.2367 & $1.8 \%$ & $1.2 \%$ \\
\hline 28 & US-28 & $\mathrm{A} 12 \mathrm{O} 3$ & 10.4914 & 10.7106 & 17.4724 & -6.9810 & -6.7619 & $-40.0 \%$ & $-38.7 \%$ \\
\hline 28 & US-28 & $\mathrm{B} 2 \mathrm{O} 3$ & 6.2386 & 6.1960 & 17.9582 & -11.7196 & -11.7622 & $-65.3 \%$ & $-65.5 \%$ \\
\hline 28 & US-28 & $\mathrm{CaO}$ & 0.9270 & 1.0127 & 0.0000 & 0.9270 & 1.0127 & & \\
\hline 28 & US-28 & $\mathrm{Cr} 2 \mathrm{O} 3$ & 0.0091 & 0.0089 & 0.0000 & 0.0091 & 0.0089 & & \\
\hline 28 & US-28 & $\mathrm{Fe} 2 \mathrm{O} 3$ & 17.5853 & 17.5690 & 5.7221 & 11.8632 & 11.8469 & $207.3 \%$ & $207.0 \%$ \\
\hline 28 & US-28 & $\mathrm{K} 2 \mathrm{O}$ & 0.0602 & 0.0534 & 0.9998 & -0.9396 & -0.9464 & $-94.0 \%$ & $-94.7 \%$ \\
\hline 28 & US-28 & $\mathrm{Li} 2 \mathrm{O}$ & 2.0205 & 2.0485 & 1.9996 & 0.0209 & 0.0489 & $1.0 \%$ & $2.4 \%$ \\
\hline 28 & US-28 & $\mathrm{MgO}$ & 0.0083 & 0.0090 & 0.0000 & 0.0083 & 0.0090 & & \\
\hline 28 & US-28 & $\mathrm{MnO}$ & 0.0646 & 0.0684 & 0.0000 & 0.0646 & 0.0684 & & \\
\hline 28 & US-28 & $\mathrm{Na} 2 \mathrm{O}$ & 19.6134 & 19.1513 & 13.3553 & 6.2581 & 5.7960 & $46.9 \%$ & $43.4 \%$ \\
\hline 28 & US-28 & $\mathrm{NiO}$ & 0.0121 & 0.0134 & 0.0000 & 0.0121 & 0.0134 & & \\
\hline 28 & US-28 & $\mathrm{PbO}$ & 0.8814 & 0.8814 & 0.0000 & 0.8814 & 0.8814 & & \\
\hline 28 & US-28 & $\mathrm{SiO} 2$ & 38.0261 & 38.7008 & 35.9739 & 2.0521 & 2.7269 & $5.7 \%$ & $7.6 \%$ \\
\hline 28 & US-28 & SO3 & 0.0624 & 0.0624 & 0.4999 & -0.4375 & -0.4375 & & \\
\hline 28 & US-28 & $\mathrm{SrO}$ & 0.0411 & 0.0411 & 2.9994 & -2.9583 & -2.9583 & $-98.6 \%$ & $-98.6 \%$ \\
\hline 28 & US-28 & $\mathrm{TiO} 2$ & 0.9378 & 1.0200 & 0.9998 & -0.0620 & 0.0202 & $-6.2 \%$ & $2.0 \%$ \\
\hline 28 & US-28 & $\mathrm{ZnO}$ & 1.7461 & 1.7461 & 1.9996 & -0.2535 & -0.2535 & $-12.7 \%$ & $-12.7 \%$ \\
\hline 28 & US-28 & $\mathrm{ZrO} 2$ & 3.4310 & 3.4310 & 0.0000 & 3.4310 & 3.4310 & & \\
\hline 28 & US-28 & Sum & 102.1565 & 102.7242 & 99.9800 & 2.1765 & 2.7442 & $2.2 \%$ & $2.7 \%$ \\
\hline $28^{*}$ & $2_{\text {US-28* }}$ & $\mathrm{Al} 2 \mathrm{O} 3$ & 17.5487 & 17.7168 & 17.4724 & 0.0763 & 0.2444 & $0.4 \%$ & $1.4 \%$ \\
\hline $28^{*}$ & US-28* & $\mathrm{B} 2 \mathrm{O} 3$ & 17.9751 & 17.6372 & 17.9582 & 0.0169 & -0.3209 & $0.1 \%$ & $-1.8 \%$ \\
\hline $28 *$ & US-28* & $\mathrm{CaO}$ & 0.0346 & 0.0378 & 0.0000 & 0.0346 & 0.0378 & & \\
\hline $28^{*}$ & US-28* & $\mathrm{Cr} 2 \mathrm{O} 3$ & 0.0073 & 0.0071 & 0.0000 & 0.0073 & 0.0071 & & \\
\hline $28 *$ & US-28* & $\mathrm{Fe} 2 \mathrm{O} 3$ & 5.5544 & 5.6178 & 5.7221 & -0.1677 & -0.1043 & $-2.9 \%$ & $-1.8 \%$ \\
\hline $28^{*}$ & US-28* & $\mathrm{K} 2 \mathrm{O}$ & 0.9489 & 0.8414 & 0.9998 & -0.0509 & -0.1584 & $-5.1 \%$ & $-15.8 \%$ \\
\hline $28^{*}$ & US-28* & $\mathrm{Li} 2 \mathrm{O}$ & 2.0253 & 2.0510 & 1.9996 & 0.0257 & 0.0514 & $1.3 \%$ & $2.6 \%$ \\
\hline $28^{*}$ & US-28* & $\mathrm{MgO}$ & 0.0083 & 0.0090 & 0.0000 & 0.0083 & 0.0090 & & \\
\hline $28^{*}$ & US-28* & $\mathrm{MnO}$ & 0.0646 & 0.0674 & 0.0000 & 0.0646 & 0.0674 & & \\
\hline $28^{*}$ & US-28* & $\mathrm{Na} 2 \mathrm{O}$ & 14.0394 & 13.7094 & 13.3553 & 0.6841 & 0.3541 & $5.1 \%$ & $2.7 \%$ \\
\hline $28^{*}$ & US-28* & $\mathrm{NiO}$ & 0.0064 & 0.0071 & 0.0000 & 0.0064 & 0.0071 & & \\
\hline $28^{*}$ & US-28* & $\mathrm{PbO}$ & 0.0048 & 0.0048 & 0.0000 & 0.0048 & 0.0048 & & \\
\hline $28^{*}$ & US-28* & $\mathrm{SiO} 2$ & 35.8868 & 36.8955 & 35.9739 & -0.0872 & 0.9216 & $-0.2 \%$ & $2.6 \%$ \\
\hline $28 *$ & US-28* & SO3 & 0.3814 & 0.3814 & 0.4999 & -0.1185 & -0.1185 & & \\
\hline $28^{*}$ & US-28* & $\mathrm{SrO}$ & 2.4805 & 2.4805 & 2.9994 & -0.5189 & -0.5189 & $-17.3 \%$ & $-17.3 \%$ \\
\hline $28^{*}$ & US-28* & $\mathrm{TiO} 2$ & 0.9366 & 1.0187 & 0.9998 & -0.0632 & 0.0189 & $-6.3 \%$ & $1.9 \%$ \\
\hline $28^{*}$ & US-28* & $\mathrm{ZnO}$ & 1.7583 & 1.7583 & 1.9996 & -0.2413 & -0.2413 & $-12.1 \%$ & $-12.1 \%$ \\
\hline $28^{*}$ & US-28* & $\mathrm{ZrO} 2$ & 0.0128 & 0.0128 & 0.0000 & 0.0128 & 0.0128 & & \\
\hline $28^{*}$ & US-28* & Sum & 99.6742 & 100.2541 & 99.9800 & -0.3058 & 0.2741 & $-0.3 \%$ & $0.3 \%$ \\
\hline 29 & US-29 & $\mathrm{A} 12 \mathrm{O} 3$ & 10.2411 & 10.4410 & 9.9980 & 0.2431 & 0.4430 & $2.4 \%$ & $4.4 \%$ \\
\hline 29 & US-29 & $\mathrm{B} 2 \mathrm{O} 3$ & 8.9916 & 8.5426 & 8.7260 & 0.2656 & -0.1834 & $3.0 \%$ & $-2.1 \%$ \\
\hline 29 & US-29 & $\mathrm{CaO}$ & 0.9399 & 1.0119 & 0.9998 & -0.0599 & 0.0121 & $-6.0 \%$ & $1.2 \%$ \\
\hline 29 & US-29 & $\mathrm{Cr} 2 \mathrm{O} 3$ & 0.5536 & 0.5416 & 0.6709 & -0.1173 & -0.1293 & $-17.5 \%$ & $-19.3 \%$ \\
\hline 29 & US-29 & $\mathrm{Fe} 2 \mathrm{O} 3$ & 4.8860 & 4.8748 & 4.9990 & -0.1130 & -0.1242 & $-2.3 \%$ & $-2.5 \%$ \\
\hline 29 & US-29 & $\mathrm{K} 2 \mathrm{O}$ & 0.9845 & 0.8735 & 0.9998 & -0.0153 & -0.1263 & $-1.5 \%$ & $-12.6 \%$ \\
\hline 29 & US-29 & $\mathrm{Li} 2 \mathrm{O}$ & 5.8936 & 5.9336 & 5.9988 & -0.1052 & -0.0652 & $-1.8 \%$ & $-1.1 \%$ \\
\hline 29 & US-29 & $\mathrm{MgO}$ & 0.4979 & 0.5372 & 0.4999 & -0.0020 & 0.0373 & & \\
\hline 29 & US-29 & $\mathrm{MnO}$ & 0.0646 & 0.0675 & 0.0000 & 0.0646 & 0.0675 & & \\
\hline 29 & US-29 & $\mathrm{Na} 2 \mathrm{O}$ & 19.5123 & 18.8136 & 18.6845 & 0.8278 & 0.1291 & $4.4 \%$ & $0.7 \%$ \\
\hline 29 & US-29 & $\mathrm{NiO}$ & 0.0064 & 0.0070 & 0.0000 & 0.0064 & 0.0070 & & \\
\hline 29 & US-29 & $\mathrm{PbO}$ & 1.3007 & 1.3007 & 0.9998 & 0.3009 & 0.3009 & $30.1 \%$ & $30.1 \%$ \\
\hline 29 & US-29 & $\mathrm{SiO} 2$ & 44.0696 & 44.1372 & 45.2634 & -1.1939 & -1.1262 & $-2.6 \%$ & $-2.5 \%$ \\
\hline 29 & US-29 & $\mathrm{SO} 3$ & 0.0624 & 0.0624 & 0.0000 & 0.0624 & 0.0624 & & \\
\hline 29 & US-29 & $\mathrm{SrO}$ & 1.0762 & 1.0762 & 1.1759 & -0.0998 & -0.0998 & $-8.5 \%$ & $-8.5 \%$ \\
\hline 29 & US-29 & $\mathrm{TiO} 2$ & 0.9091 & 0.9787 & 0.9642 & -0.0551 & 0.0145 & $-5.7 \%$ & $1.5 \%$ \\
\hline 29 & US-29 & $\mathrm{ZnO}$ & 0.0078 & 0.0078 & 0.0000 & 0.0078 & 0.0078 & & \\
\hline 29 & US-29 & $\mathrm{ZrO} 2$ & 0.0068 & 0.0068 & 0.0000 & 0.0068 & 0.0068 & & \\
\hline 29 & US-29 & Sum & 100.0038 & 99.2140 & 99.9800 & 0.0238 & -0.7660 & $0.0 \%$ & $-0.8 \%$ \\
\hline
\end{tabular}

2 Note that the compositional views with the label US-28* are considered to be representative of study glass \#28 due to an inadvertent switching of the samples for US-28 and US-37 that were used for measuring chemical composition. 
Table A4. Measured, Measured Bias-Corrected, and Targeted Compositions by Glass Number

\begin{tabular}{|c|c|c|c|c|c|c|c|c|c|}
\hline & & & & Measured & & & & & \\
\hline Glass & & & Measured & $\begin{array}{c}\text { Bias- } \\
\text { Corrected }\end{array}$ & Targeted & Diff of & Diff of & $\%$ Diff of & $\%$ Diff of \\
\hline$\#$ & GlassID & Oxide & $\left(w_{t} \%\right)$ & $(w t \%)$ & $(\mathrm{wt} \%)$ & Measured & Meas BC & Measured & Meas BC \\
\hline 30 & US-30 & $\mathrm{A} 12 \mathrm{O} 3$ & 12.9809 & 13.1649 & 12.6458 & 0.3351 & 0.5191 & $2.6 \%$ & $4.1 \%$ \\
\hline 30 & US-30 & $\mathrm{B} 2 \mathrm{O} 3$ & 18.4983 & 18.3740 & 18.1904 & 0.3079 & 0.1835 & $1.7 \%$ & $1.0 \%$ \\
\hline 30 & US-30 & $\mathrm{CaO}$ & 0.9784 & 1.0533 & 0.9998 & -0.0214 & 0.0535 & $-2.1 \%$ & $5.4 \%$ \\
\hline 30 & US-30 & Cr2O3 & 0.5814 & 0.5687 & 0.8185 & -0.2371 & -0.2497 & $-29.0 \%$ & $-30.5 \%$ \\
\hline 30 & US-30 & $\mathrm{Fe} 2 \mathrm{O} 3$ & 5.3828 & 5.4030 & 5.3285 & 0.0544 & 0.0745 & $1.0 \%$ & $1.4 \%$ \\
\hline 30 & US-30 & $\mathrm{K} 2 \mathrm{O}$ & 0.9911 & 0.8794 & 0.9998 & -0.0087 & -0.1204 & $-0.9 \%$ & $-12.0 \%$ \\
\hline 30 & US-30 & $\mathrm{Li} 2 \mathrm{O}$ & 5.9582 & 6.0530 & 5.9980 & -0.0398 & 0.0550 & $-0.7 \%$ & $0.9 \%$ \\
\hline 30 & US-30 & $\mathrm{MgO}$ & 0.0083 & 0.0089 & 0.0000 & 0.0083 & 0.0089 & & \\
\hline 30 & US-30 & $\mathrm{MnO}$ & 3.5314 & 3.7515 & 3.4970 & 0.0344 & 0.2544 & $1.0 \%$ & $7.3 \%$ \\
\hline 30 & US-30 & $\mathrm{Na} 2 \mathrm{O}$ & 5.6549 & 5.4525 & 4.9990 & 0.6559 & 0.4535 & $13.1 \%$ & $9.1 \%$ \\
\hline 30 & US-30 & $\mathrm{NiO}$ & 0.0064 & 0.0070 & 0.0000 & 0.0064 & 0.0070 & & \\
\hline 30 & US-30 & $\mathrm{PbO}$ & 0.0054 & 0.0054 & 0.0000 & 0.0054 & 0.0054 & & \\
\hline 30 & US-30 & $\mathrm{SiO} 2$ & 38.2400 & 39.0586 & 37.5242 & 0.7158 & 1.5343 & $1.9 \%$ & $4.1 \%$ \\
\hline 30 & US-30 & $\mathrm{SO} 3$ & 0.4582 & 0.4582 & 0.4999 & -0.0417 & -0.0417 & & \\
\hline 30 & US-30 & $\mathrm{SrO}$ & 2.6904 & 2.6904 & 2.9994 & -0.3090 & -0.3090 & $-10.3 \%$ & $-10.3 \%$ \\
\hline 30 & US-30 & $\mathrm{TiO} 2$ & 0.9754 & 1.0501 & 0.9998 & -0.0244 & 0.0503 & $-2.4 \%$ & $5.0 \%$ \\
\hline 30 & US-30 & $\mathrm{ZnO}$ & 0.4544 & 0.4544 & 0.4808 & -0.0264 & -0.0264 & & \\
\hline 30 & US-30 & $\mathrm{ZrO} 2$ & 3.5762 & 3.5762 & 3.9992 & -0.4230 & -0.4230 & $-10.6 \%$ & $-10.6 \%$ \\
\hline 30 & US-30 & Sum & 100.9719 & 102.0093 & 99.9800 & 0.9919 & 2.0293 & $1.0 \%$ & $2.0 \%$ \\
\hline 31 & US-31 & $\mathrm{A} 12 \mathrm{O} 3$ & 13.3446 & 13.4722 & 12.6677 & 0.6769 & 0.8045 & $5.3 \%$ & $6.4 \%$ \\
\hline 31 & US-31 & $\mathrm{B} 2 \mathrm{O} 3$ & 14.4976 & 14.2239 & 14.5213 & -0.0237 & -0.2974 & $-0.2 \%$ & $-2.0 \%$ \\
\hline 31 & US-31 & $\mathrm{CaO}$ & 0.0574 & 0.0617 & 0.0000 & 0.0574 & 0.0617 & & \\
\hline 31 & US-31 & $\mathrm{Cr} 2 \mathrm{O} 3$ & 0.0132 & 0.0129 & 0.0000 & 0.0132 & 0.0129 & & \\
\hline 31 & US-31 & $\mathrm{Fe} 2 \mathrm{O} 3$ & 14.6902 & 14.8594 & 15.4482 & -0.7581 & -0.5888 & $-4.9 \%$ & $-3.8 \%$ \\
\hline 31 & US-31 & $\mathrm{K} 2 \mathrm{O}$ & 0.0602 & 0.0534 & 0.0000 & 0.0602 & 0.0534 & & \\
\hline 31 & US-31 & $\mathrm{Li} 2 \mathrm{O}$ & 4.9301 & 4.9923 & 4.9084 & 0.0218 & 0.0839 & $0.4 \%$ & $1.7 \%$ \\
\hline 31 & US-31 & $\mathrm{MgO}$ & 0.4805 & 0.5184 & 0.4999 & -0.0194 & 0.0185 & & \\
\hline 31 & US-31 & $\mathrm{MnO}$ & 0.7437 & 0.7767 & 0.7410 & 0.0027 & 0.0357 & $0.4 \%$ & $4.8 \%$ \\
\hline 31 & US-31 & $\mathrm{Na} 2 \mathrm{O}$ & 8.3205 & 8.0216 & 7.0969 & 1.2236 & 0.9247 & $17.2 \%$ & $13.0 \%$ \\
\hline 31 & US-31 & $\mathrm{NiO}$ & 0.0223 & 0.0246 & 0.0000 & 0.0223 & 0.0246 & & \\
\hline 31 & US-31 & $\mathrm{PbO}$ & 0.8774 & 0.8774 & 0.9998 & -0.1224 & -0.1224 & $-12.2 \%$ & $-12.2 \%$ \\
\hline 31 & US-31 & $\mathrm{SiO} 2$ & 38.9353 & 40.0301 & 38.5976 & 0.3377 & 1.4325 & $0.9 \%$ & $3.7 \%$ \\
\hline 31 & US-31 & SO3 & 0.3683 & 0.3683 & 0.4999 & -0.1316 & -0.1316 & & \\
\hline 31 & US-31 & $\mathrm{SrO}$ & 2.5367 & 2.5367 & 2.9994 & -0.4627 & -0.4627 & $-15.4 \%$ & $-15.4 \%$ \\
\hline 31 & US-31 & $\mathrm{TiO} 2$ & 0.9541 & 1.0272 & 0.9998 & -0.0457 & 0.0274 & $-4.6 \%$ & $2.7 \%$ \\
\hline 31 & US-31 & $\mathrm{ZnO}$ & 0.0062 & 0.0062 & 0.0000 & 0.0062 & 0.0062 & & \\
\hline 31 & US-31 & $\mathrm{ZrO} 2$ & 0.0111 & 0.0111 & 0.0000 & 0.0111 & 0.0111 & & \\
\hline 31 & US-31 & Sum & 100.8493 & 101.8742 & 99.9800 & 0.8693 & 1.8942 & $0.9 \%$ & $1.9 \%$ \\
\hline 32 & US-32 & $\mathrm{Al} 2 \mathrm{O} 3$ & 10.4348 & 10.6526 & 9.9980 & 0.4368 & 0.6546 & $4.4 \%$ & $6.5 \%$ \\
\hline 32 & US-32 & $\mathrm{B} 2 \mathrm{O} 3$ & 19.0618 & 18.9381 & 19.0122 & 0.0496 & -0.0741 & $0.3 \%$ & $-0.4 \%$ \\
\hline 32 & US-32 & $\mathrm{CaO}$ & 0.9333 & 1.0138 & 0.9998 & -0.0665 & 0.0140 & $-6.7 \%$ & $1.4 \%$ \\
\hline 32 & US-32 & $\mathrm{Cr} 2 \mathrm{O} 3$ & 0.0164 & 0.0161 & 0.0000 & 0.0164 & 0.0161 & & \\
\hline 32 & US-32 & $\mathrm{Fe} 2 \mathrm{O} 3$ & 5.0504 & 5.0452 & 4.9990 & 0.0514 & 0.0462 & $1.0 \%$ & $0.9 \%$ \\
\hline 32 & US-32 & $\mathrm{K} 2 \mathrm{O}$ & 0.0602 & 0.0531 & 0.0000 & 0.0602 & 0.0531 & & \\
\hline 32 & US-32 & $\mathrm{Li} 2 \mathrm{O}$ & 6.1788 & 6.2675 & 5.9988 & 0.1800 & 0.2687 & $3.0 \%$ & $4.5 \%$ \\
\hline 32 & US-32 & $\mathrm{MgO}$ & 0.0083 & 0.0090 & 0.0000 & 0.0083 & 0.0090 & & \\
\hline 32 & US-32 & $\mathrm{MnO}$ & 4.3320 & 4.5935 & 3.9992 & 0.3328 & 0.5943 & $8.3 \%$ & $14.9 \%$ \\
\hline 32 & US-32 & $\mathrm{Na} 2 \mathrm{O}$ & 7.9465 & 7.8294 & 7.5029 & 0.4436 & 0.3266 & $5.9 \%$ & $4.4 \%$ \\
\hline 32 & US-32 & $\mathrm{NiO}$ & 0.0064 & 0.0071 & 0.0000 & 0.0064 & 0.0071 & & \\
\hline 32 & US-32 & $\mathrm{PbO}$ & 0.8943 & 0.8943 & 0.9998 & -0.1055 & -0.1055 & $-10.5 \%$ & $-10.5 \%$ \\
\hline 32 & US-32 & $\mathrm{SiO} 2$ & 43.7487 & 44.5335 & 43.9709 & -0.2222 & 0.5626 & $-0.5 \%$ & $1.3 \%$ \\
\hline 32 & US-32 & SO3 & 0.4114 & 0.4114 & 0.4999 & -0.0885 & -0.0885 & & \\
\hline 32 & US-32 & $\mathrm{SrO}$ & 0.0059 & 0.0059 & 0.0000 & 0.0059 & 0.0059 & & \\
\hline 32 & US-32 & $\mathrm{TiO} 2$ & 0.0083 & 0.0090 & 0.0000 & 0.0083 & 0.0090 & & \\
\hline 32 & US-32 & $\mathrm{ZnO}$ & 1.7732 & 1.7732 & 1.9996 & -0.2264 & -0.2264 & $-11.3 \%$ & $-11.3 \%$ \\
\hline 32 & US-32 & $\mathrm{ZrO} 2$ & 0.0439 & 0.0439 & 0.0000 & 0.0439 & 0.0439 & & \\
\hline 32 & US-32 & Sum & 100.9146 & 102.0966 & 99.9800 & 0.9346 & 2.1166 & $0.9 \%$ & $2.1 \%$ \\
\hline 33 & US-33 & $\mathrm{Al} 2 \mathrm{O} 3$ & 11.9794 & 12.2054 & 11.3446 & 0.6348 & 0.8608 & $5.6 \%$ & $7.6 \%$ \\
\hline 33 & US-33 & $\mathrm{B} 2 \mathrm{O} 3$ & 5.5704 & 5.3554 & 4.9990 & 0.5714 & 0.3564 & $11.4 \%$ & $7.1 \%$ \\
\hline 33 & US-33 & $\mathrm{CaO}$ & 0.0350 & 0.0380 & 0.0000 & 0.0350 & 0.0380 & & \\
\hline 33 & US-33 & $\mathrm{Cr} 2 \mathrm{O} 3$ & 0.7231 & 0.7075 & 0.9998 & -0.2767 & -0.2923 & $-27.7 \%$ & $-29.2 \%$ \\
\hline 33 & US-33 & $\mathrm{Fe} 2 \mathrm{O} 3$ & 5.0754 & 5.0332 & 4.9990 & 0.0764 & 0.0342 & $1.5 \%$ & $0.7 \%$ \\
\hline 33 & US-33 & $\mathrm{K} 2 \mathrm{O}$ & 0.0602 & 0.0531 & 0.0000 & 0.0602 & 0.0531 & & \\
\hline 33 & US-33 & $\mathrm{Li} 2 \mathrm{O}$ & 2.0630 & 2.0890 & 1.9996 & 0.0634 & 0.0894 & $3.2 \%$ & $4.5 \%$ \\
\hline 33 & US-33 & $\mathrm{MgO}$ & 0.5302 & 0.5765 & 0.4999 & 0.0303 & 0.0766 & & \\
\hline 33 & US-33 & $\mathrm{MnO}$ & 4.2480 & 4.4599 & 3.9992 & 0.2488 & 0.4607 & $6.2 \%$ & $11.5 \%$ \\
\hline 33 & US-33 & $\mathrm{Na} 2 \mathrm{O}$ & 19.7482 & 19.4583 & 19.7605 & -0.0123 & -0.3022 & $-0.1 \%$ & $-1.5 \%$ \\
\hline 33 & US-33 & $\mathrm{NiO}$ & 0.8898 & 0.9921 & 0.9652 & -0.0755 & 0.0268 & $-7.8 \%$ & $2.8 \%$ \\
\hline
\end{tabular}


Table A4. Measured, Measured Bias-Corrected, and Targeted Compositions by Glass Number

\begin{tabular}{|c|c|c|c|c|c|c|c|c|c|}
\hline & & & & Measured & & & & & \\
\hline Glass & & & Measured & $\begin{array}{c}\text { Bias- } \\
\text { Corrected }\end{array}$ & Targeted & Diff of & Diff of & $\%$ Diff of & $\%$ Diff of \\
\hline$\#$ & GlassID & Oxide & $(w t \%)$ & $(w t \%)$ & $(\mathrm{wt} \%)$ & Measured & Meas BC & Measured & Meas BC \\
\hline 33 & US-33 & $\mathrm{PbO}$ & 0.8995 & 0.8995 & 0.9998 & -0.1003 & -0.1003 & $-10.0 \%$ & $-10.0 \%$ \\
\hline 33 & US-33 & $\mathrm{SiO} 2$ & 45.9415 & 47.7953 & 45.9140 & 0.0275 & 1.8813 & $0.1 \%$ & $4.1 \%$ \\
\hline 33 & US-33 & SO3 & 0.5056 & 0.5056 & 0.4999 & 0.0057 & 0.0057 & & \\
\hline 33 & US-33 & $\mathrm{SrO}$ & 2.6076 & 2.6076 & 2.9994 & -0.3918 & -0.3918 & $-13.1 \%$ & $-13.1 \%$ \\
\hline 33 & US-33 & $\mathrm{TiO} 2$ & 0.0083 & 0.0090 & 0.0000 & 0.0083 & 0.0090 & & \\
\hline 33 & US-33 & $\mathrm{ZnO}$ & 0.1198 & 0.1198 & 0.0000 & 0.1198 & 0.1198 & & \\
\hline 33 & US-33 & $\mathrm{ZrO} 2$ & 0.0064 & 0.0064 & 0.0000 & 0.0064 & 0.0064 & & \\
\hline 33 & US-33 & Sum & 101.0117 & 102.9118 & 99.9800 & 1.0317 & 2.9317 & $1.0 \%$ & $2.9 \%$ \\
\hline 34 & US-34 & $\mathrm{A} 12 \mathrm{O} 3$ & 10.2978 & 10.4921 & 9.9980 & 0.2998 & 0.4941 & $3.0 \%$ & $4.9 \%$ \\
\hline 34 & US-34 & $\mathrm{B} 2 \mathrm{O} 3$ & 14.1595 & 13.6212 & 13.6905 & 0.4690 & -0.0694 & $3.4 \%$ & $-0.5 \%$ \\
\hline 34 & US-34 & $\mathrm{CaO}$ & 0.0325 & 0.0337 & 0.0000 & 0.0325 & 0.0337 & & \\
\hline 34 & US-34 & Cr2O3 & 0.0095 & 0.0094 & 0.0000 & 0.0095 & 0.0094 & & \\
\hline 34 & US-34 & $\mathrm{Fe} 2 \mathrm{O} 3$ & 6.4515 & 6.3978 & 6.3853 & 0.0662 & 0.0125 & $1.0 \%$ & $0.2 \%$ \\
\hline 34 & US-34 & $\mathrm{K} 2 \mathrm{O}$ & 1.0579 & 0.9289 & 0.9998 & 0.0581 & -0.0709 & $5.8 \%$ & $-7.1 \%$ \\
\hline 34 & US-34 & $\mathrm{Li} 2 \mathrm{O}$ & 2.4112 & 2.4418 & 2.4196 & -0.0084 & 0.0221 & $-0.3 \%$ & $0.9 \%$ \\
\hline 34 & US-34 & $\mathrm{MgO}$ & 0.4946 & 0.5366 & 0.4999 & -0.0053 & 0.0367 & & \\
\hline 34 & US-34 & $\mathrm{MnO}$ & 4.0899 & 4.2940 & 3.9992 & 0.0907 & 0.2948 & $2.3 \%$ & $7.4 \%$ \\
\hline 34 & US-34 & $\mathrm{Na} 2 \mathrm{O}$ & 19.4112 & 19.3388 & 19.9960 & -0.5848 & -0.6572 & $-2.9 \%$ & $-3.3 \%$ \\
\hline 34 & US-34 & $\mathrm{NiO}$ & 0.0064 & 0.0071 & 0.0000 & 0.0064 & 0.0071 & & \\
\hline 34 & US-34 & $\mathrm{PbO}$ & 0.0054 & 0.0054 & 0.0000 & 0.0054 & 0.0054 & & \\
\hline 34 & US-34 & $\mathrm{SiO} 2$ & 35.0845 & 36.5046 & 34.9930 & 0.0915 & 1.5116 & $0.3 \%$ & $4.3 \%$ \\
\hline 34 & US-34 & $\mathrm{SO} 3$ & 0.0624 & 0.0624 & 0.0000 & 0.0624 & 0.0624 & & \\
\hline 34 & US-34 & $\mathrm{SrO}$ & 2.6845 & 2.6845 & 2.9994 & -0.3149 & -0.3149 & $-10.5 \%$ & $-10.5 \%$ \\
\hline 34 & US-34 & $\mathrm{TiO} 2$ & 0.0083 & 0.0090 & 0.0000 & 0.0083 & 0.0090 & & \\
\hline 34 & US-34 & $\mathrm{ZnO}$ & 0.0062 & 0.0062 & 0.0000 & 0.0062 & 0.0062 & & \\
\hline 34 & US-34 & $\mathrm{ZrO} 2$ & 3.5053 & 3.5053 & 3.9992 & -0.4939 & -0.4939 & $-12.3 \%$ & $-12.3 \%$ \\
\hline 34 & US-34 & Sum & 99.7788 & 100.8789 & 99.9800 & -0.2012 & 0.8989 & $-0.2 \%$ & $0.9 \%$ \\
\hline 35 & US-35 & $\mathrm{A} 12 \mathrm{O} 3$ & 10.3356 & 10.4823 & 10.0161 & 0.3195 & 0.4663 & $3.2 \%$ & $4.7 \%$ \\
\hline 35 & US-35 & $\mathrm{B} 2 \mathrm{O} 3$ & 19.8265 & 19.6940 & 19.9577 & -0.1311 & -0.2637 & $-0.7 \%$ & $-1.3 \%$ \\
\hline 35 & US-35 & $\mathrm{CaO}$ & 0.9172 & 1.0224 & 0.9998 & -0.0826 & 0.0226 & $-8.3 \%$ & $2.3 \%$ \\
\hline 35 & US-35 & $\mathrm{Cr} 2 \mathrm{O} 3$ & 0.4396 & 0.4281 & 0.5175 & -0.0779 & -0.0894 & $-15.1 \%$ & $-17.3 \%$ \\
\hline 35 & US-35 & $\mathrm{Fe} 2 \mathrm{O} 3$ & 11.3018 & 11.3442 & 11.3518 & -0.0500 & -0.0076 & $-0.4 \%$ & $-0.1 \%$ \\
\hline 35 & US-35 & $\mathrm{K} 2 \mathrm{O}$ & 0.0602 & 0.0530 & 0.0000 & 0.0602 & 0.0530 & & \\
\hline 35 & US-35 & $\mathrm{Li} 2 \mathrm{O}$ & 2.0286 & 2.0608 & 1.9996 & 0.0290 & 0.0612 & $1.4 \%$ & $3.1 \%$ \\
\hline 35 & US-35 & $\mathrm{MgO}$ & 0.4979 & 0.5367 & 0.4999 & -0.0020 & 0.0368 & & \\
\hline 35 & US-35 & $\mathrm{MnO}$ & 4.0511 & 4.3035 & 3.9992 & 0.0519 & 0.3043 & $1.3 \%$ & $7.6 \%$ \\
\hline 35 & US-35 & $\mathrm{Na} 2 \mathrm{O}$ & 8.8024 & 8.4472 & 7.9410 & 0.8614 & 0.5061 & $10.8 \%$ & $6.4 \%$ \\
\hline 35 & US-35 & $\mathrm{NiO}$ & 0.0108 & 0.0119 & 0.0000 & 0.0108 & 0.0119 & & \\
\hline 35 & US-35 & $\mathrm{PbO}$ & 0.0048 & 0.0048 & 0.0000 & 0.0048 & 0.0048 & & \\
\hline 35 & US-35 & $\mathrm{SiO} 2$ & 37.9726 & 38.7851 & 37.9950 & -0.0224 & 0.7901 & $-0.1 \%$ & $2.1 \%$ \\
\hline 35 & US-35 & $\mathrm{SO} 3$ & 0.0624 & 0.0624 & 0.0000 & 0.0624 & 0.0624 & & \\
\hline 35 & US-35 & $\mathrm{SrO}$ & 2.3386 & 2.3386 & 2.7028 & -0.3642 & -0.3642 & $-13.5 \%$ & $-13.5 \%$ \\
\hline 35 & US-35 & TiO2 & 0.0083 & 0.0091 & 0.0000 & 0.0083 & 0.0091 & & \\
\hline 35 & US-35 & $\mathrm{ZnO}$ & 1.7564 & 1.7564 & 1.9996 & -0.2432 & -0.2432 & $-12.2 \%$ & $-12.2 \%$ \\
\hline 35 & US-35 & $\mathrm{ZrO} 2$ & 0.0091 & 0.0091 & 0.0000 & 0.0091 & 0.0091 & & \\
\hline 35 & US-35 & Sum & 100.4240 & 101.3497 & 99.9800 & 0.4440 & 1.3697 & $0.4 \%$ & $1.4 \%$ \\
\hline 36 & US-36 & $\mathrm{A} 12 \mathrm{O} 3$ & 12.9336 & 13.1173 & 12.8579 & 0.0757 & 0.2594 & $0.6 \%$ & $2.0 \%$ \\
\hline 36 & US-36 & $\mathrm{B} 2 \mathrm{O} 3$ & 15.4394 & 15.3366 & 15.5118 & -0.0724 & -0.1752 & $-0.5 \%$ & $-1.1 \%$ \\
\hline 36 & US-36 & $\mathrm{CaO}$ & 0.3453 & 0.3849 & 0.2074 & 0.1378 & 0.1774 & & \\
\hline 36 & US-36 & $\mathrm{Cr} 2 \mathrm{O} 3$ & 0.3928 & 0.3826 & 0.5109 & -0.1181 & -0.1284 & $-23.1 \%$ & $-25.1 \%$ \\
\hline 36 & US-36 & $\mathrm{Fe} 2 \mathrm{O} 3$ & 9.6969 & 9.7337 & 9.7610 & -0.0641 & -0.0274 & $-0.7 \%$ & $-0.3 \%$ \\
\hline 36 & US-36 & $\mathrm{K} 2 \mathrm{O}$ & 0.5613 & 0.4940 & 0.5181 & 0.0432 & -0.0241 & $8.3 \%$ & $-4.6 \%$ \\
\hline 36 & US-36 & $\mathrm{Li} 2 \mathrm{O}$ & 2.4005 & 2.4387 & 2.4222 & -0.0217 & 0.0165 & $-0.9 \%$ & $0.7 \%$ \\
\hline 36 & US-36 & $\mathrm{MgO}$ & 0.0452 & 0.0487 & 0.0455 & -0.0003 & 0.0032 & & \\
\hline 36 & US-36 & $\mathrm{MnO}$ & 1.1053 & 1.1742 & 1.2055 & -0.1003 & -0.0314 & $-8.3 \%$ & $-2.6 \%$ \\
\hline 36 & US-36 & $\mathrm{Na} 2 \mathrm{O}$ & 13.9181 & 13.3547 & 12.5191 & 1.3990 & 0.8356 & $11.2 \%$ & $6.7 \%$ \\
\hline 36 & US-36 & $\mathrm{NiO}$ & 0.5020 & 0.5526 & 0.5580 & -0.0560 & -0.0054 & $-10.0 \%$ & $-1.0 \%$ \\
\hline 36 & US-36 & $\mathrm{PbO}$ & 0.9183 & 0.9183 & 0.9998 & -0.0815 & -0.0815 & $-8.2 \%$ & $-8.2 \%$ \\
\hline 36 & US-36 & $\mathrm{SiO} 2$ & 38.0795 & 38.8950 & 38.8410 & -0.7615 & 0.0540 & $-2.0 \%$ & $0.1 \%$ \\
\hline 36 & US-36 & SO3 & 0.0624 & 0.0624 & 0.0000 & 0.0624 & 0.0624 & & \\
\hline 36 & US-36 & $\mathrm{SrO}$ & 0.0059 & 0.0059 & 0.0075 & -0.0016 & -0.0016 & & \\
\hline 36 & US-36 & $\mathrm{TiO} 2$ & 0.0225 & 0.0245 & 0.0075 & 0.0150 & 0.0170 & & \\
\hline 36 & US-36 & $\mathrm{ZnO}$ & 0.0137 & 0.0137 & 0.0075 & 0.0062 & 0.0062 & & \\
\hline 36 & US-36 & $\mathrm{ZrO} 2$ & 3.4716 & 3.4716 & 3.9992 & -0.5276 & -0.5276 & $-13.2 \%$ & $-13.2 \%$ \\
\hline 36 & US-36 & Sum & 99.9144 & 100.4092 & 99.9800 & -0.0656 & 0.4292 & $-0.1 \%$ & $0.4 \%$ \\
\hline 37 & $\begin{array}{l}\text { US-37 } \\
\end{array}$ & $\mathrm{A} 12 \mathrm{O} 3$ & 17.5487 & 17.7168 & 9.9980 & 7.5507 & 7.7188 & $75.5 \%$ & $77.2 \%$ \\
\hline 37 & US-37 & $\mathrm{B} 2 \mathrm{O} 3$ & 17.9751 & 17.6372 & 5.7703 & 12.2048 & 11.8669 & $211.5 \%$ & $205.7 \%$ \\
\hline 37 & US-37 & $\mathrm{CaO}$ & 0.0346 & 0.0378 & 0.9998 & -0.9652 & -0.9620 & $-96.5 \%$ & $-96.2 \%$ \\
\hline
\end{tabular}


Table A4. Measured, Measured Bias-Corrected, and Targeted Compositions by Glass Number

\begin{tabular}{|c|c|c|c|c|c|c|c|c|c|}
\hline & & & & Measured & & & & & \\
\hline Glass & & & Measured & $\begin{array}{c}\text { Bias- } \\
\text { Corrected }\end{array}$ & Targeted & Diff of & Diff of & $\%$ Diff of & $\%$ Diff of \\
\hline$\#$ & GlassID & Oxide & $(\mathrm{wt} \%)$ & $(w t \%)$ & $(\mathrm{wt} \%)$ & Measured & Meas BC & Measured & Meas BC \\
\hline 37 & US-37 & $\mathrm{Cr} 2 \mathrm{O} 3$ & 0.0073 & 0.0071 & 0.0000 & 0.0073 & 0.0071 & & \\
\hline 37 & US-37 & $\mathrm{Fe} 2 \mathrm{O} 3$ & 5.5544 & 5.6178 & 16.9966 & -11.4422 & -11.3788 & $-67.3 \%$ & $-66.9 \%$ \\
\hline 37 & US-37 & $\mathrm{K} 2 \mathrm{O}$ & 0.9489 & 0.8414 & 0.0000 & 0.9489 & 0.8414 & & \\
\hline 37 & US-37 & $\mathrm{Li} 2 \mathrm{O}$ & 2.0253 & 2.0510 & 1.9996 & 0.0257 & 0.0514 & $1.3 \%$ & $2.6 \%$ \\
\hline 37 & US-37 & $\mathrm{MgO}$ & 0.0083 & 0.0090 & 0.0000 & 0.0083 & 0.0090 & & \\
\hline 37 & US-37 & $\mathrm{MnO}$ & 0.0646 & 0.0674 & 0.0000 & 0.0646 & 0.0674 & & \\
\hline 37 & US-37 & $\mathrm{Na} 2 \mathrm{O}$ & 14.0394 & 13.7094 & 18.8862 & -4.8468 & -5.1768 & $-25.7 \%$ & $-27.4 \%$ \\
\hline 37 & US-37 & $\mathrm{NiO}$ & 0.0064 & 0.0071 & 0.0000 & 0.0064 & 0.0071 & & \\
\hline 37 & US-37 & $\mathrm{PbO}$ & 0.0048 & 0.0048 & 0.9998 & -0.9950 & -0.9950 & $-99.5 \%$ & $-99.5 \%$ \\
\hline 37 & US-37 & $\mathrm{SiO} 2$ & 35.8868 & 36.8955 & 37.3311 & -1.4443 & -0.4356 & $-3.9 \%$ & $-1.2 \%$ \\
\hline 37 & US-37 & SO3 & 0.3814 & 0.3814 & 0.0000 & 0.3814 & 0.3814 & & \\
\hline 37 & US-37 & $\mathrm{SrO}$ & 2.4805 & 2.4805 & 0.0000 & 2.4805 & 2.4805 & & \\
\hline 37 & US-37 & $\mathrm{TiO} 2$ & 0.9366 & 1.0187 & 0.9998 & -0.0632 & 0.0189 & $-6.3 \%$ & $1.9 \%$ \\
\hline 37 & US-37 & $\mathrm{ZnO}$ & 1.7583 & 1.7583 & 1.9996 & -0.2413 & -0.2413 & $-12.1 \%$ & $-12.1 \%$ \\
\hline 37 & US-37 & $\mathrm{ZrO} 2$ & 0.0128 & 0.0128 & 3.9992 & -3.9864 & -3.9864 & $-99.7 \%$ & $-99.7 \%$ \\
\hline 37 & US-37 & Sum & 99.6742 & 100.2541 & 99.9800 & -0.3058 & 0.2741 & $-0.3 \%$ & $0.3 \%$ \\
\hline $37 *$ & ${ }^{3}$ US-37* & $\mathrm{A} 12 \mathrm{O} 3$ & 10.4914 & 10.7106 & 9.9980 & 0.4934 & 0.7126 & $4.9 \%$ & $7.1 \%$ \\
\hline $37 *$ & US-37* & $\mathrm{B} 2 \mathrm{O} 3$ & 6.2386 & 6.1960 & 5.7703 & 0.4682 & 0.4257 & $8.1 \%$ & $7.4 \%$ \\
\hline $37^{*}$ & US-37* & $\mathrm{CaO}$ & 0.9270 & 1.0127 & 0.9998 & -0.0728 & 0.0129 & $-7.3 \%$ & $1.3 \%$ \\
\hline $37 *$ & US-37* & $\mathrm{Cr} 2 \mathrm{O} 3$ & 0.0091 & 0.0089 & 0.0000 & 0.0091 & 0.0089 & & \\
\hline $37^{*}$ & US-37* & $\mathrm{Fe} 2 \mathrm{O} 3$ & 17.5853 & 17.5690 & 16.9966 & 0.5887 & 0.5724 & $3.5 \%$ & $3.4 \%$ \\
\hline $37^{*}$ & US-37* & $\mathrm{K} 2 \mathrm{O}$ & 0.0602 & 0.0534 & 0.0000 & 0.0602 & 0.0534 & & \\
\hline $37^{*}$ & US-37* & $\mathrm{Li} 2 \mathrm{O}$ & 2.0205 & 2.0485 & 1.9996 & 0.0209 & 0.0489 & $1.0 \%$ & $2.4 \%$ \\
\hline $37 *$ & US-37* & $\mathrm{MgO}$ & 0.0083 & 0.0090 & 0.0000 & 0.0083 & 0.0090 & & \\
\hline $37 *$ & US-37* & $\mathrm{MnO}$ & 0.0646 & 0.0684 & 0.0000 & 0.0646 & 0.0684 & & \\
\hline $37^{*}$ & US-37* & $\mathrm{Na} 2 \mathrm{O}$ & 19.6134 & 19.1513 & 18.8862 & 0.7272 & 0.2650 & $3.9 \%$ & $1.4 \%$ \\
\hline $37 *$ & US-37* & $\mathrm{NiO}$ & 0.0121 & 0.0134 & 0.0000 & 0.0121 & 0.0134 & & \\
\hline $37^{*}$ & US-37* & $\mathrm{PbO}$ & 0.8814 & 0.8814 & 0.9998 & -0.1184 & -0.1184 & $-11.8 \%$ & $-11.8 \%$ \\
\hline $37^{*}$ & US-37* & $\mathrm{SiO} 2$ & 38.0261 & 38.7008 & 37.3311 & 0.6950 & 1.3697 & $1.9 \%$ & $3.7 \%$ \\
\hline $37^{*}$ & US-37* & $\mathrm{SO} 3$ & 0.0624 & 0.0624 & 0.0000 & 0.0624 & 0.0624 & & \\
\hline $37 *$ & US-37* & $\mathrm{SrO}$ & 0.0411 & 0.0411 & 0.0000 & 0.0411 & 0.0411 & & \\
\hline $37 *$ & US-37* & TiO2 & 0.9378 & 1.0200 & 0.9998 & -0.0620 & 0.0202 & $-6.2 \%$ & $2.0 \%$ \\
\hline $37^{*}$ & US-37* & $\mathrm{ZnO}$ & 1.7461 & 1.7461 & 1.9996 & -0.2535 & -0.2535 & $-12.7 \%$ & $-12.7 \%$ \\
\hline $37 *$ & US-37* & $\mathrm{ZrO} 2$ & 3.4310 & 3.4310 & 3.9992 & -0.5682 & -0.5682 & $-14.2 \%$ & $-14.2 \%$ \\
\hline $37^{*}$ & US-37* & Sum & 102.1565 & 102.7242 & 99.9800 & 2.1765 & 2.7442 & $2.2 \%$ & $2.7 \%$ \\
\hline 38 & US-38 & $\mathrm{A} 12 \mathrm{O} 3$ & 10.3686 & 10.4680 & 9.9980 & 0.3706 & 0.4700 & $3.7 \%$ & $4.7 \%$ \\
\hline 38 & US-38 & $\mathrm{B} 2 \mathrm{O} 3$ & 7.5024 & 7.3605 & 7.3139 & 0.1885 & 0.0466 & $2.6 \%$ & $0.6 \%$ \\
\hline 38 & US-38 & $\mathrm{CaO}$ & 0.7699 & 0.7984 & 0.7894 & -0.0194 & 0.0090 & $-2.5 \%$ & $1.1 \%$ \\
\hline 38 & US-38 & $\mathrm{Cr} 2 \mathrm{O} 3$ & 0.0135 & 0.0134 & 0.0000 & 0.0135 & 0.0134 & & \\
\hline 38 & US-38 & $\mathrm{Fe} 2 \mathrm{O} 3$ & 5.4150 & 5.4766 & 4.9990 & 0.4160 & 0.4776 & $8.3 \%$ & $9.6 \%$ \\
\hline 38 & US-38 & $\mathrm{K} 2 \mathrm{O}$ & 1.0092 & 0.8863 & 0.9998 & 0.0094 & -0.1135 & $0.9 \%$ & $-11.4 \%$ \\
\hline 38 & US-38 & $\mathrm{Li} 2 \mathrm{O}$ & 2.0571 & 2.0831 & 1.9996 & 0.0575 & 0.0835 & $2.9 \%$ & $4.2 \%$ \\
\hline 38 & US-38 & $\mathrm{MgO}$ & 0.0083 & 0.0090 & 0.0000 & 0.0083 & 0.0090 & & \\
\hline 38 & US-38 & $\mathrm{MnO}$ & 4.0253 & 4.2037 & 3.9992 & 0.0261 & 0.2045 & $0.7 \%$ & $5.1 \%$ \\
\hline 38 & US-38 & $\mathrm{Na} 2 \mathrm{O}$ & 18.4002 & 18.3433 & 19.7246 & -1.3244 & -1.3813 & $-6.7 \%$ & $-7.0 \%$ \\
\hline 38 & US-38 & $\mathrm{NiO}$ & 2.8345 & 3.1652 & 2.9054 & -0.0709 & 0.2598 & $-2.4 \%$ & $8.9 \%$ \\
\hline 38 & US-38 & $\mathrm{PbO}$ & 0.8825 & 0.8825 & 0.9998 & -0.1173 & -0.1173 & $-11.7 \%$ & $-11.7 \%$ \\
\hline 38 & US-38 & $\mathrm{SiO} 2$ & 39.0957 & 40.1947 & 39.7527 & -0.6569 & 0.4421 & $-1.7 \%$ & $1.1 \%$ \\
\hline 38 & US-38 & SO3 & 0.4825 & 0.4825 & 0.4999 & -0.0174 & -0.0174 & & \\
\hline 38 & US-38 & $\mathrm{SrO}$ & 2.6490 & 2.6490 & 2.9994 & -0.3504 & -0.3504 & $-11.7 \%$ & $-11.7 \%$ \\
\hline 38 & US-38 & TiO2 & 0.9391 & 1.0106 & 0.9998 & -0.0607 & 0.0108 & $-6.1 \%$ & $1.1 \%$ \\
\hline 38 & US-38 & $\mathrm{ZnO}$ & 1.7642 & 1.7642 & 1.9996 & -0.2354 & -0.2354 & $-11.8 \%$ & $-11.8 \%$ \\
\hline 38 & US-38 & $\mathrm{ZrO} 2$ & 0.0192 & 0.0192 & 0.0000 & 0.0192 & 0.0192 & & \\
\hline 38 & US-38 & Sum & 98.2362 & 99.8102 & 99.9800 & -1.7438 & -0.1698 & $-1.7 \%$ & $-0.2 \%$ \\
\hline 39 & US-39 & $\mathrm{A} 12 \mathrm{O} 3$ & 11.8472 & 12.0785 & 11.5453 & 0.3018 & 0.5331 & $2.6 \%$ & $4.6 \%$ \\
\hline 39 & US-39 & $\mathrm{B} 2 \mathrm{O} 3$ & 16.6791 & 15.8517 & 16.3478 & 0.3313 & -0.4962 & $2.0 \%$ & $-3.0 \%$ \\
\hline 39 & US-39 & $\mathrm{CaO}$ & 0.0217 & 0.0225 & 0.0000 & 0.0217 & 0.0225 & & \\
\hline 39 & US-39 & $\mathrm{Cr} 2 \mathrm{O} 3$ & 0.0110 & 0.0109 & 0.0000 & 0.0110 & 0.0109 & & \\
\hline 39 & US-39 & $\mathrm{Fe} 2 \mathrm{O} 3$ & 7.3415 & 7.3245 & 7.5019 & -0.1604 & -0.1774 & $-2.1 \%$ & $-2.4 \%$ \\
\hline 39 & US-39 & $\mathrm{K} 2 \mathrm{O}$ & 1.0558 & 0.9272 & 0.9998 & 0.0560 & -0.0726 & $5.6 \%$ & $-7.3 \%$ \\
\hline 39 & US-39 & $\mathrm{Li} 2 \mathrm{O}$ & 5.9528 & 5.9932 & 5.9978 & -0.0450 & -0.0046 & $-0.8 \%$ & $-0.1 \%$ \\
\hline 39 & US-39 & $\mathrm{MgO}$ & 0.4958 & 0.5379 & 0.4999 & -0.0041 & 0.0380 & & \\
\hline 39 & US-39 & $\mathrm{MnO}$ & 0.0646 & 0.0675 & 0.0000 & 0.0646 & 0.0675 & & \\
\hline 39 & US-39 & $\mathrm{Na} 2 \mathrm{O}$ & 5.0213 & 5.0055 & 4.9990 & 0.0223 & 0.0065 & $0.4 \%$ & $0.1 \%$ \\
\hline
\end{tabular}

3 Note that the compositional views with the label US-37* are considered to be representative of study glass \#37 due to an inadvertent switching of the samples for US-28 and US-37 that were used for measuring chemical composition. 
Table A4. Measured, Measured Bias-Corrected, and Targeted Compositions by Glass Number

\begin{tabular}{|c|c|c|c|c|c|c|c|c|c|}
\hline & & & & Measured & & & & & \\
\hline Glass & & & Measured & $\begin{array}{c}\text { Bias- } \\
\text { Corrected }\end{array}$ & Targeted & Diff of & Diff of & $\%$ Diff of & $\%$ Diff of \\
\hline$\#$ & GlassID & Oxide & $(w t \%)$ & $(w t \%)$ & $(\mathrm{wt} \%)$ & Measured & Meas BC & Measured & Meas BC \\
\hline 39 & US-39 & $\mathrm{NiO}$ & 1.7147 & 1.9147 & 1.8280 & -0.1133 & 0.0866 & $-6.2 \%$ & $4.7 \%$ \\
\hline 39 & US-39 & $\mathrm{PbO}$ & 0.9110 & 0.9110 & 0.9998 & -0.0888 & -0.0888 & $-8.9 \%$ & $-8.9 \%$ \\
\hline 39 & US-39 & $\mathrm{SiO} 2$ & 40.7537 & 40.8400 & 42.4650 & -1.7113 & -1.6249 & $-4.0 \%$ & $-3.8 \%$ \\
\hline 39 & US-39 & SO3 & 0.0624 & 0.0624 & 0.0000 & 0.0624 & 0.0624 & & \\
\hline 39 & US-39 & $\mathrm{SrO}$ & 0.7285 & 0.7285 & 0.7968 & -0.0684 & -0.0684 & $-8.6 \%$ & $-8.6 \%$ \\
\hline 39 & US-39 & $\mathrm{TiO} 2$ & 0.0083 & 0.0090 & 0.0000 & 0.0083 & 0.0090 & & \\
\hline 39 & US-39 & $\mathrm{ZnO}$ & 1.7854 & 1.7854 & 1.9996 & -0.2142 & -0.2142 & $-10.7 \%$ & $-10.7 \%$ \\
\hline 39 & US-39 & $\mathrm{ZrO} 2$ & 3.5256 & 3.5256 & 3.9992 & -0.4736 & -0.4736 & $-11.8 \%$ & $-11.8 \%$ \\
\hline 39 & US-39 & Sum & 97.9803 & 97.5959 & 99.9800 & -1.9997 & -2.3841 & $-2.0 \%$ & $-2.4 \%$ \\
\hline 40 & US-40 & $\mathrm{Al} 2 \mathrm{O} 3$ & 15.7584 & 16.0875 & 15.5178 & 0.2406 & 0.5697 & $1.6 \%$ & $3.7 \%$ \\
\hline 40 & US-40 & $\mathrm{B} 2 \mathrm{O} 3$ & 17.0816 & 16.9675 & 17.0004 & 0.0812 & -0.0329 & $0.5 \%$ & $-0.2 \%$ \\
\hline 40 & US-40 & $\mathrm{CaO}$ & 0.0455 & 0.0507 & 0.0000 & 0.0455 & 0.0507 & & \\
\hline 40 & US-40 & $\mathrm{Cr} 2 \mathrm{O} 3$ & 0.3161 & 0.3078 & 0.4253 & -0.1092 & -0.1174 & & \\
\hline 40 & US-40 & $\mathrm{Fe} 2 \mathrm{O} 3$ & 11.1660 & 11.1567 & 11.4159 & -0.2499 & -0.2592 & $-2.2 \%$ & $-2.3 \%$ \\
\hline 40 & US-40 & $\mathrm{K} 2 \mathrm{O}$ & 1.0076 & 0.8868 & 0.9998 & 0.0078 & -0.1130 & $0.8 \%$ & $-11.3 \%$ \\
\hline 40 & US-40 & $\mathrm{Li} 2 \mathrm{O}$ & 2.5189 & 2.5541 & 2.5119 & 0.0070 & 0.0422 & $0.3 \%$ & $1.7 \%$ \\
\hline 40 & US-40 & $\mathrm{MgO}$ & 0.0083 & 0.0089 & 0.0000 & 0.0083 & 0.0089 & & \\
\hline 40 & US-40 & $\mathrm{MnO}$ & 2.7793 & 2.9450 & 2.7589 & 0.0204 & 0.1861 & $0.7 \%$ & $6.7 \%$ \\
\hline 40 & US-40 & $\mathrm{Na} 2 \mathrm{O}$ & 11.1109 & 10.6610 & 10.1312 & 0.9797 & 0.5298 & $9.7 \%$ & $5.2 \%$ \\
\hline 40 & US-40 & $\mathrm{NiO}$ & 0.0064 & 0.0070 & 0.0010 & 0.0054 & 0.0060 & & \\
\hline 40 & US-40 & $\mathrm{PbO}$ & 0.9213 & 0.9213 & 0.9988 & -0.0775 & -0.0775 & $-7.8 \%$ & $-7.8 \%$ \\
\hline 40 & US-40 & $\mathrm{SiO} 2$ & 37.8121 & 38.4858 & 37.2966 & 0.5155 & 1.1893 & $1.4 \%$ & $3.2 \%$ \\
\hline 40 & US-40 & $\mathrm{SO} 3$ & 0.0624 & 0.0624 & 0.0000 & 0.0624 & 0.0624 & & \\
\hline 40 & US-40 & $\mathrm{SrO}$ & 0.0059 & 0.0059 & 0.0000 & 0.0059 & 0.0059 & & \\
\hline 40 & US-40 & $\mathrm{TiO} 2$ & 0.8686 & 0.9447 & 0.9224 & -0.0538 & 0.0223 & $-5.8 \%$ & $2.4 \%$ \\
\hline 40 & US-40 & $\mathrm{ZnO}$ & 0.0118 & 0.0118 & 0.0000 & 0.0118 & 0.0118 & & \\
\hline 40 & US-40 & $\mathrm{ZrO} 2$ & 0.0068 & 0.0068 & 0.0000 & 0.0068 & 0.0068 & & \\
\hline 40 & US-40 & Sum & 101.4878 & 102.0719 & 99.9800 & 1.5078 & 2.0919 & $1.5 \%$ & $2.1 \%$ \\
\hline 41 & US-41 & $\mathrm{A} 12 \mathrm{O} 3$ & 10.0947 & 10.2380 & 9.9980 & 0.0967 & 0.2400 & $1.0 \%$ & $2.4 \%$ \\
\hline 41 & US-41 & $\mathrm{B} 2 \mathrm{O} 3$ & 15.3267 & 15.2242 & 15.5542 & -0.2275 & -0.3300 & $-1.5 \%$ & $-2.1 \%$ \\
\hline 41 & US-41 & $\mathrm{CaO}$ & 0.9329 & 1.0191 & 0.9998 & -0.0669 & 0.0193 & $-6.7 \%$ & $1.9 \%$ \\
\hline 41 & US-41 & $\mathrm{Cr} 2 \mathrm{O} 3$ & 0.0153 & 0.0150 & 0.0000 & 0.0153 & 0.0150 & & \\
\hline 41 & US-41 & $\mathrm{Fe} 2 \mathrm{O} 3$ & 14.7617 & 14.8183 & 15.5320 & -0.7703 & -0.7137 & $-5.0 \%$ & $-4.6 \%$ \\
\hline 41 & US-41 & $\mathrm{K} 2 \mathrm{O}$ & 0.9959 & 0.8830 & 0.9988 & -0.0029 & -0.1158 & $-0.3 \%$ & $-11.6 \%$ \\
\hline 41 & US-41 & Li2O & 3.6492 & 3.7072 & 3.7243 & -0.0751 & -0.0171 & $-2.0 \%$ & $-0.5 \%$ \\
\hline 41 & US-41 & $\mathrm{MgO}$ & 0.0083 & 0.0090 & 0.0000 & 0.0083 & 0.0090 & & \\
\hline 41 & US-41 & $\mathrm{MnO}$ & 0.0646 & 0.0686 & 0.0000 & 0.0646 & 0.0686 & & \\
\hline 41 & US-41 & $\mathrm{Na} 2 \mathrm{O}$ & 7.0500 & 6.8835 & 6.5193 & 0.5308 & 0.3642 & $8.1 \%$ & $5.6 \%$ \\
\hline 41 & US-41 & $\mathrm{NiO}$ & 0.0121 & 0.0134 & 0.0000 & 0.0121 & 0.0134 & & \\
\hline 41 & US-41 & $\mathrm{PbO}$ & 0.8941 & 0.8941 & 0.9998 & -0.1057 & -0.1057 & $-10.6 \%$ & $-10.6 \%$ \\
\hline 41 & US-41 & $\mathrm{SiO} 2$ & 40.8071 & 41.6808 & 42.6545 & -1.8473 & -0.9737 & $-4.3 \%$ & $-2.3 \%$ \\
\hline 41 & US-41 & $\mathrm{SO} 3$ & 0.0624 & 0.0624 & 0.0000 & 0.0624 & 0.0624 & & \\
\hline 41 & US-41 & $\mathrm{SrO}$ & 2.5929 & 2.5929 & 2.9994 & -0.4065 & -0.4065 & $-13.6 \%$ & $-13.6 \%$ \\
\hline 41 & US-41 & $\mathrm{TiO} 2$ & 0.0083 & 0.0091 & 0.0000 & 0.0083 & 0.0091 & & \\
\hline 41 & US-41 & $\mathrm{ZnO}$ & 0.0062 & 0.0062 & 0.0000 & 0.0062 & 0.0062 & & \\
\hline 41 & US-41 & $\mathrm{ZrO} 2$ & 0.0125 & 0.0125 & 0.0000 & 0.0125 & 0.0125 & & \\
\hline 41 & US-41 & Sum & 97.2949 & 98.1372 & 99.9800 & -2.6851 & -1.8428 & $-2.7 \%$ & $-1.8 \%$ \\
\hline 42 & US-42 & $\mathrm{A} 12 \mathrm{O} 3$ & 20.4066 & 20.8334 & 19.9960 & 0.4106 & 0.8374 & $2.1 \%$ & $4.2 \%$ \\
\hline 42 & US-42 & $\mathrm{B} 2 \mathrm{O} 3$ & 6.4237 & 6.3809 & 6.1871 & 0.2366 & 0.1938 & $3.8 \%$ & $3.1 \%$ \\
\hline 42 & US-42 & $\mathrm{CaO}$ & 0.8742 & 0.9745 & 0.9998 & -0.1256 & -0.0253 & $-12.6 \%$ & $-2.5 \%$ \\
\hline 42 & US-42 & $\mathrm{Cr} 2 \mathrm{O} 3$ & 0.0073 & 0.0071 & 0.0000 & 0.0073 & 0.0071 & & \\
\hline 42 & US-42 & $\mathrm{Fe} 2 \mathrm{O} 3$ & 5.1255 & 5.1218 & 4.9990 & 0.1265 & 0.1228 & $2.5 \%$ & $2.5 \%$ \\
\hline 42 & US-42 & $\mathrm{K} 2 \mathrm{O}$ & 0.9778 & 0.8606 & 0.9998 & -0.0220 & -0.1392 & $-2.2 \%$ & $-13.9 \%$ \\
\hline 42 & US-42 & $\mathrm{Li} 2 \mathrm{O}$ & 5.9582 & 6.0435 & 5.9988 & -0.0406 & 0.0447 & $-0.7 \%$ & $0.7 \%$ \\
\hline 42 & US-42 & $\mathrm{MgO}$ & 0.0083 & 0.0089 & 0.0000 & 0.0083 & 0.0089 & & \\
\hline 42 & US-42 & $\mathrm{MnO}$ & 4.1641 & 4.4147 & 3.9992 & 0.1649 & 0.4155 & $4.1 \%$ & $10.4 \%$ \\
\hline 42 & US-42 & $\mathrm{Na} 2 \mathrm{O}$ & 19.5123 & 18.7203 & 18.8199 & 0.6924 & -0.0995 & $3.7 \%$ & $-0.5 \%$ \\
\hline 42 & US-42 & $\mathrm{NiO}$ & 0.0064 & 0.0070 & 0.0000 & 0.0064 & 0.0070 & & \\
\hline 42 & US-42 & $\mathrm{PbO}$ & 0.8871 & 0.8871 & 0.9998 & -0.1127 & -0.1127 & $-11.3 \%$ & $-11.3 \%$ \\
\hline 42 & US-42 & $\mathrm{SiO} 2$ & 35.9402 & 36.5826 & 35.4919 & 0.4483 & 1.0907 & $1.3 \%$ & $3.1 \%$ \\
\hline 42 & US-42 & $\mathrm{SO} 3$ & 0.0593 & 0.0593 & 0.0000 & 0.0593 & 0.0593 & & \\
\hline 42 & US-42 & $\mathrm{SrO}$ & 0.0059 & 0.0059 & 0.0000 & 0.0059 & 0.0059 & & \\
\hline 42 & US-42 & $\mathrm{TiO} 2$ & 0.0083 & 0.0091 & 0.0000 & 0.0083 & 0.0091 & & \\
\hline 42 & US-42 & $\mathrm{ZnO}$ & 1.3693 & 1.3693 & 1.4887 & -0.1195 & -0.1195 & $-8.0 \%$ & $-8.0 \%$ \\
\hline 42 & US-42 & $\mathrm{ZrO} 2$ & 0.0122 & 0.0122 & 0.0000 & 0.0122 & 0.0122 & & \\
\hline 42 & US-42 & Sum & 101.7466 & 102.2982 & 99.9800 & 1.7666 & 2.3182 & $1.8 \%$ & $2.3 \%$ \\
\hline 43 & US-43 & $\mathrm{A} 12 \mathrm{O} 3$ & 17.5960 & 17.9637 & 17.1673 & 0.4286 & 0.7964 & $2.5 \%$ & $4.6 \%$ \\
\hline 43 & US-43 & $\mathrm{B} 2 \mathrm{O} 3$ & 8.7501 & 8.6917 & 8.6447 & 0.1054 & 0.0470 & $1.2 \%$ & $0.5 \%$ \\
\hline
\end{tabular}


Table A4. Measured, Measured Bias-Corrected, and Targeted Compositions by Glass Number

\begin{tabular}{|c|c|c|c|c|c|c|c|c|c|}
\hline & & & & Measured & & & & & \\
\hline Glass & & & Measured & $\begin{array}{c}\text { Bias- } \\
\text { Corrected }\end{array}$ & Targeted & Diff of & Diff of & $\%$ Diff of & $\%$ Diff of \\
\hline$\#$ & GlassID & Oxide & $(w t \%)$ & $(w t \%)$ & $\left(w t^{\%} \%\right)$ & Measured & Meas BC & Measured & Meas BC \\
\hline 43 & US-43 & $\mathrm{CaO}$ & 0.9035 & 0.9869 & 0.9998 & -0.0963 & -0.0129 & $-9.6 \%$ & $-1.3 \%$ \\
\hline 43 & US-43 & $\mathrm{Cr} 2 \mathrm{O} 3$ & 0.0073 & 0.0071 & 0.0000 & 0.0073 & 0.0071 & & \\
\hline 43 & US-43 & $\mathrm{Fe} 2 \mathrm{O} 3$ & 9.1072 & 9.0990 & 8.8568 & 0.2504 & 0.2423 & $2.8 \%$ & $2.7 \%$ \\
\hline 43 & US-43 & $\mathrm{K} 2 \mathrm{O}$ & 0.9766 & 0.8658 & 0.9988 & -0.0222 & -0.1330 & $-2.2 \%$ & $-13.3 \%$ \\
\hline 43 & US-43 & $\mathrm{Li} 2 \mathrm{O}$ & 4.8978 & 4.9677 & 4.9049 & -0.0070 & 0.0628 & $-0.1 \%$ & $1.3 \%$ \\
\hline 43 & US-43 & $\mathrm{MgO}$ & 0.4859 & 0.5293 & 0.4999 & -0.0140 & 0.0294 & & \\
\hline 43 & US-43 & $\mathrm{MnO}$ & 0.7105 & 0.7523 & 0.8120 & -0.1016 & -0.0598 & $-12.5 \%$ & $-7.4 \%$ \\
\hline 43 & US-43 & $\mathrm{Na} 2 \mathrm{O}$ & 11.2794 & 11.0135 & 10.2945 & 0.9849 & 0.7190 & $9.6 \%$ & $7.0 \%$ \\
\hline 43 & US-43 & $\mathrm{NiO}$ & 0.1826 & 0.2026 & 0.1981 & -0.0155 & 0.0044 & & \\
\hline 43 & US-43 & $\mathrm{PbO}$ & 0.8882 & 0.8882 & 0.9998 & -0.1116 & -0.1116 & $-11.2 \%$ & $-11.2 \%$ \\
\hline 43 & US-43 & $\mathrm{SiO} 2$ & 38.1865 & 38.8648 & 39.7012 & -1.5146 & -0.8364 & $-3.8 \%$ & $-2.1 \%$ \\
\hline 43 & US-43 & SO3 & 0.4089 & 0.4089 & 0.4999 & -0.0910 & -0.0910 & & \\
\hline 43 & US-43 & $\mathrm{SrO}$ & 0.4157 & 0.4157 & 0.4724 & -0.0567 & -0.0567 & & \\
\hline 43 & US-43 & $\mathrm{TiO} 2$ & 0.4545 & 0.4944 & 0.4773 & -0.0228 & 0.0170 & & \\
\hline 43 & US-43 & $\mathrm{ZnO}$ & 0.4407 & 0.4407 & 0.4534 & -0.0127 & -0.0127 & & \\
\hline 43 & US-43 & $\mathrm{ZrO} 2$ & 3.3669 & 3.3669 & 3.9992 & -0.6323 & -0.6323 & $-15.8 \%$ & $-15.8 \%$ \\
\hline 43 & US-43 & Sum & 99.0582 & 99.9591 & 99.9800 & -0.9218 & -0.0209 & $-0.9 \%$ & $0.0 \%$ \\
\hline 44 & US-44 & $\mathrm{A} 12 \mathrm{O} 3$ & 13.6327 & 13.8261 & 12.8029 & 0.8299 & 1.0233 & $6.5 \%$ & $8.0 \%$ \\
\hline 44 & US-44 & $\mathrm{B} 2 \mathrm{O} 3$ & 12.4610 & 12.3775 & 12.5864 & -0.1253 & -0.2088 & $-1.0 \%$ & $-1.7 \%$ \\
\hline 44 & US-44 & $\mathrm{CaO}$ & 0.5439 & 0.6064 & 0.5553 & -0.0113 & 0.0511 & $-2.0 \%$ & $9.2 \%$ \\
\hline 44 & US-44 & $\mathrm{Cr} 2 \mathrm{O} 3$ & 0.2616 & 0.2548 & 0.3051 & -0.0434 & -0.0502 & & \\
\hline 44 & US-44 & $\mathrm{Fe} 2 \mathrm{O} 3$ & 8.7426 & 8.7753 & 8.8598 & -0.1172 & -0.0845 & $-1.3 \%$ & $-1.0 \%$ \\
\hline 44 & US-44 & $\mathrm{K} 2 \mathrm{O}$ & 0.6445 & 0.5672 & 0.6397 & 0.0048 & -0.0725 & $0.8 \%$ & $-11.3 \%$ \\
\hline 44 & US-44 & $\mathrm{Li} 2 \mathrm{O}$ & 4.3919 & 4.4618 & 4.0488 & 0.3431 & 0.4130 & $8.5 \%$ & $10.2 \%$ \\
\hline 44 & US-44 & $\mathrm{MgO}$ & 0.2550 & 0.2748 & 0.2525 & 0.0025 & 0.0224 & & \\
\hline 44 & US-44 & $\mathrm{MnO}$ & 1.7560 & 1.8655 & 1.8339 & -0.0778 & 0.0316 & $-4.2 \%$ & $1.7 \%$ \\
\hline 44 & US-44 & $\mathrm{Na} 2 \mathrm{O}$ & 13.9181 & 13.3541 & 12.7286 & 1.1895 & 0.6255 & $9.3 \%$ & $4.9 \%$ \\
\hline 44 & US-44 & $\mathrm{NiO}$ & 0.3394 & 0.3736 & 0.3587 & -0.0192 & 0.0150 & & \\
\hline 44 & US-44 & $\mathrm{PbO}$ & 0.6070 & 0.6070 & 0.6665 & -0.0595 & -0.0595 & $-8.9 \%$ & $-8.9 \%$ \\
\hline 44 & US-44 & $\mathrm{SiO} 2$ & 37.4378 & 38.2395 & 39.5480 & -2.1103 & -1.3085 & $-5.3 \%$ & $-3.3 \%$ \\
\hline 44 & US-44 & $\mathrm{SO} 3$ & 0.2278 & 0.2278 & 0.2222 & 0.0057 & 0.0057 & & \\
\hline 44 & US-44 & $\mathrm{SrO}$ & 1.4073 & 1.4073 & 1.6195 & -0.2122 & -0.2122 & $-13.1 \%$ & $-13.1 \%$ \\
\hline 44 & US-44 & $\mathrm{TiO} 2$ & 0.4487 & 0.4880 & 0.4650 & -0.0163 & 0.0230 & & \\
\hline 44 & US-44 & $\mathrm{ZnO}$ & 0.9653 & 0.9653 & 1.0237 & -0.0584 & -0.0584 & $-5.7 \%$ & $-5.7 \%$ \\
\hline 44 & US-44 & $\mathrm{ZrO} 2$ & 1.3076 & 1.3076 & 1.4636 & -0.1560 & -0.1560 & $-10.7 \%$ & $-10.7 \%$ \\
\hline 44 & US-44 & Sum & 99.3483 & 99.9798 & 99.9800 & -0.6317 & -0.0002 & $-0.6 \%$ & $0.0 \%$ \\
\hline 45 & US-45 & $\mathrm{A} 12 \mathrm{O} 3$ & 13.2123 & 13.4881 & 12.8029 & 0.4095 & 0.6853 & $3.2 \%$ & $5.4 \%$ \\
\hline 45 & US-45 & $\mathrm{B} 2 \mathrm{O} 3$ & 12.8635 & 12.7786 & 12.5864 & 0.2771 & 0.1923 & $2.2 \%$ & $1.5 \%$ \\
\hline 45 & US-45 & $\mathrm{CaO}$ & 0.5422 & 0.5837 & 0.5553 & -0.0131 & 0.0284 & $-2.4 \%$ & $5.1 \%$ \\
\hline 45 & US-45 & $\mathrm{Cr} 2 \mathrm{O} 3$ & 0.2558 & 0.2502 & 0.3051 & -0.0493 & -0.0548 & & \\
\hline 45 & US-45 & $\mathrm{Fe} 2 \mathrm{O} 3$ & 8.9321 & 8.9240 & 8.8598 & 0.0722 & 0.0642 & $0.8 \%$ & $0.7 \%$ \\
\hline 45 & US-45 & $\mathrm{K} 2 \mathrm{O}$ & 0.6640 & 0.5892 & 0.6397 & 0.0244 & -0.0505 & $3.8 \%$ & $-7.9 \%$ \\
\hline 45 & US-45 & $\mathrm{Li} 2 \mathrm{O}$ & 4.0636 & 4.1216 & 4.0488 & 0.0148 & 0.0728 & $0.4 \%$ & $1.8 \%$ \\
\hline 45 & US-45 & $\mathrm{MgO}$ & 0.2467 & 0.2661 & 0.2525 & -0.0058 & 0.0136 & & \\
\hline 45 & US-45 & $\mathrm{MnO}$ & 1.7915 & 1.8984 & 1.8339 & -0.0423 & 0.0645 & $-2.3 \%$ & $3.5 \%$ \\
\hline 45 & US-45 & $\mathrm{Na} 2 \mathrm{O}$ & 13.4396 & 12.9576 & 12.7286 & 0.7109 & 0.2289 & $5.6 \%$ & $1.8 \%$ \\
\hline 45 & US-45 & $\mathrm{NiO}$ & 0.3321 & 0.3664 & 0.3587 & -0.0265 & 0.0077 & & \\
\hline 45 & US-45 & $\mathrm{PbO}$ & 0.5911 & 0.5911 & 0.6665 & -0.0754 & -0.0754 & $-11.3 \%$ & $-11.3 \%$ \\
\hline 45 & US-45 & $\mathrm{SiO} 2$ & 39.6305 & 40.3297 & 39.5480 & 0.0825 & 0.7816 & $0.2 \%$ & $2.0 \%$ \\
\hline 45 & US-45 & $\mathrm{SO} 3$ & 0.2222 & 0.2222 & 0.2222 & 0.0000 & 0.0000 & & \\
\hline 45 & US-45 & $\mathrm{SrO}$ & 1.4339 & 1.4339 & 1.6195 & -0.1856 & -0.1856 & $-11.5 \%$ & $-11.5 \%$ \\
\hline 45 & US-45 & $\mathrm{TiO} 2$ & 0.4529 & 0.4876 & 0.4650 & -0.0121 & 0.0226 & & \\
\hline 45 & US-45 & $\mathrm{ZnO}$ & 0.9607 & 0.9607 & 1.0237 & -0.0631 & -0.0631 & $-6.2 \%$ & $-6.2 \%$ \\
\hline 45 & US-45 & $\mathrm{ZrO} 2$ & 1.3130 & 1.3130 & 1.4636 & -0.1506 & -0.1506 & $-10.3 \%$ & $-10.3 \%$ \\
\hline 45 & US-45 & Sum & 100.9477 & 101.5621 & 99.9800 & 0.9677 & 1.5821 & $1.0 \%$ & $1.6 \%$ \\
\hline
\end{tabular}




\section{Exhibit A1. PSAL Oxide Measurements in Analytical Sequence for Samples Prepared Using the LM Method}
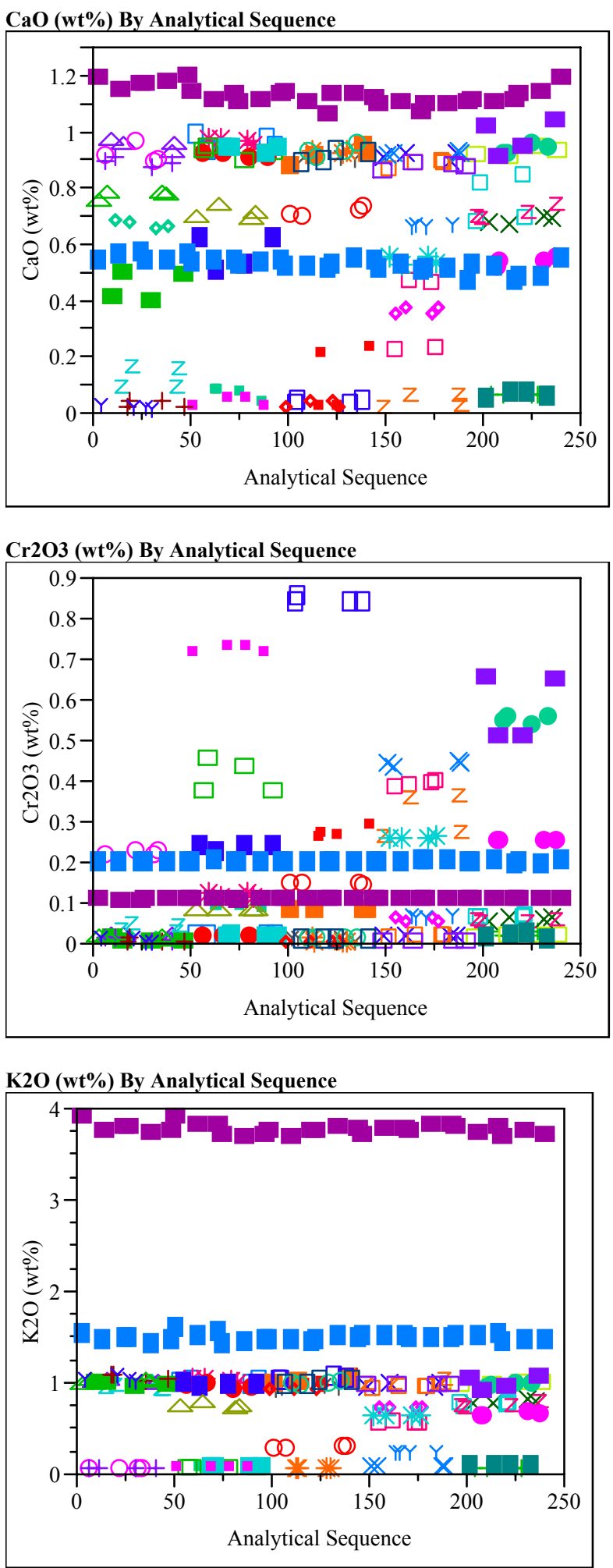
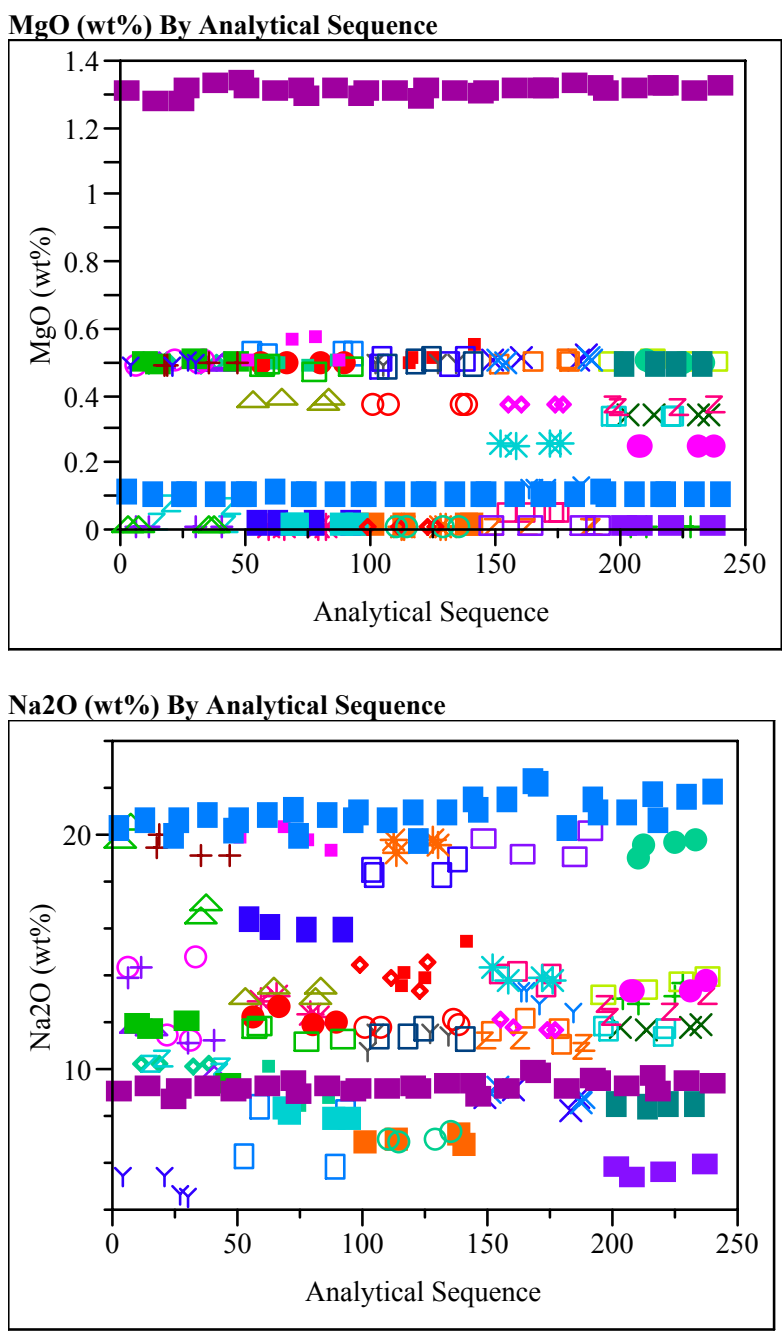

NiO (wt\%) By Analytical Sequence

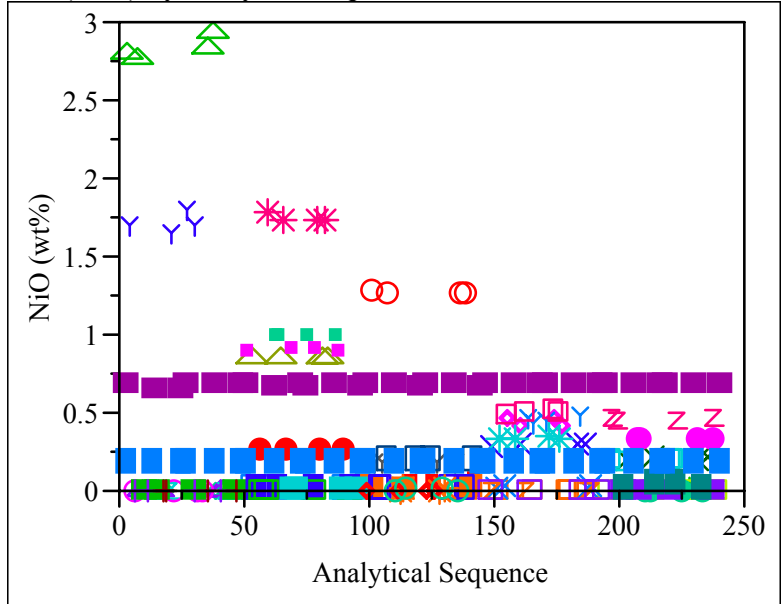




\section{Exhibit A1. PSAL Oxide Measurements in Analytical Sequence for Samples Prepared Using the LM Method}

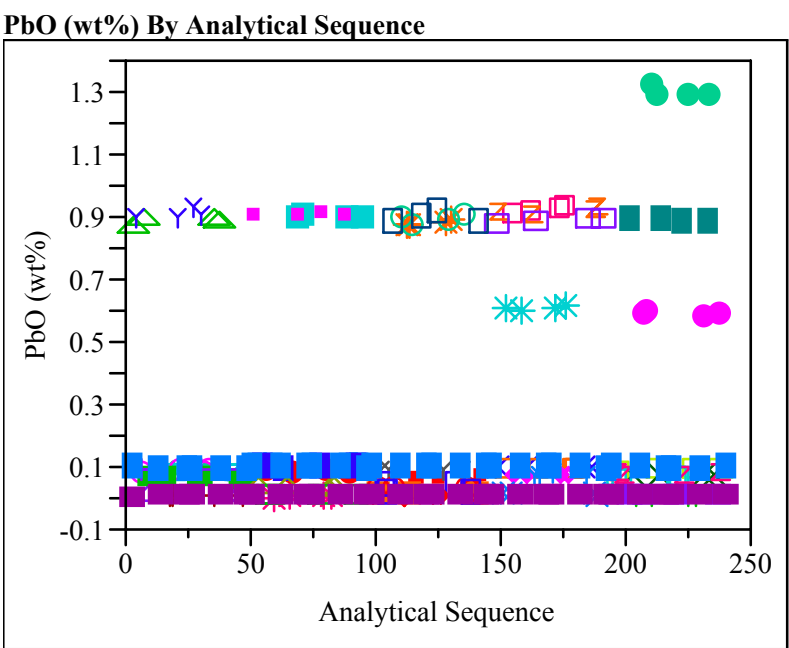

SO3 (wt\%) By Analytical Sequence

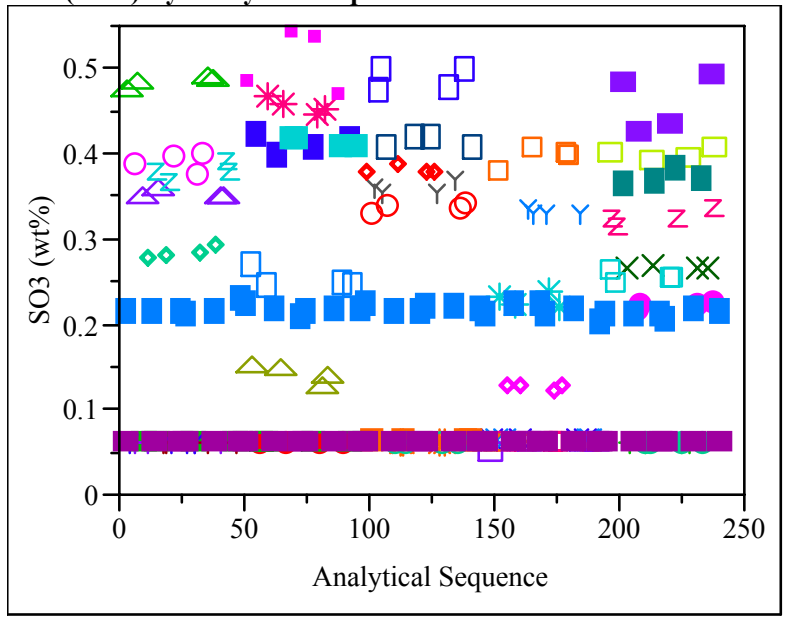

SrO (wt\%) By Analytical Sequence

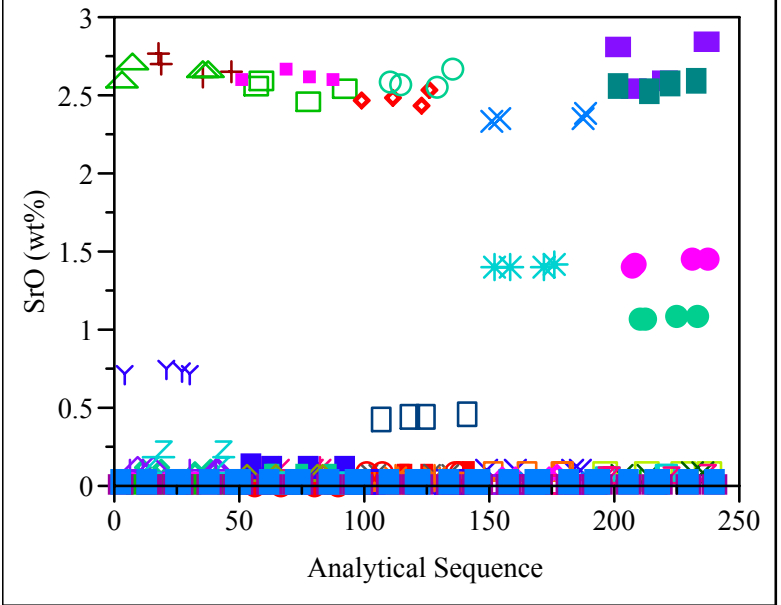

TiO2 (wt\%) By Analytical Sequence

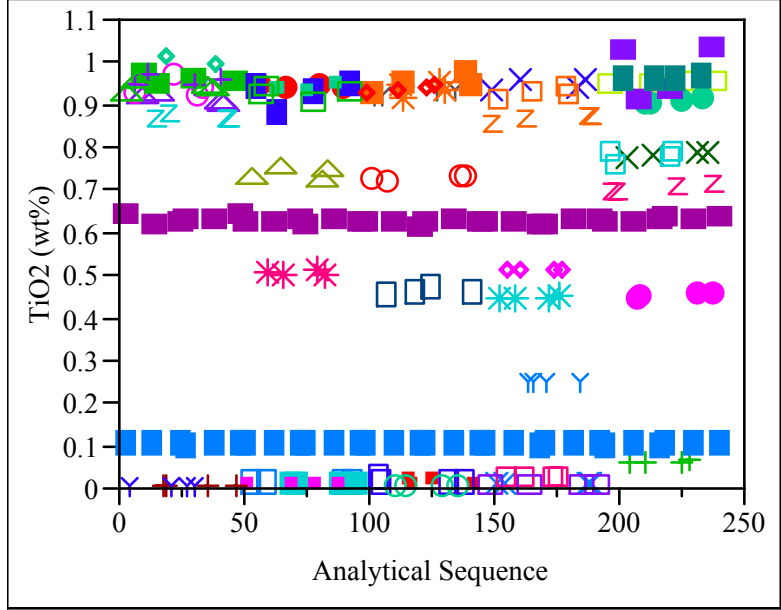

$\mathrm{ZnO}(w \mathrm{t} \%)$ By Analytical Sequence

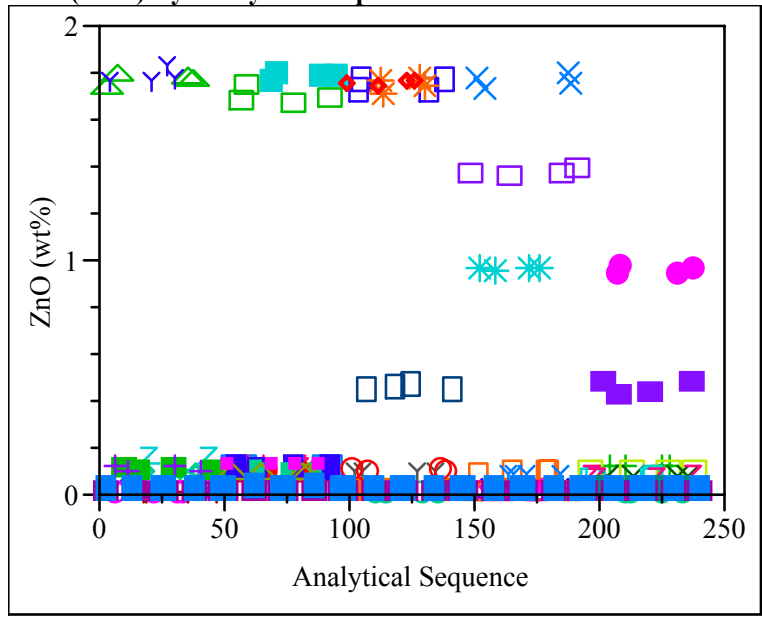

ZrO2 (wt\%) By Analytical Sequence

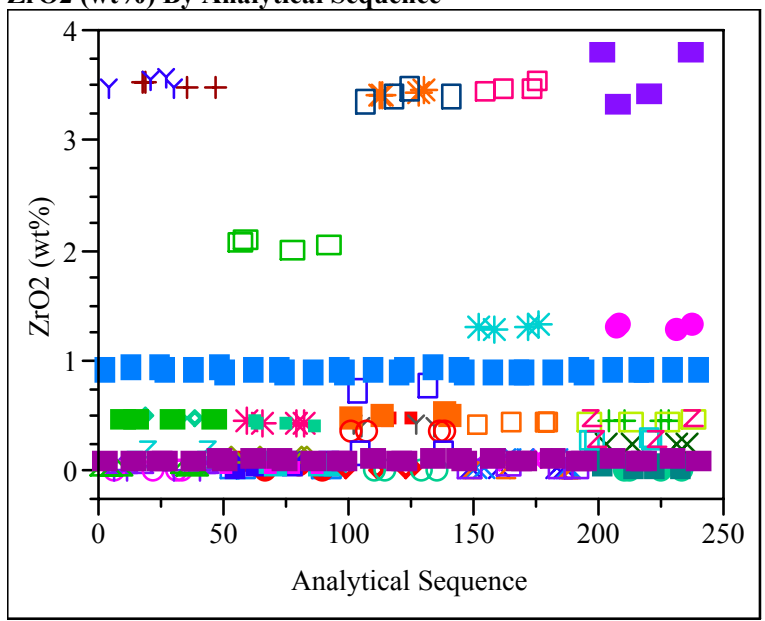




\section{Exhibit A2. PSAL Oxide Measurements in Analytical Sequence for Samples Prepared Using the PF Method}
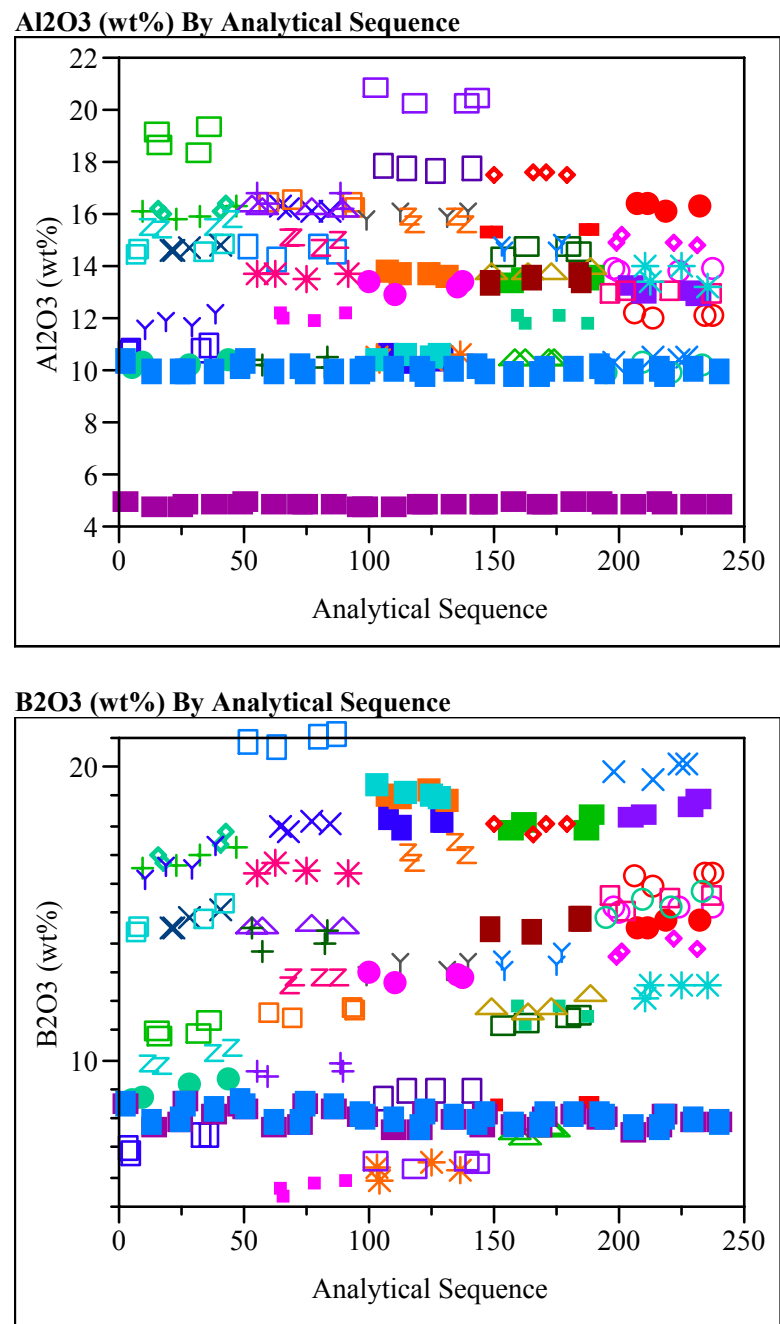

Fe2O3 (wt\%) By Analytical Sequence

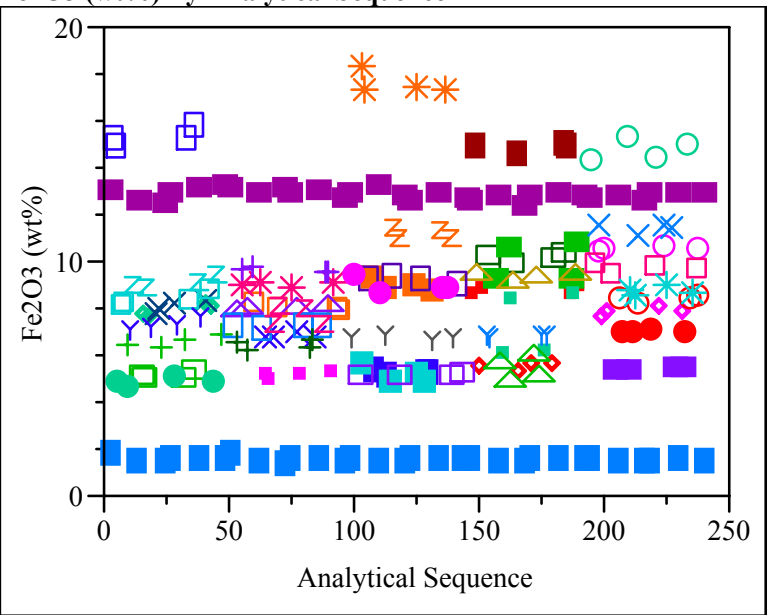

Li2O (wt\%) By Analytical Sequence

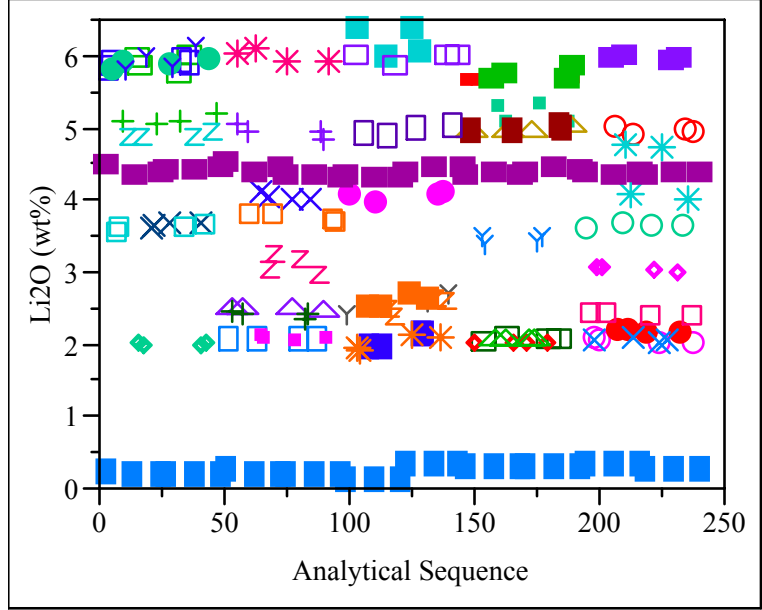

MnO (wt\%) By Analytical Sequence

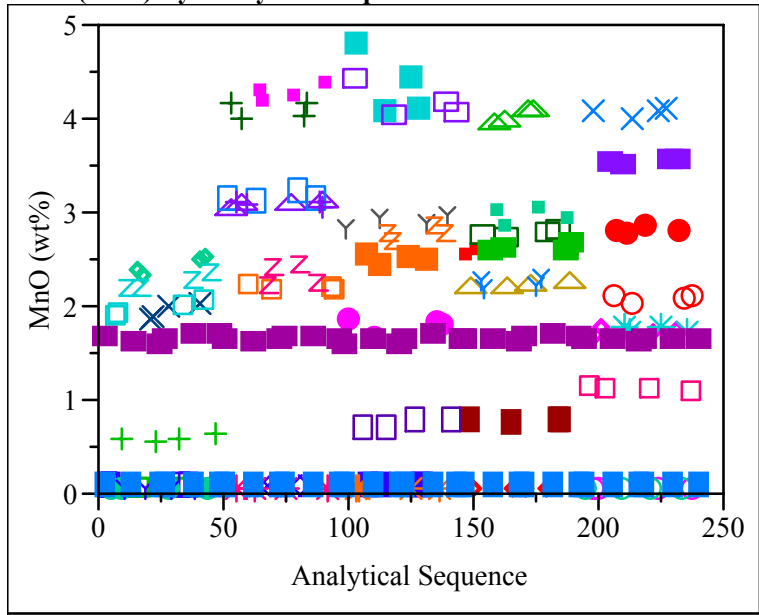

$\mathrm{SiO2}(\mathrm{wt} \%)$ By Analytical Sequence

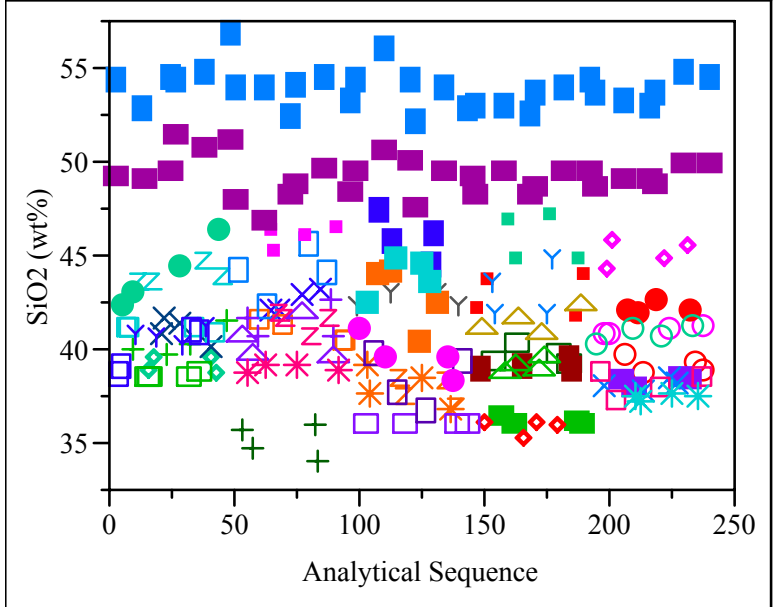




\section{Exhibit A3. PSAL Oxide Measurements by Analytical Block for Samples of the} Standard Glasses Prepared Using the LM Method

Oneway Analysis of $\mathrm{CaO}(\mathrm{wt} \%)$ By Block/Sub-Block Glass ID=Batch 1, Reference Value $=1.220 \mathrm{wt} \%$

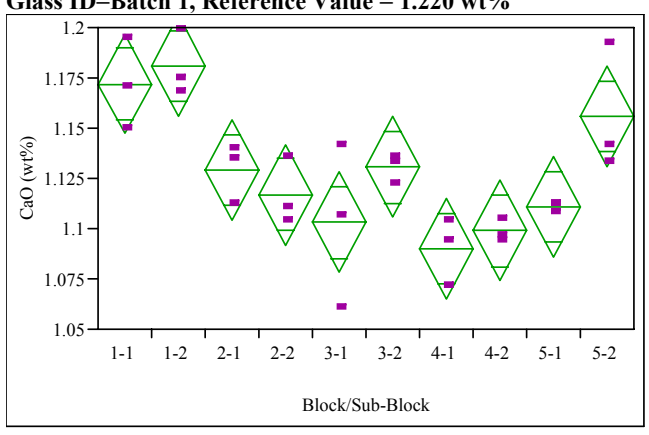

\section{Oneway Anova}

Summary of Fit

Rsquare

Q 0.02074

$\begin{array}{lr}\text { Mean of Response } & 1.128828 \\ \text { Observations (or Sum Wgts) } & 30\end{array}$

Analysis of Variance

Source DF Sum of Squares Mean Square F Ratio Prob $>$ F

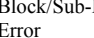

Error

$\begin{array}{ll}20 & 0.00860371 \\ 29 & 0.03513273\end{array}$

Means for Oneway Anova

$\begin{array}{lrrrrr}\text { Level } & \text { Number } & \text { Mean } & \text { Std Error } & \text { Lower 95\% } & \text { Upper 95\% } \\ 1-1 & 3 & 1.17206 & 0.01197 & 1.1471 & 1.1970 \\ 1-2 & 3 & 1.18092 & 0.01197 & 1.1559 & 1.2059 \\ 2-1 & 3 & 1.12915 & 0.01197 & 1.1042 & 1.1541 \\ 2-2 & 3 & 1.11703 & 0.01197 & 1.0920 & 1.1420 \\ 3-1 & 3 & 1.10304 & 0.01197 & 1.0781 & 1.1280 \\ 3-2 & 3 & 1.13055 & 0.01197 & 1.1056 & 1.1555 \\ 4-1 & 3 & 1.08998 & 0.01197 & 1.0650 & 1.1150 \\ 4-2 & 3 & 1.09884 & 0.01197 & 1.0739 & 1.1238 \\ 5-1 & 3 & 1.11096 & 0.01197 & 1.0860 & 1.1359 \\ 5-2 & 3 & 1.15574 & 0.01197 & 1.1308 & 1.1807\end{array}$

Std Error uses a pooled estimate of error variance
Oneway Analysis of Cr2O3 (wt\%) By Block/Sub-Block

Glass ID $=$ Batch 1, Reference Value $=\mathbf{0 . 1 0 7} \mathbf{w t} \%$

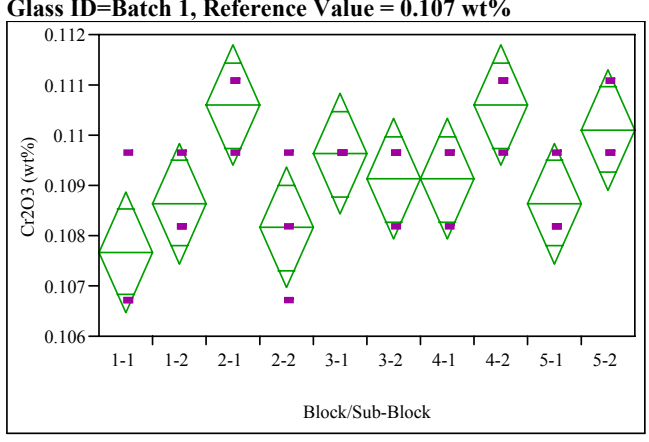

Oneway Anova

Summary of Fit

Rsquare
Adj Rsquare

\subsection{381098
0.00998}

Root Mean Square Error $\quad 0.000998$ $\begin{array}{lr}\text { Mean of Response } & 0.10923 \\ \text { Observations (or Sum Wgts) } & 30\end{array}$

Analysis of Variance

Source DF Sum of Squares Mean Square F Ratio Prob $>$ F $\begin{array}{lllllll}\text { Block/Sub-Block } & 9 & 0.00002677 & 2.975 \mathrm{e}-6 & 2.9841 & 0.0200\end{array}$ Error
C. Total $\begin{array}{ll}20 & 0.00001994 \\ 29 & 0.00004671\end{array}$

Means for Oneway Anova

Level Number Mean Std Error Lower 95\% Upper 95\%

$\begin{array}{lllllll}1-1 & & 3 & 0.107671 & 0.00058 & 0.10647 & 0.10887 \\ 1-2 & & 3 & 0.10846 & 0.00058 & 0.10744 & 0.1985\end{array}$

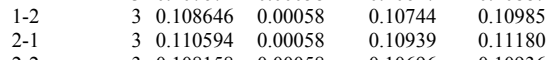

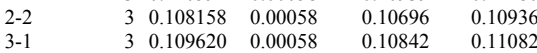

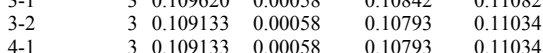

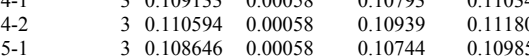

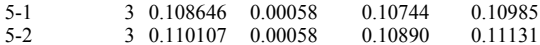

Std Error uses a pooled estimate of error variance
Oneway Analysis of K2O (wt\%) By Block/Sub-Block Glass ID=Batch 1, Reference Value $=3.327 \mathrm{wt} \%$

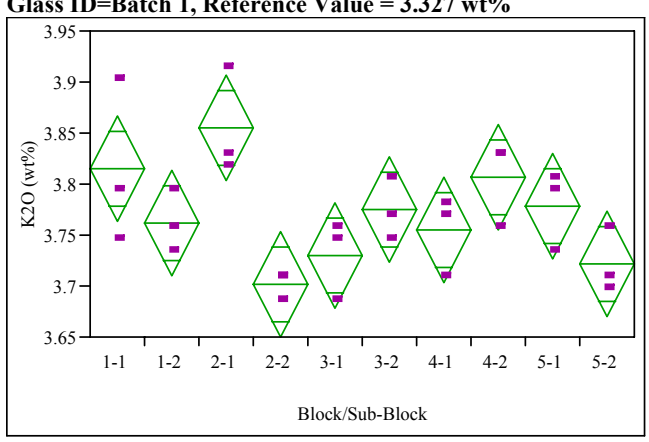

Oneway Anova Summary of Fit

Rsquare
Adj Rsquare

\subsection{6}

Root Mean Square Error $\quad 0.042985$ $\begin{array}{lr}\text { Mean of Response } & 3.769996 \\ \text { Observations (or Sum Wgts) } & 30\end{array}$

Analysis of Variance

Source DF Sum of Squares Mean Square F Ratio Prob $>F$ $\begin{array}{llllll}\text { Block/Sub-Block } & 0.05808598 & 0.006454 & 3.4930 & 0.0095\end{array}$ $\begin{array}{lll}\text { Error } & 20 & 0.03695369 \\ \text { C. Total } & 29 & 0.09503967\end{array}$

Means for Oneway Anova

\begin{tabular}{|c|c|c|c|c|c|}
\hline & & & & & t \\
\hline 1-1 & & 81 & 0.02 & & \\
\hline 1-2 & & 3.76 & & & \\
\hline & & 3.85472 & & & \\
\hline & & 3.70214 & & & \\
\hline & & 3.73024 & & & \\
\hline & & 3.77 & & & \\
\hline & & 3.75434 & 0.02 & & 3.80 \\
\hline & & 3.80654 & 0.02 & 8 & 3 \\
\hline & & 3.77843 & 0.02482 & 3.7 & 3. \\
\hline & & & & & \\
\hline
\end{tabular}

Std Error uses a pooled estimate of error variance 


\section{Exhibit A3. PSAL Oxide Measurements by Analytical Block for Samples of the} Standard Glasses Prepared Using the LM Method

Oneway Analysis of $\mathrm{MgO}(\mathrm{wt} \%)$ By Block/Sub-Block Glass ID=Batch 1, Reference Value $=1.419 \mathrm{wt} \%$

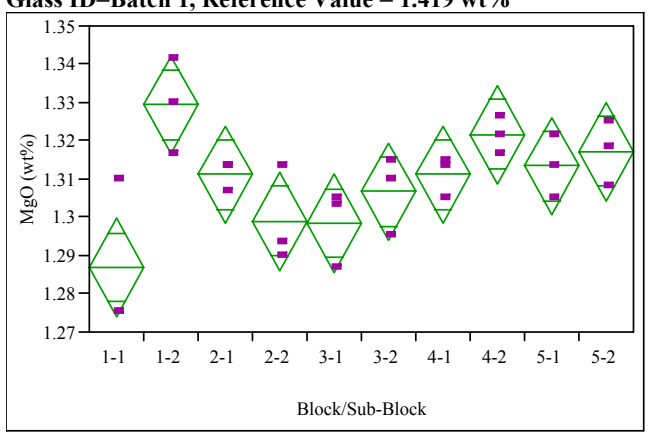

Oneway Anova

Summary of Fit

Rsquare
Adj Rsquare

0.486891
1.01064

Mean of Response $\quad 1.309504$

Ansersis of Variance

Source DF Sum of Squares Mean Square F Ratio Prob $>$ F $\begin{array}{llrrrr}\text { Block/Sub-Block } & 9 & 0.00413410 & 0.000459 & 4.0576 & 0.0044\end{array}$ $\begin{array}{lll}\text { Error } & 20 & 0.00226413 \\ \text { C. Total } & 29 & 0.00639824\end{array}$

Means for Oneway Anova

\begin{tabular}{|c|c|c|c|c|c|}
\hline & umber & Mean & Std Error & & \\
\hline & & 1.28684 & 0.00614 & 1.2740 & \\
\hline & & 1.32940 & 0.00614 & 1.3166 & \\
\hline & & 1.31116 & & & \\
\hline & & 1.29900 & & & \\
\hline & & 1.29 & & & \\
\hline & & $\begin{array}{l}1.29 \\
1.30\end{array}$ & & & \\
\hline & & $\begin{array}{l}1.30 \\
1.31\end{array}$ & 0.0 & & \\
\hline & & 1.3 & 0. & & \\
\hline & & 1.31337 & 0.00614 & 1.3006 & \\
\hline & & $\begin{array}{l}1.31724 \\
1.31724\end{array}$ & $\begin{array}{l}0.00014 \\
0.00614\end{array}$ & $\begin{array}{l}1.5000 \\
1.3044\end{array}$ & $\begin{array}{l}1.3202 \\
1.3301\end{array}$ \\
\hline
\end{tabular}

Std Error uses a pooled estimate of error variance
Oneway Analysis of $\mathrm{Na2O}$ (wt\%) By Block/Sub-Block Glass ID $=$ Batch 1, Reference Value $=9.003 \mathrm{wt} \%$

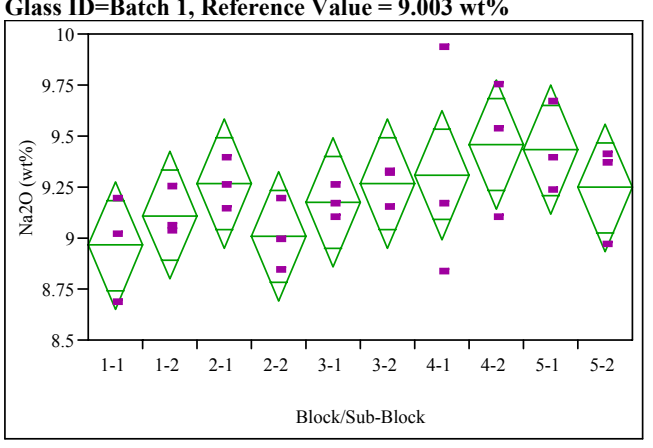

Oneway Anova

Summary of Fit

Rsquare
Adj Rsquare

0.343291

$\begin{array}{ll}\text { Adj Rsquare } & 0.047772 \\ \text { Root Mean Square Error } & 0.261259 \\ 9.223915\end{array}$

Observations (or Sum Wgts) $\begin{array}{r}9.223915 \\ 30\end{array}$

Analysis of Variance $\begin{array}{lllrrr}\text { Source } & \text { DF } & \text { Sum of Squares } & \text { Mean Square } & \text { F Ratio } & \text { Prob > F } \\ \text { Block/Sub-Block } & 9 & 0.7136131 & 0.079290 & 1.1617 & 0.3692\end{array}$ $\begin{array}{llll}\text { Error } & 20 & 1.3651297 & 0.068256 \\ \text { C. Total } & 29 & 2.0787427 & \end{array}$

Means for Oneway Anova

\begin{tabular}{|c|c|c|c|c|}
\hline $\mathrm{Nu}$ & Mean & Std Error & & er $95 \%$ \\
\hline & 8.96420 & 0.15084 & 8.6496 & 9.2788 \\
\hline & 9.11248 & 0.15084 & 8.7978 & 9.4271 \\
\hline & 9.26525 & 0.15084 & 8.9506 & 9.5799 \\
\hline & 9.00913 & 0.15084 & 8.6945 & 9.3238 \\
\hline 3 & 9.17539 & 0.15084 & 8.8607 & 9.4900 \\
\hline 3 & 9.26525 & 0.15084 & 8.9506 & 9.5799 \\
\hline 3 & 9.31019 & 0.15084 & 8.9955 & 9.6248 \\
\hline $4-2$ & 9.45847 & 0.15084 & 9.1438 & 9.7731 \\
\hline 5-1 & 9.43151 & 0.15084 & 9.1169 & 9.7461 \\
\hline $5-2$ & 9.24728 & 0.15084 & 8.9326 & 9.5619 \\
\hline
\end{tabular}

Std Error uses a pooled estimate of error variance
Oneway Analysis of $\mathrm{NiO}(\mathrm{wt} \%)$ By Block/Sub-Block Glass ID $=$ Batch 1, Reference Value $=\mathbf{0 . 7 5 1} \mathbf{w t} \%$

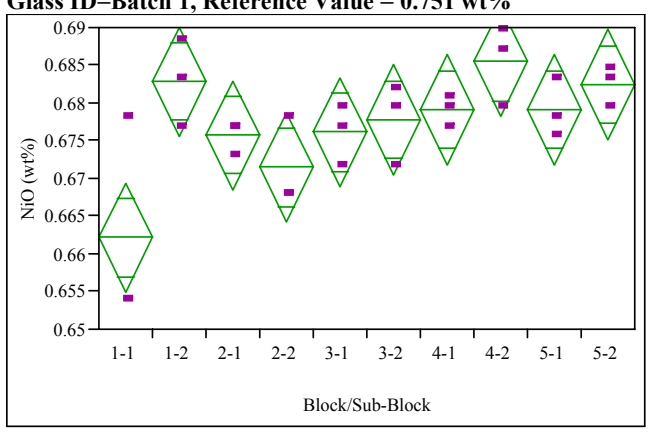

Oneway Anova

Summary of Fit

Rsquare

Error $\quad 0.006036$ $\begin{array}{lr}\text { Mean of Response } & 0.677225 \\ \text { Observations (or Sum Wgts) } & 30\end{array}$

Analysis of Variance

Source DF Sum of Squares Mean Square F Ratio Prob > F $\begin{array}{lllll}0.00119955 & 0.000133 & 3.6583 & 0.0075\end{array}$

$\begin{array}{lll}\text { Error } & 20 & 0.00072867 \\ \text { C. Total } & 29 & 0.00192821\end{array}$

Means for Oneway Anova

Level Number Mean Std Error Lower 95\% Upper 95\%

$\begin{array}{lllllll}1-1 & & 3 & 0.662124 & 0.00348 & 0.65485 & 0.66939\end{array}$

$\begin{array}{lllllll}1-2 & & 3 & 0.682908 & 0.00348 & 0.67564 & 0.69018 \\ 2-1 & & 3 & 0.675698 & 0.00348 & 0.66843 & 0.68297\end{array}$

$\begin{array}{lllllll}2-2 & & 3 & 0.671456 & 0.00348 & 0.66419 & 0.67873 \\ 3-1 & & 3 & 0.676122 & 0.00348 & 0.66885 & 0.68339\end{array}$

$\begin{array}{lllllll}3-2 & & 3 & 0.677818 & 0.00348 & 0.67055 & 0.68509 \\ 4-1 & & 3 & 0.679091 & 0.00348 & 0.67182 & 0.68636\end{array}$

$\begin{array}{lllllll}4-2 & 3 & 0.685453 & 0.00348 & 0.67818 & 0.68272 \\ 5-1 & & 3 & 0.679091 & 0.00348 & 0.67182 & 0.68636\end{array}$

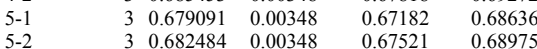

Std Error uses a pooled estimate of error variance 


\section{Exhibit A3. PSAL Oxide Measurements by Analytical Block for Samples of the} Standard Glasses Prepared Using the LM Method

Oneway Analysis of PbO (wt\%) By Block/Sub-Block Glass ID $=$ Batch 1 , Reference Value $=0 \mathrm{wt} \%$

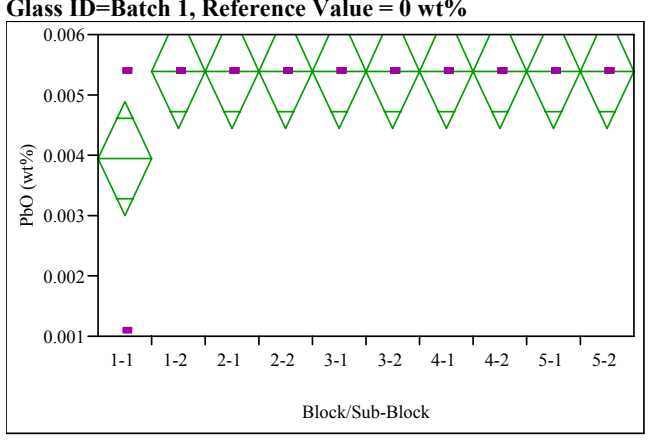

Oneway Anova

Summary of Fit

Rsquare
Adj Rsquare

are Error $\quad 0.000787$

$\begin{array}{lr}\text { Mean of Response } & 0.005242 \\ \text { Observations (or Sum Wgts) } & 30\end{array}$

Analysis of Variance

Source DF Sum of Squares Mean Square F Ratio Prob $>$ F

$\begin{array}{llllllll} & 0.0000557 & 6.1886-7 & 1.000 & 0.4711\end{array}$

Error
C. Total

$\begin{array}{ll}20 & 0.00001238 \\ 29 & 0.00001795\end{array}$

Means for Oneway Anova

Level Number Mean Std Error Lower 95\% Upper 95\%

$\begin{array}{lllllll}1-1 & 3 & 0.003950 & 0.00045 & 0.00300 & 0.0049\end{array}$

$\begin{array}{lllllll}1-2 & & 3 & 0.005386 & 0.00045 & 0.00444 & 0.00633 \\ 2-1 & & 3 & 0.005386 & 0.00045 & 0.0044 & 0.0633\end{array}$

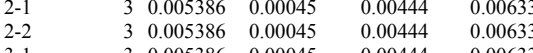

$\begin{array}{lllll}3 & 0.005386 & 0.00045 & 0.00444 & 0.00633 \\ 3 & 0.005386 & 0.00045 & 0.00444 & 0.00633 \\ 3 & 0.00536 & 0.0045 & 0.0044 & 0.00632\end{array}$

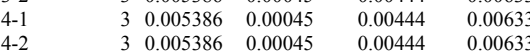

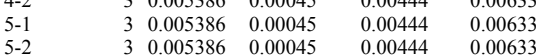

Std Error uses a pooled estimate of error variance
Oneway Analysis of SO3 (wt \%) By Block/Sub-Block

Glass ID=Batch 1, Reference Value $=0 \mathrm{wt} \%$

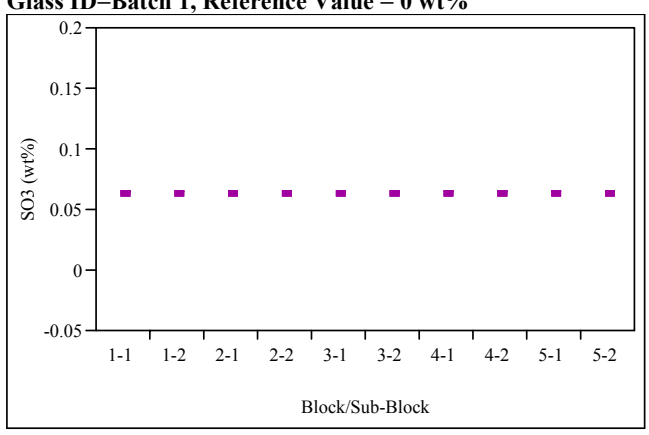

Oneway Anova

Summary of Fit

Rsquare

Root Mean Square Erro

$\begin{array}{lr}\text { Mean of Response } & 0.062423 \\ \text { Observations (or Sum Wgts) } & 30\end{array}$

Analysis of Variance

Source DF Sum of Squares Mean Square F Ratio Prob $>F$ $\begin{array}{lllll}1.4444 \mathrm{e}-33 & 1.605 \mathrm{e}-34 & -2.2222 & 0.0000\end{array}$ Error
C. Total $29-1.444 \mathrm{e}-33$

Means for Oneway Anova

Level Number Mean Std Error Lower 95\% Upper 95\%

$\begin{array}{lll}1-1 & 3 & 0.062423 \\ 1-2 & 3 & 0.06223\end{array}$

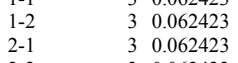

$\begin{array}{llll}2-2 & 3 & 0.062423 \\ 3-1 & 3 & 0.062423\end{array}$

$\begin{array}{lll}3-2 & 3 & 0.062423 \\ 4-1 & 3 & 0.062423 \\ 4-2 & 3 & 0.062423\end{array}$

$\begin{array}{llll}4-2 & 3 & 0.062423 \\ 5-1 & 3 & 0.06223\end{array}$

$\begin{array}{llll}5-2 & 3 & 0.062423\end{array}$

Std Error uses a pooled estimate of error variance
Oneway Analysis of SrO (wt\%) By Block/Sub-Block Glass ID $=$ Batch 1, Reference Value $=0 \mathbf{~ w t} \%$

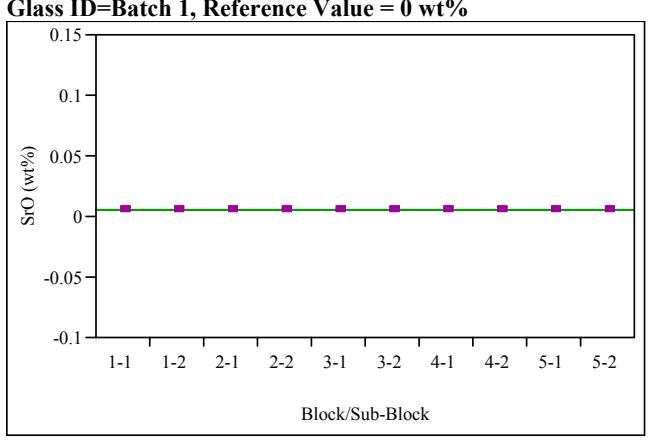

Oneway Anova

Summary of Fit

Rsquare
Adj Rsquare

Root Mean Square Erro

$\begin{array}{lr}\text { Mean of Response } & 0.005913 \\ \text { Observations (or Sum Wgts) } & 30\end{array}$

Analysis of Variance

Source DF Sum of Squares Mean Square F Ratio Prob $>F$ Block/Sub-Block 9
Error

Error
C. Total

Means for Oneway Anova

Level Number Mean Std Error Lower 95\% Upper 95\%

$\begin{array}{llllll}1-1 & 3 & 0.005913 & 0 & 0.00591 & 0.0059\end{array}$

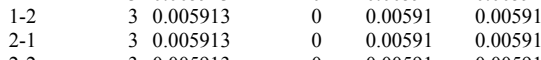

$\begin{array}{llllll}2-2 & 3 & 0.005913 & 0 & 0.00591 & 0.00591 \\ 3-1 & 3 & 0.005913 & 0 & 0.00591 & 0.00591\end{array}$

$3-2 \quad 30.005913$

$\begin{array}{lll}4-1 & 3 & 0.005913 \\ 4-2 & 3 & 0.005913 \\ 5-1 & 3 & 0.05913\end{array}$

$\begin{array}{lll}0 & 0.00591 & 0.00591 \\ 0 & 0.00591 & 0.00591\end{array}$

Std Error uses a pooled estimate of error variance 


\section{Exhibit A3. PSAL Oxide Measurements by Analytical Block for Samples of the} Standard Glasses Prepared Using the LM Method

Oneway Analysis of TiO2 (wt\%) By Block/Sub-Block Glass ID=Batch 1 , Reference Value $=\mathbf{0 . 6 7 7} \mathbf{w t} \%$

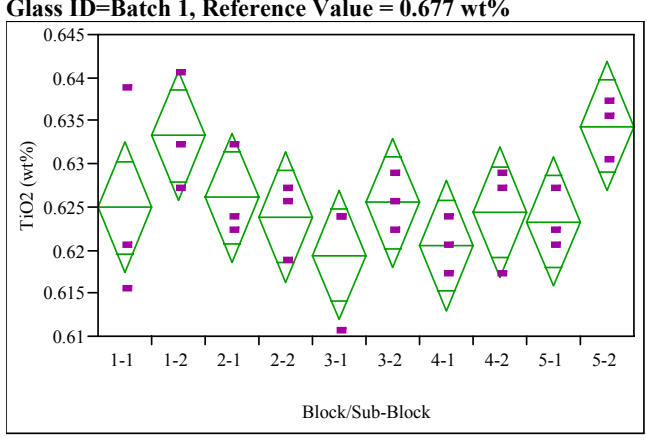

\section{Oneway Anova}

Summary of Fit

Rsquare
Adj Rsquare

\subsection{6}

0.200406
Root Mean Square Error $\quad 0.006249$

$\begin{array}{lr}\text { Mean of Response } & 0.625556 \\ \text { Observations (or Sum Wgts) } & 30\end{array}$

Analysis of Variance

Source DF Sum of Squares Mean Square F Ratio Prob $>$ F

Block/Sub-Bic
Error

Error
C. Total

$\begin{array}{ll}20 & 0.00078088 \\ 29 & 0.00141606\end{array}$

Means for Oneway Anova

Level Number Mean Std Error Lower 95\% Upper 95\%

$\begin{array}{lllllll}1-1 & & 3 & 0.624944 & 0.00361 & 0.61742 & 0.63247 \\ 1-2 & & 3 & 0.633284 & 0.00361 & 0.6257 & 0.6378\end{array}$

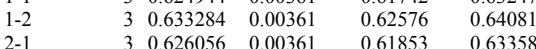

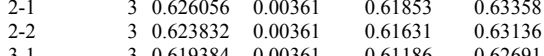

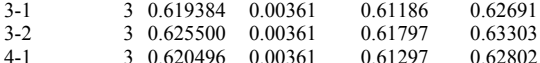

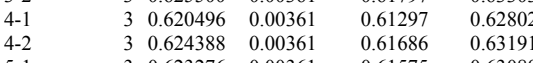

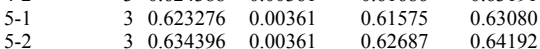

Std Error uses a pooled estimate of error variance
Oneway Analysis of $\mathrm{ZnO}$ (wt\%) By Block/Sub-Block

Glass ID=Batch 1, Reference Value $=0 \mathrm{wt} \%$

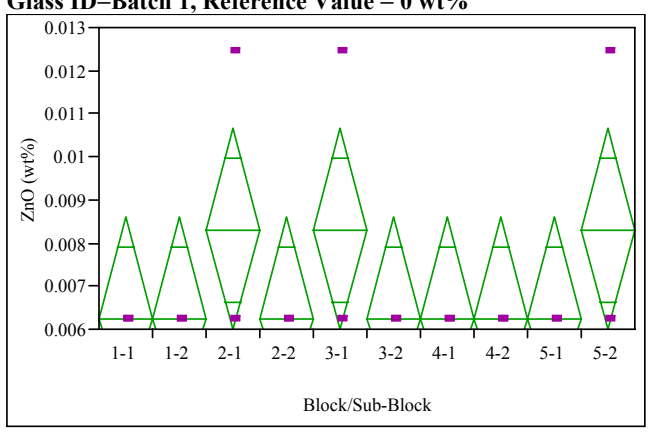

Oneway Anova

Summary of Fit

Rsquare

$\begin{array}{ll} & 0.259259 \\ \text { Root Mean Square Error } \quad 0.07407 & 0.001968\end{array}$ Mean of Response $\quad 0.006846$

Observations (or Sum Wgts) $\quad 30$

Analysis of Variance

Source DF Sum of Squares Mean Square F Ratio Prob $>$ F $\begin{array}{llllll}\text { Block/Sub-Block } & 9 & 0.00002712 & 3.013 \mathrm{e}-6 & 0.7778 & 0.6387\end{array}$ $\begin{array}{llll}\text { Error } & 20 & 0.00007748 & 3.8738 \mathrm{e}-6 \\ \text { C. Total } & 29 & 0.00010459 & \end{array}$

Means for Oneway Anova

Level Number Mean Std Error Lower 95\% Upper 95\%

$\begin{array}{lllllll}1-1 & 3 & 0.006224 & 0.00114 & 0.00385 & 0.0085\end{array}$

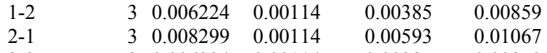

$\begin{array}{lllllll}2-2 & & 3 & 0.006224 & 0.00114 & 0.00385 & 0.00859 \\ 3-1 & & 3 & 0.008299 & 0.00114 & 0.00593 & 0.01067\end{array}$

$\begin{array}{lllllll}3-2 & & 3 & 0.006224 & 0.00114 & 0.00385 & 0.00859 \\ 4-1 & & 3 & 0.006224 & 0.00114 & 0.00385 & 0.00859\end{array}$

$\begin{array}{lllllll}4-2 & & 3 & 0.006224 & 0.00114 & 0.00385 & 0.00859 \\ 5-1 & & 3 & 0.006224 & 0.00114 & 0.00385 & 0.00859 \\ 5-2 & & 3 & 0.006224 & 0.00114 & 0.00385 & 0.0059\end{array}$

$\begin{array}{lllllll}5-1 & & 3 & 0.006224 & 0.00114 & 0.00385 & 0.00859 \\ 5-2 & & 3 & 0.008299 & 0.00114 & 0.00593 & 0.01067\end{array}$

Std Error uses a pooled estimate of error variance
Oneway Analysis of ZrO2 (wt\%) By Block/Sub-Block Glass ID $=$ Batch 1, Reference Value $=\mathbf{0 . 0 9 8} \mathbf{w t} \%$

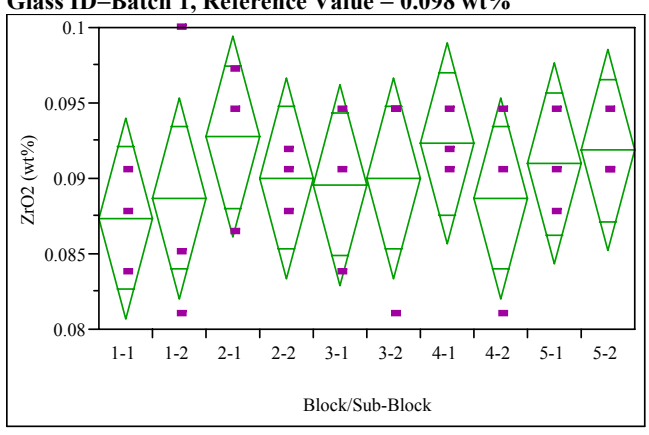

Oneway Anova

Summary of Fit

Rsquare

$\begin{array}{ll} & 0.117647 \\ \text { Root Mean Square Error } & -0.27941 \\ 0 & 0.005537\end{array}$ Mean of Response $\quad 0.090233$ Observations (or Sum Wgts) $\quad 30$

Analysis of Variance
Source
DF Sum of Squares Mean Square F Ratio Prob $>$ F $\begin{array}{llllll}9 & 0.00008174 & 9.083 \mathrm{e}-6 & 0.2963 & 0.9674\end{array}$

$\begin{array}{lll}\text { Error } & 20 & 0.00061309 \\ \text { C. Total } & 29 & 0.00069483\end{array}$

Means for Oneway Anova

Level Number Mean Std Error Lower 95\% Upper 95\%

$\begin{array}{llllll}1-1 & 3 & 0.087352 & 0.00320 & 0.08068 & 0.09402\end{array}$

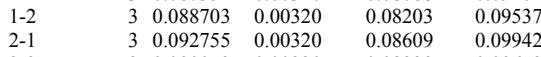

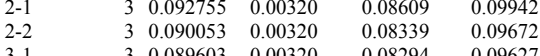

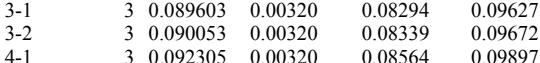

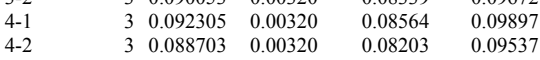

$\begin{array}{lllll}3 & 0.090954 & 0.00320 & 0.08429 & 0.09762\end{array}$

Std Error uses a pooled estimate of error variance 


\section{Exhibit A3. PSAL Oxide Measurements by Analytical Block for Samples of the} Standard Glasses Prepared Using the LM Method

Oneway Analysis of $\mathrm{CaO}(\mathrm{wt} \%)$ By Block/Sub-Block Glass ID $=$ LRM, Reference Value $=0.5 \mathbf{w t} \%$

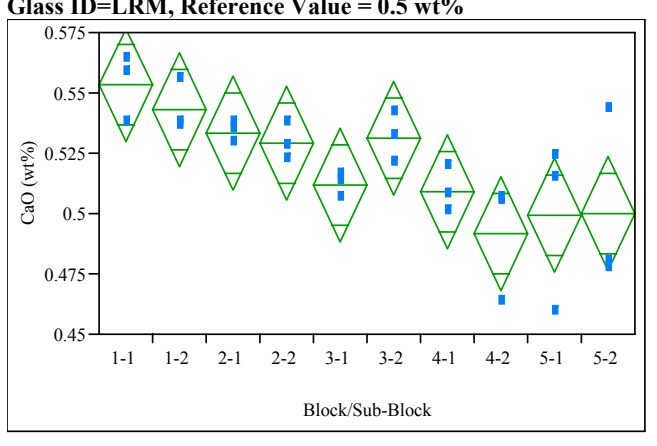

Oneway Anova

Summary of Fit

Rsquare

\subsection{3}

$\begin{array}{ll}\text { Adj Rsquare } & 0.420555 \\ \text { Root Mean Square Error } \quad 0.019569\end{array}$

$\begin{array}{lr}\text { Mean of Response } & 0.520176 \\ \text { Observations (or Sum Wgts) } & 30\end{array}$

Analysis of Variance

Source DF Sum of Squares Mean Square F Ratio Prob $>$ F

$\begin{array}{llllll}\text { Block/Sub-Block } & 9 & 0.01150648 & 0.001278 & 3.3387 & 0.0118\end{array}$

Error
C. Total

$\begin{array}{ll}20 & 0.00765876 \\ 29 & 0.01916524\end{array}$

Means for Oneway Anova

Level Number Mean Std Error Lower 95\% Upper 95\%

$\begin{array}{lllllll}1-1 & & 3 & 0.553150 & 0.01130 & 0.52958 & 0.57672\end{array}$

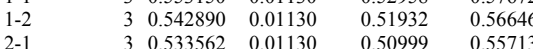

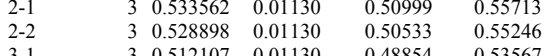

$\begin{array}{lllllll}3-1 & 3 & 0.512107 & 0.01130 & 0.48854 & 0.53567 \\ 3-2 & & 3 & 0.531230 & 0.01130 & 0.50766 & 0.55480\end{array}$

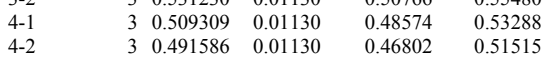

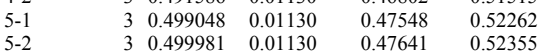

Std Error uses a pooled estimate of error variance
Oneway Analysis of Cr2O3 (wt\%) By Block/Sub-Block

Glass ID $=$ LRM, Reference Value $=\mathbf{0 . 2} \mathbf{w t} \%$

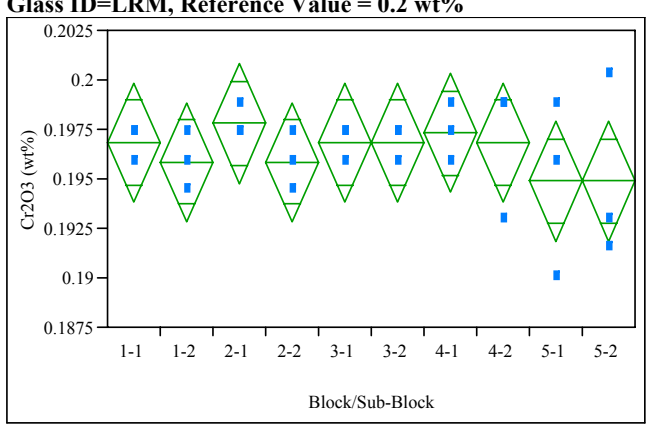

Oneway Anova

Summary of Fit

Rsquare

0.173321
-0.19868

Root Mean Square Error $\quad 0.002503$

$\begin{array}{lr}\text { Mean of Response } & 0.19639 \\ \text { Observations (or Sum Wgts) } & 30\end{array}$

Analysis of Variance

Source DF Sum of Squares Mean Square F Ratio Prob $>F$

$\begin{array}{lllllll} & 0.00002628 & 2.9196 \mathrm{e}-6 & 0.4659 & 0.8804\end{array}$

$\begin{array}{llll}\text { Error } & 20 & 0.00012533 & 6.2664 \mathrm{e}-6 \\ \text { C. Total } & 29 & 0.00015160 & \end{array}$

Means for Oneway Anova

Level Number Mean Std Error Lower 95\% Upper 95\%

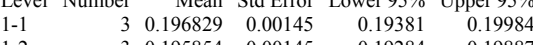

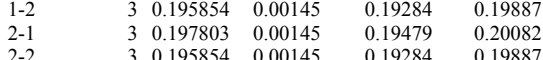

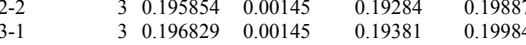

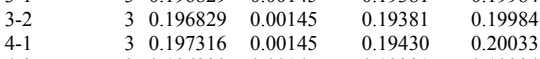

$\begin{array}{lllllll}4-2 & & 3 & 0.196829 & 0.00145 & 0.19381 & 0.19984 \\ 5-1 & & 3 & 0.194880 & 0.00145 & 0.19187 & 0.19789 \\ 5-2 & & 3 & 0.194880 & 0.00145 & 0.19187 & 0.19789\end{array}$

Std Error uses a pooled estimate of error variance
Oneway Analysis of K2O (wt\%) By Block/Sub-Block

Glass ID=LRM, Reference Value $=\mathbf{1 . 5} \mathbf{w t} \%$

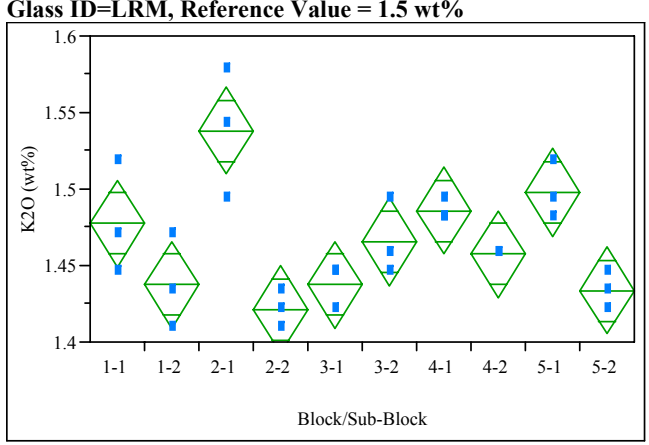

Oneway Anova

Summary of Fit

Rsquare
Adj Rsquare

0.755032
0.644797

$\begin{array}{ll}\text { Rdj Rsquare } & 0.644797 \\ \text { Root Mean Square Error } & 0.023585 \\ & 1.465195\end{array}$

Observations (or Sum Wgts) $\begin{array}{r}1.465195 \\ 30\end{array}$

Analysis of Variance

Source DF Sum of Squares Mean Square F Ratio Prob $>$ F $\begin{array}{lllll}0.03428858 & 0.003810 & 6.8493 & 0.0002\end{array}$ $\begin{array}{lll}\text { Error } & 20 & 0.01112480 \\ \text { C. Total } & 29 & 0.04541338\end{array}$

Means for Oneway Anova

\begin{tabular}{|c|c|c|c|c|}
\hline $\mathrm{Nu}$ & M & Std Erro & & fer $95 \%$ \\
\hline & 1.47764 & 0.01362 & 1.4492 & 1.5060 \\
\hline 3 & 1.43749 & 0.01362 & 1.4091 & 1.4659 \\
\hline 3 & 1.53787 & 0.01362 & 1.5095 & 1.5663 \\
\hline 3 & 1.42143 & 0.01362 & 1.3930 & 1.4498 \\
\hline 3 & 1.43749 & 0.01362 & 1.4091 & 1.4659 \\
\hline 3 & 1.46560 & 0.01362 & 1.4372 & 1.4940 \\
\hline 3 & 1.48567 & 0.01362 & 1.4573 & 1.5141 \\
\hline $4-2$ & 1.45757 & 0.01362 & 1.4292 & 1.4860 \\
\hline $5-1$ & 1.49772 & 0.01362 & 1.4693 & 1.5261 \\
\hline 5-2 & 1.43347 & 0.01362 & 1.4051 & 1.4619 \\
\hline
\end{tabular}

Std Error uses a pooled estimate of error variance 


\section{Exhibit A3. PSAL Oxide Measurements by Analytical Block for Samples of the} Standard Glasses Prepared Using the LM Method

Oneway Analysis of $\mathrm{MgO}(\mathrm{wt} \%)$ By Block/Sub-Block Glass ID $=$ LRM, Reference Value $=\mathbf{0 . 1} \mathbf{w t} \%$

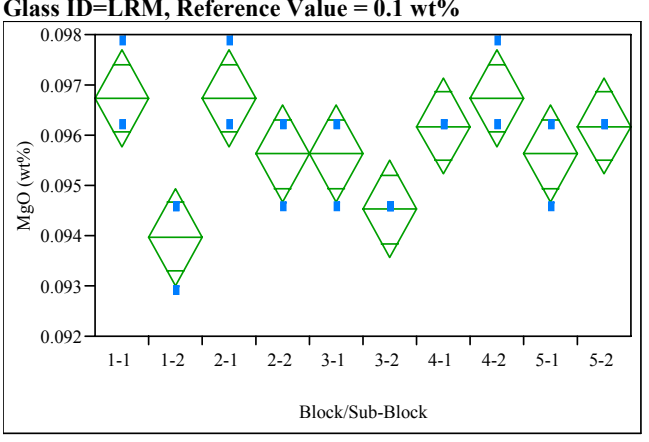

Oneway Anova

Summary of Fit

Rsquare

\subsection{3}

Adj Rsquare $\quad 0.493766$

$\begin{array}{lr}\text { Mean of Response } & 0.095794 \\ \text { Observations (or Sum Wgts) } & 30\end{array}$

Analysis of Variance

Source DF Sum of Squares Mean Square F Ratio Prob $>$ F

$0.00002392-2.6583 \mathrm{e}-6-4.1429-0.0039$

Error
C. Total

$\begin{array}{ll}20 & 0.00001283 \\ 29 & 0.00003676\end{array}$

Means for Oneway Anova

Level Number Mean Std Error Lower 95\% Upper 95\%

$\begin{array}{lllllll}1-1 & & 3 & 0.096734 & 0.00046 & 0.09577 & 0.09770\end{array}$

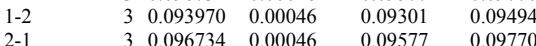

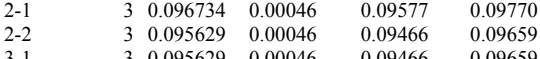

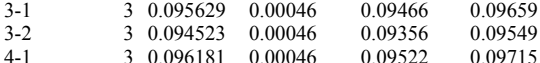

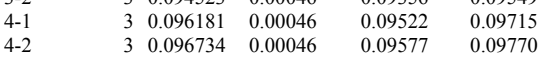

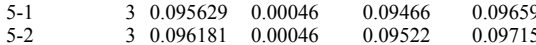

Std Error uses a pooled estimate of error variance
Oneway Analysis of $\mathrm{Na2O}$ (wt\%) By Block/Sub-Block Glass ID $=$ LRM, Reference Value $=\mathbf{2 0 . 0} \mathbf{w t} \%$

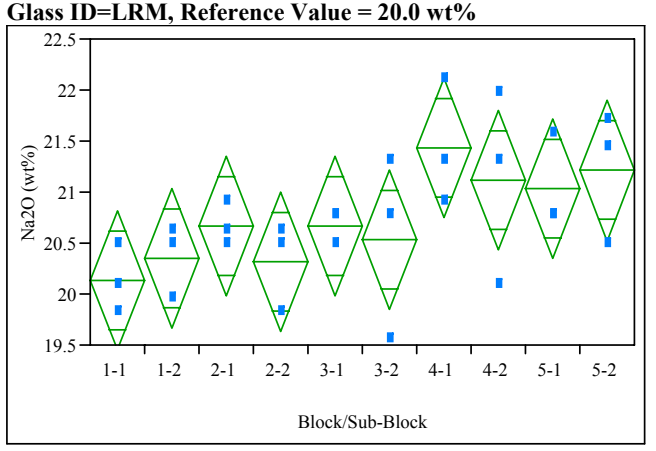

Oneway Anova

Summary of Fit

Rsquare

\subsection{9}

$\begin{array}{ll}\text { Adj Rsquare } & 0.187187 \\ \text { Rot Mean Square Error } & 0.567656\end{array}$

\begin{tabular}{lr} 
Root Mean Square Error & 0.567656 \\
Mean of Response & 20.74572 \\
\hline
\end{tabular}

Analysis of Variance

Source DF Sum of Squares Mean Square F Ratio Prob $>F$ $\begin{array}{llrrrr}\text { Block/Sub-Block } & 9 & 5.052155 & 0.561351 & 1.7421 & 0.1443\end{array}$ $\begin{array}{llr}\text { Error } & 20 & 6.444662 \\ \text { C. Total } & 29 & 11.496817\end{array}$

Means for Oneway Anova

\begin{tabular}{|c|c|c|c|c|}
\hline r & $\mathrm{Me}$ & Std Error & 1 & er $95 \%$ \\
\hline & 0.1301 & 0.32774 & 19.446 & 20.814 \\
\hline & 20.3548 & 0.32774 & 19.671 & 21.038 \\
\hline & 20.6693 & 0.32774 & 19.986 & 21.353 \\
\hline & 20.3099 & 0.32774 & 19.626 & 20.994 \\
\hline & 20.6693 & 0.32774 & 19.986 & 21.353 \\
\hline & 20.5345 & 0.32774 & 19.851 & 21.218 \\
\hline 3 & 21.4332 & 0.32774 & 20.750 & 22.117 \\
\hline $4-2$ & 21.1187 & 0.32774 & 20.435 & 21.802 \\
\hline $5-1 \quad$ & 21.0288 & 0.32774 & 20.345 & \\
\hline $5-2$ & 21.2085 & 0.32774 & 20.525 & 21.892 \\
\hline
\end{tabular}

Std Error uses a pooled estimate of error variance
Oneway Analysis of $\mathrm{NiO}(\mathrm{wt} \%)$ By Block/Sub-Block Glass ID=LRM, Reference Value $=0.1 \mathrm{wt} \%$

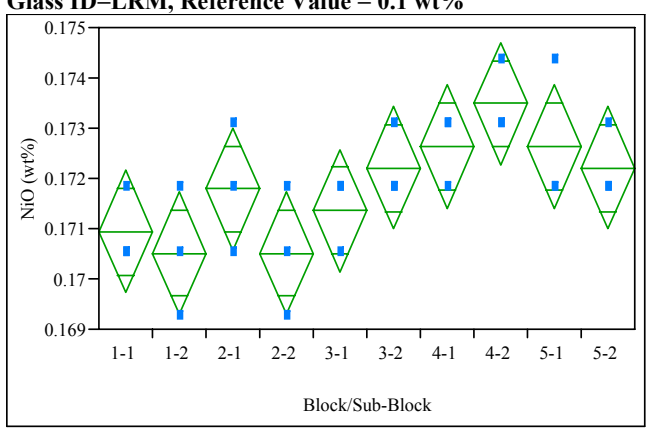

Oneway Anova

Summary of Fit

Rsquare

Error $\quad 0.001013$ $\begin{array}{lr}\text { Mean of Response } & 0.17183 \\ \text { Observations (or Sum Wgts) } & 30\end{array}$

Analysis of Variance

Source DF Sum of Squares Mean Square F Ratio Prob > F $\begin{array}{lllll}0.00002639 & 2.9327 \mathrm{e}-6 & 2.8596 & 0.024\end{array}$

$\begin{array}{lll}\text { Error } & 20 & 0.00002051 \\ \text { C. Total } & 29 & 0.00004690\end{array}$

Means for Oneway Anova

Level Number Mean Std Error Lower 95\% Upper 95\%

$\begin{array}{lllllll}1-1 & & 3 & 0.170939 & 0.00058 & 0.16972 & 0.17216 \\ 1-2 & & 3 & 0.170515 & 0.00058 & 0.1970 & 0.1733\end{array}$

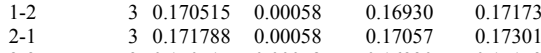

$\begin{array}{lllllll}2-2 & & 3 & 0.170515 & 0.00058 & 0.16930 & 0.17173 \\ 3-1 & & 3 & 0.171363 & 0.00058 & 0.17014 & 0.17258\end{array}$

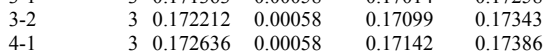

$\begin{array}{lllllll}4-2 & 3 & 0.173484 & 0.00058 & 0.17226 & 0.17470 \\ 5-1 & & 3 & 0.172636 & 0.00058 & 0.17142 & 0.17386\end{array}$

$\begin{array}{lllllll}5-1 & & 3 & 0.172636 & 0.00058 & 0.17142 & 0.17386 \\ 5-2 & & 3 & 0.172212 & 0.00058 & 0.17099 & 0.17343\end{array}$

Std Error uses a pooled estimate of error variance 


\section{Exhibit A3. PSAL Oxide Measurements by Analytical Block for Samples of the} Standard Glasses Prepared Using the LM Method

Oneway Analysis of $\mathrm{PbO}(\mathrm{wt} \%)$ By Block/Sub-Block Glass ID=LRM, Reference Value $=0.1 \mathrm{wt} \%$

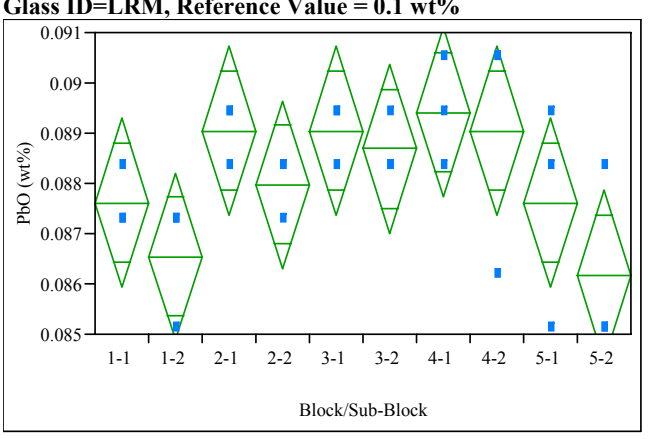

Oneway Anova

Summary of Fit

Rsquare
Adj Rsquare

\subsection{4}

Adj Rsquare $\quad 0.23036$

$\begin{array}{lr}\text { Mean of Response } & 0.088115 \\ \text { Observations (or Sum Wgts) } & 30\end{array}$

Analysis of Variance

Source DF Sum of Squares Mean Square F Ratio Prob $>$ F

$\begin{array}{lllll}9 & 0.00003419 & 0.0000038 & 1.9644 & 0.100\end{array}$

Error

$\begin{array}{ll}20 & 0.00003868 \\ 29 & 0.00007287\end{array}$

Means for Oneway Anova

Level Number Mean Std Error Lower 95\% Upper 95\%

$\begin{array}{lllllll}1-1 & & 3 & 0.087612 & 0.00080 & 0.08594 & 0.08929\end{array}$

$\begin{array}{lllllll}1-2 & & 3 & 0.086535 & 0.00080 & 0.08486 & 0.0882 \\ 2-1 & & 3 & 0.089049 & 0.00080 & 0.08737 & 0.09072\end{array}$

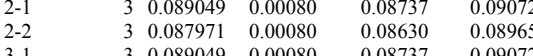

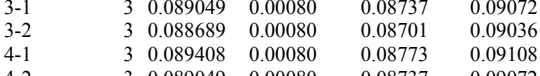

$\begin{array}{llllll}5-1 & 3 & 0.087612 & 0.00080 & 0.08737 & 0.09072 \\ 5 & 3 & 0.0864 & 0.08929\end{array}$
Oneway Analysis of SO3 (wt \%) By Block/Sub-Block Glass ID $=$ LRM, Reference Value $=0.2 \mathrm{wt} \%$

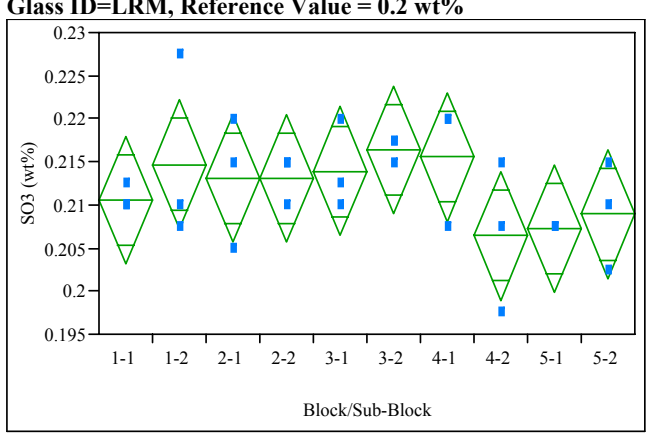

\section{Oneway Anova}

Summary of Fit

Rsquare

0.302018
Root Mean Square Error $\quad-0.01207$
0.0062 $\begin{array}{lr}\text { Mean of Response } & 0.211987 \\ \text { Observations (or Sum Wgts) } & 30\end{array}$

Analysis of Variance

Source DF Sum of Squares Mean Square F Ratio Prob $>F$ Block/Sub-Block $\begin{array}{llll}\text { Error } & 20 & 0.00076892 & 0.000038 \\ \text { C. Total } & 29 & 0.00110164 & \end{array}$

Means for Oneway Anova

$\begin{array}{lrrrrr}\text { Level } & \text { Number } & \text { Mean } & \text { Std Error } & \text { Lower } 95 \% & \text { Upper } 95 \% \\ 1-1 & 3 & 0.210572 & 0.00358 & 0.20310 & 0.21804 \\ 1-2 & 3 & 0.214733 & 0.00358 & 0.20727 & 0.222220 \\ 2-1 & 3 & 0.213069 & 0.00358 & 0.20560 & 0.22054 \\ 2-2 & 3 & 0.213069 & 0.00358 & 0.20560 & 0.22054 \\ 3-1 & 3 & 0.213091 & 0.00358 & 0.20643 & 0.22137 \\ 3-2 & 3 & 0.216398 & 0.00358 & 0.20893 & 0.22387 \\ 4-1 & 3 & 0.215566 & 0.00358 & 0.20810 & 0.22303 \\ 4-2 & 3 & 0.206410 & 0.00358 & 0.19894 & 0.21388 \\ 5-1 & 3 & 0.207243 & 0.00358 & 0.19978 & 0.21471 \\ 5-2 & 3 & 0.208907 & 0.00358 & 0.20144 & 0.21637\end{array}$

Std Error uses a pooled estimate of error variance
Oneway Analysis of SrO (wt\%) By Block/Sub-Block Glass ID $=$ LRM, Reference Value $=0.0 \mathrm{wt} \%$

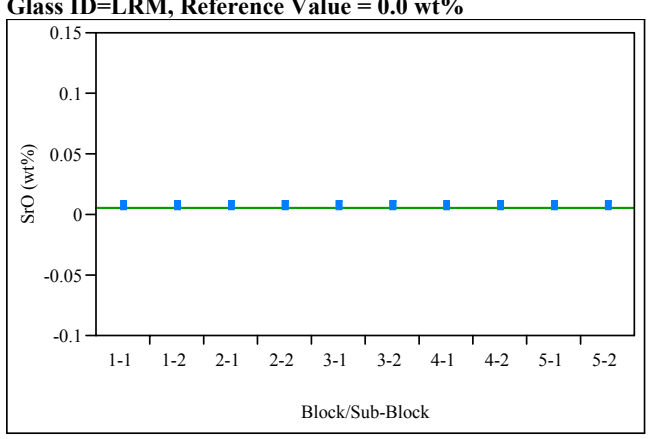

Oneway Anova

Summary of Fit

Rsquare

Root Mean Square Erro

$\begin{array}{lr}\text { Mean of Response } & 0.005913 \\ \text { Observations (or Sum Wgts) } & 30\end{array}$

Analysis of Variance

Source DF Sum of Squares Mean Square F Ratio Prob $>F$ \begin{tabular}{lr} 
Block/Sub-Block & 9 \\
Error & 20 \\
\hline C. Total & 29
\end{tabular}

Means for Oneway Anova

$\begin{array}{lrrrrr}\text { Level } & \text { Number } & \text { Mean } & \text { Std Error } & \text { Lower } 95 \% & \text { Upper } 95 \% \\ 1-1 & 3 & 0.005913 & 0 & 0.00591 & 0.00591 \\ 1-2 & 3 & 0.005913 & 0 & 0.00591 & 0.00591 \\ 2-1 & 3 & 0.005913 & 0 & 0.00591 & 0.00591 \\ 2-2 & 3 & 0.005913 & 0 & 0.00591 & 0.00591 \\ 3-1 & 3 & 0.005913 & 0 & 0.0591 & 0.00591 \\ 3-2 & 3 & 0.005913 & 0 & 0.00591 & 0.00591 \\ 4-1 & 3 & 0.005913 & 0 & 0.00591 & 0.00591 \\ 4-2 & 3 & 0.005913 & 0 & 0.00591 & 0.00591 \\ 5-1 & 3 & 0.005913 & 0 & 0.00591 & 0.00591 \\ 5-2 & 3 & 0.005913 & 0 & 0.00591 & 0.00591\end{array}$

Std Error uses a pooled estimate of error variance 


\section{Exhibit A3. PSAL Oxide Measurements by Analytical Block for Samples of the} Standard Glasses Prepared Using the LM Method

Oneway Analysis of TiO2 (wt\%) By Block/Sub-Block Glass ID $=$ LRM, Reference Value $=0.1 \mathrm{wt} \%$

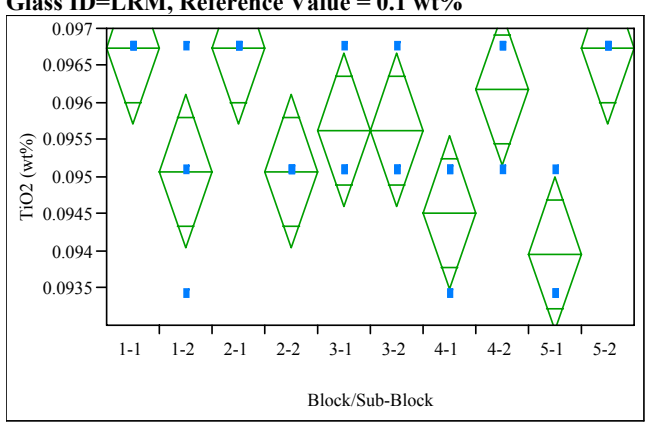

Oneway Anova

Summary of Fit

Rsquare
Adj Rsquare

\subsection{4}

\begin{tabular}{ll} 
Adj Rsquare & 0.472727 \\
Root Mean Square Error & 0.000861 \\
\hline
\end{tabular}

Mean of Response $\quad 0.095632$

Sum Wgts)

Analysis of Variance
Source $\quad$ DF Sum of Squares Mean Square F Ratio Prob > F

$\begin{array}{llrrrr}\text { Source } & \text { DF } & \text { Sum of Squares } & \text { Mean Square } & \text { F Ratio } & \text { Prob }>\text { F } \\ \text { Block/Sub-Block } & 9 & 0.00002597 & 2.8853 \mathrm{e}-6 & 3.8889 & 0.0055\end{array}$

$\begin{array}{llll}\text { Error } & 20 & 0.00001484 & 7.4193 \mathrm{e}-7 \\ \text { C. Total } & 29 & 0.00004081 & \end{array}$

Means for Oneway Anova

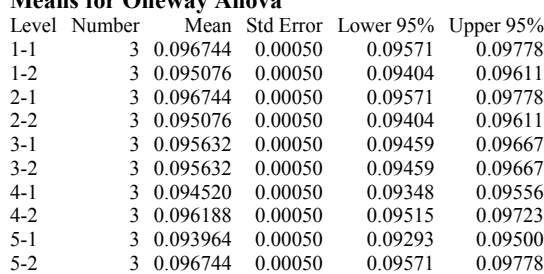

Std Error uses a pooled estimate of error variance
Oneway Analysis of $\mathrm{ZnO}$ (wt\%) By Block/Sub-Block Glass ID $=$ LRM, Reference Value $=0.0 \mathrm{wt} \%$

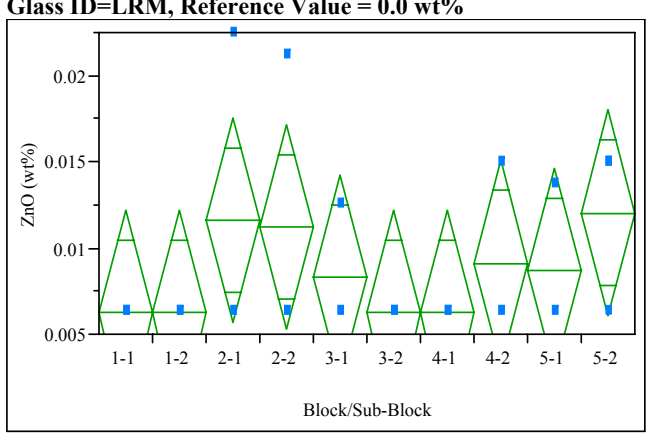

Oneway Anova

Summary of Fit

Rsquare
Adj Rsquare

Root Mean Square Error $\quad \begin{array}{r}-0.10556 \\ 0.04938\end{array}$

$\begin{array}{lr}\text { Mean of Response } & 0.008589 \\ \text { Observations (or Sum Wgts) } & 30\end{array}$

Analysis of Variance

Source DF Sum of Squares Mean Square F Ratio Prob $>$ F $\begin{array}{lllllll}\text { Block/Sub-Block } \quad 9 & 0.00015191 & 0.000017 & 0.6923 & 0.708\end{array}$ $\begin{array}{llll}\text { Error } & 20 & 0.00048758 & 0.000024 \\ \text { C. Total } & 29 & 0.00063949 & \end{array}$

Means for Oneway Anova

\begin{tabular}{|c|c|c|c|c|}
\hline & & & & \\
\hline & 066224 & 0.00285 & 0.00028 & 0.01 \\
\hline & 0.006224 & 0.00285 & 0.00028 & \\
\hline & 18 & 0.00 & & \\
\hline & 3 & 0.00 & & \\
\hline & 99 & 0.00 & & \\
\hline & 0.006 & 0.00 & 0.0 & \\
\hline & 4 & 0.0 & 0 & \\
\hline & 009129 & 0.00 & 0.0 & \\
\hline & 0.008714 & 0.00285 & 0.00277 & 0.014 \\
\hline & 0.012033 & 0.00285 & 09 & 0.01798 \\
\hline
\end{tabular}

Std Error uses a pooled estimate of error variance
Oneway Analysis of ZrO2 (wt\%) By Block/Sub-Block Glass ID=LRM, Reference Value $=1.0 \mathrm{wt} \%$

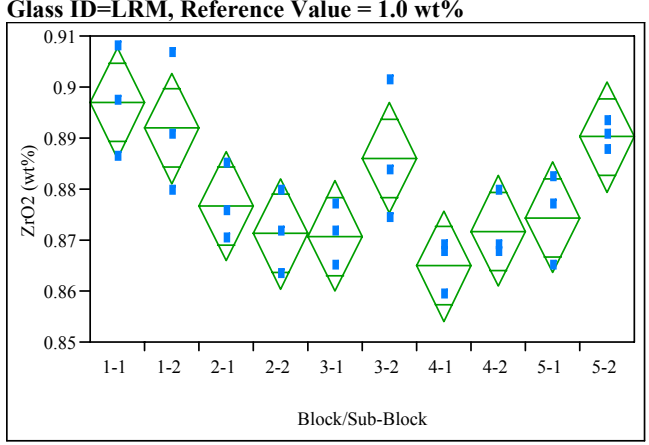

Oneway Anova Summary of Fit

Rsquare
Adj Rsquare

0.664481

$\begin{array}{ll} & 0.513498 \\ \text { Root Mean Square Error } & 0.008987\end{array}$ $\begin{array}{lr}\text { Mean of Response } & 0.879506 \\ \text { Observations (or Sum Wgts) } & 30\end{array}$

Analysis of Variance

Source DF Sum of Squares Mean Square F Ratio Prob $>$ F $\begin{array}{lllll}\text { Block/Sub-Block } & 0.00319930 & 0.000355 & 4.4010 & 0.002\end{array}$ $\begin{array}{lll}\text { Error } & 20 & 0.00161543 \\ \text { C. Total } & 29 & 0.00481473\end{array}$

Means for Oneway Anova

Level Number

$\begin{array}{lrrrrr}\text { Level } & \text { Number } & \text { Mean } & \text { Std Error } & \text { Lower 95\% } & \text { Upper 95\% } \\ 1-1 & 3 & 0.896931 & 0.00519 & 0.88611 & 0.90775 \\ 1-2 & 3 & 0.891978 & 0.00519 & 0.88115 & 0.90280 \\ 2-1 & 3 & 0.876669 & 0.00519 & 0.86585 & 0.88749 \\ 2-2 & 3 & 0.871266 & 0.00519 & 0.86044 & 0.88209 \\ 3-1 & 3 & 0.870816 & 0.00519 & 0.85999 & 0.88164 \\ 3-2 & 3 & 0.886125 & 0.00519 & 0.87530 & 0.89695 \\ 4-1 & 3 & 0.864962 & 0.00519 & 0.85414 & 0.87579 \\ 4-2 & 3 & 0.871176 & 0.00519 & 0.86089 & 0.88254 \\ 5-1 & 3 & 0.874418 & 0.00519 & 0.86359 & 0.82524 \\ 5-2 & 3 & 0.890177 & 0.00519 & 0.87935 & 0.90100\end{array}$

Std Error uses a pooled estimate of error variance 


\section{Exhibit A4: PSAL Oxide Measurements by Analytical Block for Samples of the} Standard Glasses Prepared Using the PF Method

Oneway Analysis of Al2O3 (wt\%) By Block/Sub-Block Glass ID=Batch 1, Reference Value $=4.877 \mathrm{wt} \%$

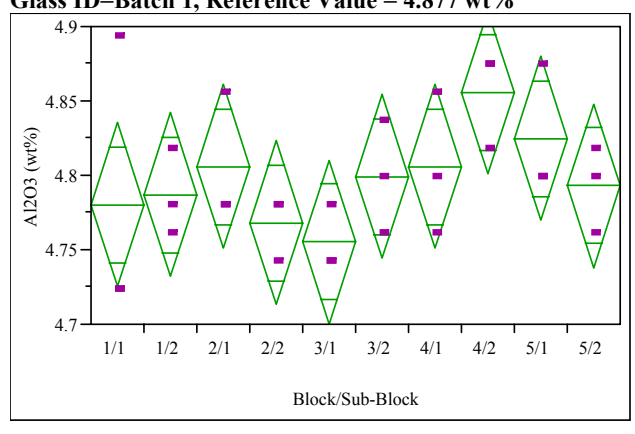

Oneway Anova

Summary of Fit

Rsquare

$$
0.347137
$$

0.053348
Adj Rsquare

Mean of Response

0.045636
4.797441

Observations (or Sum Wgts) $\quad 30$

$\begin{array}{lccccc}\text { Analysis of Variance } & \text { DF } & & & \\ \text { Source } & \text { Sum of Squares } & \text { Mean Square } & \text { F Ratio } & \text { Prob }>\text { F } \\ \text { Block/Sub-Block } & 9 & 0.02214720 & 0.002261 & 1.1816 & 0.3579\end{array}$

$\begin{array}{lrrr}\text { Block/Sub-Block } & 9 & 0.02214720 & 0.00246 \\ \text { Error } & 20 & 0.04165245 & 0.002083 \\ \text { C. Total } & 29 & 0.06379966 & \end{array}$

Means for Oneway Anova

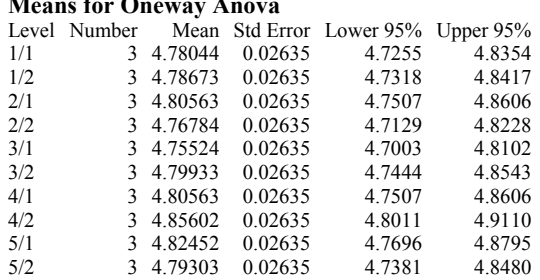

Std Error uses a pooled estimate of error variance

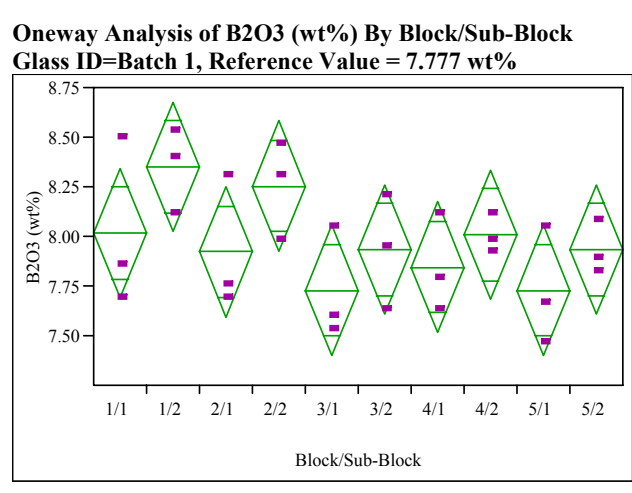

Oneway Anova

Summary of Fit

Rsquare
Adj Rsqu

0.426703
0.168719

$\begin{array}{ll}\text { Adj Rsquare } & 0.168719 \\ \text { Root Mean Square Error } & 0.271886 \\ \text { Mer } & 7.971390\end{array}$

$\begin{array}{lr}\text { Mean of Response } & 7.971399 \\ \text { Observations (or Sum Wgts) } & 30\end{array}$

Analysis of Variance

Source DF Sum of Squares Mean Square F Ratio Prob $>$ F

$\begin{array}{lrrrrr}\text { Block/Sub-Block } & 9 & 1.1003991 & 0.122267 & 1.6540 & 0.1668\end{array}$

Error
C. Total

$\begin{array}{ll}20 & 1.4784420 \\ 29 & 2.5788411\end{array}$

Means for Oneway Anova

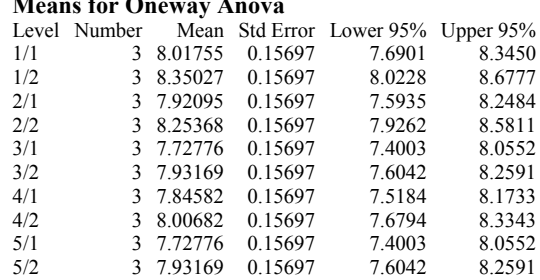

Std Error uses a pooled estimate of error variance
Oneway Analysis of Fe2O3 (wt\%) By Block/Sub-Block Glass ID $=$ Batch 1, Reference Value $=\mathbf{1 2 . 8 3 9} \mathbf{w t} \%$

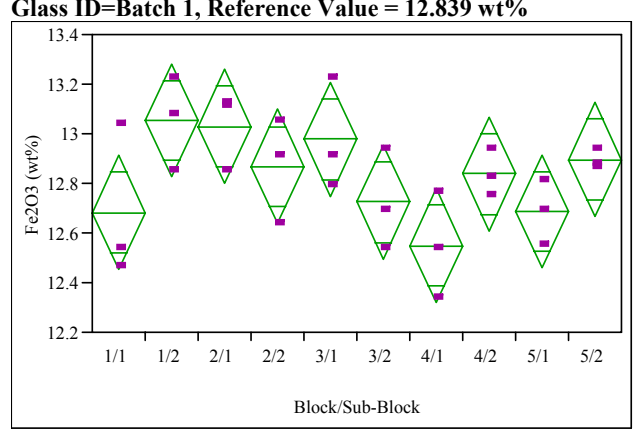

Oneway Anova

Summary of Fit

Rsquare

0.508572

$\begin{array}{ll}\text { Adj Rsquare } & 0.287429 \\ \text { Root Mean Square Error } & 0.190496\end{array}$

$\begin{array}{lr}\text { Mean of Response } & 12.83013 \\ \text { Observations (or Sum Wgts) } & 30\end{array}$

Analysis of Variance

Source DF Sum of Squares Mean Square F Ratio Prob $>$ F \begin{tabular}{lrllll} 
Block/Sub-Block & 9 & 0.7510901 & 0.083454 & 2.2997 & 0.0580 \\
\hline C. Tow & 20 & 0.7257712 & 0.036289 & &
\end{tabular} $\begin{array}{llll}\text { Error } & 20 & 0.7257712 & 0.036289 \\ \text { C. Total } & 29 & 1.4768613 & \end{array}$

Means for Oneway Anova

Level Number Mean Std Error Lower 95\% Upper 95\%

$\begin{array}{lrrrrr}1 / 1 & 3 & 12.6814 & 0.10998 & 12.452 & 12.911 \\ 1 / 2 & 3 & 13.0532 & 0.10998 & 12.824 & 13.283 \\ 2 / 1 & 3 & 13.0293 & 0.10998 & 12.800 & 13.259 \\ 2 / 2 & 3 & 12.8673 & 0.10998 & 12.638 & 13.097 \\ 3 / 1 & 3 & 12.979 & 0.10998 & 12.747 & 13.206 \\ 3 / 2 & 3 & 12.7243 & 0.10998 & 12.495 & 12.954 \\ 3 / 2 & 3 & 12.5480 & 0.10998 & 12.319 & 12.777 \\ 4 / 1 & 3 & 12.8837 & 0.10998 & 12.609 & 13.068 \\ 4 / 2 & 3 & 12.6862 & 0.10998 & 12.457 & 12.916 \\ 5 / 1 & 3 & 12.8959 & 0.10998 & 12.666 & 13.125\end{array}$

Std Error uses a pooled estimate of error variance 


\section{Exhibit A4: PSAL Oxide Measurements by Analytical Block for Samples of the} Standard Glasses Prepared Using the PF Method

Oneway Analysis of Li2O (wt\%) By Block/Sub-Block Glass ID=Batch 1, Reference Value $=4.429 \mathrm{wt} \%$

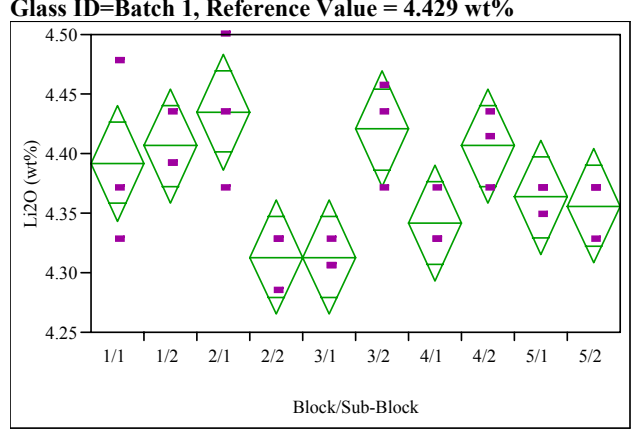

Oneway Anova

Summary of Fit

Rsquare

\subsection{9}

$\begin{array}{ll}0.443953 \\ \text { Root Rean Square Error } & 0.040085\end{array}$

Mean of Response 4.374693

Observations (or Sum Wgts)

$\begin{array}{lccccc}\text { Analysis of Variance } & \text { DF } & & & \\ \text { Source } & \text { Dum of Squares } & \text { Mean Square } & \text { F Ratio } & \text { Prob }>\text { F } \\ \text { Block/Sub-Block } & 9 & 0.05166456 & 0.005741 & 3.5726 & 0.0085\end{array}$

$\begin{array}{lrrr}\text { Block/Sub-Block } & 9 & 0.05166456 & 0.00574 \\ \text { Error } & 20 & 0.03213585 & 0.001607 \\ \text { C. Total } & 29 & 0.08380041 & \end{array}$

Means for Oneway Anova

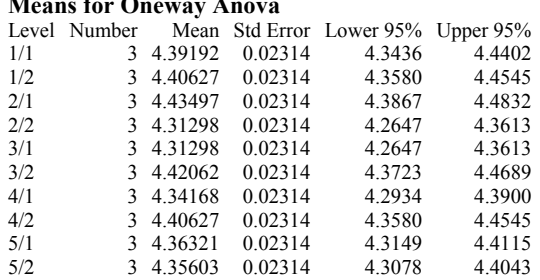

Std Error uses a pooled estimate of error variance

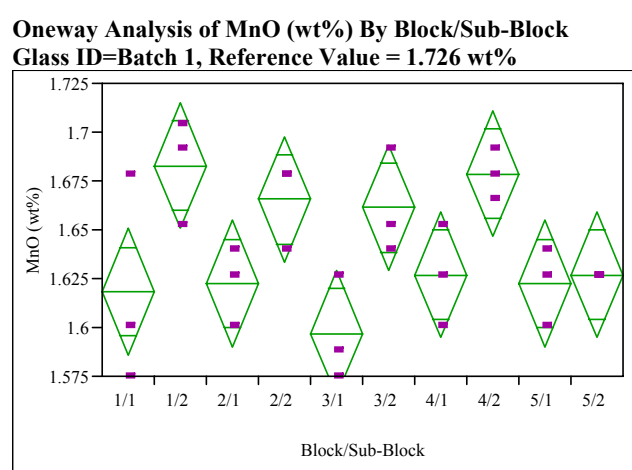

\section{Oneway Anova}

Summary of Fit

$\begin{array}{lr}\text { Rsquare } & 0.617721 \\ \text { Adj Rsquare } & 0.445696 \\ \text { Root Mean Square Error } & 0.026775 \\ \text { Mean of Response } & 1.640254 \\ \text { Observations (or Sum Wgts) } & 30\end{array}$

Analysis of Variance

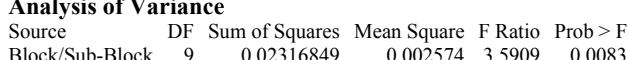

$\begin{array}{lrrr}\text { Block/Sub-Block } & 9 & 0.02316849 & 0.002574 \\ \text { Error } & 20 & 0.01433790 & 0.000717 \\ \text { C. Total } & 29 & 0.03750639 & \end{array}$

Means for Oneway Anova

Level Number Mean Std Error Lower 95\% Upper 95\%

\begin{tabular}{|c|c|c|c|c|}
\hline $1 / 1$ & 31.61830 & 0.01546 & 1.5861 & 1.6505 \\
\hline in & $\begin{array}{ll}31.68286 \\
\end{array}$ & 0.01546 & 1.6506 & 1.7151 \\
\hline$z / 1$ & 1.62261 & 0.01546 & 1.5904 & 1.6549 \\
\hline & $\begin{array}{ll}3 & 1.66565\end{array}$ & 0.01546 & 1.6334 & 1.6979 \\
\hline & 1.59678 & 0.01546 & 1.5645 & 1.6290 \\
\hline & $\begin{array}{ll}3 & 1.66134\end{array}$ & 0.01546 & 1.6291 & 1.6936 \\
\hline 71 & $\begin{array}{ll}3 & 1.62691\end{array}$ & 0.01546 & 1.5947 & 1.6592 \\
\hline $4 / 2$ & $\begin{array}{ll}3 & 1.67856\end{array}$ & 0.01546 & 1.6463 & 1.7108 \\
\hline & 1.62261 & 0.01546 & 1.5904 & 1.6549 \\
\hline & 1.62691 & 0.01546 & 1.5947 & 1.6592 \\
\hline
\end{tabular}

Std Error uses a pooled estimate of error variance
Oneway Analysis of $\mathrm{SiO} 2$ (wt\%) By Block/Sub-Block Glass ID=Batch 1, Reference Value $=\mathbf{5 0 . 2 2} \mathbf{w t} \%$

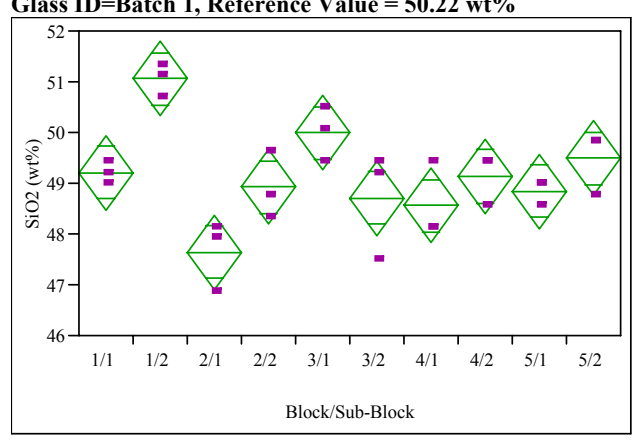

Oneway Anova

Summary of Fit

Rsquare
Adj Rsqu

\begin{tabular}{ll} 
Adj Rsquare & 0.751553 \\
Root Mean Square Error & 0.639752 \\
\hline & 0.607601
\end{tabular} Mean of Response $\quad 49.15398$

Observations (or Sum Wgts)

Analysis of Variance

$\begin{array}{lrrrrr}\text { Source } & \text { DF } & \text { Sum of Squares } & \text { Mean Square } & \text { F Ratio } & \text { Prob }>\text { F } \\ \text { Block/Sub-Block } & 9 & 22.335355 & 2.48171 & 6.7222 & 0.0002\end{array}$ $\begin{array}{llr}\text { Error } & 20 & 7.383589 \\ \text { C. Total } & 29 & 29.718944\end{array}$

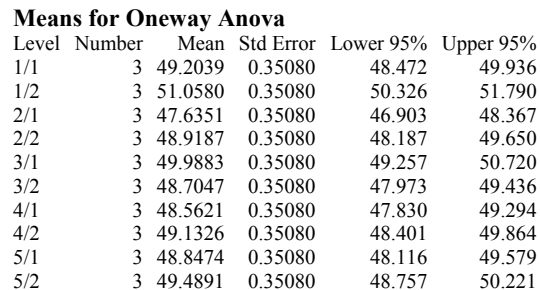

Std Error uses a pooled estimate of error variance 


\section{Exhibit A4: PSAL Oxide Measurements by Analytical Block for Samples of the} Standard Glasses Prepared Using the PF Method

Oneway Analysis of Al2O3 (wt\%) By Block/Sub-Block Glass ID $=$ LRM, Reference Value $=\mathbf{1 0 . 0} \mathbf{w t} \%$

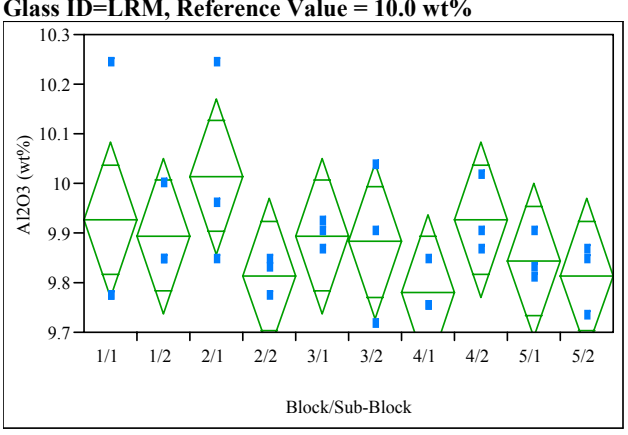

Oneway Anova

Summary of Fit

Rsquare

\subsection{3}

$\begin{array}{ll}-0.05091 \\ \text { Adj Rsquare } & 0.129996\end{array}$

$\begin{array}{lr}\text { Mean of Response } & 9.878936 \\ \text { Observations (or Sum Wgts) } & 30\end{array}$

$\begin{array}{llrrrr}\text { Analysis of Variance } \\ \text { Source } & \text { DF } & \text { Sum of Squares } & \text { Mean Square } & \text { F Ration } & \text { Prob > F } \\ \text { Block/Sub-Block } & 9 & 0.12834906 & 0.014261 & 0.8439 & 0.5862\end{array}$

Error

$\begin{array}{ll}20 & 0.33797990 \\ 29 & 0.46632896\end{array}$

Means for Oneway Anova

\begin{tabular}{lrrrrr}
\multicolumn{7}{c}{ Level } & Number & Mean & Std Error & Lower $95 \%$ & Upper $95 \%$ \\
$1 / 1$ & 3 & 9.9262 & 0.07505 & 9.7696 & 10.083 \\
$1 / 2$ & 3 & 9.8947 & 0.07505 & 9.7381 & 10.051 \\
$2 / 1$ & 3 & 10.0144 & 0.07505 & 9.8578 & 10.171 \\
$2 / 2$ & 3 & 9.8128 & 0.07505 & 9.6562 & 9.969 \\
$3 / 1$ & 3 & 9.8947 & 0.07505 & 9.7381 & 10.051 \\
$3 / 2$ & 3 & 9.8821 & 0.07505 & 9.7255 & 10.039 \\
$4 / 1$ & 3 & 9.7813 & 0.07505 & 9.6248 & 9.938 \\
$4 / 2$ & 3 & 9.9262 & 0.057505 & 9.7696 & 10.083 \\
$5 / 1$ & 3 & 9.8443 & 0.07505 & 9.6877 & 10.001 \\
$5 / 2$ & 3 & 9.8128 & 0.07505 & 9.6562 & 9.969
\end{tabular}

Std Error uses a pooled estimate of error variance
Oneway Analysis of B2O3 (wt\%) By Block/Sub-Block Glass ID $=$ LRM, Reference Value $=8.0 \mathrm{wt} \%$

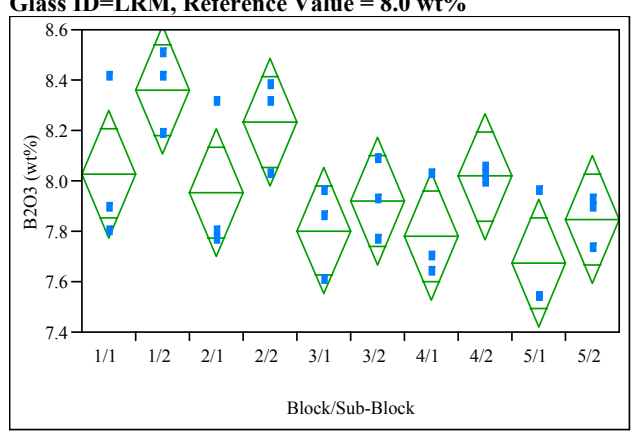

Oneway Anova

Summary of Fit

$\begin{array}{ll}\text { Rsquare } & 0.575516 \\ \text { Adj Rsquare } & 0.384498 \\ \text { Root Mean Square Error } & 0.209252\end{array}$ $\begin{array}{lr}\text { Mean of Response } & 7.961739 \\ \text { Observations (or Sum Wgts) } & 30\end{array}$

Analysis of Variance

Source DF Sum of Squares Mean Square F Ratio Prob $>$ F $\begin{array}{llll}0.131924 & 3.0129 & 0.019\end{array}$

Error

2.0630452

Means for Oneway Anova

Level Number Mean Std Error Lower 95\% Upper 95\%

$\begin{array}{llllll}1 / 1 & 3 & 8.02828 & 0.12081 & 7.7763 & 8.2803\end{array}$

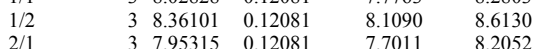

$\begin{array}{llllll}2 / 1 & 3 & 7.95315 & 0.12081 & 7.7011 & 8.2052 \\ 2 / 2 & 3 & 8.23221 & 0.12081 & 7.9802 & 8.4842 \\ 3 / 1 & 3 & 7.80289 & 0.12081 & 7.5509 & 8.0549\end{array}$

$3 / 2 \quad 37.920950 .1208$

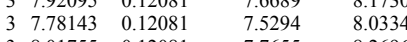

$\begin{array}{lllllll}4 / 2 & & 3 & 8.01755 & 0.12081 & 7.7655 & 8.2696 \\ 5 / 1 & & 3 & 7.67410 & 0.12081 & 7.4221 & 7.9261\end{array}$

Std Error uses a pooled estimate of error variance
Oneway Analysis of Fe2O3 (wt\%) By Block/Sub-Block Glass ID $=$ LRM, Reference Value $=1.0 \mathrm{wt} \%$

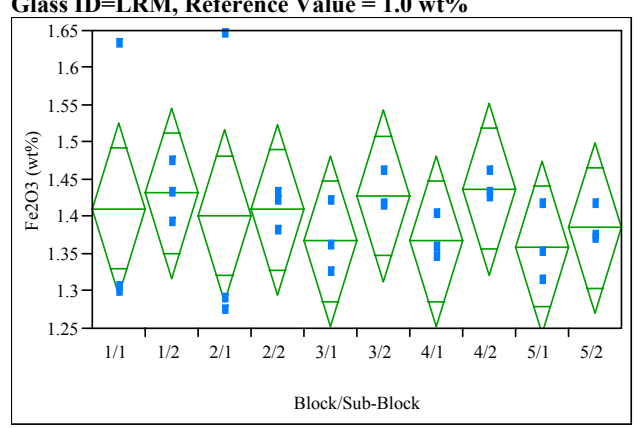

Oneway Anova

Summary of Fit

Rsquare

0.10846
-0.29273

Root Mean Square Error $\quad 0.095227$

Mean of Response
Observations (or Sum Wgts)

Analysis of Variance

$\begin{array}{llll}\text { Source } & \text { DF Sum of Squares Mean Square F Ratio Prob }>\text { F }\end{array}$

$\begin{array}{llll}\text { Error } & 20 & 0.18136377 & 0.009068 \\ \text { C. Total } & 29 & 0.20342750 & \end{array}$

Means for Oneway Anova

Level Number Mean Std Error Lower 95\% Upper 95\%

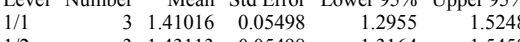

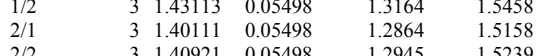

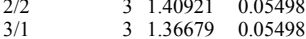

$\begin{array}{llll}3 / 2 & 3 & 1.42779 & 0.05498 \\ 4 / 1 & 3 & 1.36727 & 0.05498\end{array}$

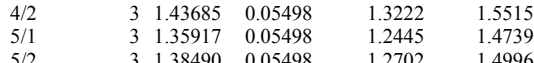

Std Error uses a pooled estimate of error variance 


\section{Exhibit A4: PSAL Oxide Measurements by Analytical Block for Samples of the} Standard Glasses Prepared Using the PF Method

Oneway Analysis of Li2O (wt\%) By Block/Sub-Block Glass ID=LRM, Reference Value $=0.1 \mathbf{w t} \%$

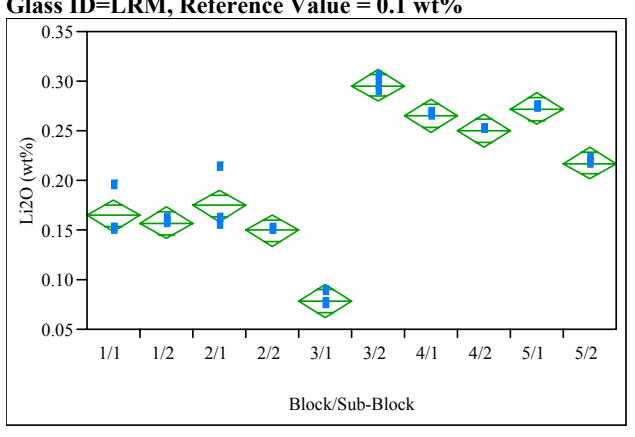

Oneway Anova

Summary of Fit

Rsquare

\subsection{8}

0.960383
Root Mean Square Error $\quad 0.01337$

$\begin{array}{lr}\text { Mean of Response } & 0.202301 \\ \text { Observations (or Sum Wgts) } & 30\end{array}$

$\begin{array}{llllll}\text { Analysis of Variance } & \text { DF } & & & \\ \text { Source } & \text { Dum of Squares } & \text { Mean Square } & \text { F Ratio } & \text { Prob }>\text { F } \\ \text { Block/Sub-Block } & 9 & 0.12727481 & 0.014142 & 79.1116 & <.0001\end{array}$

Error

$\begin{array}{ll}20 & 0.00357511 \\ 29 & 0.13084992\end{array}$

Means for Oneway Anova

$\begin{array}{lrrrrr}\text { Level } & \text { Number } & \text { Mean } & \text { Std Error } & \text { Lower 95\% } & \text { Upper 95\% } \\ 1 / 1 & 3 & 0.164338 & 0.00772 & 0.14824 & 0.18044 \\ 1 & 3 & 0.157162 & 0.00772 & 0.11106 & 0.17326\end{array}$

$\begin{array}{lllllll}1 / 2 & & 3 & 0.157162 & 0.00772 & 0.14106 & 0.17326 \\ 2 / 1 & & 3 & 0.174385 & 0.00772 & 0.15828 & 0.19049 \\ 2 & & 3 & 0.149268 & 0.00772 & 0.13317 & 0.16537\end{array}$

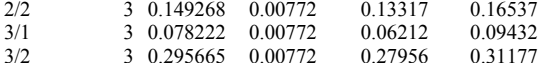

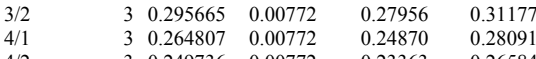

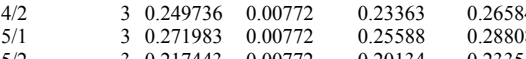

Std Error uses a pooled estimate of error variance
Oneway Analysis of $\mathrm{MnO}(\mathrm{wt} \%)$ By Block/Sub-Block Glass ID $=$ LRM, Reference Value $=0.1 \mathrm{wt} \%$

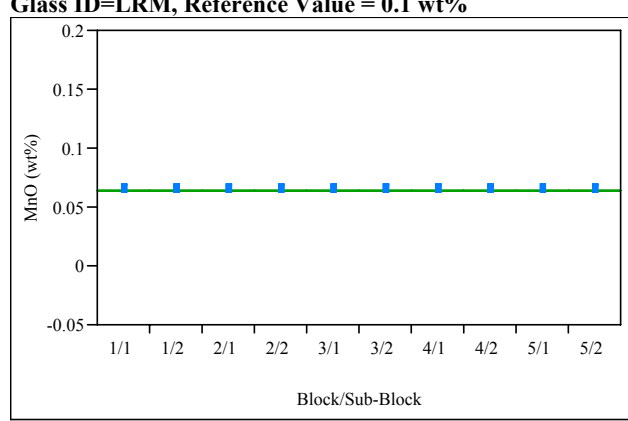

Oneway Anova

Summary of Fit

Rsquare

Root Mean Square Error

$\begin{array}{lr}\text { Mean of Response } & 0.06456 \\ \text { Observations (or Sum Wgts) } & 30\end{array}$

Analysis of Variance

Source DF Sum of Squares Mean Square F Ratio Prob $>F$

Block/Sub-Block 99

C. Total

$$
\begin{aligned}
& 20 \\
& 29
\end{aligned}
$$

Means for Oneway Anova

Level Number Mean Std Error Lower 95\% Upper 95\%

$\begin{array}{llllll}1 / 1 & 3 & 0.064560 & 0 & 0.06456 & 0.05456\end{array}$

$\begin{array}{lllllll}1 / 2 & & 3 & 0.064560 & 0 & 0.06456 & 0.06456 \\ 2 / 1 & & 3 & 0.064560 & 0 & 0.06456 & 0.06456\end{array}$

$\begin{array}{lllll}3 & 0.064560 & 0 & 0.06456 & 0.06456\end{array}$

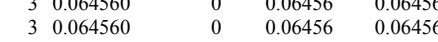

$\begin{array}{llllll}4 / 1 & 3 & 0.064560 & 0 & 0.06456 & 0.06456 \\ 4 / 2 & 3 & 0.064560 & 0 & 0.06456 & 0.06456 \\ 5 / 1 & 3 & 0.064560 & 0 & 0.06456 & 0.06456\end{array}$

$\begin{array}{lllllll}5 / 2 & 3 & 0.064560 & 0 & 0.06456 & 0.06456\end{array}$

Std Error uses a pooled estimate of error variance
Oneway Analysis of $\mathrm{SiO} 2(\mathrm{wt} \%)$ By Block/Sub-Block Glass ID $=$ LRM, Reference Value $=\mathbf{5 4 . 3 7} \mathbf{~ w t} \%$

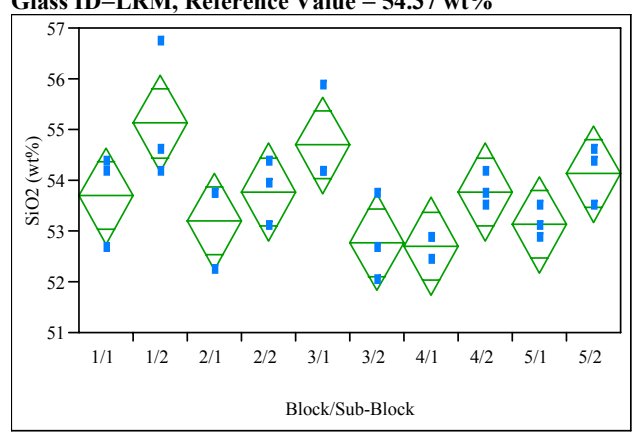

Oneway Anova

Summary of Fit

Rsquare

$\begin{array}{ll}\text { Adj Rsquare } & 0.575569 \\ \text { Root Mean Square En } & 0.384576\end{array}$ Mean of Response $\quad \begin{aligned} & 0.790866 \\ & 53.69643\end{aligned}$

Observations (or Sum Wgts)

Analysis of Variance

$\begin{array}{lrrrrr}\text { Source } & \text { DF } & \text { Sum of Squares } & \text { Mean Square } & \text { F Ratio } & \text { Prob }>\text { F } \\ \text { Block/Sub-Block } & 9 & 16.963947 & 1.88488 & 3.0136 & 0.0191\end{array}$ $\begin{array}{lrrr}\text { Block/Sub-Block } & 9 & 16.963947 & 1.88488 \\ \text { Error } & 20 & 12.509386 & 0.62547 \\ \text { C. Total } & 29 & 29.473333 & \end{array}$

Means for Oneway Anova

$\begin{array}{lrrrrr}\text { Level } & \text { Number } & \text { Mean } & \text { Std Error } & \text { Lower 95\% } & \text { Upper } 95 \% \\ 1 / 1 & 3 & 53.6964 & 0.45661 & 52.744 & 54.649 \\ 1 / 2 & 3 & 55.1226 & 0.45661 & 54.170 & 56.075 \\ 2 / 1 & 3 & 53.1973 & 0.45661 & 52.245 & 54.150 \\ 2 / 2 & 3 & 53.7677 & 0.45661 & 52.815 & 54.720 \\ 3 / 1 & 3 & 54.6948 & 0.45661 & 53.742 & 55.647 \\ 3 / 2 & 3 & 52.7694 & 0.45661 & 51.817 & 53.722 \\ 4 / 1 & 3 & 52.6981 & 0.45661 & 51.746 & 53.651 \\ 4 / 2 & 3 & 53.7677 & 0.45661 & 52.815 & 54.720 \\ 5 / 1 & 3 & 53.1260 & 0.45661 & 52.173 & 54.078 \\ 5 / 2 & 3 & 54.1243 & 0.45661 & 53.172 & 55.077\end{array}$

Std Error uses a pooled estimate of error variance 


\section{Exhibit A5. Measured and Measured Bias-Corrected Oxide Weight Percents by Lab ID for the Samples Prepared Using the} LM Method

Group $=1$, Oxide $=\mathrm{CaO}($ wt $\%)$

Variability Chart for Measured (wt\%)

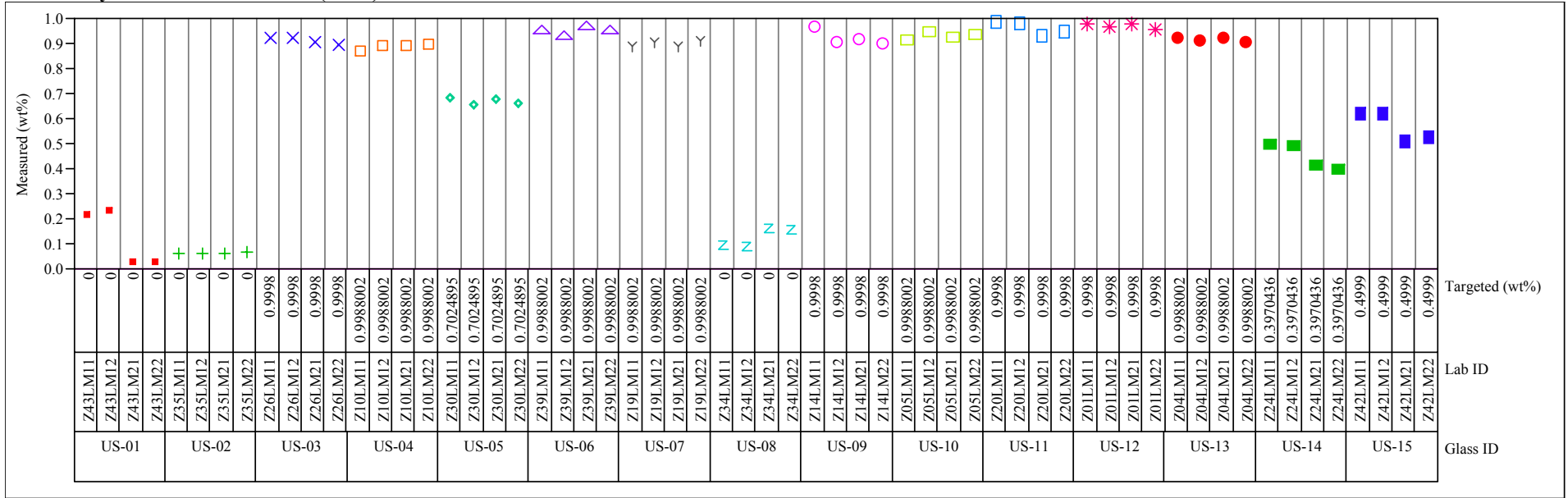

Group $=1$, Oxide $=\mathrm{CaO}($ wt $\%)$

Variability Chart for Measured bias-corrected (wt\%)

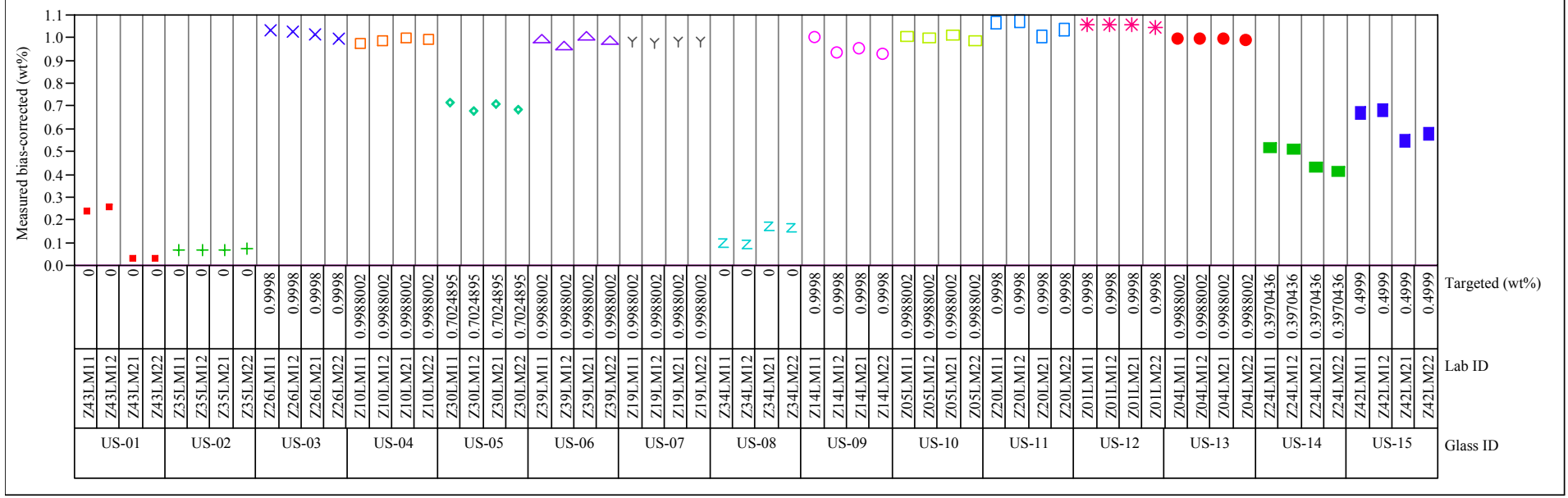


Exhibit A5. Measured and Measured Bias-Corrected Oxide Weight Percents by Lab ID for the Samples Prepared Using the LM Method

Group $=1$, Oxide $=\mathbf{C r} 203(w t \%)$

Variability Chart for Measured (wt\%)

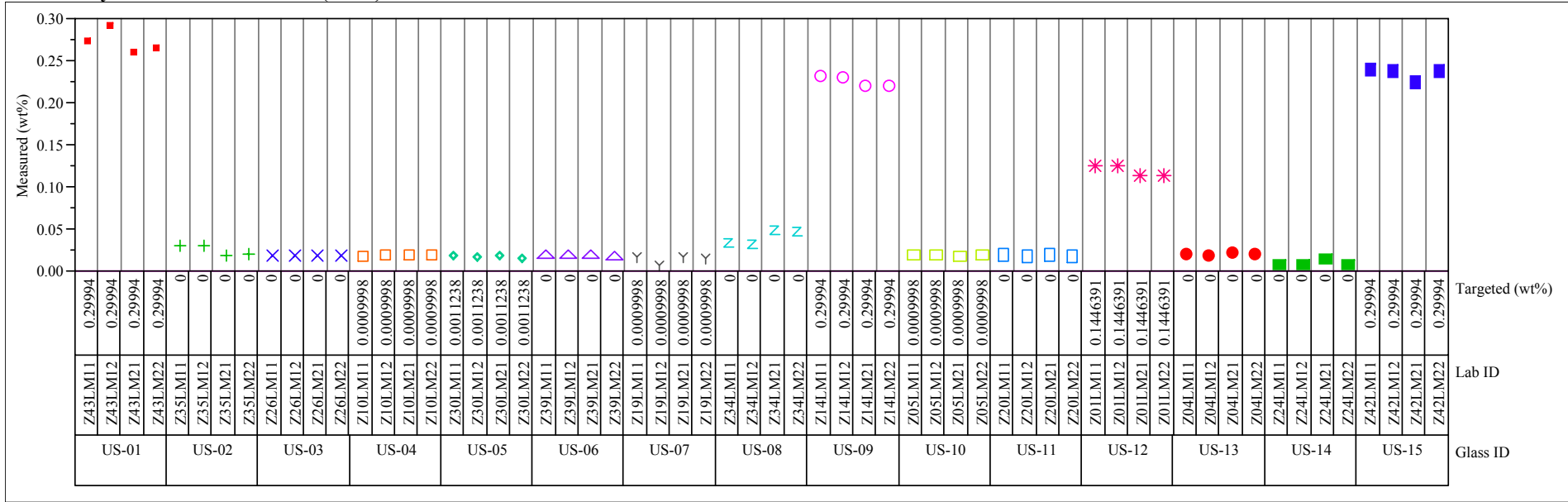

Group $=1$, Oxide $=\mathbf{C r} 203(w t \%)$

Variability Chart for Measured bias-corrected (wt $\%$ )

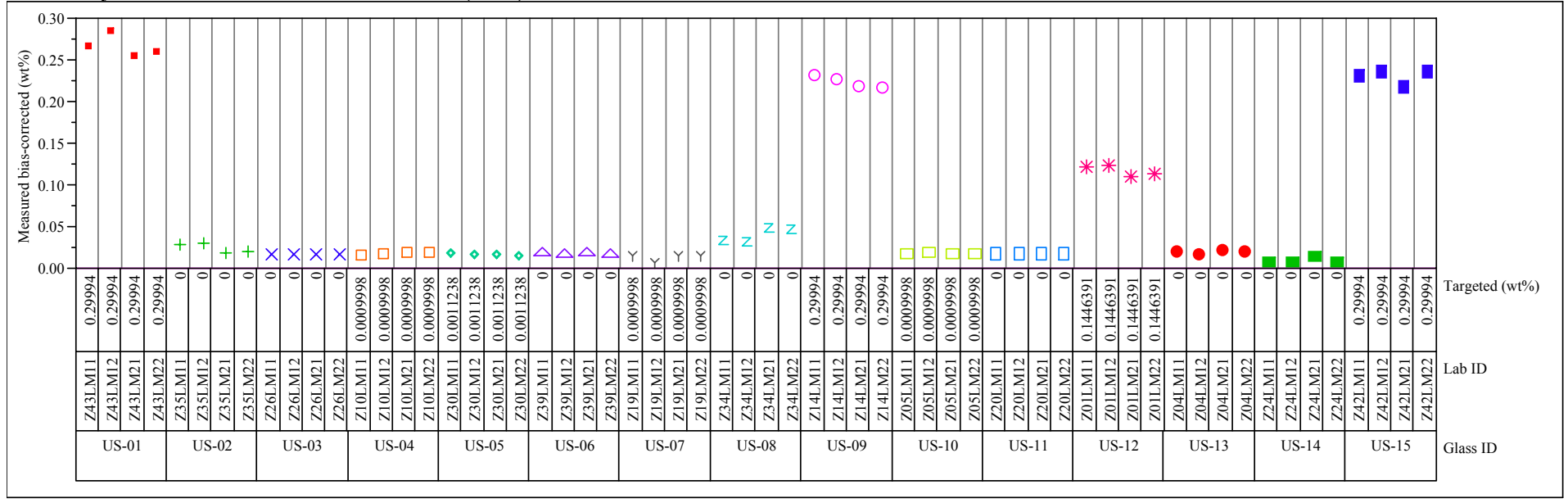


Exhibit A5. Measured and Measured Bias-Corrected Oxide Weight Percents by Lab ID for the Samples Prepared Using the LM Method

Group $=1$, Oxide $=K 20(w t \%)$

Variability Chart for Measured (wt\%)

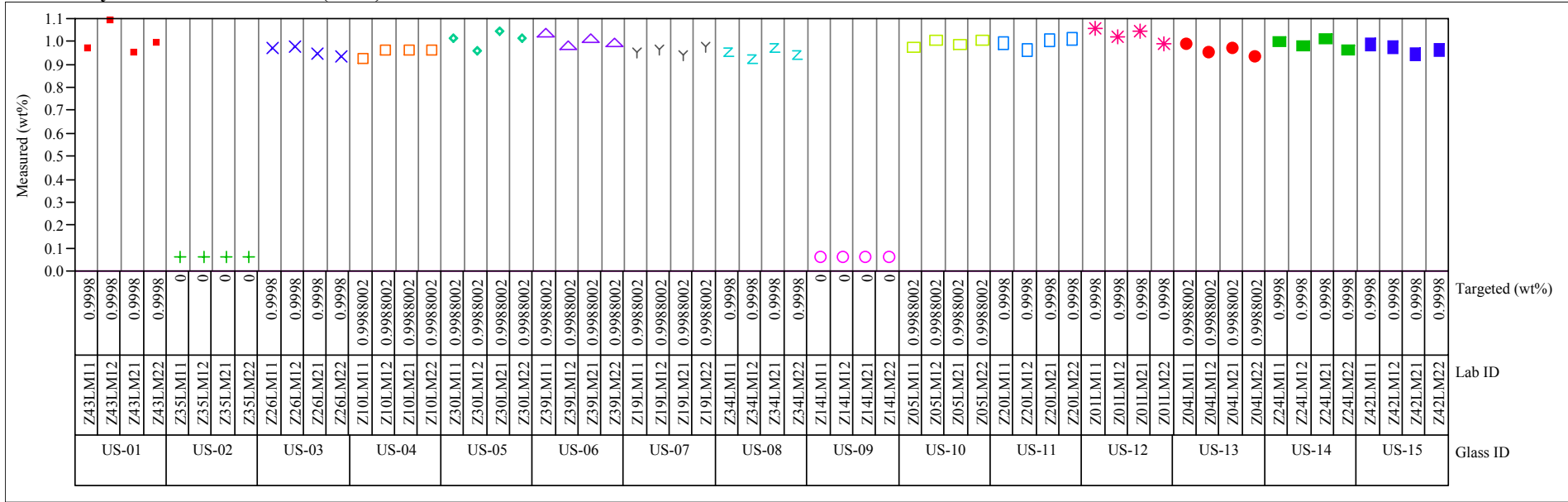

Group $=1$, Oxide $=\mathrm{K} 2 \mathrm{O}(\mathrm{wt} \%)$

Variability Chart for Measured bias-corrected (wt\%)

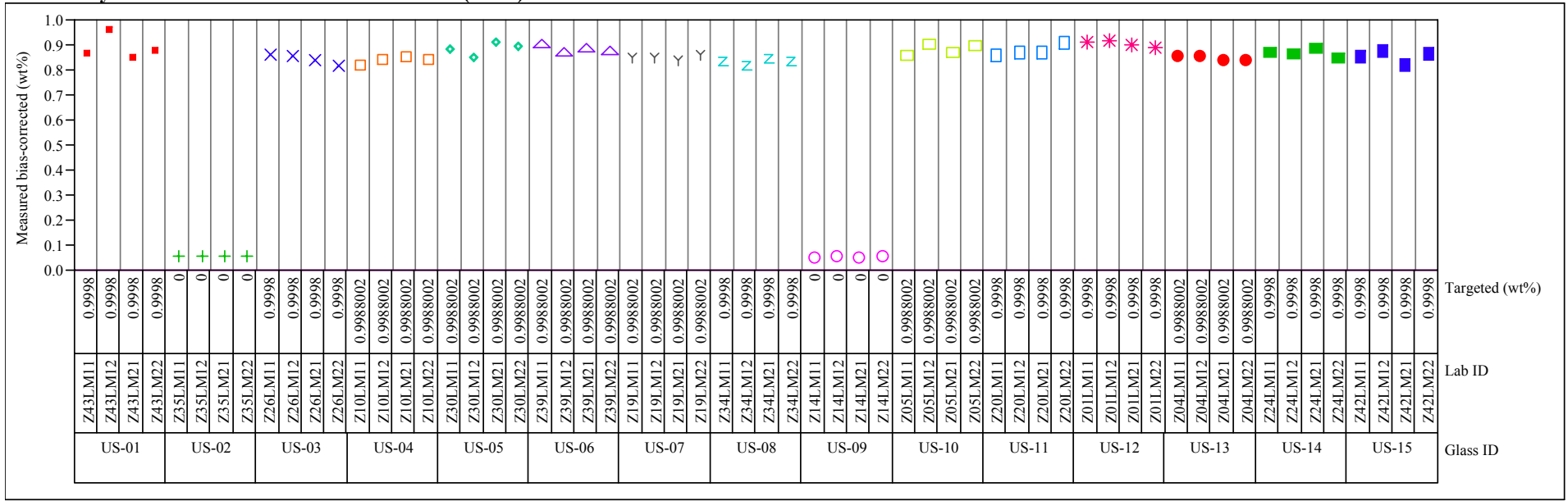


Exhibit A5. Measured and Measured Bias-Corrected Oxide Weight Percents by Lab ID for the Samples Prepared Using the LM Method

Group $=1$, Oxide $=\mathrm{MgO}(\mathrm{wt} \%)$

Variability Chart for Measured (wt\%)

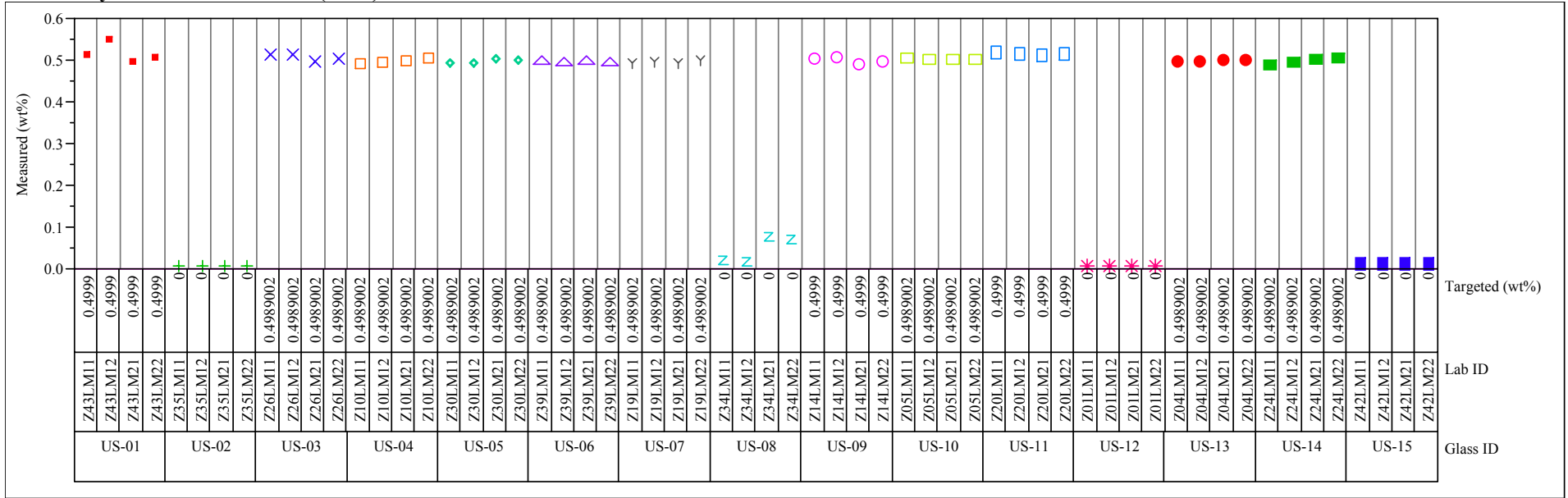

Group $=1$, Oxide $=\operatorname{MgO}(w t \%)$

Variability Chart for Measured bias-corrected (wt\%)

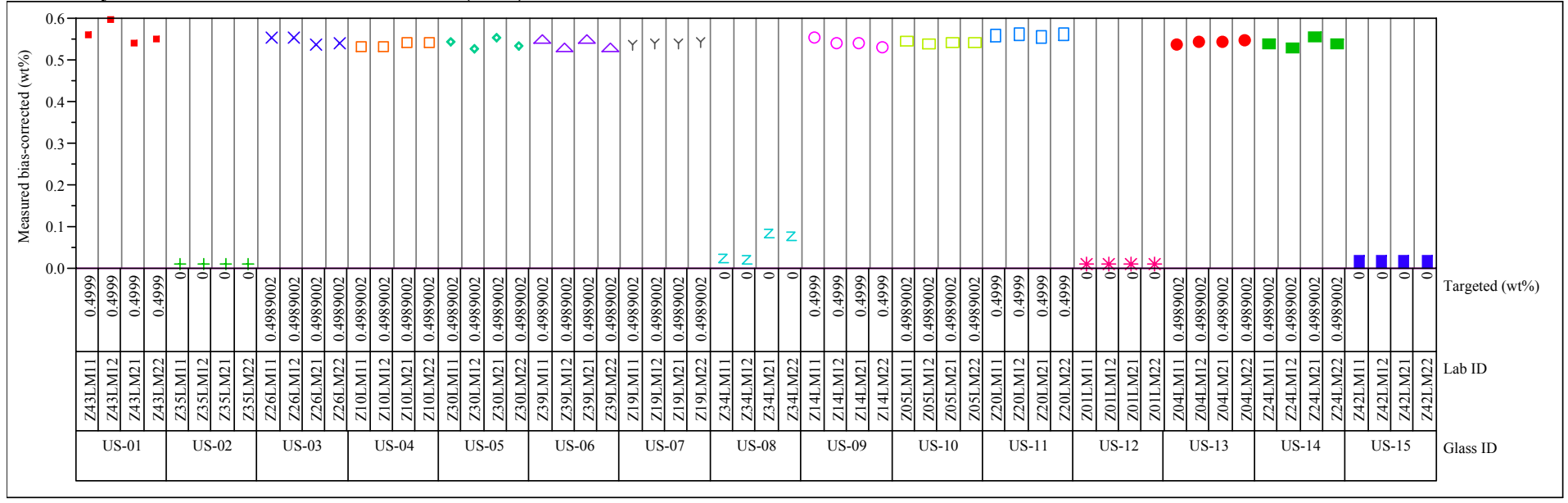




\section{Exhibit A5. Measured and Measured Bias-Corrected Oxide Weight Percents by Lab ID for the Samples Prepared Using the} LM Method

Group $=1$, Oxide $=\mathrm{Na2O}(\mathrm{wt} \%)$

Variability Chart for Measured (wt\%)

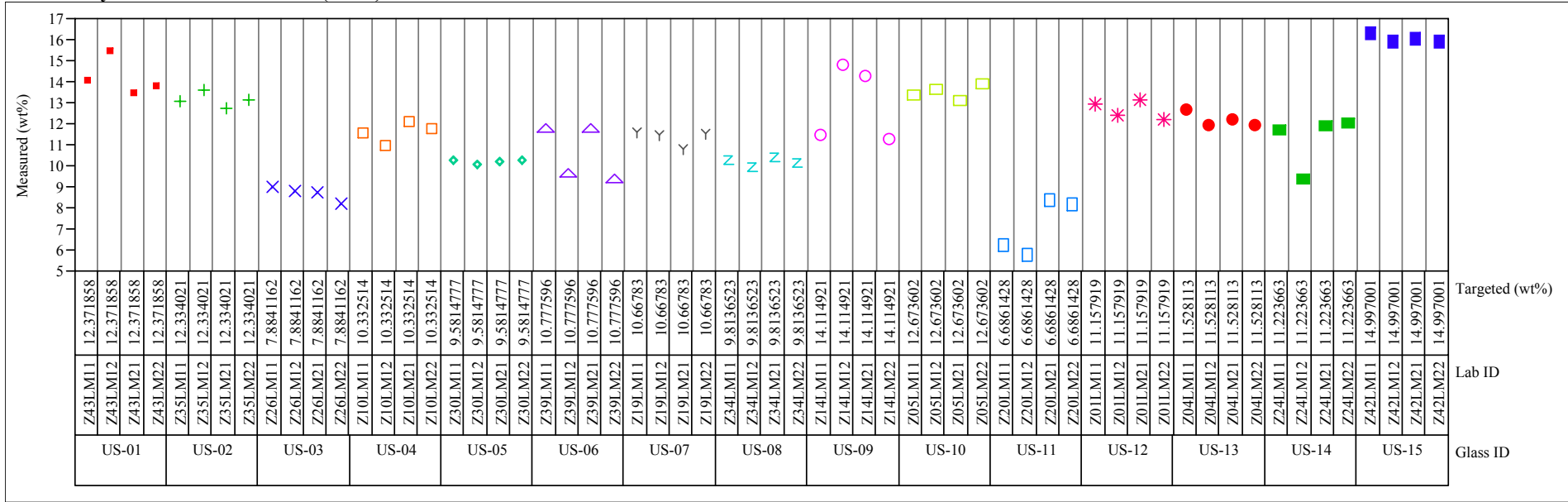

Group $=1$, Oxide $=\mathrm{Na2O}(\mathrm{wt} \%)$

Variability Chart for Measured bias-corrected (wt $\%$ )

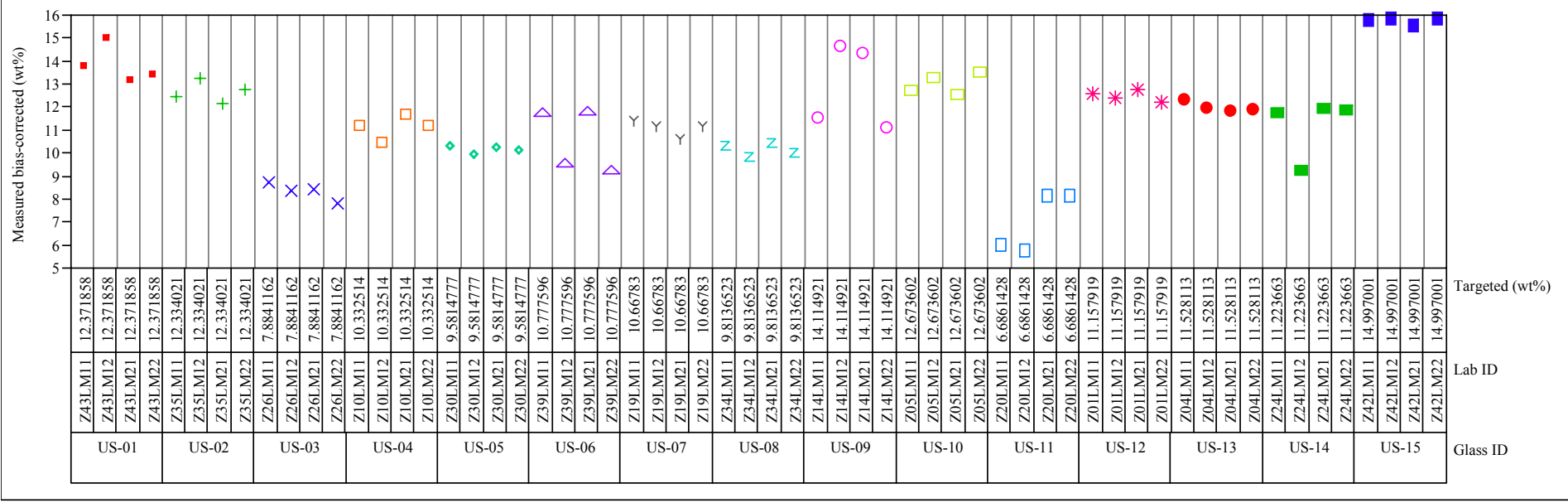


Exhibit A5. Measured and Measured Bias-Corrected Oxide Weight Percents by Lab ID for the Samples Prepared Using the LM Method

Group $=1$, Oxide $=\mathrm{NiO}(\mathrm{wt} \%)$

Variability Chart for Measured (wt\%)

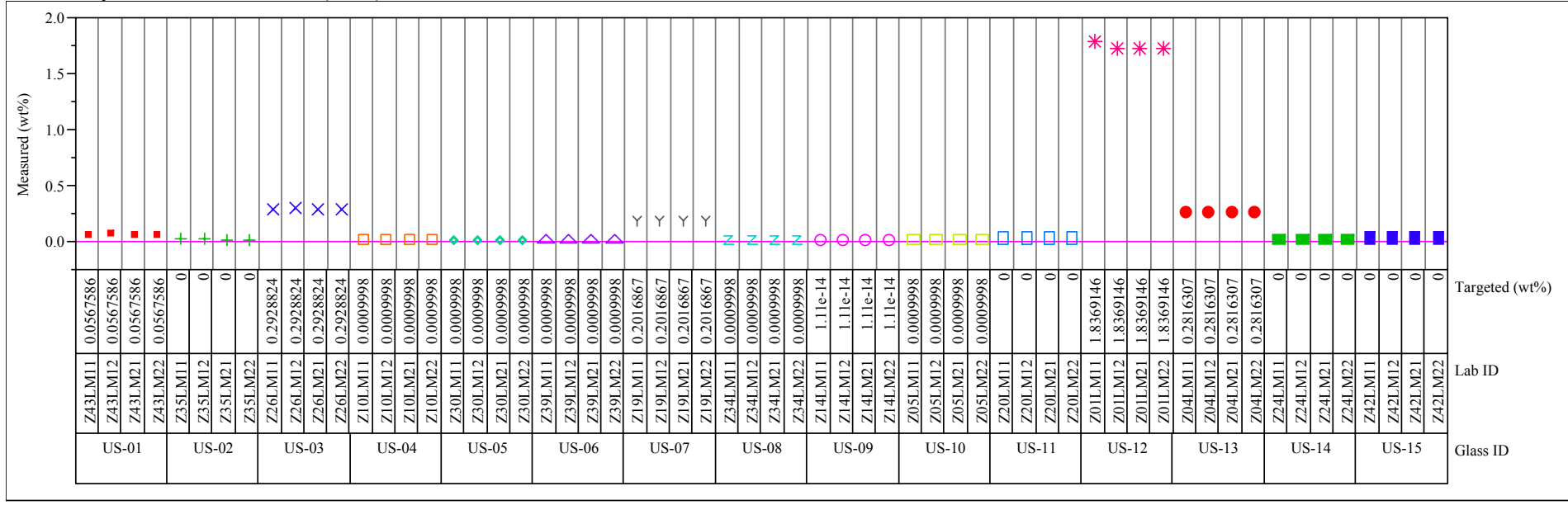

Group $=1$, Oxide $=\mathrm{NiO}(\mathrm{wt} \%)$

Variability Chart for Measured bias-corrected (wt\%)

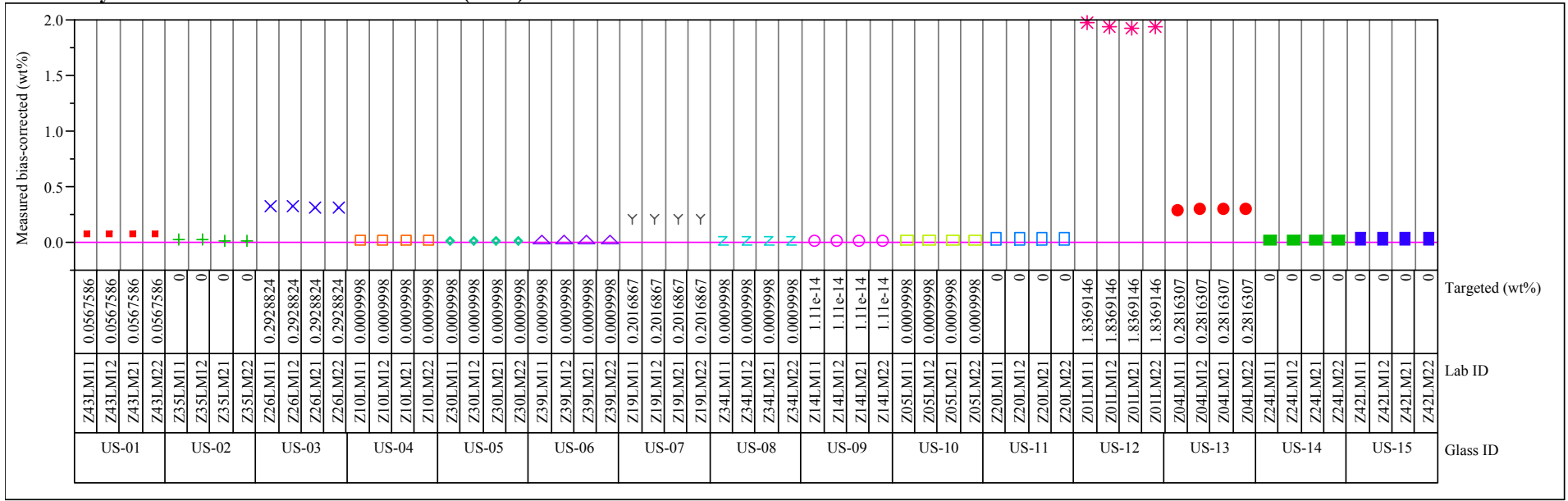


Exhibit A5. Measured and Measured Bias-Corrected Oxide Weight Percents by Lab ID for the Samples Prepared Using the LM Method

Group $=1$, Oxide $=\mathbf{P b O}($ wt $\%)$

Variability Chart for Measured (wt\%)

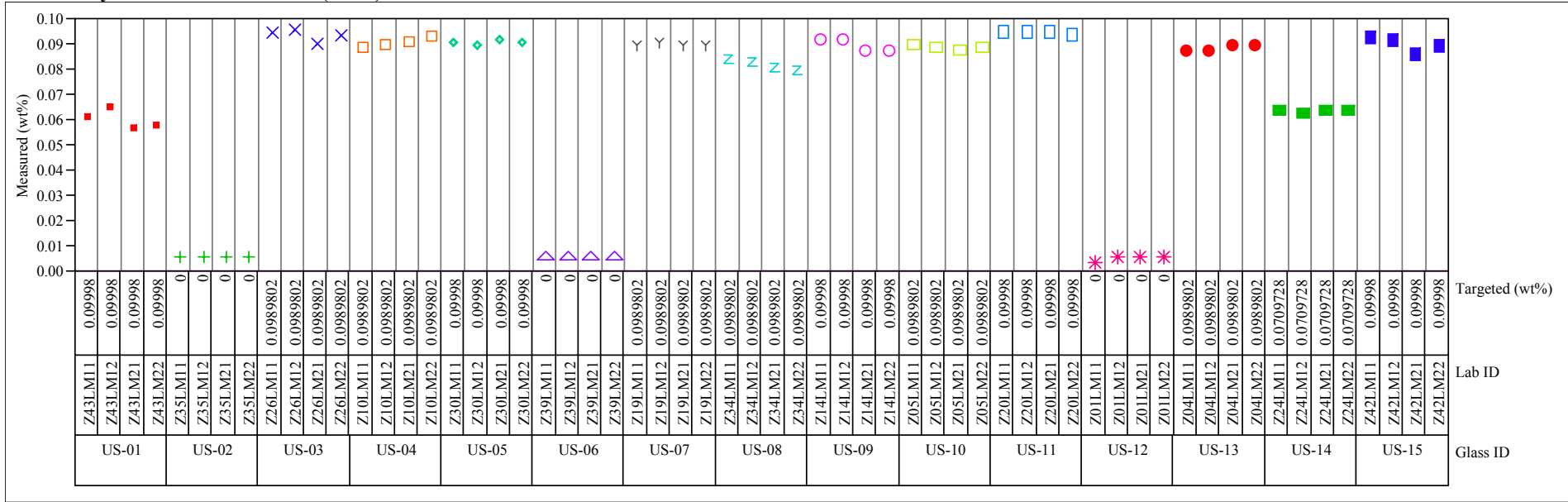

Group $=1$, Oxide $=\operatorname{PbO}($ wt $\%)$

Variability Chart for Measured bias-corrected (wt\%)

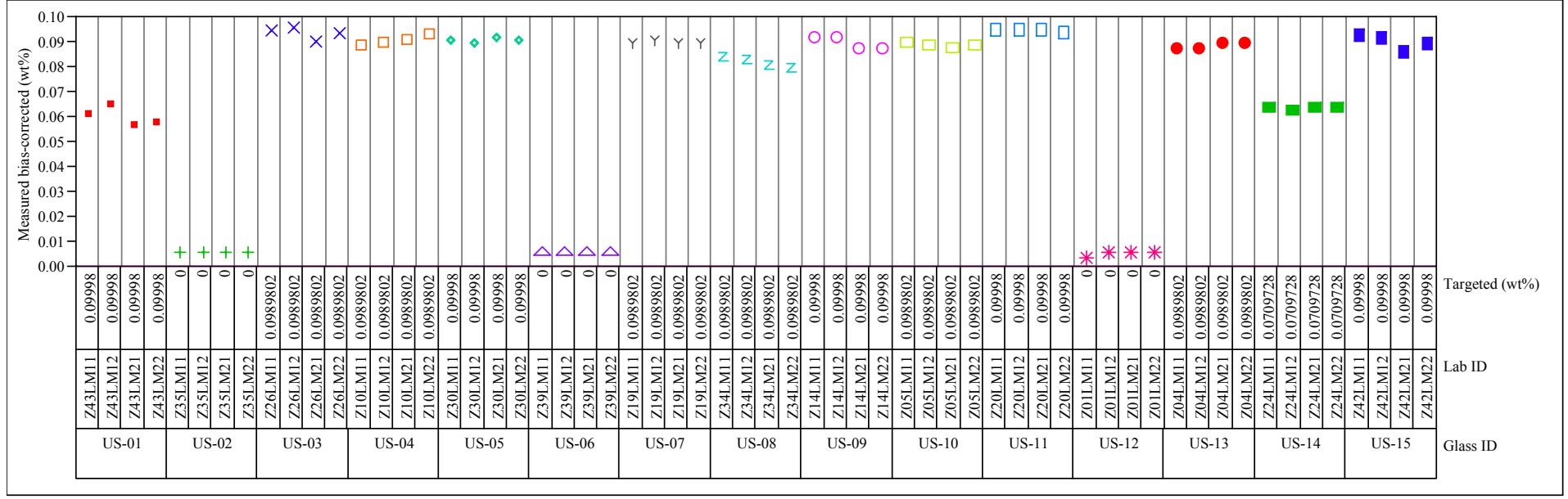


Exhibit A5. Measured and Measured Bias-Corrected Oxide Weight Percents by Lab ID for the Samples Prepared Using the LM Method

Group $=1$, Oxide $=\mathrm{SO3}(\mathrm{wt} \%)$

Variability Chart for Measured (wt\%)

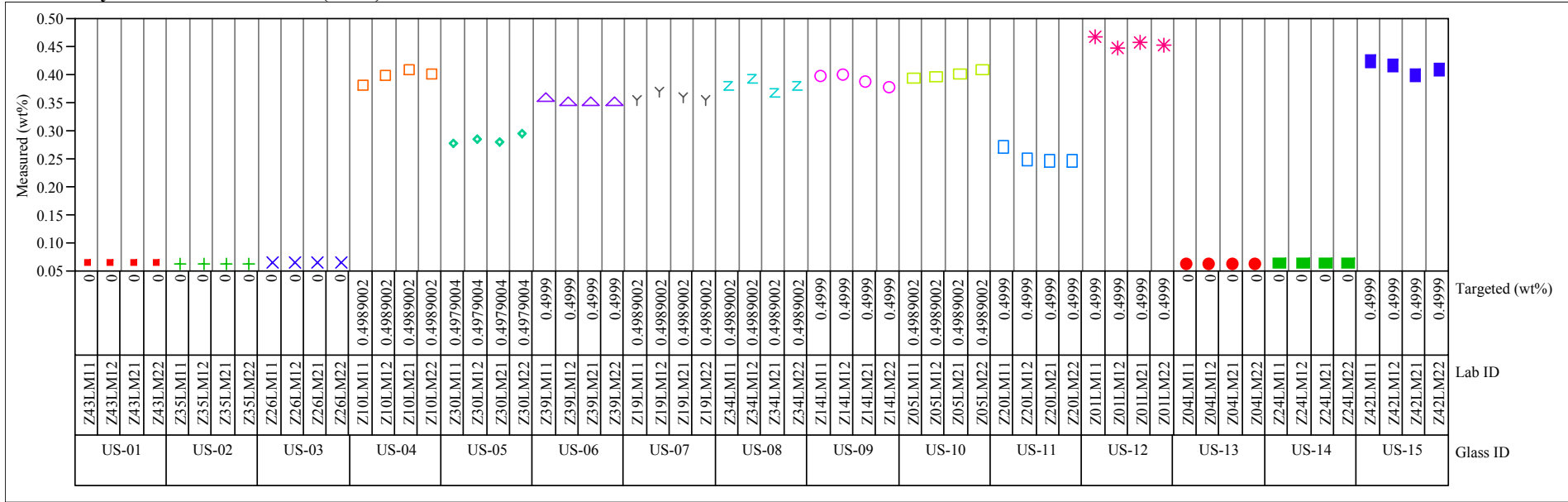

Group $=1$, Oxide $=\mathrm{SO3}(\mathrm{wt} \%)$

Variability Chart for Measured bias-corrected ( $\mathbf{w t} \%)$

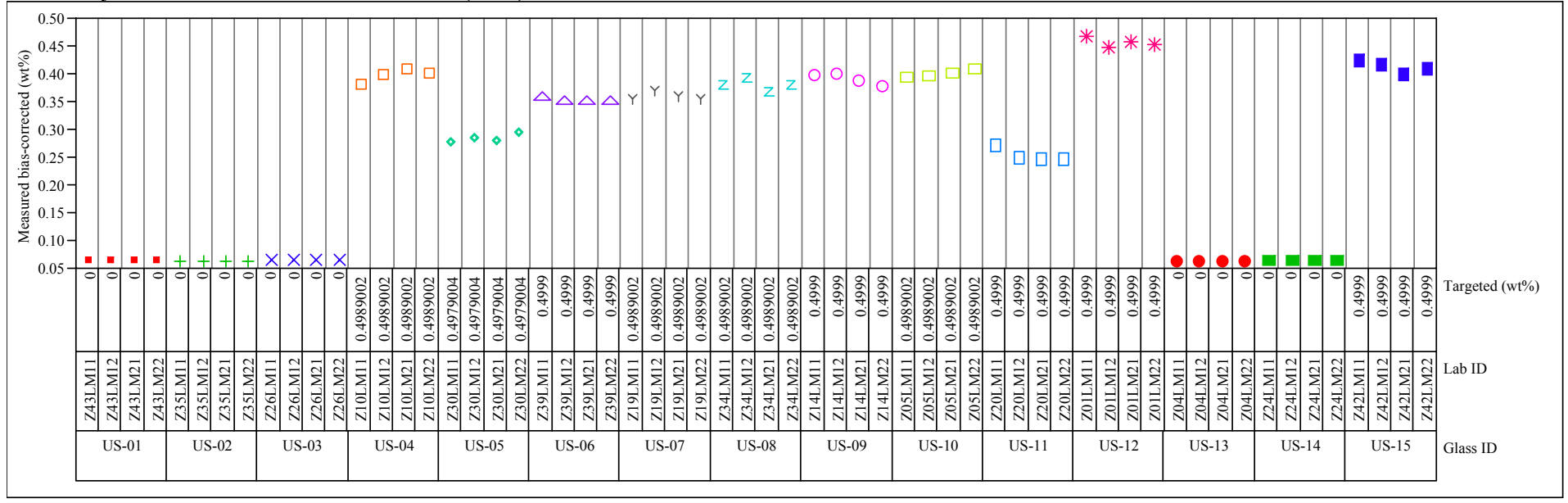


Exhibit A5. Measured and Measured Bias-Corrected Oxide Weight Percents by Lab ID for the Samples Prepared Using the LM Method

Group $=1$, Oxide $=\operatorname{SrO}($ wt $\%)$

Variability Chart for Measured (wt\%)

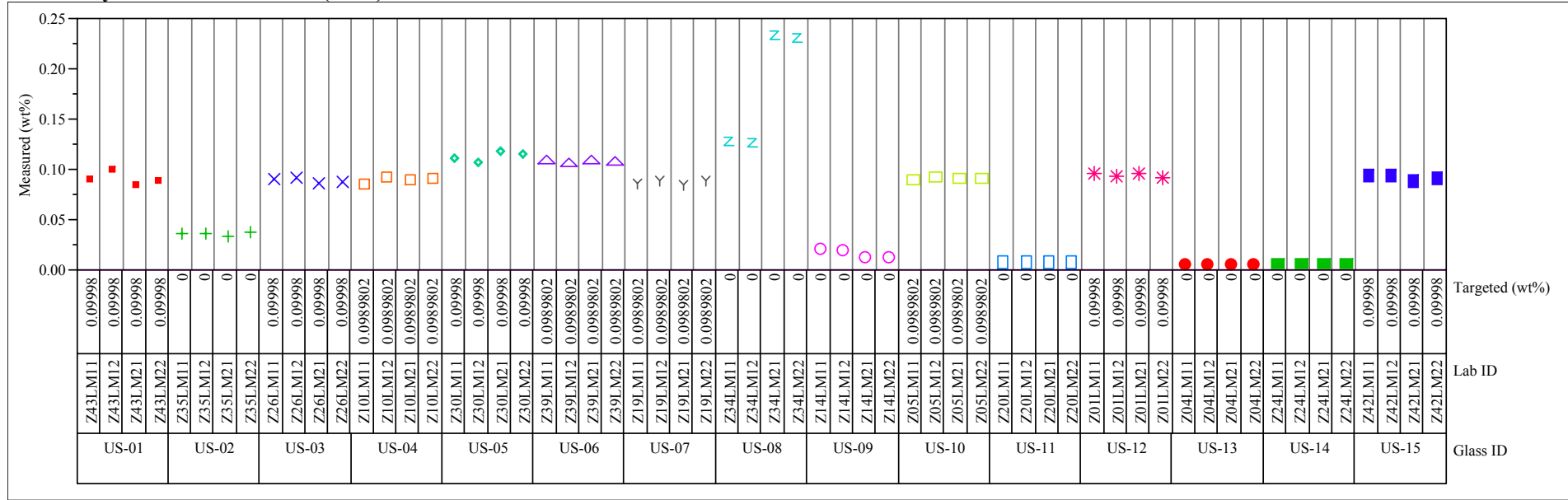

Group $=1$, Oxide $=\operatorname{SrO}($ wt $\%)$

Variability Chart for Measured bias-corrected ( $\mathbf{w t} \%$ )

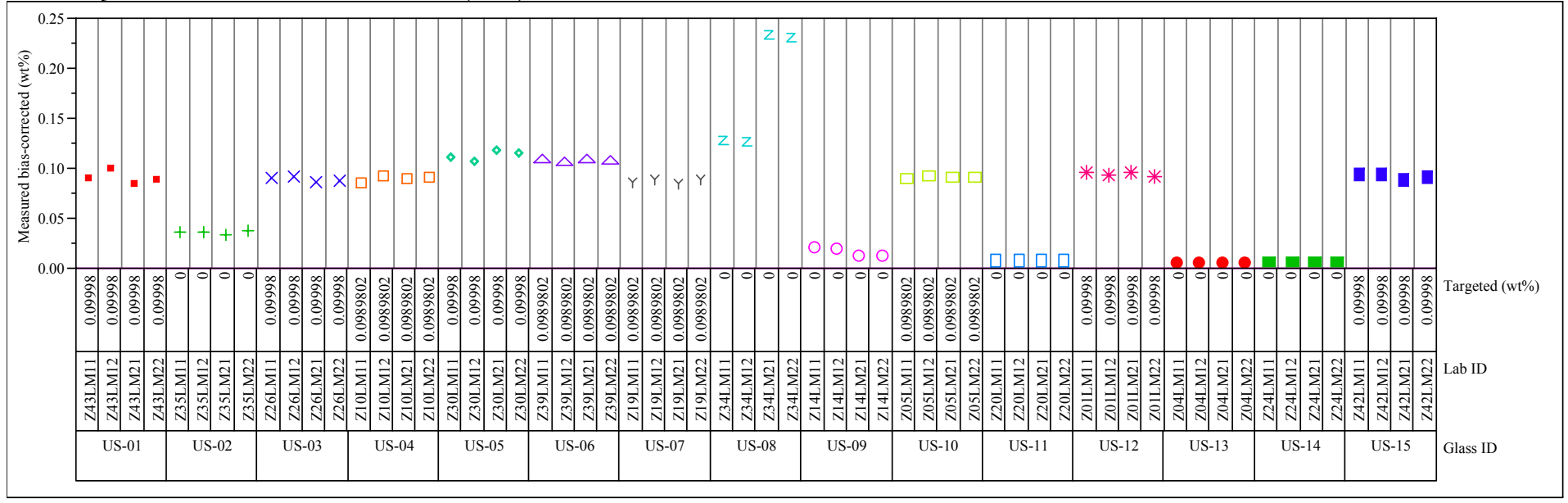




\section{Exhibit A5. Measured and Measured Bias-Corrected Oxide Weight Percents by Lab ID for the Samples Prepared Using the} LM Method

Group $=1$, Oxide $=\mathrm{TiO} 2(\mathrm{wt} \%)$

Variability Chart for Measured (wt\%)

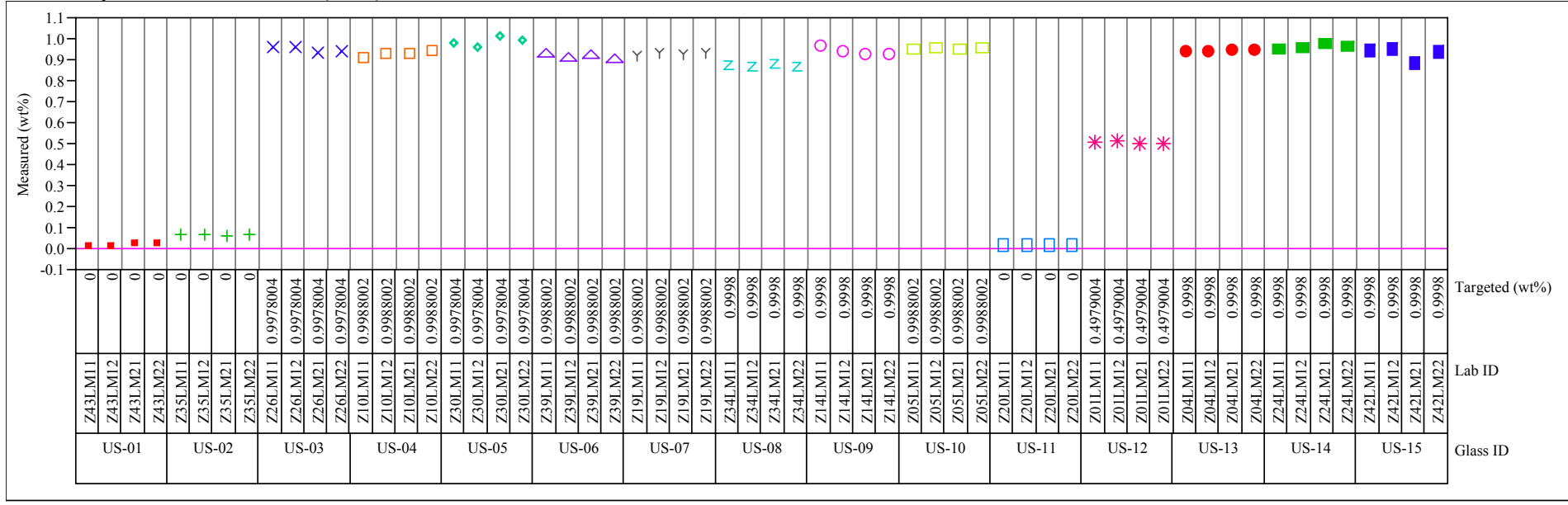

Group $=1$, Oxide $=\mathrm{TiO} 2($ wt $\%)$

Variability Chart for Measured bias-corrected (wt\%)

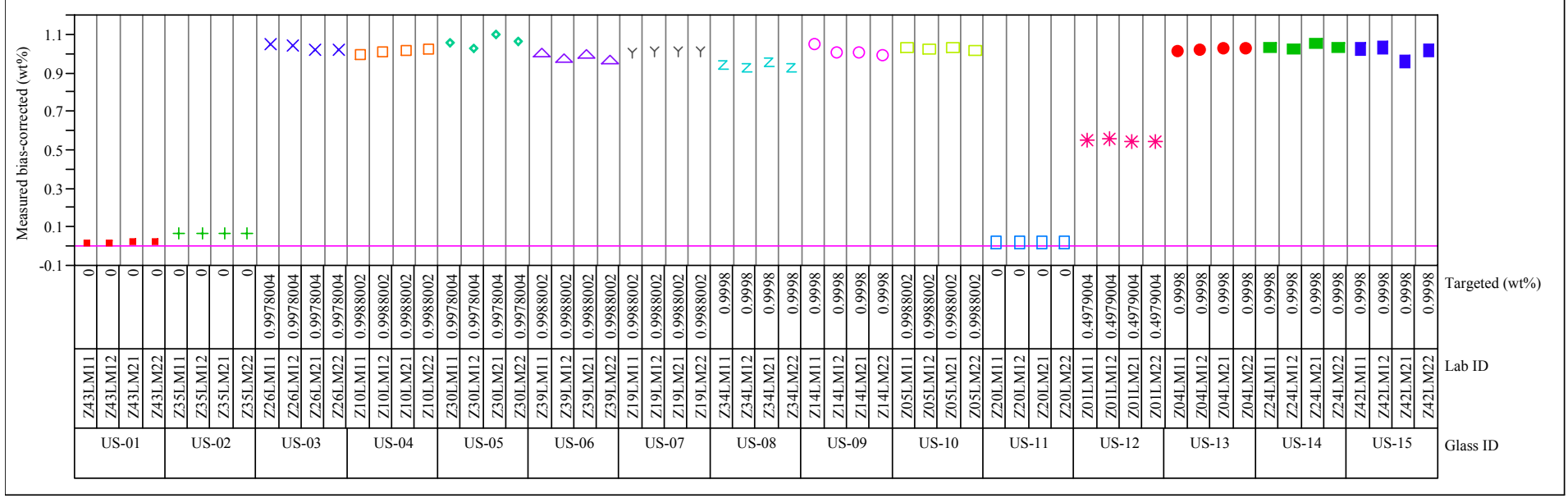


Exhibit A5. Measured and Measured Bias-Corrected Oxide Weight Percents by Lab ID for the Samples Prepared Using the LM Method

Group $=1$, Oxide $=\mathrm{ZnO}($ wt $\%)$

Variability Chart for Measured (wt\%)

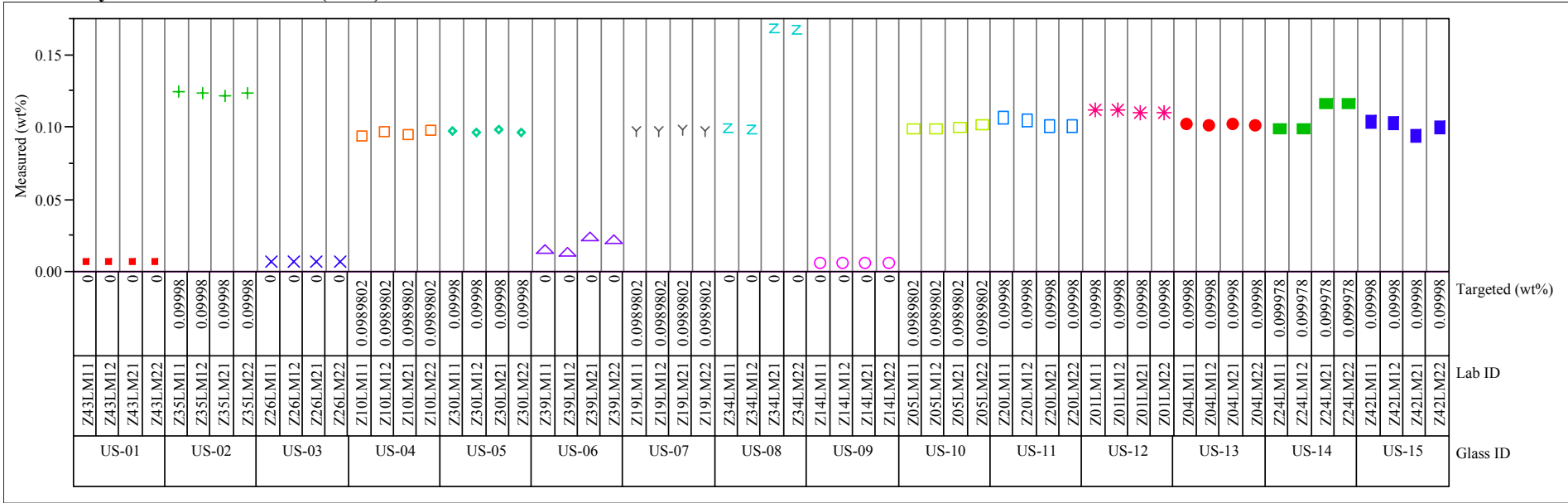

Group $=1$, Oxide $=\mathrm{ZnO}($ wt $\%)$

Variability Chart for Measured bias-corrected (wt\%)

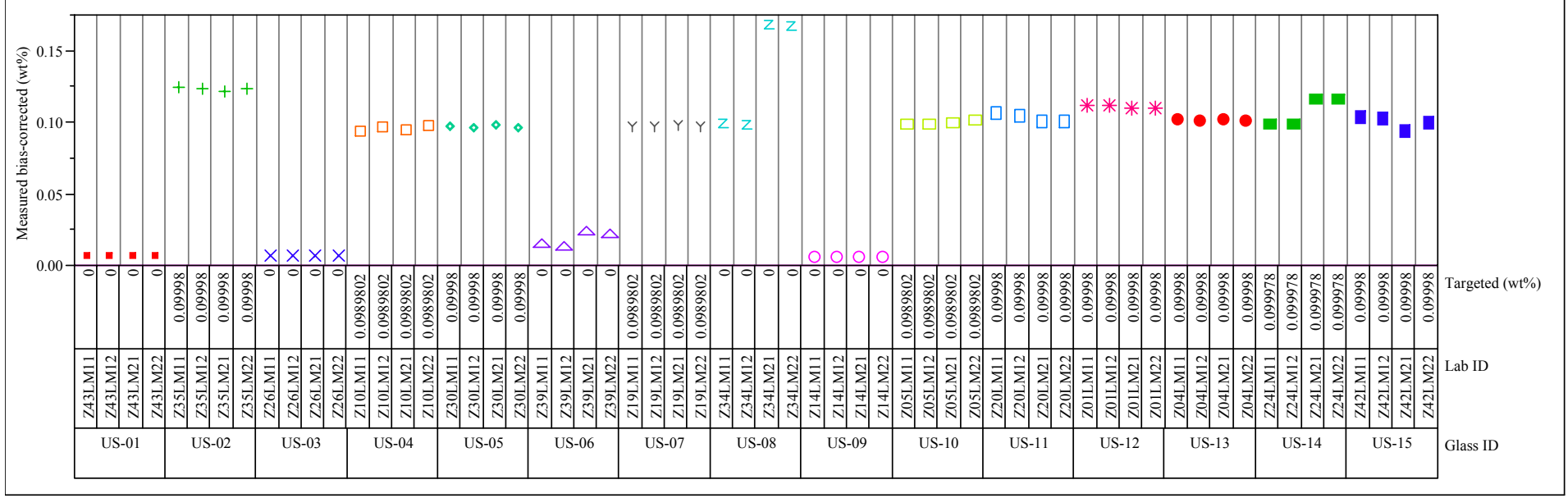


Exhibit A5. Measured and Measured Bias-Corrected Oxide Weight Percents by Lab ID for the Samples Prepared Using the LM Method

Group $=1$, Oxide $=\mathrm{ZrO} 2(\mathbf{w t} \%)$

Variability Chart for Measured (wt\%)

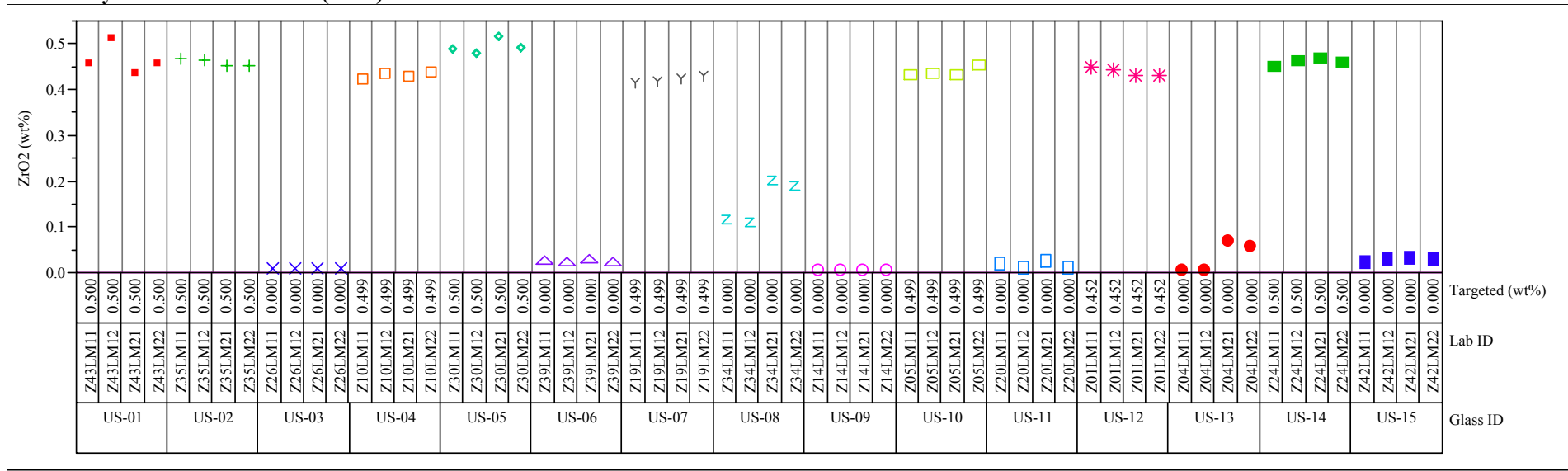

Group=1, Oxide $=\mathrm{ZrO2}(\mathrm{wt} \%)$

Variability Chart for Measured bias-corrected (wt\%)

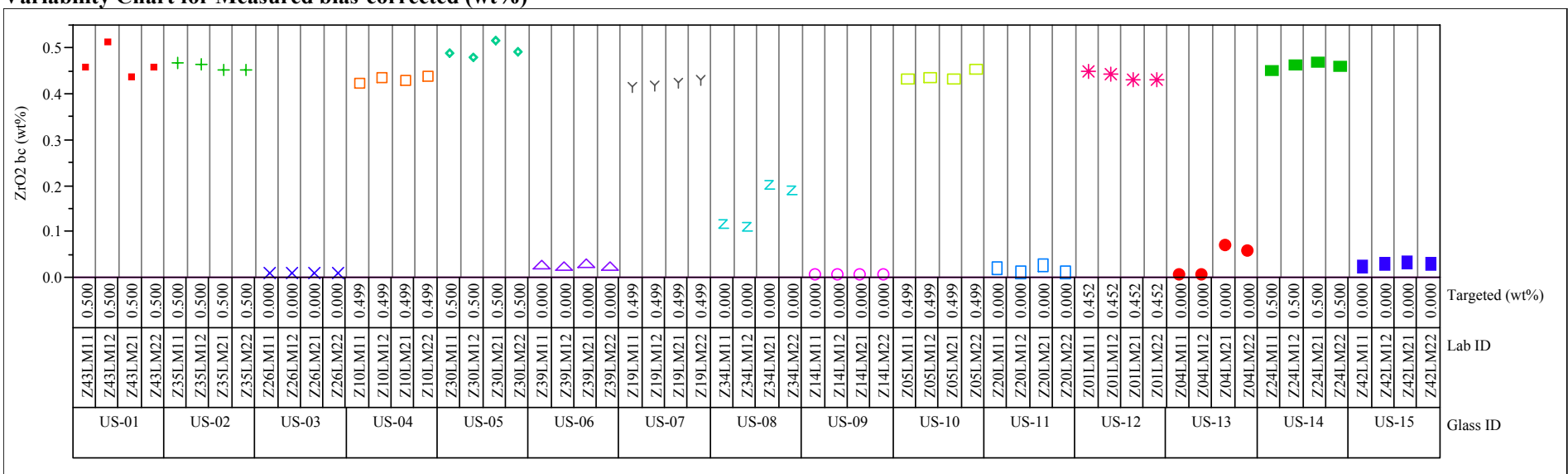


Exhibit A5. Measured and Measured Bias-Corrected Oxide Weight Percents by Lab ID for the Samples Prepared Using the LM Method

Group $=2$, Oxide $=\mathrm{CaO}(\mathrm{wt} \%)$

Variability Chart for Measured (wt\%)

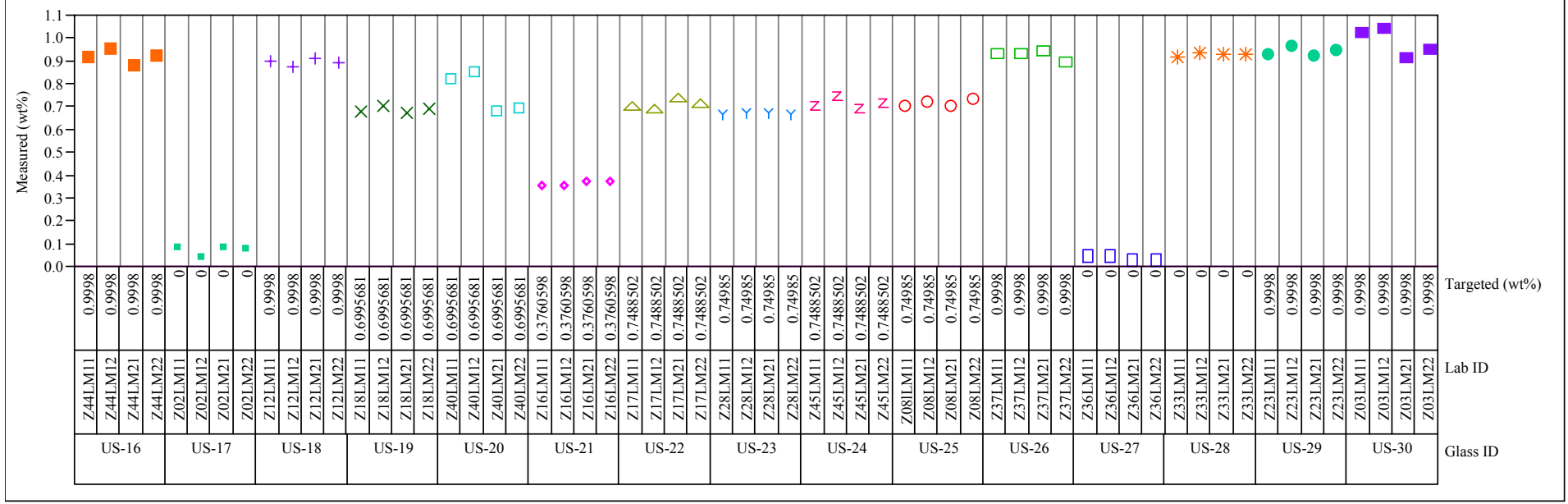

Group $=2$, Oxide $=\mathrm{CaO}(\mathrm{wt} \%)$

Variability Chart for Measured bias-corrected (wt\%)

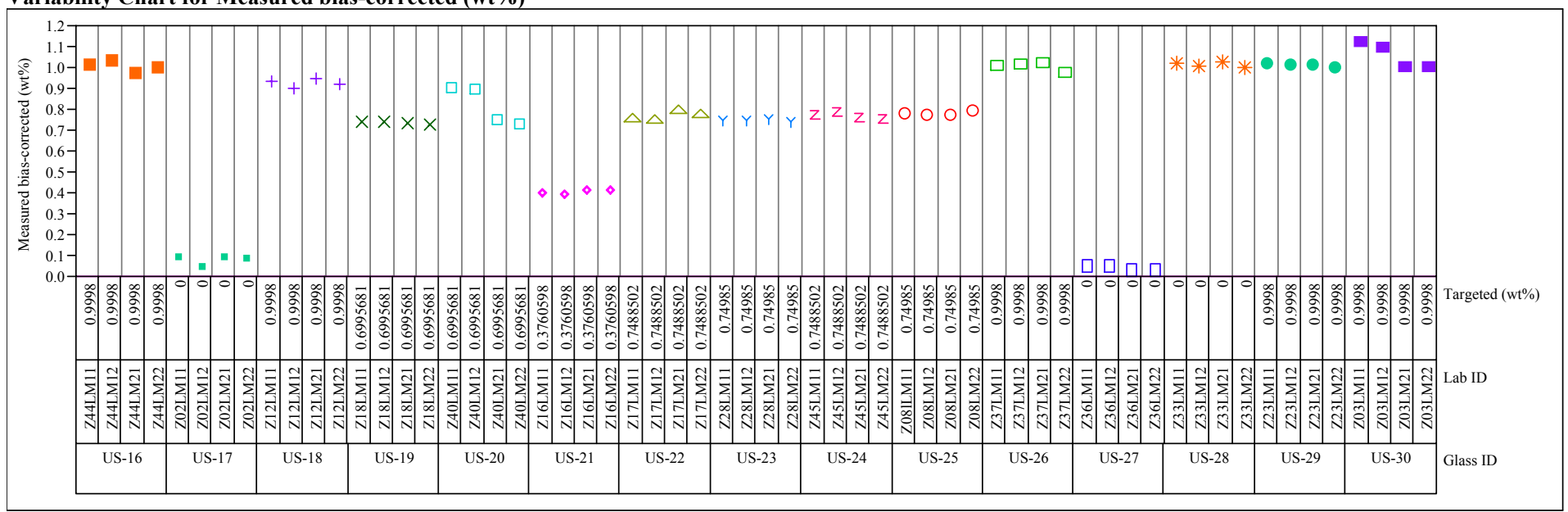




\section{Exhibit A5. Measured and Measured Bias-Corrected Oxide Weight Percents by Lab ID for the Samples Prepared Using the} LM Method

Group $=2$, Oxide $=\mathbf{C r} 203(w t \%)$

Variability Chart for Measured (wt\%)

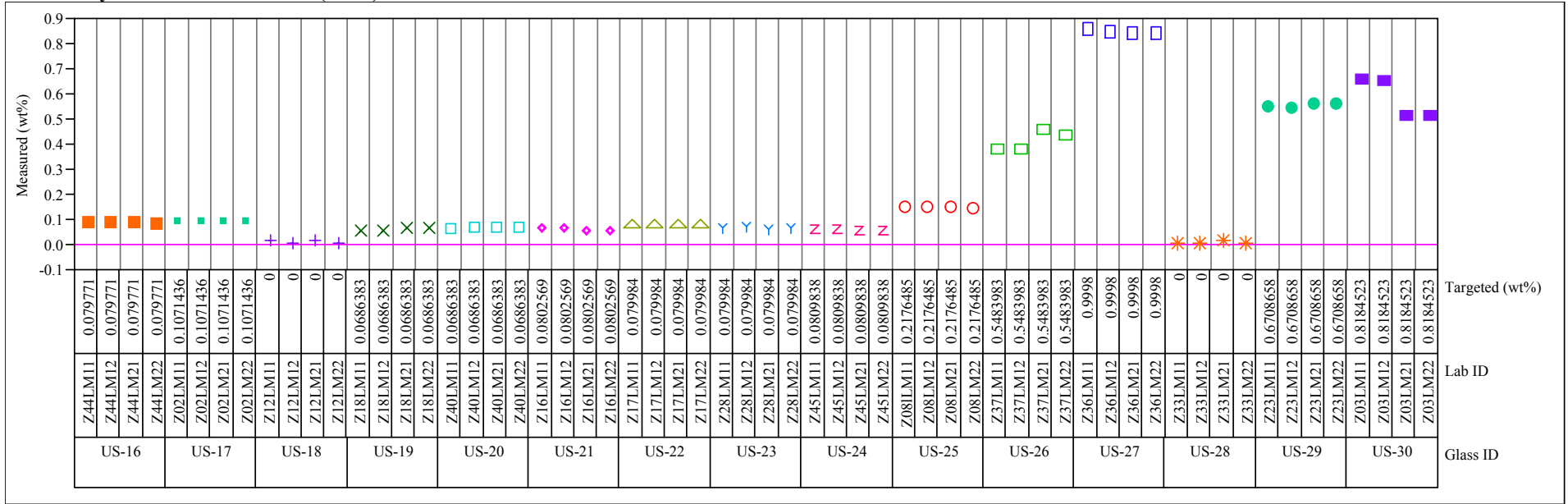

Group $=2$, Oxide $=\operatorname{Cr2O3}($ wt $\%)$

Variability Chart for Measured bias-corrected (wt\%)

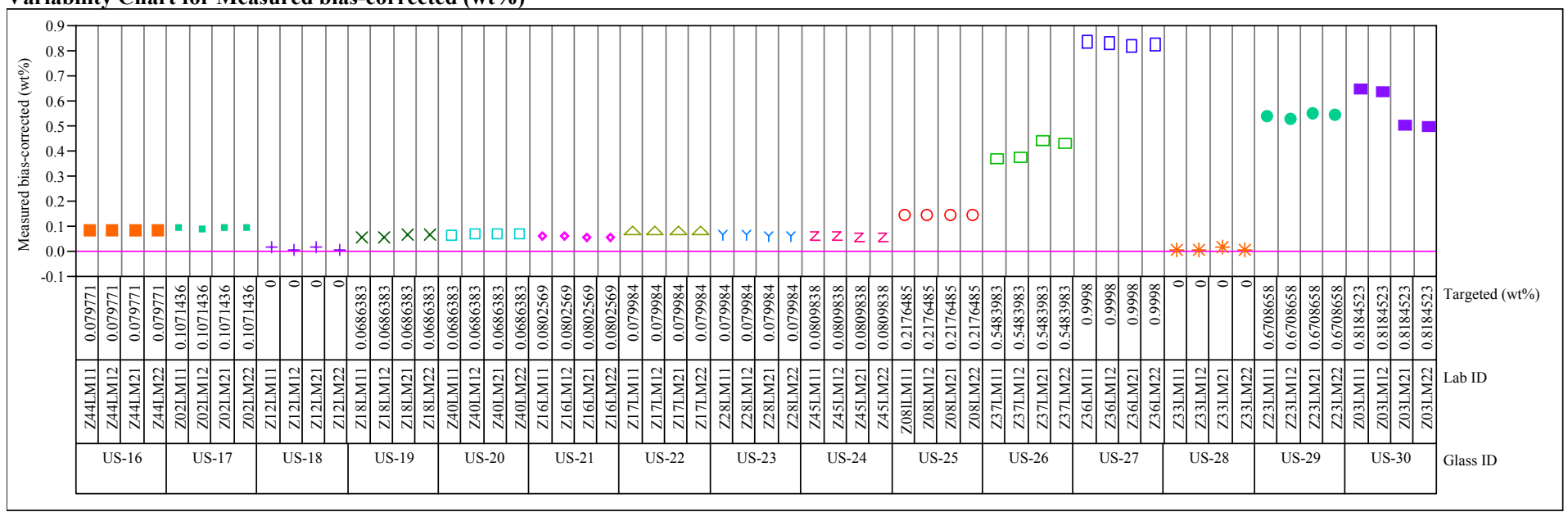


Exhibit A5. Measured and Measured Bias-Corrected Oxide Weight Percents by Lab ID for the Samples Prepared Using the LM Method

Group $=2$, Oxide $=\mathbf{K} 20($ wt $\%)$

Variability Chart for Measured (wt\%)

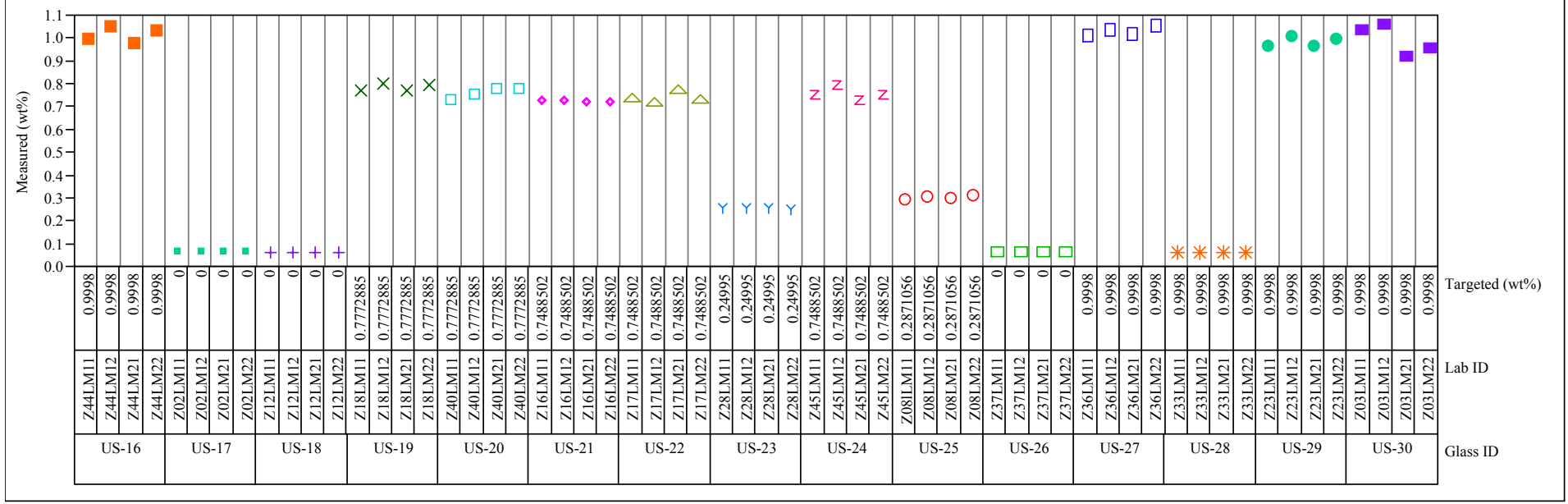

Group $=2$, Oxide $=K 20(w t \%)$

Variability Chart for Measured bias-corrected (wt\%)

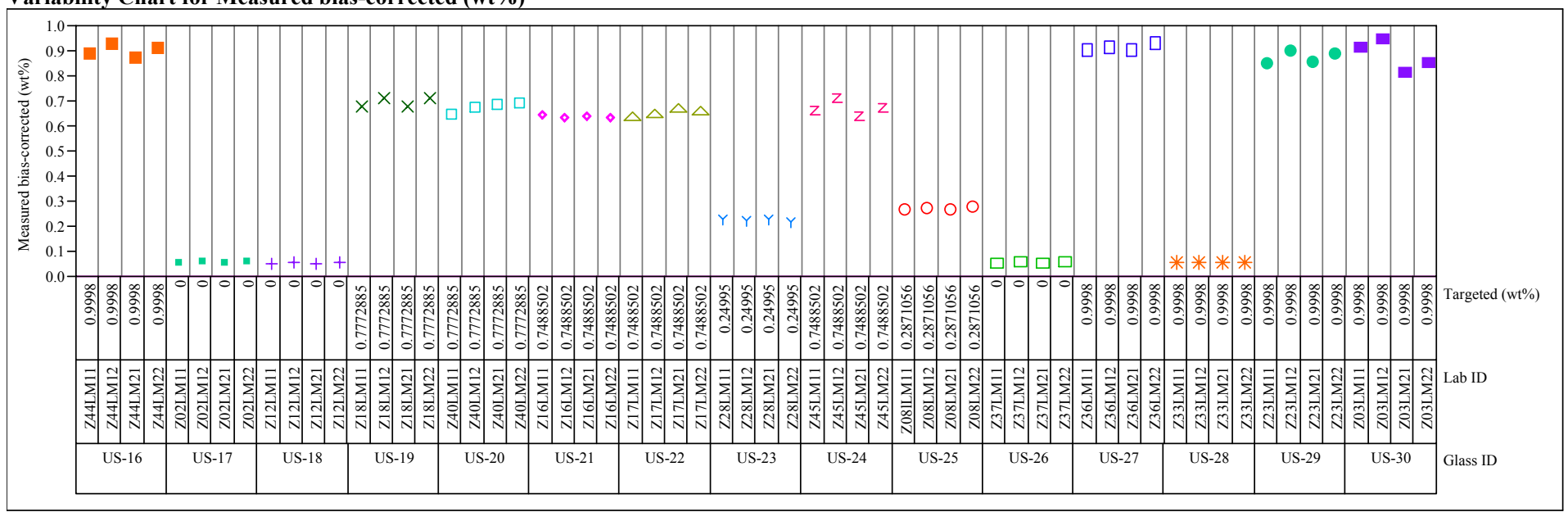




\section{Exhibit A5. Measured and Measured Bias-Corrected Oxide Weight Percents by Lab ID for the Samples Prepared Using the} LM Method

Group $=2$, Oxide $=\mathrm{MgO}(\mathrm{wt} \%)$

Variability Chart for Measured (wt\%)

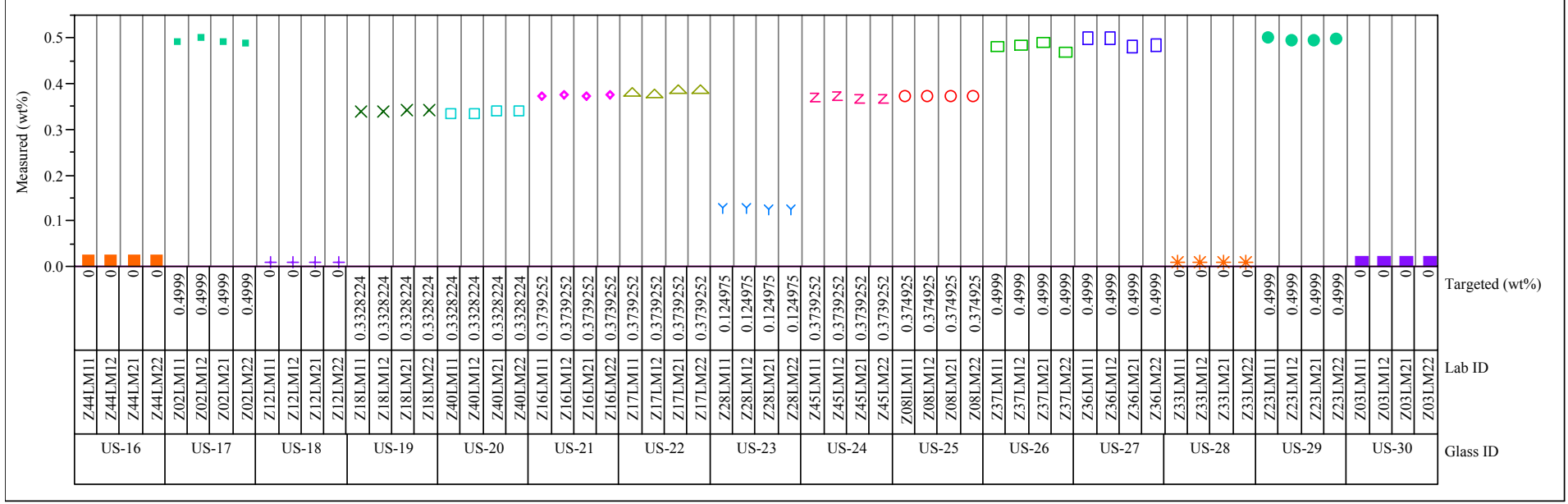

Group $=2$, Oxide $=\operatorname{MgO}(\mathbf{w t} \%)$

Variability Chart for Measured bias-corrected (wt\%)

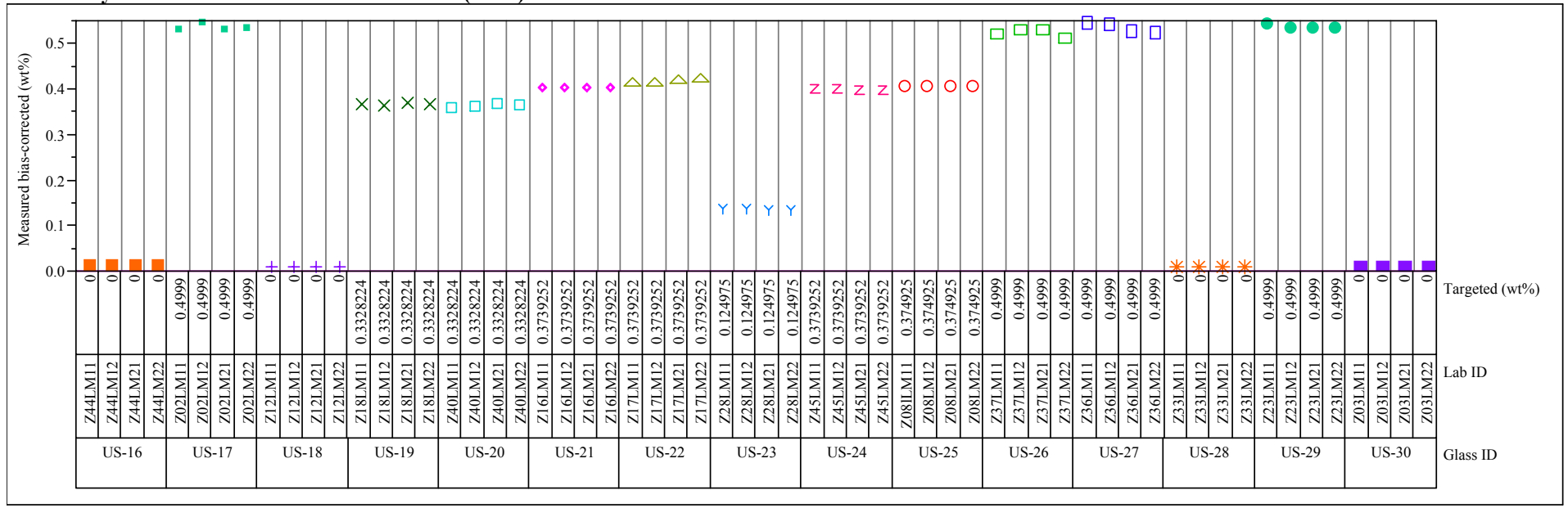




\section{Exhibit A5. Measured and Measured Bias-Corrected Oxide Weight Percents by Lab ID for the Samples Prepared Using the} LM Method

Group $=2$, Oxide $=\mathrm{Na2O}(\mathrm{wt} \%)$

Variability Chart for Measured (wt\%)

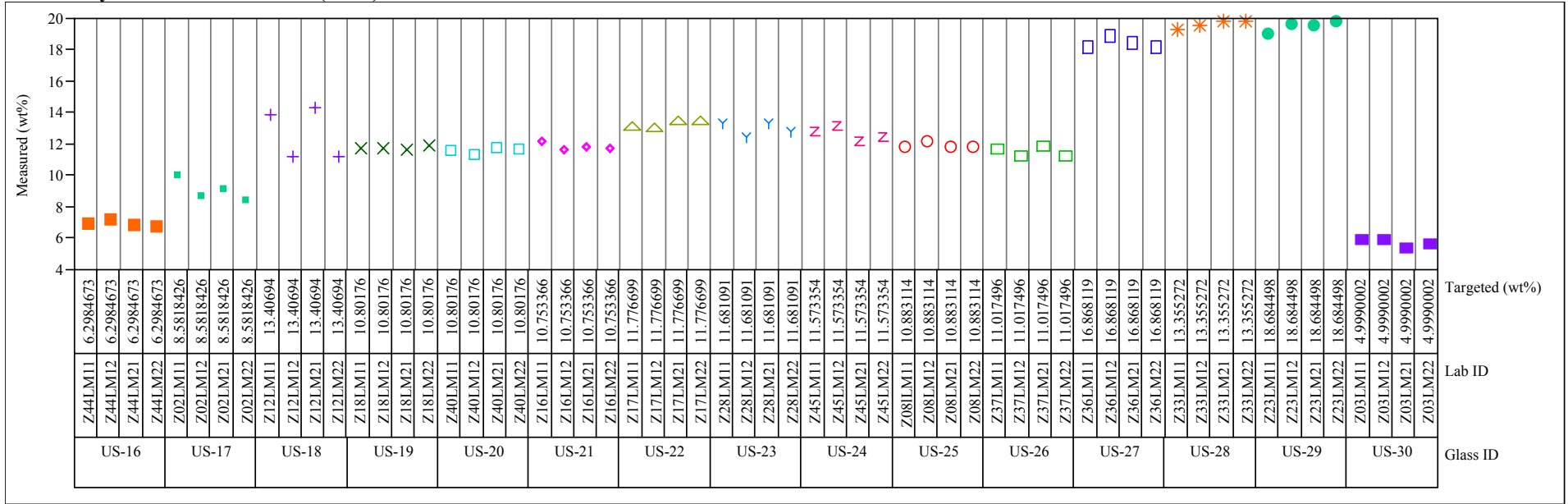

Group $=2$, Oxide $=\mathrm{Na2O}(\mathrm{wt} \%)$

Variability Chart for Measured bias-corrected (wt\%)

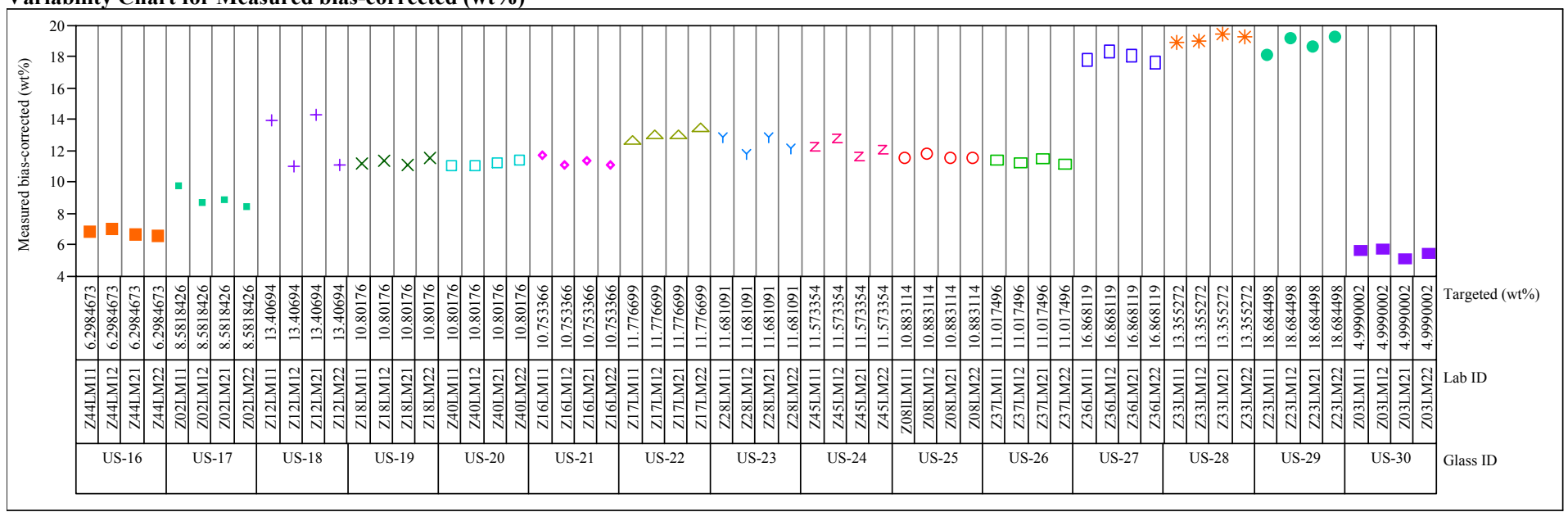




\section{Exhibit A5. Measured and Measured Bias-Corrected Oxide Weight Percents by Lab ID for the Samples Prepared Using the} LM Method

Group $=2$, Oxide $=\mathrm{NiO}($ wt $\%)$

Variability Chart for Measured (wt\%)

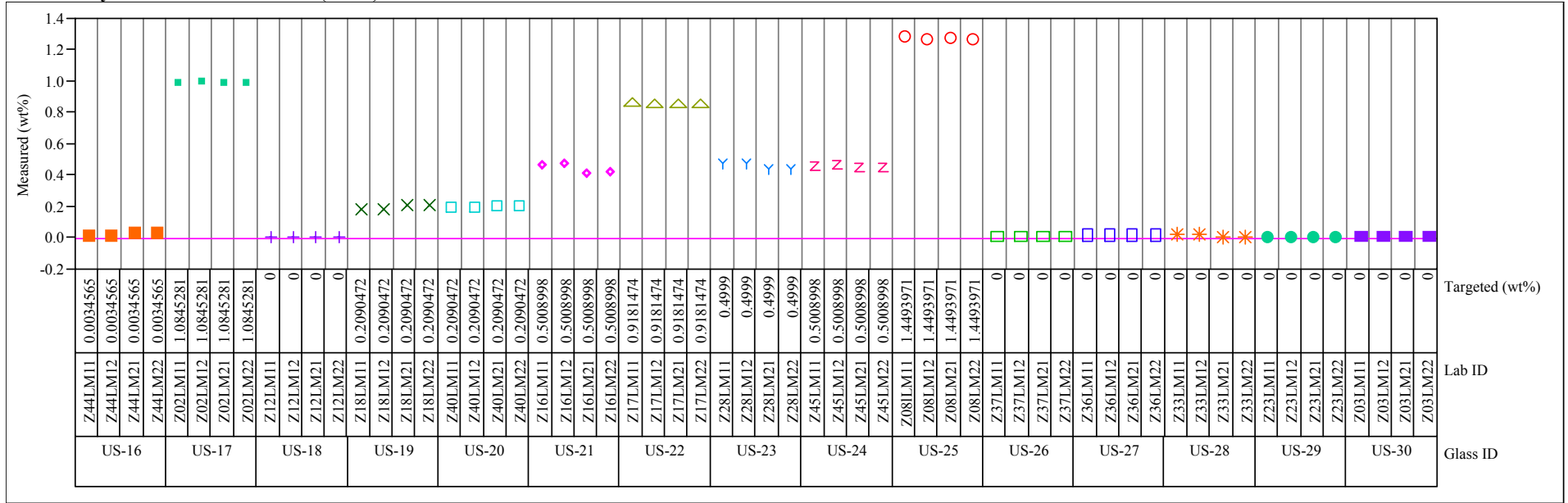

Group $=2$, Oxide=NiO $($ wt $\%)$

Variability Chart for Measured bias-corrected (wt\%)

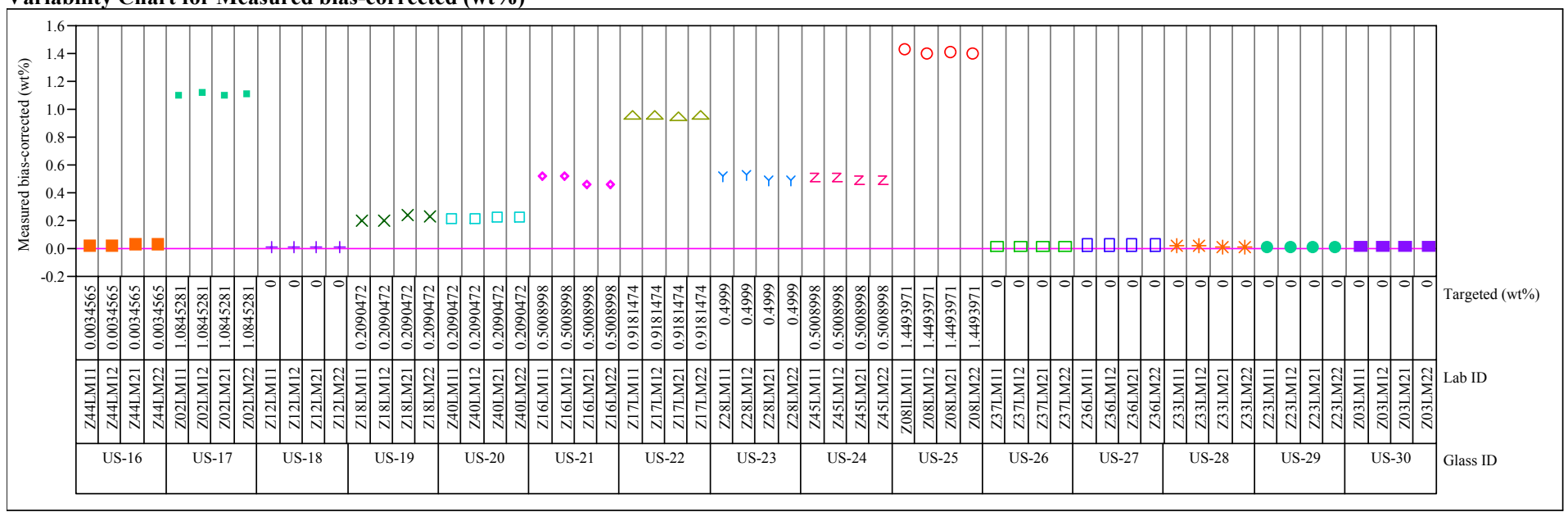


Exhibit A5. Measured and Measured Bias-Corrected Oxide Weight Percents by Lab ID for the Samples Prepared Using the LM Method

Group $=2$, Oxide $=\mathbf{P b O}($ wt $\%)$

Variability Chart for Measured (wt\%)

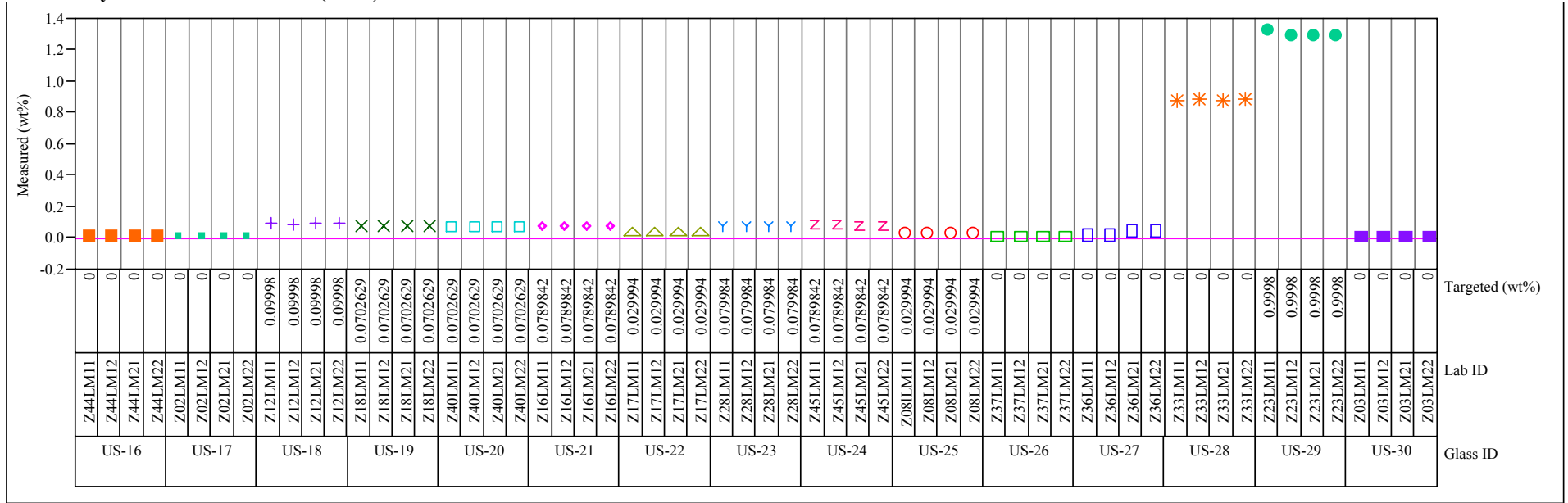

Group $=2$, Oxide $=\mathbf{P b O}(\mathbf{w t} \%)$

Variability Chart for Measured bias-corrected (wt\%)

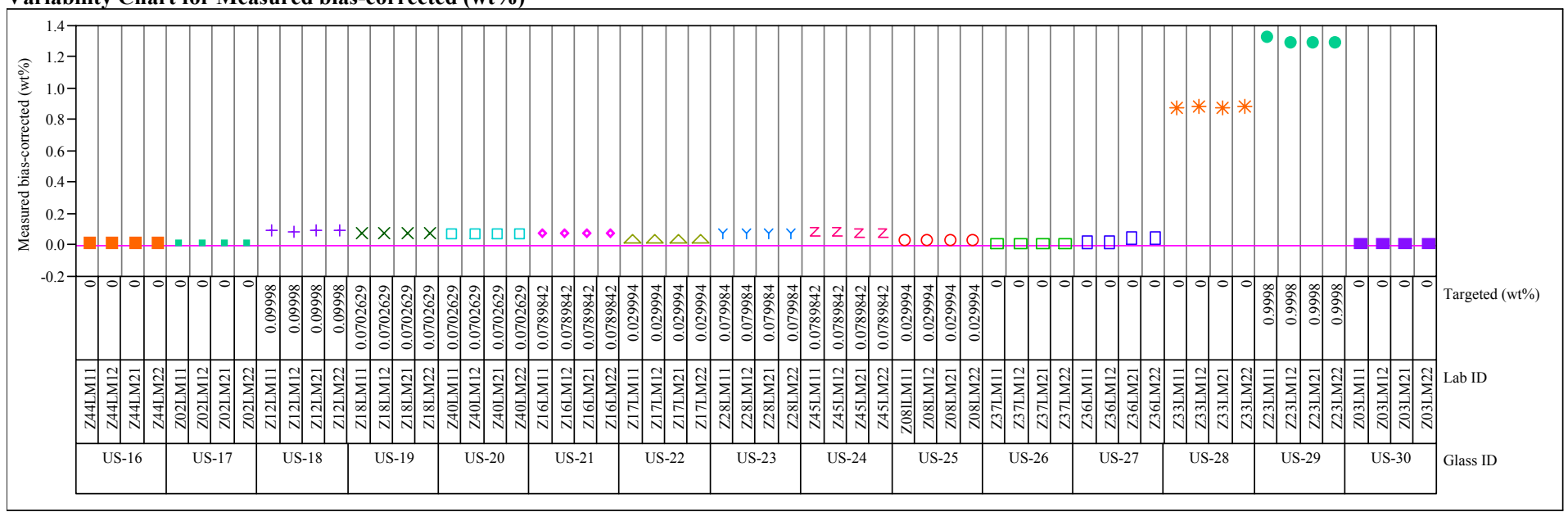




\section{Exhibit A5. Measured and Measured Bias-Corrected Oxide Weight Percents by Lab ID for the Samples Prepared Using the} LM Method

Group $=2$, Oxide $=\mathrm{SO3}(\mathrm{wt} \%)$

Variability Chart for Measured (wt\%)

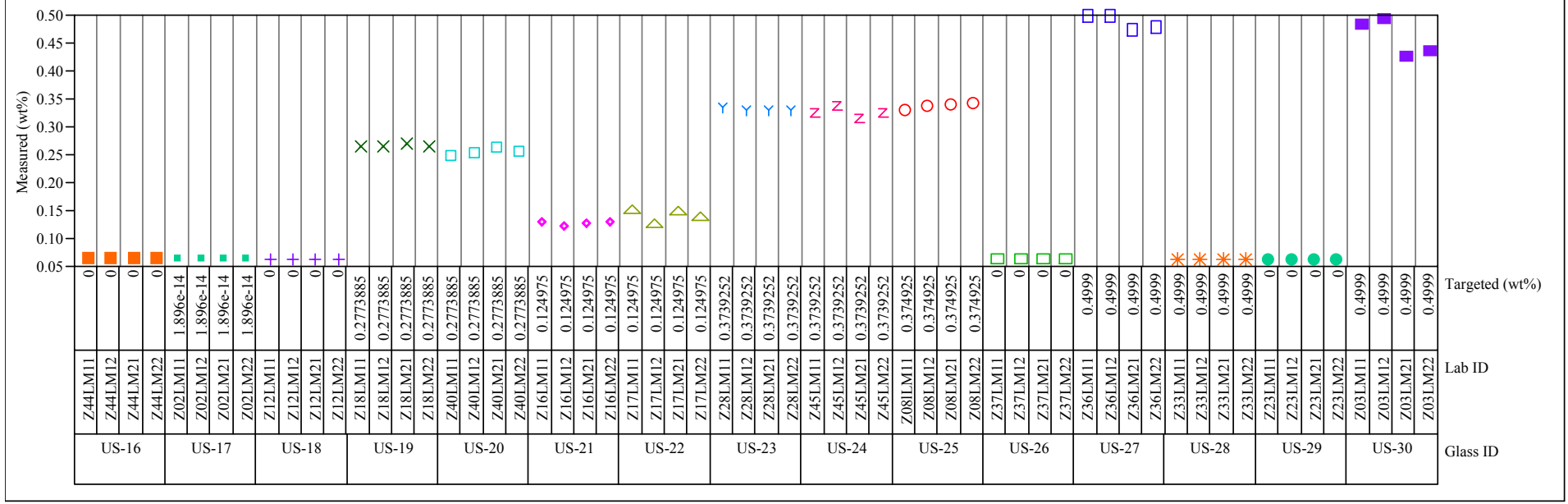

Group $=2$, Oxide $=\mathrm{SO3}($ wt $\%)$

Variability Chart for Measured bias-corrected (wt\%)

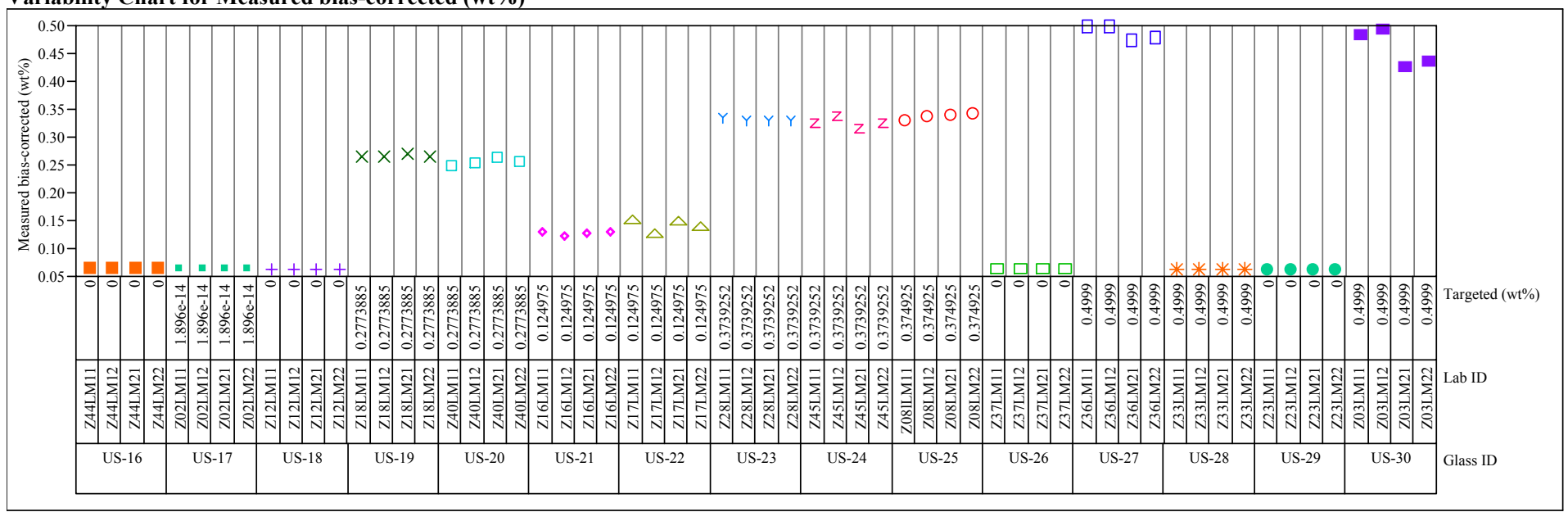


Exhibit A5. Measured and Measured Bias-Corrected Oxide Weight Percents by Lab ID for the Samples Prepared Using the LM Method

Group $=2$, Oxide $=$ SrO $($ wt $\%)$

Variability Chart for Measured (wt\%)

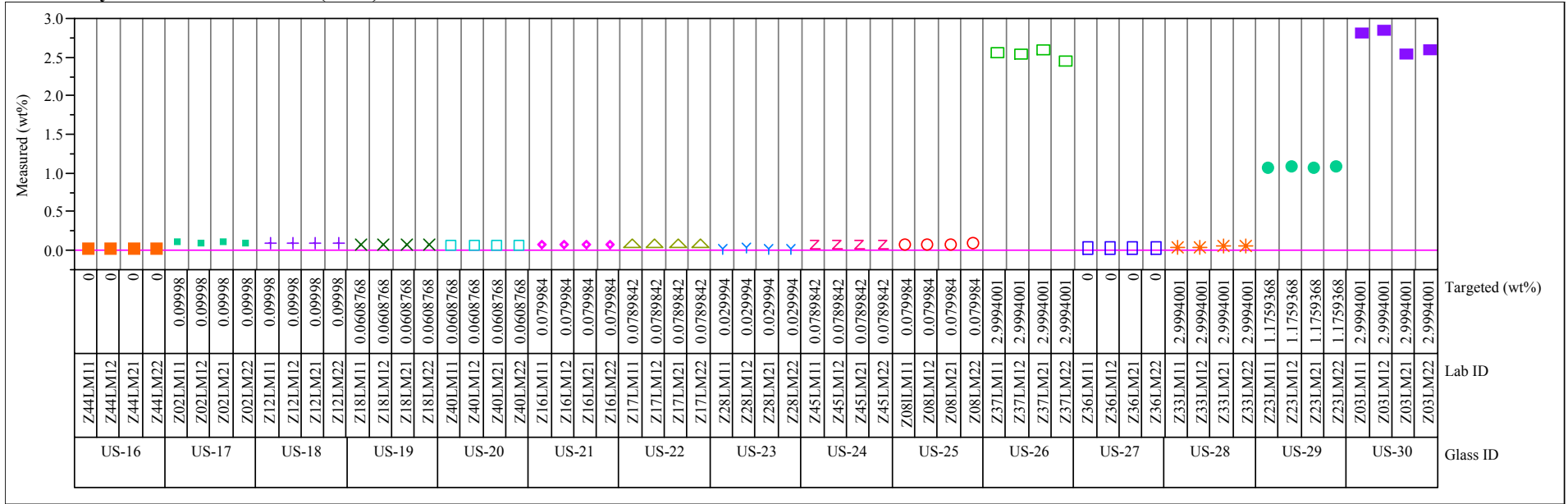

Group $=2$, Oxide $=\operatorname{SrO}($ wt $\%)$

Variability Chart for Measured bias-corrected (wt\%)

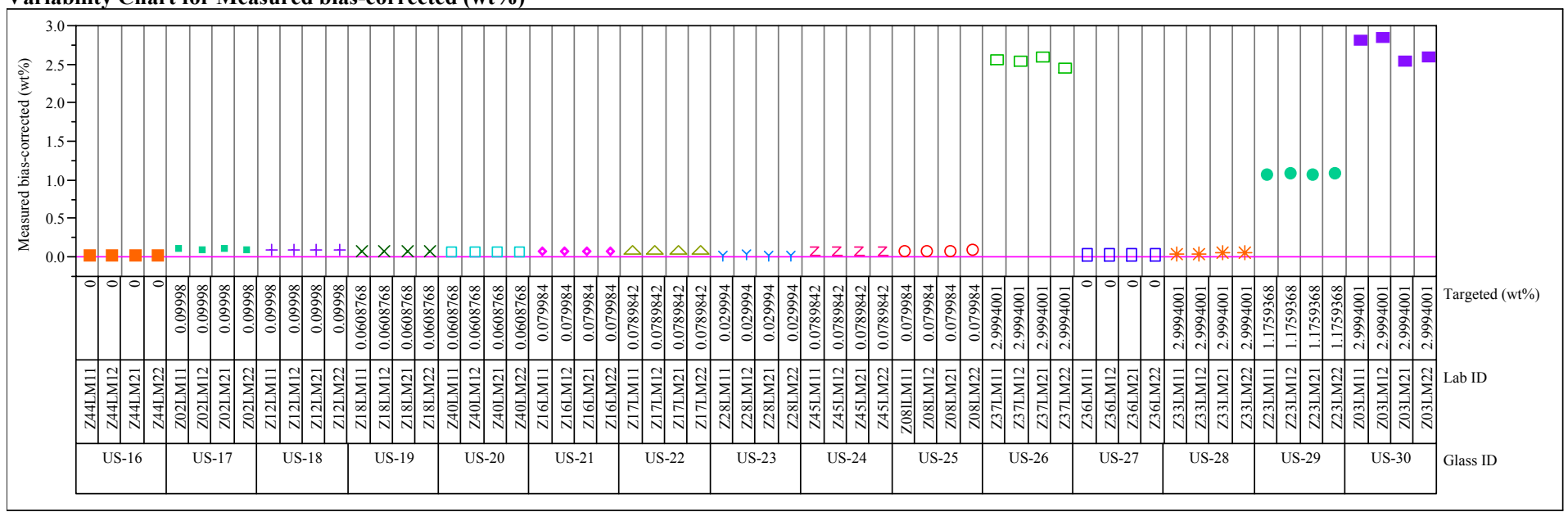




\section{Exhibit A5. Measured and Measured Bias-Corrected Oxide Weight Percents by Lab ID for the Samples Prepared Using the} LM Method

Group $=2$, Oxide $=\mathrm{TiO} 2($ wt $\%)$

Variability Chart for Measured (wt\%)

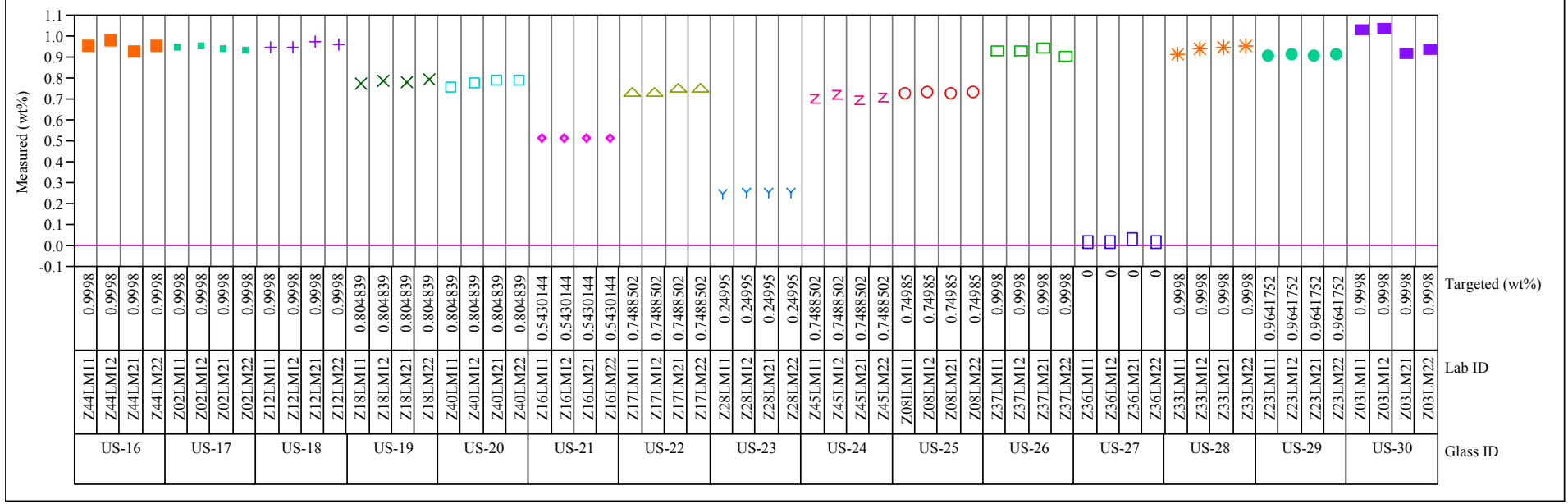

Group=2, Oxide $=$ TiO2 $($ wt $\%)$

Variability Chart for Measured bias-corrected (wt\%)

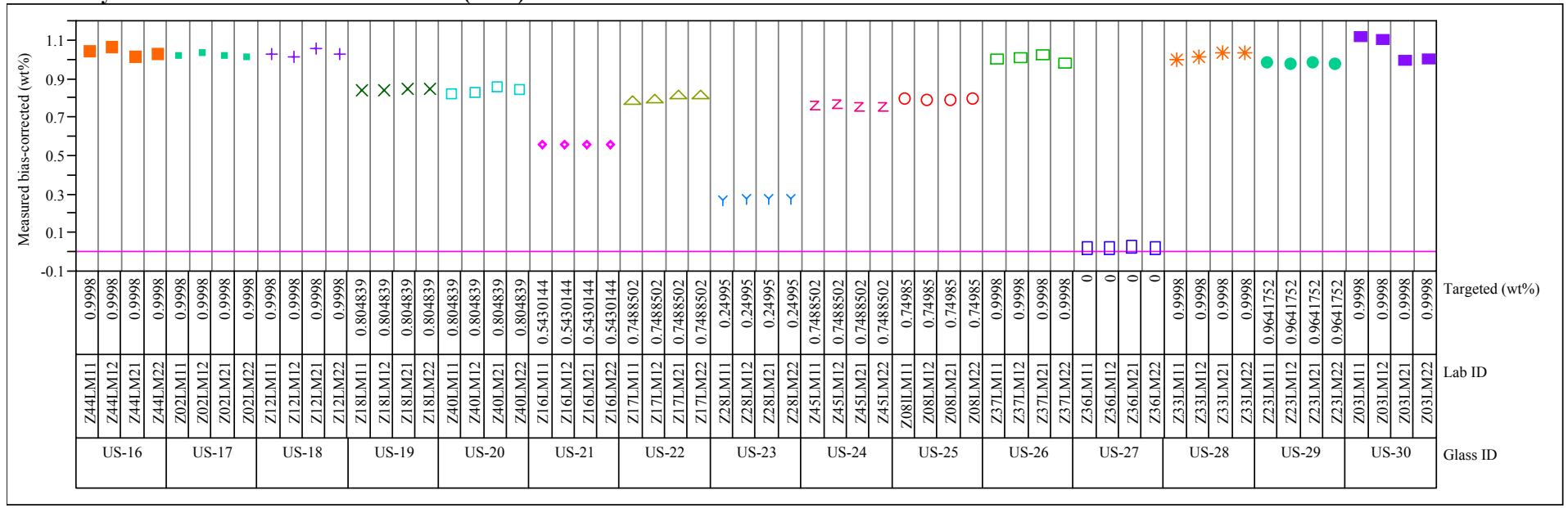


Exhibit A5. Measured and Measured Bias-Corrected Oxide Weight Percents by Lab ID for the Samples Prepared Using the LM Method

Group $=2$, Oxide $=\mathrm{ZnO}($ wt $\%)$

Variability Chart for Measured (wt\%)

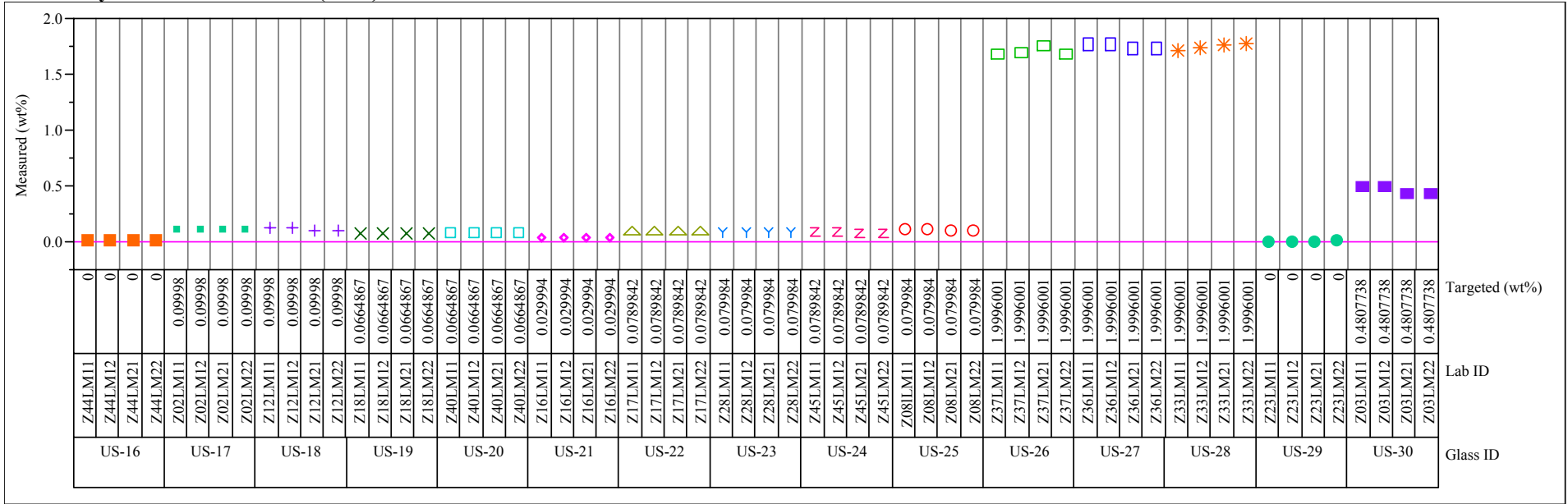

Group $=2$, Oxide $=\mathbf{Z n O}($ wt $\%)$

Variability Chart for Measured bias-corrected (wt\%)

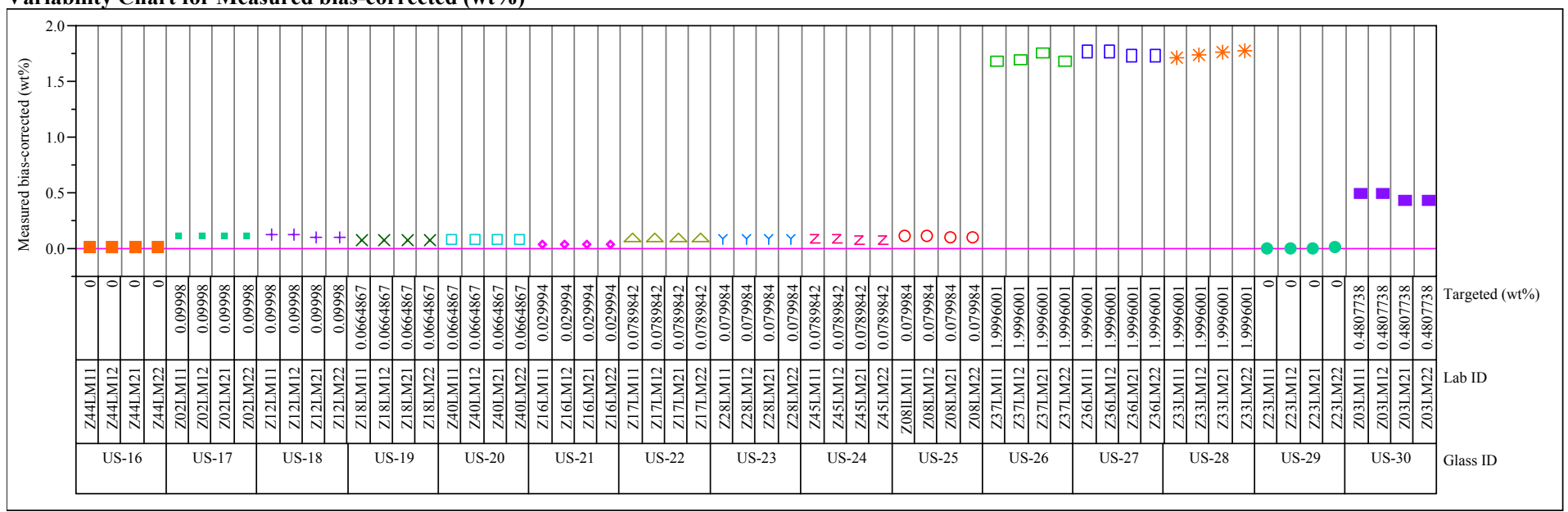




\section{Exhibit A5. Measured and Measured Bias-Corrected Oxide Weight Percents by Lab ID for the Samples Prepared Using the} LM Method

Group $=2$, Oxide $=\mathrm{ZrO} 2(\mathbf{w t} \%)$

Variability Chart for Measured (wt\%)

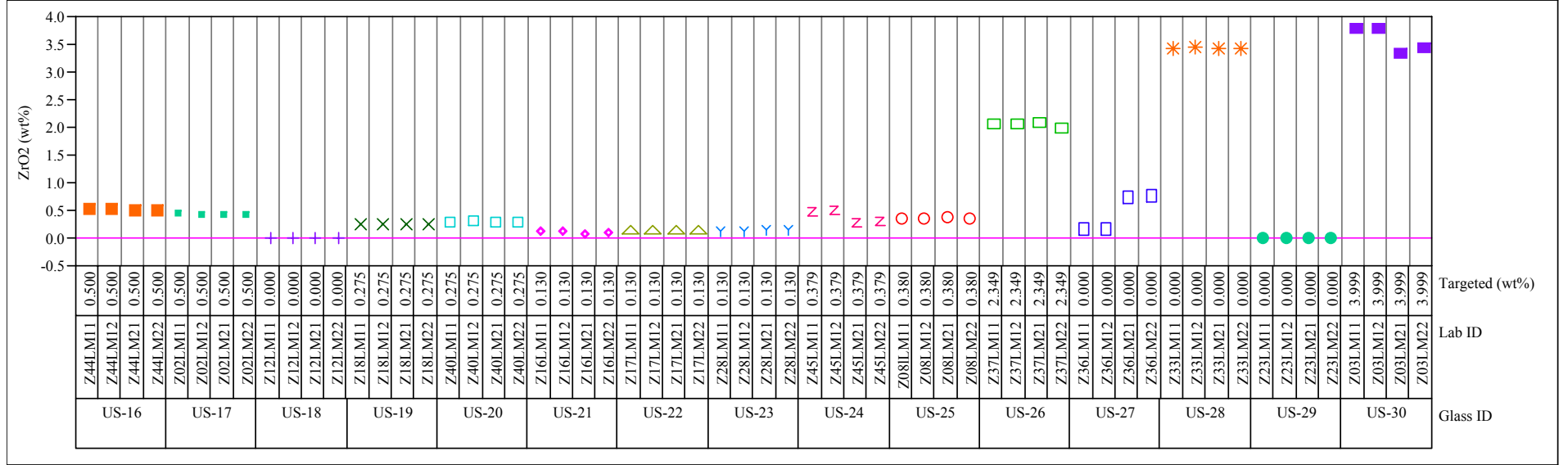

Group $=2$, Oxide $=\mathrm{ZrO} 2(w t \%)$

Variability Chart for Measured bias-corrected (wt\%)

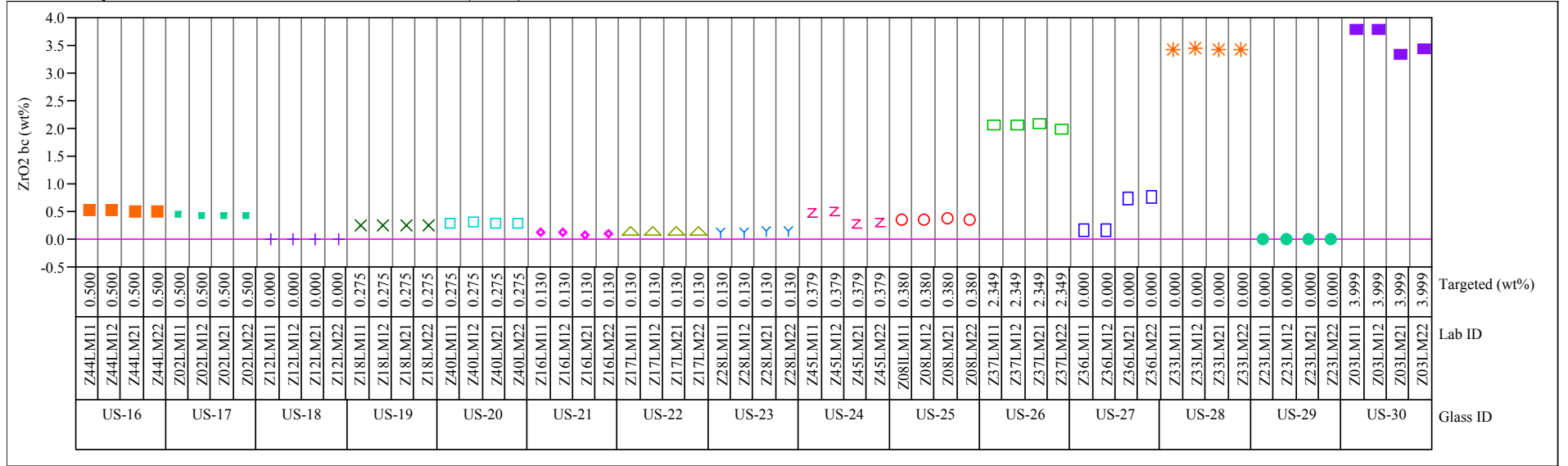




\section{Exhibit A5. Measured and Measured Bias-Corrected Oxide Weight Percents by Lab ID for the Samples Prepared Using the} LM Method

Group $=3$, Oxide $=\mathrm{CaO}($ wt $\%)$

Variability Chart for Measured (wt\%)

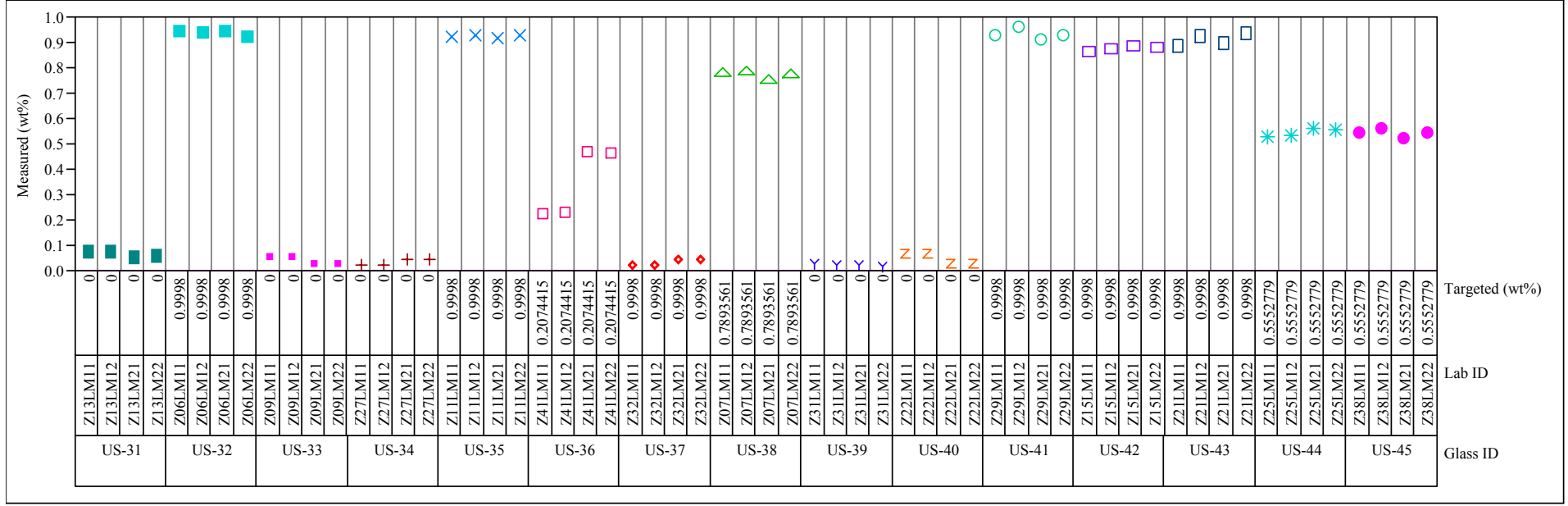

Group $=3$, Oxide $=\mathrm{CaO}($ wt $\%)$

Variability Chart for Measured bias-corrected (wt\%)

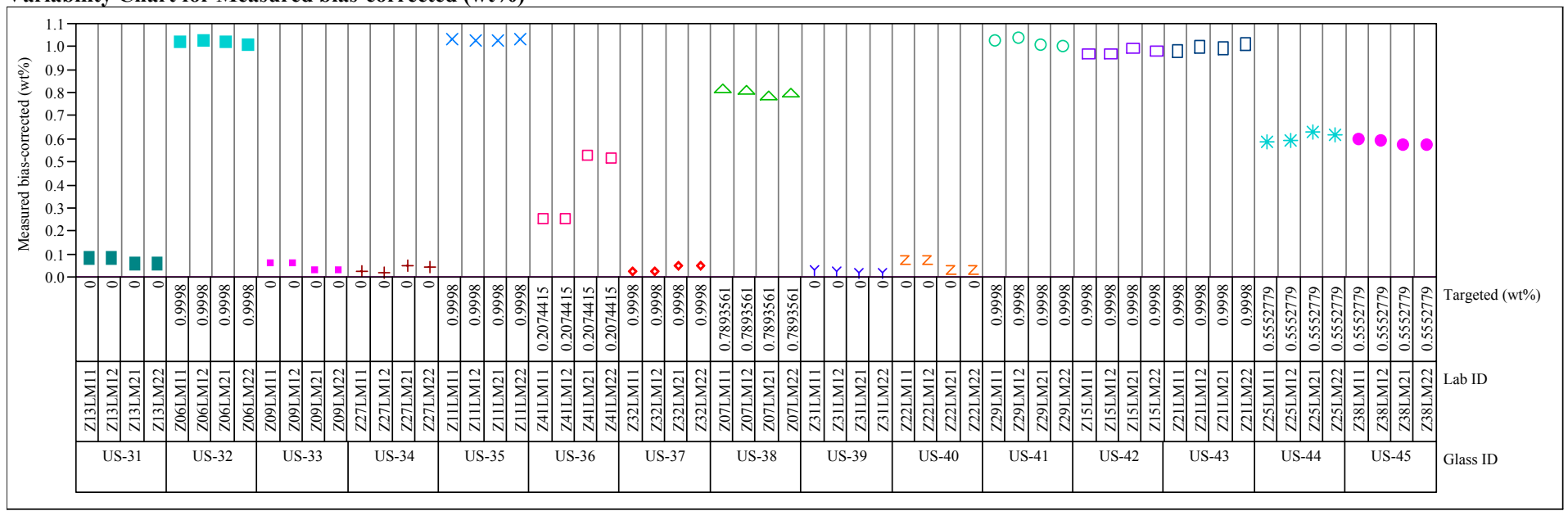


Exhibit A5. Measured and Measured Bias-Corrected Oxide Weight Percents by Lab ID for the Samples Prepared Using the LM Method

Group=3, Oxide $=\mathbf{C r 2 O 3}(\mathrm{wt} \%)$

Variability Chart for Measured (wt\%)

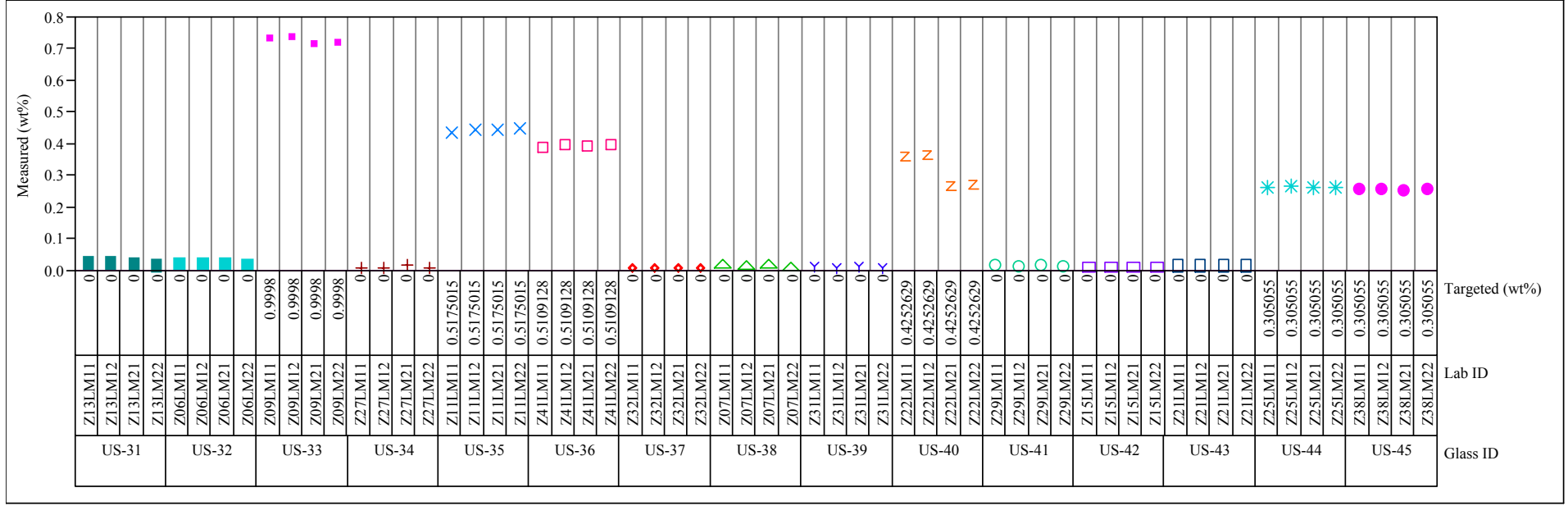

Group $=3$, Oxide $=\mathbf{C r 2 O 3}($ wt $\%)$

Variability Chart for Measured bias-corrected (wt\%)

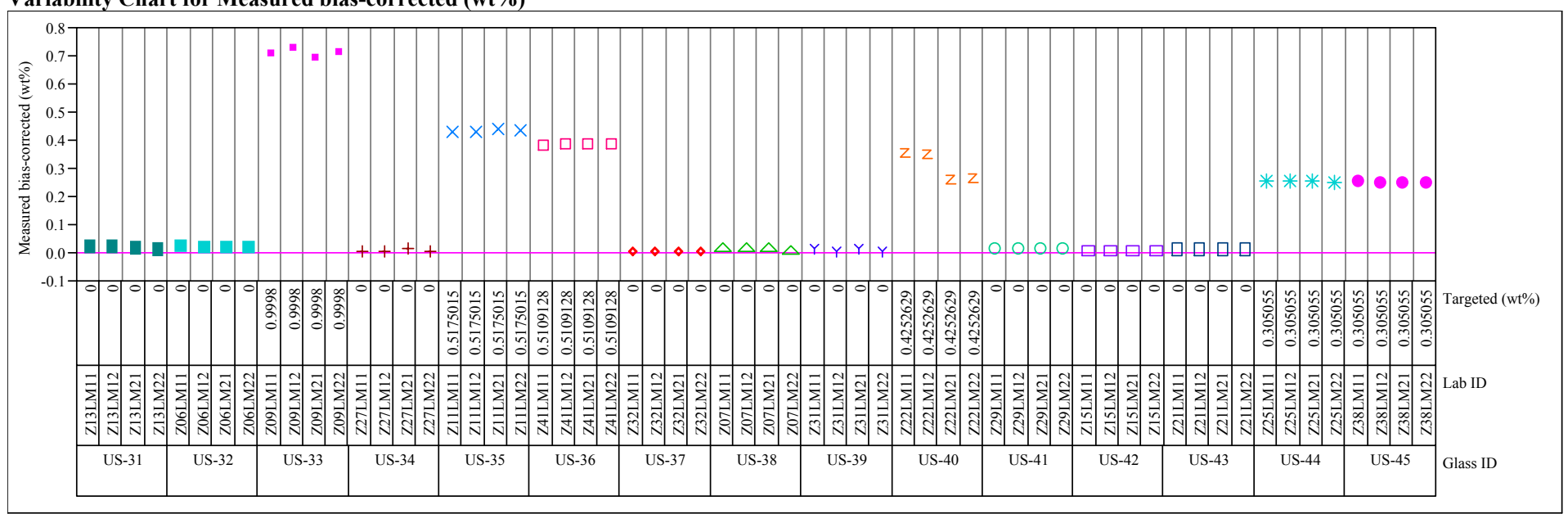




\section{Exhibit A5. Measured and Measured Bias-Corrected Oxide Weight Percents by Lab ID for the Samples Prepared Using the} LM Method

Group $=3$, Oxide $=K 20(w t \%)$

Variability Chart for Measured (wt\%)

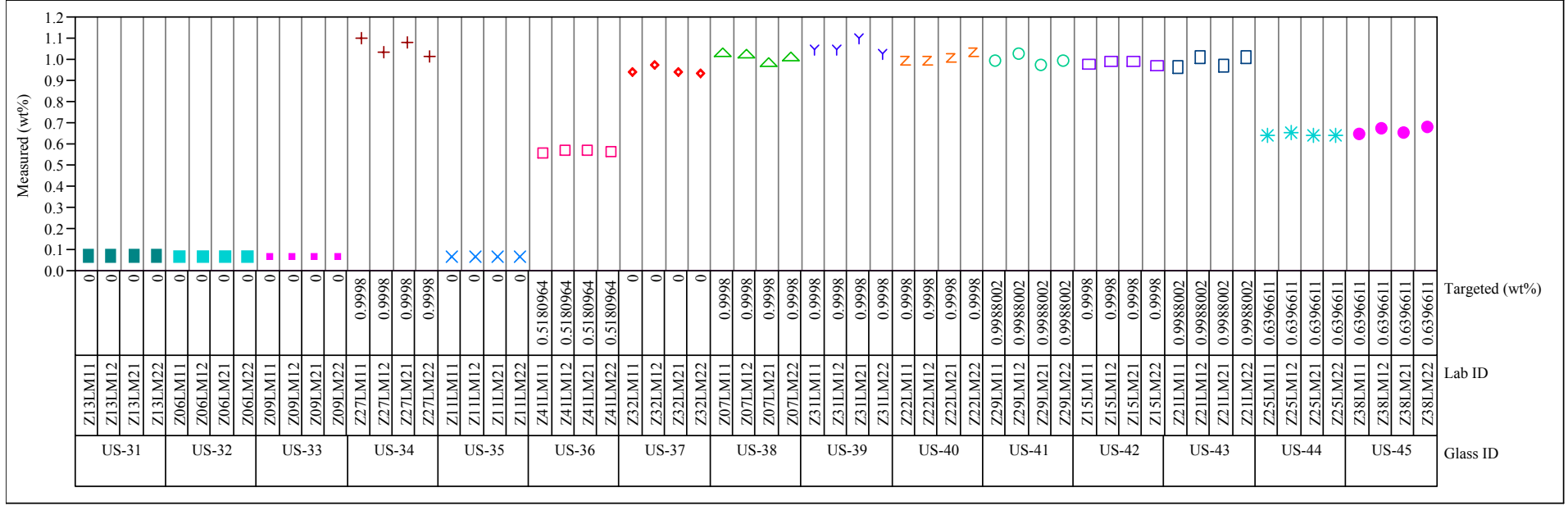

Group $=3$, Oxide $=K 20(w t \%)$

Variability Chart for Measured bias-corrected (wt\%)

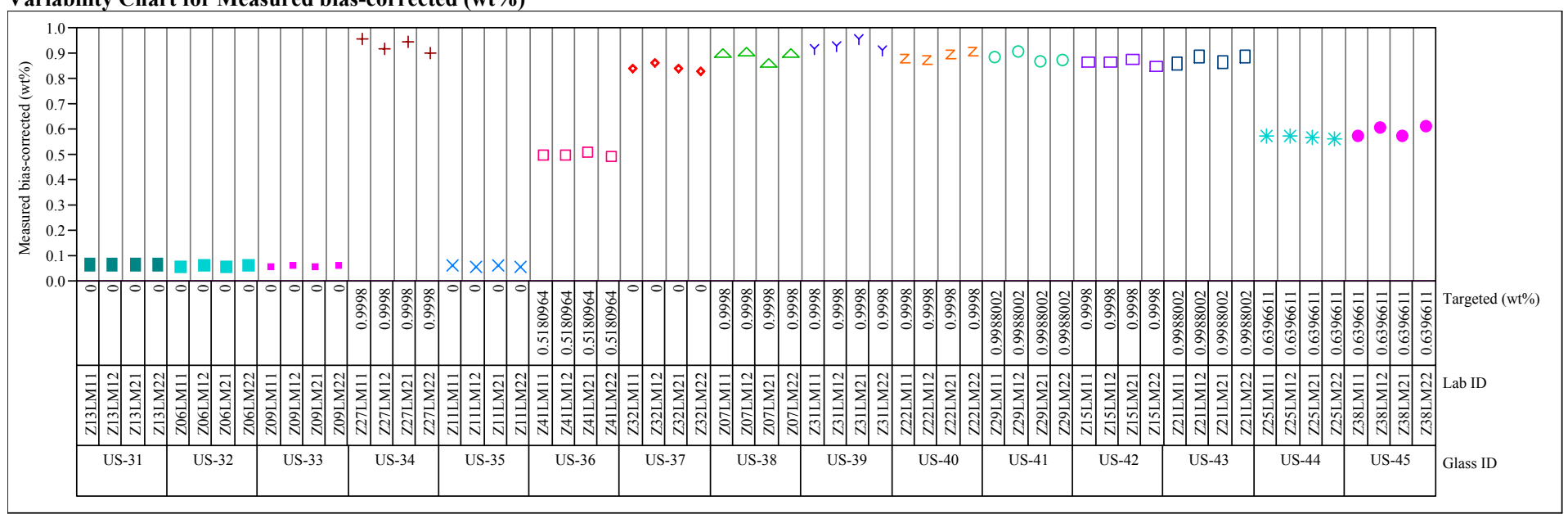


Exhibit A5. Measured and Measured Bias-Corrected Oxide Weight Percents by Lab ID for the Samples Prepared Using the LM Method

Group $=3$, Oxide $=\mathrm{MgO}($ wt $\%)$

Variability Chart for Measured (wt\%)

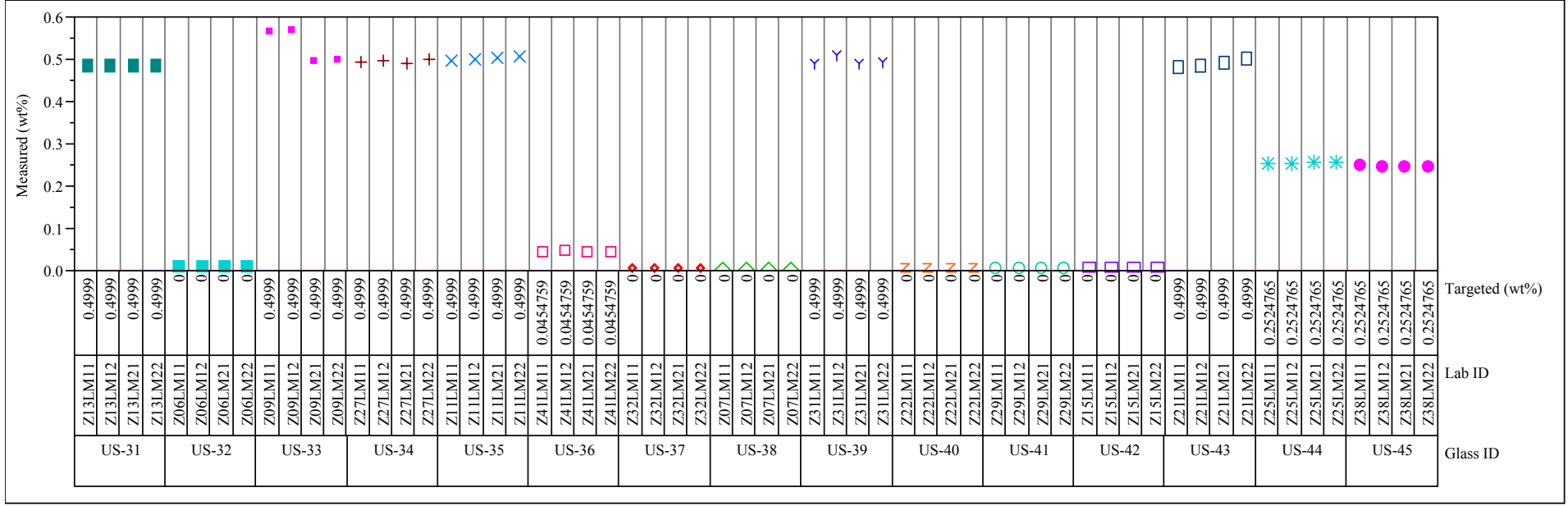

Group $=3$, Oxide $=\mathrm{MgO}(\mathrm{wt} \%)$

Variability Chart for Measured bias-corrected (wt\%)

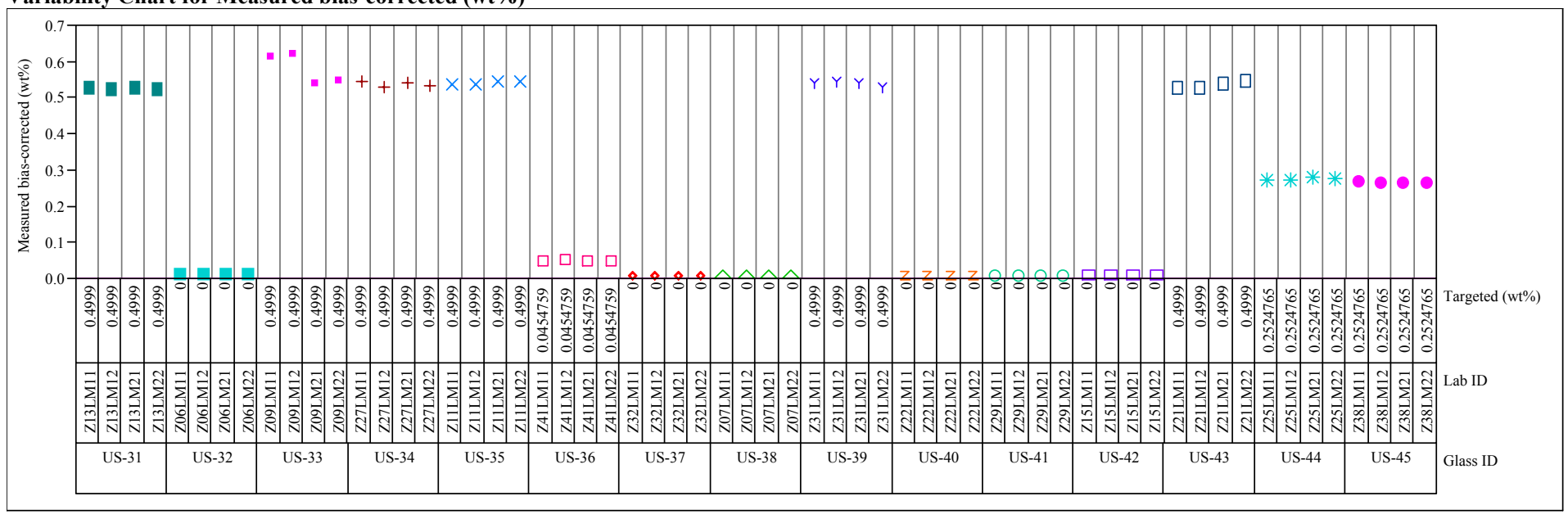




\section{Exhibit A5. Measured and Measured Bias-Corrected Oxide Weight Percents by Lab ID for the Samples Prepared Using the} LM Method

Group $=3$, Oxide $=\mathrm{Na2O}(\mathrm{wt} \%)$

Variability Chart for Measured (wt\%)

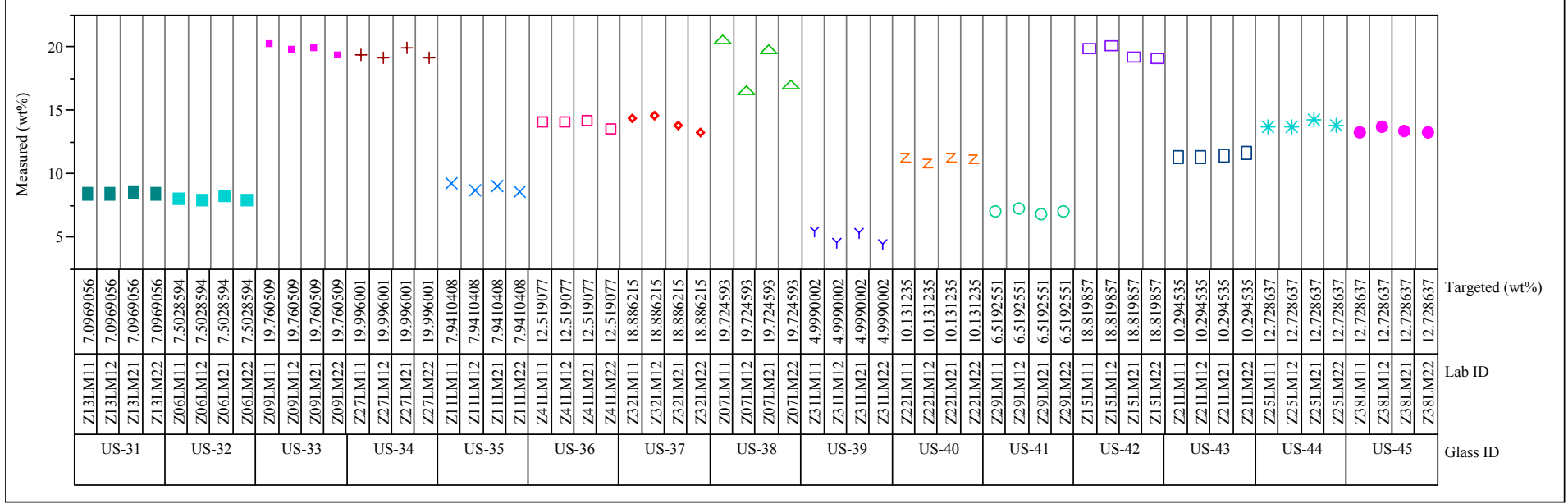

Group=3, Oxide=Na2O (wt\%)

Variability Chart for Measured bias-corrected (wt\%)

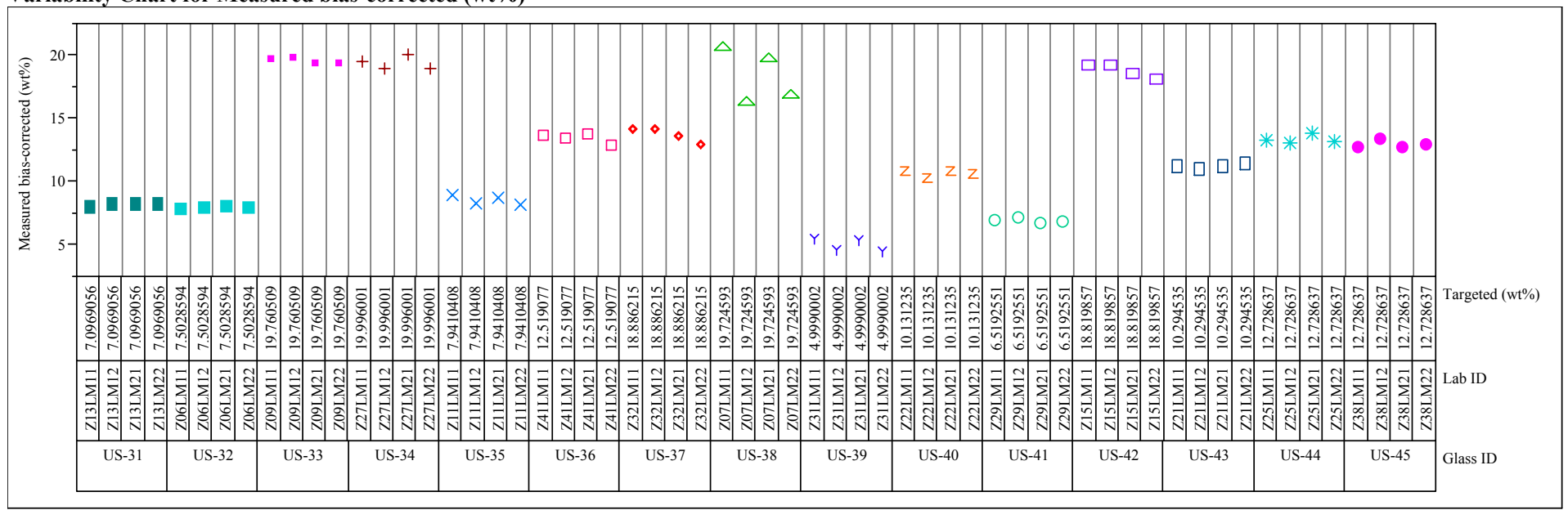


Exhibit A5. Measured and Measured Bias-Corrected Oxide Weight Percents by Lab ID for the Samples Prepared Using the LM Method

Group=3, Oxide $=\mathrm{NiO}(\mathbf{w t} \%)$

Variability Chart for Measured (wt\%)

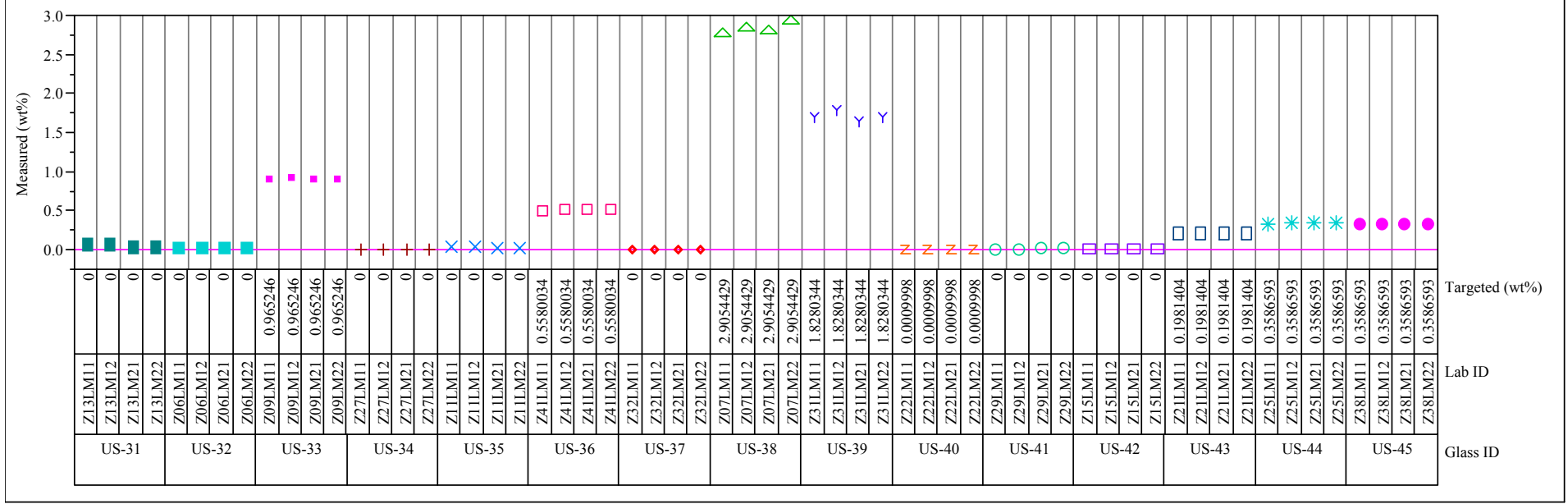

Group $=3$, Oxide= $=\mathrm{NiO}($ wt $\%)$

Variability Chart for Measured bias-corrected (wt $\%)$

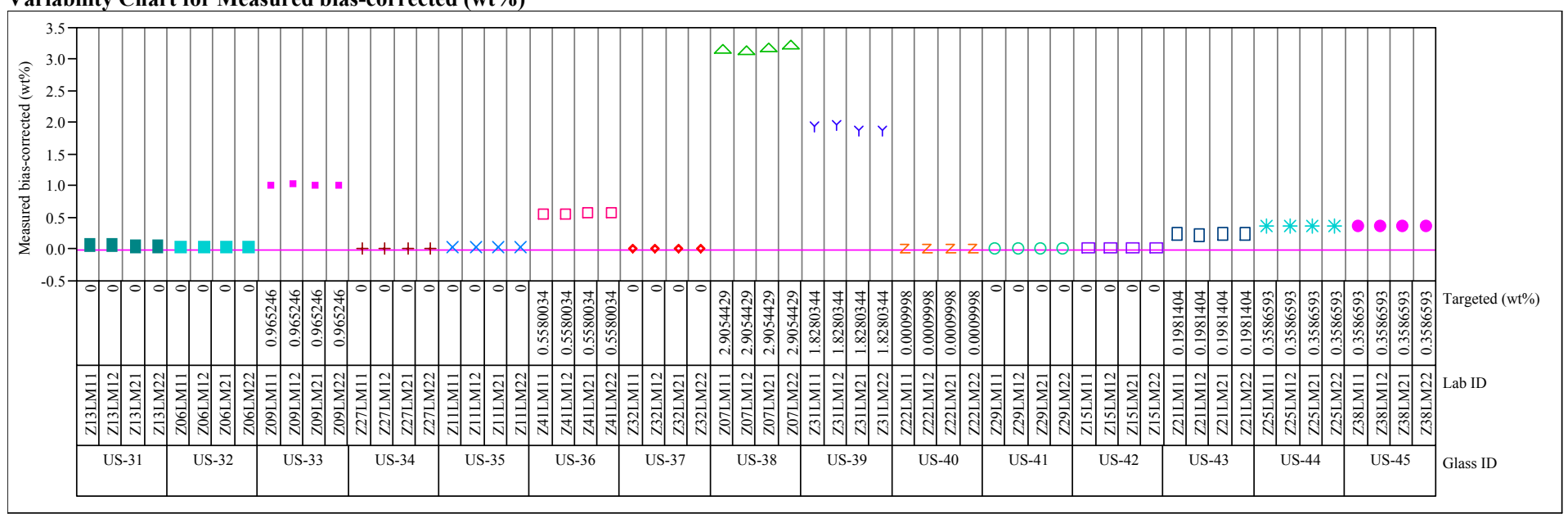


Exhibit A5. Measured and Measured Bias-Corrected Oxide Weight Percents by Lab ID for the Samples Prepared Using the LM Method

Group $=3$, Oxide $=$ PbO $($ wt $\%)$

Variability Chart for Measured (wt\%)

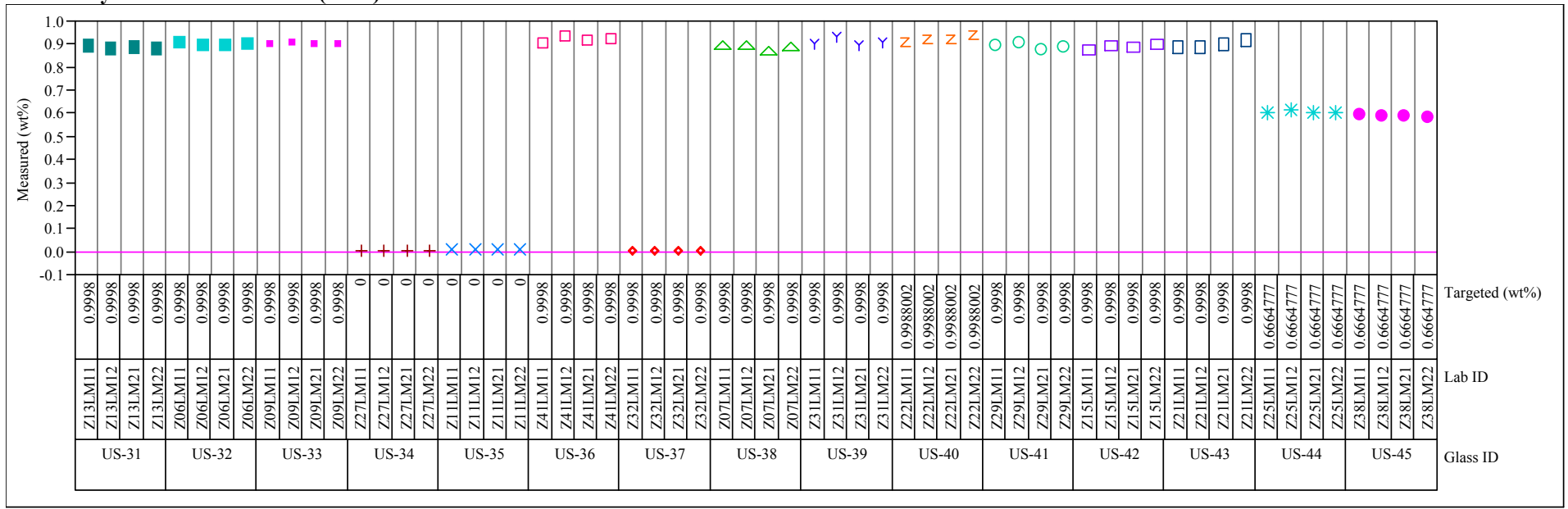

Group $=3$, Oxide $=$ PbO $($ wt $\%)$

Variability Chart for Measured bias-corrected (wt\%)

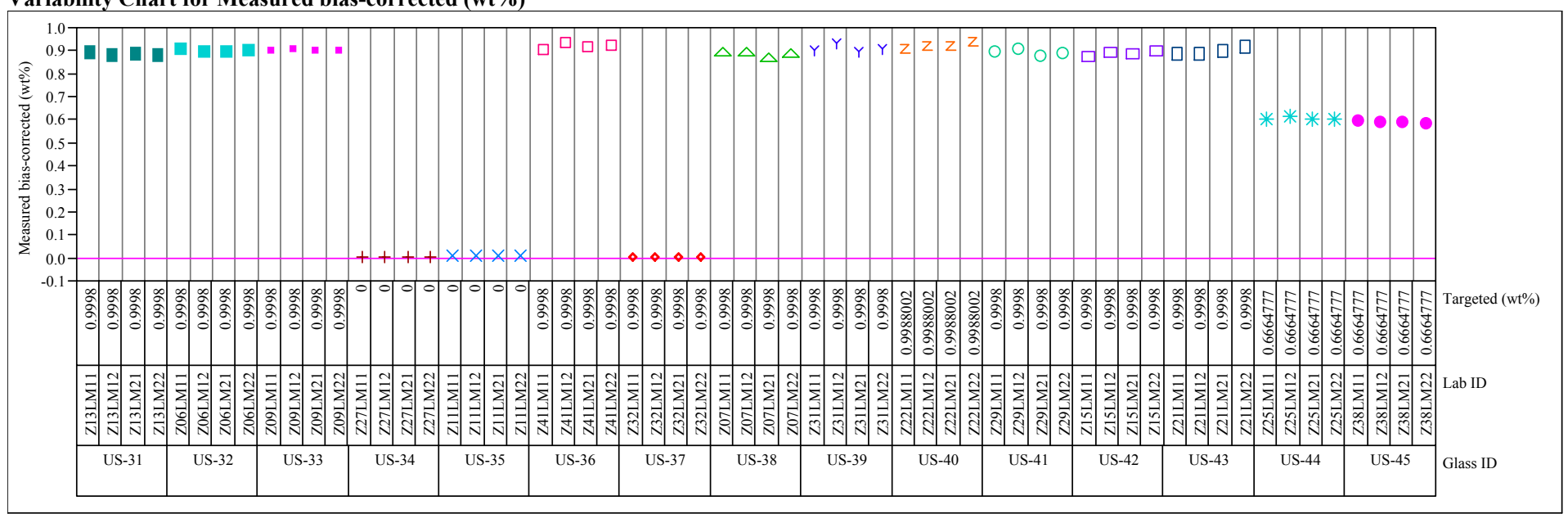


Exhibit A5. Measured and Measured Bias-Corrected Oxide Weight Percents by Lab ID for the Samples Prepared Using the LM Method

Group=3, Oxide $=\mathrm{SO3}(\mathbf{w t} \%)$

Variability Chart for Measured (wt\%)

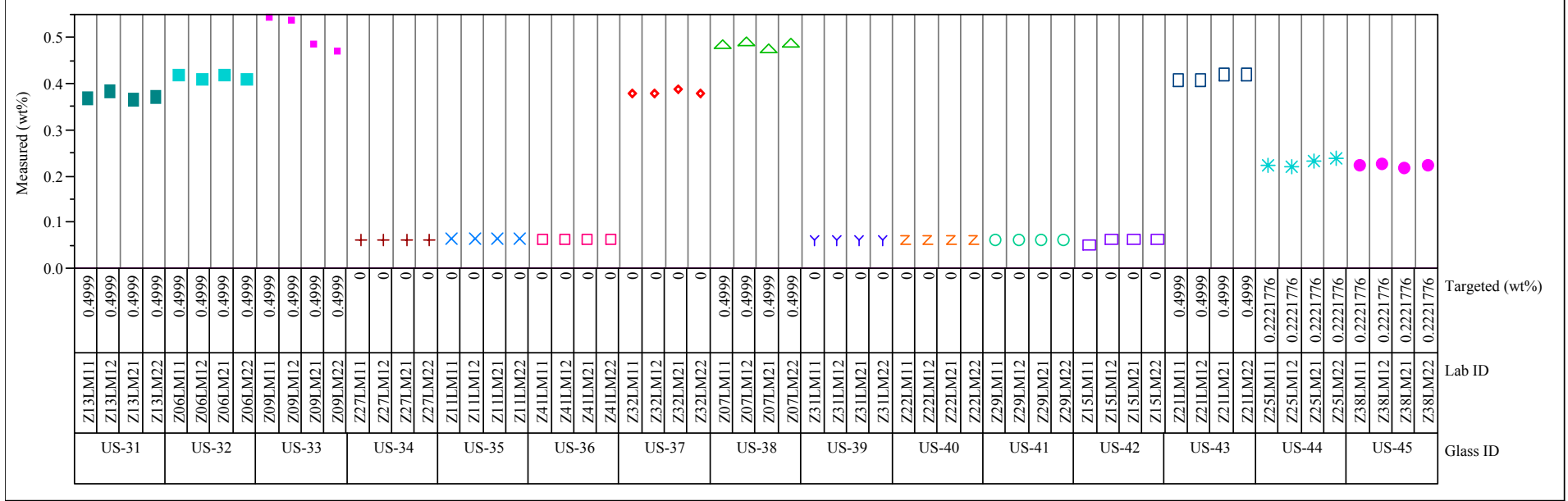

Group $=3$, Oxide=SO3 (wt $\%)$

Variability Chart for Measured bias-corrected (wt\%)

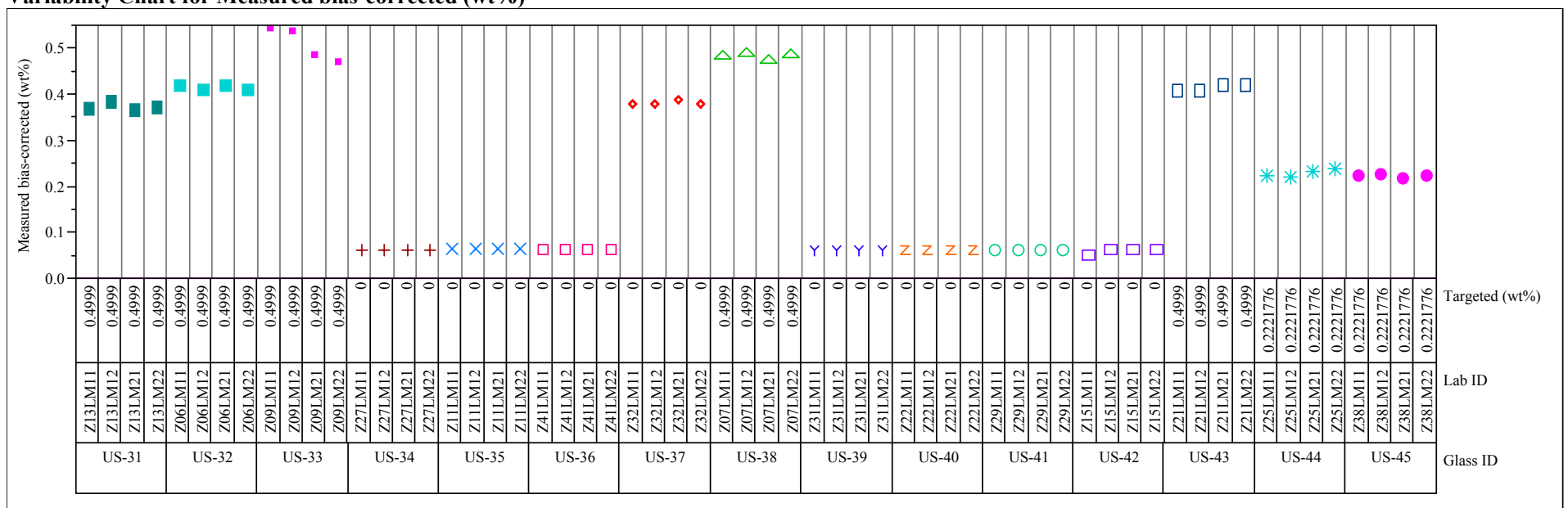


Exhibit A5. Measured and Measured Bias-Corrected Oxide Weight Percents by Lab ID for the Samples Prepared Using the LM Method

Group=3, Oxide=SrO (wt\%)

Variability Chart for Measured (wt\%)

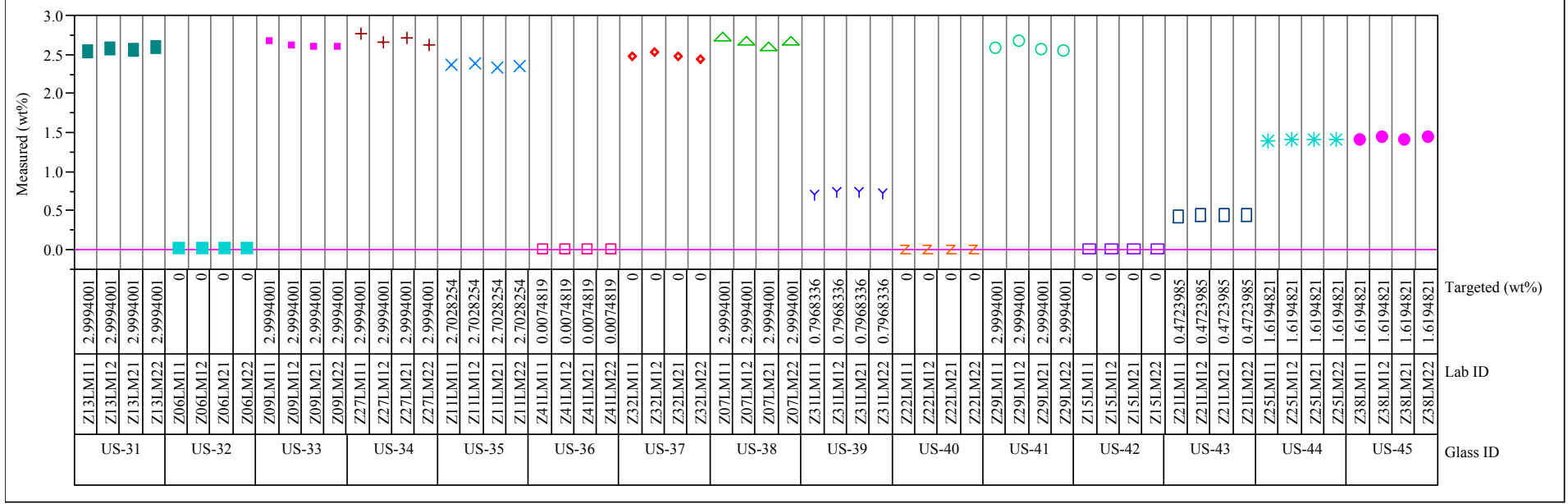

Group $=3$, Oxide $=\operatorname{SrO}($ wt $\%)$

Variability Chart for Measured bias-corrected (wt\%)

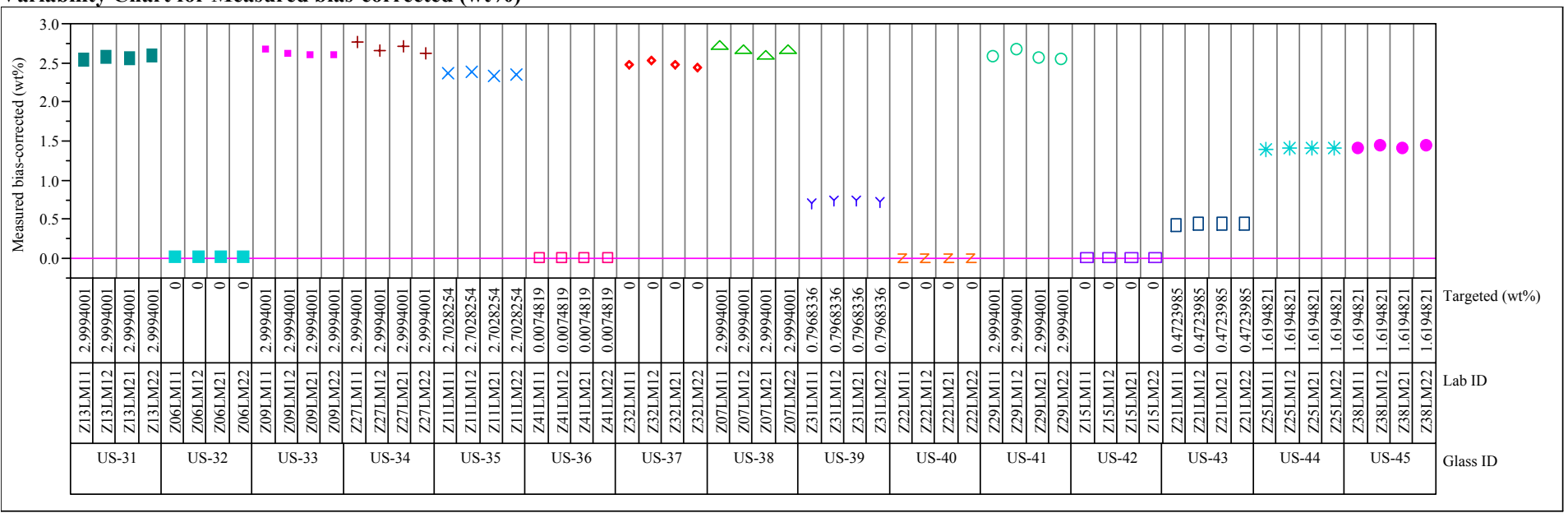




\section{Exhibit A5. Measured and Measured Bias-Corrected Oxide Weight Percents by Lab ID for the Samples Prepared Using the} LM Method

Group $=3$, Oxide $=\mathrm{TiO} 2($ wt $\%)$

Variability Chart for Measured (wt\%)

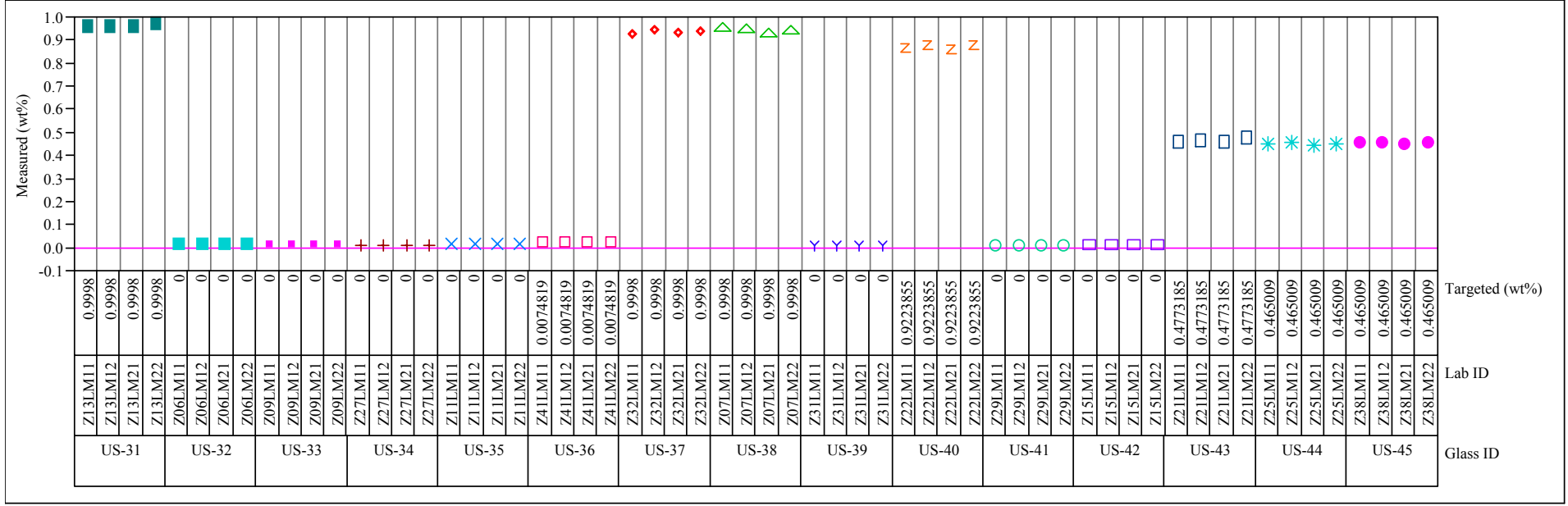

Group $=3$, Oxide $=\mathrm{TiO} 2($ wt $\%)$

Variability Chart for Measured bias-corrected (wt\%)

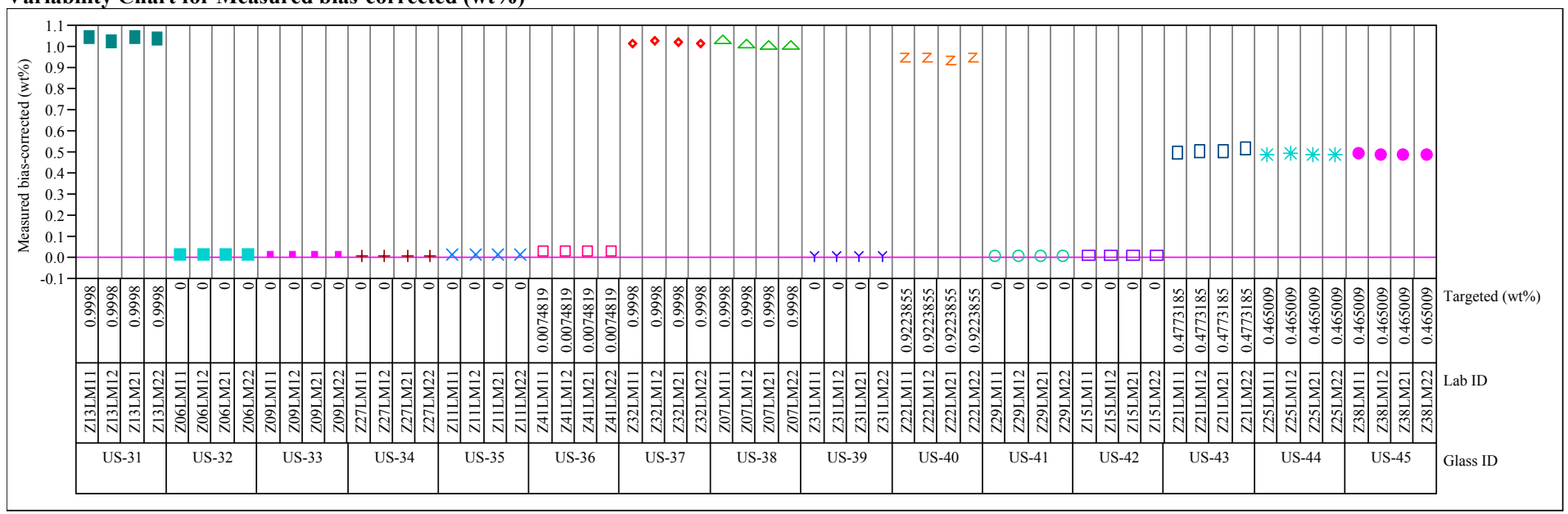


Exhibit A5. Measured and Measured Bias-Corrected Oxide Weight Percents by Lab ID for the Samples Prepared Using the LM Method

Group $=3$, Oxide $=\mathrm{ZnO}($ wt $\%)$

Variability Chart for Measured (wt\%)

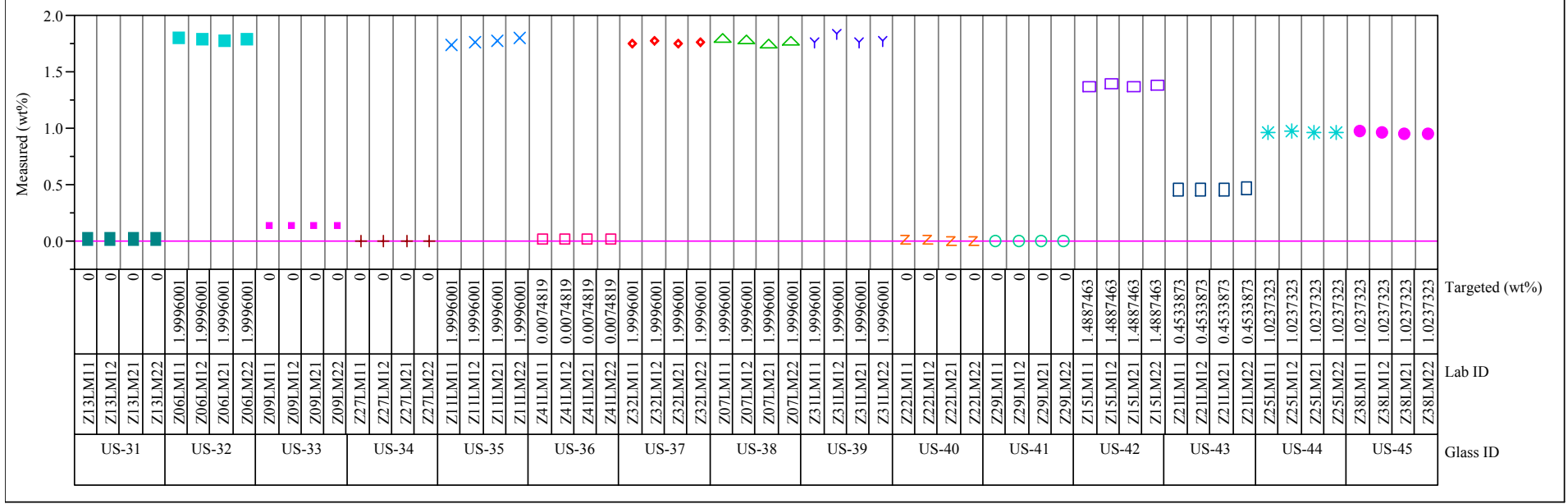

Group $=3$, Oxide $=\mathrm{ZnO}(\mathrm{wt} \%)$

Variability Chart for Measured bias-corrected (wt\%)

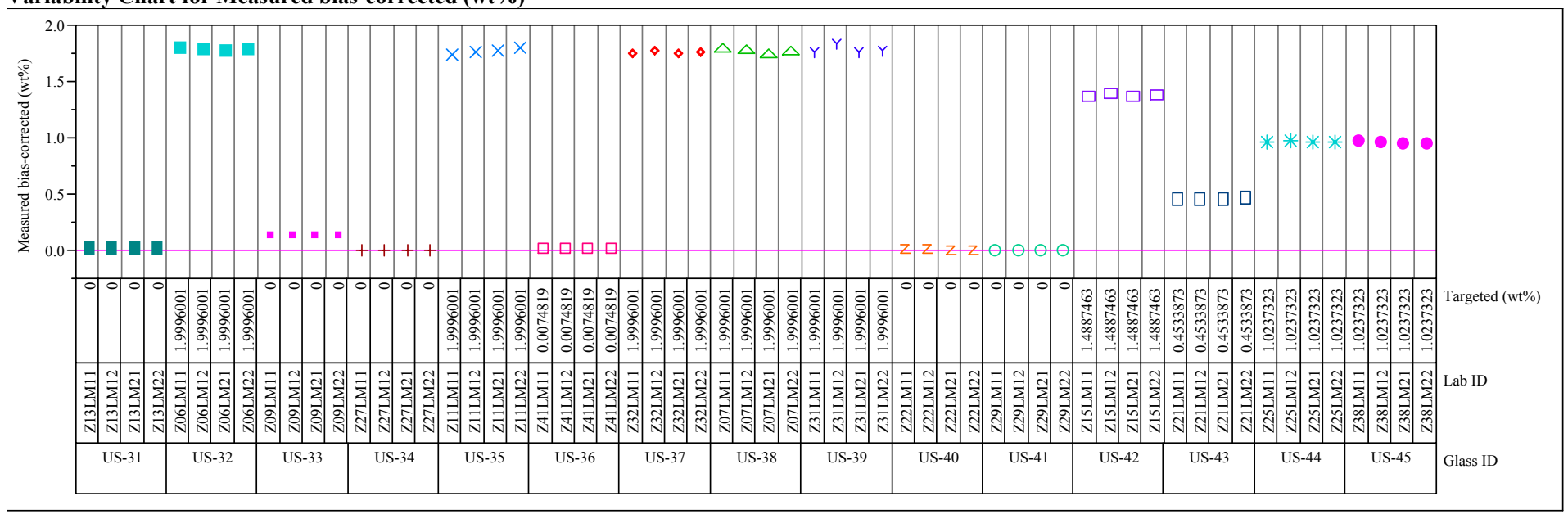




\section{Exhibit A5. Measured and Measured Bias-Corrected Oxide Weight Percents by Lab ID for the Samples Prepared Using the} LM Method

Group $=3$, Oxide $=\mathrm{ZrO2}(\mathbf{w t} \%)$

Variability Chart for Measured (wt\%)

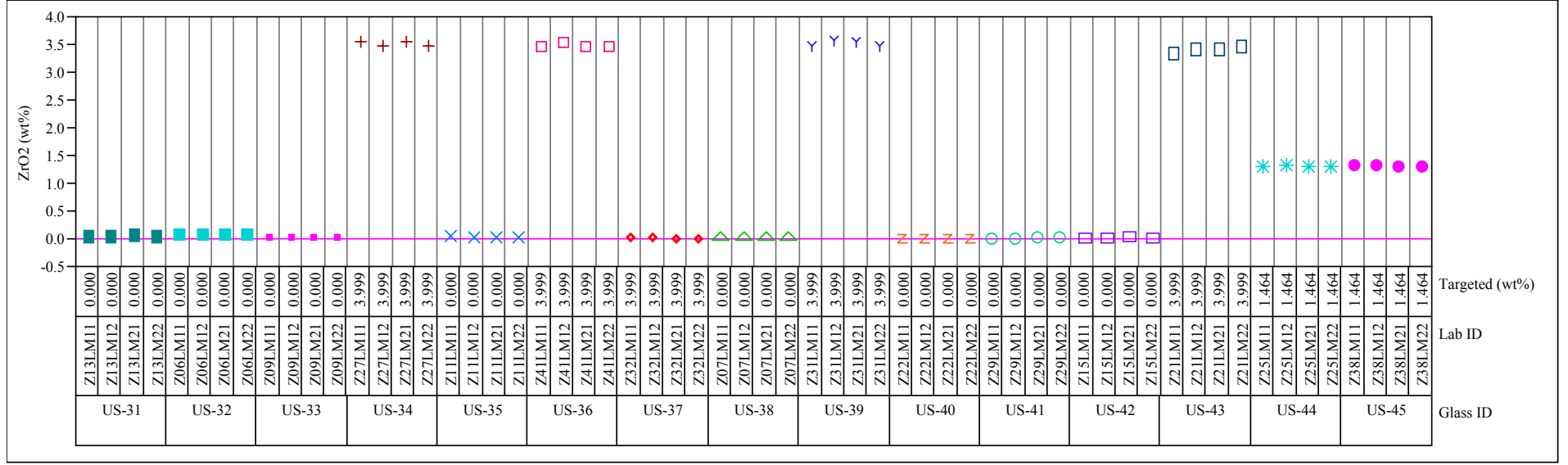

Group $=3$, Oxide $=\mathrm{ZrO2}(\mathrm{wt} \%)$

Variability Chart for Measured bias-corrected (wt\%)

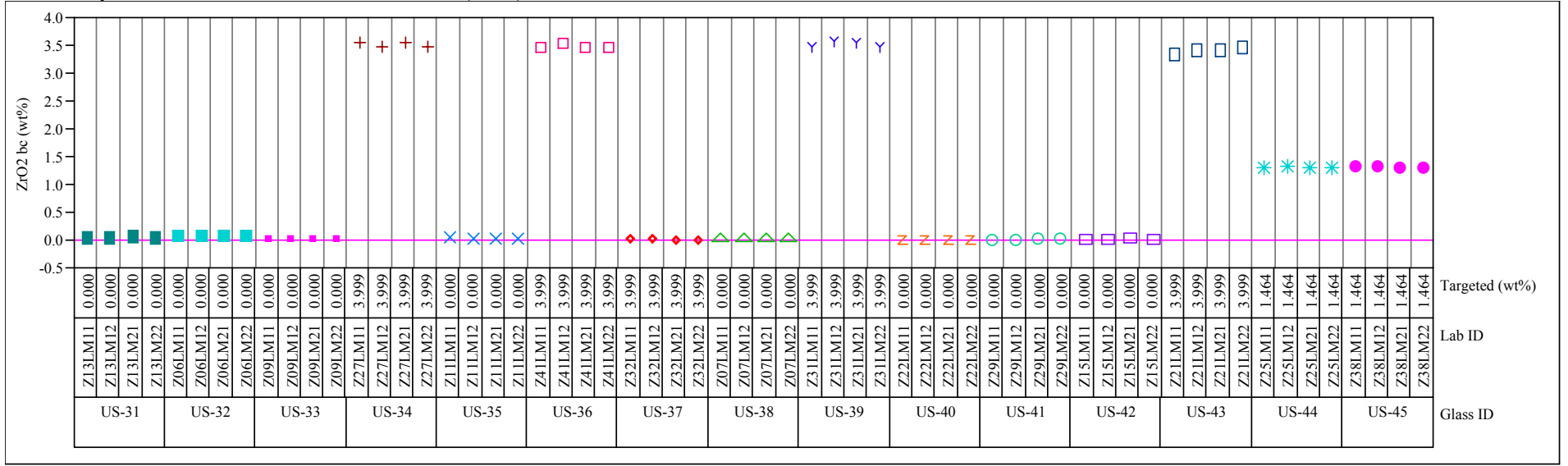


Exhibit A5. Measured and Measured Bias-Corrected Oxide Weight Percents by Lab ID for the Samples Prepared Using the LM Method

Group $=4$, Oxide $=\mathrm{CaO}(\mathrm{wt} \%)$

Variability Chart for Measured (wt\%)

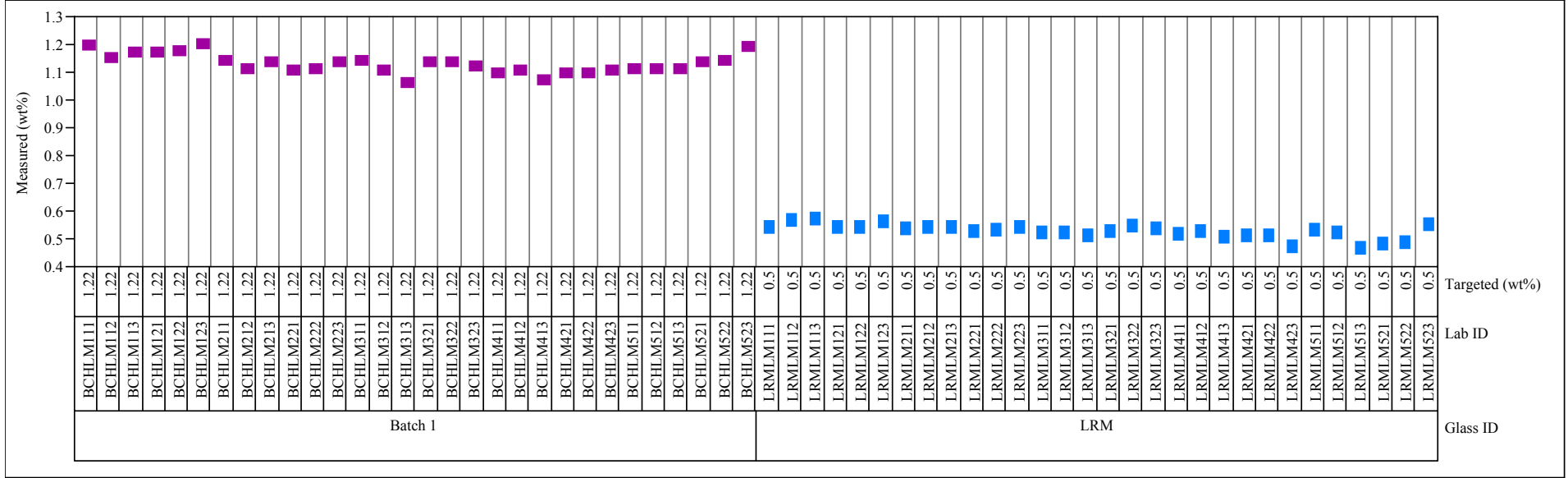

Group $=4$, Oxide $=\mathrm{CaO}\left(\mathrm{wt}_{\mathrm{t}} \mathrm{)}\right)$

Variability Chart for Measured bias-corrected (wt $\%$ )

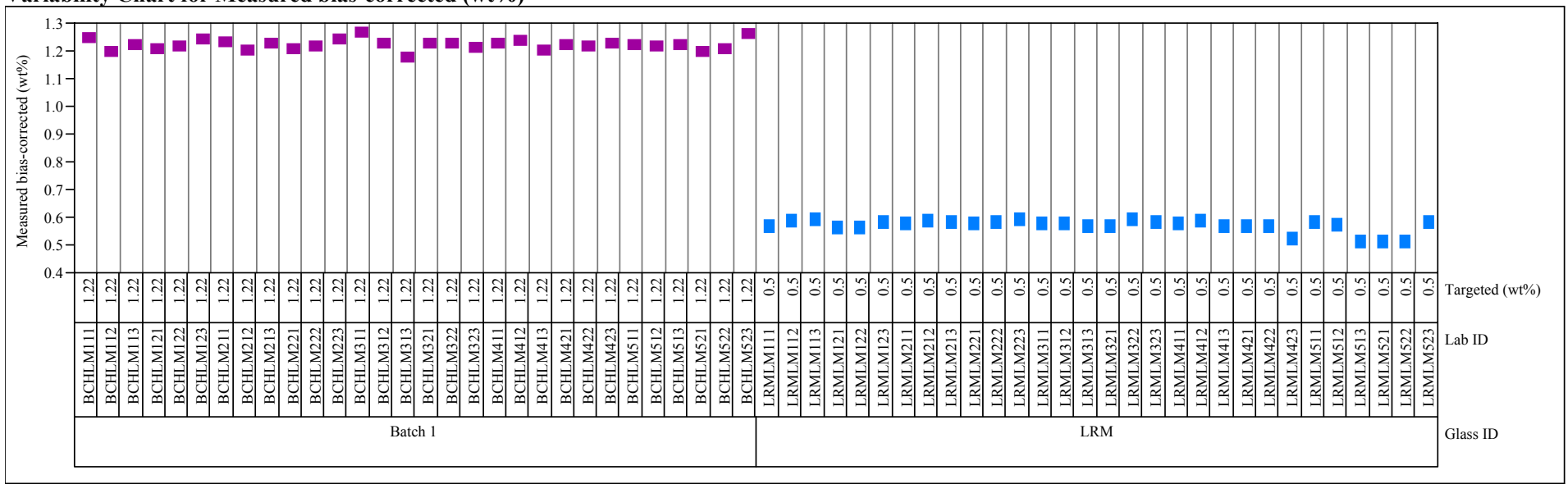


Exhibit A5. Measured and Measured Bias-Corrected Oxide Weight Percents by Lab ID for the Samples Prepared Using the LM Method

Group $=4$, Oxide $=\mathbf{C r} 203(w t \%)$

Variability Chart for Measured (wt\%)

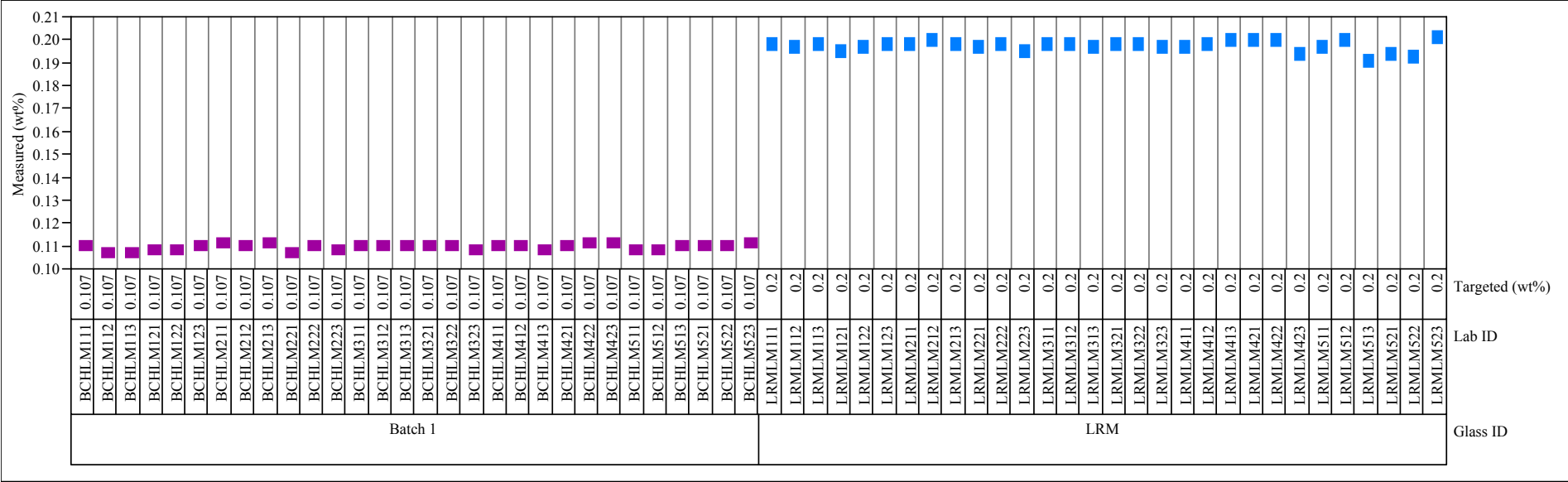

Group $=4$, Oxide $=\mathrm{Cr} 2 \mathrm{O} 3(\mathrm{wt} \%)$

Variability Chart for Measured bias-corrected (wt\%)

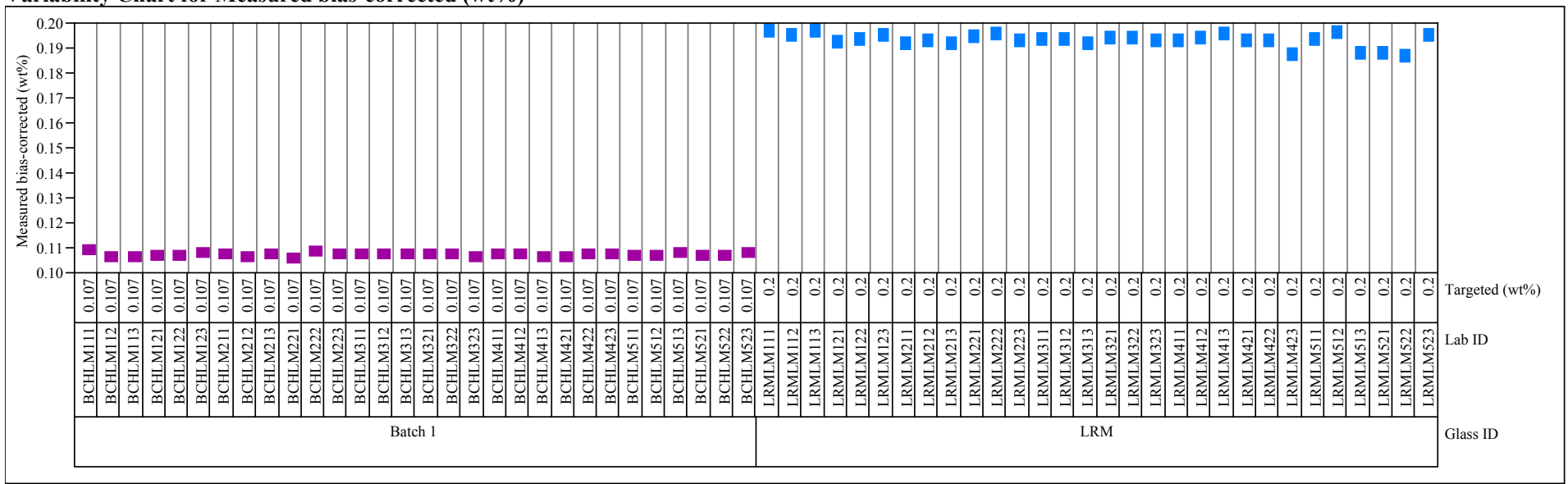


Exhibit A5. Measured and Measured Bias-Corrected Oxide Weight Percents by Lab ID for the Samples Prepared Using the LM Method

Group $=4$, Oxide $=K 20(w t \%)$

Variability Chart for Measured (wt\%)

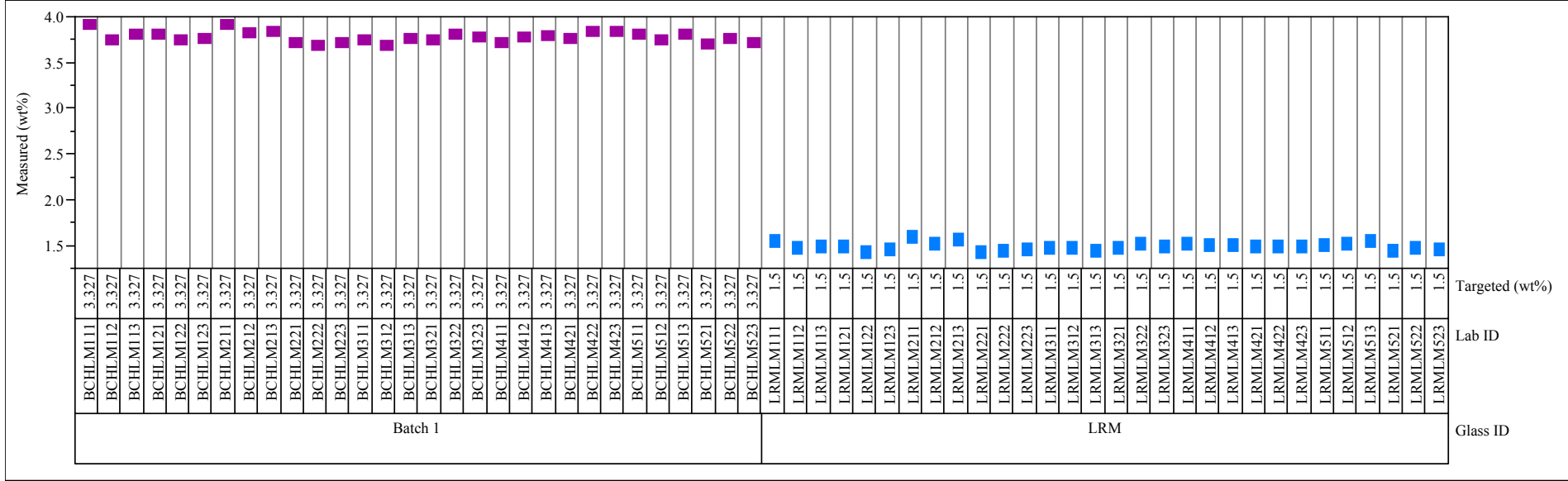

Group $=4$, Oxide $=\mathrm{K} 2 \mathrm{O}(\mathrm{wt} \%)$

Variability Chart for Measured bias-corrected (wt\%)

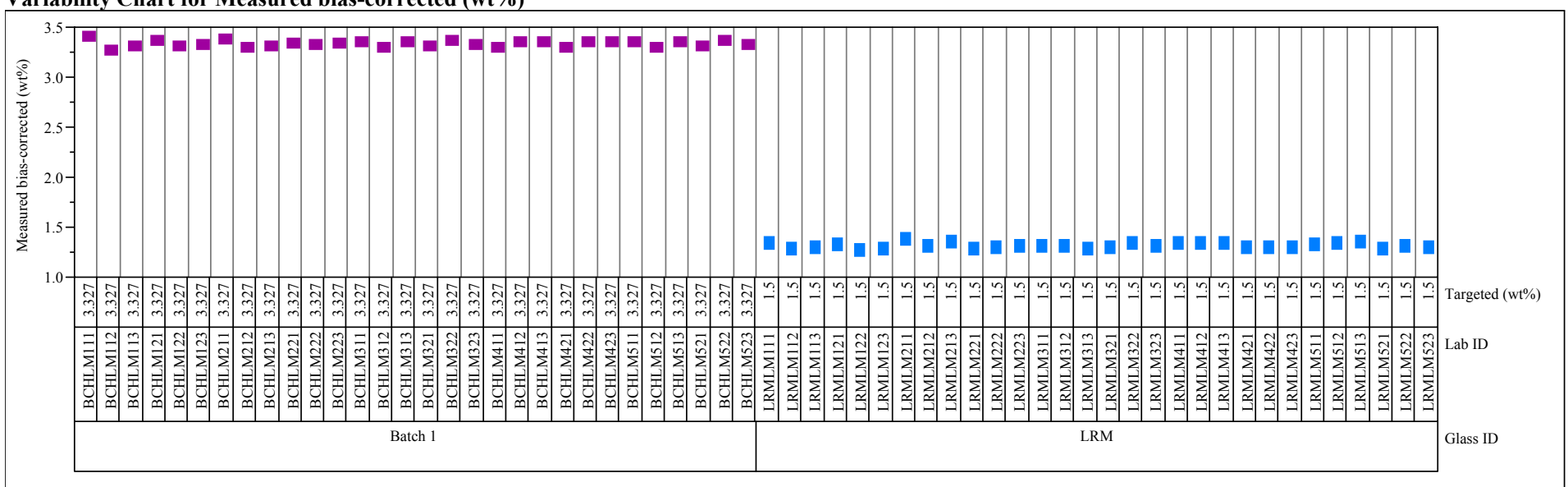


Exhibit A5. Measured and Measured Bias-Corrected Oxide Weight Percents by Lab ID for the Samples Prepared Using the LM Method

Group $=4$, Oxide $=\mathrm{MgO}(\mathrm{wt} \%)$

Variability Chart for Measured (wt\%)

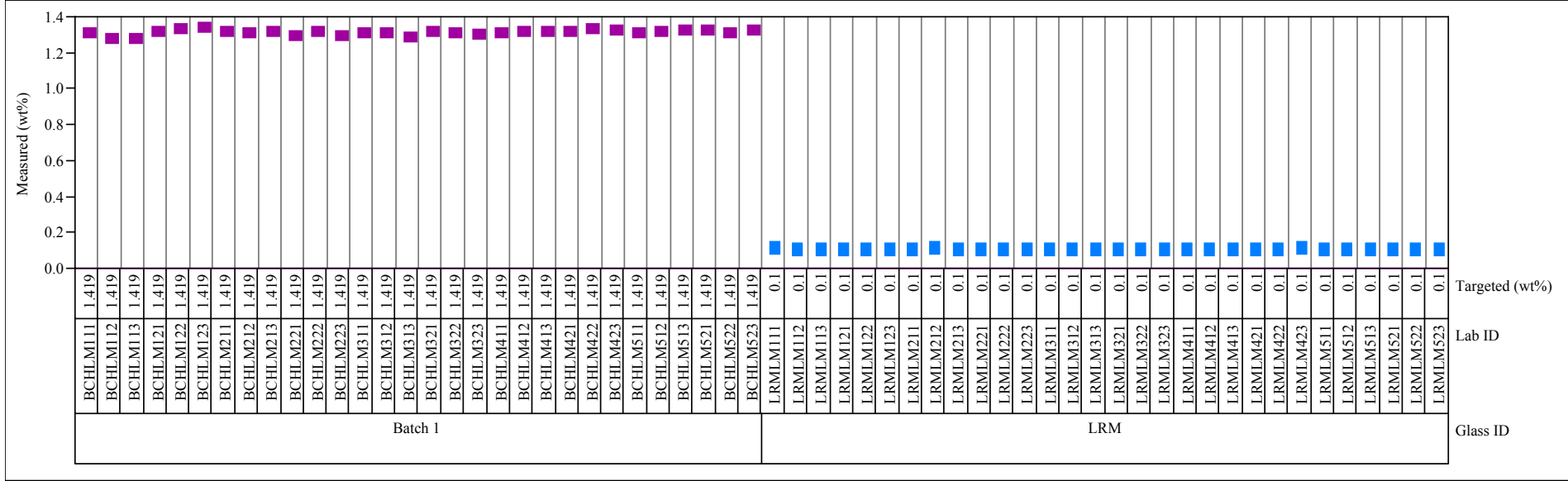

Group $=4$, Oxide $=\operatorname{MgO}($ wt $\%)$

Variability Chart for Measured bias-corrected (wt\%)

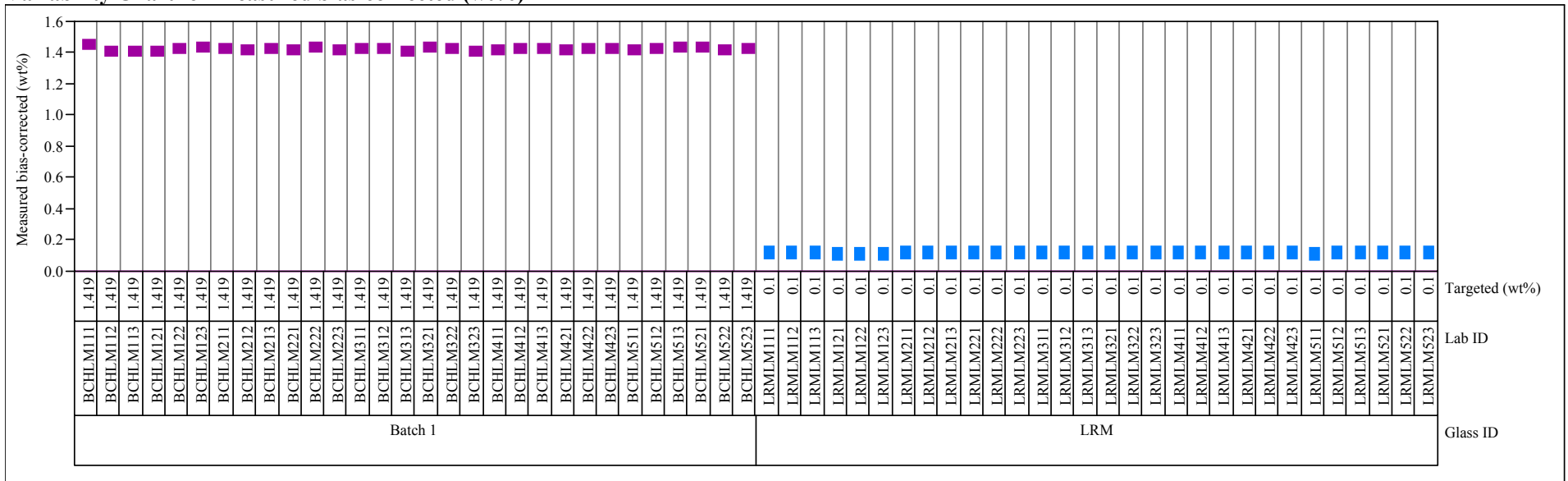


Exhibit A5. Measured and Measured Bias-Corrected Oxide Weight Percents by Lab ID for the Samples Prepared Using the LM Method

Group=4, Oxide= $\mathrm{Na2O}(\mathrm{wt} \%)$

Variability Chart for Measured (wt\%)

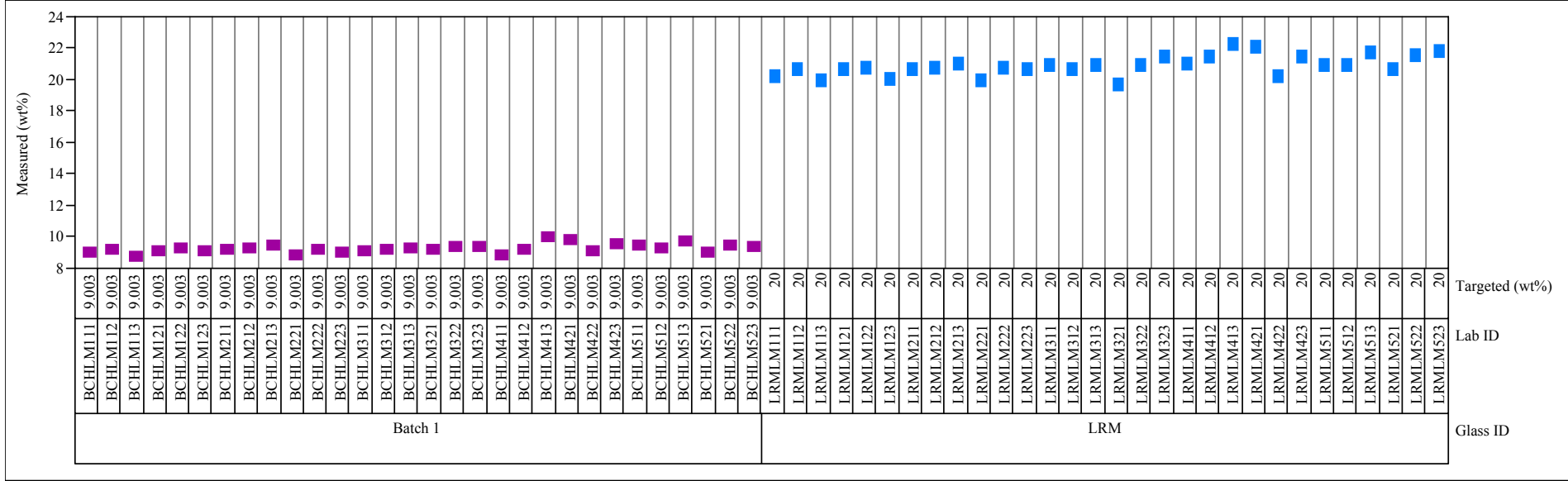

Group $=4$, Oxide $=\mathrm{Na} 2 \mathrm{O}(\mathrm{wt} \%)$

Variability Chart for Measured bias-corrected ( wt $\%$ )

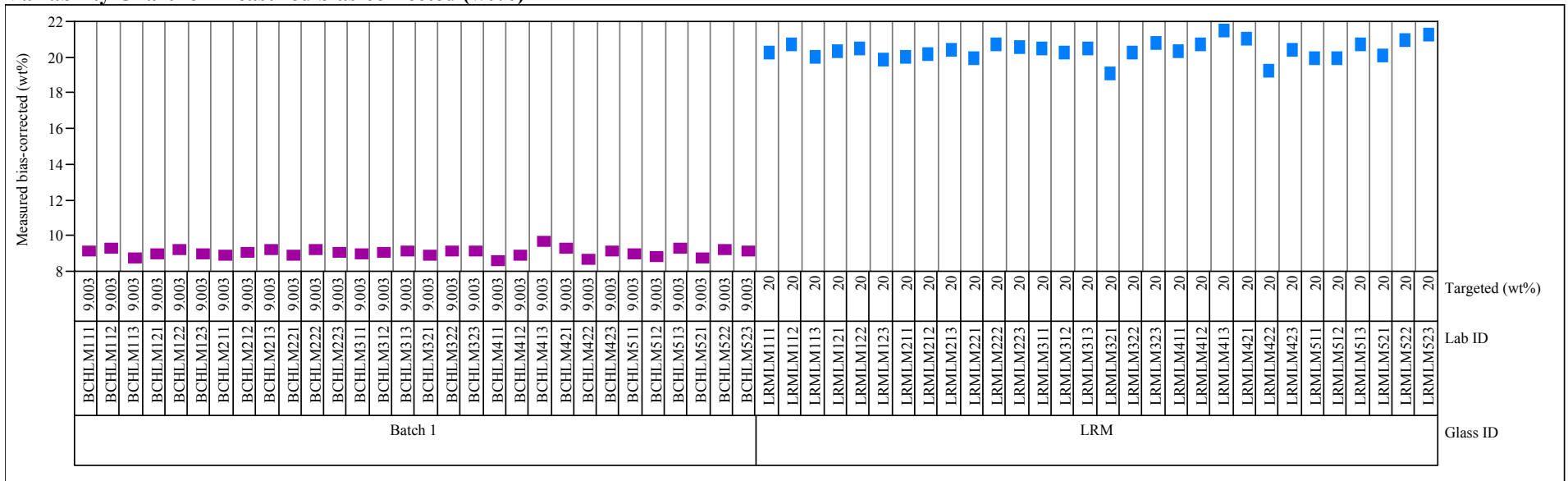


Exhibit A5. Measured and Measured Bias-Corrected Oxide Weight Percents by Lab ID for the Samples Prepared Using the LM Method

Group=4, Oxide $=\mathrm{NiO}($ wt $\%)$

Variability Chart for Measured (wt\%)

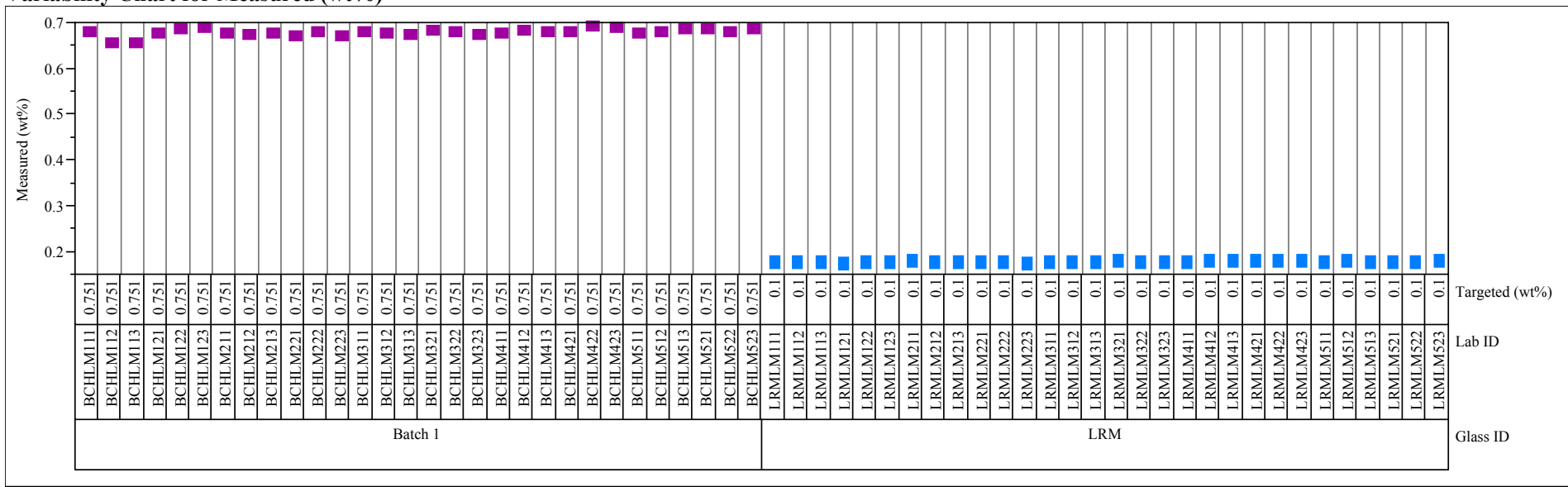

Group $=4$, Oxide $=\mathrm{NiO}($ wt $\%)$

Variability Chart for Measured bias-corrected (wt $\%$ )

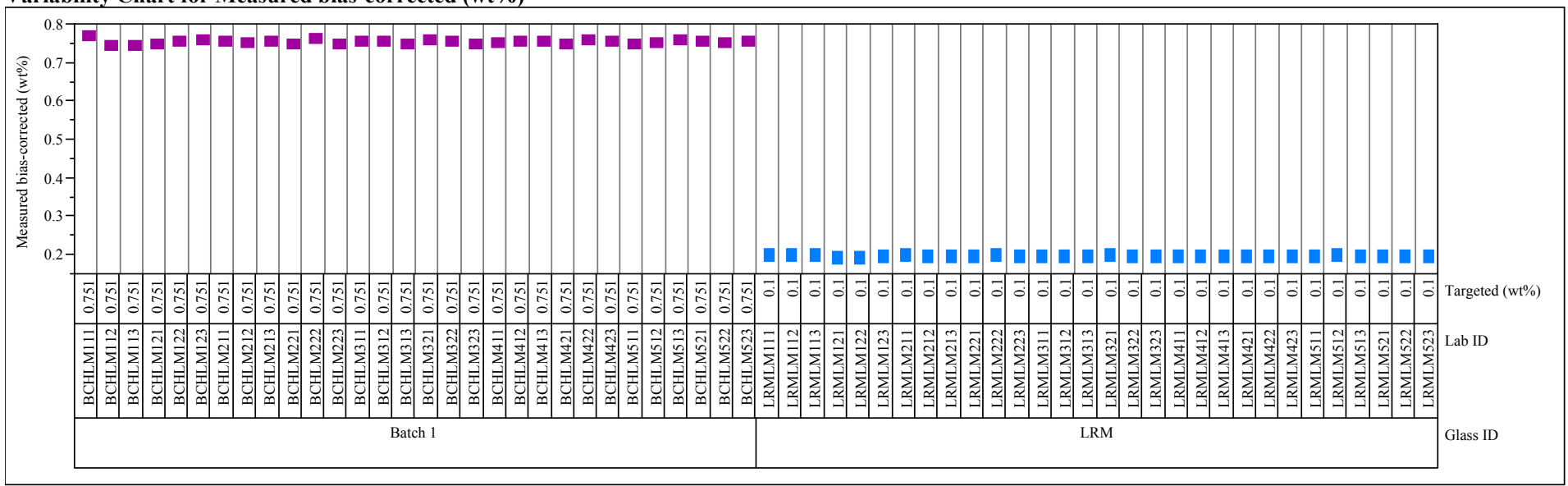


Exhibit A5. Measured and Measured Bias-Corrected Oxide Weight Percents by Lab ID for the Samples Prepared Using the LM Method

Group $=4$, Oxide $=\mathrm{PbO}(\mathrm{wt} \%)$

Variability Chart for Measured (wt\%)

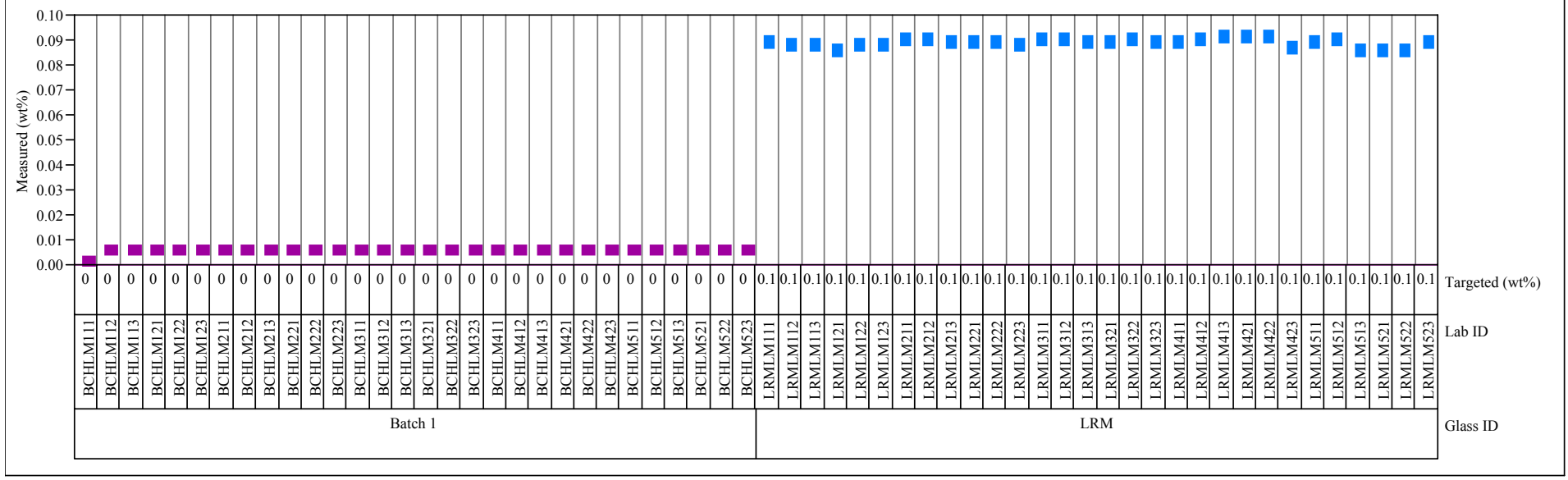

Group $=4$, Oxide $=\mathbf{P b O}($ wt $\%)$

Variability Chart for Measured bias-corrected (wt $\%)$

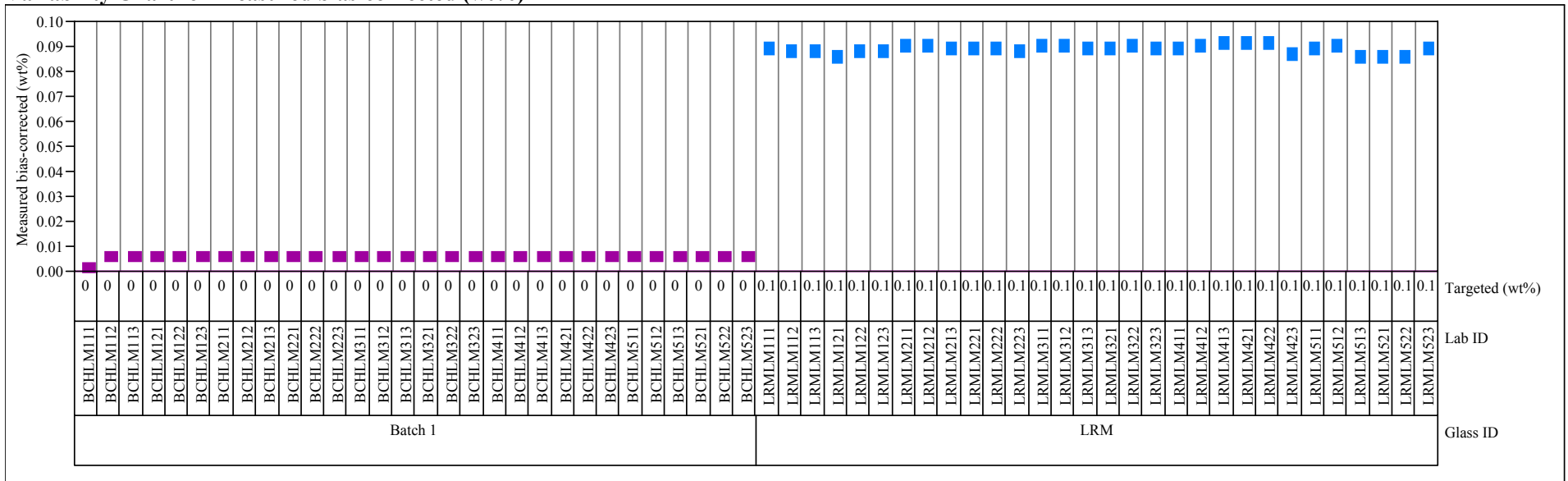


Exhibit A5. Measured and Measured Bias-Corrected Oxide Weight Percents by Lab ID for the Samples Prepared Using the LM Method

Group=4, Oxide=SO3 $(w t \%)$

Variability Chart for Measured (wt\%)

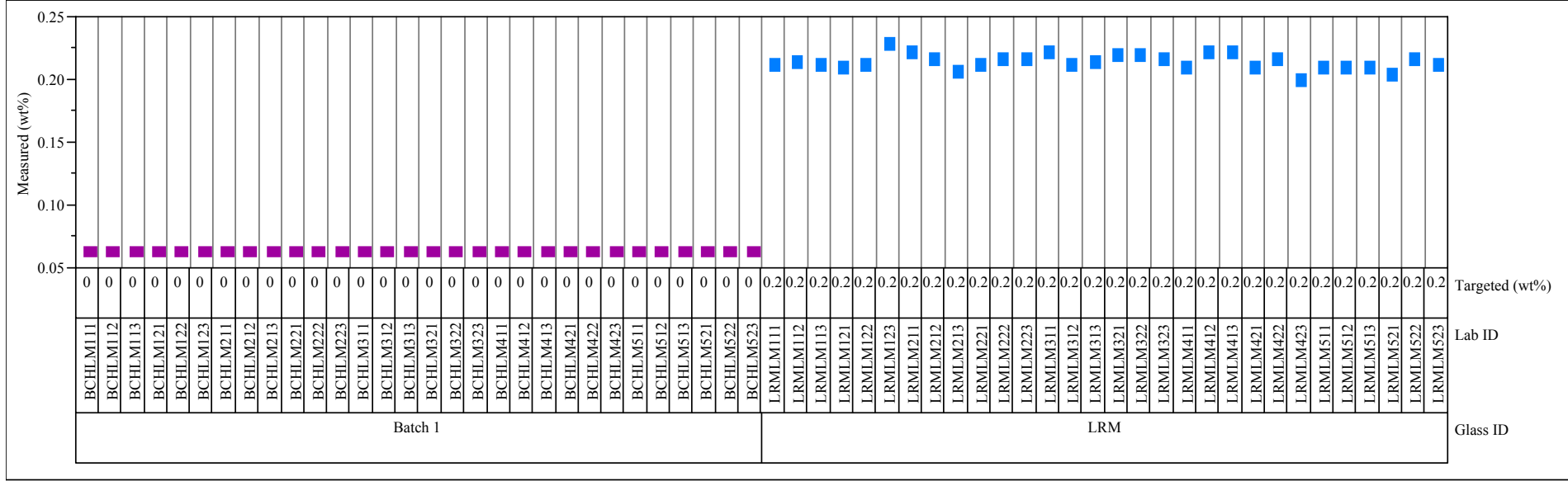

Group $=4$, Oxide=SO3 $($ wt $\%)$

Variability Chart for Measured bias-corrected ( wt $\%$

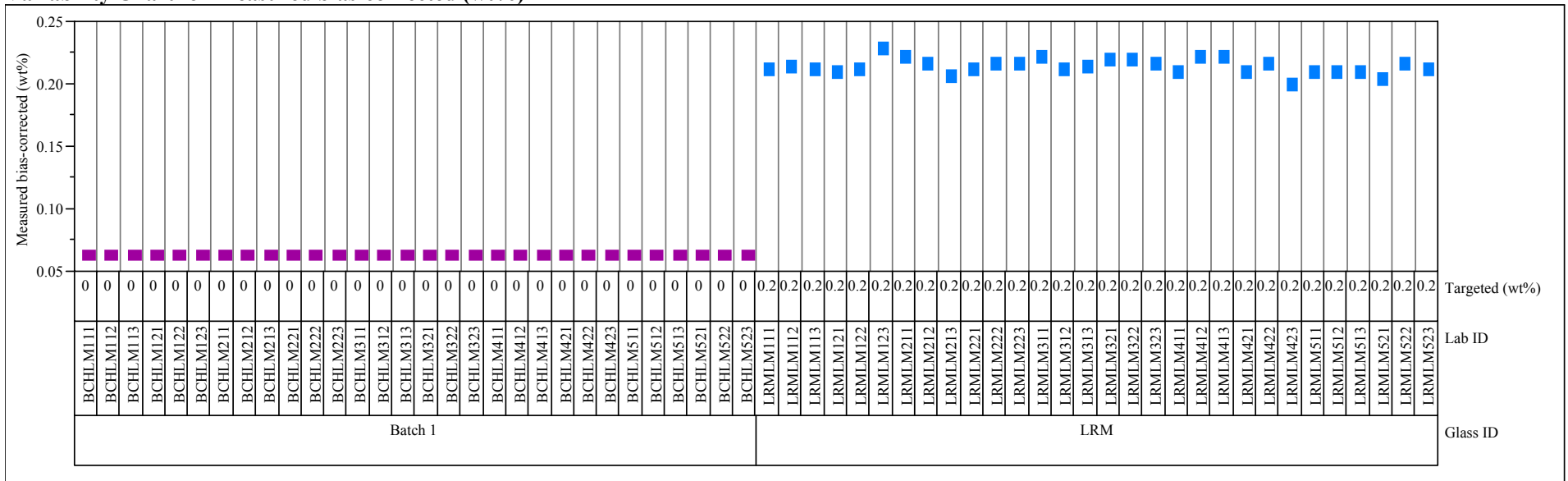


Exhibit A5. Measured and Measured Bias-Corrected Oxide Weight Percents by Lab ID for the Samples Prepared Using the LM Method

Group $=4$, Oxide $=\operatorname{SrO}(\mathbf{w t} \%)$

Variability Chart for Measured (wt\%)

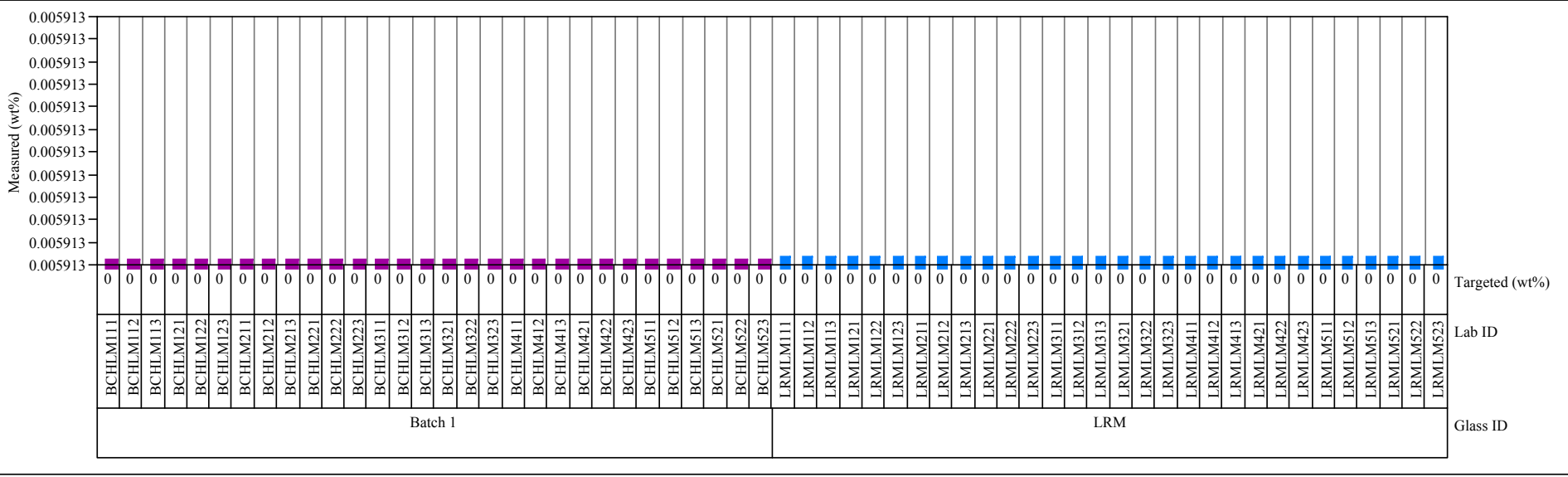

Group $=4$, Oxide=SrO $(w t \%)$

Variability Chart for Measured bias-corrected (wt\%)

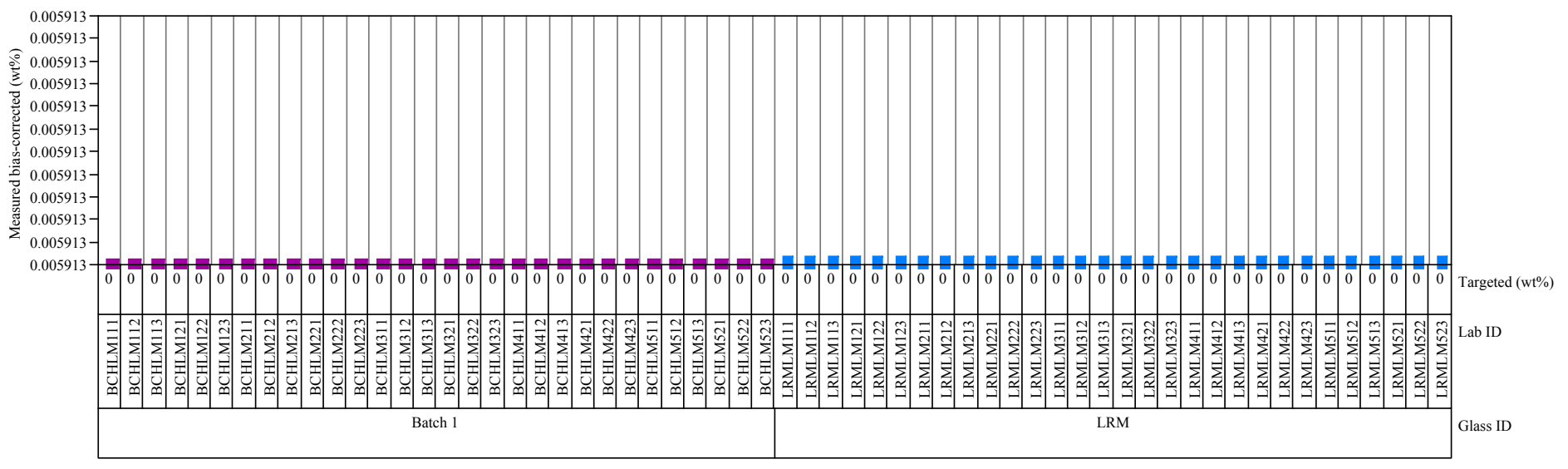


Exhibit A5. Measured and Measured Bias-Corrected Oxide Weight Percents by Lab ID for the Samples Prepared Using the LM Method

Group $=4$, Oxide $=\mathrm{TiO} 2($ wt $\%)$

Variability Chart for Measured (wt\%)

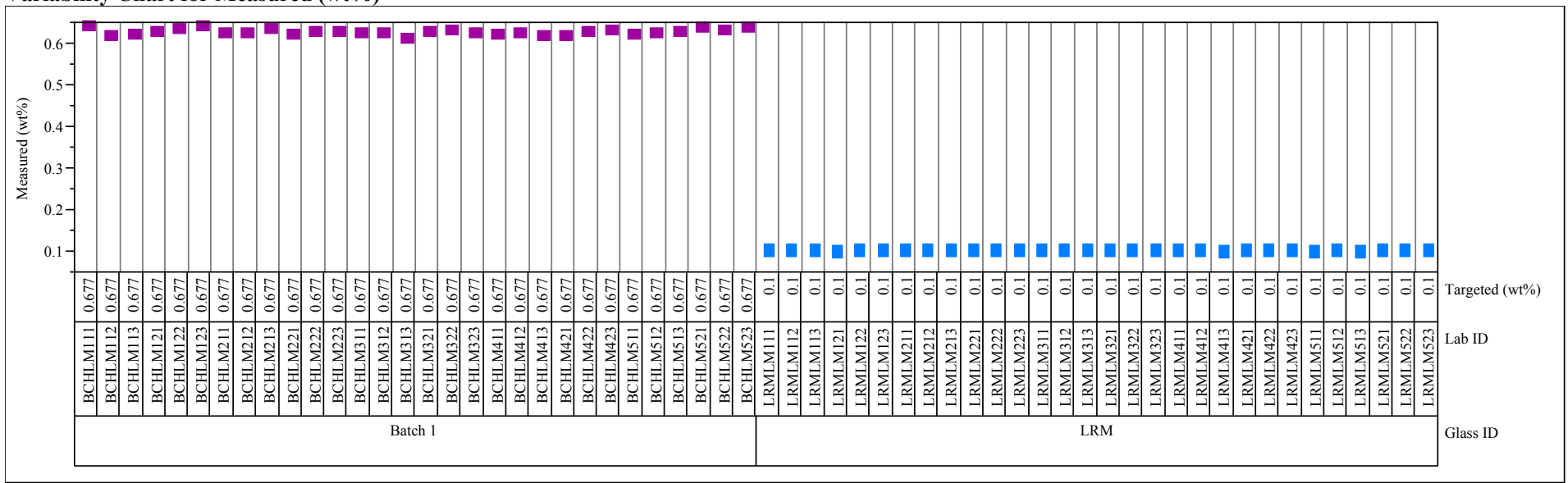

Group=4, Oxide=TiO2 $($ wt $\%)$

Variability Chart for Measured bias-corrected ( $\mathbf{w t} \%$

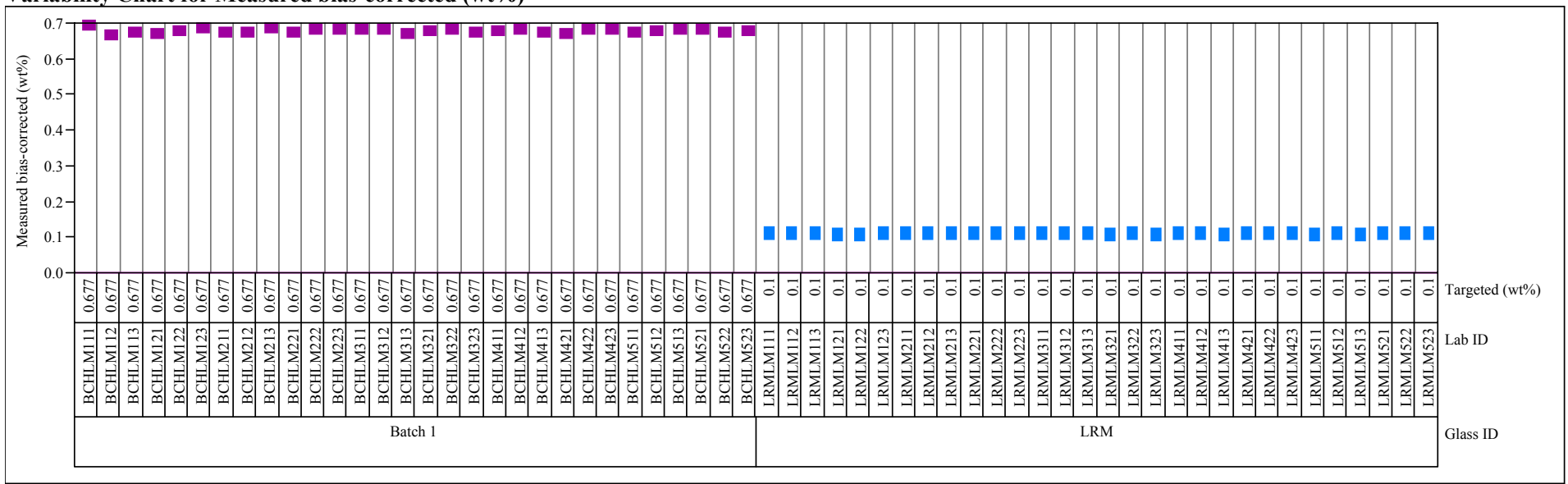


Exhibit A5. Measured and Measured Bias-Corrected Oxide Weight Percents by Lab ID for the Samples Prepared Using the LM Method

Group $=4$, Oxide $=\mathrm{ZnO}($ wt $\%)$

Variability Chart for Measured (wt\%)

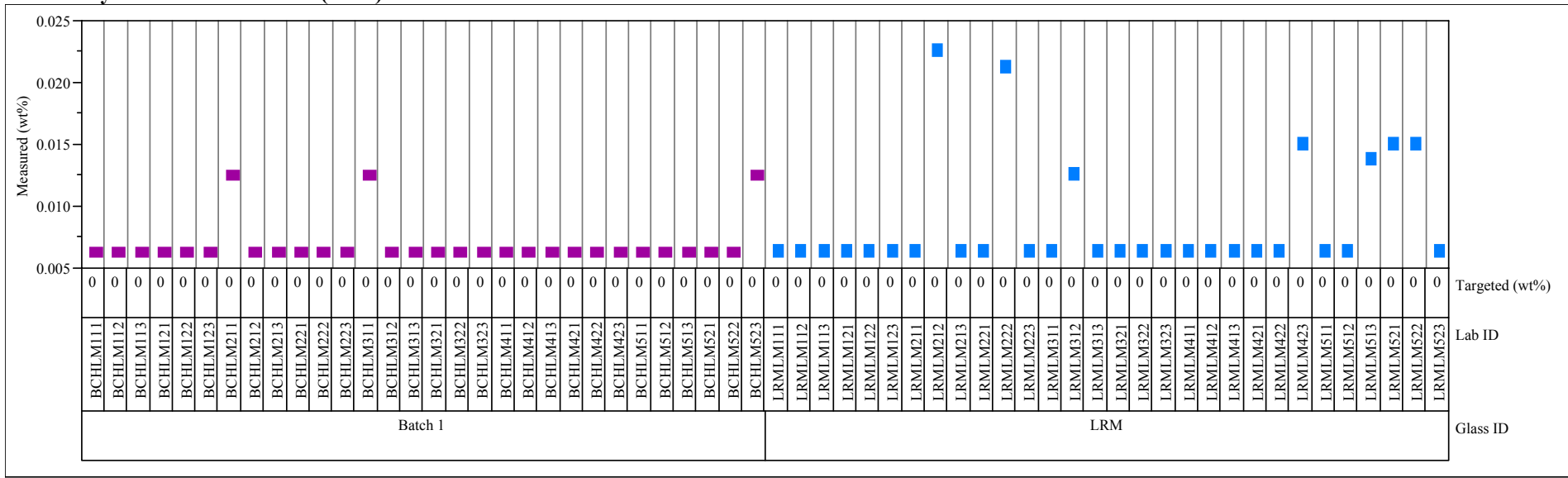

Group $=4$, Oxide $=\mathrm{ZnO}($ wt $\%)$

Variability Chart for Measured bias-corrected (wt\%)

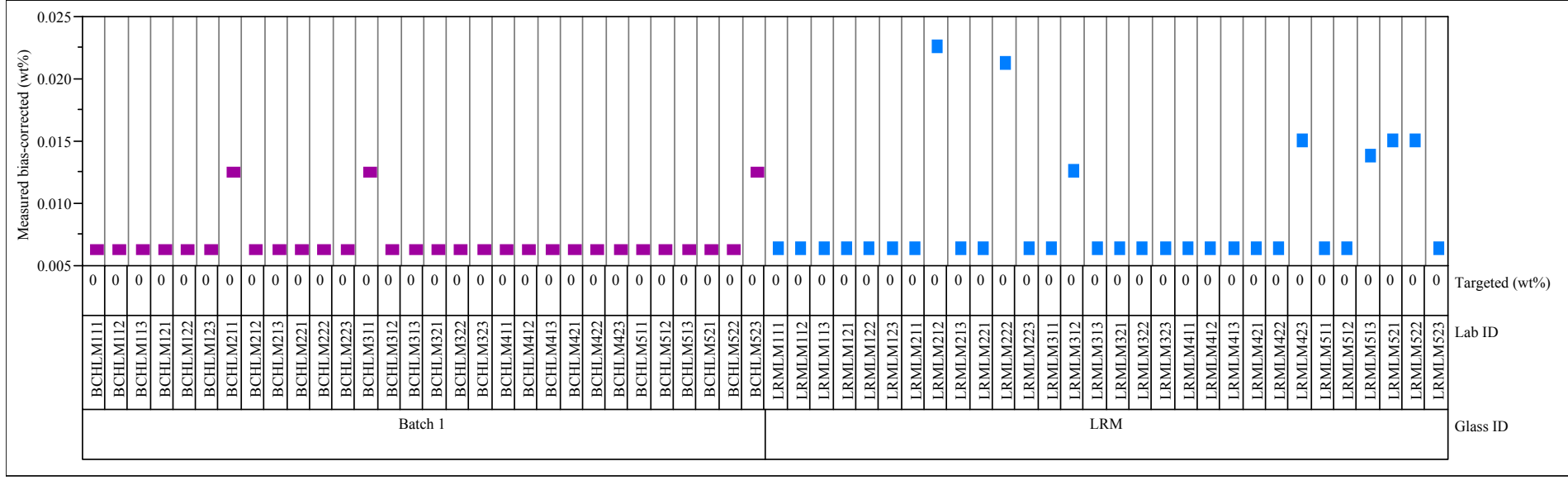


Exhibit A5. Measured and Measured Bias-Corrected Oxide Weight Percents by Lab ID for the Samples Prepared Using the LM Method

Group $=4$, Oxide $=\mathrm{ZrO2}(\mathrm{wt} \%)$

Variability Chart for Measured (wt\%)

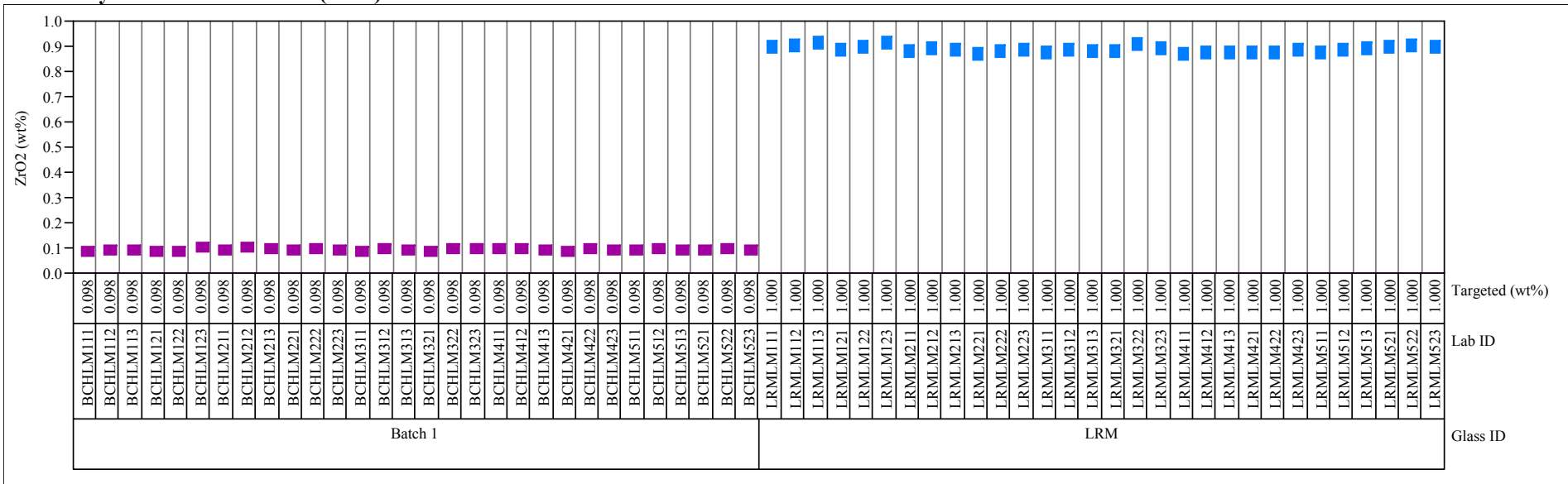

Group $=4$, Oxide $=\mathrm{ZrO2}\left(\mathrm{wt}^{\mathrm{\%}} \mathrm{)}\right)$

Variability Chart for Measured bias-corrected (wt\%)

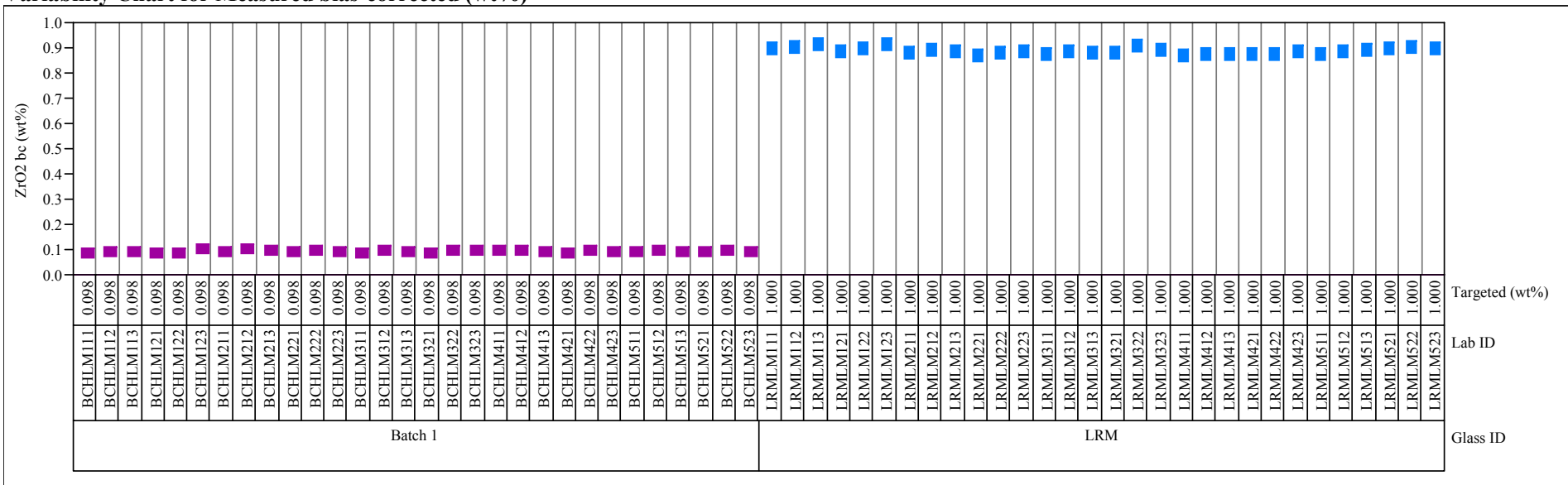


Exhibit A6. Measured and Measured Bias-Corrected Oxide Weight Percents by Lab ID for the Samples Prepared Using the PF Method

Group $=1$, Oxide $=\mathrm{Al} 2 \mathrm{O} 3(\mathrm{wt} \%)$

Variability Chart for Measured (wt\%)

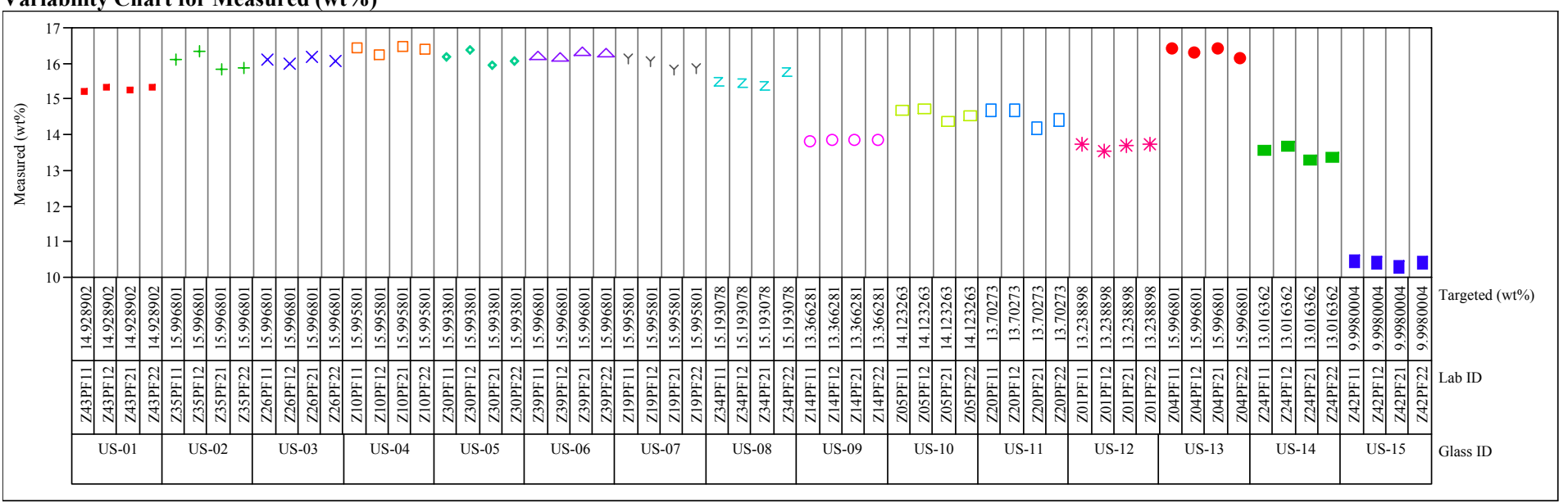

Group $=1$, Oxide $=\mathrm{Al2O}(\mathrm{wt} \%)$

Variability Chart for Measured bias-corrected (wt\%)

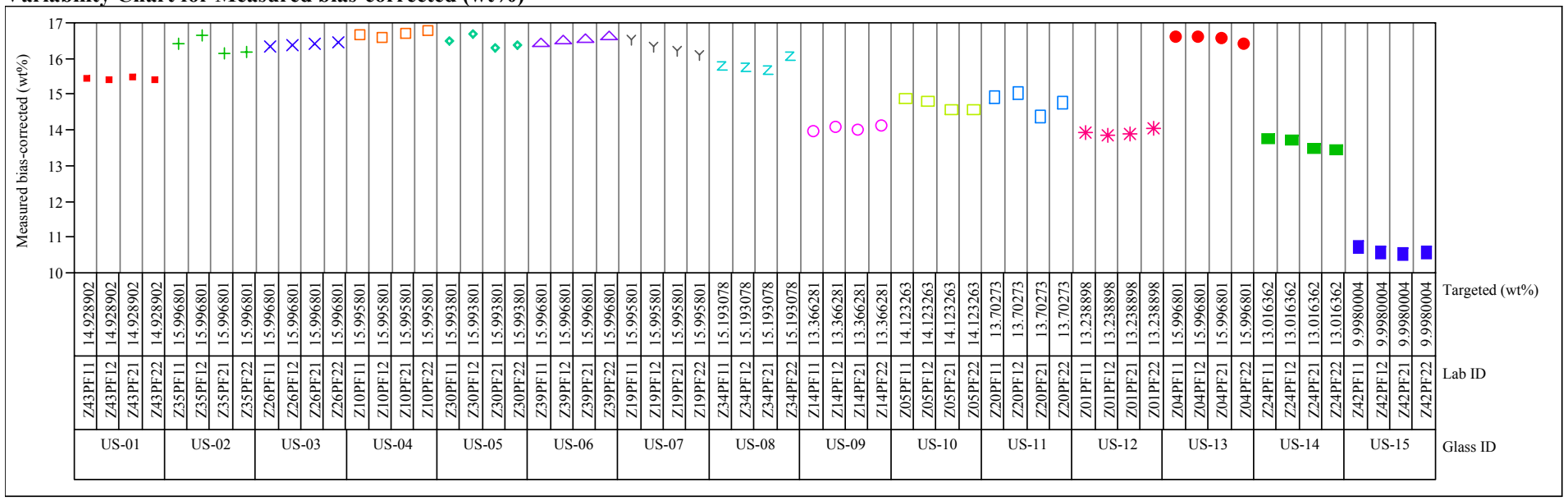


Exhibit A6. Measured and Measured Bias-Corrected Oxide Weight Percents by Lab ID for the Samples Prepared Using the PF Method

Group $=1$, Oxide $=\mathbf{B 2 O 3}($ wt $\%)$

Variability Chart for Measured (wt \%)

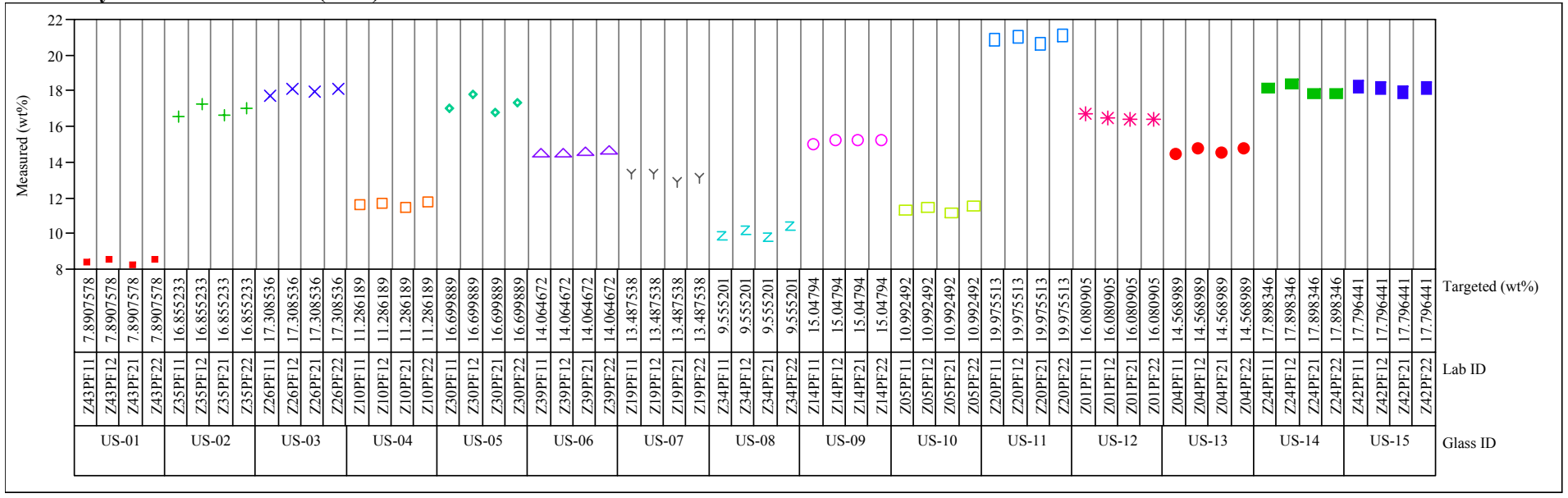

Group $=1$, Oxide $=B 203(w t \%)$

Variability Chart for Measured bias-corrected (wt\%)

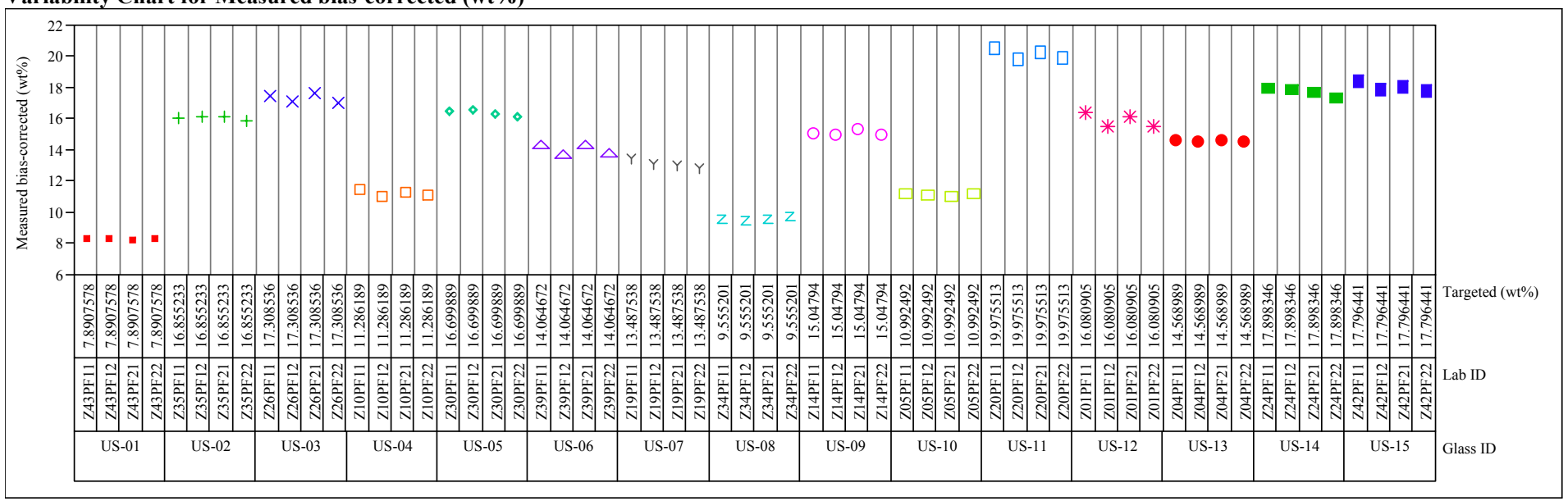


Exhibit A6. Measured and Measured Bias-Corrected Oxide Weight Percents by Lab ID for the Samples Prepared Using the PF Method

Group $=1$, Oxide $=\mathrm{Fe} 2 \mathrm{O} 3(\mathrm{wt} \%)$

Variability Chart for Measured (wt \%)

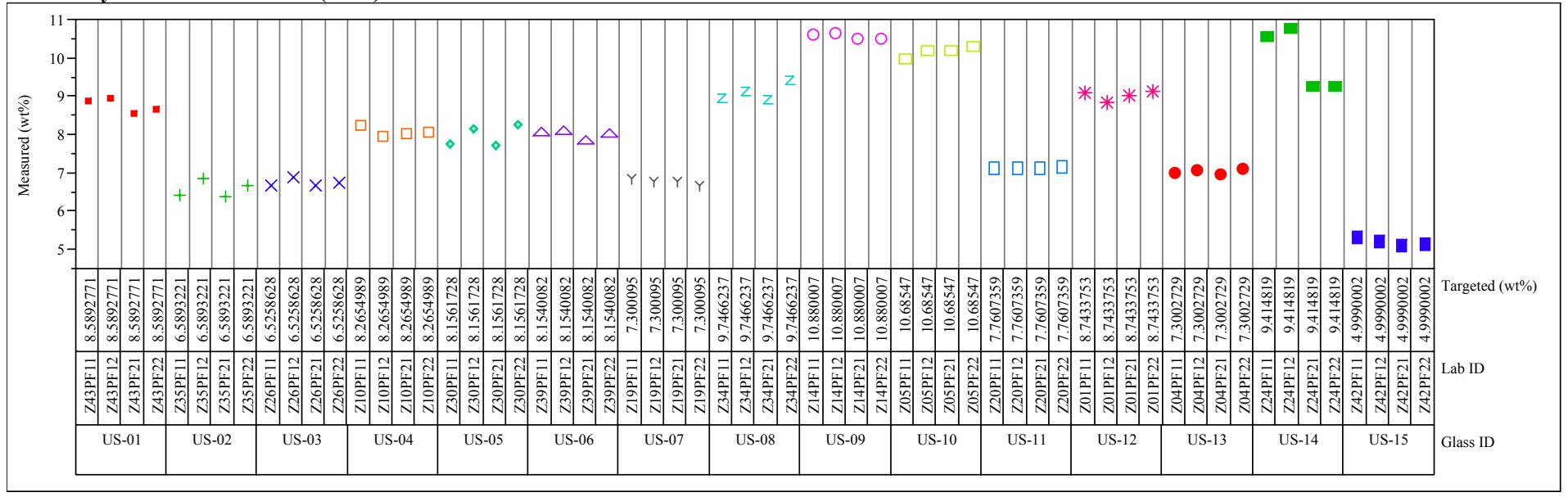

Group=1, Oxide $=\mathrm{Fe} 2 \mathrm{O} 3(\mathrm{wt} \%)$

Variability Chart for Measured bias-corrected (wt\%)

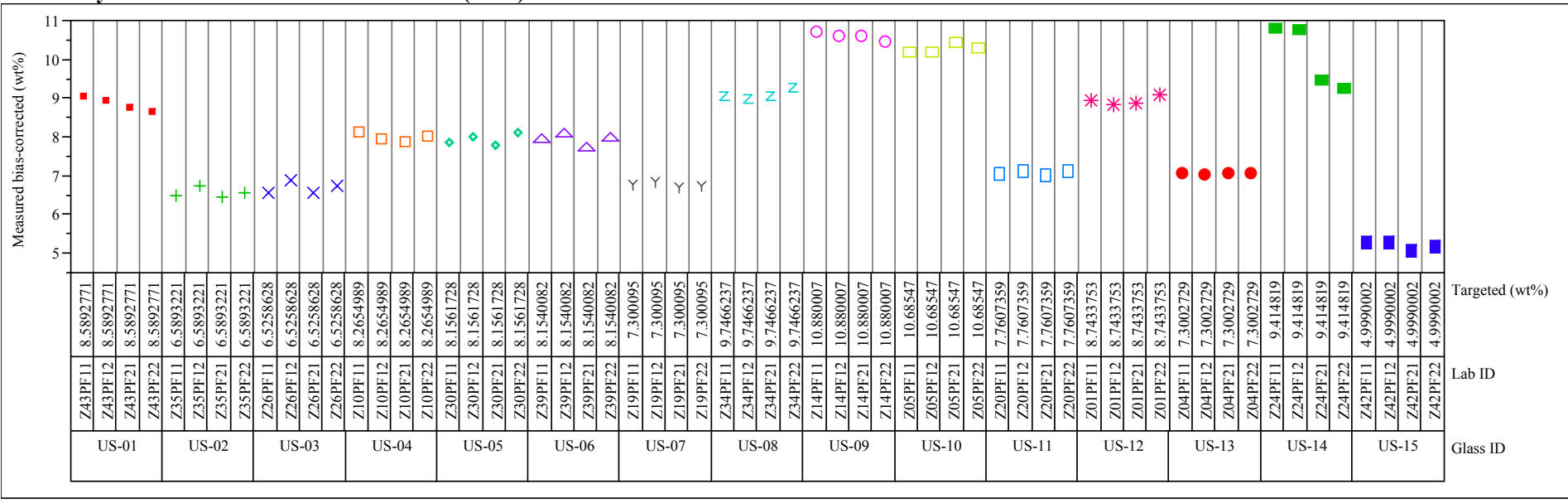


Exhibit A6. Measured and Measured Bias-Corrected Oxide Weight Percents by Lab ID for the Samples Prepared Using the PF Method

Group $=1$, Oxide $=\mathrm{Li} 20($ wt $\%)$

Variability Chart for Measured (wt \%)

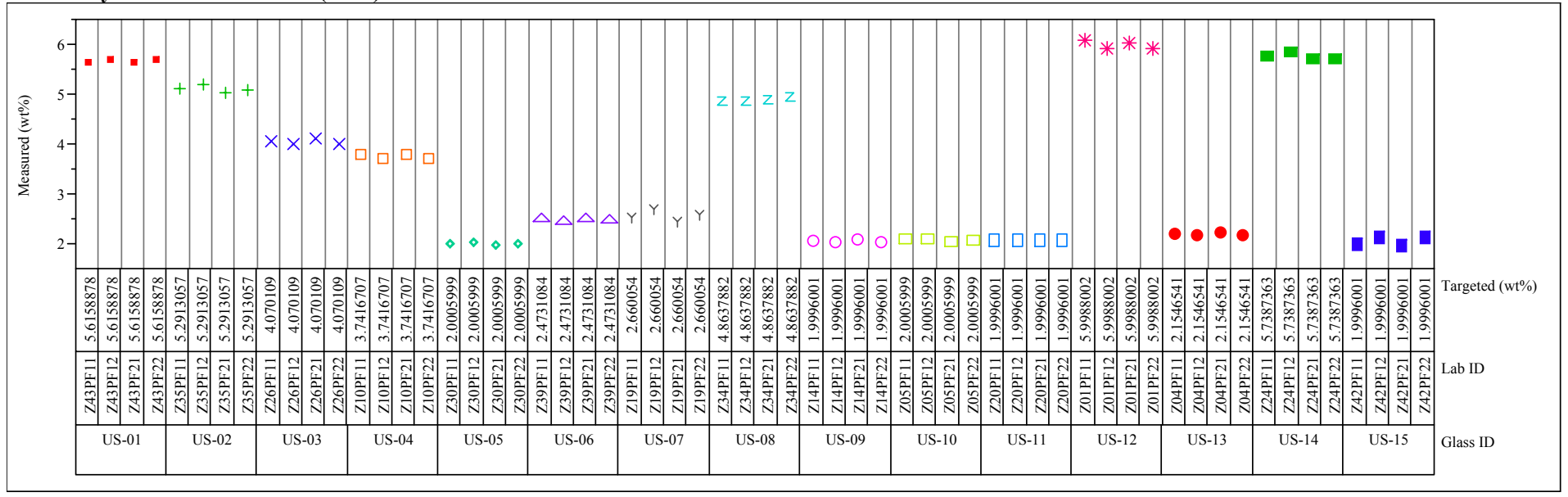

Group $=1$, Oxide $=$ Li2O (wt $\%)$

Variability Chart for Measured bias-corrected (wt\%)

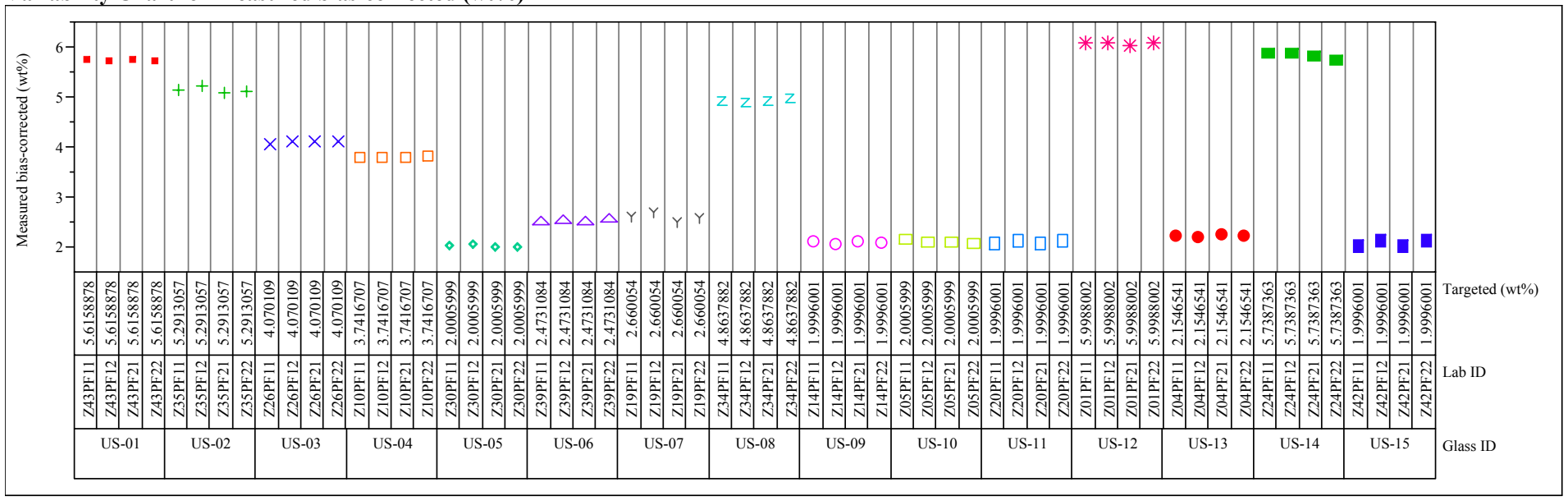


Exhibit A6. Measured and Measured Bias-Corrected Oxide Weight Percents by Lab ID for the Samples Prepared Using the PF Method

Group $=1$, Oxide $=\mathrm{MnO}(\mathrm{wt} \%)$

Variability Chart for Measured (wt \%)

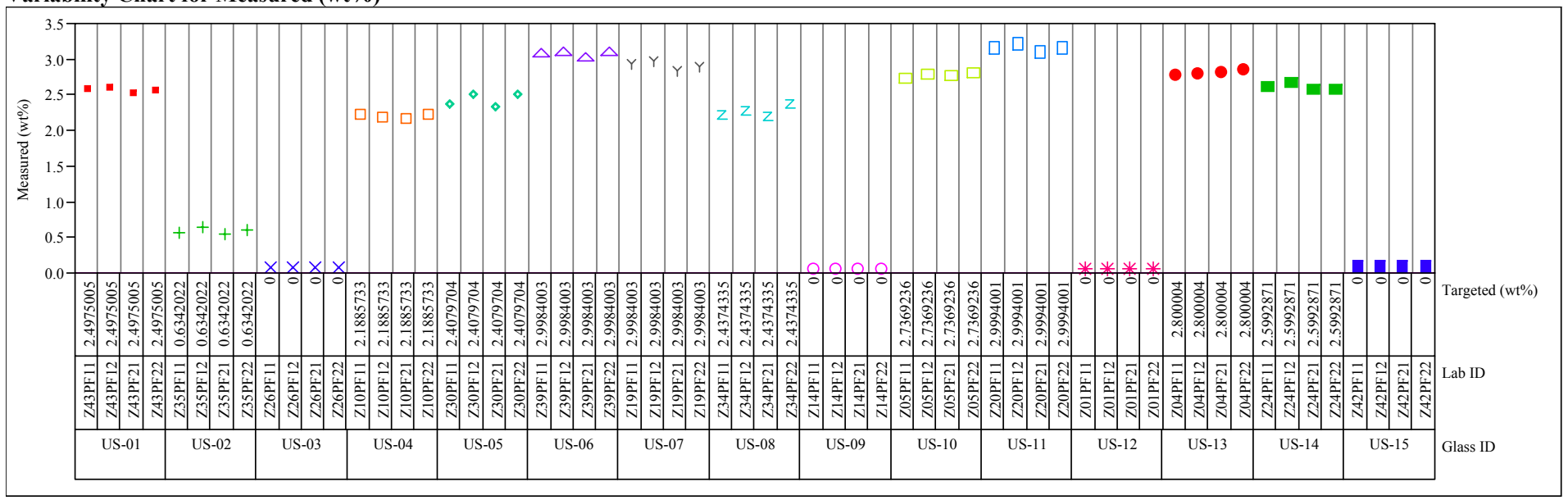

Group $=1$, Oxide $=\mathrm{MnO}(\mathbf{w t} \%)$

Variability Chart for Measured bias-corrected (wt\%)

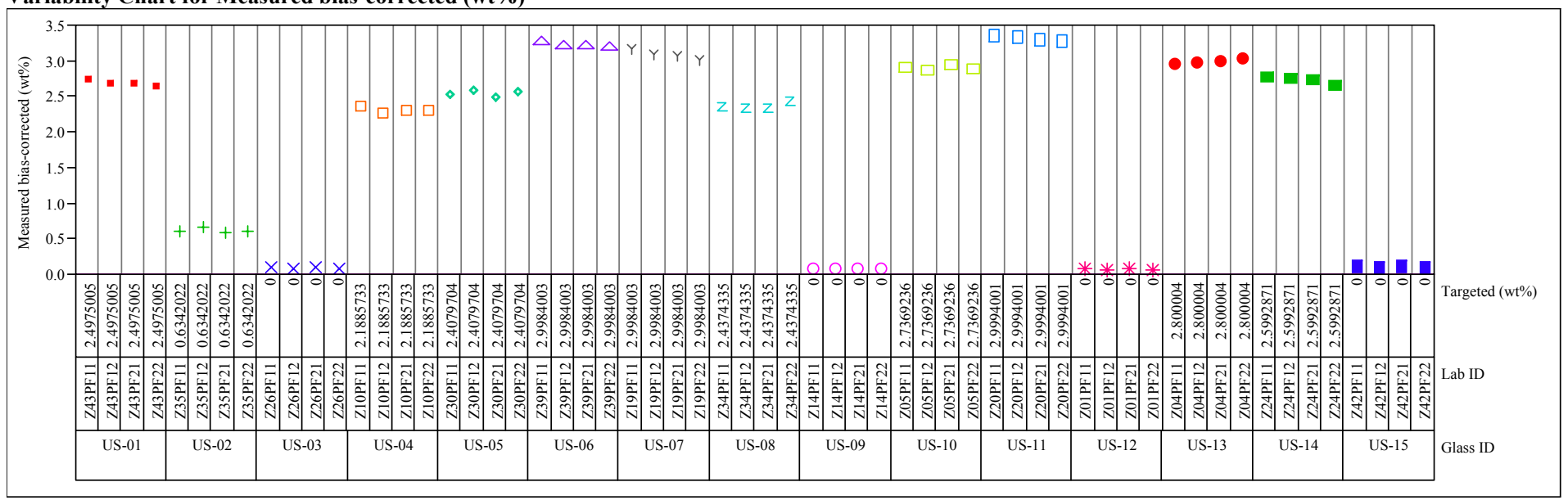


Exhibit A6. Measured and Measured Bias-Corrected Oxide Weight Percents by Lab ID for the Samples Prepared Using the PF Method

Group $=1$, Oxide $=\mathrm{SiO} 2($ wt $\%)$

Variability Chart for Measured (wt \%)

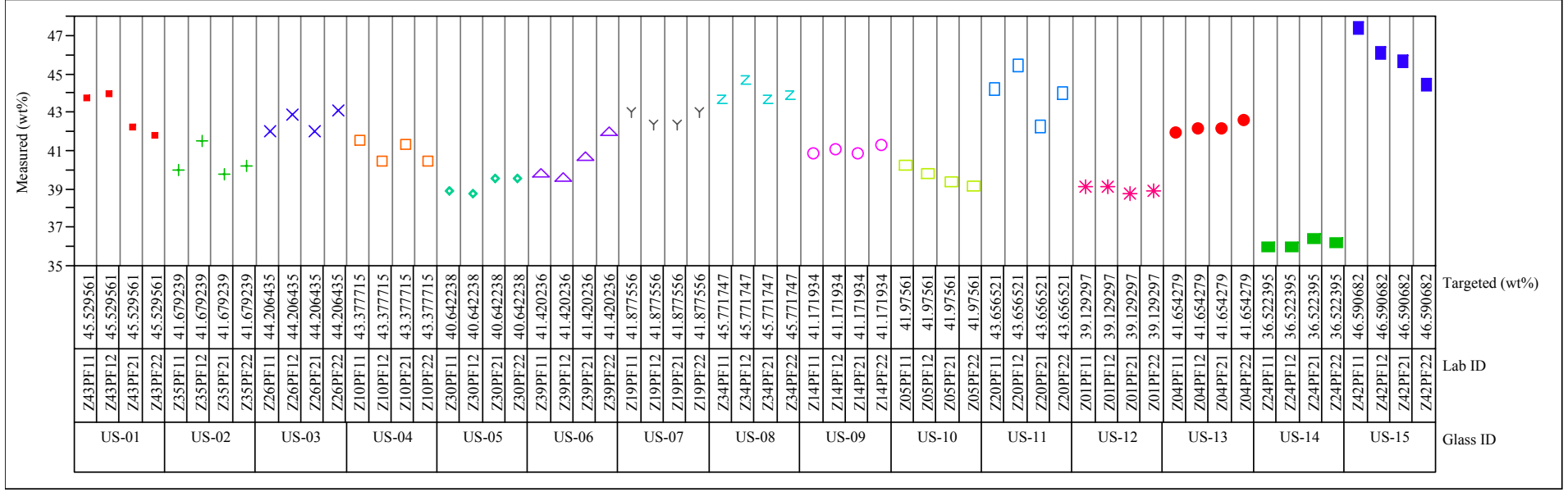

Group $=1$, Oxide $=\mathrm{SiO} 2(\mathrm{wt} \%)$

Variability Chart for Measured bias-corrected (wt\%)

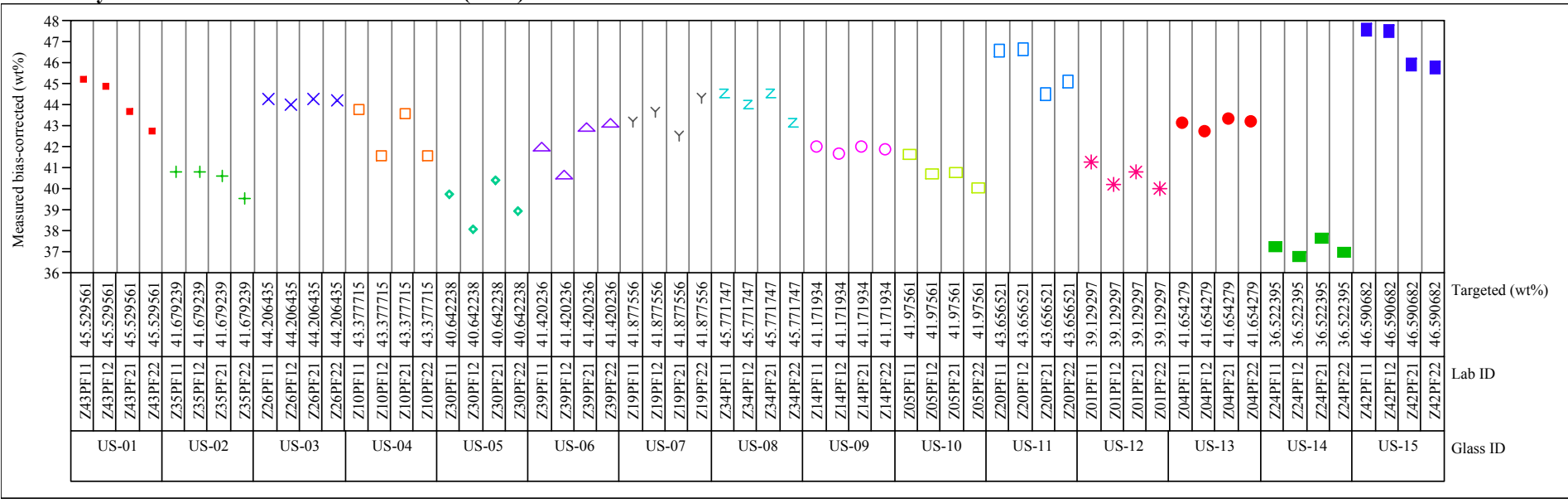


Exhibit A6. Measured and Measured Bias-Corrected Oxide Weight Percents by Lab ID for the Samples Prepared Using the PF Method

Group $=2$, Oxide $=\mathrm{Al} 2 \mathrm{O} 3(\mathrm{wt} \%)$

Variability Chart for Measured (wt\%)

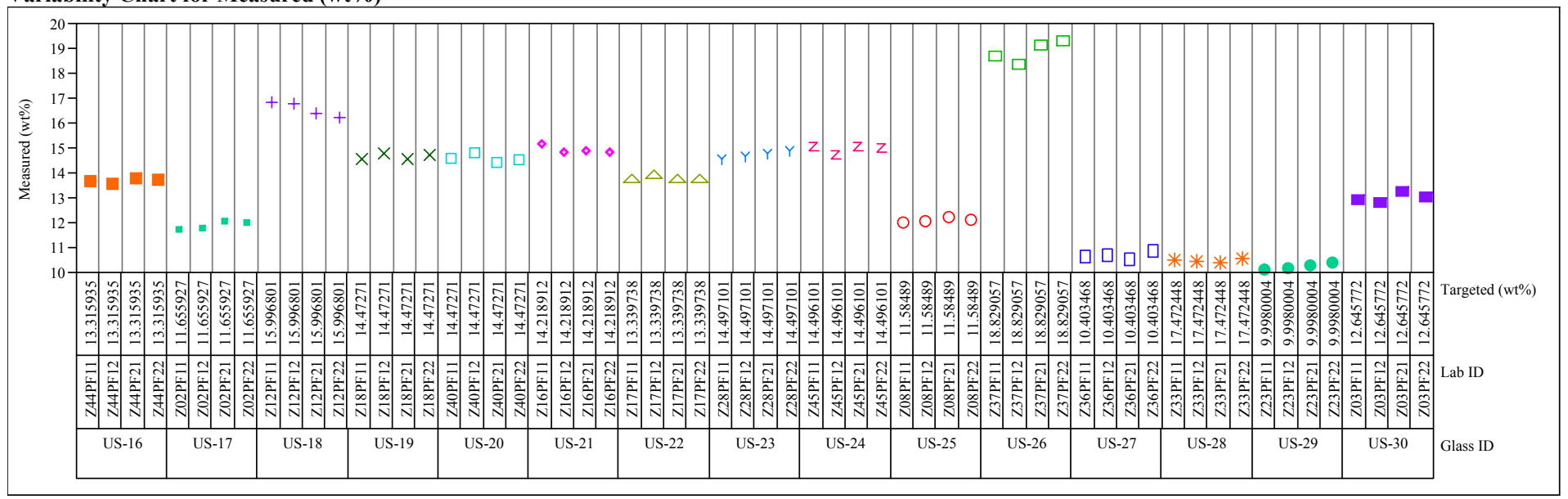

Group $=2$, Oxide $=\mathrm{Al} 203(\mathrm{wt} \%)$

Variability Chart for Measured bias-corrected (wt\%)

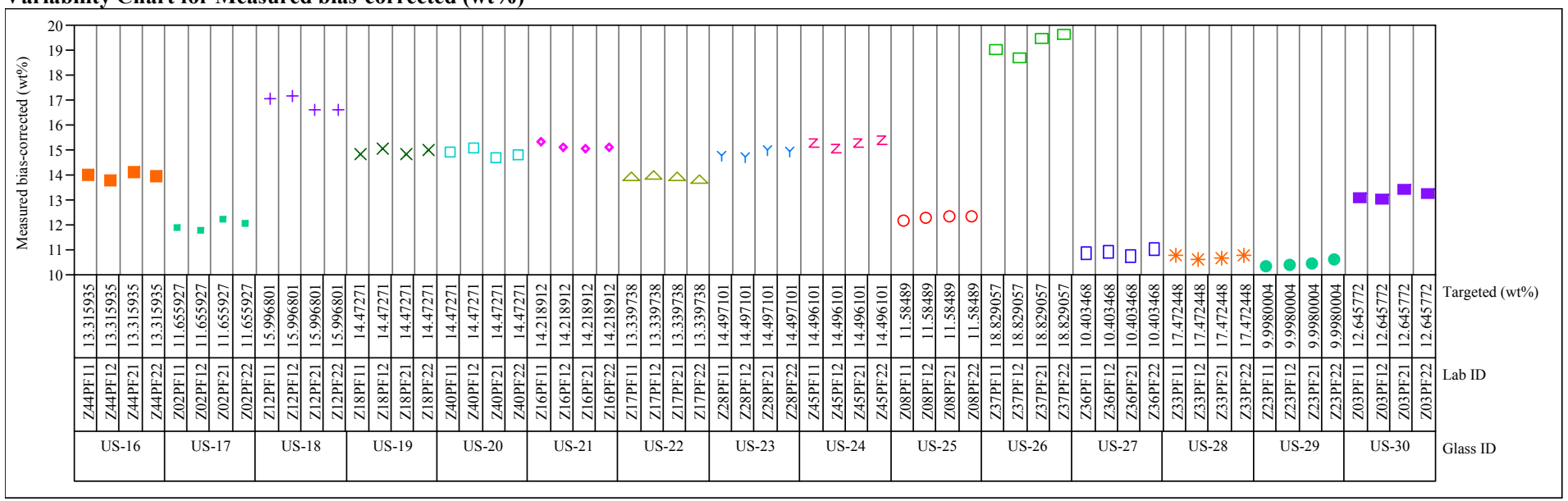


Exhibit A6. Measured and Measured Bias-Corrected Oxide Weight Percents by Lab ID for the Samples Prepared Using the PF Method

Group $=2$, Oxide $=\mathbf{B 2 O 3}($ wt $\%)$

Variability Chart for Measured (wt \%)

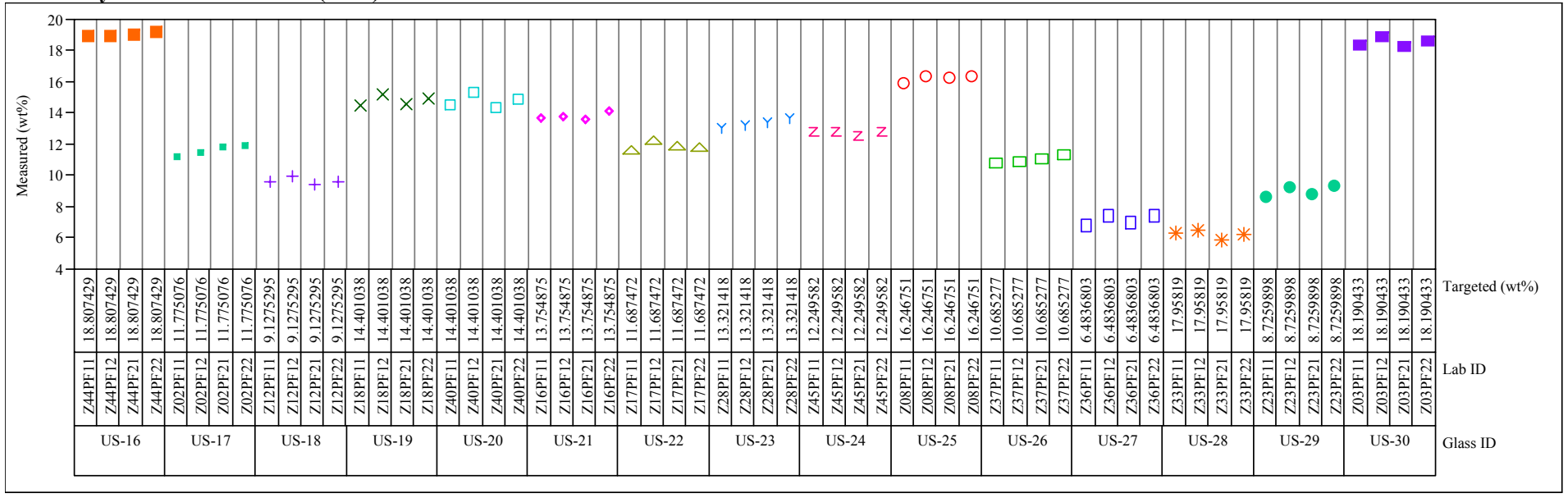

Group $=2$, Oxide $=\mathbf{B 2 O 3}(\mathbf{w t} \%)$

Variability Chart for Measured bias-corrected (wt\%)

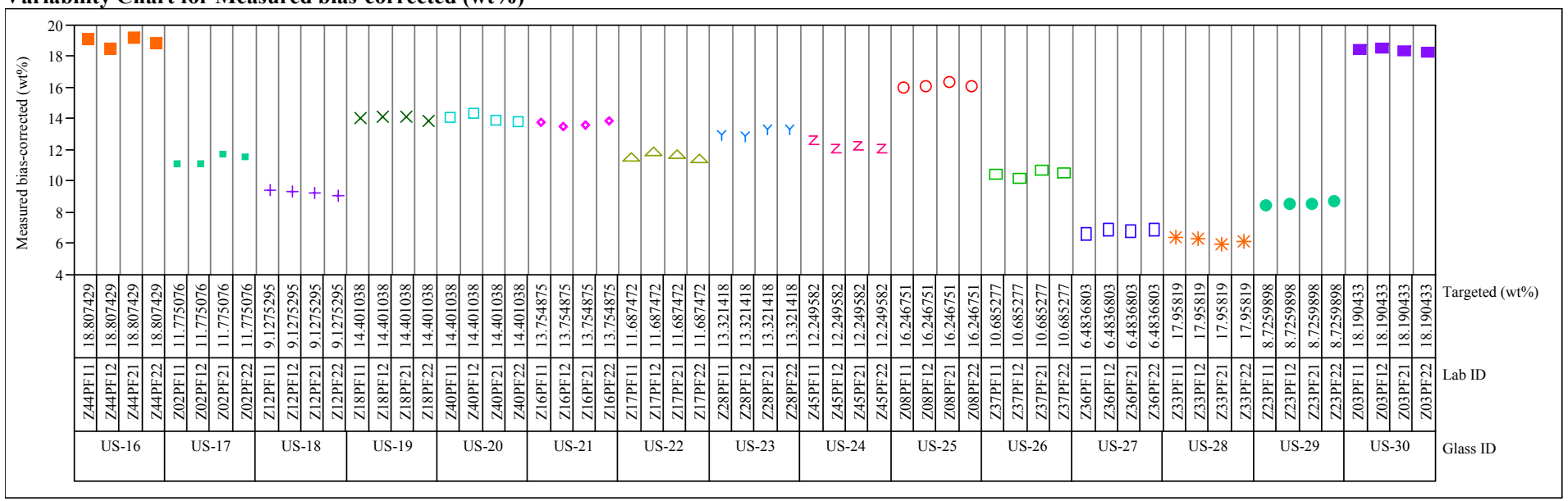


Exhibit A6. Measured and Measured Bias-Corrected Oxide Weight Percents by Lab ID for the Samples Prepared Using the PF Method

Group $=2$, Oxide $=\mathrm{Fe} 2 \mathrm{O} 3(\mathrm{wt} \%)$

Variability Chart for Measured (wt \%)

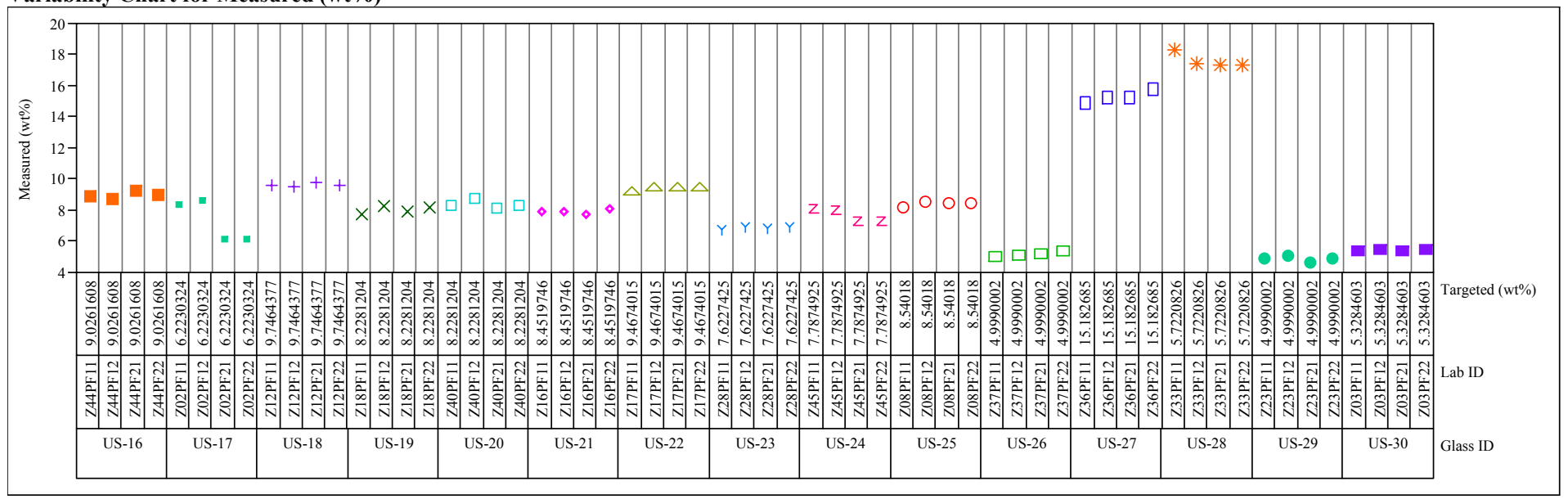

Group $=2$, Oxide $=$ Fe2O3 $($ wt $\%)$

Variability Chart for Measured bias-corrected (wt\%)

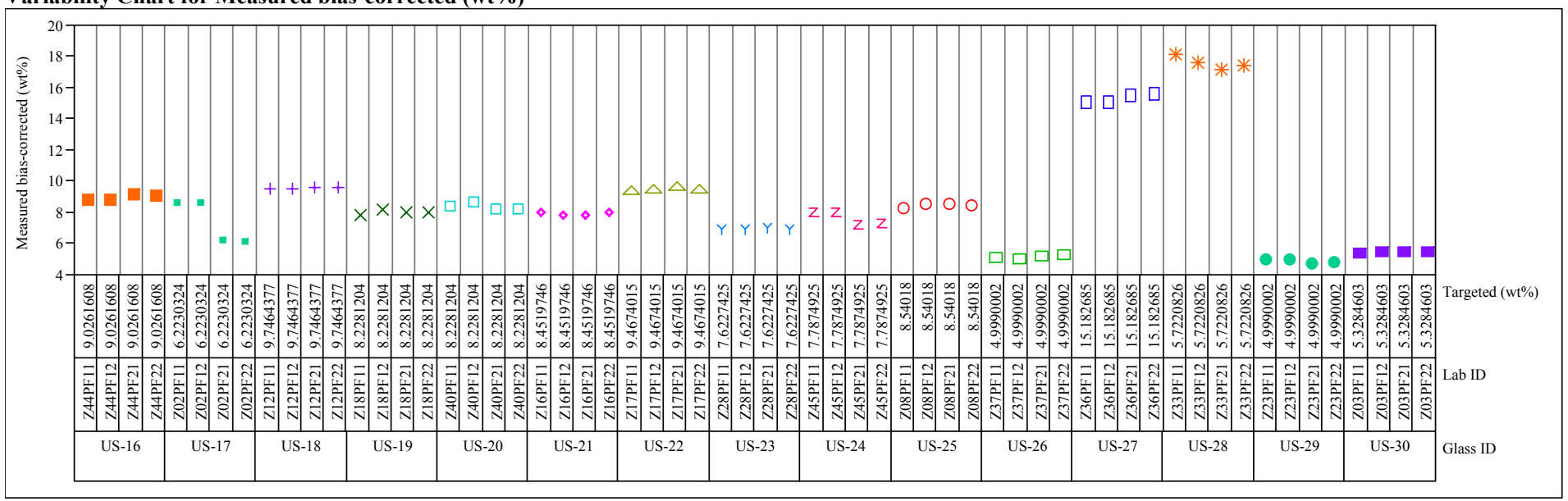


Exhibit A6. Measured and Measured Bias-Corrected Oxide Weight Percents by Lab ID for the Samples Prepared Using the PF Method

Group $=2$, Oxide $=\mathrm{Li} 20(\mathrm{wt} \%)$

Variability Chart for Measured (wt \%)

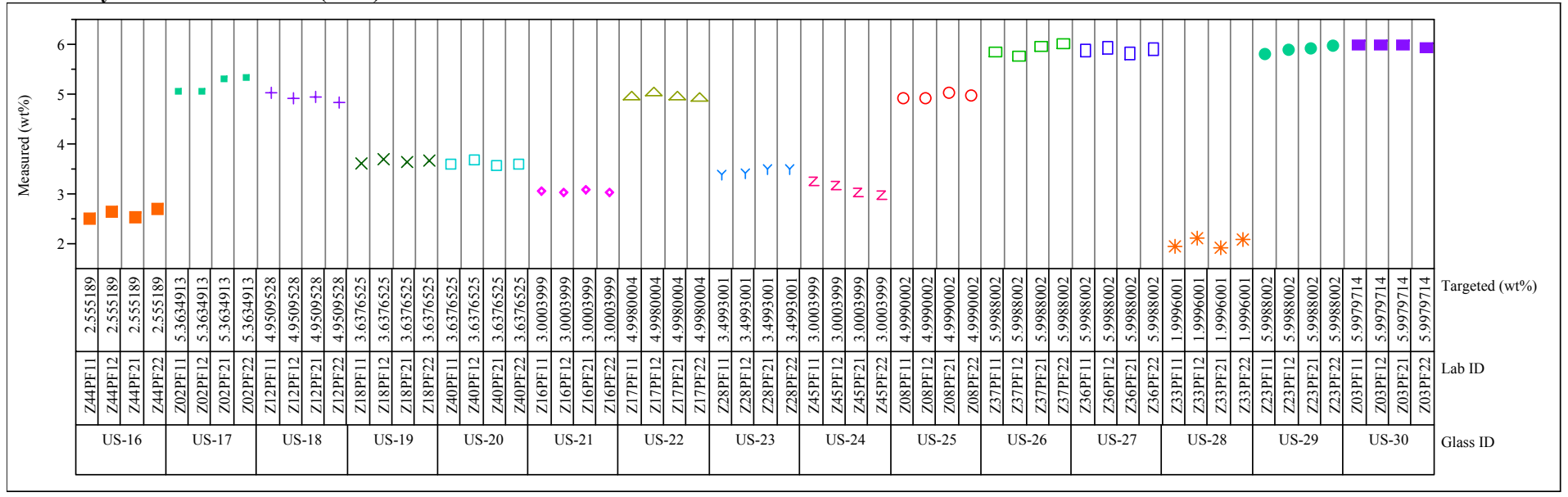

Group $=2$, Oxide $=$ Li2O (wt \%)

Variability Chart for Measured bias-corrected (wt\%)

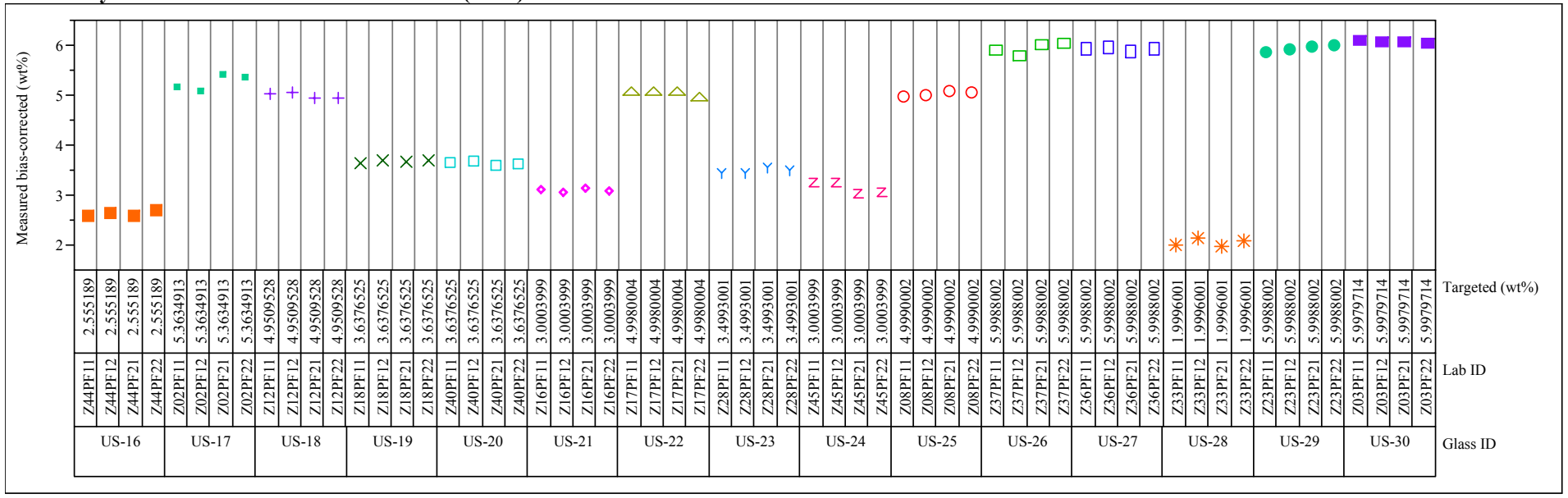


Exhibit A6. Measured and Measured Bias-Corrected Oxide Weight Percents by Lab ID for the Samples Prepared Using the PF Method

Group $=2$, Oxide $=\mathrm{MnO}($ wt $\%)$

Variability Chart for Measured (wt \%)

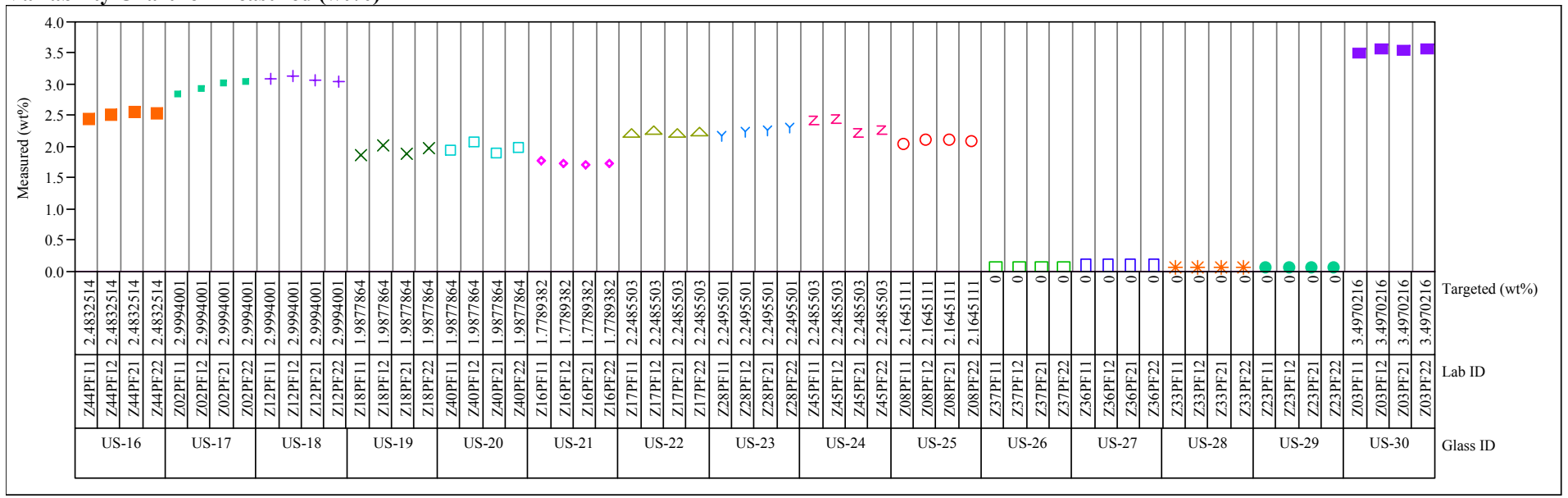

Group $=2$, Oxide $=\mathrm{MnO}(\mathrm{wt} \%)$

Variability Chart for Measured bias-corrected (wt\%)

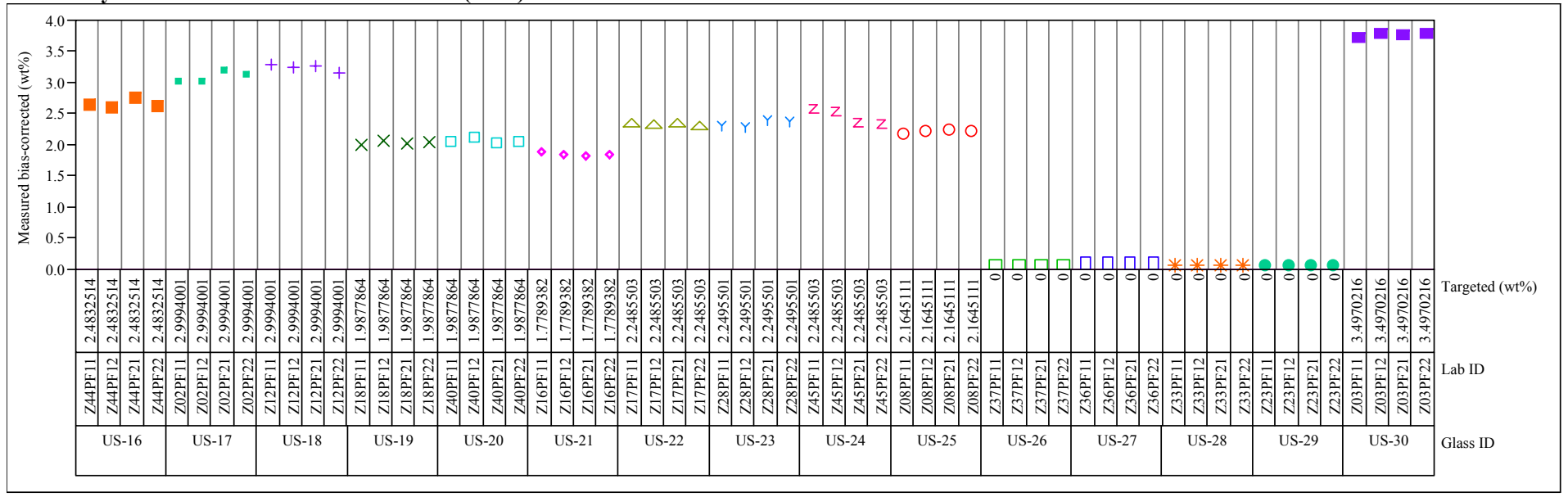


Exhibit A6. Measured and Measured Bias-Corrected Oxide Weight Percents by Lab ID for the Samples Prepared Using the PF Method

Group $=2$, Oxide $=\mathrm{SiO} 2(\mathrm{wt} \%)$

Variability Chart for Measured (wt \%)

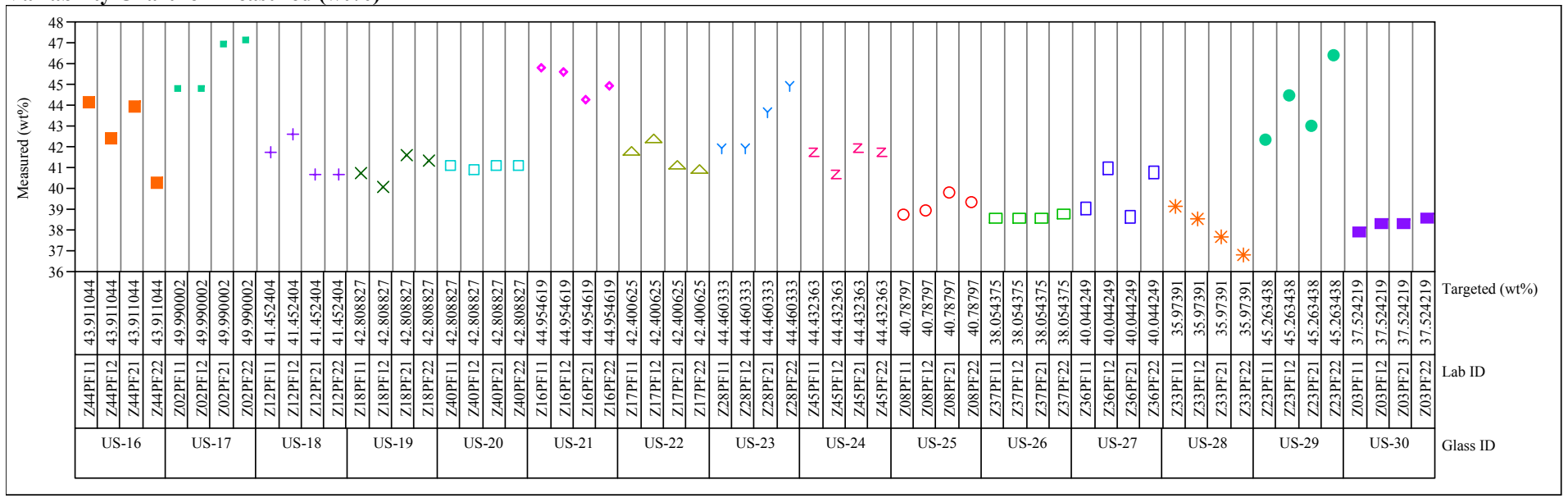

Group $=2$, Oxide $=\mathrm{SiO} 2(\mathrm{wt} \%)$

Variability Chart for Measured bias-corrected (wt\%)

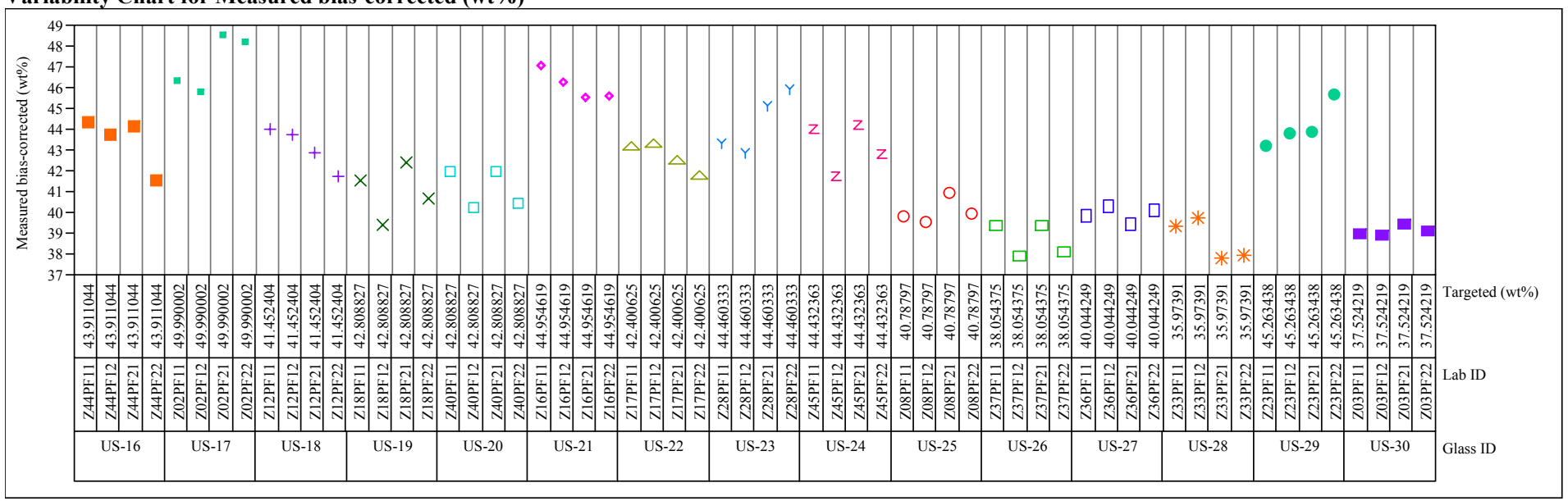




\section{Exhibit A6. Measured and Measured Bias-Corrected Oxide Weight Percents by Lab ID} for the Samples Prepared Using the PF Method

Group $=3$, Oxide $=\mathrm{Al} 2 \mathrm{O} 3(\mathrm{wt} \%)$

Variability Chart for Measured (wt\%)

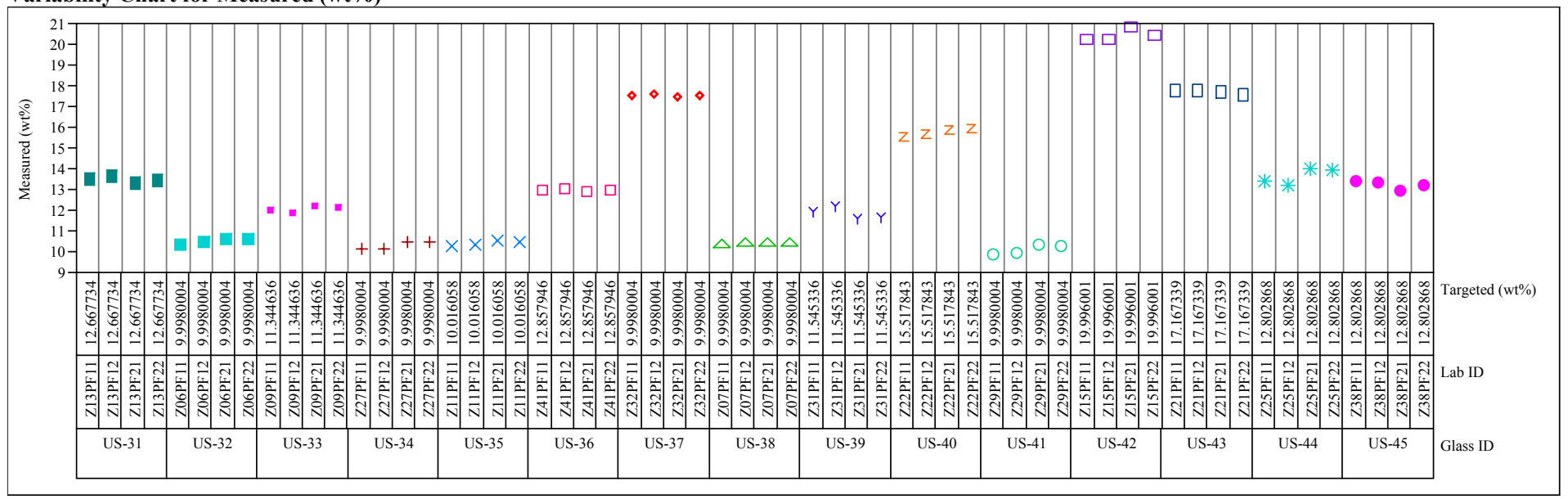

Group $=3$, Oxide $=\mathrm{Al} 203(\mathrm{wt} \%)$

Variability Chart for Measured bias-corrected (wt\%)

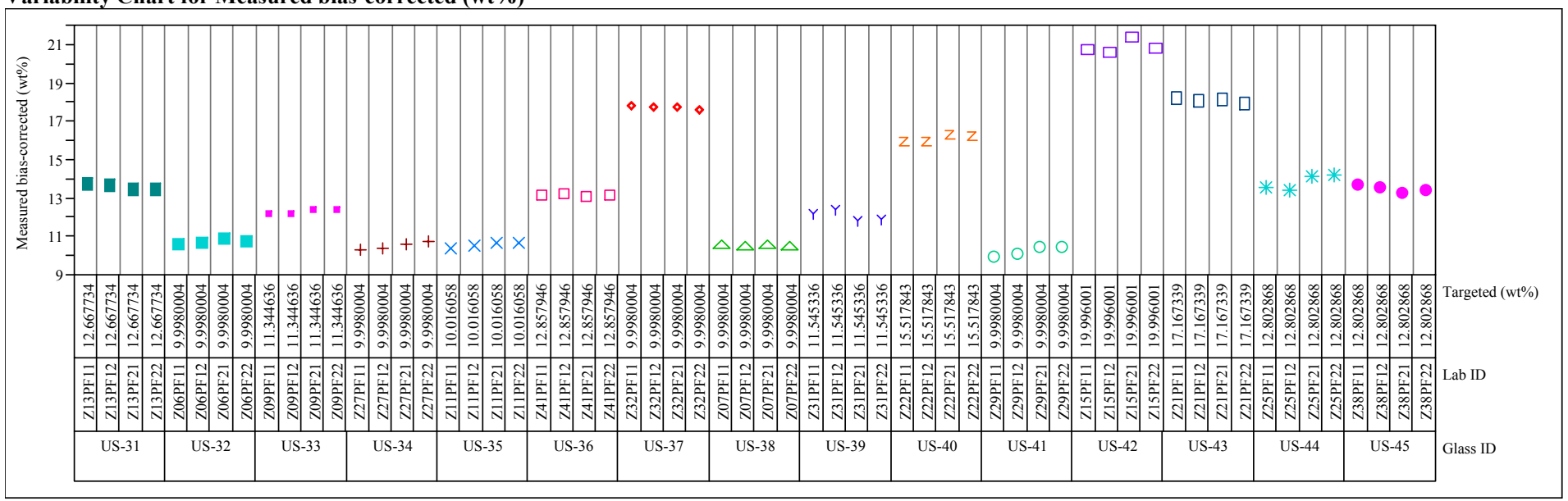


Exhibit A6. Measured and Measured Bias-Corrected Oxide Weight Percents by Lab ID for the Samples Prepared Using the PF Method

Group $=3$, Oxide $=\mathbf{B 2 O 3}(\mathrm{wt} \%)$

Variability Chart for Measured (wt\%)

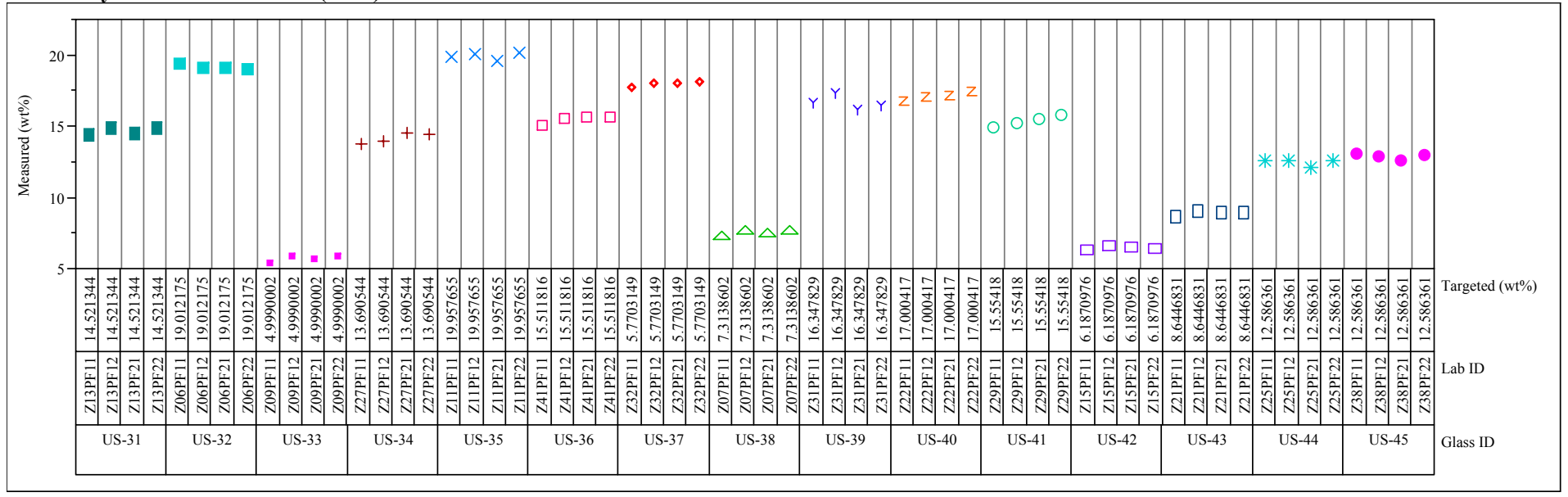

Group $=3$, Oxide $=$ B2O3 $($ wt $\%)$

Variability Chart for Measured bias-corrected (wt\%)

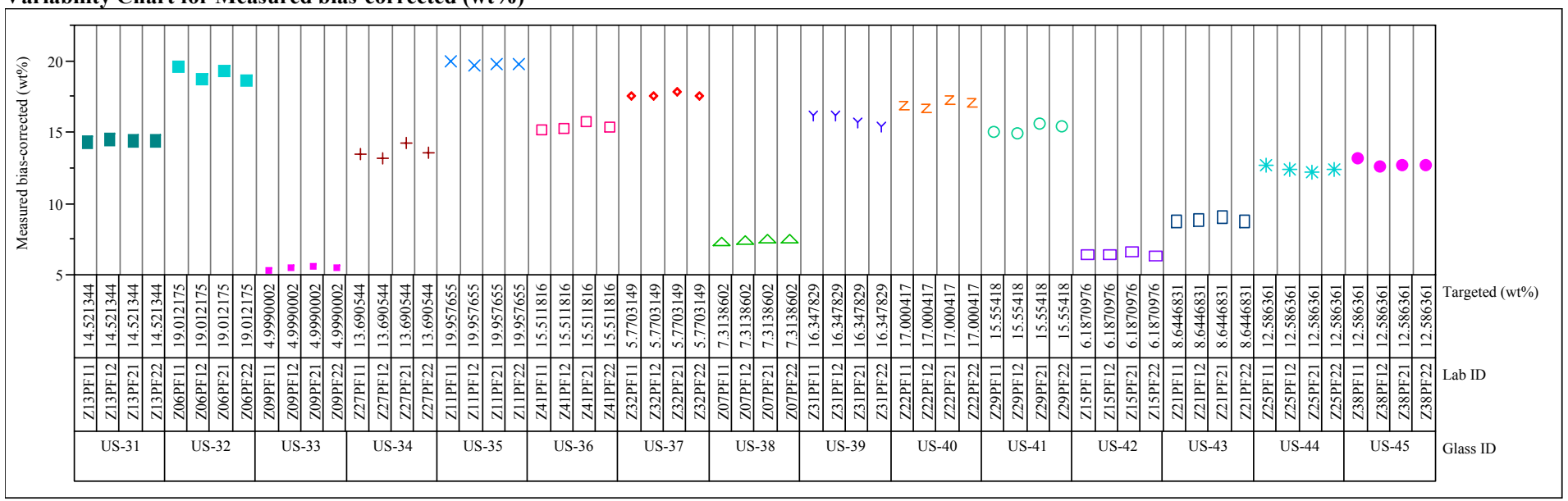


Exhibit A6. Measured and Measured Bias-Corrected Oxide Weight Percents by Lab ID for the Samples Prepared Using the PF Method

Group $=3$, Oxide $=\mathrm{Fe} 2 \mathrm{O} 3(\mathrm{wt} \%)$

Variability Chart for Measured (wt\%)

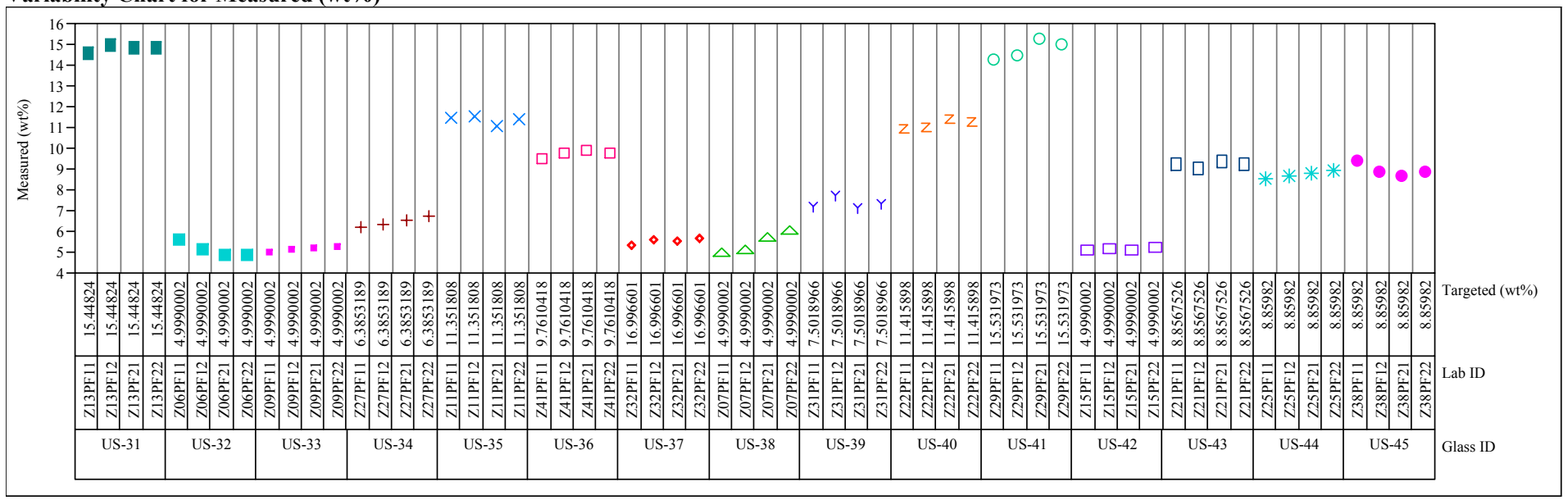

Group $=3$, Oxide $=\mathrm{Fe} 2 \mathrm{O} 3(\mathrm{wt} \%)$

Variability Chart for Measured bias-corrected (wt\%)

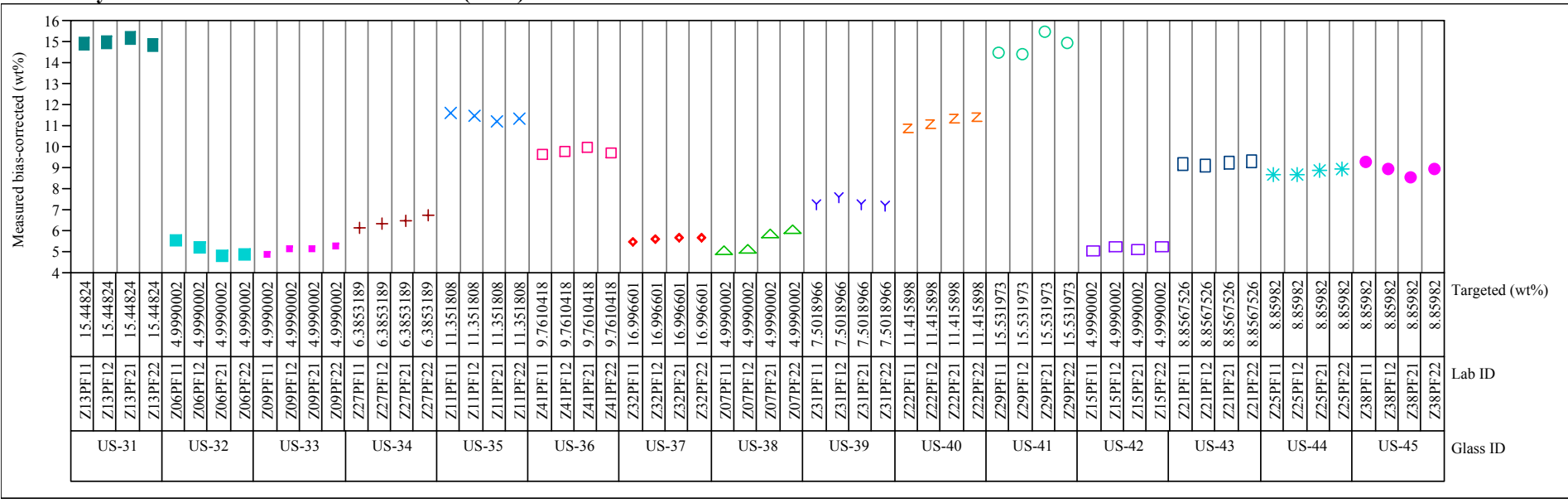


Exhibit A6. Measured and Measured Bias-Corrected Oxide Weight Percents by Lab ID for the Samples Prepared Using the PF Method

Group $=3$, Oxide $=\mathrm{Li} 20(\mathrm{wt} \%)$

Variability Chart for Measured (wt\%)

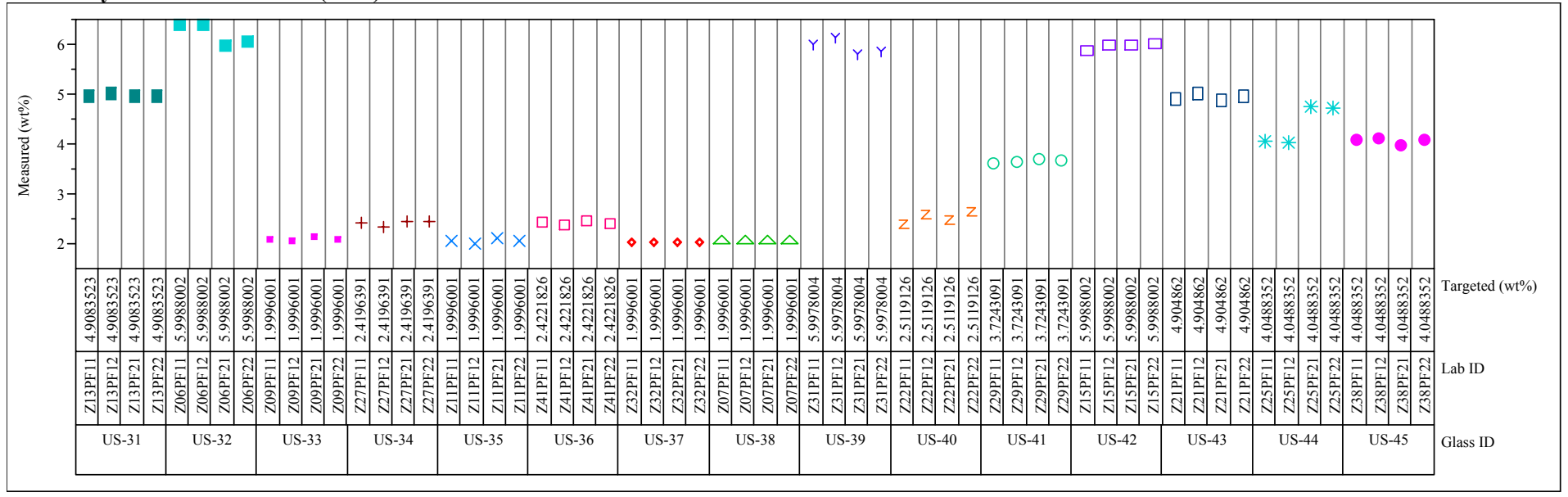

Group $=3$, Oxide $=$ Li2O (wt \%)

Variability Chart for Measured bias-corrected (wt\%)

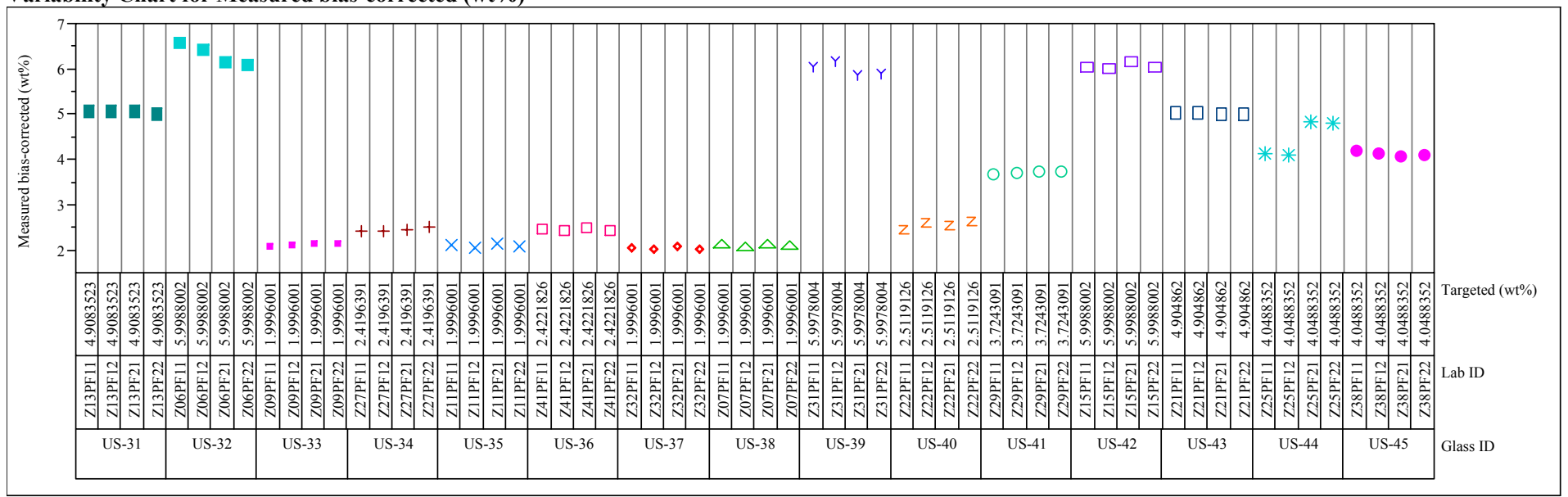


Exhibit A6. Measured and Measured Bias-Corrected Oxide Weight Percents by Lab ID for the Samples Prepared Using the PF Method

Group $=3$, Oxide $=\mathrm{MnO}(\mathrm{wt} \%)$

Variability Chart for Measured (wt\%)

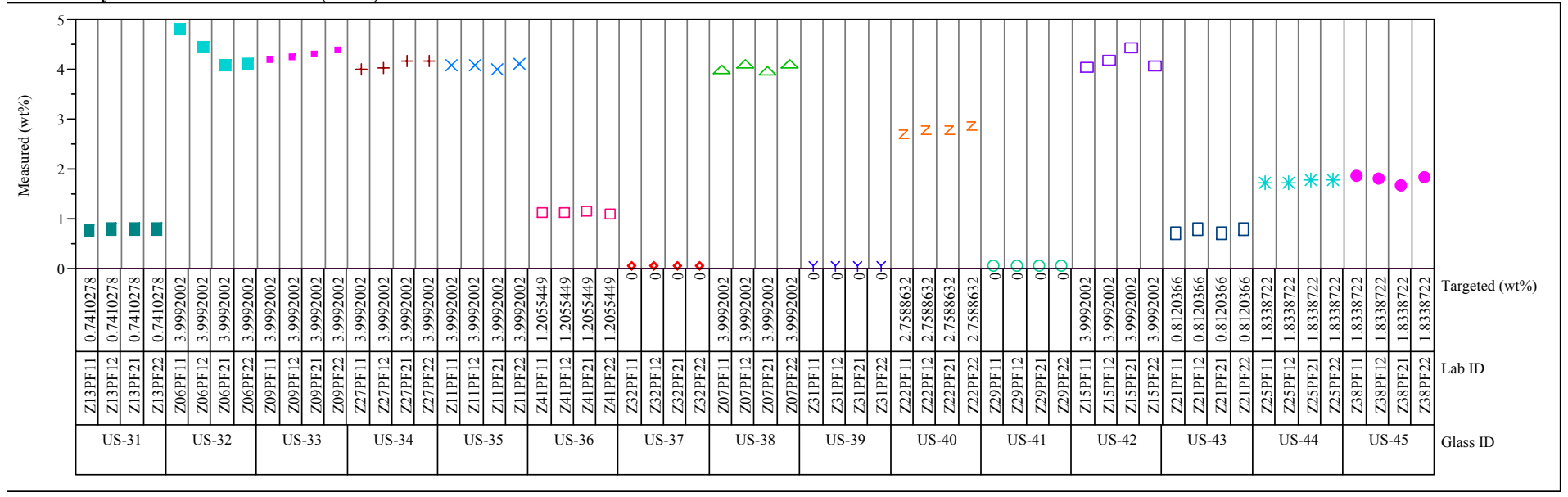

Group $=3$, Oxide $=\mathrm{MnO}(\mathrm{wt} \%)$

Variability Chart for Measured bias-corrected (wt\%)

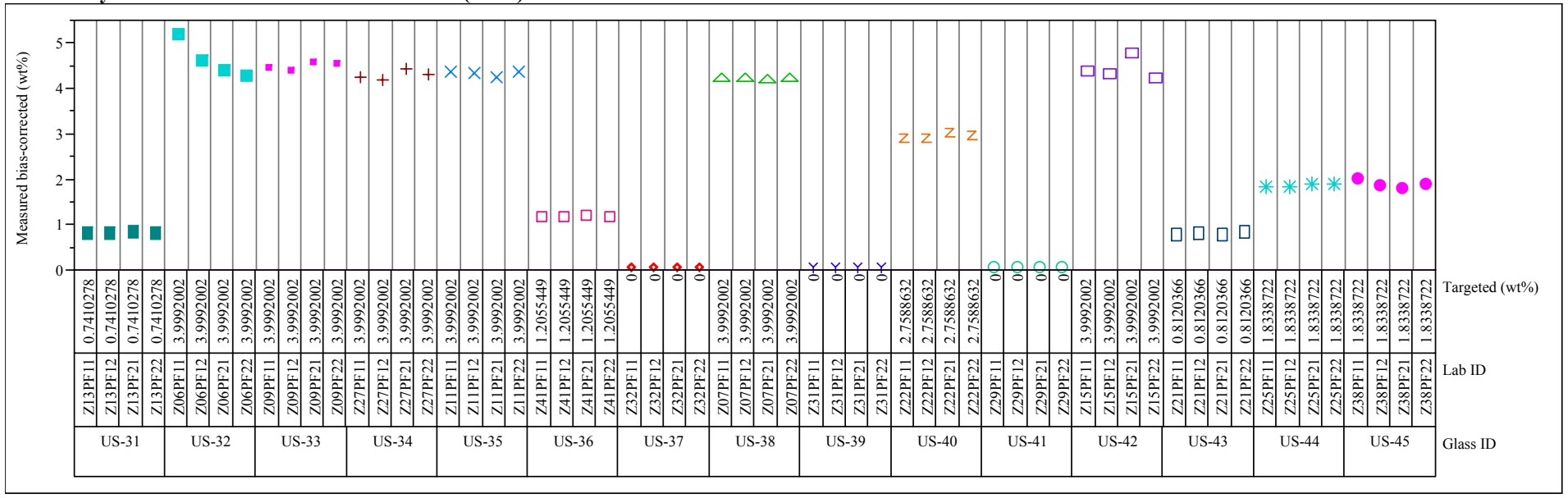


Exhibit A6. Measured and Measured Bias-Corrected Oxide Weight Percents by Lab ID for the Samples Prepared Using the PF Method

Group $=3$, Oxide $=\mathrm{SiO} 2($ wt $\%)$

Variability Chart for Measured (wt \%)

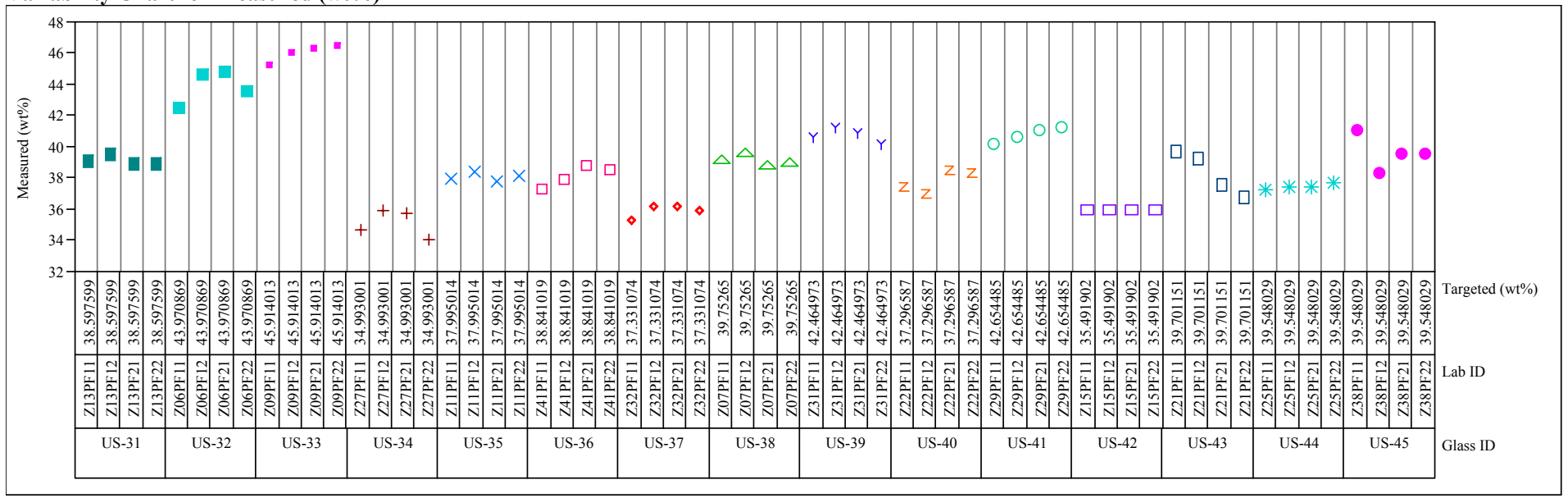

Group $=3$, Oxide $=\mathrm{SiO} 2(\mathrm{wt} \%)$

Variability Chart for Measured bias-corrected (wt\%)

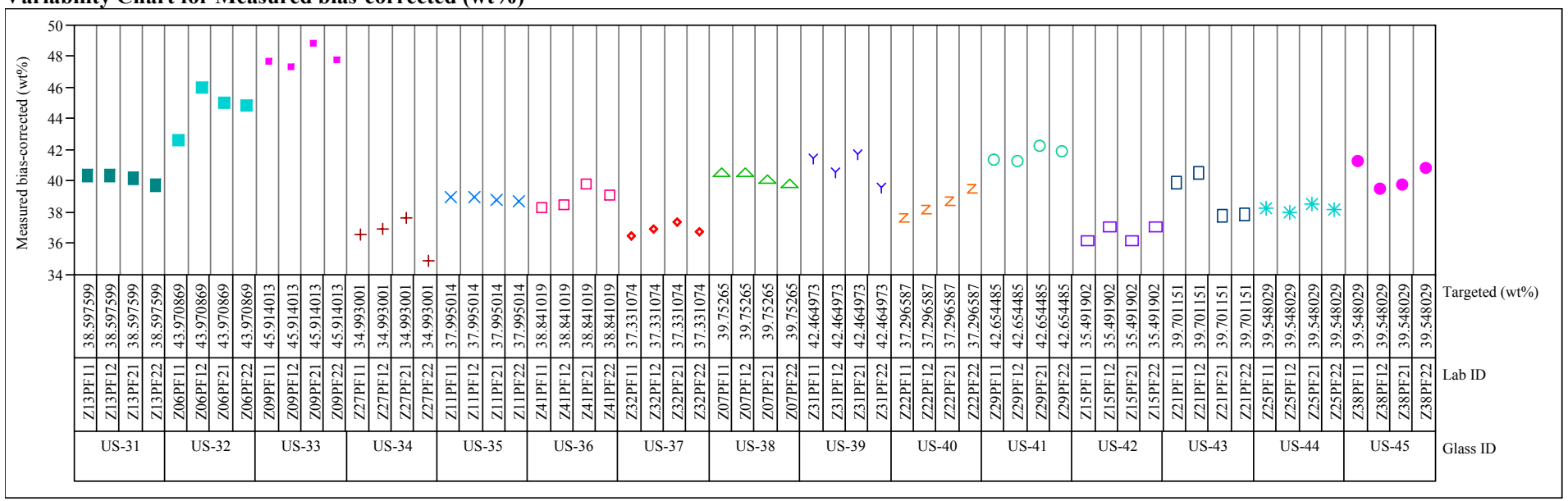


Exhibit A6. Measured and Measured Bias-Corrected Oxide Weight Percents by Lab ID for the Samples Prepared Using the PF Method

Group $=4$, Oxide $=\mathrm{Al2O}(\mathrm{wt} \%)$

Variability Chart for Measured (wt \%)

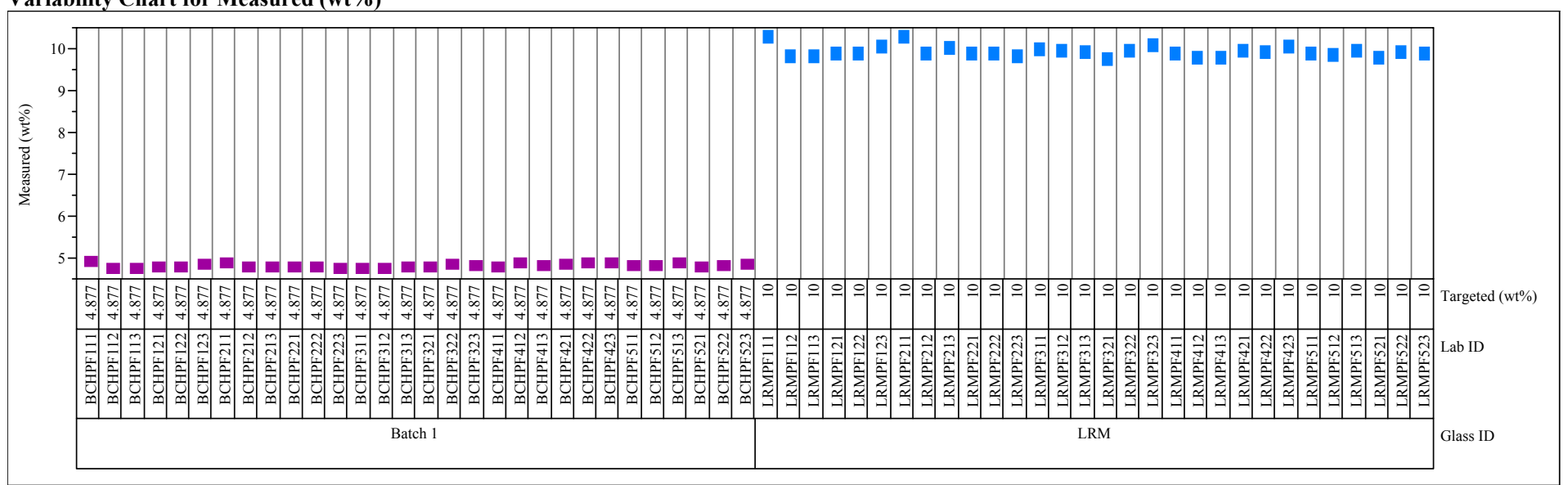

Group $=4$, Oxide $=\mathbf{A l 2 O}(\mathbf{w t} \%)$

Variability Chart for Measured bias-corrected (wt\%)

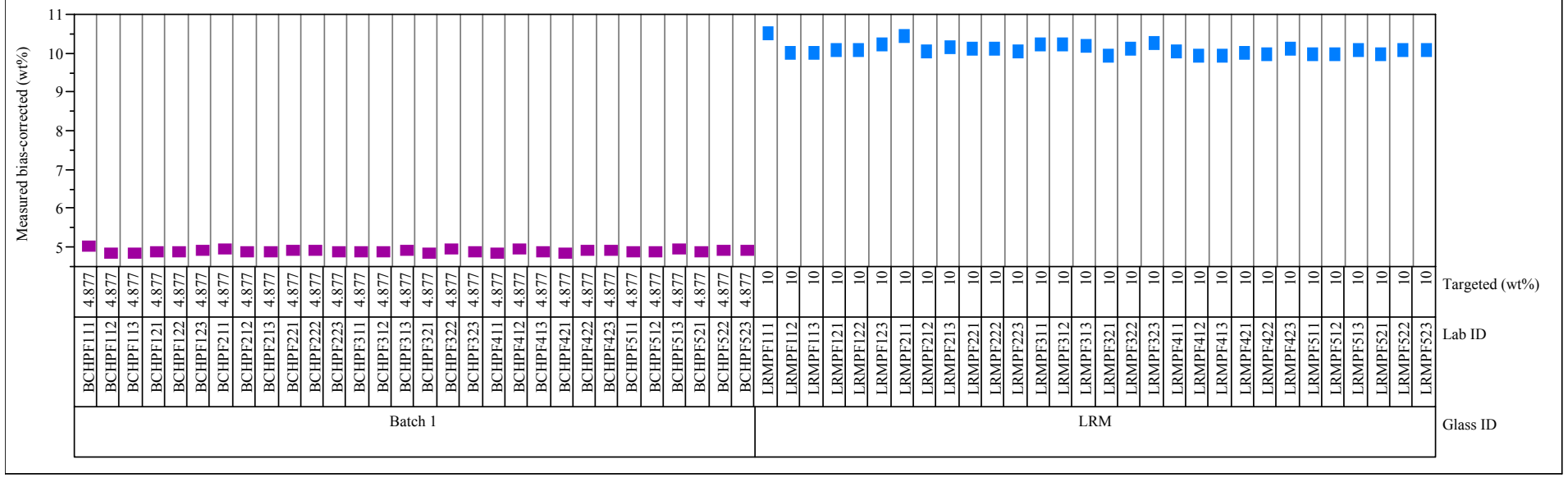


Exhibit A6. Measured and Measured Bias-Corrected Oxide Weight Percents by Lab ID for the Samples Prepared Using the PF Method

Group $=4$, Oxide $=\mathrm{B} 2 \mathrm{O3}(\mathrm{wt} \%)$

Variability Chart for Measured (wt \%)

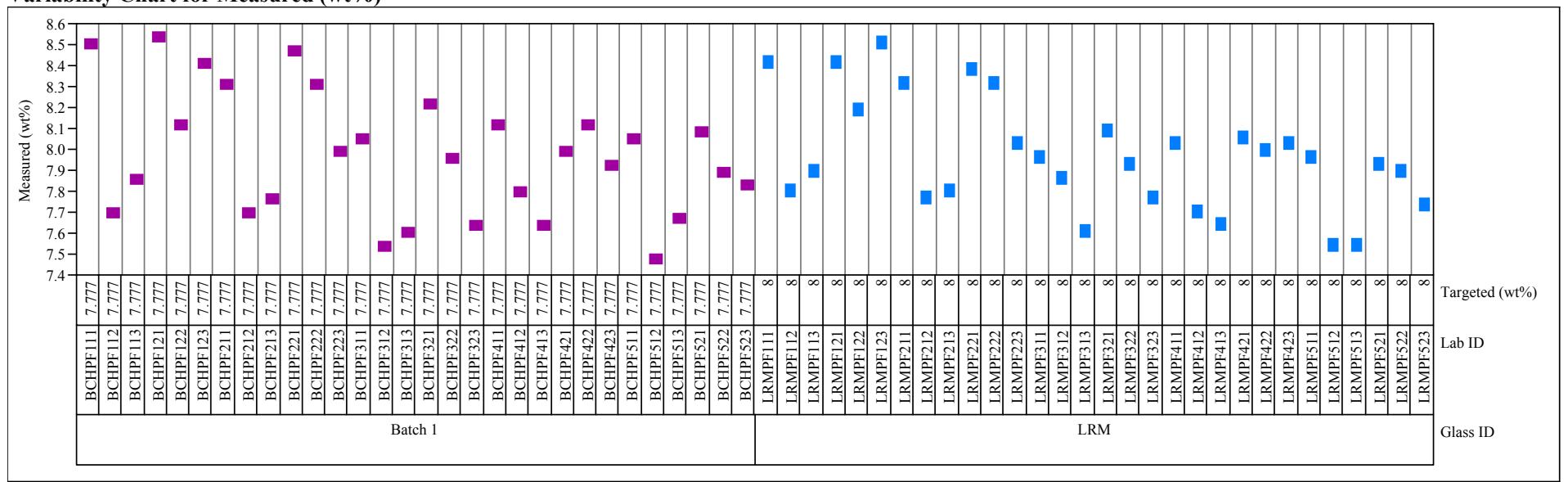

Group=4, Oxide $=\mathbf{B 2 O 3}(\mathrm{wt} \%)$

Variability Chart for Measured bias-corrected (wt\%)

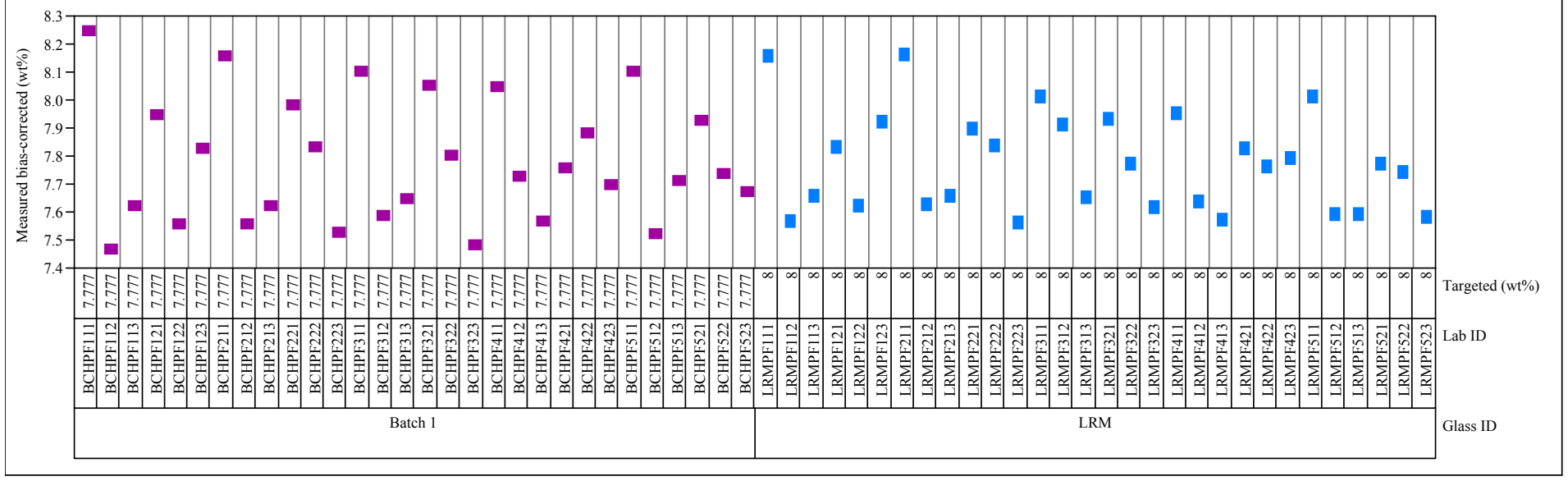


Exhibit A6. Measured and Measured Bias-Corrected Oxide Weight Percents by Lab ID for the Samples Prepared Using the PF Method

Group $=4$, Oxide $=\mathrm{Fe} 2 \mathrm{O} 3(\mathrm{wt} \%)$

Variability Chart for Measured (wt\%)

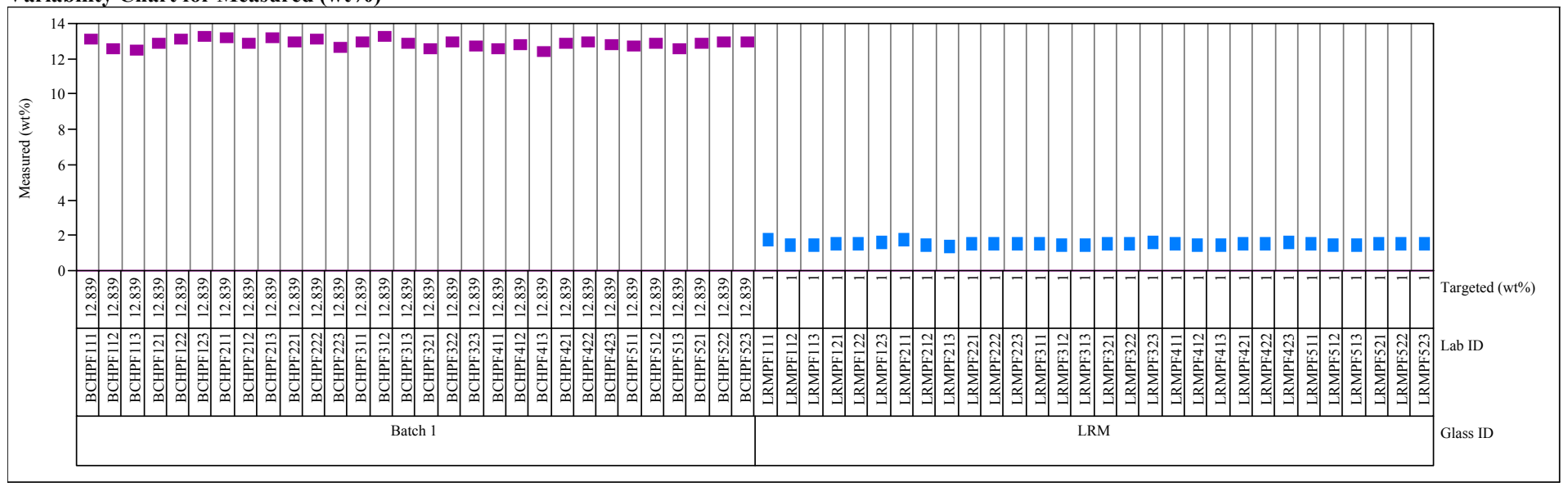

Group=4, Oxide=Fe2O3 $(w t \%)$

Variability Chart for Measured bias-corrected (wt\%)

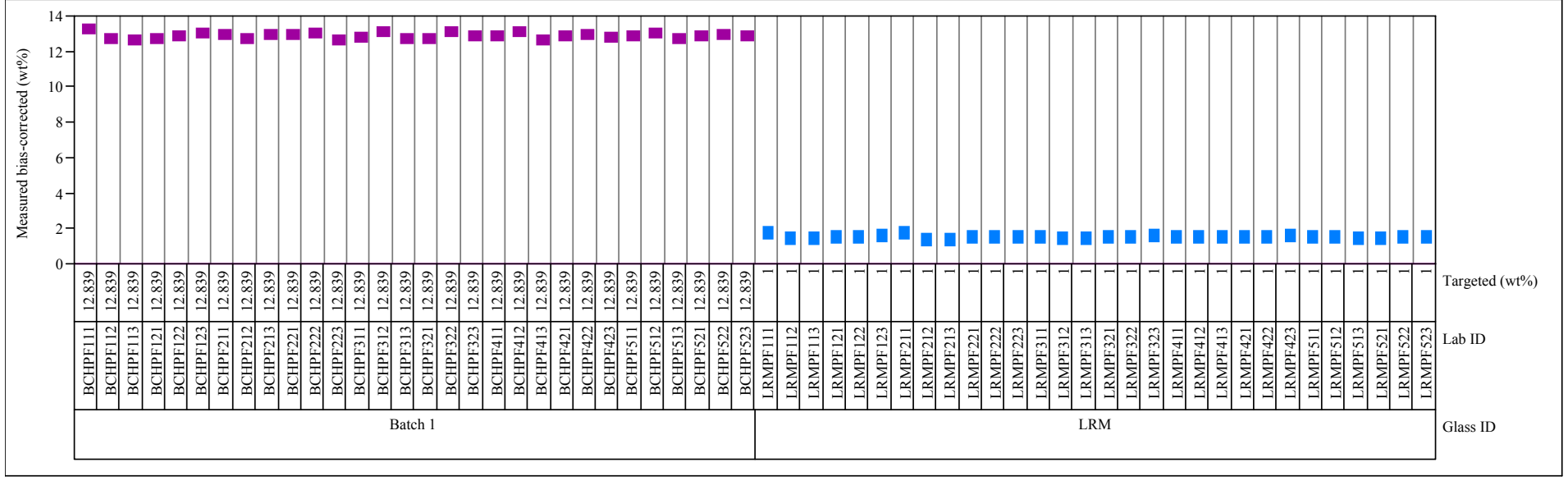


Exhibit A6. Measured and Measured Bias-Corrected Oxide Weight Percents by Lab ID for the Samples Prepared Using the PF Method

Group $=4$, Oxide $=\mathrm{Li} 20($ wt $\%)$

Variability Chart for Measured (wt\%)

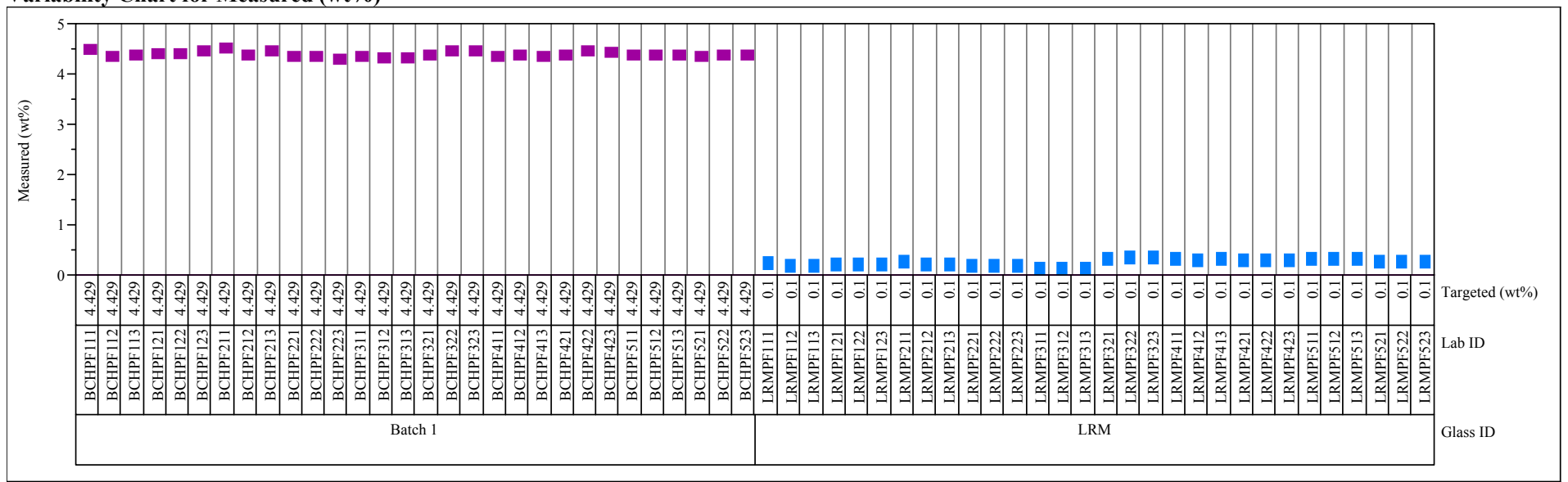

Group $=4$, Oxide $=$ Li2O $($ wt $\%)$

Variability Chart for Measured bias-corrected (wt\%)

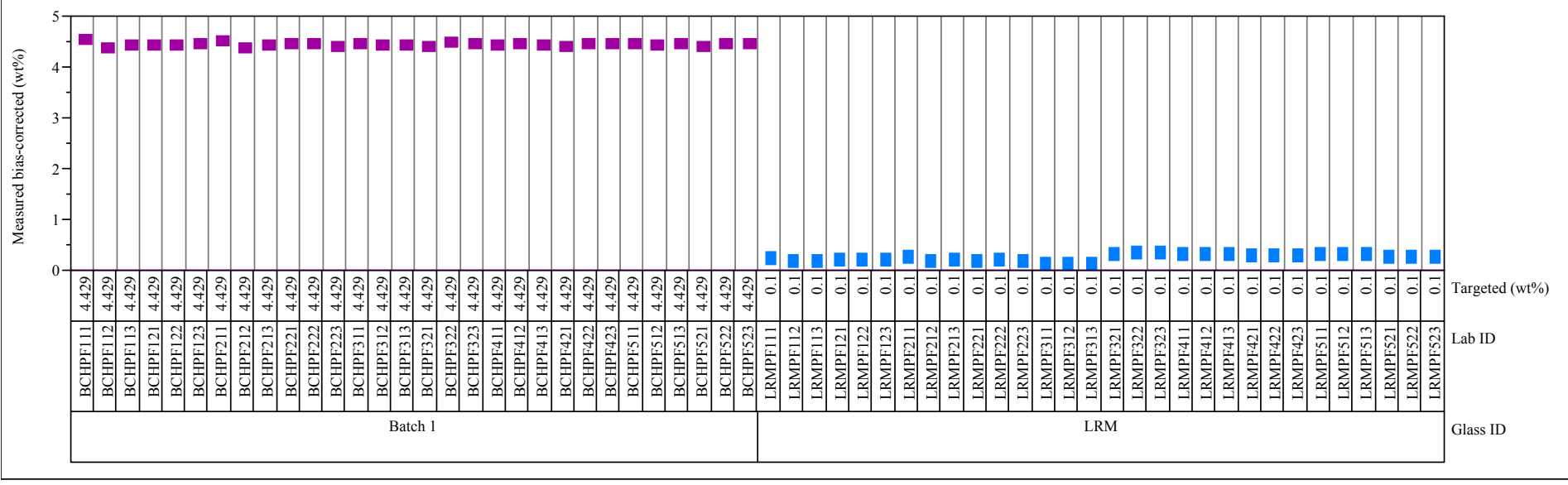


Exhibit A6. Measured and Measured Bias-Corrected Oxide Weight Percents by Lab ID for the Samples Prepared Using the PF Method

Group $=4$, Oxide $=\mathrm{MnO}($ wt $\%)$

Variability Chart for Measured (wt \%)

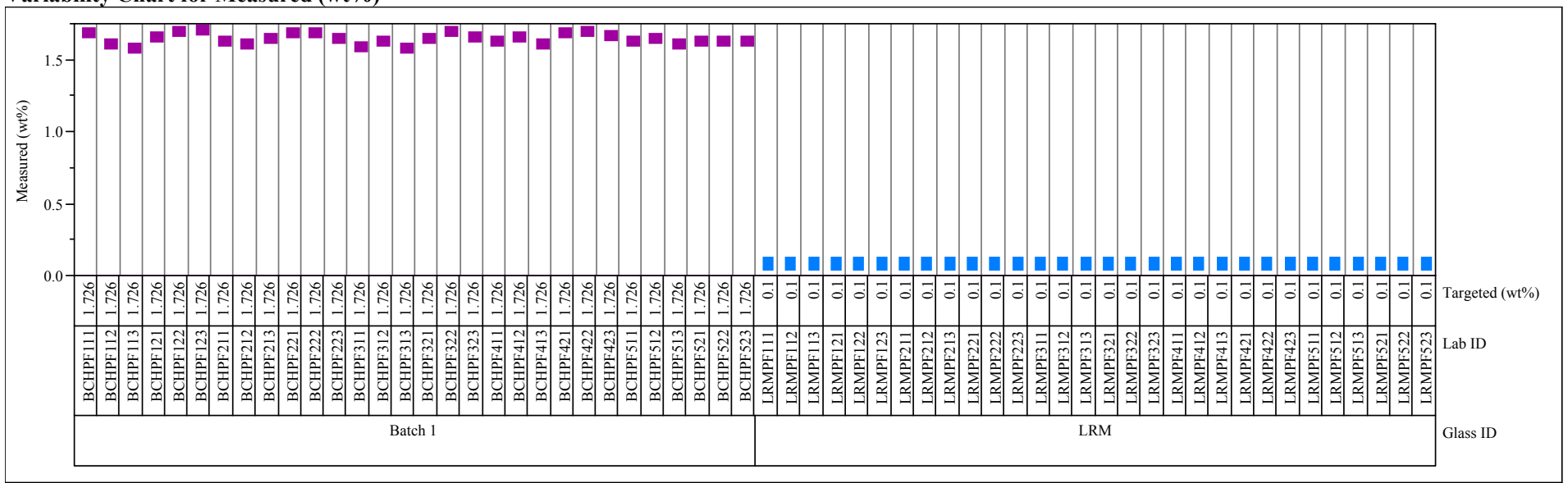

Group $=4$, Oxide $=\mathrm{MnO}(\mathrm{wt} \%)$

Variability Chart for Measured bias-corrected (wt\%)

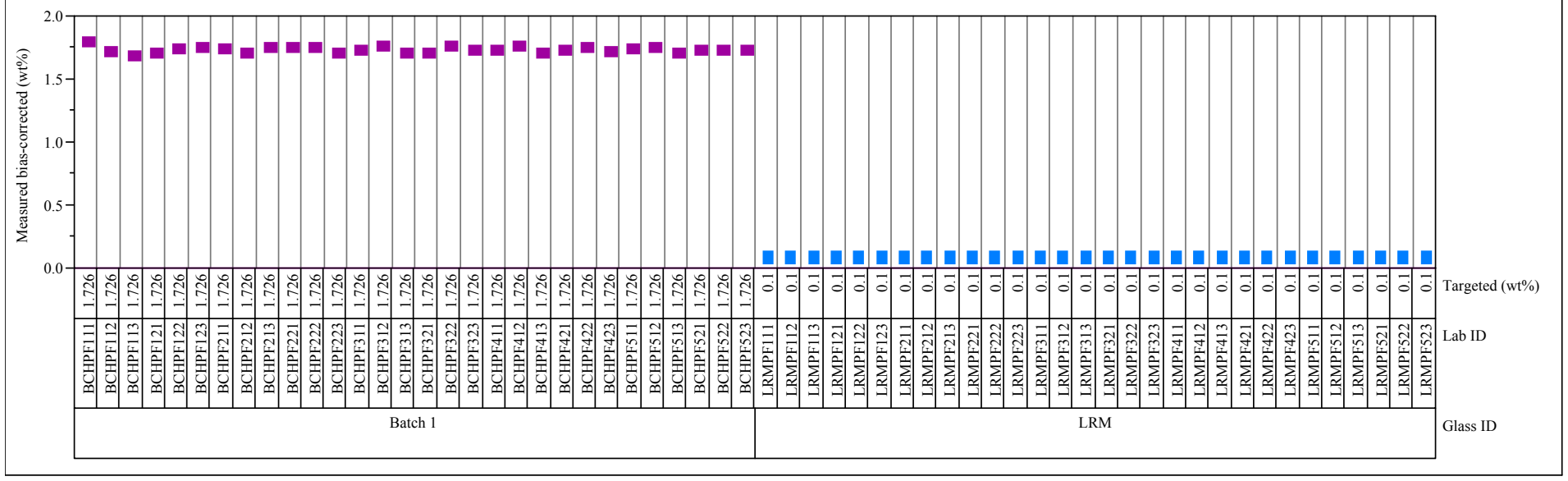


Exhibit A6. Measured and Measured Bias-Corrected Oxide Weight Percents by Lab ID for the Samples Prepared Using the PF Method

Group $=4$, Oxide $=\mathrm{SiO} 2(\mathrm{wt} \%)$

Variability Chart for Measured (wt\%)

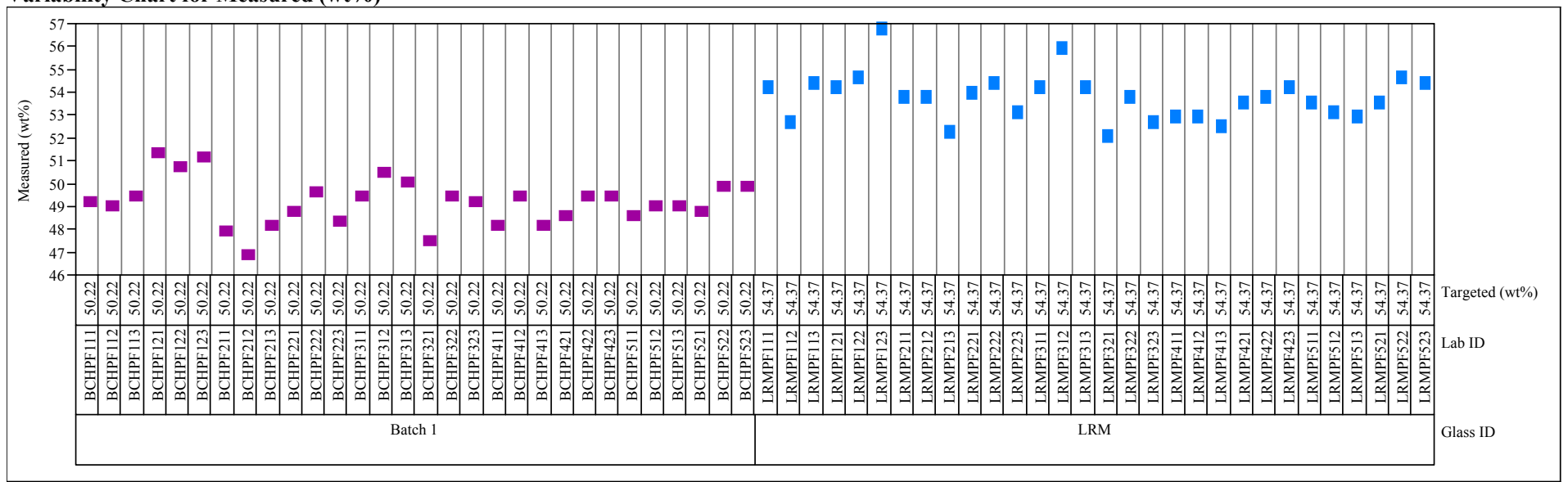

Group $=4$, Oxide $=\mathrm{SiO} 2($ wt $\%)$

Variability Chart for Measured bias-corrected (wt\%)

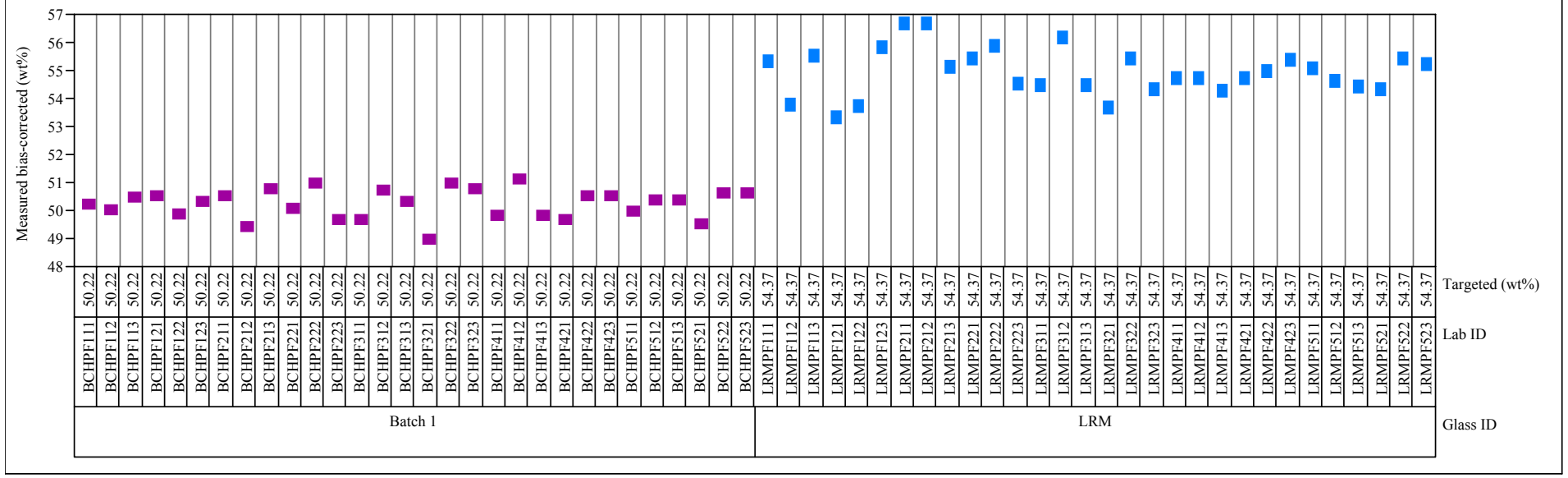




\section{Exhibit A7. Plots of the Targeted, Measured, and Measured Bias-Corrected Values by Glass ID for Each Oxide}

\section{Overlay Plot Oxide=A12O3}

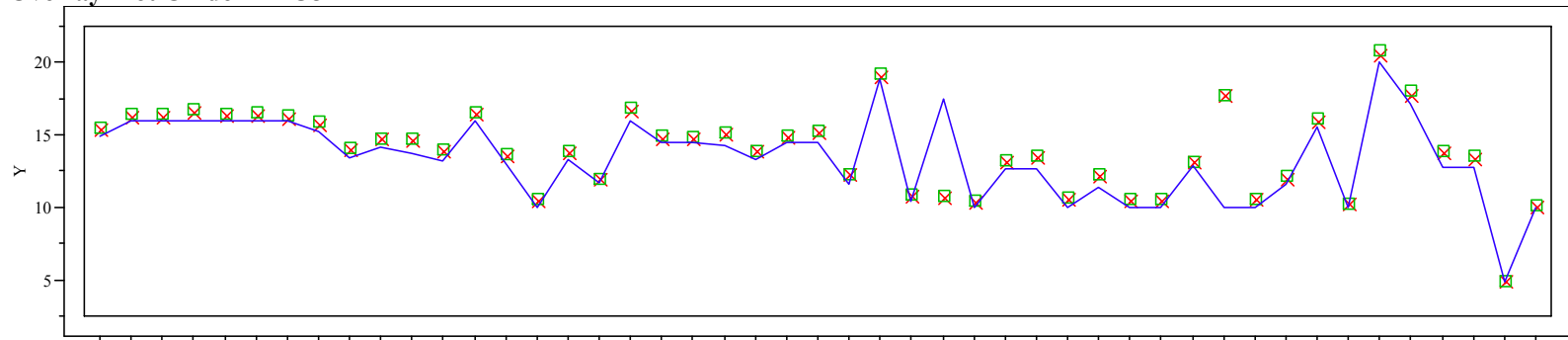

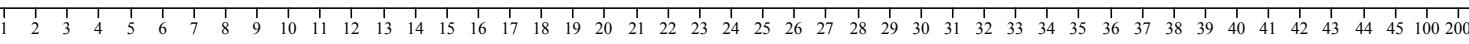
Glass

Overlay Plot Oxide $=\mathbf{B 2 O 3}$

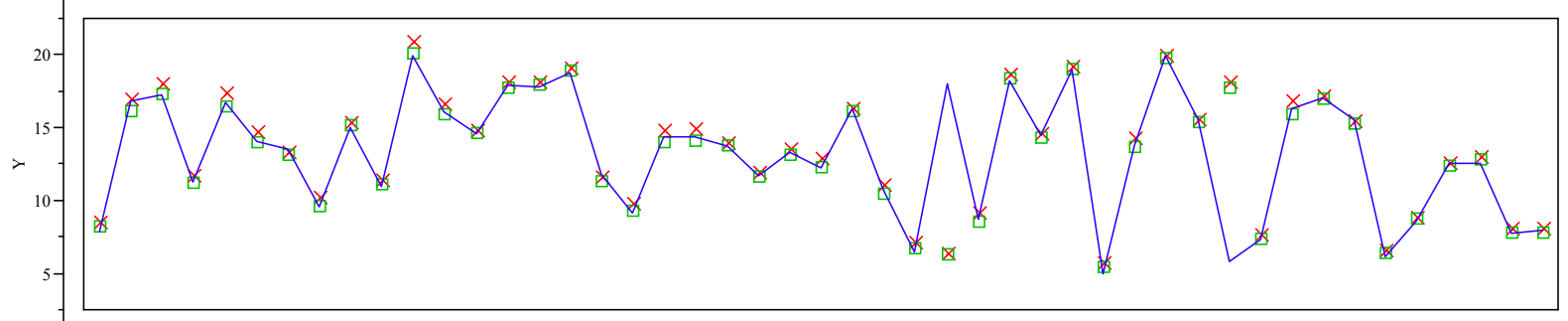

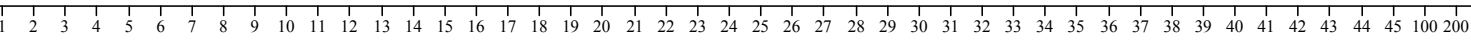
Glass \#

Overlay Plot Oxide $=\mathrm{CaO}$

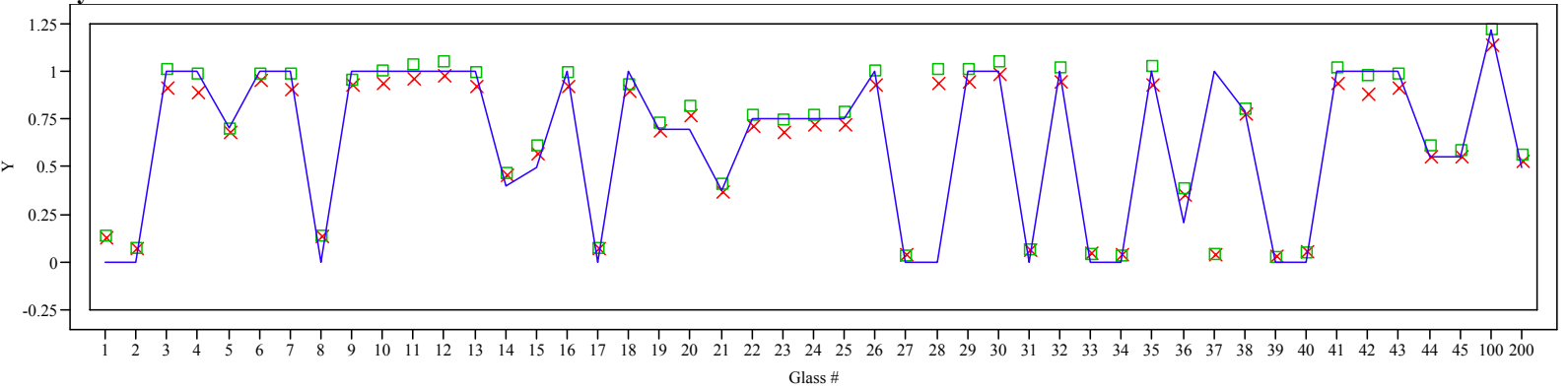

\section{Overlay Plot Oxide $=\mathrm{Cr} 2 \mathrm{O3}$}

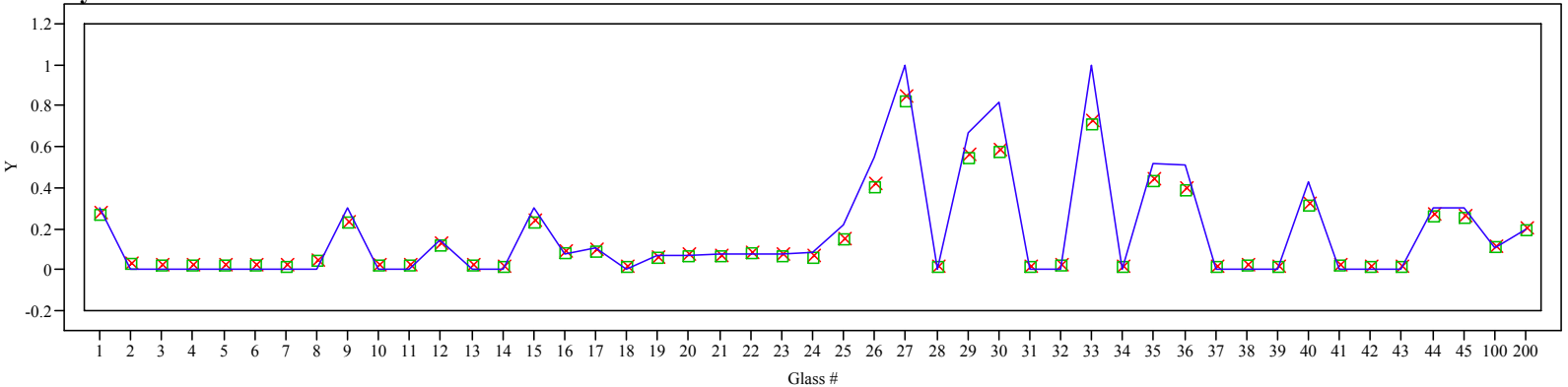

Y $\times$ Measured

- Measured bc - Targeted 


\section{Exhibit A7. Plots of the Targeted, Measured, and Measured Bias-Corrected Values by Glass ID for Each Oxide}
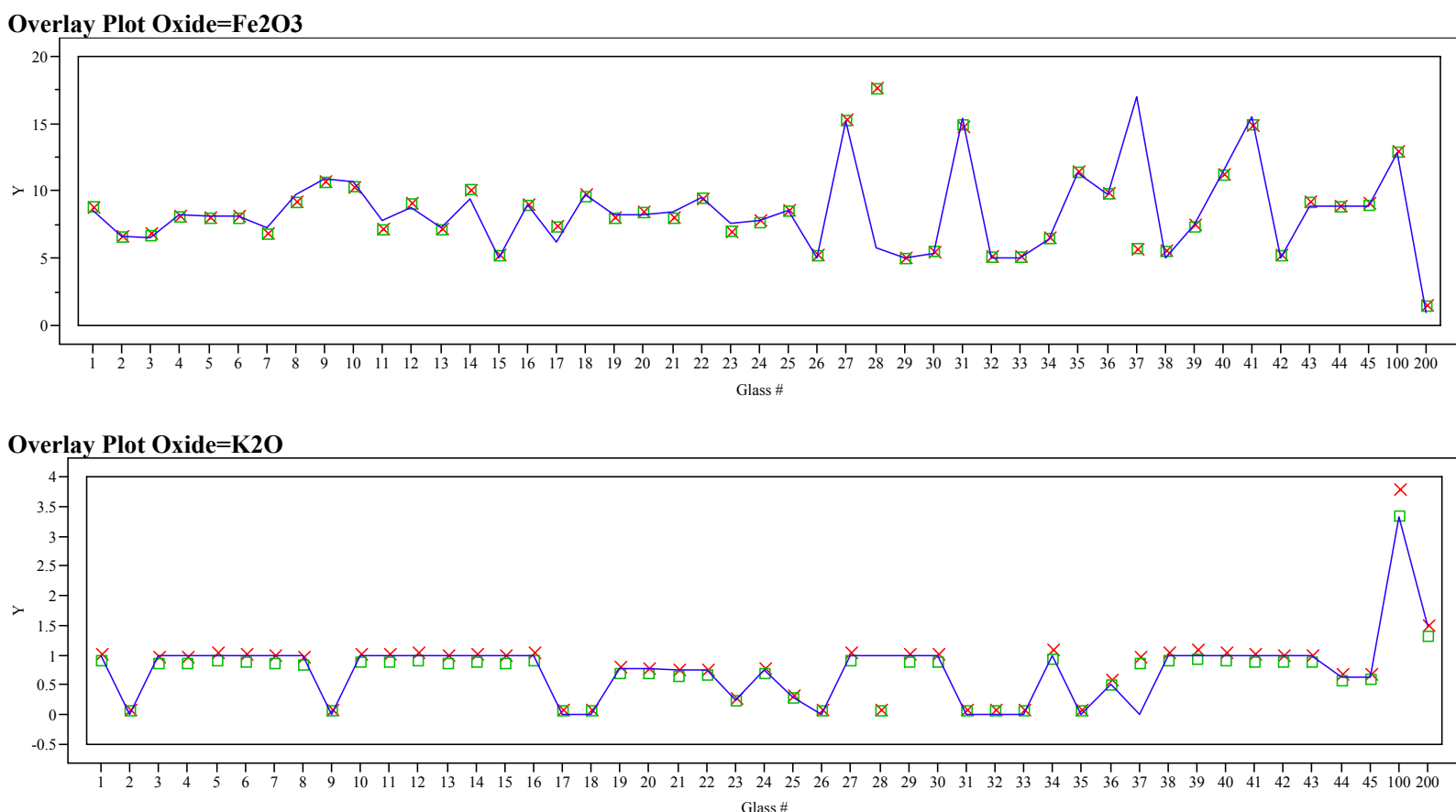

Overlay Plot Oxide $=$ Li2O

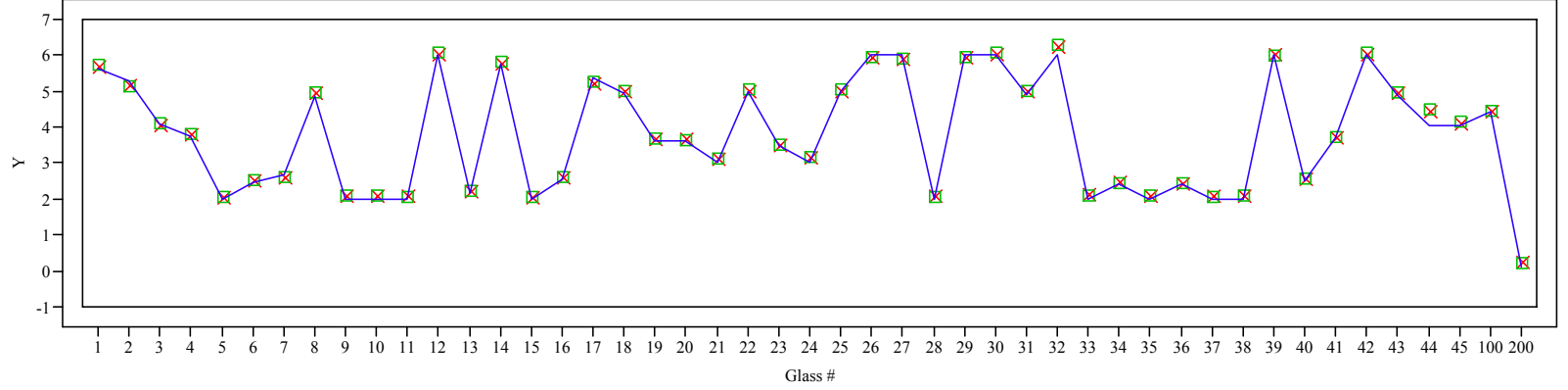

Overlay Plot Oxide $=$ MgO

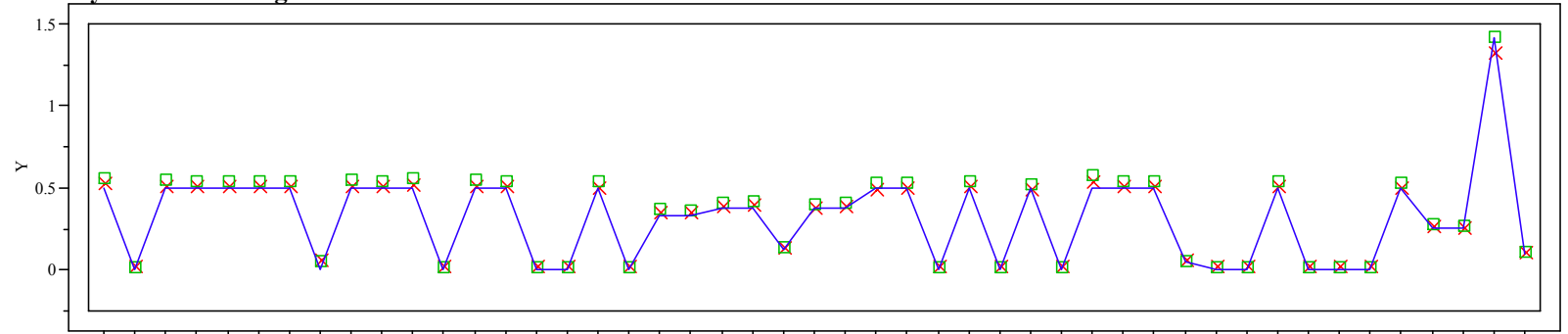

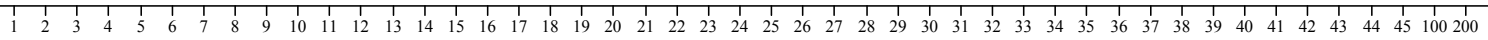
Glass \#

Y $\times$ Measured $\quad$ Measured bc Targeted 


\section{Exhibit A7. Plots of the Targeted, Measured, and Measured Bias-Corrected Values by Glass ID for Each Oxide}

Overlay Plot Oxide $=\mathrm{MnO}$

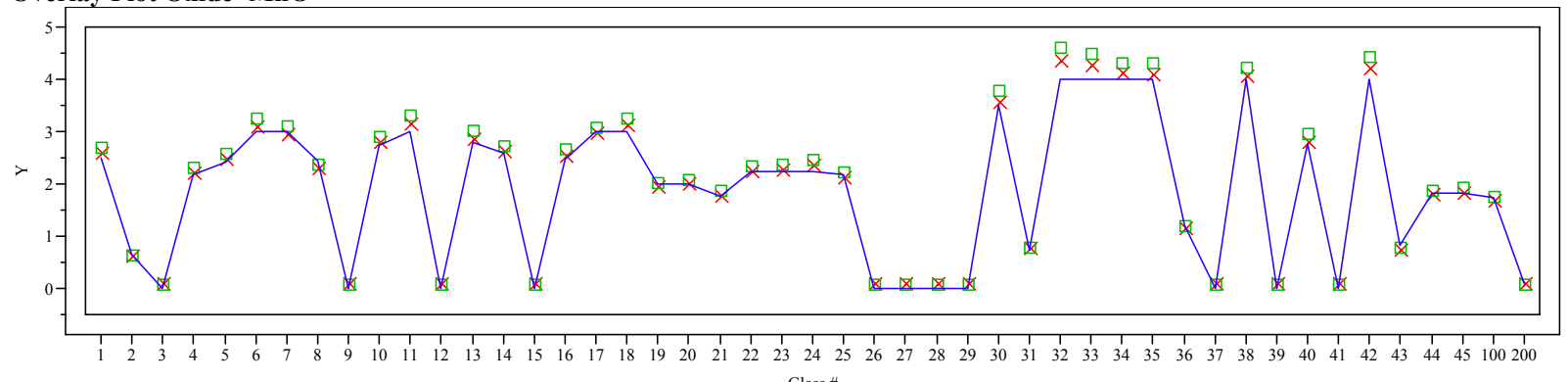
Glass \#

Overlay Plot Oxide $=$ Na2O

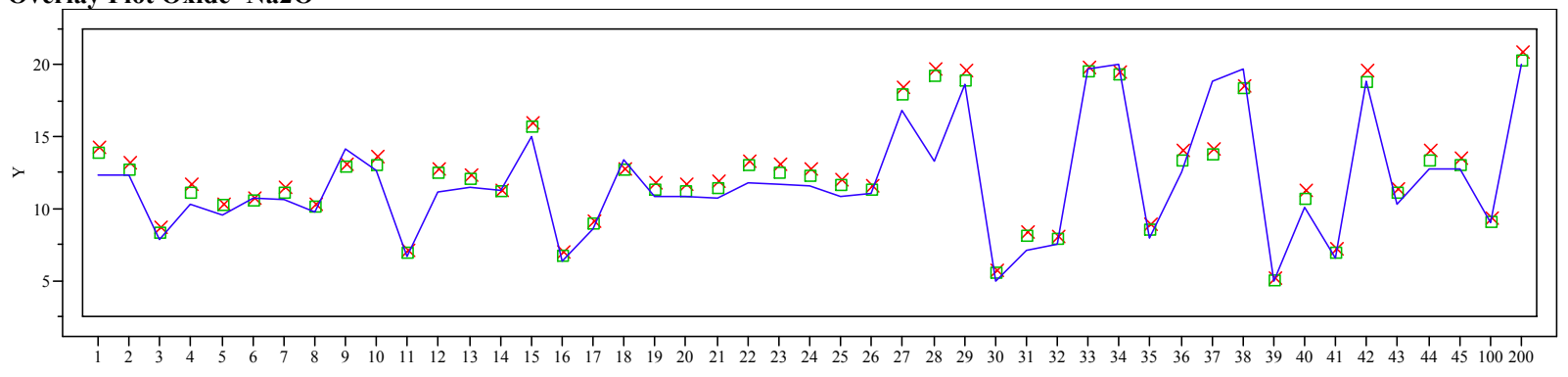
Glass \#

Overlay Plot Oxide=NiO

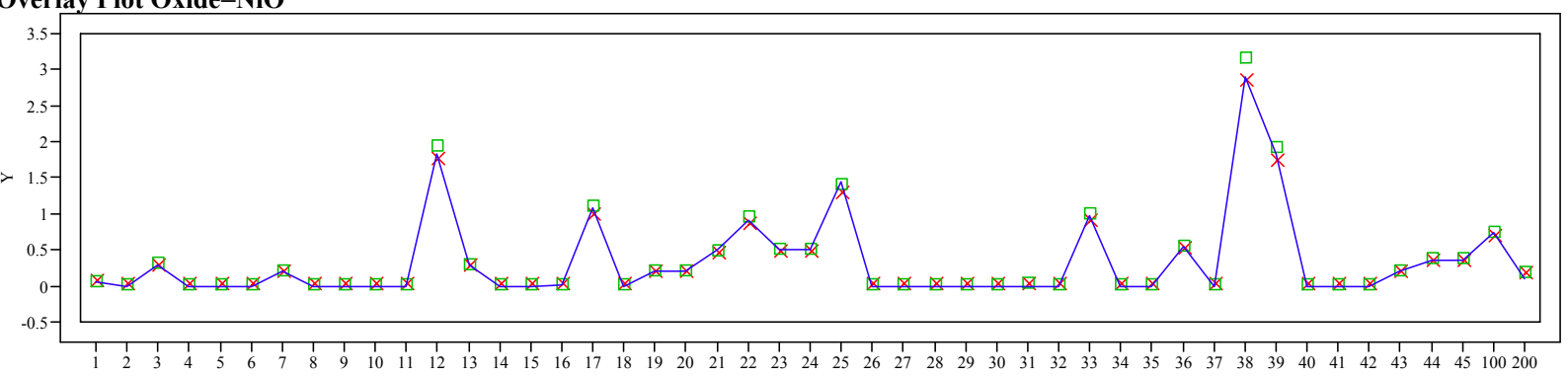
Glass \#

Overlay Plot Oxide $=$ PbO

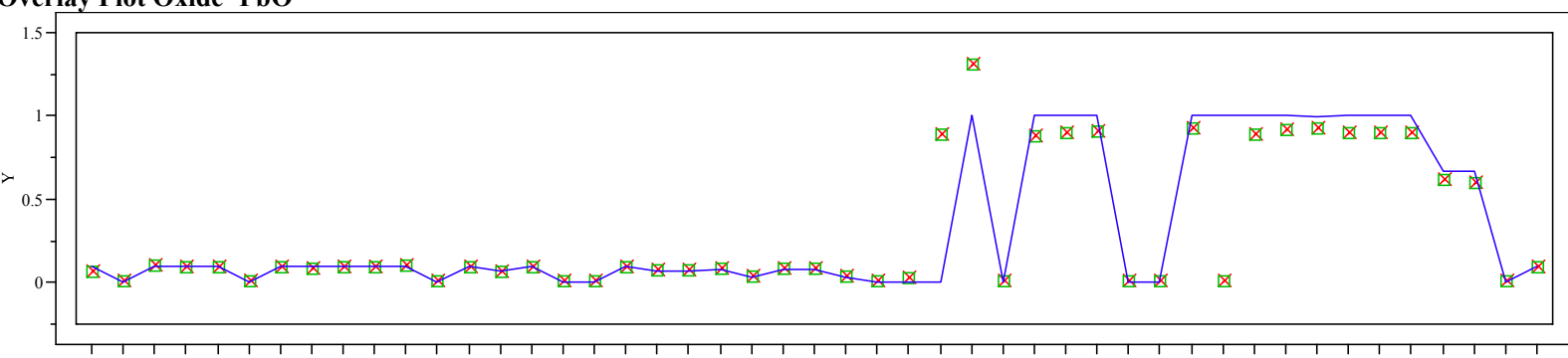

$\begin{array}{llllllllllllllllllllllllllllllllllllllllllllllllll}1 & 1 & 1 & 1 & 1 & 1 & 1 & 1 & 1 & 1 & 1 & 1 & 1 & 1 & 1 & 1 & 1 & 1 & 1 & 1 & 1 & 1 & 1 & 1 & 1 & 1 & 1 & 1 & 1 & 1 & 1 & 1 & 1 & 1 & 1 & 1 & 1 & 1 & 1 & 1 & 1 & 1 & 1 & 1 & 1 & 1 & 1 & 1 \\ 1 & 1 & & & 5 & 6 & 7 & 8 & 9 & 10 & 11 & 12 & 13 & 14 & 15 & 16 & 17 & 18 & 19 & 20 & 21 & 22 & 23 & 24 & 25 & 26 & 27 & 28 & 29 & 30 & 31 & 32 & 33 & 34 & 35 & 36 & 37 & 38 & 39 & 40 & 41 & 42 & 43 & 44 & 45 & 100 & 200\end{array}$ Glass \#

Y X Measured

口 Measured bc Targeted 


\section{Exhibit A7. Plots of the Targeted, Measured, and Measured Bias-Corrected Values by Glass ID for Each Oxide}

Overlay Plot Oxide $=\mathrm{SiO} 2$

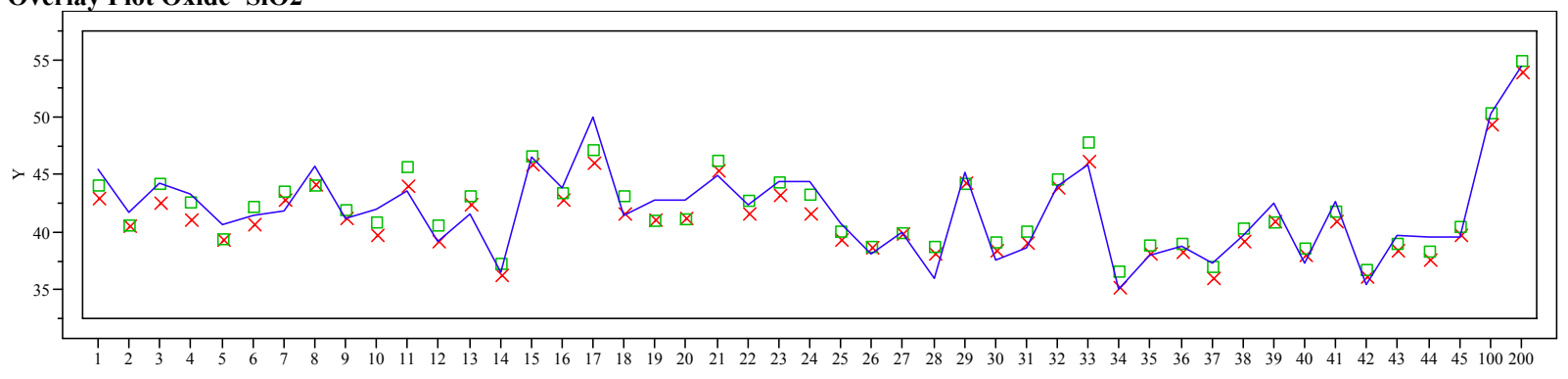
Glass \#

\section{Overlay Plot Oxide $=$ SO3}

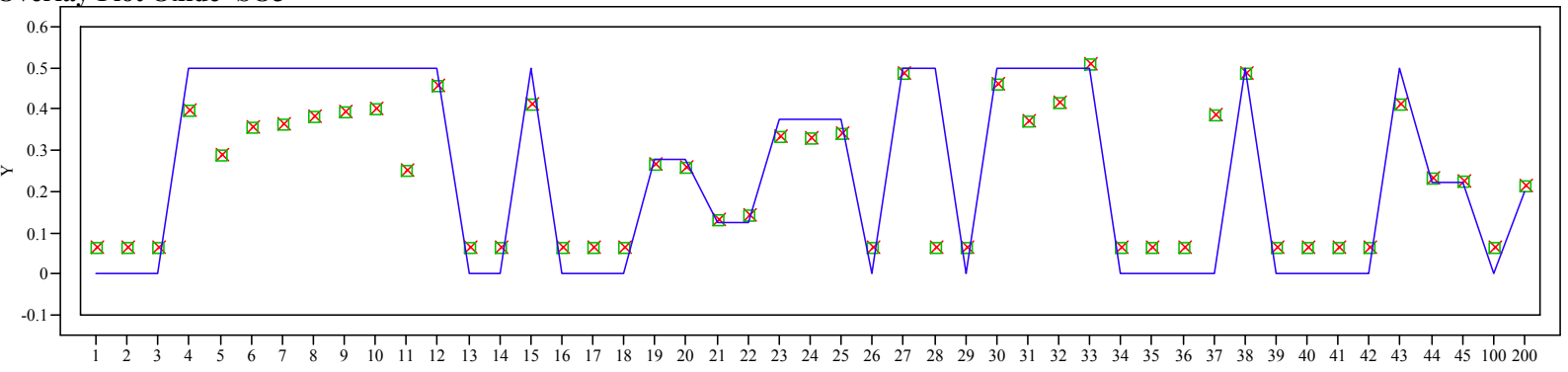
Glass \#

Overlay Plot Oxide $=$ SrO

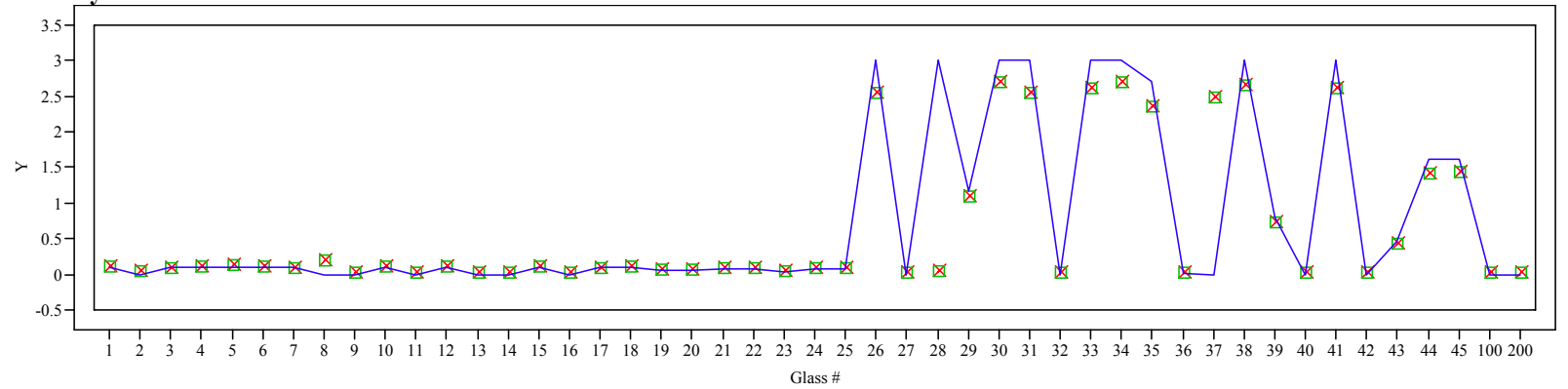

Overlay Plot Oxide $=\mathrm{TiO2}$

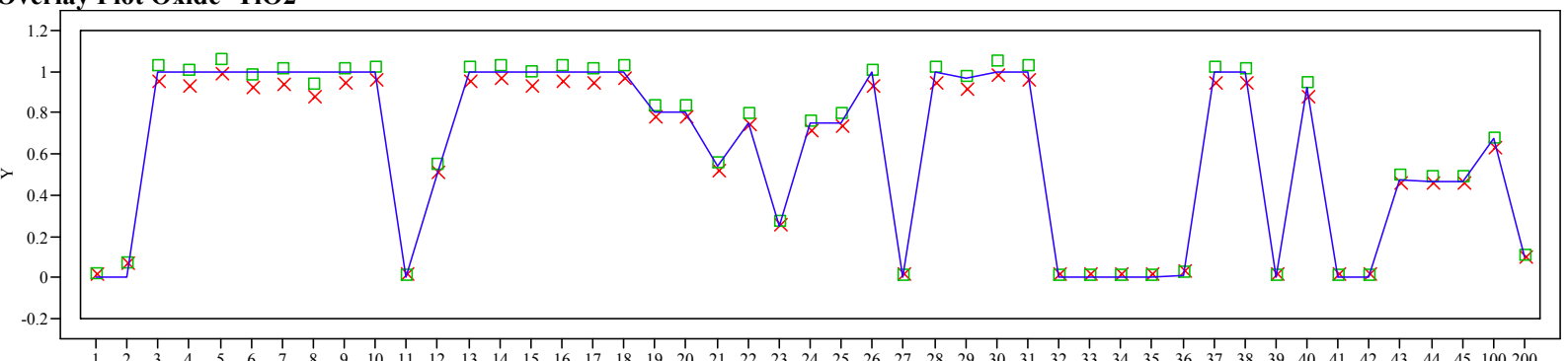




\section{Exhibit A7. Plots of the Targeted, Measured, and Measured Bias-Corrected Values by Glass ID for Each Oxide}

Overlay Plot Oxide $=\mathrm{ZnO}$

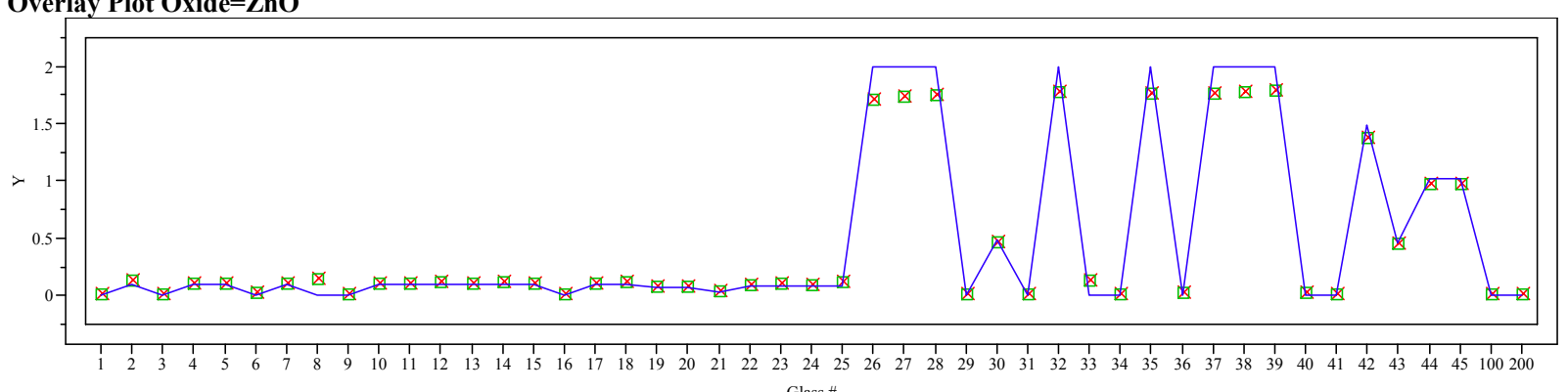

Overlay Plot Oxide $=\mathrm{ZrO} 2$

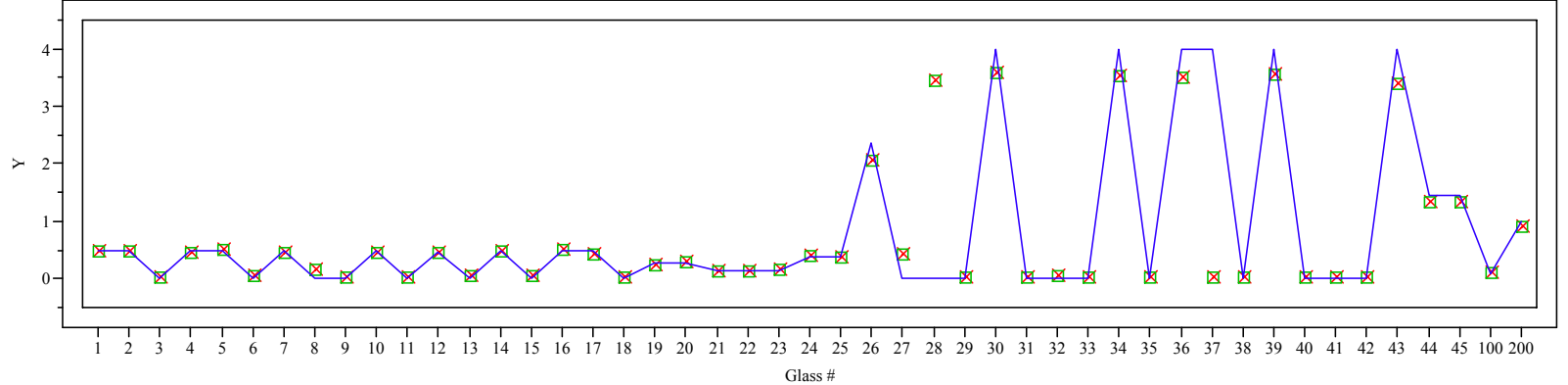

Overlay Plot Oxide $=$ Sum of Oxides

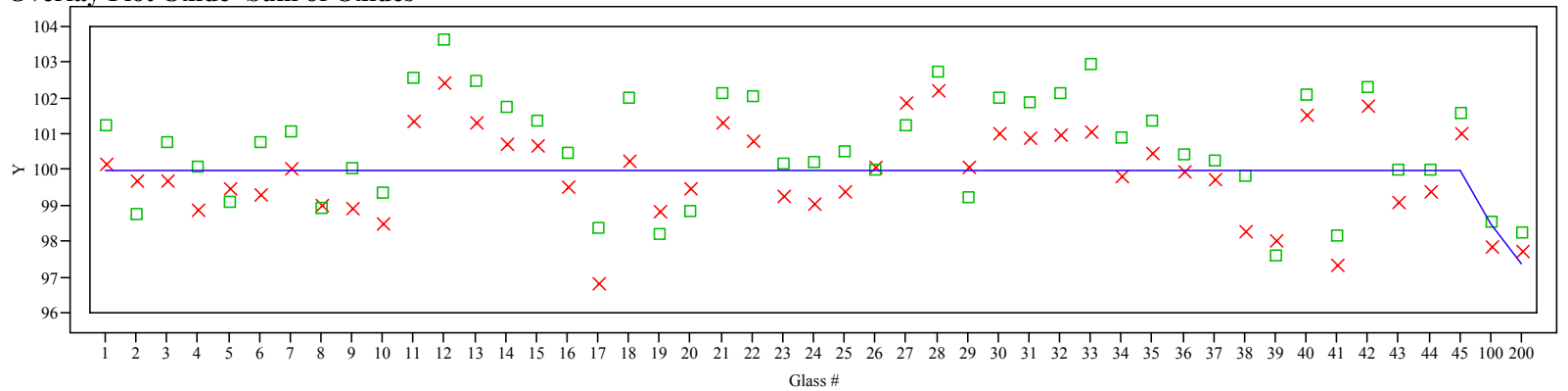

Y $\times$ Measured $\square$ Measured bc - Targeted 
This page is intentionally left blank. 


\section{Appendix B:}

\section{Tables and Exhibits Supporting the Analysis of the PCT Results for the US Study Glasses}


Table B1. Laboratory Measurements of the PCT Solutions for the US Study Glasses

\begin{tabular}{|c|c|c|c|c|c|c|c|c|c|c|c|c|c|}
\hline Set & Type & Glass ID & Heat Treatment & Block & Seq & $\begin{array}{l}\text { Analytical } \\
\text { Sequence }\end{array}$ & Lab ID & $\mathrm{Al}$ ar & $\mathrm{B}$ ar & $\mathrm{Fe}$ ar & $\mathrm{Li}$ ar & $\mathrm{Na}$ ar & Si ar \\
\hline 1 & Soln Std & Soln Std & & 1 & 1 & 1 & STD-B1-1 & 3.75 & 20.6 & 4.07 & 9.88 & 82 & 50.2 \\
\hline 1 & ARM & ARM & & 1 & 2 & 2 & W73 & 2.46 & 10.5 & $<0.100$ & 8.08 & 21.3 & 37.2 \\
\hline 1 & US & US-01 & quenched & 1 & 3 & 3 & W88 & 20.5 & 7.32 & 9.84 & 9.16 & 30.7 & 54.7 \\
\hline 1 & US & US-01ccc & $\mathrm{ccc}$ & 1 & 4 & 4 & W45 & 19 & 6.82 & 8.19 & 8.5 & 27.7 & 50.3 \\
\hline 1 & US & US-02 & quenched & 1 & 5 & 5 & W76 & 17.3 & 53.7 & 8.06 & 22.2 & 48.1 & 39.4 \\
\hline 1 & US & US-02 $\mathrm{ccc}$ & $\mathrm{ccc}$ & 1 & 6 & 6 & W68 & 17.6 & 45.2 & 8.33 & 18.7 & 42.5 & 41.6 \\
\hline 1 & US & US-11 & quenched & 1 & 7 & 7 & W96 & 11.2 & 10.2 & 0.152 & 3.15 & 10.6 & 24.4 \\
\hline 1 & US & US-05ccc & $\mathrm{ccc}$ & 1 & 8 & 8 & W55 & 14.8 & 14.2 & 0.478 & 3.72 & 14.7 & 33 \\
\hline 1 & US & US-08 & quenched & 1 & 9 & 9 & W85 & 18.2 & 7.53 & 10 & 8.06 & 13.6 & 47.5 \\
\hline 1 & Soln Std & Soln Std & & 1 & 10 & 10 & STD-B1-2 & 3.95 & 19.6 & 3.91 & 9.81 & 82.9 & 49.1 \\
\hline 1 & US & US-08ccc & $\mathrm{ccc}$ & 1 & 11 & 11 & W90 & 18.8 & 7.81 & 10.9 & 7.7 & 14.8 & 49.5 \\
\hline 1 & US & US-10 & quenched & 1 & 12 & 12 & W51 & 11.8 & 7.48 & 0.154 & 2.81 & 21.8 & 29 \\
\hline 1 & US & US-10ccc & $\mathrm{ccc}$ & 1 & 13 & 13 & W75 & 11.4 & 7.3 & 0.192 & 2.69 & 21.3 & 28.5 \\
\hline 1 & US & US-09 $\mathrm{ccc}$ & $\mathrm{ccc}$ & 1 & 14 & 14 & W95 & 8.09 & 13.8 & $<0.100$ & 3.35 & 6.84 & 22.9 \\
\hline 1 & US & US-11ccc & $\mathrm{ccc}$ & 1 & 15 & 15 & W08 & 10 & 30.9 & 0.64 & 5.32 & 15.9 & 28.8 \\
\hline 1 & US & US-13 & quenched & 1 & 16 & 16 & W74 & 13.1 & 9.84 & 0.338 & 3.09 & 17.6 & 29.2 \\
\hline 1 & US & US-13ccc & $\mathrm{ccc}$ & 1 & 17 & 17 & W58 & 11.9 & 8.19 & 0.491 & 2.65 & 15.4 & 26.2 \\
\hline 1 & US & US-14 & quenched & 1 & 18 & 18 & W80 & 14.9 & 98.9 & 1.98 & 41.1 & 91.4 & 33.7 \\
\hline 1 & US & US-14ccc & $\mathrm{ccc}$ & 1 & 19 & 19 & W91 & 15.2 & 88.2 & 1.35 & 36.8 & 82.2 & 34.2 \\
\hline 1 & Soln Std & Soln Std & & 1 & 20 & 20 & STD-B1-3 & 3.83 & 20.2 & 4.17 & 9.8 & 83.5 & 48.8 \\
\hline 1 & Soln Std & Soln Std & & 2 & 1 & 21 & STD-B2-1 & 3.87 & 20.3 & 5.25 & 9.77 & 82.8 & 49.5 \\
\hline 1 & ARM & ARM & & 2 & 2 & 22 & W26 & 2.57 & 10.6 & $<0.100$ & 7.89 & 21.9 & 35.4 \\
\hline 1 & US & US-01 & quenched & 2 & 3 & 23 & W37 & 18.8 & 6.75 & 8.27 & 8.38 & 28.4 & 49.6 \\
\hline 1 & US & US-01ccc & $\mathrm{ccc}$ & 2 & 4 & 24 & W24 & 18.9 & 6.75 & 7.84 & 8.4 & 27.9 & 49.9 \\
\hline 1 & US & US-02 & quenched & 2 & 5 & 25 & W05 & 17.1 & 53.1 & 7.69 & 21.9 & 49 & 38.7 \\
\hline 1 & US & US-02ccc & $\mathrm{ccc}$ & 2 & 6 & 26 & W79 & 17.3 & 43.7 & 7.88 & 18.1 & 42.9 & 39.8 \\
\hline 1 & US & US-05 & quenched & 2 & 7 & 27 & W89 & 11.9 & 10.5 & $<0.100$ & 3.17 & 11.4 & 25.1 \\
\hline 1 & US & US-05ccc & $\mathrm{ccc}$ & 2 & 8 & 28 & W09 & 13.8 & 15.6 & 0.344 & 3.78 & 15.6 & 29.9 \\
\hline 1 & US & US-08 & quenched & 2 & 9 & 29 & W67 & 19.2 & 7.61 & 10.2 & 8.16 & 14.5 & 48.5 \\
\hline 1 & Soln Std & Soln Std & & 2 & 10 & 30 & STD-B2-2 & 3.71 & 19.2 & 3.45 & 9.58 & 83.8 & 48 \\
\hline 1 & US & US-08ccc & $\mathrm{ccc}$ & 2 & 11 & 31 & W83 & 16.9 & 6.97 & 8.4 & 6.9 & 14 & 42.9 \\
\hline 1 & US & US-10 & quenched & 2 & 12 & 32 & W07 & 11.9 & 7.14 & $<0.100$ & 2.72 & 22.5 & 28.4 \\
\hline 1 & US & US-10ccc & $\mathrm{ccc}$ & 2 & 13 & 33 & W78 & 11.3 & 6.91 & $<0.100$ & 2.61 & 21.7 & 26.8 \\
\hline 1 & US & US-11 & quenched & 2 & 14 & 34 & W31 & 7.63 & 13.5 & $<0.100$ & 3.2 & 5.87 & 23.5 \\
\hline 1 & US & US-11 ccc & $\mathrm{ccc}$ & 2 & 15 & 35 & W21 & 9.74 & 29.1 & 0.564 & 4.93 & 14.3 & 27.9 \\
\hline 1 & US & US-13 & quenched & 2 & 16 & 36 & W47 & 12.4 & 9.54 & 0.204 & 2.96 & 16.3 & 27.8 \\
\hline 1 & US & US-13ccc & $\mathrm{ccc}$ & 2 & 17 & 37 & W44 & 11.5 & 8.11 & 0.418 & 2.55 & 14.3 & 26.1 \\
\hline 1 & US & US-14 & quenched & 2 & 18 & 38 & W63 & 14.5 & 104 & 2.07 & 42 & 89.5 & 34.6 \\
\hline 1 & US & US-14ccc & $\operatorname{ccc}$ & 2 & 19 & 39 & W01 & 14.6 & 85.6 & 1.87 & 34.7 & 75.6 & 34.3 \\
\hline 1 & Soln Std & Soln Std & & 2 & 20 & 40 & STD-B2-3 & 3.57 & 20.3 & 3.65 & 9.64 & 80.3 & 48.5 \\
\hline
\end{tabular}


Table B1. Laboratory Measurements of the PCT Solutions for the US Study Glasses

\begin{tabular}{|c|c|c|c|c|c|c|c|c|c|c|c|c|c|}
\hline Set & Type & Glass ID & Heat Treatment & Block & Seq & $\begin{array}{c}\text { Analytical } \\
\text { Sequence }\end{array}$ & Lab ID & $\mathrm{Al}$ ar & $\mathrm{B}$ ar & $\mathrm{Fe}$ ar & $\mathrm{Li}$ ar & $\mathrm{Na}$ ar & Si ar \\
\hline 1 & Soln Std & Soln Std & & 3 & 1 & 41 & STD-B3-1 & 3.83 & 20.4 & 4.98 & 9.77 & 82.2 & 49.5 \\
\hline 1 & ARM & ARM & & 3 & 2 & 42 & W82 & 2.65 & 11.1 & $<0.100$ & 8.12 & 22.5 & 36.3 \\
\hline 1 & blank & blank & & 3 & 3 & 43 & W17 & $<0.100$ & $<0.100$ & $<0.100$ & $<0.500$ & $<0.100$ & $<0.100$ \\
\hline 1 & US & US-01 & quenched & 3 & 4 & 44 & W18 & 19.2 & 6.71 & 8.74 & 8.49 & 29.1 & 50.8 \\
\hline 1 & US & US-01ccc & $\mathrm{ccc}$ & 3 & 5 & 45 & W28 & 19.2 & 6.83 & 8.31 & 8.53 & 28 & 50.8 \\
\hline 1 & US & US-02 & quenched & 3 & 6 & 46 & W52 & 18.3 & 56.6 & 8.65 & 23 & 50.2 & 42.4 \\
\hline 1 & US & US-02cec & $\mathrm{ccc}$ & 3 & 7 & 47 & W56 & 16.7 & 42.8 & 7.85 & 17.5 & 40.1 & 39.3 \\
\hline 1 & US & US-05 & quenched & 3 & 8 & 48 & W65 & 11.9 & 10.5 & $<0.100$ & 3.2 & 10.9 & 25.8 \\
\hline 1 & US & US- $05 \mathrm{ccc}$ & $\mathrm{ccc}$ & 3 & 9 & 49 & W13 & 13.8 & 16 & 0.279 & 3.85 & 15.6 & 30.6 \\
\hline 1 & Soln Std & Soln Std & & 3 & 10 & 50 & STD-B3-2 & 3.85 & 19.7 & 3.67 & 9.72 & 82 & 49.1 \\
\hline 1 & US & US-08 & quenched & 3 & 11 & 51 & W23 & 19.8 & 8.03 & 11 & 8.48 & 14.5 & 50.6 \\
\hline 1 & US & US-08ccc & $\mathrm{ccc}$ & 3 & 12 & 52 & W98 & 17.4 & 7.06 & 9.13 & 7.12 & 13.7 & 45 \\
\hline 1 & US & US-10 & quenched & 3 & 13 & 53 & W71 & 12 & 7.22 & 0.112 & 2.77 & 21.8 & 28.8 \\
\hline 1 & US & US-10 ccc & $\mathrm{ccc}$ & 3 & 14 & 54 & W29 & 11.6 & 7.22 & 0.163 & 2.71 & 21.1 & 28.9 \\
\hline 1 & US & US-11 & quenched & 3 & 15 & 55 & W53 & 8.32 & 13.3 & $<0.100$ & 3.29 & 6.96 & 23.4 \\
\hline 1 & US & US-11 ccc & $\mathrm{ccc}$ & 3 & 16 & 56 & W86 & 9.89 & 29.1 & 0.543 & 5.03 & 15.5 & 27.9 \\
\hline 1 & US & US-13 & quenched & 3 & 17 & 57 & W69 & 12.6 & 9.28 & 0.194 & 2.96 & 16.9 & 27.6 \\
\hline 1 & US & US-13ccc & $\mathrm{ccc}$ & 3 & 18 & 58 & W11 & 11.9 & 8.05 & 0.505 & 2.62 & 15.2 & 26.1 \\
\hline 1 & US & US-14 & quenched & 3 & 19 & 59 & W60 & 15.6 & 110 & 1.93 & 44.1 & 94.3 & 36.1 \\
\hline 1 & US & US-14ccc & $\mathrm{ccc}$ & 3 & 20 & 60 & W36 & 15.3 & 88.2 & 2.04 & 35.4 & 77.3 & 35.4 \\
\hline 1 & Soln Std & Soln Std & & 3 & 21 & 61 & STD-B3-3 & 4.28 & 20.9 & 3.92 & 9.95 & 82.6 & 50.8 \\
\hline 1 & Soln Std & Soln Std & & 4 & 1 & 62 & STD-B4-1 & 3.84 & 20.8 & 5.47 & 9.72 & 81.9 & 50.3 \\
\hline 1 & EA & EA & & 4 & 2 & 63 & W92 & $<0.100$ & 37.2 & $<0.100$ & 11 & 99.2 & 52.4 \\
\hline 1 & US & US-03 & quenched & 4 & 3 & 64 & W14 & 13.4 & 11.2 & 4.7 & 5.74 & 4.69 & 32.4 \\
\hline 1 & US & US- $03 \mathrm{ccc}$ & $\mathrm{ccc}$ & 4 & 4 & 65 & W39 & 13 & 10.5 & 4.12 & 5.21 & 5.66 & 31.4 \\
\hline 1 & US & US-04 & quenched & 4 & 5 & 66 & W42 & 13.2 & 7.16 & 0.278 & 4.88 & 12.6 & 29.4 \\
\hline 1 & US & US-04ccc & $\operatorname{ccc}$ & 4 & 6 & 67 & W10 & 12.6 & 6.77 & $<0.100$ & 4.41 & 12.8 & 27.7 \\
\hline 1 & US & US-06 & quenched & 4 & 7 & 68 & W35 & 12.3 & 8.83 & $<0.100$ & 3.31 & 13.5 & 26 \\
\hline 1 & US & US-06 ccc & $\mathrm{ccc}$ & 4 & 8 & 69 & W06 & 11.4 & 7.9 & $<0.100$ & 2.86 & 12.7 & 24.8 \\
\hline 1 & US & US-07 & quenched & 4 & 9 & 70 & W19 & 12.2 & 8.66 & $<0.100$ & 3.56 & 13.2 & 26.4 \\
\hline 1 & Soln Std & Soln Std & & 4 & 10 & 71 & STD-B4-2 & 4.14 & 20.1 & 3.72 & 9.64 & 82.9 & 49.7 \\
\hline 1 & US & US-07ccc & $\mathrm{ccc}$ & 4 & 11 & 72 & W93 & 11.6 & 8.03 & $<0.100$ & 3.12 & 12.3 & 25.5 \\
\hline 1 & US & US-09 & quenched & 4 & 12 & 73 & W66 & 12.8 & 12.1 & 6.72 & 2.96 & 26.4 & 32.2 \\
\hline 1 & US & US-09ccc & $\mathrm{ccc}$ & 4 & 13 & 74 & W16 & 11.9 & 11.5 & 4.7 & 2.68 & 24.5 & 30.7 \\
\hline 1 & US & US-12 & quenched & 4 & 14 & 75 & W46 & 15.1 & 36.6 & 2.61 & 17.9 & 43.9 & 37.3 \\
\hline 1 & US & US-12ccc & $\mathrm{ccc}$ & 4 & 15 & 76 & W48 & 16.4 & 51.1 & 6.43 & 24.4 & 53.6 & 40.6 \\
\hline 1 & US & US-15 & quenched & 4 & 16 & 77 & W84 & 5.81 & 145 & 2.9 & 20.4 & 168 & 26.4 \\
\hline 1 & US & US-15ccc & $\mathrm{ccc}$ & 4 & 17 & 78 & W70 & 6.13 & 117 & 3.39 & 16.6 & 137 & 29 \\
\hline 1 & Soln Std & Soln Std & & 4 & 18 & 79 & STD-B4-3 & 3.8 & 20.8 & 4.53 & 9.77 & 82.1 & 50.2 \\
\hline 1 & Soln Std & Soln Std & & 5 & 1 & 80 & STD-B5-1 & 3.98 & 20.7 & 3.93 & 9.81 & 82.6 & 50 \\
\hline
\end{tabular}


Table B1. Laboratory Measurements of the PCT Solutions for the US Study Glasses

\begin{tabular}{|c|c|c|c|c|c|c|c|c|c|c|c|c|c|}
\hline Set & Type & Glass ID & Heat Treatment & Block & Seq & $\begin{array}{c}\text { Analytical } \\
\text { Sequence }\end{array}$ & Lab ID & $\mathrm{Al}$ ar & $\mathrm{B}$ ar & $\mathrm{Fe}$ ar & $\mathrm{Li}$ ar & $\mathrm{Na}$ ar & $\mathrm{Si}$ ar \\
\hline 1 & EA & EA & & 5 & 2 & 81 & W64 & $<0.100$ & 37.4 & $<0.100$ & 11.4 & 99.5 & 53.2 \\
\hline 1 & US & US-05 & quenched & 5 & 3 & 82 & W97 & 13.1 & 10.8 & 4.85 & 5.72 & 4.83 & 31.8 \\
\hline 1 & US & US-03ccc & $\mathrm{ccc}$ & 5 & 4 & 83 & W38 & 13.2 & 10.4 & 4.32 & 5.34 & 5.86 & 31.8 \\
\hline 1 & US & US-04 & quenched & 5 & 5 & 84 & W54 & 13.7 & 7.22 & 0.634 & 5.14 & 13.7 & 30.3 \\
\hline 1 & US & US-04ccc & $\mathrm{ccc}$ & 5 & 6 & 85 & W77 & 13.5 & 6.9 & 0.314 & 4.75 & 13.1 & 30.5 \\
\hline 1 & US & US-06 & quenched & 5 & 7 & 86 & W22 & 12.6 & 8.8 & 0.207 & 3.47 & 14.4 & 26.7 \\
\hline 1 & US & US-06 ccc & $\mathrm{ccc}$ & 5 & 8 & 87 & W57 & 12.3 & 8.24 & 0.439 & 3.12 & 13.6 & 27.1 \\
\hline 1 & US & US-07 & quenched & 5 & 9 & 88 & W81 & 12.7 & 8.5 & $<0.100$ & 3.72 & 13.7 & 27 \\
\hline 1 & Soln Std & Soln Std & & 5 & 10 & 89 & STD-B5-2 & 4.01 & 19.9 & 4.81 & 9.78 & 83.4 & 49.7 \\
\hline 1 & US & US-07ccc & $\mathrm{ccc}$ & 5 & 11 & 90 & W33 & 12.1 & 8.13 & $<0.100$ & 3.34 & 12.7 & 27 \\
\hline 1 & US & US-09 & quenched & 5 & 12 & 91 & W61 & 11.8 & 11.8 & 6.7 & 3 & 24.4 & 32.1 \\
\hline 1 & US & US-09 & $\mathrm{ccc}$ & 5 & 13 & 92 & W72 & 12.3 & 12 & 5.02 & 2.9 & 26.5 & 32.1 \\
\hline 1 & US & US-12 & quenched & 5 & 14 & 93 & W27 & 15.6 & 38.4 & 2.73 & 18.9 & 44.8 & 38.9 \\
\hline 1 & US & US-12ccc & $\mathrm{ccc}$ & 5 & 15 & 94 & W32 & 16.5 & 51.5 & 6.84 & 24.8 & 53.7 & 41.3 \\
\hline 1 & US & US-15 & quenched & 5 & 16 & 95 & W03 & 6.43 & 155 & 3.4 & 22.3 & 178 & 28.5 \\
\hline 1 & US & US-15ccc & $\mathrm{ccc}$ & 5 & 17 & 96 & W50 & 6.27 & 120 & 3.72 & 17.3 & 141 & 30.2 \\
\hline 1 & Soln Std & Soln Std & & 5 & 18 & 97 & STD-B5-3 & 3.99 & 21.3 & 4.05 & 9.76 & 82.6 & 49.6 \\
\hline 1 & Soln Std & Soln Std & & 6 & 1 & 98 & STD-B6-1 & 4.07 & 20.5 & 3.69 & 9.88 & 81.3 & 50.1 \\
\hline 1 & blank & blank & & 6 & 2 & 99 & W40 & $<0.100$ & 0.403 & $<0.100$ & $<0.500$ & $<0.100$ & $<0.100$ \\
\hline 1 & EA & EA & & 6 & 3 & 100 & W02 & 0.123 & 39.1 & $<0.100$ & 12 & 102 & 55.9 \\
\hline 1 & US & US-03 & quenched & 6 & 4 & 101 & W62 & 14 & 11.4 & 4.85 & 6.3 & 5.45 & 34.3 \\
\hline 1 & US & US-03ccc & $\mathrm{ccc}$ & 6 & 5 & 102 & W12 & 13.3 & 10.5 & 4.07 & 5.63 & 5.83 & 32 \\
\hline 1 & US & US-04 & quenched & 6 & 6 & 103 & W43 & 14.6 & 7.66 & 0.398 & 5.64 & 13.2 & 32.4 \\
\hline 1 & US & US-04ccc & $\mathrm{ccc}$ & 6 & 7 & 104 & W30 & 13.6 & 7.23 & $<0.100$ & 5.16 & 13.2 & 30.9 \\
\hline 1 & US & US-06 & quenched & 6 & 8 & 105 & W15 & 12.6 & 8.83 & $<0.100$ & 3.75 & 13.9 & 27 \\
\hline 1 & US & US-06 ccc & $\mathrm{ccc}$ & 6 & 9 & 106 & W87 & 11.6 & 7.98 & $<0.100$ & 3.3 & 13.1 & 26.1 \\
\hline 1 & Soln Std & Soln Std & & 6 & 10 & 107 & STD-B6-2 & 4.15 & 20.3 & 3.77 & 10 & 81.7 & 50.5 \\
\hline 1 & US & US-07 & quenched & 6 & 11 & 108 & W49 & 13.1 & 9.53 & $<0.100$ & 4.2 & 15 & 29.4 \\
\hline 1 & US & US-07ccc & $\mathrm{ccc}$ & 6 & 12 & 109 & W25 & 12 & 8.11 & $<0.100$ & 3.6 & 13.3 & 27 \\
\hline 1 & US & US-09 & quenched & 6 & 13 & 110 & W41 & 11.9 & 12.5 & 6.23 & 3.38 & 25 & 32.5 \\
\hline 1 & US & US-03 & quenched & 6 & 14 & 111 & W97 & 12.6 & 11.9 & 5.42 & 3.15 & 24.4 & 33.9 \\
\hline 1 & US & US-12 & quenched & 6 & 15 & 112 & W04 & 16.6 & 39.2 & 3.18 & 19.3 & 44.6 & 40.7 \\
\hline 1 & US & US-12ccc & $\mathrm{ccc}$ & 6 & 16 & 113 & W34 & 18.2 & 54.2 & 7.07 & 25.8 & 55.9 & 44.6 \\
\hline 1 & US & US-15 & quenched & 6 & 17 & 114 & W20 & 6.79 & 163 & 3.64 & 23 & 180 & 30.5 \\
\hline 1 & US & US- $15 \mathrm{ccc}$ & $\mathrm{ccc}$ & 6 & 18 & 115 & W59 & 6.68 & 126 & 3.82 & 17.9 & 142 & 32 \\
\hline 1 & Soln Std & Soln Std & & 6 & 19 & 116 & STD-B6-3 & 4.02 & 21.6 & 3.69 & 9.83 & 80.3 & 50.3 \\
\hline 2 & Soln Std & Soln Std & & 1 & 1 & 117 & STD-B1-1 & 3.92 & 21.5 & 3.9 & 10.1 & 81.2 & 50.6 \\
\hline 2 & US & US-23 & quenched & 1 & 2 & 118 & X005 & 16.2 & 11.5 & 4.72 & 6.06 & 18.9 & 42.6 \\
\hline 2 & US & US-24ccc & $\operatorname{ccc}$ & 1 & 3 & 119 & X065 & 14.3 & 9.46 & 2.28 & 4.73 & 18.6 & 37.1 \\
\hline 2 & US & US-25 & quenched & 1 & 4 & 120 & X093 & 13 & 35.3 & 1.47 & 14.8 & 38.5 & 39.2 \\
\hline
\end{tabular}


Table B1. Laboratory Measurements of the PCT Solutions for the US Study Glasses

\begin{tabular}{|c|c|c|c|c|c|c|c|c|c|c|c|c|c|}
\hline Set & Type & Glass ID & Heat Treatment & Block & Seq & $\begin{array}{l}\text { Analytical } \\
\text { Sequence }\end{array}$ & Lab ID & $\mathrm{Al}$ ar & $\mathrm{B}$ ar & $\mathrm{Fe}$ ar & $\mathrm{Li}$ ar & $\mathrm{Na}$ ar & Si ar \\
\hline 2 & US & US-21 & quenched & 1 & 5 & 121 & X021 & 15.1 & 11.5 & 5.9 & 5.38 & 15.2 & 41.3 \\
\hline 2 & US & US-28 & quenched & 1 & 6 & 122 & $\mathrm{X} 012$ & 15.9 & 59.2 & 4.13 & 8.74 & 64.7 & 28 \\
\hline 2 & US & US-25ccc & $\operatorname{ccc}$ & 1 & 7 & 123 & $\mathrm{X} 088$ & 13.3 & 43.4 & 3.04 & 17.5 & 44.8 & 40.4 \\
\hline 2 & US & US-30 & quenched & 1 & 8 & 124 & $\mathrm{X} 097$ & 10.6 & 19.1 & 0.585 & 9.6 & 9.21 & 24 \\
\hline 2 & US & US-21 ccc & $\operatorname{ccc}$ & 1 & 9 & 125 & X009 & 13.6 & 10.7 & 3.17 & 4.82 & 14.6 & 37.6 \\
\hline 2 & US & US-24 & quenched & 1 & 10 & 126 & $\begin{array}{l}\mathrm{X} 029 \\
\end{array}$ & 14 & 9.6 & 2.18 & $\begin{array}{l}4.8 \\
\end{array}$ & 17.5 & 36.6 \\
\hline 2 & Soln Std & Soln Std & & 1 & 11 & 127 & STD-B1-2 & 3.86 & 20.5 & 3.69 & 9.94 & 81 & 49.6 \\
\hline 2 & US & US- $23 \mathrm{ccc}$ & $\mathrm{ccc}$ & 1 & 12 & 128 & X092 & 15.3 & 11.5 & 3.78 & 5.64 & 18.9 & 40.2 \\
\hline 2 & US & US-27 & quenched & 1 & 13 & 129 & $\mathrm{X} 032$ & 28.9 & 15.7 & 35.4 & 17.5 & 118 & 95.1 \\
\hline 2 & EA & EA & & 1 & 14 & 130 & $\mathrm{X} 069$ & 0.48 & 38.2 & 0.202 & 11.8 & 103 & 54.9 \\
\hline 2 & US & US-20 ccc & $\operatorname{ccc}$ & 1 & 15 & 131 & $\mathrm{X} 030$ & 14.5 & 12.1 & 2.19 & 5.74 & 17 & 36.1 \\
\hline 2 & US & US-27ccc & $\operatorname{ccc}$ & 1 & 16 & 132 & $\mathrm{X} 049$ & 8.12 & 205 & $<0.100$ & 122 & 568 & 267 \\
\hline 2 & US & US- $28 \mathrm{ccc}$ & $\operatorname{ccc}$ & 1 & 17 & 133 & $\mathrm{X} 071$ & 15.6 & 46.6 & 4.65 & 7.1 & 53.8 & 27.8 \\
\hline 2 & US & US-30 ccc & $\mathrm{ccc}$ & 1 & 18 & 134 & $\mathrm{X} 013$ & 11.1 & 22.5 & 0.269 & 10.4 & 9.84 & 26 \\
\hline 2 & US & US-20 & quenched & 1 & 19 & 135 & $\mathrm{X} 048$ & 15 & 12.8 & 3.59 & 6.1 & 16.8 & 36.9 \\
\hline 2 & blank & Blank 1 (ACTL-14) & & 1 & 20 & 136 & $\mathrm{X} 051$ & 4.03 & 1.23 & 0.197 & 0.709 & 5.41 & 22.7 \\
\hline 2 & Soln Std & Soln Std & & 1 & 21 & 137 & STD-B1-3 & 3.85 & 20.3 & 3.57 & 9.89 & 81 & 49.6 \\
\hline 2 & Soln Std & Soln Std & & 2 & 1 & 138 & STD-B2-1 & 3.86 & 20.9 & 3.86 & 9.9 & 81.1 & 50.1 \\
\hline 2 & US & US-23ccc & $\mathrm{ccc}$ & 2 & 2 & 139 & X026 & 15.7 & 11.2 & 3.59 & 5.58 & 18.6 & 41.2 \\
\hline 2 & US & US- $21 \mathrm{ccc}$ & $\operatorname{ccc}$ & 2 & 3 & 140 & $\mathrm{X} 006$ & 13.4 & 9.93 & 3.42 & 4.65 & 14 & 37.6 \\
\hline 2 & US & US-20 & quenched & 2 & 4 & 141 & $\mathrm{X} 010$ & 15.3 & 11.5 & 3.87 & 6.11 & 18.8 & 38.5 \\
\hline 2 & US & US-23 & quenched & 2 & 5 & 142 & $\mathrm{X} 003$ & 16.4 & 11.4 & 3.71 & 6.17 & 19.4 & 43.7 \\
\hline 2 & US & US-27ccc & $\mathrm{ccc}$ & 2 & 6 & 143 & $\mathrm{X} 082$ & 7.77 & 201 & $<0.100$ & 121 & 571 & 260 \\
\hline 2 & US & US-30 & quenched & 2 & 7 & 144 & $\mathrm{X} 075$ & 10.4 & 20.4 & $<0.100$ & 9.76 & 8.69 & 24 \\
\hline 2 & US & US-28ccc & $\operatorname{ccc}$ & 2 & 8 & 145 & $\mathrm{X} 035$ & 15.9 & 46.5 & 5.19 & 7.07 & 54.7 & 28.5 \\
\hline 2 & US & US-24ccc & $\mathrm{ccc}$ & 2 & 9 & 146 & $\mathrm{X} 062$ & 14.7 & 9.77 & 1.81 & 4.61 & 17.9 & 37.2 \\
\hline 2 & US & US-24 & quenched & 2 & 10 & 147 & $\mathrm{X} 095$ & 13.6 & 9.06 & 1.71 & 4.65 & 16.9 & 35.9 \\
\hline 2 & Soln Std & Soln Std & & 2 & 11 & 148 & STD-B-2-2 & 3.76 & 20 & 3.72 & 9.83 & 81.3 & 49.4 \\
\hline 2 & US & US-27 & quenched & 2 & 12 & 149 & $\mathrm{X} 057$ & 28.2 & 15.2 & 33.5 & 17.3 & 118 & 92.1 \\
\hline 2 & EA & EA & & 2 & 13 & 150 & $\mathrm{X} 053$ & 0.292 & 37.5 & $<0.100$ & 11.6 & 102 & 55.1 \\
\hline 2 & US & US-20 ccc & $\mathrm{ccc}$ & 2 & 14 & 151 & $\mathrm{X} 045$ & 14.1 & 11.1 & 2.12 & 5.55 & 15.9 & 35.6 \\
\hline 2 & US & US-25 & quenched & 2 & 15 & 152 & X096 & 12.3 & 34.2 & 1.48 & 14.5 & 37.4 & 38.7 \\
\hline 2 & US & US-21 & quenched & 2 & 16 & 153 & $\mathrm{X} 023$ & 25.4 & 10.4 & 4.9 & 5.06 & 16.3 & 60 \\
\hline 2 & US & US-25ccc & $\mathrm{ccc}$ & 2 & 17 & 154 & X081 & 30 & 42.7 & 3.51 & 17.4 & 46.8 & 71.3 \\
\hline 2 & US & US-30 ccc & $\mathrm{ccc}$ & 2 & 18 & 155 & $\mathrm{X} 028$ & 11.2 & 21.5 & $<0.100$ & 10.6 & 9.68 & 26.7 \\
\hline 2 & US & US-28 & quenched & 2 & 19 & 156 & $\mathrm{X} 077$ & 14.8 & 55.3 & 4.14 & 8.24 & 62.1 & 26.2 \\
\hline 2 & Soln Std & Soln Std & & 2 & 20 & 157 & STD-B2-3 & 3.74 & 20 & 3.62 & 9.74 & 81.1 & 48.9 \\
\hline 2 & Soln Std & Soln Std & & 3 & 1 & 158 & STD-B3-1 & 4.03 & 20.6 & 3.51 & 9.81 & 81.1 & 49.6 \\
\hline 2 & US & US-21 & quenched & 3 & 2 & 159 & X060 & 13.8 & 10.2 & 4.23 & 4.77 & 14.2 & 37.4 \\
\hline 2 & US & US-23 & quenched & 3 & 3 & 160 & X094 & 17.6 & 10.6 & 4.01 & 5.59 & 18 & 45.3 \\
\hline
\end{tabular}


Table B1. Laboratory Measurements of the PCT Solutions for the US Study Glasses

\begin{tabular}{|c|c|c|c|c|c|c|c|c|c|c|c|c|c|}
\hline Set & Type & Glass ID & Heat Treatment & Block & Seq & $\begin{array}{l}\text { Analytical } \\
\text { Sequence }\end{array}$ & Lab ID & $\mathrm{Al}$ ar & $\mathrm{B}$ ar & $\mathrm{Fe}$ ar & $\mathrm{Li}$ ar & $\mathrm{Na}$ ar & $\mathrm{Si}$ ar \\
\hline 2 & US & US-23ccc & $\mathrm{ccc}$ & 3 & 4 & 161 & X085 & 14.9 & 10 & 2.96 & 5.29 & 18.2 & 39 \\
\hline 2 & US & US-20ccc & $\mathrm{ccc}$ & 3 & 5 & 162 & $\mathrm{X} 061$ & 14.9 & 10.5 & 2.94 & 5.44 & 16.8 & 36.1 \\
\hline 2 & US & US-27ccc & $\mathrm{ccc}$ & 3 & 6 & 163 & $\mathrm{X} 055$ & 8.06 & 190 & $<0.100$ & 115 & 541 & 249 \\
\hline 2 & US & US-25 & quenched & 3 & 7 & 164 & $\mathrm{X} 084$ & 14 & 34.6 & 1.14 & 13.9 & 37.5 & 39.4 \\
\hline 2 & blank & Blank 2 (ACTL -14) & & 3 & 8 & 165 & $\mathrm{X} 054$ & $<0.100$ & 0.79 & $<0.100$ & $<0.500$ & $<0.100$ & $<0.100$ \\
\hline 2 & US & US-28 & quenched & 3 & 9 & 166 & $\mathrm{X} 047$ & 15.1 & 56.9 & 4.28 & 8.18 & 60.5 & 26.5 \\
\hline 2 & US & US-30 & quenched & 3 & 10 & 167 & X090 & 10.7 & 19 & $<0.100$ & 9.32 & 8.17 & 23.9 \\
\hline 2 & Soln Std & Soln Std & & 3 & 11 & 168 & STD-B3-2 & 4 & 21.4 & 3.93 & 10.1 & 80.8 & 52.5 \\
\hline 2 & US & US-25ccc & $\mathrm{ccc}$ & 3 & 12 & 169 & $\mathrm{X} 011$ & 14.9 & 42.2 & 2.79 & 16.8 & 42.7 & 39.7 \\
\hline 2 & US & US-21ccc & $\mathrm{ccc}$ & 3 & 13 & 170 & X004 & 14.3 & 9.86 & 4.23 & 4.59 & 15.7 & 38.5 \\
\hline 2 & US & US-30 ccc & $\mathrm{ccc}$ & 3 & 14 & 171 & $\mathrm{X} 001$ & 11.7 & 21.1 & $<0.100$ & 10.2 & 10 & 27.4 \\
\hline 2 & $\overline{\mathrm{US}}$ & US-28ccc & $\mathrm{ccc}$ & 3 & 15 & 172 & $\mathrm{X} 076$ & 15.9 & 45.1 & 5.41 & 6.85 & 53.4 & 28 \\
\hline 2 & US & US-20 & quenched & 3 & 16 & 173 & X039 & 14.6 & 11.6 & 3.13 & 5.83 & 15.7 & 37.1 \\
\hline 2 & EA & EA & & 3 & 17 & 174 & $\mathrm{X} 031$ & 0.132 & 37.4 & $<0.100$ & 11.3 & 101 & 53.6 \\
\hline 2 & US & US-24ccc & $\mathrm{ccc}$ & 3 & 18 & 175 & X014 & 14.1 & 8.72 & 1.78 & 4.38 & 18.1 & 36.6 \\
\hline 2 & US & US-24 & quenched & 3 & 19 & 176 & X034 & 13.9 & 8.46 & 1.66 & 4.53 & 17.8 & 36.1 \\
\hline 2 & US & US-27 & quenched & 3 & 20 & 177 & X037 & 28.4 & 14.4 & 36 & 16.8 & 116 & 92.5 \\
\hline 2 & Soln Std & Soln Std & & 3 & 21 & 178 & STD-B3-3 & 3.92 & 20.2 & 3.7 & 9.86 & 80.7 & 51.1 \\
\hline 2 & Soln Std & Soln Std & & 4 & 1 & 179 & STD-B4-1 & 3.82 & 21.3 & 4.02 & 10 & 81.4 & 50.9 \\
\hline 2 & US & US-19 & quenched & 4 & 2 & 180 & X089 & 15 & 12.4 & 3.69 & 6.03 & 16.4 & 38.1 \\
\hline 2 & US & US-26 & quenched & 4 & 3 & 181 & $\mathrm{X} 033$ & 18.1 & 8.7 & 0.29 & 8.87 & 20.6 & 28.7 \\
\hline 2 & US & US-17 & quenched & 4 & 4 & 182 & $\mathrm{X} 083$ & 14.8 & 11.4 & 3.71 & 9.61 & 11.2 & 56.8 \\
\hline 2 & ARM & ARM & & 4 & 5 & 183 & X066 & 2.96 & 11 & $<0.100$ & 8.25 & 22.1 & 37.6 \\
\hline 2 & US & US-18 & quenched & 4 & 6 & 184 & X043 & 22.7 & 9.07 & 5.44 & 8.38 & 35.1 & 48 \\
\hline 2 & US & US-18ccc & $\mathrm{ccc}$ & 4 & 7 & 185 & $\mathrm{X} 036$ & 29.7 & 187 & 10.9 & 116 & 203 & 100 \\
\hline 2 & US & US-17ccc & $\mathrm{ccc}$ & 4 & 8 & 186 & X041 & 15.3 & 11.7 & 4.52 & 8.46 & 13.2 & 54.1 \\
\hline 2 & US & US-26ccc & $\mathrm{ccc}$ & 4 & 9 & 187 & $\begin{array}{l}\mathrm{X} 024 \\
\end{array}$ & 15.6 & 71.5 & 0.194 & 41.7 & 57.5 & 34.5 \\
\hline 2 & US & US-29ccc & $\mathrm{ccc}$ & 4 & 10 & 188 & $\mathrm{X} 018$ & 23.3 & 25.4 & 9.73 & 23 & 146 & 94.8 \\
\hline 2 & Soln Std & Soln Std & & 4 & 11 & 189 & STD-B4-2 & 3.89 & 21.7 & 4.09 & 10.1 & 82.4 & 51.4 \\
\hline 2 & US & US-16 & quenched & 4 & 12 & 190 & $\mathrm{X019}$ & 10.7 & 22 & 2.7 & 5.61 & 8.19 & 30.9 \\
\hline 2 & US & US-22 & quenched & 4 & 13 & 191 & $\mathrm{X} 017$ & 16.2 & 12.3 & 3.52 & 8.07 & 27.1 & 43.8 \\
\hline 2 & US & US-29 & quenched & 4 & 14 & 192 & $\mathrm{X} 016$ & 24.9 & 29.5 & 10.1 & 24.5 & 174 & 96.9 \\
\hline 2 & blank & Blank 1 (ACTL -15) & & 4 & 15 & 193 & $\mathrm{X} 067$ & $<0.100$ & 0.24 & $<0.100$ & 0.533 & $<0.100$ & 0.564 \\
\hline 2 & US & US-16ccc & $\mathrm{ccc}$ & 4 & 16 & 194 & X059 & 11.7 & 30.8 & 2.3 & 6.82 & 12.9 & 33.7 \\
\hline 2 & US & US-19cec & $\mathrm{ccc}$ & 4 & 17 & 195 & $\mathrm{X} 022$ & 14.2 & 11.2 & 2.68 & 5.47 & 16 & 35.8 \\
\hline 2 & US & US-22ccc & $\mathrm{ccc}$ & 4 & 18 & 196 & $\mathrm{X} 070$ & 27.4 & 11.3 & 4.57 & 7.71 & 39.5 & 67.7 \\
\hline 2 & Soln Std & Soln Std & & 4 & 19 & 197 & STD-B4-3 & 3.86 & 20.7 & 4.08 & 10 & 82.4 & 51.1 \\
\hline 2 & Soln Std & Soln Std & & 5 & 1 & 198 & STD-B5-1 & 3.98 & 20.9 & 4.02 & 10 & 81.4 & 50.5 \\
\hline 2 & US & US-16 & quenched & 5 & 2 & 199 & $\mathrm{X} 078$ & 11.1 & 21.9 & 2.58 & 5.8 & 9.12 & 30.7 \\
\hline 2 & US & US-19ccc & $\mathrm{ccc}$ & 5 & 3 & 200 & $\mathrm{X} 050$ & 13.9 & 11 & 2.39 & 5.45 & 16.2 & 34.1 \\
\hline
\end{tabular}


Table B1. Laboratory Measurements of the PCT Solutions for the US Study Glasses

\begin{tabular}{|c|c|c|c|c|c|c|c|c|c|c|c|c|c|}
\hline Set & Type & Glass ID & Heat Treatment & Block & Seq & $\begin{array}{c}\text { Analytical } \\
\text { Sequence }\end{array}$ & Lab ID & $\mathrm{Al}$ ar & $\mathrm{B}$ ar & $\mathrm{Fe}$ ar & $\mathrm{Li}$ ar & $\mathrm{Na}$ ar & $\mathrm{Si}$ ar \\
\hline 2 & US & US-18 & quenched & 5 & 4 & 201 & X068 & 22.1 & 9.01 & 4.88 & 8.21 & 34.8 & 45.5 \\
\hline 2 & US & US-18ccc & $\mathrm{ccc}$ & 5 & 5 & 202 & $\mathrm{X} 080$ & 29.4 & 178 & 10.7 & 112 & 197 & 99.7 \\
\hline 2 & US & US-22ccc & $\mathrm{ccc}$ & 5 & 6 & 203 & $\mathrm{X} 074$ & 15.8 & 12.5 & 3.23 & 7.76 & 25.7 & 42.6 \\
\hline 2 & US & US-22 & quenched & 5 & 7 & 204 & $\mathrm{X} 072$ & 16.1 & 12.7 & 2.93 & 8.13 & 27.1 & 42.7 \\
\hline 2 & US & US-26ccc & $\mathrm{ccc}$ & 5 & 8 & 205 & $\mathrm{X} 046$ & 16.6 & 71.9 & 0.757 & 42.9 & 60.7 & 35.7 \\
\hline 2 & US & US-16ccc & $\mathrm{ccc}$ & 5 & 9 & 206 & $\mathrm{X} 027$ & 11.3 & 30 & 1.82 & 6.74 & 12.3 & 32.1 \\
\hline 2 & ARM & ARM & & 5 & 10 & 207 & $\mathrm{X} 058$ & 2.94 & 10.8 & $<0.100$ & 7.9 & 21.7 & 34.8 \\
\hline 2 & Soln Std & Soln Std & & 5 & 11 & 208 & STD-B5-2 & 4.16 & 20.7 & 4 & 10 & 81.9 & 50.4 \\
\hline 2 & US & US-29 & quenched & 5 & 12 & 209 & X098 & 24.8 & 30.3 & 9.78 & 24.7 & 173 & 98.2 \\
\hline 2 & US & US-29ccc & $\mathrm{ccc}$ & 5 & 13 & 210 & $\mathrm{X} 091$ & 22.9 & 25 & 9.7 & 22.8 & 145 & 93.6 \\
\hline 2 & US & US-26 & quenched & 5 & 14 & 211 & $\mathrm{X} 073$ & 17.9 & 8.57 & 0.305 & 8.81 & 20.2 & 28.2 \\
\hline 2 & US & US-17ccc & $\mathrm{ccc}$ & 5 & 15 & 212 & $\mathrm{X} 044$ & 14 & 10.6 & 2.93 & 8.62 & 11.2 & 52.8 \\
\hline 2 & US & US-17 & quenched & 5 & 16 & 213 & $\mathrm{X} 063$ & 15 & 11 & 3.2 & 9.52 & 11.1 & 54.4 \\
\hline 2 & US & US-19 & quenched & 5 & 17 & 214 & $\mathrm{X} 056$ & 17.3 & 11.5 & 3.73 & 6.03 & 16.9 & 42.2 \\
\hline 2 & Soln Std & Soln Std & & 5 & 18 & 215 & STD-B5-3 & 4.05 & 20.5 & 4.13 & 10.1 & 82.6 & 51.2 \\
\hline 2 & Soln Std & Soln Std & & 6 & 1 & 216 & STD-B6-1 & 3.63 & 20.4 & 3.25 & 9.81 & 80.5 & 51.4 \\
\hline 2 & US & US-29 & quenched & 6 & 2 & 217 & X064 & 25.8 & 29.4 & 9.44 & 24.5 & 174 & 98.6 \\
\hline 2 & US & US-19ccc & $\mathrm{ccc}$ & 6 & 3 & 218 & X008 & 15.2 & 11.1 & 1.82 & 5.32 & 17.1 & 37.7 \\
\hline 2 & US & US-16 ccc & $\mathrm{ccc}$ & 6 & 4 & 219 & $\mathrm{X} 040$ & 11.1 & 30.1 & 0.904 & 6.54 & 12.9 & 32.5 \\
\hline 2 & US & US-16 & quenched & 6 & 5 & 220 & $\mathrm{X} 007$ & 10.9 & 23.4 & 1.66 & 5.71 & 8.82 & 31.2 \\
\hline 2 & ARM & ARM & & 6 & 6 & 221 & $\mathrm{X} 052$ & 2.88 & 10.2 & $<0.100$ & 7.74 & 21.2 & 36.5 \\
\hline 2 & blank & Blank 2 (ACTL -15) & & 6 & 7 & 222 & $\mathrm{X} 002$ & 0.159 & $<0.100$ & $<0.100$ & $<0.500$ & $<0.100$ & $<0.100$ \\
\hline 2 & US & US-29ccc & $\mathrm{ccc}$ & 6 & 8 & 223 & X079 & 22.7 & 24.5 & 9.16 & 23.1 & 145 & 94 \\
\hline 2 & US & US-17 & quenched & 6 & 9 & 224 & X099 & 14 & 10.1 & 2.87 & 8.91 & 10.7 & 53.5 \\
\hline 2 & US & US-17ccc & $\mathrm{ccc}$ & 6 & 10 & 225 & $\mathrm{X} 025$ & 13.5 & 9.55 & 1.74 & 8.21 & 11.5 & 51.5 \\
\hline 2 & Soln Std & Soln Std & & 6 & 11 & 226 & STD-B6-2 & 3.67 & 19.9 & 3.15 & 9.73 & 81.1 & 50.9 \\
\hline 2 & US & US-26 & quenched & 6 & 12 & 227 & X086 & 17.8 & 7.8 & $<0.100$ & 8.42 & 20.8 & 28.1 \\
\hline 2 & US & US-18 & quenched & 6 & 13 & 228 & $\mathrm{X} 020$ & 21.9 & 8.66 & 4.16 & 8.08 & 34.4 & 47.4 \\
\hline 2 & US & US-26ccc & $\mathrm{ccc}$ & 6 & 14 & 229 & $\mathrm{X} 015$ & 16.4 & 71.8 & $<0.100$ & 43.1 & 60.2 & 35.5 \\
\hline 2 & US & US-22ccc & $\mathrm{ccc}$ & 6 & 15 & 230 & X087 & 15.5 & 11 & 2.13 & 7.42 & 26.4 & 42.1 \\
\hline 2 & US & US-18ccc & $\mathrm{ccc}$ & 6 & 16 & 231 & X100 & 29.9 & 179 & 10.6 & 112 & 196 & 101 \\
\hline 2 & US & US-19 & quenched & 6 & 17 & 232 & $\mathrm{X} 038$ & 14.8 & 11.9 & 2.35 & 5.6 & 16.2 & 35.4 \\
\hline 2 & US & US-22 & quenched & 6 & 18 & 233 & $\mathrm{X} 042$ & 15.5 & 12 & 1.88 & 7.7 & 26.8 & 41.8 \\
\hline 2 & Soln Std & Soln Std & & 6 & 19 & 234 & STD-B6-3 & 3.71 & 20.4 & 3.09 & 9.69 & 80.7 & 50.5 \\
\hline 3 & Soln Std & Soln Std & & 1 & 1 & 235 & STD-B1-1 & 3.9 & 18.5 & 3.49 & 10.1 & 79.5 & 50.6 \\
\hline 3 & EA & EA & & 1 & 2 & 236 & Y85 & $<0.100$ & 37.3 & $<0.010$ & 12 & 99.7 & 56.1 \\
\hline 3 & US & US-31 & quenched & 1 & 3 & 237 & Y15 & 12 & 12.3 & $<0.010$ & 8.74 & 9.42 & 31 \\
\hline 3 & US & US-31ccc & $\mathrm{ccc}$ & 1 & 4 & 238 & Y89 & 9.98 & 7.12 & 0.698 & 6.81 & 6.2 & 27.5 \\
\hline 3 & US & US-33 & quenched & 7 & 5 & 239 & Y81 & 12.1 & 2.15 & 0.162 & 2.98 & 78.8 & 47.3 \\
\hline 3 & US & US-33ccc & $\mathrm{ccc}$ & 1 & 6 & 240 & Y66 & 12.4 & 1.81 & 1.32 & 3.11 & 70.5 & 46.7 \\
\hline
\end{tabular}


Table B1. Laboratory Measurements of the PCT Solutions for the US Study Glasses

\begin{tabular}{|c|c|c|c|c|c|c|c|c|c|c|c|c|c|}
\hline Set & Type & Glass ID & Heat Treatment & Block & Seq & $\begin{array}{l}\text { Analytical } \\
\text { Sequence }\end{array}$ & Lab ID & $\mathrm{Al}$ ar & $\mathrm{B}$ ar & $\mathrm{Fe}$ ar & $\mathrm{Li}$ ar & $\mathrm{Na}$ ar & Si ar \\
\hline 3 & US & US-35 & quenched & 1 & 7 & 241 & Y46 & 6.36 & 29.4 & $<0.010$ & 5.27 & 22.7 & 22.3 \\
\hline 3 & US & US-35ccc & $\mathrm{ccc}$ & 1 & 8 & 242 & Y45 & 5.75 & 149 & 0.34 & 20 & 88.2 & 23.7 \\
\hline 3 & US & US-37 & quenched & 1 & 9 & 243 & Y73 & 14.9 & 6.22 & $<0.010$ & 3.65 & 75.9 & 41.1 \\
\hline 3 & US & US-37ccc & $\operatorname{ccc}$ & 1 & 10 & 244 & Y38 & 9.62 & 20.9 & 0.801 & 9.17 & 114 & 54.5 \\
\hline 3 & Soln Std & Soln Std & & 1 & 11 & 245 & STD-B1-2 & 3.76 & 17.3 & 3.5 & 9.69 & 76.7 & 48.5 \\
\hline 3 & US & US-39 & quenched & 1 & 12 & 246 & $\mathrm{Y} 75$ & 10.1 & 13.8 & $<0.010$ & 9.36 & 5.02 & 33.1 \\
\hline 3 & US & US-39ccc & $\mathrm{ccc}$ & 1 & 13 & 247 & Y80 & 10.8 & 11.8 & 0.206 & 8.76 & 4.28 & 33.1 \\
\hline 3 & US & US-41 & quenched & 1 & 14 & 248 & Y51 & 6.53 & 7.78 & 0.159 & 5.08 & 6 & 25.1 \\
\hline 3 & US & US-41ccc & $\mathrm{ccc}$ & 1 & 15 & 249 & Y84 & 6.29 & 6.62 & 0.959 & 5.11 & 5.18 & 24.5 \\
\hline 3 & US & US-43 & quenched & 1 & 16 & 250 & Y88 & 18.1 & 4.56 & $<0.010$ & 8.12 & 16.1 & 31.1 \\
\hline 3 & US & US-43ccc & $\operatorname{ccc}$ & 1 & 17 & 251 & Y92 & 18.4 & 26.2 & 0.503 & 24 & 23.1 & 37.8 \\
\hline 3 & US & US-45 & quenched & 1 & 18 & 252 & Y97 & 12.5 & 9.52 & $<0.010$ & 6.44 & 26.8 & 31.9 \\
\hline 3 & US & US-45ccc & $\operatorname{ccc}$ & 1 & 19 & 253 & Y70 & 12.1 & 8.89 & $<0.010$ & 6.17 & 25.2 & 31 \\
\hline 3 & Soln Std & Soln Std & & 1 & 20 & 254 & STD-B1-3 & 3.79 & 17 & 3.56 & 9.75 & 75.6 & 48.8 \\
\hline 3 & Soln Std & Soln Std & & 2 & 1 & 255 & STD-B2-1 & 3.84 & 20.7 & 3.61 & 9.99 & 82 & 50.1 \\
\hline 3 & EA & EA & & 2 & 2 & 256 & Y27 & $<0.100$ & 40.1 & 0.248 & 12 & 104 & 55.8 \\
\hline 3 & US & US-31 & quenched & 2 & 3 & 257 & Y06 & 12.3 & 14.2 & $<0.010$ & 8.73 & 9.6 & 31.1 \\
\hline 3 & US & US-31ccc & $\mathrm{ccc}$ & 2 & 4 & 258 & Y41 & 9.69 & 8.74 & 0.776 & 6.54 & 5.75 & 26.3 \\
\hline 3 & US & US-33 & quenched & 2 & 5 & 259 & Y79 & 12 & 4.1 & 0.325 & 2.83 & 79.7 & 45.6 \\
\hline 3 & US & US-33 ccc & $\operatorname{ccc}$ & 2 & 6 & 260 & Y30 & 12.8 & 4.12 & 1.38 & 3.19 & 78.4 & 48.8 \\
\hline 3 & US & US-35 & quenched & 2 & 7 & 261 & Y53 & 6.03 & 31.3 & $<0.010$ & 5.11 & 23.2 & 22.5 \\
\hline 3 & US & US-35ccc & $\mathrm{ccc}$ & 2 & 8 & 262 & Y33 & 5.46 & 153 & 0.616 & 20.3 & 92 & 23.9 \\
\hline 3 & US & US-37 & quenched & 2 & 9 & 263 & Y20 & 15.1 & 8.7 & $<0.010$ & 3.64 & 79.8 & 42.3 \\
\hline 3 & US & US-37ccc & $\mathrm{ccc}$ & 2 & 10 & 264 & Y82 & 10.6 & 24.5 & 1.44 & 9.52 & 124 & 55.9 \\
\hline 3 & Soln Std & Soln Std & & 2 & 11 & 265 & STD-B2-2 & 3.76 & 20.2 & 3.52 & 9.79 & 80.7 & 49.3 \\
\hline 3 & US & US-39 & quenched & 2 & 12 & 266 & Y34 & 10.1 & 15.8 & $<0.010$ & 9.2 & 5.11 & 32.8 \\
\hline 3 & US & US-39ccc & ccc & 2 & 13 & 267 & Y86 & 10.4 & 14 & 0.347 & 8.51 & 4.08 & 32.7 \\
\hline 3 & US & US-41 & quenched & 2 & 14 & 268 & Y72 & 6.57 & 10.5 & 0.303 & 5.13 & 6.22 & 26.2 \\
\hline 3 & US & US-41 ccc & $\mathrm{ccc}$ & 2 & 15 & 269 & Y96 & 6.08 & 8.92 & 1.05 & 5.08 & 5.22 & 25.1 \\
\hline 3 & US & US-43 & quenched & 2 & 16 & 270 & Y18 & 18.5 & 8.55 & $<0.010$ & 8.22 & 17.1 & 31.9 \\
\hline 3 & US & US- $43 \mathrm{ccc}$ & $\operatorname{ccc}$ & 2 & 17 & 271 & Y24 & 19 & 30 & 0.634 & 24.8 & 24.9 & 39.5 \\
\hline 3 & US & US-45 & quenched & 2 & 18 & 272 & Y61 & 12.9 & 12.1 & 0.035 & 6.52 & 29.4 & 32.5 \\
\hline 3 & US & US-45ccc & $\mathrm{ccc}$ & 2 & 19 & 273 & Y83 & 12.7 & 11.7 & 0.228 & 6.26 & 27.6 & 32 \\
\hline 3 & Soln Std & Soln Std & & 2 & 20 & 274 & STD-B2-3 & 3.77 & 19.5 & 3.35 & 9.75 & 81.8 & 48.9 \\
\hline 3 & Soln Std & Soln Std & & 3 & 1 & 275 & STD-B3-1 & 3.81 & 20.2 & 3.48 & 9.88 & 80.3 & 48.8 \\
\hline 3 & blank & blank & & 3 & 2 & 276 & Y12 & $<0.100$ & $<0.500$ & $<0.010$ & $<1.00$ & $<0.100$ & $<0.100$ \\
\hline 3 & EA & EA & & 3 & 3 & 277 & Y04 & 0.255 & 38 & $<0.010$ & 11.7 & 102.1 & 54.4 \\
\hline 3 & US & US-31 & quenched & 3 & 4 & 278 & Y94 & 12.2 & 14 & $<0.010$ & 8.62 & 9.05 & 30.5 \\
\hline 3 & US & US-31ccc & ccc & 3 & 5 & 279 & Y71 & $\begin{array}{l}10.1 \\
\end{array}$ & 9.23 & 1.24 & 6.93 & 7.22 & 27.4 \\
\hline 3 & US & US-33 & quenched & 3 & 6 & 280 & Y36 & 11.6 & 4.35 & 0.294 & 2.89 & 78.3 & 44.5 \\
\hline
\end{tabular}


Table B1. Laboratory Measurements of the PCT Solutions for the US Study Glasses

\begin{tabular}{|c|c|c|c|c|c|c|c|c|c|c|c|c|c|}
\hline Set & Type & Glass ID & Heat Treatment & Block & Seq & $\begin{array}{l}\text { Analytical } \\
\text { Sequence }\end{array}$ & Lab ID & $\mathrm{Al}$ ar & $\mathrm{B}$ ar & $\mathrm{Fe}$ ar & $\mathrm{Li}$ ar & $\mathrm{Na}$ ar & Si ar \\
\hline 3 & US & US-33ccc & $\mathrm{ccc}$ & 3 & 7 & 281 & Y76 & 13.2 & 4.42 & 4.33 & 3.21 & 75.8 & 47.6 \\
\hline 3 & US & US-35 & quenched & 3 & 8 & 282 & Y57 & 5.71 & 28.8 & $<0.010$ & 4.85 & 21.4 & 21.4 \\
\hline 3 & US & US-35ccc & $\mathrm{ccc}$ & 3 & 9 & 283 & Y56 & 5.49 & 140 & 0.563 & 19 & 86.9 & 22.5 \\
\hline 3 & US & US-37 & quenched & 3 & 10 & 284 & Y13 & 15.1 & 8.78 & 0.295 & 3.76 & 79.3 & 41.7 \\
\hline 3 & Soln Std & Soln Std & & 3 & 11 & 285 & STD-B3-2 & 3.87 & 20.5 & 3.45 & 9.93 & 81 & 49.3 \\
\hline 3 & US & US-37ccc & $\operatorname{ccc}$ & 3 & 12 & 286 & Y63 & 9.45 & 23.4 & 0.946 & 9.08 & 119 & 53.3 \\
\hline 3 & US & US-39 & quenched & 3 & 13 & 287 & Y29 & 9.74 & 15.8 & $<0.010$ & 9.09 & 4.58 & 32 \\
\hline 3 & US & US-39ccc & $\mathrm{ccc}$ & 3 & 14 & 288 & Y37 & 10.5 & 14.3 & 0.337 & 8.71 & 3.84 & 32.9 \\
\hline 3 & US & $\overline{\text { US-41 }}$ & quenched & 3 & 15 & 289 & Y08 & 6.35 & 10.3 & 0.171 & 5.08 & 5.96 & 24.7 \\
\hline 3 & US & US-41ccc & ccc & 3 & 16 & 290 & Y77 & 6.07 & 9.14 & 1.01 & 5.09 & 5.19 & 24.3 \\
\hline 3 & US & US-43 & quenched & 3 & 17 & 291 & Y62 & 17.9 & 7.11 & $<0.010$ & 8.04 & 16.2 & 30.7 \\
\hline 3 & US & US-43ccc & $\mathrm{ccc}$ & 3 & 18 & 292 & Y26 & 18.5 & 28.7 & 0.62 & 24.1 & 24.3 & 37.7 \\
\hline 3 & US & US-45 & quenched & 3 & 19 & 293 & Y22 & 12.3 & 11.9 & 0.028 & 6.3 & 27.7 & 30.8 \\
\hline 3 & US & US-45 ccc & $\mathrm{ccc}$ & 3 & 20 & 294 & Y10 & 12.1 & 11.5 & 0.222 & 6.1 & 25.5 & 31.2 \\
\hline 3 & Soln Std & Soln Std & & 3 & 21 & 295 & STD-B3-3 & 3.84 & 20 & 3.5 & 9.9 & 80.6 & 49.3 \\
\hline 3 & Soln Std & Soln Std & & 4 & 1 & 296 & STD-B4-1 & 3.7 & 20.3 & 3.68 & 9.8 & 81.7 & 49.5 \\
\hline 3 & $\overline{\text { ARM }}$ & ARM-1 & & 4 & 2 & 297 & Y11 & 2.48 & 12.2 & $<0.010$ & 8.21 & 20.9 & 36.5 \\
\hline 3 & US & US-32 & quenched & 4 & 3 & 298 & Y40 & 8.23 & 124 & 1.7 & 49.9 & 63 & 42.3 \\
\hline 3 & US & US-32ccc & $\mathrm{ccc}$ & 4 & 4 & 299 & Y07 & 8.46 & 94.6 & 1.49 & 37.7 & 49.1 & 41.4 \\
\hline 3 & US & US-34 & quenched & 4 & 5 & 300 & Y95 & 15.8 & 105 & 0.191 & 22.6 & 263 & 45.9 \\
\hline 3 & US & US-34ccc & $\mathrm{ccc}$ & 4 & 6 & 301 & Y47 & 15.4 & 96.4 & 0.133 & 21.2 & 244 & 44 \\
\hline 3 & US & US-36 & quenched & 4 & 7 & 302 & Y58 & 9.99 & 18.1 & 0.214 & 4.51 & 22.6 & 24 \\
\hline 3 & US & US-36ccc & $\mathrm{ccc}$ & 4 & 8 & 303 & Y74 & 10.3 & 22.8 & 0.682 & 5.21 & 27.3 & 25.5 \\
\hline 3 & US & US-38 & quenched & 4 & 9 & 304 & Y78 & 12.4 & 10.5 & 0.616 & 3.93 & 95.9 & 47.5 \\
\hline 3 & US & US-38ccc & ccc & 4 & 10 & 305 & Y98 & 14 & 11.1 & 1.55 & 4.54 & 101 & 55.3 \\
\hline 3 & Soln Std & Soln Std & & 4 & 11 & 306 & STD-B4-2 & 3.69 & 20.4 & 3.9 & 9.79 & 81 & 49.7 \\
\hline 3 & US & US-40 & quenched & 4 & 12 & 307 & Y87 & 15.3 & 36.1 & 3.37 & 7.73 & 29.3 & 32 \\
\hline 3 & US & US-40ccc & $\mathrm{ccc}$ & 4 & 13 & 308 & Y09 & 15.2 & 49.3 & 3.03 & 9.81 & 38.1 & 32.5 \\
\hline 3 & US & US-42 & quenched & 4 & 14 & 309 & Y50 & 49.6 & 15 & 5.27 & 18.2 & 140 & 70.9 \\
\hline 3 & US & US-42 $\mathrm{ccc}$ & $\mathrm{ccc}$ & 4 & 15 & 310 & Y42 & 1.74 & 10.8 & $<0.010$ & 33.7 & 309 & 19.6 \\
\hline 3 & US & US-44 & quenched & 4 & 16 & 311 & Y01 & 11.9 & 12 & 0.437 & 6.21 & 27.8 & 30.4 \\
\hline 3 & US & US-44ccc & $\mathrm{ccc}$ & 4 & 17 & 312 & Y16 & 12.5 & 11.9 & 0.693 & 6.25 & 27.1 & 31.8 \\
\hline 3 & Soln Std & Soln Std & & 4 & 18 & 313 & STD-B4-3 & 3.75 & 19.9 & 3.84 & 9.87 & 83.1 & 49.5 \\
\hline 3 & Soln Std & Soln Std & & 5 & 1 & 314 & STD-B5-1 & 3.8 & 20.3 & 3.63 & 9.8 & 80.2 & 49.2 \\
\hline 3 & ARM & ARM-1 & & 5 & 2 & 315 & Y02 & 2.54 & 11.1 & $<0.010$ & 8.36 & 21.5 & 37 \\
\hline 3 & US & US-32 & quenched & 5 & 3 & 316 & Y14 & 7.83 & 124 & 1.16 & 48.4 & 60.4 & 41.5 \\
\hline 3 & US & US-32ccc & $\mathrm{ccc}$ & 5 & 4 & 317 & Y39 & 8.02 & 103 & 1.92 & 40.3 & 51.2 & 42 \\
\hline 3 & US & US-34 & quenched & 5 & 5 & 318 & Y67 & 16.1 & 107 & $<0.010$ & 23.1 & 262 & 46.2 \\
\hline 3 & US & US-34ccc & $\operatorname{ccc}$ & 5 & 6 & 319 & Y64 & 15.1 & 98.8 & $<0.010$ & 21.6 & 240 & 44 \\
\hline 3 & US & US-36 & quenched & 5 & 7 & 320 & Y25 & 10.3 & 19.4 & $<0.010$ & 4.78 & 23.8 & 24.8 \\
\hline
\end{tabular}


Table B1. Laboratory Measurements of the PCT Solutions for the US Study Glasses

\begin{tabular}{|c|c|c|c|c|c|c|c|c|c|c|c|c|c|}
\hline Set & Type & Glass ID & Heat Treatment & Block & Seq & $\begin{array}{c}\text { Analytical } \\
\text { Sequence }\end{array}$ & Lab ID & $\mathrm{Al}$ ar & $\mathrm{B}$ ar & $\mathrm{Fe}$ ar & $\mathrm{Li}$ ar & $\mathrm{Na}$ ar & $\mathrm{Si}$ ar \\
\hline 3 & US & US-36ccc & $\mathrm{ccc}$ & 5 & 8 & 321 & $\mathrm{Y} 05$ & 10.3 & 23 & 0.311 & 5.26 & 26.8 & 25.8 \\
\hline 3 & US & US-38 & quenched & 5 & 9 & 322 & Y91 & 11.1 & 11.1 & 0.137 & 4.02 & 94.4 & 49.3 \\
\hline 3 & US & US-38ccc & $\mathrm{ccc}$ & 5 & 10 & 323 & Y19 & 14.2 & 11.3 & 1.62 & 4.62 & 98.8 & 56.3 \\
\hline 3 & Soln Std & Soln Std & & 5 & 11 & 324 & STD-B5-2 & 3.82 & 20.8 & 3.79 & 9.89 & 80.3 & 50.1 \\
\hline 3 & US & US-40 & quenched & 5 & 12 & 325 & Y43 & 15.1 & 34.4 & 3.03 & 7.42 & 28.4 & 31.4 \\
\hline 3 & US & US-40ccc & $\mathrm{ccc}$ & 5 & 13 & 326 & Y23 & 13.6 & 48.4 & 2.38 & 9.42 & 35.7 & 29.7 \\
\hline 3 & US & US-42 & quenched & 5 & 14 & 327 & Y44 & 48.6 & 15.3 & 5.05 & 18.1 & 135 & 71 \\
\hline 3 & US & US-42ccc & $\mathrm{ccc}$ & 5 & 15 & 328 & Y90 & 3.3 & 10.7 & $<0.010$ & 33.1 & 303 & 19.3 \\
\hline 3 & US & US-44 & quenched & 5 & 16 & 329 & Y49 & 12.3 & 12.4 & 0.137 & 6.41 & 28.4 & 31.6 \\
\hline 3 & US & US-44ccc & $\mathrm{ccc}$ & 5 & 17 & 330 & Y48 & 12.7 & 12.3 & 0.216 & 6.46 & 27.9 & 32.9 \\
\hline 3 & Soln Std & Soln Std & & 5 & 18 & 331 & STD-B5-3 & 3.77 & 20.3 & 3.7 & 9.84 & 80 & 49.7 \\
\hline 3 & Soln Std & Soln Std & & 6 & 1 & 332 & STD-B6-1 & 3.88 & 20.1 & 3.53 & 9.86 & 80.5 & 49.3 \\
\hline 3 & ARM & ARM-1 & & 6 & 2 & 333 & Y21 & 2.59 & 10.4 & $<0.010$ & 8.16 & 20.9 & 36 \\
\hline 3 & blank & blank & & 6 & 3 & 334 & Y17 & $<0.100$ & $<0.500$ & $<0.010$ & $<1.00$ & $<0.100$ & $<0.100$ \\
\hline 3 & US & US-32 & quenched & 6 & 4 & 335 & Y35 & 8.1 & 131 & 1.1 & 51 & 63.5 & 42.6 \\
\hline 3 & US & US-32ccc & $\mathrm{ccc}$ & 6 & 5 & 336 & Y68 & 8.14 & 102 & 0.726 & 40.4 & 50.7 & 41.6 \\
\hline 3 & US & US-34 & quenched & 6 & 6 & 337 & Y28 & 16.2 & 106 & $<0.010$ & 23.5 & 267 & 46.2 \\
\hline 3 & US & US-34ccc & $\mathrm{ccc}$ & 6 & 7 & 338 & Y69 & 15.1 & 99.1 & $<0.010$ & 21.9 & 242 & 44.1 \\
\hline 3 & US & US-36 & quenched & 6 & 8 & 339 & Y59 & 10.4 & 18.9 & $<0.010$ & 4.75 & 24.1 & 25.1 \\
\hline 3 & US & US-36ccc & $\mathrm{ccc}$ & 6 & 9 & 340 & Y65 & 10.8 & 23.8 & 0.232 & 5.5 & 28.4 & 26.7 \\
\hline 3 & US & US-38 & quenched & 6 & 10 & 341 & Y54 & 11.1 & 10.7 & $<0.010$ & 4.04 & 94.8 & 48.3 \\
\hline 3 & Soln Std & Soln Std & & 6 & 11 & 342 & STD-B6-2 & 3.87 & 20.5 & 3.58 & 9.98 & 80.5 & 49.4 \\
\hline 3 & US & US-38ccc & $\mathrm{ccc}$ & 6 & 12 & 343 & Y93 & 13.7 & 10.3 & 1.5 & 4.45 & 95.8 & 53.3 \\
\hline 3 & US & US-40 & quenched & 6 & 13 & 344 & Y03 & 15.1 & 33.5 & 2.45 & 7.43 & 28.8 & 30.6 \\
\hline 3 & US & US-40 ccc & $\mathrm{ccc}$ & 6 & 14 & 345 & Y32 & 14.3 & 48.7 & 2.09 & 9.94 & 39.3 & 29.5 \\
\hline 3 & US & US-42 & quenched & 6 & 15 & 346 & Y55 & 50.1 & 14.6 & 4.91 & 18.7 & 141 & 70.6 \\
\hline 3 & US & US-42ccc & $\mathrm{ccc}$ & 6 & 16 & 347 & Y31 & 1.76 & 10.5 & $<0.010$ & 33.6 & 304 & 19.6 \\
\hline 3 & US & US-44 & quenched & 6 & 17 & 348 & Y52 & 12.4 & 12.4 & 0.028 & 6.54 & 28.8 & 31.9 \\
\hline 3 & US & US-44ccc & $\mathrm{ccc}$ & 6 & 18 & 349 & Y60 & 12.9 & 11.8 & 0.112 & 6.54 & 29.1 & 32.2 \\
\hline 3 & Soln Std & Soln Std & & 6 & 19 & 350 & STD-B6-3 & 3.9 & 19.7 & 3.46 & 9.93 & 81.6 & 49.1 \\
\hline
\end{tabular}


Table B2. PSAL Measurements of the PCT Solutions for the US Study Glasses After Appropriate Adjustments

\begin{tabular}{|c|c|c|c|c|c|c|c|c|c|c|c|c|c|}
\hline Set & Type & Glass ID & $\begin{array}{c}\text { Heat } \\
\text { Treatment }\end{array}$ & Block & Seq & $\begin{array}{c}\text { Analytical } \\
\text { Sequence }\end{array}$ & Lab ID & $\mathrm{Al}$ (ppm) & $\mathrm{B}(\mathrm{ppm})$ & $\mathrm{Fe}(\mathrm{ppm})$ & $\mathrm{Li}(\mathrm{ppm})$ & $\mathrm{Na}(\mathrm{ppm})$ & $\mathrm{Si}(\mathrm{ppm})$ \\
\hline 1 & Soln Std & Soln Std & & 1 & 1 & 1 & STD-B1-1 & 3.750 & 20.600 & 4.070 & 9.880 & 82.000 & 50.200 \\
\hline 1 & ARM & ARM & & 1 & 2 & 2 & W73 & 4.100 & 17.500 & 0.083 & 13.467 & 35.501 & 62.001 \\
\hline 1 & US & US-01 & quenched & 1 & 3 & 3 & W88 & 34.167 & 12.200 & 16.400 & 15.267 & 51.168 & 91.168 \\
\hline 1 & US & US-01ccc & $\mathrm{ccc}$ & 1 & 4 & 4 & W45 & 31.667 & 11.367 & 13.650 & 14.167 & 46.168 & 83.835 \\
\hline 1 & US & US-02 & quenched & 1 & 5 & 5 & W76 & 28.834 & 89.502 & 13.434 & 37.001 & 80.168 & 65.668 \\
\hline 1 & US & US-02 $\mathrm{ccc}$ & $\mathrm{ccc}$ & 1 & 6 & 6 & W68 & 29.334 & 75.335 & 13.884 & 31.167 & 70.835 & 69.335 \\
\hline 1 & US & US-11 & quenched & 1 & 7 & 7 & W96 & 18.667 & 17.000 & 0.253 & 5.250 & 17.667 & 40.667 \\
\hline 1 & US & US- $05 \mathrm{ccc}$ & $\mathrm{ccc}$ & 1 & 8 & 8 & W55 & 24.667 & 23.667 & 0.797 & 6.200 & 24.500 & 55.001 \\
\hline 1 & US & US-08 & quenched & 1 & 9 & 9 & W85 & 30.334 & 12.550 & 16.667 & 13.434 & 22.667 & 79.168 \\
\hline 1 & Soln Std & Soln Std & & 1 & 10 & 10 & STD-B1-2 & 3.950 & 19.600 & 3.910 & 9.810 & 82.900 & 49.100 \\
\hline 1 & US & US- $08 \mathrm{ccc}$ & $\mathrm{ccc}$ & 1 & 11 & 11 & W90 & 31.334 & 13.017 & 18.167 & 12.834 & 24.667 & 82.502 \\
\hline 1 & US & US-10 & quenched & 1 & 12 & 12 & W51 & 19.667 & 12.467 & 0.257 & 4.683 & 36.334 & 48.334 \\
\hline 1 & US & US-10ccc & $\mathrm{ccc}$ & 1 & 13 & 13 & W75 & 19.000 & 12.167 & 0.320 & 4.483 & 35.501 & 47.501 \\
\hline 1 & US & US-09cec & $\mathrm{ccc}$ & $\overline{1}$ & 14 & 14 & W95 & 13.484 & 23.000 & 0.083 & 5.583 & 11.400 & 38.167 \\
\hline 1 & US & US-11ccc & $\mathrm{ccc}$ & 1 & 15 & 15 & W08 & 16.667 & 51.501 & 1.067 & 8.867 & 26.501 & 48.001 \\
\hline 1 & US & US-13 & quenched & 1 & 16 & 16 & W74 & 21.834 & 16.400 & 0.563 & 5.150 & 29.334 & 48.668 \\
\hline 1 & US & US-13ccc & $\mathrm{ccc}$ & 1 & 17 & 17 & W58 & 19.834 & 13.650 & 0.818 & 4.417 & 25.667 & 43.668 \\
\hline 1 & US & US-14 & quenched & 1 & 18 & 18 & W80 & 24.834 & 164.837 & 3.300 & 68.501 & 152.336 & 56.168 \\
\hline 1 & US & US-14ccc & $\mathrm{ccc}$ & 1 & 19 & 19 & W91 & 25.334 & 147.003 & 2.250 & 61.335 & 137.003 & 57.001 \\
\hline 1 & Soln Std & Soln Std & & 1 & 20 & 20 & STD-B1-3 & 3.830 & 20.200 & 4.170 & 9.800 & 83.500 & 48.800 \\
\hline 1 & Soln Std & Soln Std & & 2 & 1 & 21 & STD-B2-1 & 3.870 & 20.300 & 5.250 & 9.770 & 82.800 & 49.500 \\
\hline 1 & ARM & $\overline{\text { ARM }}$ & & 2 & 2 & 22 & W26 & 4.283 & 17.667 & 0.083 & 13.150 & 36.501 & 59.001 \\
\hline 1 & US & US-01 & quenched & 2 & 3 & 23 & W37 & 31.334 & 11.250 & 13.784 & 13.967 & 47.334 & 82.668 \\
\hline 1 & US & US-01 ccc & $\mathrm{ccc}$ & 2 & 4 & 24 & W24 & 31.501 & 11.250 & 13.067 & 14.000 & 46.501 & 83.168 \\
\hline 1 & US & US-02 & quenched & 2 & 5 & 25 & W05 & 28.501 & 88.502 & 12.817 & 36.501 & 81.668 & 64.501 \\
\hline 1 & US & US- $02 \mathrm{ccc}$ & $\mathrm{ccc}$ & 2 & 6 & 26 & W79 & 28.834 & 72.835 & 13.134 & 30.167 & 71.501 & 66.335 \\
\hline 1 & US & US-05 & quenched & 2 & 7 & 27 & W89 & 19.834 & 17.500 & 0.083 & 5.283 & 19.000 & 41.834 \\
\hline 1 & US & US- $05 \mathrm{ccc}$ & $\mathrm{ccc}$ & 2 & 8 & 28 & W09 & 23.000 & 26.001 & 0.573 & 6.300 & 26.001 & 49.834 \\
\hline 1 & US & US- 08 & quenched & 2 & 9 & 29 & W67 & 32.001 & 12.684 & 17.000 & 13.600 & 24.167 & 80.835 \\
\hline 1 & Soln Std & Soln Std & & 2 & 10 & 30 & STD-B2-2 & 3.710 & 19.200 & 3.450 & 9.580 & 83.800 & 48.000 \\
\hline 1 & US & US- $08 \mathrm{ccc}$ & $\mathrm{ccc}$ & 2 & 11 & 31 & W83 & 28.167 & 11.617 & 14.000 & 11.500 & 23.334 & 71.501 \\
\hline 1 & US & US-10 & quenched & 2 & 12 & 32 & W07 & 19.834 & 11.900 & 0.083 & 4.533 & 37.501 & 47.334 \\
\hline 1 & US & US-10ccc & $\mathrm{ccc}$ & 2 & 13 & 33 & W78 & 18.834 & 11.517 & 0.083 & 4.350 & 36.167 & 44.668 \\
\hline 1 & US & US-11 & quenched & 2 & 14 & 34 & W31 & 12.717 & 22.500 & 0.083 & 5.333 & 9.784 & 39.167 \\
\hline 1 & US & US- $11 \mathrm{ccc}$ & $\mathrm{ccc}$ & 2 & 15 & 35 & W21 & 16.234 & 48.501 & 0.940 & 8.217 & 23.834 & 46.501 \\
\hline 1 & US & US-13 & quenched & 2 & 16 & 36 & W47 & 20.667 & 15.900 & 0.340 & 4.933 & 27.167 & 46.334 \\
\hline 1 & US & US-13ccc & $\mathrm{ccc}$ & 2 & 17 & 37 & W44 & 19.167 & 13.517 & 0.697 & 4.250 & 23.834 & 43.501 \\
\hline 1 & US & US-14 & quenched & 2 & 18 & 38 & W63 & 24.167 & 173.337 & 3.450 & 70.001 & 149.170 & 57.668 \\
\hline 1 & US & US-14cec & $\mathrm{ccc}$ & 2 & 19 & 39 & W01 & 24.334 & 142.670 & 3.117 & 57.834 & 126.003 & 57.168 \\
\hline 1 & Soln Std & Soln Std & & 2 & 20 & 40 & STD-B2-3 & 3.570 & 20.300 & 3.650 & 9.640 & 80.300 & 48.500 \\
\hline
\end{tabular}


Table B2. PSAL Measurements of the PCT Solutions for the US Study Glasses After Appropriate Adjustments

\begin{tabular}{|c|c|c|c|c|c|c|c|c|c|c|c|c|c|}
\hline Set & Type & Glass ID & $\begin{array}{c}\text { Heat } \\
\text { Treatment }\end{array}$ & Block & Seq & $\begin{array}{l}\text { Analytical } \\
\text { Sequence }\end{array}$ & Lab ID & $\mathrm{Al}(\mathrm{ppm})$ & B (ppm) & $\mathrm{Fe}(\mathrm{ppm})$ & $\mathrm{Li}(\mathrm{ppm})$ & $\mathrm{Na}(\mathrm{ppm})$ & $\mathrm{Si}(\mathrm{ppm})$ \\
\hline 1 & Soln Std & Soln Std & & 3 & 1 & 41 & STD-B3-1 & 3.830 & 20.400 & 4.980 & 9.770 & 82.200 & 49.500 \\
\hline 1 & ARM & ARM & & 3 & 2 & 42 & W82 & 4.417 & 18.500 & 0.083 & 13.534 & 37.501 & 60.501 \\
\hline 1 & blank & blank & & 3 & 3 & 43 & W17 & 0.083 & 0.083 & 0.083 & 0.417 & 0.083 & 0.083 \\
\hline 1 & US & US-01 & quenched & 3 & 4 & 44 & W18 & 32.001 & 11.184 & 14.567 & 14.150 & 48.501 & 84.668 \\
\hline 1 & US & US-01 ccc & $\operatorname{ccc}$ & 3 & 5 & 45 & W28 & 32.001 & 11.384 & 13.850 & 14.217 & 46.668 & 84.668 \\
\hline 1 & US & US-02 & quenched & 3 & 6 & 46 & W52 & 30.501 & 94.335 & 14.417 & 38.334 & 83.668 & 70.668 \\
\hline 1 & US & US-02ccc & $\mathrm{ccc}$ & 3 & 7 & 47 & W56 & 27.834 & 71.335 & 13.084 & 29.167 & 66.835 & 65.501 \\
\hline 1 & US & US-05 & quenched & 3 & 8 & 48 & W65 & 19.834 & 17.500 & 0.083 & 5.333 & 18.167 & 43.001 \\
\hline 1 & US & US-05 ccc & $\mathrm{ccc}$ & 3 & 9 & 49 & W13 & 23.000 & 26.667 & 0.465 & 6.417 & 26.001 & 51.001 \\
\hline 1 & Soln Std & Soln Std & & 3 & 10 & 50 & STD-B3-2 & 3.850 & 19.700 & 3.670 & 9.720 & 82.000 & 49.100 \\
\hline 1 & US & US-08 & quenched & 3 & 11 & 51 & W23 & 33.001 & 13.384 & 18.334 & 14.134 & 24.167 & 84.335 \\
\hline 1 & US & US-08ccc & $\operatorname{ccc}$ & 3 & 12 & 52 & W98 & 29.001 & 11.767 & 15.217 & 11.867 & 22.834 & 75.002 \\
\hline 1 & US & US-10 & quenched & 3 & 13 & 53 & W71 & 20.000 & 12.034 & 0.187 & 4.617 & 36.334 & 48.001 \\
\hline 1 & US & US-10 ccc & $\mathrm{ccc}$ & 3 & 14 & 54 & W29 & 19.334 & 12.034 & 0.272 & 4.517 & 35.167 & 48.168 \\
\hline 1 & US & US-11 & quenched & 3 & 15 & 55 & W53 & 13.867 & 22.167 & 0.083 & 5.483 & 11.600 & 39.001 \\
\hline 1 & US & US-11 ccc & $\mathrm{ccc}$ & 3 & 16 & 56 & W86 & 16.484 & 48.501 & 0.905 & 8.384 & 25.834 & 46.501 \\
\hline 1 & US & US-13 & quenched & 3 & 17 & 57 & W69 & 21.000 & 15.467 & 0.323 & 4.933 & 28.167 & 46.001 \\
\hline 1 & US & US-13ccc & $\mathrm{ccc}$ & 3 & 18 & 58 & W11 & 19.834 & 13.417 & 0.842 & 4.367 & 25.334 & 43.501 \\
\hline 1 & US & US-14 & quenched & 3 & 19 & 59 & W60 & 26.001 & 183.337 & 3.217 & 73.501 & 157.170 & 60.168 \\
\hline 1 & US & US-14ccc & $\mathrm{ccc}$ & 3 & 20 & 60 & W36 & 25.501 & 147.003 & 3.400 & 59.001 & 128.836 & 59.001 \\
\hline 1 & Soln Std & Soln Std & & 3 & 21 & 61 & STD-B3-3 & 4.280 & 20.900 & 3.920 & 9.950 & 82.600 & 50.800 \\
\hline 1 & Soln Std & Soln Std & & 4 & 1 & 62 & STD-B4-1 & 3.840 & 20.800 & 5.470 & 9.720 & 81.900 & 50.300 \\
\hline 1 & EA & EA & & 4 & 2 & 63 & W92 & 0.833 & 620.001 & 0.833 & 183.334 & 1653.337 & 873.335 \\
\hline 1 & US & US-03 & quenched & 4 & 3 & 64 & W14 & 22.334 & 18.667 & 7.833 & 9.567 & 7.817 & 54.001 \\
\hline 1 & US & US-03ccc & $\mathrm{ccc}$ & 4 & 4 & 65 & W39 & 21.667 & 17.500 & 6.867 & 8.684 & 9.434 & 52.334 \\
\hline 1 & US & US-04 & quenched & 4 & 5 & 66 & W42 & 22.000 & 11.934 & 0.463 & 8.133 & 21.000 & 49.001 \\
\hline 1 & US & US-04ccc & ccc & 4 & 6 & 67 & W10 & 21.000 & 11.284 & 0.083 & 7.350 & 21.334 & 446.168 \\
\hline 1 & US & US-06 & quenched & 4 & 7 & 68 & W35 & 20.500 & 14.717 & 0.083 & 5.517 & 22.500 & 43.334 \\
\hline 1 & US & US-06 ccc & ccc & 4 & 8 & 69 & W06 & 19.000 & 13.167 & 0.083 & 4.767 & 21.167 & 41.334 \\
\hline 1 & US & US-07 & quenched & 4 & 9 & 70 & W19 & 20.334 & 14.434 & 0.083 & 5.933 & 22.000 & 44.001 \\
\hline 1 & Soln Std & Soln Std & & 4 & 10 & 71 & STD-B4-2 & 4.140 & 20.100 & 3.720 & 9.640 & 82.900 & 49.700 \\
\hline 1 & US & US-07ccc & $\mathrm{ccc}$ & 4 & 11 & 72 & W93 & 19.334 & 13.384 & 0.083 & 5.200 & 20.500 & 42.501 \\
\hline 1 & US & US-09 & quenched & 4 & 12 & 73 & W66 & 21.334 & 20.167 & 11.200 & 4.933 & 44.001 & 53.668 \\
\hline 1 & US & US-09 $\mathrm{ccc}$ & $\mathrm{ccc}$ & 4 & 13 & 74 & W16 & 19.834 & 19.167 & 7.833 & 4.467 & 40.834 & 51.168 \\
\hline 1 & US & US-12 & quenched & 4 & 14 & 75 & W46 & 25.167 & 61.001 & 4.350 & 29.834 & 73.168 & 62.168 \\
\hline 1 & US & US-12ccc & $\mathrm{ccc}$ & 4 & 15 & 76 & W48 & 27.334 & 85.168 & 10.717 & 40.667 & 89.335 & 67.668 \\
\hline 1 & US & US-15 & quenched & 4 & 16 & 77 & W84 & 9.684 & 241.672 & 4.833 & 34.001 & 280.006 & 44.001 \\
\hline 1 & US & US-15ccc & $\operatorname{ccc}$ & 4 & 17 & 78 & W70 & 10.217 & 195.004 & 5.650 & 27.667 & 228.338 & 48.334 \\
\hline 1 & Soln Std & Soln Std & & 4 & 18 & 79 & STD-B4-3 & 3.800 & 20.800 & 4.530 & 9.770 & 82.100 & 50.200 \\
\hline 1 & Soln Std & Soln Std & & 5 & 1 & 80 & STD-B5-1 & 3.980 & 20.700 & 3.930 & 9.810 & 82.600 & 50.000 \\
\hline
\end{tabular}


Table B2. PSAL Measurements of the PCT Solutions for the US Study Glasses After Appropriate Adjustments

\begin{tabular}{|c|c|c|c|c|c|c|c|c|c|c|c|c|c|}
\hline Set & Type & Glass ID & $\begin{array}{c}\text { Heat } \\
\text { Treatment }\end{array}$ & Block & Seq & $\begin{array}{l}\text { Analytical } \\
\text { Sequence }\end{array}$ & Lab ID & $\mathrm{Al}$ (ppm) & B (ppm) & $\mathrm{Fe}(\mathrm{ppm})$ & Li (ppm) & $\mathrm{Na}(\mathrm{ppm})$ & $\mathrm{Si}(\mathrm{ppm})$ \\
\hline 1 & EA & EA & & 5 & 2 & 81 & W64 & 0.833 & 623.335 & 0.833 & 190.000 & 1658.337 & 886.668 \\
\hline 1 & US & US-05 & quenched & 5 & 3 & 82 & W97 & 21.834 & 18.000 & 8.083 & 9.534 & 8.050 & 53.001 \\
\hline 1 & US & US-03ccc & $\mathrm{ccc}$ & 5 & 4 & 83 & W38 & 22.000 & 17.334 & 7.200 & 8.900 & 9.767 & 53.001 \\
\hline 1 & US & US-04 & quenched & 5 & 5 & 84 & W54 & 22.834 & 12.034 & 1.057 & 8.567 & 22.834 & 50.501 \\
\hline 1 & US & US-04ccc & $\mathrm{ccc}$ & 5 & 6 & 85 & W77 & 22.500 & 11.500 & 0.523 & 7.917 & 21.834 & 50.834 \\
\hline 1 & US & US-06 & quenched & 5 & 7 & 86 & W22 & 21.000 & 14.667 & 0.345 & 5.783 & 24.000 & 44.501 \\
\hline 1 & US & US-06ccc & $\mathrm{ccc}$ & 5 & 8 & 87 & W57 & 20.500 & 13.734 & 0.732 & 5.200 & 22.667 & 45.168 \\
\hline 1 & US & US-07 & quenched & 5 & 9 & 88 & W81 & 21.167 & 14.167 & 0.083 & 6.200 & 22.834 & 45.001 \\
\hline 1 & Soln Std & Soln Std & & 5 & 10 & 89 & STD-B5-2 & 4.010 & 19.900 & 4.810 & 9.780 & 83.400 & 49.700 \\
\hline 1 & US & US-07ccc & $\mathrm{ccc}$ & 5 & 11 & 90 & W33 & 20.167 & 13.550 & 0.083 & 5.567 & 21.167 & 45.001 \\
\hline 1 & US & US-09 & quenched & 5 & 12 & 91 & W61 & 19.667 & 19.667 & 11.167 & 5.000 & 40.667 & 53.501 \\
\hline 1 & US & US-09 & $\mathrm{ccc}$ & 5 & 13 & 92 & W72 & 20.500 & 20.000 & 8.367 & 4.833 & 44.168 & 53.501 \\
\hline 1 & US & US-12 & quenched & 5 & 14 & 93 & W27 & 26.001 & 64.001 & 4.550 & 31.501 & 74.668 & 64.835 \\
\hline 1 & US & US-12ccc & $\mathrm{ccc}$ & 5 & 15 & 94 & W32 & 27.501 & 85.835 & 11.400 & 41.334 & 89.502 & 68.835 \\
\hline 1 & US & US-15 & quenched & 5 & 16 & 95 & W03 & 10.717 & 258.339 & 5.667 & 37.167 & 296.673 & 47.501 \\
\hline 1 & US & US-15ccc & $\operatorname{ccc}$ & 5 & 17 & 96 & W50 & 10.450 & 200.004 & 6.200 & 28.834 & 235.005 & 50.334 \\
\hline 1 & Soln Std & Soln Std & & 5 & 18 & 97 & STD-B5-3 & 3.990 & 21.300 & 4.050 & 9.760 & 82.600 & 49.600 \\
\hline 1 & Soln Std & Soln Std & & 6 & 1 & 98 & STD-B6-1 & 4.070 & 20.500 & 3.690 & 9.880 & 81.300 & 50.100 \\
\hline 1 & blank & blank & & 6 & 2 & 99 & W40 & 0.083 & 0.672 & 0.083 & 0.417 & 0.083 & 0.083 \\
\hline 1 & EA & EA & & 6 & 3 & 100 & W02 & 2.050 & 651.668 & 0.833 & 200.000 & 1700.003 & 931.669 \\
\hline 1 & US & US-03 & quenched & 6 & 4 & 101 & W62 & 23.334 & 19.000 & 8.083 & 10.500 & 9.084 & 57.168 \\
\hline 1 & US & US-03ccc & $\mathrm{ccc}$ & 6 & 5 & 102 & W12 & 22.167 & 17.500 & 6.783 & 9.384 & 9.717 & 53.334 \\
\hline 1 & US & US-04 & quenched & 6 & 6 & 103 & W43 & 24.334 & 12.767 & 0.663 & 9.400 & 22.000 & 54.001 \\
\hline 1 & US & US-04ccc & $\operatorname{ccc}$ & 6 & 7 & 104 & W30 & 22.667 & 12.050 & 0.083 & 8.600 & 22.000 & 51.501 \\
\hline 1 & US & US-06 & quenched & 6 & 8 & 105 & W15 & 21.000 & 14.717 & 0.083 & 6.250 & 23.167 & 45.001 \\
\hline 1 & US & US-06ccc & $\mathrm{ccc}$ & 6 & 9 & 106 & W87 & 19.334 & 13.300 & 0.083 & 5.500 & 21.834 & 43.501 \\
\hline 1 & Soln Std & Soln Std & & 6 & 10 & 107 & STD-B6-2 & 4.150 & 20.300 & 3.770 & 10.000 & 81.700 & 50.500 \\
\hline 1 & US & US-07 & quenched & 6 & 11 & 108 & W49 & 21.834 & 15.884 & 0.083 & 7.000 & 25.001 & 49.001 \\
\hline 1 & US & US-07 ccc & $\mathrm{ccc}$ & 6 & 12 & 109 & W25 & 20.000 & 13.517 & 0.083 & 6.000 & 22.167 & 45.001 \\
\hline 1 & US & US-09 & quenched & 6 & 13 & 110 & W41 & 19.834 & 20.834 & 10.384 & 5.633 & 41.668 & 54.168 \\
\hline 1 & US & US-03 & quenched & 6 & 14 & 111 & W97 & 21.000 & 19.834 & 9.034 & 5.250 & 40.667 & 56.501 \\
\hline 1 & US & US-12 & quenched & 6 & 15 & 112 & W04 & 27.667 & 65.335 & 5.300 & 32.167 & 74.335 & 67.835 \\
\hline 1 & US & US-12ccc & $\mathrm{ccc}$ & 6 & 16 & 113 & W34 & 30.334 & 90.335 & 11.784 & 43.001 & 93.169 & 74.335 \\
\hline 1 & US & US-15 & quenched & 6 & 17 & 114 & W20 & 11.317 & 271.672 & 6.067 & 38.334 & 300.006 & 50.834 \\
\hline 1 & US & US-15ccc & ccc & 6 & 18 & 115 & W59 & 11.134 & 210.004 & 6.367 & 29.834 & 236.671 & 53.334 \\
\hline 1 & Soln Std & Soln Std & & 6 & 19 & 116 & STD-B6-3 & 4.020 & 21.600 & 3.690 & 9.830 & 80.300 & 50.300 \\
\hline 2 & Soln Std & Soln Std & & 1 & 1 & 117 & STD-B1-1 & 3.920 & 21.500 & 3.900 & 10.100 & 81.200 & 50.600 \\
\hline 2 & US & US-23 & quenched & 1 & 2 & 118 & X005 & 27.001 & 19.167 & 7.867 & 10.100 & 31.501 & 71.001 \\
\hline 2 & US & US-24ccc & ccc & 1 & 3 & 119 & X065 & 23.834 & 15.767 & 3.800 & 7.883 & 31.001 & 61.835 \\
\hline 2 & US & US-25 & quenched & 1 & 4 & 120 & X093 & 21.667 & 58.835 & 2.450 & 24.667 & 64.168 & 65.335 \\
\hline
\end{tabular}


Table B2. PSAL Measurements of the PCT Solutions for the US Study Glasses After Appropriate Adjustments

\begin{tabular}{|c|c|c|c|c|c|c|c|c|c|c|c|c|c|}
\hline Set & Type & Glass ID & $\begin{array}{c}\text { Heat } \\
\text { Treatment }\end{array}$ & Block & Seq & $\begin{array}{l}\text { Analytical } \\
\text { Sequence }\end{array}$ & Lab ID & $\mathrm{Al}(\mathrm{ppm})$ & B (ppm) & $\mathrm{Fe}(\mathrm{ppm})$ & Li (ppm) & $\mathrm{Na}(\mathrm{ppm})$ & $\mathrm{Si}(\mathrm{ppm})$ \\
\hline 2 & US & US-21 & quenched & 1 & 5 & 121 & $\mathrm{X} 021$ & 25.167 & 19.167 & 9.834 & 8.967 & 25.334 & 68.835 \\
\hline 2 & US & US-28 & quenched & 1 & 6 & 122 & $\mathrm{X} 012$ & 26.501 & 98.669 & 6.883 & 14.567 & 107.835 & 46.668 \\
\hline 2 & US & US- $25 \mathrm{ccc}$ & $\operatorname{ccc}$ & 1 & 7 & 123 & $\mathrm{X088}$ & 22.167 & 72.335 & 5.067 & 29.167 & 74.668 & 67.335 \\
\hline 2 & US & US-30 & quenched & 1 & 8 & 124 & X097 & 17.667 & 31.834 & 0.975 & 16.000 & 15.350 & 40.001 \\
\hline 2 & US & US-21 ccc & $\mathrm{ccc}$ & 1 & 9 & 125 & X009 & 22.667 & 17.834 & 5.283 & 8.033 & 24.334 & 62.668 \\
\hline 2 & US & US-24 & quenched & 1 & 10 & 126 & $\mathrm{X} 029$ & 23.334 & 16.000 & 3.633 & 8.000 & 29.167 & 61.001 \\
\hline 2 & Soln Std & Soln Std & & 1 & 11 & 127 & STD-B1-2 & 3.860 & 20.500 & 3.690 & 9.940 & 81.000 & 49.600 \\
\hline 2 & US & US-23ccc & $\mathrm{ccc}$ & 1 & 12 & 128 & X092 & 25.501 & 19.167 & 6.300 & 9.400 & 31.501 & 67.001 \\
\hline 2 & US & US-27 & quenched & 1 & 13 & 129 & $\mathrm{X} 032$ & 48.168 & 26.167 & 59.001 & 29.167 & 196.671 & 158.503 \\
\hline 2 & EA & EA & & 1 & 14 & 130 & X069 & 8.000 & 636.668 & 3.367 & 196.667 & 1716.670 & 915.002 \\
\hline 2 & US & US-20 $\mathrm{ccc}$ & $\operatorname{ccc}$ & 1 & 15 & 131 & $\mathrm{X} 030$ & 24.167 & 20.167 & 3.650 & 9.567 & 28.334 & 60.168 \\
\hline 2 & US & US-27ccc & $\mathrm{ccc}$ & 1 & 16 & 132 & X049 & 13.534 & 341.674 & 0.083 & 203.337 & 946.686 & 445.009 \\
\hline 2 & US & US-28ccc & $\mathrm{ccc}$ & 1 & 17 & 133 & $\mathrm{X} 071$ & 26.001 & 77.668 & 7.750 & 11.834 & 89.668 & 46.334 \\
\hline 2 & US & US-30 ccc & $\mathrm{ccc}$ & 1 & 18 & 134 & $\mathrm{X} 013$ & 18.500 & 37.501 & 0.448 & 17.334 & 16.400 & 43.334 \\
\hline 2 & US & US-20 & quenched & 1 & 19 & 135 & $\mathrm{X} 048$ & 25.001 & 21.334 & 5.983 & 10.167 & 28.001 & 61.501 \\
\hline 2 & blank & \multicolumn{2}{|c|}{ Blank 1 (ACTL-14) } & 1 & 20 & 136 & X051 & 6.717 & 2.050 & 0.328 & 1.182 & 9.017 & 37.834 \\
\hline 2 & Soln Std & Soln Std & & 1 & 21 & 137 & STD-B1-3 & 3.850 & 20.300 & 3.570 & 9.890 & 81.000 & 49.600 \\
\hline 2 & Soln Std & Soln Std & & 2 & 1 & 138 & STD-B2-1 & 3.860 & 20.900 & 3.860 & 9.900 & 81.100 & 50.100 \\
\hline 2 & US & US-23ccc & $\mathrm{ccc}$ & 2 & 2 & 139 & $\mathrm{X} 026$ & 26.167 & 18.667 & 5.983 & 9.300 & 31.001 & 68.668 \\
\hline 2 & US & US-21 ccc & $\mathrm{ccc}$ & 2 & 3 & 140 & $\mathrm{X} 006$ & 22.334 & 16.550 & 5.700 & 7.750 & 23.334 & 62.668 \\
\hline 2 & US & US-20 & quenched & 2 & 4 & 141 & $\mathrm{X} 010$ & 25.501 & 19.167 & 6.450 & 10.184 & 31.334 & 64.168 \\
\hline 2 & US & US-23 & quenched & 2 & 5 & 142 & $\mathrm{X} 003$ & 27.334 & 19.000 & 6.183 & 10.284 & 32.334 & 72.835 \\
\hline 2 & US & US-27ccc & $\mathrm{ccc}$ & 2 & 6 & 143 & $\mathrm{X} 082$ & 12.950 & 335.007 & 0.083 & 201.671 & 951.686 & 433.342 \\
\hline 2 & US & US-30 & quenched & 2 & 7 & 144 & $\mathrm{X} 075$ & 17.334 & 34.001 & 0.083 & 16.267 & 14.484 & 40.001 \\
\hline 2 & US & US-28ccc & $\mathrm{ccc}$ & 2 & 8 & 145 & $\mathrm{X} 035$ & 26.501 & 77.502 & 8.650 & 11.784 & 91.168 & 47.501 \\
\hline 2 & US & US-24ccc & $\mathrm{ccc}$ & 2 & 9 & 146 & $\mathrm{X} 062$ & 24.500 & 16.284 & 3.017 & 7.683 & 29.834 & 62.001 \\
\hline 2 & US & US-24 & quenched & 2 & 10 & 147 & X095 & 22.667 & $\begin{array}{l}15.100 \\
\end{array}$ & 2.850 & 7.750 & 28.167 & 59.835 \\
\hline 2 & Soln Std & Soln Std & & 2 & 11 & 148 & STD-B-2-2 & 3.760 & 20.000 & 3.720 & 9.830 & 81.300 & 49.400 \\
\hline 2 & US & US-27 & quenched & 2 & 12 & 149 & $\mathrm{X} 057$ & 47.001 & 25.334 & 55.834 & 28.834 & 196.671 & 153.503 \\
\hline 2 & EA & EA & & 2 & 13 & 150 & $\mathrm{X} 053$ & 4.867 & 625.001 & 0.833 & 193.334 & 1700.003 & 918.335 \\
\hline 2 & US & US-20 ccc & $\mathrm{ccc}$ & 2 & 14 & 151 & $\mathrm{X} 045$ & 23.500 & 18.500 & 3.533 & 9.250 & 26.501 & 59.335 \\
\hline 2 & US & US-25 & quenched & 2 & 15 & 152 & X096 & 20.500 & 57.001 & 2.467 & 24.167 & 62.335 & 64.501 \\
\hline 2 & US & US-21 & quenched & 2 & 16 & 153 & $\mathrm{X} 023$ & 42.334 & 17.334 & 8.167 & 8.434 & 27.167 & 100.002 \\
\hline 2 & US & US- $25 \mathrm{ccc}$ & $\mathrm{ccc}$ & 2 & 17 & 154 & X081 & 50.001 & 71.168 & 5.850 & 29.001 & 78.002 & 118.836 \\
\hline 2 & US & US-30ccc & $\mathrm{ccc}$ & 2 & 18 & 155 & $\mathrm{X} 028$ & 18.667 & 35.834 & 0.083 & 17.667 & 16.134 & 44.501 \\
\hline 2 & US & US-28 & quenched & 2 & 19 & 156 & X077 & 24.667 & 92.169 & 6.900 & 13.734 & 103.502 & 43.668 \\
\hline 2 & Soln Std & Soln Std & & 2 & 20 & 157 & STD-B2-3 & 3.740 & 20.000 & 3.620 & 9.740 & 81.100 & 48.900 \\
\hline 2 & Soln Std & Soln Std & & 3 & 1 & 158 & STD-B3-1 & 4.030 & 20.600 & 3.510 & 9.810 & 81.100 & 49.600 \\
\hline 2 & US & US-21 & quenched & 3 & 2 & 159 & X060 & 23.000 & 17.000 & 7.050 & 7.950 & 23.667 & 62.335 \\
\hline 2 & US & US-23 & quenched & 3 & 3 & 160 & X094 & 29.334 & 17.667 & 6.683 & 9.317 & 30.001 & 75.502 \\
\hline
\end{tabular}


Table B2. PSAL Measurements of the PCT Solutions for the US Study Glasses After Appropriate Adjustments

\begin{tabular}{|c|c|c|c|c|c|c|c|c|c|c|c|c|c|}
\hline Set & Type & Glass ID & $\begin{array}{c}\text { Heat } \\
\text { Treatment }\end{array}$ & Block & Seq & $\begin{array}{l}\text { Analytical } \\
\text { Sequence }\end{array}$ & Lab ID & $\mathrm{Al}(\mathrm{ppm})$ & B (ppm) & $\mathrm{Fe}(\mathrm{ppm})$ & $\mathrm{Li}(\mathrm{ppm})$ & $\mathrm{Na}(\mathrm{ppm})$ & $\mathrm{Si}(\mathrm{ppm})$ \\
\hline 2 & US & US- $23 \mathrm{ccc}$ & $\mathrm{ccc}$ & 3 & 4 & 161 & $\mathrm{X} 085$ & 24.834 & 16.667 & 4.933 & 8.817 & 30.334 & 65.001 \\
\hline 2 & US & US-20 ccc & $\mathrm{ccc}$ & 3 & 5 & 162 & $\mathrm{X} 061$ & 24.834 & 17.500 & 4.900 & 9.067 & 28.001 & 60.168 \\
\hline 2 & US & US-27ccc & $\mathrm{ccc}$ & 3 & 6 & 163 & $\mathrm{X} 055$ & 13.434 & 316.673 & 0.083 & 191.671 & 901.685 & 415.008 \\
\hline 2 & US & US-25 & quenched & 3 & 7 & 164 & $\mathrm{X} 084$ & 23.334 & 57.668 & 1.900 & 23.167 & 62.501 & 65.668 \\
\hline 2 & blank & \multicolumn{2}{|c|}{ Blank 2 (ACTL -14) } & 3 & 8 & 165 & $\mathrm{X} 054$ & 0.083 & 1.317 & 0.083 & 0.417 & 0.083 & 0.083 \\
\hline 2 & US & US-28 & quenched & 3 & 9 & 166 & $\mathrm{X} 047$ & 25.167 & 94.835 & 7.133 & 13.634 & 100.835 & 44.168 \\
\hline 2 & US & US-30 & quenched & 3 & 10 & 167 & $\mathrm{X} 090$ & 17.834 & 31.667 & 0.083 & 15.534 & 13.617 & 39.834 \\
\hline 2 & Soln Std & Soln Std & & 3 & 11 & 168 & STD-B3-2 & 4.000 & 21.400 & 3.930 & 10.100 & 80.800 & 52.500 \\
\hline 2 & US & US- $25 \mathrm{ccc}$ & $\mathrm{ccc}$ & 3 & 12 & 169 & $\mathrm{X} 011$ & 24.834 & 70.335 & 4.650 & 28.001 & 71.168 & 66.168 \\
\hline 2 & US & US- $21 \mathrm{ccc}$ & $\mathrm{ccc}$ & 3 & 13 & 170 & $\mathrm{X} 004$ & 23.834 & 16.434 & 7.050 & 7.650 & 26.167 & 64.168 \\
\hline 2 & US & US-30 ccc & $\operatorname{ccc}$ & 3 & 14 & 171 & $\mathrm{X} 001$ & 19.500 & 35.167 & 0.083 & 17.000 & 16.667 & 45.668 \\
\hline 2 & US & US- $28 \mathrm{ccc}$ & $\mathrm{ccc}$ & 3 & 15 & 172 & $\mathrm{X} 076$ & 26.501 & 75.168 & 9.017 & 11.417 & 89.002 & 46.668 \\
\hline 2 & US & US-20 & quenched & 3 & 16 & 173 & X039 & 24.334 & 19.334 & 5.217 & 9.717 & 26.167 & 61.835 \\
\hline 2 & EA & EA & & 3 & 17 & 174 & $\mathrm{X} 031$ & 2.200 & 623.335 & 0.833 & 188.334 & 1683.337 & 893.335 \\
\hline 2 & US & US-24ccc & $\mathrm{ccc}$ & 3 & 18 & 175 & $\mathrm{X} 014$ & 23.500 & 14.534 & 2.967 & 7.300 & 30.167 & 61.001 \\
\hline 2 & US & US-24 & quenched & 3 & 19 & 176 & $\mathrm{X} 034$ & 23.167 & 14.100 & 2.767 & 7.550 & 29.667 & 60.168 \\
\hline 2 & US & US-27 & quenched & 3 & 20 & 177 & $\mathrm{X} 037$ & 47.334 & 24.000 & 60.001 & 28.001 & 193.337 & 154.170 \\
\hline 2 & Soln Std & Soln Std & & 3 & 21 & 178 & STD-B3-3 & 3.920 & 20.200 & 3.700 & 9.860 & 80.700 & 51.100 \\
\hline 2 & Soln Std & Soln Std & & 4 & 1 & 179 & STD-B4-1 & 3.820 & 21.300 & 4.020 & 10.000 & 81.400 & 50.900 \\
\hline 2 & US & US-19 & quenched & 4 & 2 & 180 & X089 & 25.001 & 20.667 & 6.150 & 10.050 & 27.334 & 63.501 \\
\hline 2 & US & US-26 & quenched & 4 & 3 & 181 & $\mathrm{X} 033$ & 30.167 & 14.500 & 0.483 & 14.784 & 34.334 & 47.834 \\
\hline 2 & US & US-17 & quenched & 4 & 4 & 182 & X083 & 24.667 & 19.000 & 6.183 & 16.017 & 18.667 & 94.669 \\
\hline 2 & ARM & ARM & & 4 & 5 & 183 & X066 & 4.933 & 18.334 & 0.083 & 13.750 & 36.834 & 62.668 \\
\hline 2 & US & US-18 & quenched & 4 & 6 & 184 & X043 & 37.834 & 15.117 & 9.067 & 13.967 & 58.501 & 80.002 \\
\hline 2 & US & US-18ccc & $\mathrm{ccc}$ & 4 & 7 & 185 & $\mathrm{X} 036$ & 49.501 & 311.673 & 18.167 & 193.337 & 338.340 & 166.670 \\
\hline 2 & US & US-17ccc & $\mathrm{ccc}$ & 4 & 8 & 186 & X041 & 25.501 & 19.500 & 7.533 & 14.100 & 22.000 & 90.168 \\
\hline 2 & US & US-26ccc & $\operatorname{ccc}$ & 4 & 9 & 187 & $\mathrm{X} 024$ & 26.001 & 119.169 & 0.323 & 69.501 & 95.835 & 57.501 \\
\hline 2 & US & US-29ccc & $\mathrm{ccc}$ & 4 & 10 & 188 & $\mathrm{X} 018$ & 38.834 & 42.334 & 16.217 & 38.334 & 243.338 & 158.003 \\
\hline 2 & Soln Std & Soln Std & & 4 & 11 & 189 & STD-B4-2 & 3.890 & 21.700 & 4.090 & 10.100 & 82.400 & 51.400 \\
\hline 2 & US & US-16 & quenched & 4 & 12 & 190 & X019 & 17.834 & 36.667 & 4.500 & 9.350 & 13.650 & 51.501 \\
\hline 2 & US & US-22 & quenched & 4 & 13 & 191 & X017 & 27.001 & 20.500 & 5.867 & 13.450 & 45.168 & 73.001 \\
\hline 2 & US & US-29 & quenched & 4 & 14 & 192 & $\mathrm{X} 016$ & 41.501 & 49.168 & 16.834 & 40.834 & 290.006 & 161.503 \\
\hline 2 & blank & \multicolumn{2}{|c|}{ Blank 1 (ACTL -15) } & 4 & 15 & 193 & X067 & 0.083 & 0.400 & 0.083 & 0.888 & 0.083 & 0.940 \\
\hline 2 & US & US-16ccc & $\mathrm{ccc}$ & 4 & 16 & 194 & X059 & 19.500 & 51.334 & 3.833 & 11.367 & 21.500 & 56.168 \\
\hline 2 & US & US-19cec & $\operatorname{ccc}$ & 4 & 17 & 195 & $\mathrm{X} 022$ & 23.667 & 18.667 & 4.467 & 9.117 & 26.667 & 59.668 \\
\hline 2 & US & US-22ccc & $\mathrm{ccc}$ & 4 & 18 & 196 & $\mathrm{X} 070$ & 45.668 & 18.834 & 7.617 & 12.850 & 65.835 & 112.836 \\
\hline 2 & Soln Std & Soln Std & & 4 & 19 & 197 & STD-B4-3 & 3.860 & 20.700 & 4.080 & 10.000 & 82.400 & 51.100 \\
\hline 2 & Soln Std & Soln Std & & 5 & 1 & 198 & STD-B5-1 & 3.980 & 20.900 & 4.020 & 10.000 & 81.400 & 50.500 \\
\hline 2 & US & US-16 & quenched & 5 & 2 & 199 & $\mathrm{X} 078$ & 18.500 & 36.501 & 4.300 & 9.667 & 15.200 & 51.168 \\
\hline 2 & US & US-19cec & $\mathrm{ccc}$ & 5 & 3 & 200 & $\mathrm{X} 050$ & 23.167 & 18.334 & 3.983 & 9.084 & 27.001 & 56.834 \\
\hline
\end{tabular}


Table B2. PSAL Measurements of the PCT Solutions for the US Study Glasses After Appropriate Adjustments

\begin{tabular}{|c|c|c|c|c|c|c|c|c|c|c|c|c|c|}
\hline Set & Type & Glass ID & $\begin{array}{c}\text { Heat } \\
\text { Treatment }\end{array}$ & Block & Seq & $\begin{array}{l}\text { Analytical } \\
\text { Sequence }\end{array}$ & Lab ID & $\mathrm{Al}(\mathrm{ppm})$ & B (ppm) & $\mathrm{Fe}(\mathrm{ppm})$ & $\mathrm{Li}(\mathrm{ppm})$ & $\mathrm{Na}(\mathrm{ppm})$ & $\mathrm{Si}(\mathrm{ppm})$ \\
\hline 2 & US & US-18 & quenched & 5 & 4 & 201 & $\mathrm{X} 068$ & 36.834 & 15.017 & 8.133 & 13.684 & 58.001 & 75.835 \\
\hline 2 & US & US-18ccc & $\mathrm{ccc}$ & 5 & 5 & 202 & $\mathrm{X} 080$ & 49.001 & 296.673 & 17.834 & 186.670 & 328.340 & 166.170 \\
\hline 2 & US & US-22ccc & $\mathrm{ccc}$ & 5 & 6 & 203 & $\mathrm{X} 074$ & 26.334 & 20.834 & 5.383 & 12.934 & 42.834 & 71.001 \\
\hline 2 & US & US-22 & quenched & 5 & 7 & 204 & $\mathrm{X} 072$ & 26.834 & 21.167 & 4.883 & 13.550 & 45.168 & 71.168 \\
\hline 2 & US & US-26ccc & $\mathrm{ccc}$ & 5 & 8 & 205 & X046 & 27.667 & 119.836 & 1.262 & 71.501 & 101.169 & 59.501 \\
\hline 2 & US & US-16 ccc & $\mathrm{ccc}$ & 5 & 9 & 206 & $\mathrm{X} 027$ & 18.834 & 50.001 & 3.033 & 11.234 & 20.500 & 53.501 \\
\hline 2 & ARM & ARM & & 5 & 10 & 207 & X058 & 4.900 & 18.000 & 0.083 & 13.167 & 36.167 & 58.001 \\
\hline 2 & Soln Std & Soln Std & & 5 & 11 & 208 & STD-B5-2 & 4.160 & 20.700 & 4.000 & 10.000 & 81.900 & 50.400 \\
\hline 2 & US & US-29 & quenched & 5 & 12 & 209 & X098 & 41.334 & 50.501 & 16.300 & 41.167 & 288.339 & 163.670 \\
\hline 2 & US & US-29ccc & $\mathrm{ccc}$ & 5 & 13 & 210 & X091 & 38.167 & 41.668 & 16.167 & 38.001 & 241.672 & 156.003 \\
\hline 2 & US & US-26 & quenched & 5 & 14 & 211 & $\mathrm{X} 073$ & 29.834 & 14.284 & 0.508 & 14.684 & 33.667 & 47.001 \\
\hline 2 & US & US-17ccc & $\mathrm{ccc}$ & 5 & 15 & 212 & X044 & 23.334 & 17.667 & 4.883 & 14.367 & 18.667 & 88.002 \\
\hline 2 & US & US-17 & quenched & 5 & 16 & 213 & X063 & 25.001 & 18.334 & 5.333 & 15.867 & 18.500 & 90.668 \\
\hline 2 & US & US-19 & quenched & 5 & 17 & 214 & X056 & 28.834 & 19.167 & 6.217 & 10.050 & 28.167 & 70.335 \\
\hline 2 & Soln Std & Soln Std & & 5 & 18 & 215 & STD-B5-3 & 4.050 & 20.500 & 4.130 & 10.100 & 82.600 & 51.200 \\
\hline 2 & Soln Std & Soln Std & & 6 & 1 & 216 & STD-B6-1 & 3.630 & 20.400 & 3.250 & 9.810 & 80.500 & 51.400 \\
\hline 2 & US & US-29 & quenched & 6 & 2 & 217 & $\mathrm{X} 064$ & 43.001 & 49.001 & 15.734 & 40.834 & 290.006 & 164.337 \\
\hline 2 & US & US-19ccc & $\mathrm{ccc}$ & 6 & 3 & 218 & $\mathrm{X} 008$ & 25.334 & 18.500 & 3.033 & 8.867 & 28.501 & 62.835 \\
\hline 2 & US & US-16 $\mathrm{ccc}$ & $\mathrm{ccc}$ & 6 & 4 & 219 & $\mathrm{X} 040$ & 18.500 & 50.168 & 1.507 & 10.900 & 21.500 & 54.168 \\
\hline 2 & US & US-16 & quenched & 6 & 5 & 220 & $\mathrm{X} 007$ & 18.167 & 39.001 & 2.767 & 9.517 & 14.700 & 52.001 \\
\hline 2 & ARM & ARM & & 6 & 6 & 221 & $\mathrm{X} 052$ & 4.800 & 17.000 & 0.083 & 12.900 & 35.334 & 60.835 \\
\hline 2 & blank & \multicolumn{2}{|c|}{ Blank 2 (ACTL -15) } & 6 & 7 & 222 & $\mathrm{X} 002$ & 0.265 & 0.083 & 0.083 & 0.417 & 0.083 & 0.083 \\
\hline 2 & US & US-29ccc & $\mathrm{ccc}$ & 6 & 8 & 223 & $\mathrm{X} 079$ & 37.834 & 40.834 & 15.267 & 38.501 & 241.672 & 156.670 \\
\hline 2 & US & US-17 & quenched & 6 & 9 & 224 & X099 & 23.334 & 16.834 & 4.783 & 14.850 & 17.834 & 89.168 \\
\hline 2 & US & US-17ccc & $\mathrm{ccc}$ & 6 & 10 & 225 & $\mathrm{X} 025$ & 22.500 & 15.917 & 2.900 & 13.684 & 19.167 & 85.835 \\
\hline 2 & Soln Std & Soln Std & & 6 & 11 & 226 & STD-B6-2 & 3.670 & 19.900 & 3.150 & 9.730 & 81.100 & 50.900 \\
\hline 2 & US & US-26 & quenched & 6 & 12 & 227 & $\mathrm{X} 086$ & 29.667 & 13.000 & 0.083 & 14.034 & 34.667 & 46.834 \\
\hline 2 & US & US-18 & quenched & 6 & 13 & 228 & $\mathrm{X} 020$ & 36.501 & 14.434 & 6.933 & 13.467 & 57.334 & 79.002 \\
\hline 2 & US & US-26ccc & $\mathrm{ccc}$ & 6 & 14 & 229 & $\mathrm{X} 015$ & 27.334 & 119.669 & 0.083 & 71.835 & 100.335 & 59.168 \\
\hline 2 & US & US-22ccc & $\mathrm{ccc}$ & 6 & 15 & 230 & $\mathrm{X} 087$ & 25.834 & 18.334 & 3.550 & 12.367 & 44.001 & 70.168 \\
\hline 2 & US & US- $18 \mathrm{ccc}$ & $\mathrm{ccc}$ & 6 & 16 & 231 & $\mathrm{X} 100$ & 49.834 & 298.339 & 17.667 & 186.670 & 326.673 & 168.337 \\
\hline 2 & US & US-19 & quenched & 6 & 17 & 232 & $\mathrm{X} 038$ & 24.667 & 19.834 & 3.917 & 9.334 & 27.001 & 59.001 \\
\hline 2 & US & US-22 & quenched & 6 & 18 & 233 & $\mathrm{X} 042$ & 25.834 & 20.000 & 3.133 & 12.834 & 44.668 & 69.668 \\
\hline 2 & Soln Std & Soln Std & & 6 & 19 & 234 & STD-B6-3 & 3.710 & 20.400 & 3.090 & 9.690 & 80.700 & 50.500 \\
\hline 3 & Soln Std & Soln Std & & 1 & 1 & 235 & STD-B1-1 & 3.900 & 18.500 & 3.490 & 10.100 & 79.500 & 50.600 \\
\hline 3 & EA & EA & & 1 & 2 & 236 & Y85 & 0.833 & 621.668 & 0.083 & 200.000 & 1661.670 & 935.002 \\
\hline 3 & US & US-31 & quenched & 1 & 3 & 237 & Y15 & 20.000 & 20.500 & 0.008 & 14.567 & 15.700 & 51.668 \\
\hline 3 & US & US-31ccc & $\mathrm{ccc}$ & 1 & 4 & 238 & Y89 & 16.634 & 11.867 & 1.163 & 11.350 & 10.334 & 45.834 \\
\hline 3 & US & US-33 & quenched & 1 & 5 & 239 & Y81 & 20.167 & 3.583 & 0.270 & 4.967 & 131.336 & 78.835 \\
\hline 3 & US & US-33 $\mathrm{ccc}$ & $\mathrm{ccc}$ & 1 & 6 & 240 & Y66 & 20.667 & 3.017 & 2.200 & 5.183 & 117.502 & 77.835 \\
\hline
\end{tabular}


Table B2. PSAL Measurements of the PCT Solutions for the US Study Glasses After Appropriate Adjustments

\begin{tabular}{|c|c|c|c|c|c|c|c|c|c|c|c|c|c|}
\hline Set & Type & Glass ID & $\begin{array}{c}\text { Heat } \\
\text { Treatment }\end{array}$ & Block & Seq & $\begin{array}{l}\text { Analytical } \\
\text { Sequence }\end{array}$ & Lab ID & $\mathrm{Al}(\mathrm{ppm})$ & B (ppm) & $\mathrm{Fe}(\mathrm{ppm})$ & $\mathrm{Li}(\mathrm{ppm})$ & $\mathrm{Na}(\mathrm{ppm})$ & $\mathrm{Si}(\mathrm{ppm})$ \\
\hline 3 & US & US-35 & quenched & 1 & 7 & 241 & Y46 & 10.600 & 49.001 & 0.008 & 8.784 & 37.834 & 37.167 \\
\hline 3 & US & US-35ccc & $\mathrm{ccc}$ & 1 & 8 & 242 & Y45 & 9.584 & 248.338 & 0.567 & 33.334 & 147.003 & 39.501 \\
\hline 3 & US & US-37 & quenched & 1 & 9 & 243 & Y73 & 24.834 & 10.367 & 0.008 & 6.083 & 126.503 & 68.501 \\
\hline 3 & US & US-37 $\mathrm{ccc}$ & $\mathrm{ccc}$ & 1 & 10 & 244 & Y38 & 16.034 & 34.834 & 1.335 & 15.284 & 190.004 & 90.835 \\
\hline 3 & Soln Std & Soln Std & & 1 & 11 & 245 & STD-B1-2 & 3.760 & 17.300 & 3.500 & 9.690 & 76.700 & 48.500 \\
\hline 3 & US & US-39 & quenched & 1 & 12 & 246 & $\mathrm{Y75}$ & 16.834 & 23.000 & 0.008 & 15.600 & 8.367 & 55.168 \\
\hline 3 & US & US-39cec & $\mathrm{ccc}$ & 1 & 13 & 247 & Y80 & 18.000 & 19.667 & 0.343 & 14.600 & 7.133 & 55.168 \\
\hline 3 & US & US-41 & quenched & 1 & 14 & 248 & Y51 & 10.884 & 12.967 & 0.265 & 8.467 & 10.000 & 41.834 \\
\hline 3 & US & US-41ccc & $\mathrm{ccc}$ & 1 & 15 & 249 & Y84 & 10.484 & 11.034 & 1.598 & 8.517 & 8.634 & 40.834 \\
\hline 3 & US & US-43 & quenched & 1 & 16 & 250 & Y88 & 30.167 & 7.600 & 0.008 & 13.534 & 26.834 & 51.834 \\
\hline 3 & US & US-43ccc & $\operatorname{ccc}$ & 1 & 17 & 251 & Y92 & 30.667 & 43.668 & 0.838 & 40.001 & 38.501 & 63.001 \\
\hline 3 & US & US-45 & quenched & 1 & 18 & 252 & Y97 & 20.834 & 15.867 & 0.008 & 10.734 & 44.668 & 53.168 \\
\hline 3 & US & US- $45 \mathrm{ccc}$ & $\mathrm{ccc}$ & 1 & 19 & 253 & Y70 & 20.167 & 14.817 & 0.008 & 10.284 & 42.001 & 51.668 \\
\hline 3 & Soln Std & Soln Std & & 1 & 20 & 254 & STD-B1-3 & 3.790 & 17.000 & 3.560 & 9.750 & 75.600 & 48.800 \\
\hline 3 & Soln Std & Soln Std & & 2 & 1 & 255 & STD-B2-1 & 3.840 & 20.700 & 3.610 & 9.990 & 82.000 & 50.100 \\
\hline 3 & EA & EA & & 2 & 2 & 256 & Y27 & 0.833 & 668.335 & 4.133 & 200.000 & 1733.337 & 930.002 \\
\hline 3 & US & US-31 & quenched & 2 & 3 & 257 & Y06 & 20.500 & 23.667 & 0.008 & 14.550 & 16.000 & 51.834 \\
\hline 3 & US & US-31 ccc & $\mathrm{ccc}$ & 2 & 4 & 258 & Y41 & 16.150 & 14.567 & 1.293 & 10.900 & 9.584 & 43.834 \\
\hline 3 & US & US-33 & quenched & 2 & 5 & 259 & Y79 & 20.000 & 6.833 & 0.542 & 4.717 & 132.836 & 76.002 \\
\hline 3 & US & US-33ccc & $\mathrm{ccc}$ & 2 & 6 & 260 & Y30 & 21.334 & 6.867 & 2.300 & 5.317 & 130.669 & 81.335 \\
\hline 3 & US & US-35 & quenched & 2 & 7 & 261 & Y53 & 10.050 & 52.168 & 0.008 & 8.517 & 38.667 & 37.501 \\
\hline 3 & US & US-35 ccc & $\mathrm{ccc}$ & 2 & 8 & 262 & Y33 & 9.100 & 255.005 & 1.027 & 33.834 & 153.336 & 39.834 \\
\hline 3 & US & US-37 & quenched & 2 & 9 & 263 & Y20 & 25.167 & 14.500 & 0.008 & 6.067 & 133.003 & 70.501 \\
\hline 3 & US & US-37ccc & $\mathrm{ccc}$ & 2 & 10 & 264 & Y82 & 17.667 & 40.834 & 2.400 & 15.867 & 206.671 & 93.169 \\
\hline 3 & Soln Std & Soln Std & & 2 & 11 & 265 & STD-B2-2 & 3.760 & 20.200 & 3.520 & 9.790 & 80.700 & 49.300 \\
\hline 3 & US & US-39 & quenched & 2 & 12 & 266 & Y34 & 16.834 & 26.334 & 0.008 & 15.334 & 8.517 & 54.668 \\
\hline 3 & US & US-39ccc & $\mathrm{ccc}$ & 2 & 13 & 267 & Y86 & 17.334 & 23.334 & 0.578 & 14.184 & 6.800 & 54.501 \\
\hline 3 & US & US-41 & quenched & 2 & 14 & 268 & Y72 & 10.950 & 17.500 & 0.505 & 8.550 & 10.367 & 43.668 \\
\hline 3 & US & US-41 ccc & $\mathrm{ccc}$ & 2 & 15 & 269 & Y96 & 10.134 & 14.867 & 1.750 & 8.467 & 8.700 & 41.834 \\
\hline 3 & US & US-43 & quenched & 2 & 16 & 270 & Y18 & 30.834 & 14.250 & 0.008 & 13.700 & 28.501 & 53.168 \\
\hline 3 & US & US- $43 \mathrm{ccc}$ & $\mathrm{ccc}$ & 2 & 17 & 271 & Y24 & 31.667 & 50.001 & 1.057 & 41.334 & 41.501 & 65.835 \\
\hline 3 & US & US-45 & quenched & 2 & 18 & 272 & Y61 & 21.500 & 20.167 & 0.058 & 10.867 & 49.001 & 54.168 \\
\hline 3 & US & US- $45 \mathrm{ccc}$ & $\mathrm{ccc}$ & 2 & 19 & 273 & Y83 & 21.167 & 19.500 & 0.380 & 10.434 & 46.001 & 53.334 \\
\hline 3 & Soln Std & Soln Std & & 2 & 20 & 274 & STD-B2-3 & 3.770 & 19.500 & 3.350 & 9.750 & 81.800 & 48.900 \\
\hline 3 & Soln Std & Soln Std & & 3 & 1 & 275 & STD-B3-1 & 3.810 & 20.200 & 3.480 & 9.880 & 80.300 & 48.800 \\
\hline 3 & blank & blank & & 3 & 2 & 276 & Y12 & 0.083 & 0.417 & 0.008 & 0.833 & 0.083 & 0.083 \\
\hline 3 & EA & EA & & 3 & 3 & 277 & Y04 & 4.250 & 633.335 & 0.083 & 195.000 & 1701.670 & 906.668 \\
\hline 3 & US & US-31 & quenched & 3 & 4 & 278 & Y94 & 20.334 & 23.334 & 0.008 & 14.367 & 15.084 & 50.834 \\
\hline 3 & US & US-31 ccc & $\mathrm{ccc}$ & 3 & 5 & 279 & Y71 & 16.834 & 15.384 & 2.067 & 11.550 & 12.034 & 45.668 \\
\hline 3 & US & US-33 & quenched & 3 & 6 & 280 & Y36 & 19.334 & 7.250 & 0.490 & 4.817 & 130.503 & 74.168 \\
\hline
\end{tabular}


Table B2. PSAL Measurements of the PCT Solutions for the US Study Glasses After Appropriate Adjustments

\begin{tabular}{|c|c|c|c|c|c|c|c|c|c|c|c|c|c|}
\hline Set & Type & Glass ID & $\begin{array}{c}\text { Heat } \\
\text { Treatment }\end{array}$ & Block & Seq & $\begin{array}{l}\text { Analytical } \\
\text { Sequence }\end{array}$ & Lab ID & $\mathrm{Al}(\mathrm{ppm})$ & B (ppm) & $\mathrm{Fe}(\mathrm{ppm})$ & $\mathrm{Li}(\mathrm{ppm})$ & $\mathrm{Na}(\mathrm{ppm})$ & $\mathrm{Si}(\mathrm{ppm})$ \\
\hline 3 & US & US-33ccc & $\mathrm{ccc}$ & 3 & 7 & 281 & Y76 & 22.000 & 7.367 & 7.217 & 5.350 & 126.336 & 79.335 \\
\hline 3 & US & US-35 & quenched & 3 & 8 & 282 & Y57 & 9.517 & 48.001 & 0.008 & 8.083 & 35.667 & 35.667 \\
\hline 3 & US & US-35 $\mathrm{ccc}$ & $\mathrm{ccc}$ & 3 & 9 & 283 & Y56 & 9.150 & 233.338 & 0.938 & 31.667 & 144.836 & 37.501 \\
\hline 3 & US & US-37 & quenched & 3 & 10 & 284 & Y13 & 25.167 & 14.634 & 0.492 & 6.267 & 132.169 & 69.501 \\
\hline 3 & Soln Std & Soln Std & & 3 & 11 & 285 & STD-B3-2 & 3.870 & 20.500 & 3.450 & 9.930 & 81.000 & 49.300 \\
\hline 3 & US & US-37ccc & $\mathrm{ccc}$ & 3 & 12 & 286 & Y63 & 15.750 & 39.001 & 1.577 & 15.134 & 198.337 & 88.835 \\
\hline 3 & US & US-39 & quenched & 3 & 13 & 287 & Y29 & 16.234 & 26.334 & 0.008 & 15.150 & 7.633 & 53.334 \\
\hline 3 & US & US-39ccc & $\mathrm{ccc}$ & 3 & 14 & 288 & Y37 & 17.500 & 23.834 & 0.562 & 14.517 & 6.400 & 54.834 \\
\hline 3 & US & US-41 & quenched & 3 & 15 & 289 & Y08 & 10.584 & 17.167 & 0.285 & 8.467 & 9.934 & 41.167 \\
\hline 3 & US & US-41 ccc & $\mathrm{ccc}$ & 3 & 16 & 290 & Y77 & 10.117 & 15.234 & 1.683 & 8.484 & 8.650 & 40.501 \\
\hline 3 & US & US-43 & quenched & 3 & 17 & 291 & Y62 & 29.834 & 11.850 & 0.008 & 13.400 & 27.001 & 51.168 \\
\hline 3 & US & US-43ccc & $\operatorname{ccc}$ & 3 & 18 & 292 & Y26 & 30.834 & 47.834 & 1.033 & 40.167 & 40.501 & 62.835 \\
\hline 3 & US & US-45 & quenched & 3 & 19 & 293 & Y22 & 20.500 & 19.834 & 0.047 & 10.500 & 46.168 & 51.334 \\
\hline 3 & US & US- $45 \mathrm{ccc}$ & $\mathrm{ccc}$ & 3 & 20 & 294 & Y10 & 20.167 & 19.167 & 0.370 & 10.167 & 42.501 & 52.001 \\
\hline 3 & Soln Std & Soln Std & & 3 & 21 & 295 & STD-B3-3 & 3.840 & 20.000 & 3.500 & 9.900 & 80.600 & 49.300 \\
\hline 3 & Soln Std & Soln Std & & 4 & 1 & 296 & STD-B4-1 & 3.700 & 20.300 & 3.680 & 9.800 & 81.700 & 49.500 \\
\hline 3 & ARM & ARM-1 & & 4 & 2 & 297 & Y11 & 4.133 & 20.334 & 0.008 & 13.684 & 34.834 & 60.835 \\
\hline 3 & US & US-32 & quenched & 4 & 3 & 298 & Y40 & 13.717 & 206.671 & 2.833 & 83.168 & 105.002 & 70.501 \\
\hline 3 & US & US-32ccc & $\mathrm{ccc}$ & 4 & 4 & 299 & Y07 & 14.100 & 157.670 & 2.483 & 62.835 & 81.835 & 69.001 \\
\hline 3 & US & US-34 & quenched & 4 & 5 & 300 & Y95 & 26.334 & 175.004 & 0.318 & 37.667 & 438.342 & 76.502 \\
\hline 3 & US & US-34ccc & $\operatorname{ccc}$ & 4 & 6 & 301 & Y47 & 25.667 & 160.670 & 0.222 & 35.334 & 406.675 & 73.335 \\
\hline 3 & US & US-36 & quenched & 4 & 7 & 302 & Y58 & 16.650 & 30.167 & 0.357 & 7.517 & 37.667 & 40.001 \\
\hline 3 & US & US-36ccc & $\mathrm{ccc}$ & 4 & 8 & 303 & Y74 & 17.167 & 38.001 & 1.137 & 8.684 & 45.501 & 42.501 \\
\hline 3 & US & US-38 & quenched & 4 & 9 & 304 & Y78 & 20.667 & 17.500 & 1.027 & 6.550 & 159.837 & 79.168 \\
\hline 3 & US & US-38ccc & $\mathrm{ccc}$ & 4 & 10 & 305 & Y98 & 23.334 & 18.500 & 2.583 & 7.567 & 168.337 & 92.169 \\
\hline 3 & Soln Std & Soln Std & & 4 & 11 & 306 & STD-B4-2 & 3.690 & 20.400 & 3.900 & 9.790 & 81.000 & 49.700 \\
\hline 3 & US & US-40 & quenched & 4 & 12 & 307 & Y87 & 25.501 & 60.168 & 5.617 & 12.884 & 48.834 & 53.334 \\
\hline 3 & US & US-40 ccc & ccc & 4 & 13 & 308 & Y09 & 25.334 & 82.168 & 5.050 & 16.350 & 63.501 & 54.168 \\
\hline 3 & US & US-42 & quenched & 4 & 14 & 309 & Y50 & 82.668 & 25.001 & 8.784 & 30.334 & 233.338 & 118.169 \\
\hline 3 & US & US-42ccc & ccc & 4 & 15 & 310 & Y42 & 2.900 & 18.000 & 0.008 & 56.168 & 515.010 & 32.667 \\
\hline 3 & US & US-44 & quenched & 4 & 16 & 311 & Y01 & 19.834 & 20.000 & 0.728 & 10.350 & 46.334 & 50.668 \\
\hline 3 & US & US-44ccc & $\mathrm{ccc}$ & 4 & 17 & 312 & Y16 & 20.834 & 19.834 & 1.155 & 10.417 & 45.168 & 53.001 \\
\hline 3 & Soln Std & Soln Std & & 4 & 18 & 313 & STD-B4-3 & 3.750 & 19.900 & 3.840 & 9.870 & 83.100 & 49.500 \\
\hline 3 & Soln Std & Soln Std & & 5 & 1 & 314 & STD-B5-1 & 3.800 & 20.300 & 3.630 & 9.800 & 80.200 & 49.200 \\
\hline 3 & ARM & ARM-1 & & 5 & 2 & 315 & Y02 & 4.233 & 18.500 & 0.008 & 13.934 & 35.834 & 61.668 \\
\hline 3 & US & US-32 & quenched & 5 & 3 & 316 & Y14 & 13.050 & 206.671 & 1.933 & 80.668 & 100.669 & 69.168 \\
\hline 3 & US & US-32 $\mathrm{ccc}$ & $\operatorname{ccc}$ & 5 & 4 & 317 & Y39 & 13.367 & 171.670 & 3.200 & 67.168 & 85.335 & 70.001 \\
\hline 3 & US & US-34 & quenched & 5 & 5 & 318 & Y67 & 26.834 & $\begin{array}{l}178.337 \\
\end{array}$ & 0.008 & 38.501 & 436.675 & 77.002 \\
\hline 3 & US & US-34ccc & $\mathrm{ccc}$ & 5 & 6 & 319 & Y64 & 25.167 & 164.670 & 0.008 & 36.001 & 400.008 & 73.335 \\
\hline 3 & US & US-36 & quenched & 5 & 7 & 320 & Y25 & 17.167 & 32.334 & 0.008 & 7.967 & 39.667 & 41.334 \\
\hline
\end{tabular}


Table B2. PSAL Measurements of the PCT Solutions for the US Study Glasses After Appropriate Adjustments

\begin{tabular}{|c|c|c|c|c|c|c|c|c|c|c|c|c|c|}
\hline Set & Type & Glass ID & $\begin{array}{c}\text { Heat } \\
\text { Treatment }\end{array}$ & Block & Seq & $\begin{array}{l}\text { Analytical } \\
\text { Sequence }\end{array}$ & Lab ID & $\mathrm{Al}$ (ppm) & B (ppm) & $\mathrm{Fe}(\mathrm{ppm})$ & $\mathrm{Li}(\mathrm{ppm})$ & $\mathrm{Na}(\mathrm{ppm})$ & $\mathrm{Si}(\mathrm{ppm})$ \\
\hline 3 & US & US-36ccc & $\mathrm{ccc}$ & 5 & 8 & 321 & Y05 & 17.167 & 38.334 & 0.518 & 8.767 & 44.668 & 43.001 \\
\hline 3 & US & US-38 & quenched & 5 & 9 & 322 & Y91 & 18.500 & 18.500 & 0.228 & 6.700 & 157.336 & 82.168 \\
\hline 3 & US & US-38ccc & ccc & 5 & 10 & 323 & Y19 & 23.667 & 18.834 & 2.700 & 7.700 & 164.670 & 93.835 \\
\hline 3 & Soln Std & Soln Std & & 5 & 11 & 324 & STD-B5-2 & 3.820 & 20.800 & 3.790 & 9.890 & 80.300 & 50.100 \\
\hline 3 & US & US-40 & quenched & 5 & 12 & 325 & Y43 & 25.167 & 57.334 & 5.050 & 12.367 & 47.334 & 52.334 \\
\hline 3 & US & US-40ccc & $\mathrm{ccc}$ & 5 & 13 & 326 & Y23 & 22.667 & 80.668 & 3.967 & 15.700 & 59.501 & 49.501 \\
\hline 3 & US & US-42 & quenched & 5 & 14 & 327 & Y44 & 81.002 & 25.501 & 8.417 & 30.167 & 225.005 & 118.336 \\
\hline 3 & US & US-42ccc & $\mathrm{ccc}$ & 5 & 15 & 328 & Y90 & 5.500 & 17.834 & 0.008 & 55.168 & 505.010 & 32.167 \\
\hline 3 & US & US-44 & quenched & 5 & 16 & 329 & Y49 & 20.500 & 20.667 & 0.228 & 10.684 & 47.334 & 52.668 \\
\hline 3 & US & US-44ccc & $\mathrm{ccc}$ & 5 & 17 & 330 & Y48 & 21.167 & 20.500 & 0.360 & 10.767 & 46.501 & 54.834 \\
\hline 3 & Soln Std & Soln Std & & 5 & 18 & 331 & STD-B5-3 & 3.770 & 20.300 & 3.700 & 9.840 & 80.000 & 49.700 \\
\hline 3 & Soln Std & Soln Std & & 6 & 1 & 332 & STD-B6-1 & 3.880 & 20.100 & 3.530 & 9.860 & 80.500 & 49.300 \\
\hline 3 & ARM & ARM-1 & & 6 & 2 & 333 & Y21 & 4.317 & 17.334 & 0.008 & 13.600 & 34.834 & 60.001 \\
\hline 3 & blank & blank & & 6 & 3 & 334 & Y17 & 0.083 & 0.417 & 0.008 & 0.833 & 0.083 & 0.083 \\
\hline 3 & US & US-32 & quenched & 6 & 4 & 335 & Y35 & 13.500 & 218.338 & 1.833 & 85.002 & 105.835 & 71.001 \\
\hline 3 & US & US-32 ccc & $\mathrm{ccc}$ & 6 & 5 & 336 & Y68 & 13.567 & 170.003 & 1.210 & 67.335 & 84.502 & 69.335 \\
\hline 3 & US & US-34 & quenched & 6 & 6 & 337 & Y28 & 27.001 & 176.670 & 0.008 & 39.167 & 445.009 & 77.002 \\
\hline 3 & US & US-34ccc & $\mathrm{ccc}$ & 6 & 7 & 338 & Y69 & 25.167 & 165.170 & 0.008 & 36.501 & 403.341 & 73.501 \\
\hline 3 & US & US-36 & quenched & 6 & 8 & 339 & Y59 & 17.334 & 31.501 & 0.008 & 7.917 & 40.167 & 41.834 \\
\hline 3 & US & US-36 ccc & $\mathrm{ccc}$ & 6 & 9 & 340 & Y65 & 18.000 & 39.667 & 0.387 & 9.167 & 47.334 & 44.501 \\
\hline 3 & US & US-38 & quenched & 6 & 10 & 341 & Y54 & 18.500 & 17.834 & 0.008 & 6.733 & 158.003 & 80.502 \\
\hline 3 & Soln Std & Soln Std & & 6 & 11 & 342 & STD-B6-2 & 3.870 & 20.500 & 3.580 & 9.980 & 80.500 & 49.400 \\
\hline 3 & US & US-38ccc & $\mathrm{ccc}$ & 6 & 12 & 343 & Y93 & 22.834 & 17.167 & 2.500 & 7.417 & 159.670 & 88.835 \\
\hline 3 & US & US-40 & quenched & 6 & 13 & 344 & $\mathrm{Y} 03$ & 25.167 & 55.834 & 4.083 & 12.384 & 48.001 & 51.001 \\
\hline 3 & US & US-40cce & $\mathrm{ccc}$ & 6 & 14 & 345 & Y32 & 23.834 & 81.168 & 3.483 & 16.567 & 65.501 & 49.168 \\
\hline 3 & US & US-42 & quenched & 6 & 15 & 346 & Y55 & 83.502 & 24.334 & 8.183 & 31.167 & 235.005 & 117.669 \\
\hline 3 & US & US-42ccc & $\mathrm{ccc}$ & 6 & 16 & 347 & Y31 & 2.933 & 17.500 & 0.008 & 56.001 & 506.677 & 32.667 \\
\hline 3 & US & US-44 & quenched & 6 & 17 & 348 & Y52 & 20.667 & 20.667 & 0.047 & 10.900 & 48.001 & 53.168 \\
\hline 3 & US & US-44ccc & $\mathrm{ccc}$ & 6 & 18 & 349 & Y60 & 21.500 & 19.667 & 0.187 & 10.900 & 48.501 & 53.668 \\
\hline 3 & Soln Std & Soln Std & & 6 & 19 & 350 & STD-B6-3 & 3.900 & 19.700 & 3.460 & 9.930 & 81.600 & 49.100 \\
\hline
\end{tabular}


Exhibit B1. Laboratory PCT Measurements in Analytical Sequence for Study Glasses, EA, ARM, Blanks, and Solution Standards

Al (ppm) By Analytical Sequence

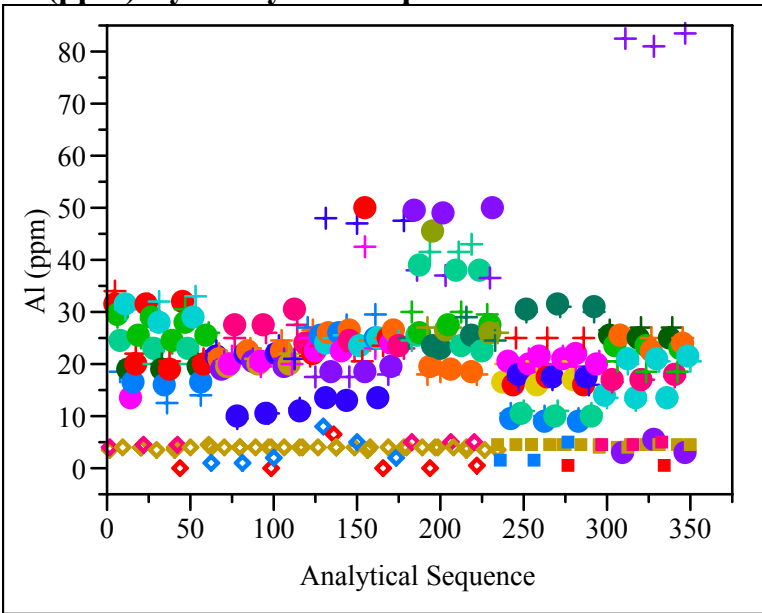

B (ppm) By Analytical Sequence

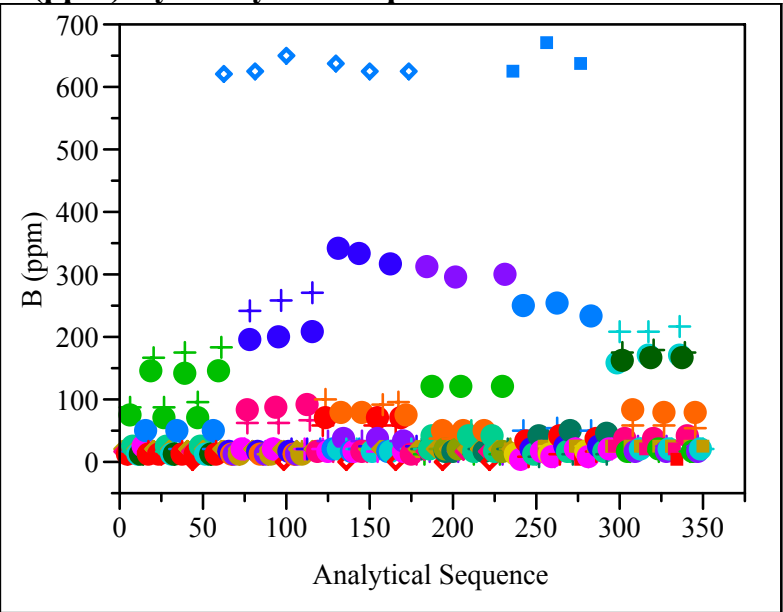

Fe (ppm) By Analytical Sequence

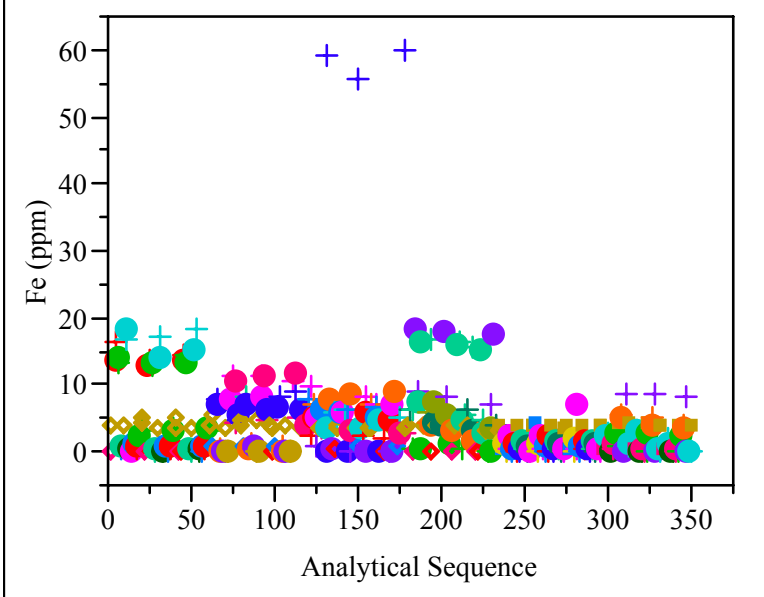

Li (ppm) By Analytical Sequence

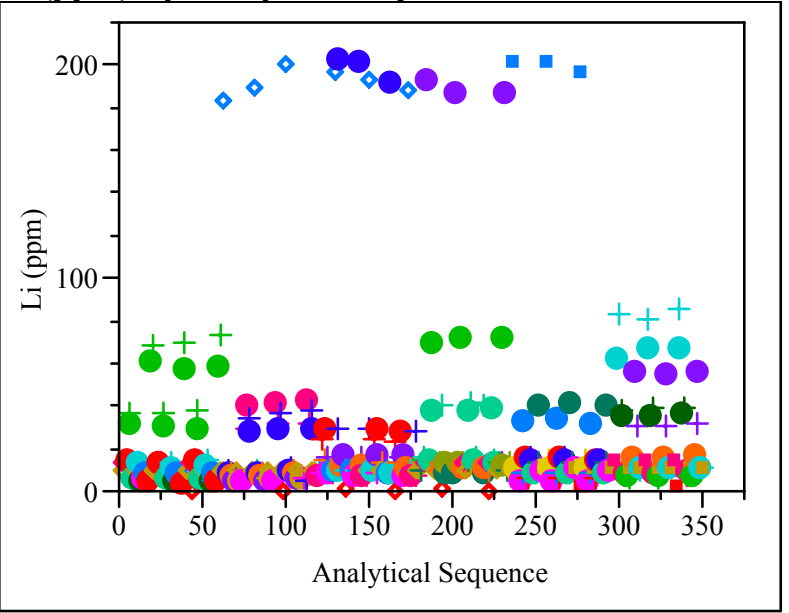

Na (ppm) By Analytical Sequence

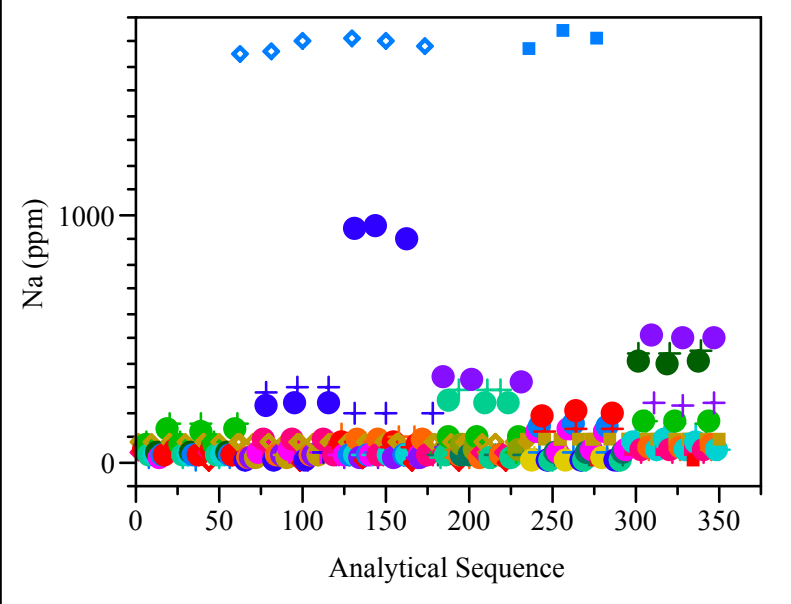

Si (ppm) By Analytical Sequence

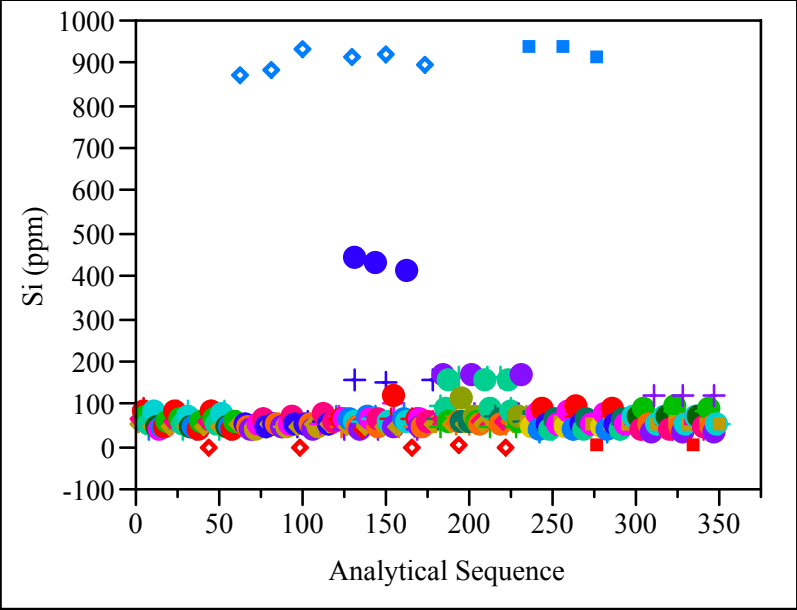


Exhibit B2. Laboratory PCT Measurements in Analytical Sequence for Study Glasses

Al (ppm) By Analytical Sequence
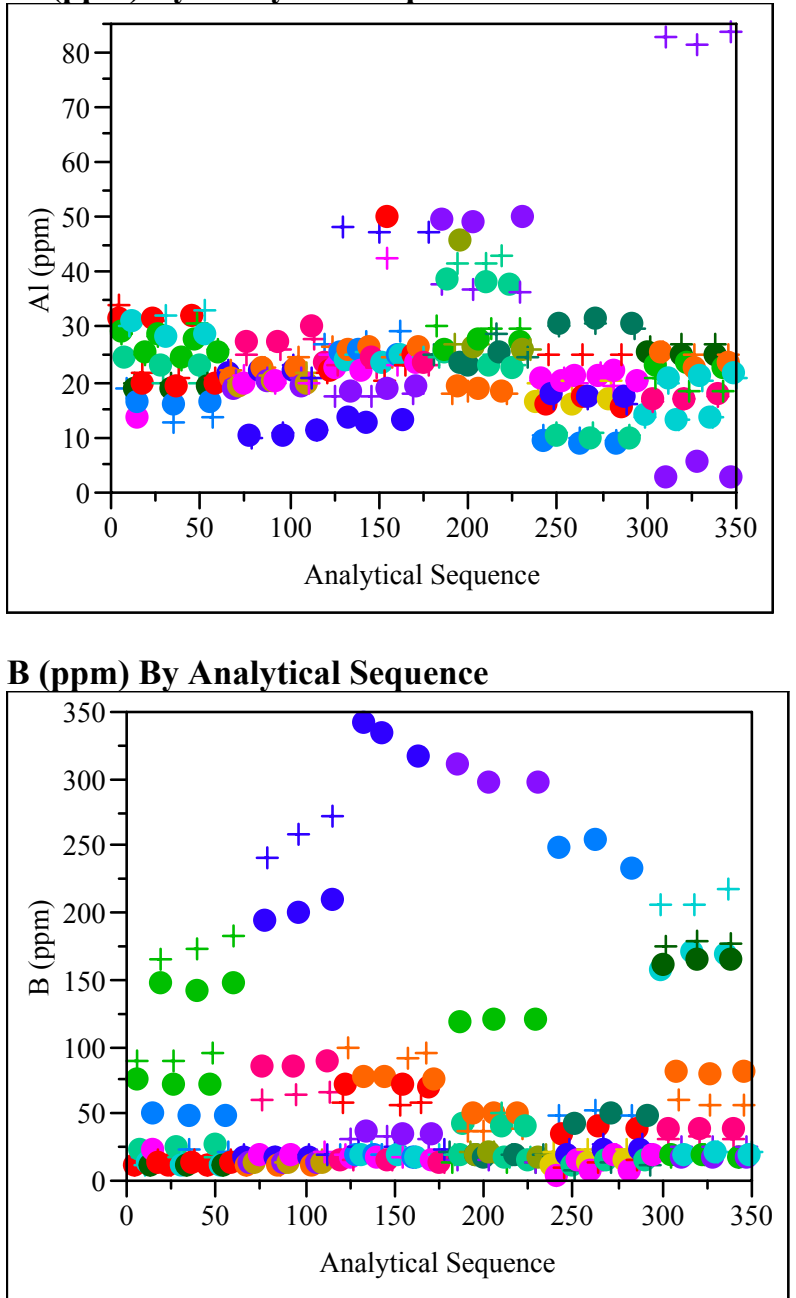

Fe (ppm) By Analytical Sequence

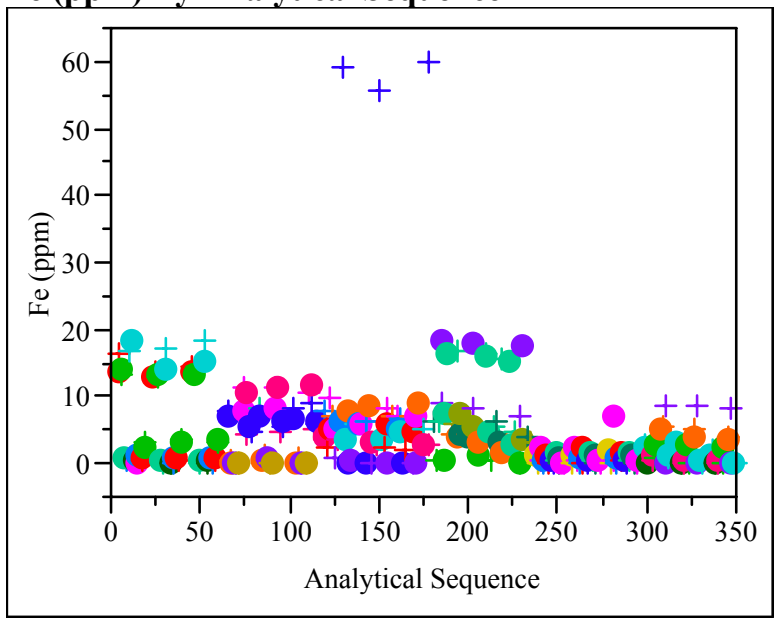

Li (ppm) By Analytical Sequence

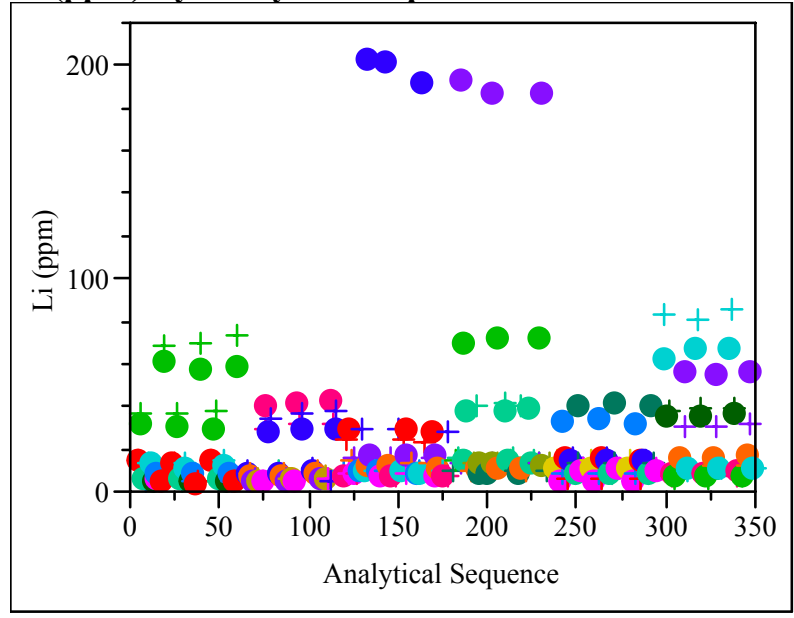

Na (ppm) By Analytical Sequence

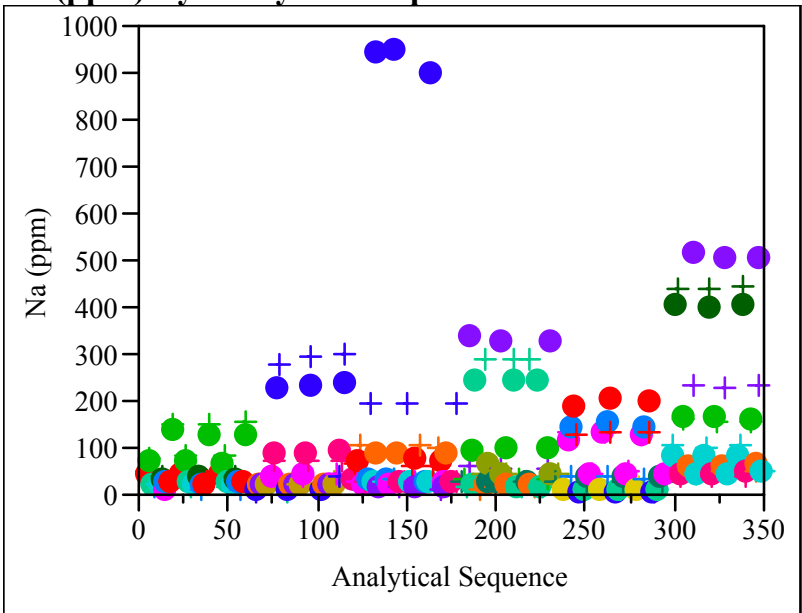

Si (ppm) By Analytical Sequence

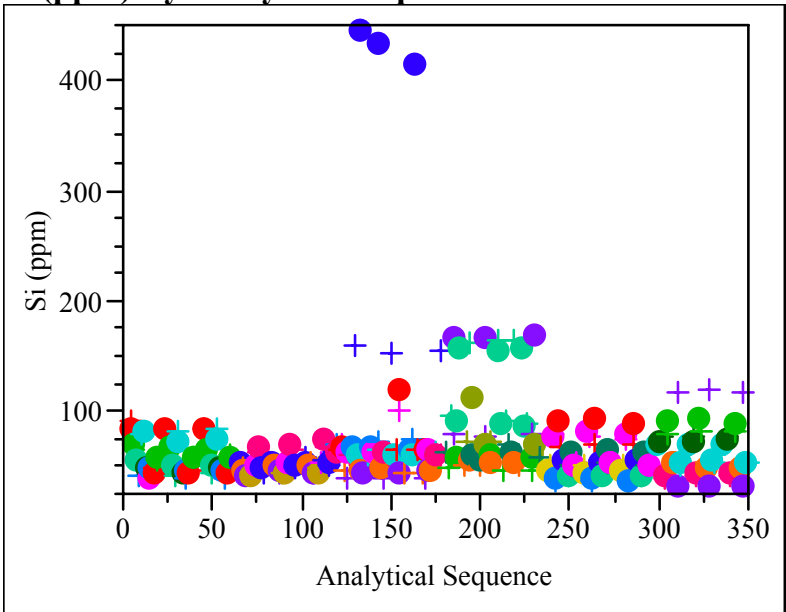


Exhibit B3. Measurements of the Multi-Element Solution Standard

Oneway Analysis of Al (ppm) By Set/Block

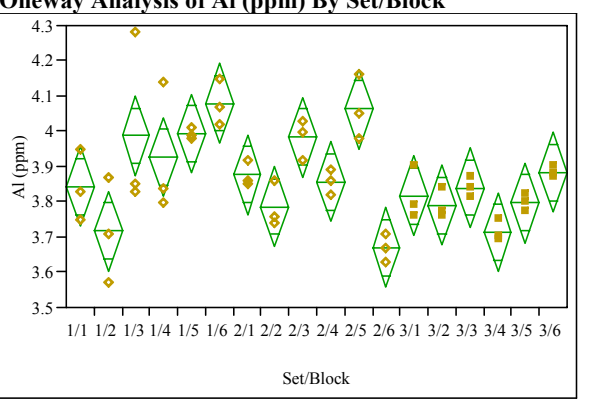

Oneway Anova/Summary of Fit $\begin{array}{ll}\text { Rsquare } & 0.682044 \\ \text { Adj Rsquare } & 0.531898\end{array}$ Root Mean Square Error $\quad 0.096138$ $\begin{array}{lr}\text { Mean of Response } & 3.867963 \\ \text { Observations (or Sum Wgts) } & 54\end{array}$

Analysis of Variance

Source DF Sum of Squares Mean Square F Ratio Prob $>F$ Set/Block $17-0.7137426$ $\begin{array}{llll}\text { Error } & 36 & 0.3327333 & 0.009243 \\ \text { C. Total } & 53 & 1.0464759 & \end{array}$

Means for Oneway Anova

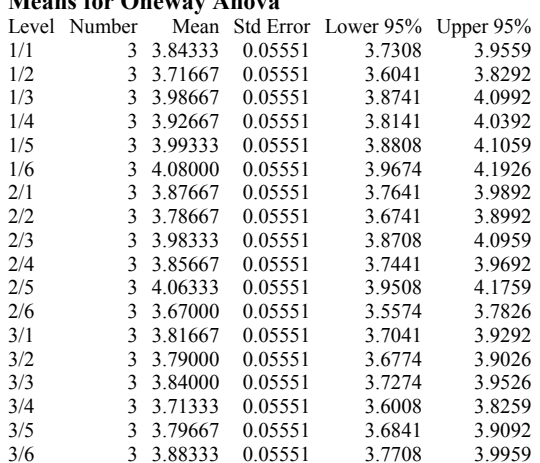

Std Error uses a pooled estimate of error variance by Set and ICP Block

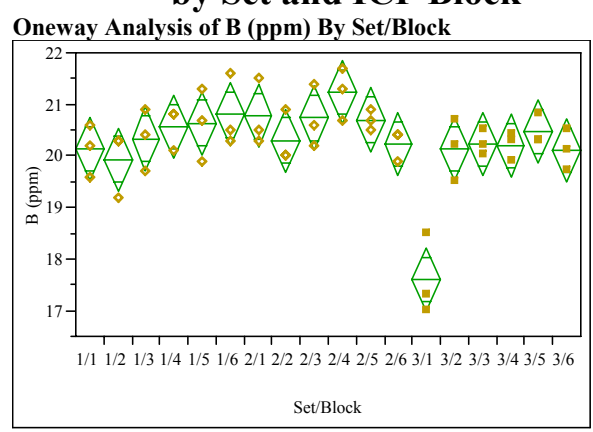

Oneway Anova/Summary of Fit

$\begin{array}{ll}\text { Rsquare } & 0.740029 \\ \text { Adj Rsquare } & 0.617265\end{array}$

$\begin{array}{ll}\text { Adj Rsquare } & 0.61726 \\ \text { Root Mean Square Error } & 0.525463\end{array}$

Mean of Response
Observations (or Sum Wgts)

Analysis of Variance

Source DF Sum of Squares Mean Square F Ratio Prob $>F$

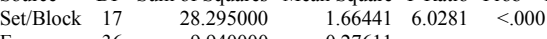

$\begin{array}{lrr}\text { Error } & 36 & 9.940000 \\ \text { C. Total } & 53 & 38.235000\end{array}$

\section{Means for Oneway Anova}

\begin{tabular}{|c|c|c|c|c|}
\hline \multicolumn{5}{|l|}{ Means for On } \\
\hline & ean & Std Error & & per $\mathrm{s}$ \\
\hline $1 / 1$ & 20.1333 & 0.30338 & 19.518 & 20.749 \\
\hline $1 / 2$ & 19.9333 & 0.30338 & 19.318 & 20.549 \\
\hline $1 / 3$ & 20.3333 & 0.30338 & 19.718 & 20.949 \\
\hline $1 / 4$ & 20.5667 & 0.30338 & 19.951 & 21.182 \\
\hline $1 / 5$ & 20.6333 & 0.30338 & 20.018 & 21.249 \\
\hline $1 / 6$ & 20.8000 & 0.30338 & 20.185 & 21.415 \\
\hline $2 / 1$ & 20.7667 & 0.30338 & 20.151 & 21.382 \\
\hline $2 / 2$ & 20.3000 & 0.30338 & 19.685 & 20.915 \\
\hline $2 / 3$ & 20.7333 & 0.30338 & 20.118 & 21.349 \\
\hline $2 / 4$ & 21.2333 & 0.30338 & 20.618 & 21.849 \\
\hline $2 / 5$ & 20.7000 & 0.30338 & 20.085 & 21.315 \\
\hline $2 / 6$ & 20.2333 & 0.30338 & 19.618 & 20.849 \\
\hline $3 / 1$ & 17.6000 & 0.30338 & 16.985 & 18.215 \\
\hline $3 / 2$ & 20.1333 & 0.30338 & 19.518 & 20.749 \\
\hline $3 / 3$ & 20.2333 & 0.30338 & 19.618 & 20.849 \\
\hline $3 / 4$ & 20.2000 & 0.30338 & 19.585 & 20.815 \\
\hline $3 / 5$ & 20.4667 & 0.30338 & 19.851 & 21.082 \\
\hline $3 / 6$ & 20.1000 & 0.30338 & 19.485 & 20.715 \\
\hline
\end{tabular}

Std Error uses a pooled estimate of error variance

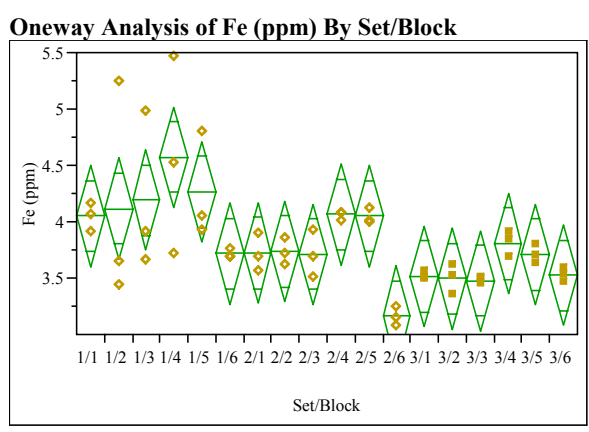

Oneway Anova/Summary of Fit$$
\text { Rsquare }
$$

$\begin{array}{ll}\text { Adj Rsquare } & 0.32216 \\ \text { Root Mean Square Error } & 0.38102\end{array}$ $\begin{array}{lr}\text { Mean of Response } & 3.826481 \\ \text { Observations (or Sum Wgts) } & 54\end{array}$

Analysis of Variance

Source DF Sum of Squares Mean Square F Ratio Prob $>F$

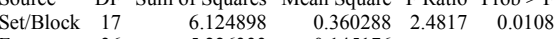

$\begin{array}{lrr}\text { Crror } & 36 & 5.226333 \\ \text { C. Total } & 53 & 11.351231\end{array}$

Means for Oneway Anova

Level Number $\quad$ Mean Std Error Lower 95\% Upper 95\%

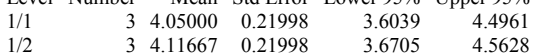

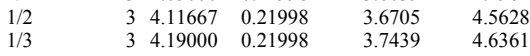

$\begin{array}{lllllll}1 / 4 & & 3 & 4.57333 & 0.21998 & 4.1272 & 5.0195 \\ 1 / 5 & & 3 & 4.26333 & 0.21998 & 3.8172 & 4.7095\end{array}$

$\begin{array}{llllll}1 / 6 & 3 & 3.71667 & 0.21998 & 3.2705 & 4.1628 \\ 2 / 1 & 3 & 3.72000 & 0.21998 & 3.2739 & 4.1661\end{array}$

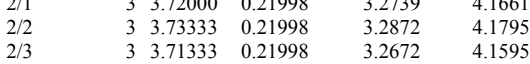

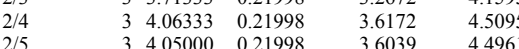

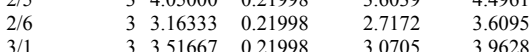

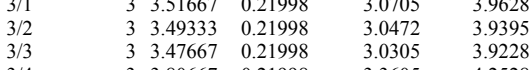

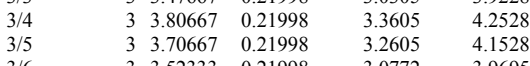

Std Error uses a pooled estimate of error variance 
Exhibit B3. Measurements of the Multi-Element Solution Standard

Oneway Analysis of Li (ppm) By Set/Block

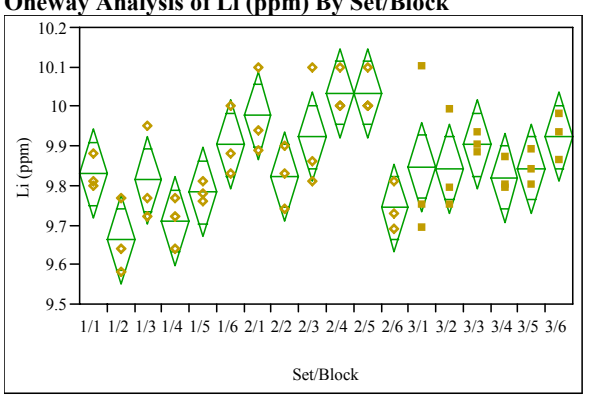

Oneway Anova/Summary of Fit Adj Rsquare

0.095801

$\begin{array}{lr}\text { Mean of Response } & 9.856481 \\ \text { Observations (or Sum Wgts) } & 54\end{array}$

Analysis of Variance

Source DF Sum of Squares Mean Square F Ratio Prob $>F$

$\begin{array}{lll}0.030472 & 3.3202 & 0.0012\end{array}$

$\begin{array}{lll}\text { Error } & 36 & 0.33040000 \\ \text { C. Total } & 53 & 0.84843148\end{array}$

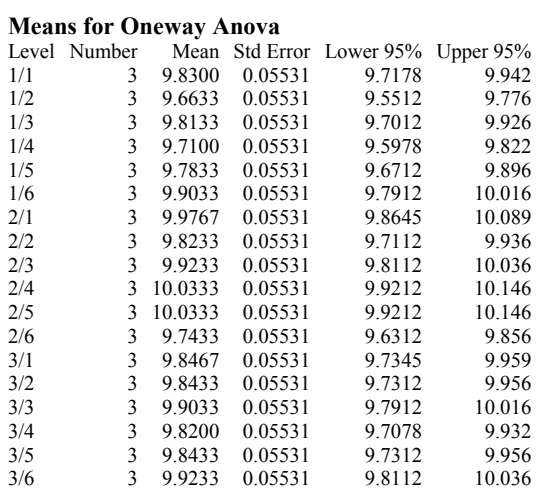

Std Error uses a pooled estimate of error variance by Set and ICP Block

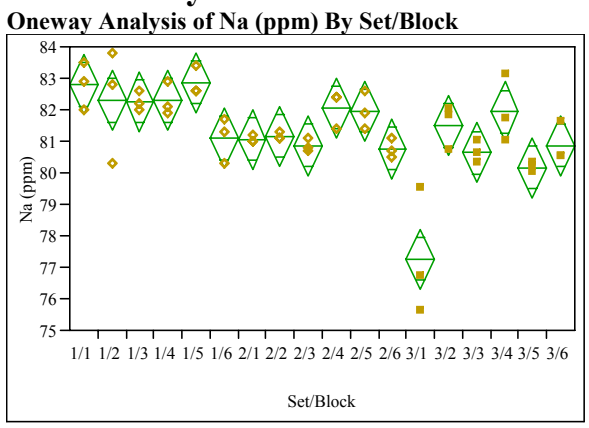

Oneway Anova/Summary of Fit

$\begin{array}{ll}\text { Rsquare } & 0.776763 \\ \text { Adj Rsquare } & 0.671345\end{array}$

$\begin{array}{lr}\text { Adj Rsquare } & 0.671345 \\ \text { Root Mean Square Error } \quad 0.81695\end{array}$

$\begin{array}{lr}\text { Mean of Response } & 81.32778 \\ \text { Observations (or Sum Wgts) } & 54\end{array}$

Analysis of Variance

Source DF Sum of Squares Mean Square F Ratio Prob $>F$

$\begin{array}{llll}4.91775 & 7.3684 & <000 \\ 0.66741 & 0 & 0\end{array}$

$\begin{array}{lrr}\text { Error } & 36 & 24.02667 \\ \text { C. Total } & 53 & 107.62833\end{array}$

Means for Oneway Anova

\begin{tabular}{|c|c|c|c|c|}
\hline vel & Mean & Std Err & & per 95\% \\
\hline & 382.8000 & 0.47167 & 81.843 & 83.757 \\
\hline 3 & $\begin{array}{l}382.3000\end{array}$ & 0.47167 & 81.343 & 83.257 \\
\hline & $\begin{array}{lll}3 & 82.2667\end{array}$ & 0.47 & 81.310 & 83.223 \\
\hline 3 & $\begin{array}{l}382.3000\end{array}$ & 0.47 & 81.343 & 83.257 \\
\hline & $\begin{array}{lll}3 & 82.8667\end{array}$ & 0.47167 & 81.910 & 83.823 \\
\hline 3 & $\begin{array}{ll}3 & 81.1000\end{array}$ & 0.47167 & 0.143 & 82.057 \\
\hline $2 / 1$ & $\begin{array}{ll}3 & 81.0667\end{array}$ & 0.47167 & 0.110 & 82.023 \\
\hline $2 / 2$ & $\begin{array}{ll}3 & 81.1667\end{array}$ & 0.47167 & 0.210 & 82.123 \\
\hline $2 / 3$ & 380.8667 & 0.47167 & 9.910 & 81.82. \\
\hline $2 / 4$ & $3 \quad 82.0667$ & 0.47167 & 110 & 83.023 \\
\hline $2 / 5$ & $\begin{array}{l}381.9667 \\
\end{array}$ & 0.47167 & 1.010 & 82.923 \\
\hline $2 / 6$ & 380.7667 & 0.47167 & 79.810 & 81.723 \\
\hline & $\begin{array}{ll}3 & 77.2667\end{array}$ & 0.47167 & 76.310 & 78.223 \\
\hline & 381.5000 & 0.47167 & 80.543 & 82.457 \\
\hline & 380.6333 & 0.47167 & 79.677 & 81.590 \\
\hline $3 /$ & 381.9333 & 0.47167 & 80.977 & 82.890 \\
\hline $3 / 5$ & $\begin{array}{ll}380.1667\end{array}$ & 0.47167 & 79.210 & 81.123 \\
\hline & 380.8667 & 0.47167 & 79.910 & 81.823 \\
\hline
\end{tabular}

Std Error uses a pooled estimate of error variance

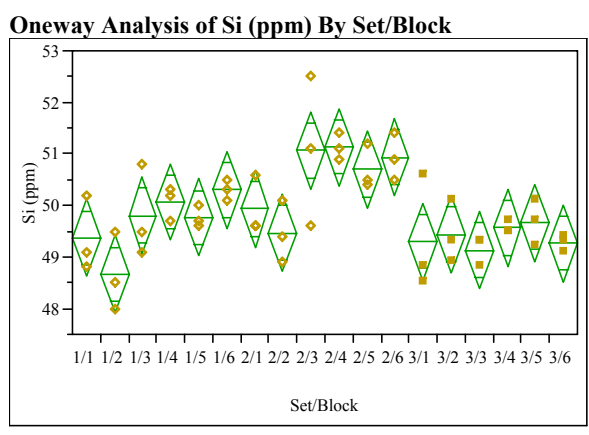

Oneway Anova/Summary of Fit Rsquare

$\begin{array}{ll}\text { Adj Rsquare } & 0.462204 \\ \text { Root Mean Square Error } & 0.638865\end{array}$ $\begin{array}{lr}\text { Mean of Response } & 49.86481 \\ \text { Observations (or Sum Wgts) } & 54\end{array}$

Analysis of Variance

Source DF Sum of Squares Mean Square F Ratio Prob $>$ F \begin{tabular}{lll} 
Set/Block & 17 & 25.529815 \\
Error & 36 & 14.693 \\
\hline
\end{tabular} $\begin{array}{lll}\text { Error } & 36 & 14.693333 \\ \text { C. Total } & 53 & 40.223148\end{array}$

Means for Oneway Anova

Level Number Mean Std Error Lower 95\% Upper 95\%

$\begin{array}{llllll}1 / 1 & 3 & 49.3667 & 0.36885 & 48.619 & 50.115 \\ 1 / 2 & 3 & 48.6667 & 0.3685 & 47.919 & 49.415\end{array}$

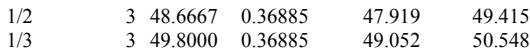

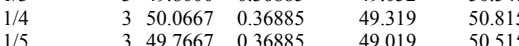

$\begin{array}{llllll}1 / 6 & 3 & 49.3000 & 0.36885 & 49.519 & 50.515 \\ 1 / 6 & & 3 & 50.3003 & 51.048\end{array}$

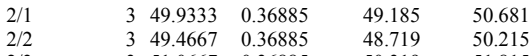

$\begin{array}{lllllll}2 / 3 & 3 & 51.0667 & 0.36885 & 50.319 & 51.815 \\ 2 / 4 & 3 & 51.1333 & 0.36885 & 50.38 & 51.81\end{array}$

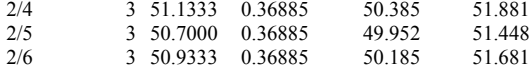

$\begin{array}{llllll}3 / 1 & 3 & 49.3000 & 0.36885 & 48.552 & 50.048\end{array}$

$\begin{array}{lllllll}3 / 2 & 3 & 49.4333 & 0.36885 & 48.685 & 50.181 \\ 3 / 3 & & 3 & 49.1333 & 0.36885 & 48.385 & 49.881\end{array}$

$\begin{array}{lllllll}3 / 4 & 3 & 49.5667 & 0.36885 & 48.819 & 50.315 \\ 3 / 5 & & 3 & 49.6667 & 0.36885 & 48.919 & 50.415\end{array}$

$\begin{array}{llllll}3 / 5 & 3 & 49.6667 & 0.36885 & 48.919 & 50.415 \\ 3 / 6 & 3 & 49.2667 & 0.36885 & 48.519 & 50.015\end{array}$

Std Error uses a pooled estimate of error variance 
Exhibit B4. Laboratory PCT Measurements by Glass Number for Study Glasses and Standards

(100 - Solution Standard; 101 - EA; 102 - ARM; 103 - Blanks)

Al (ppm) By Glass \#

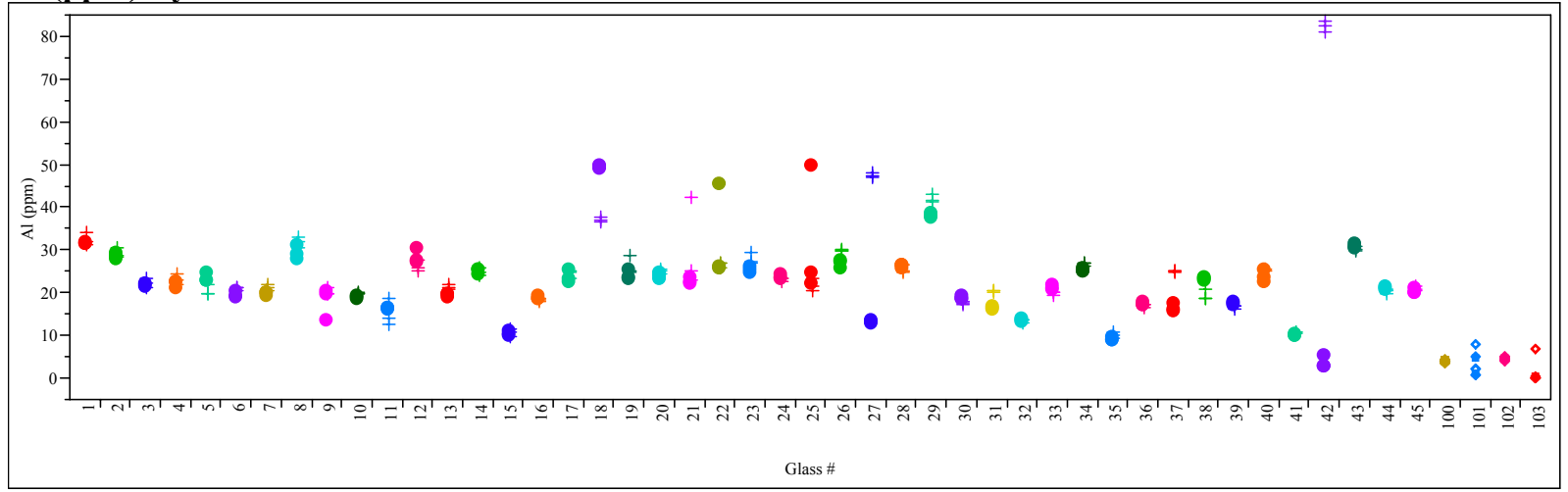

B (ppm) By Glass \#

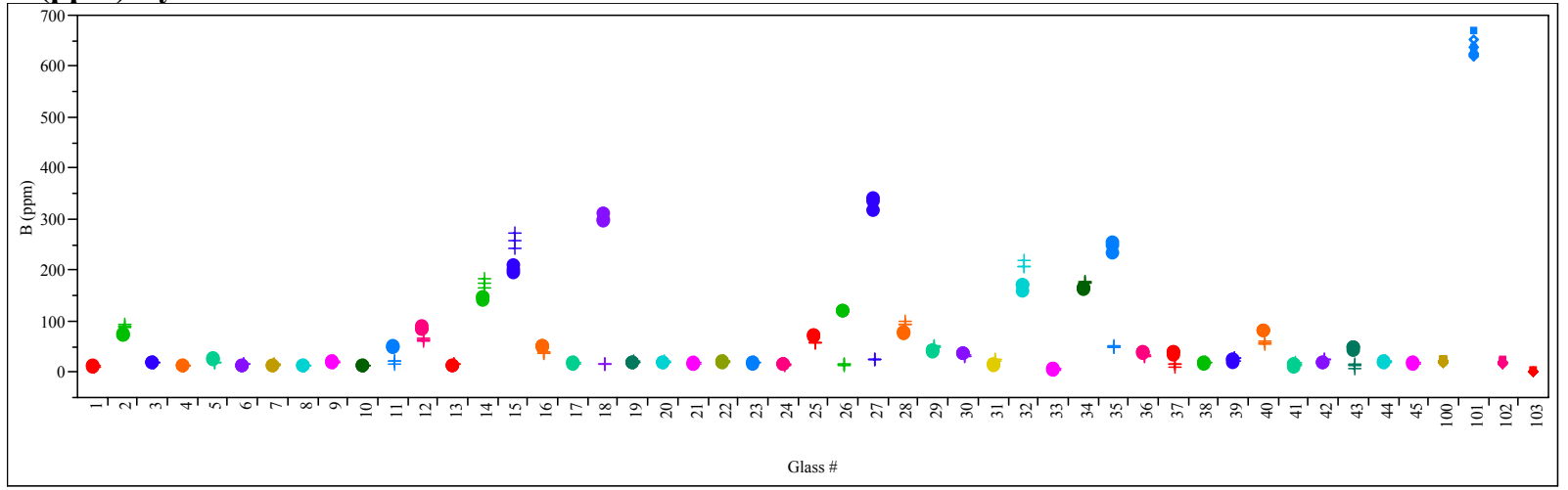

Fe (ppm) By Glass \#

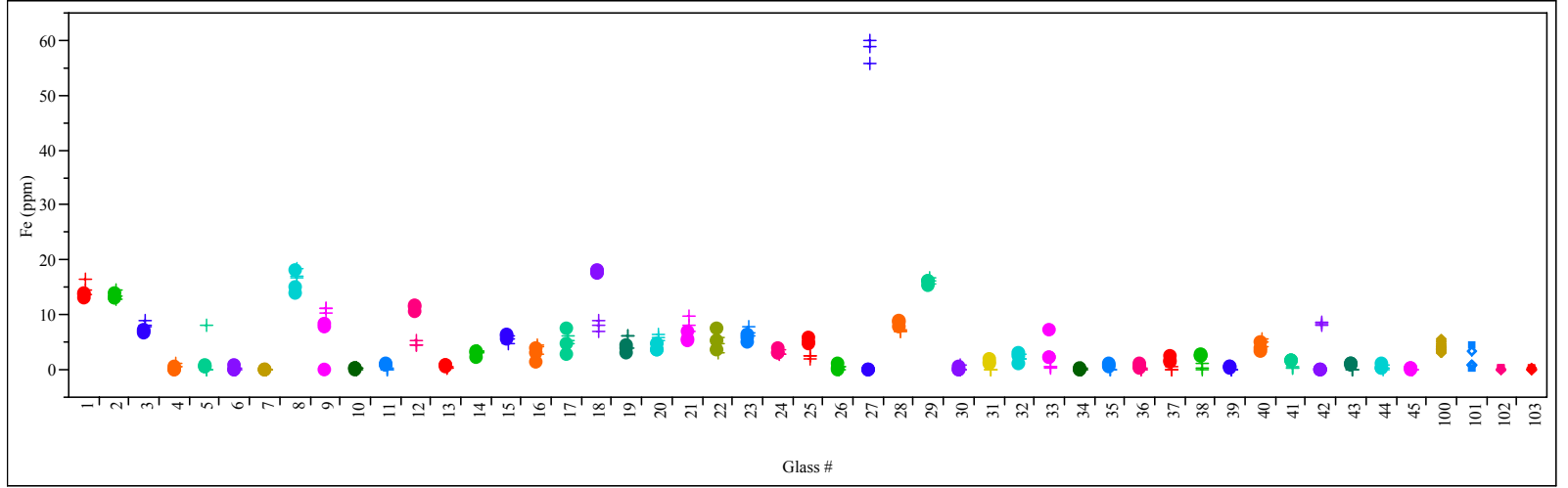


Exhibit B4. Laboratory PCT Measurements by Glass Number for Study Glasses and Standards

(100 - Solution Standard; 101 - EA; 102 - ARM; 103 - Blanks)

\section{Li (ppm) By Glass \#}

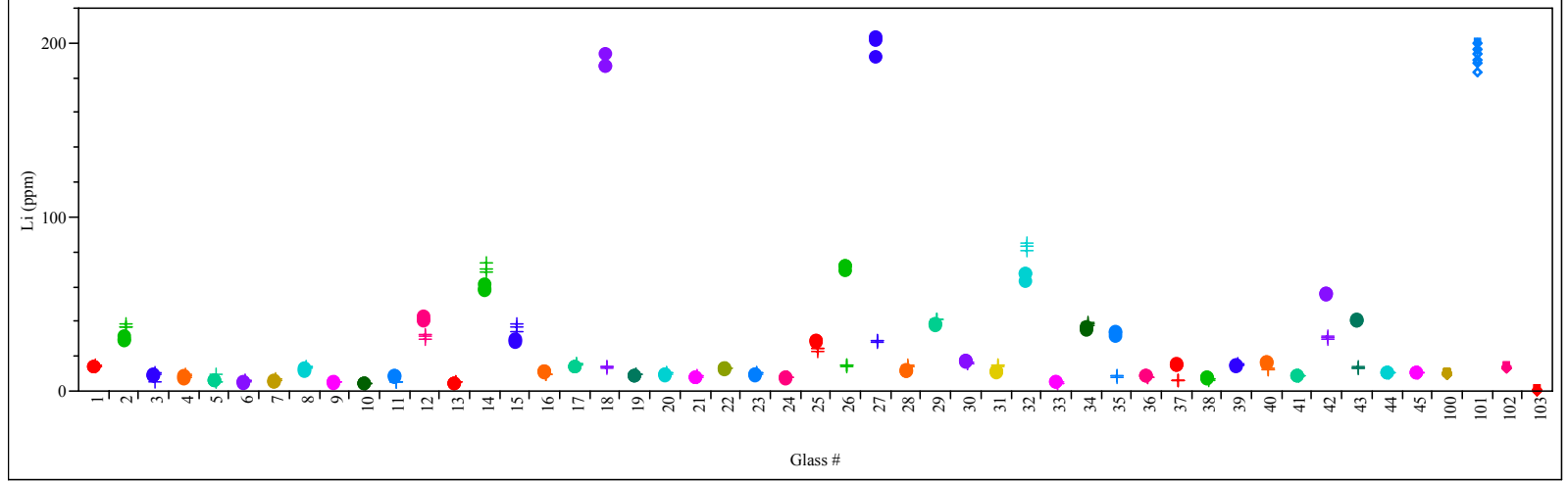

Na (ppm) By Glass \#

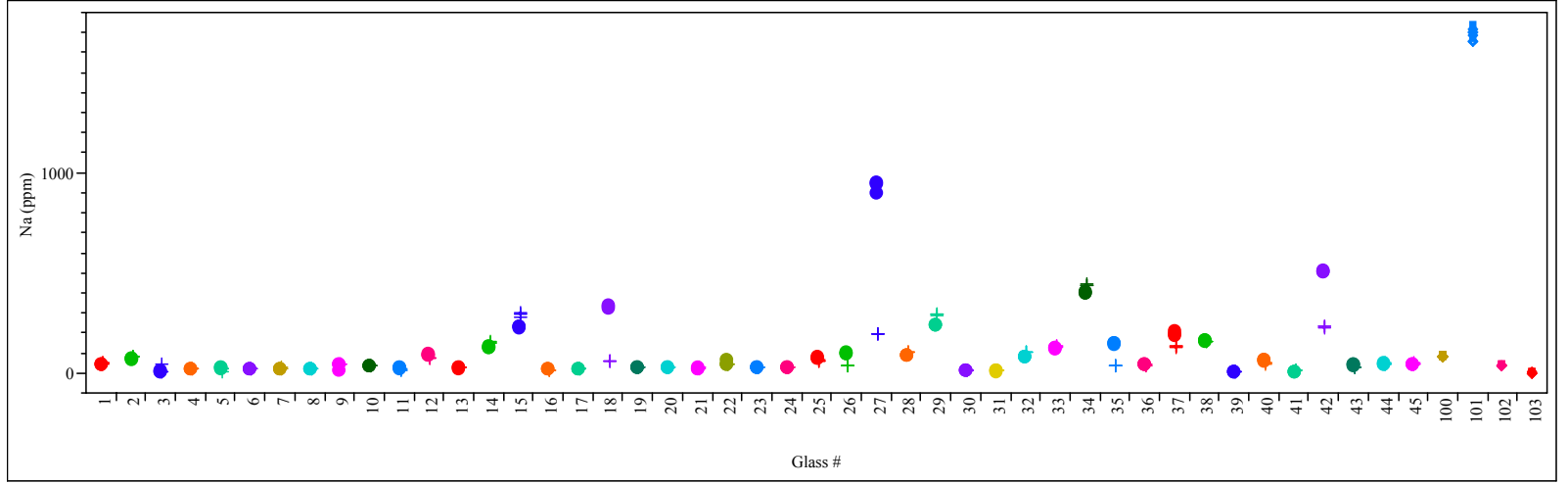

\section{Si (ppm) By Glass \#}

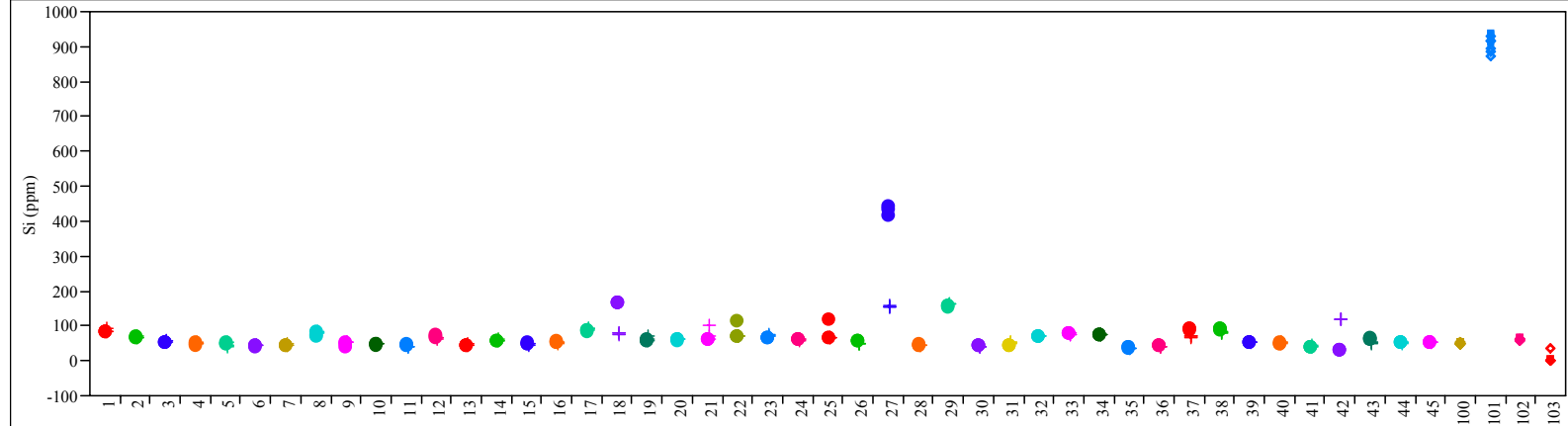


Exhibit B5. Laboratory PCT Measurements by Glass Number for Study Glasses

Set $=1$ Variability Chart for Al (ppm)

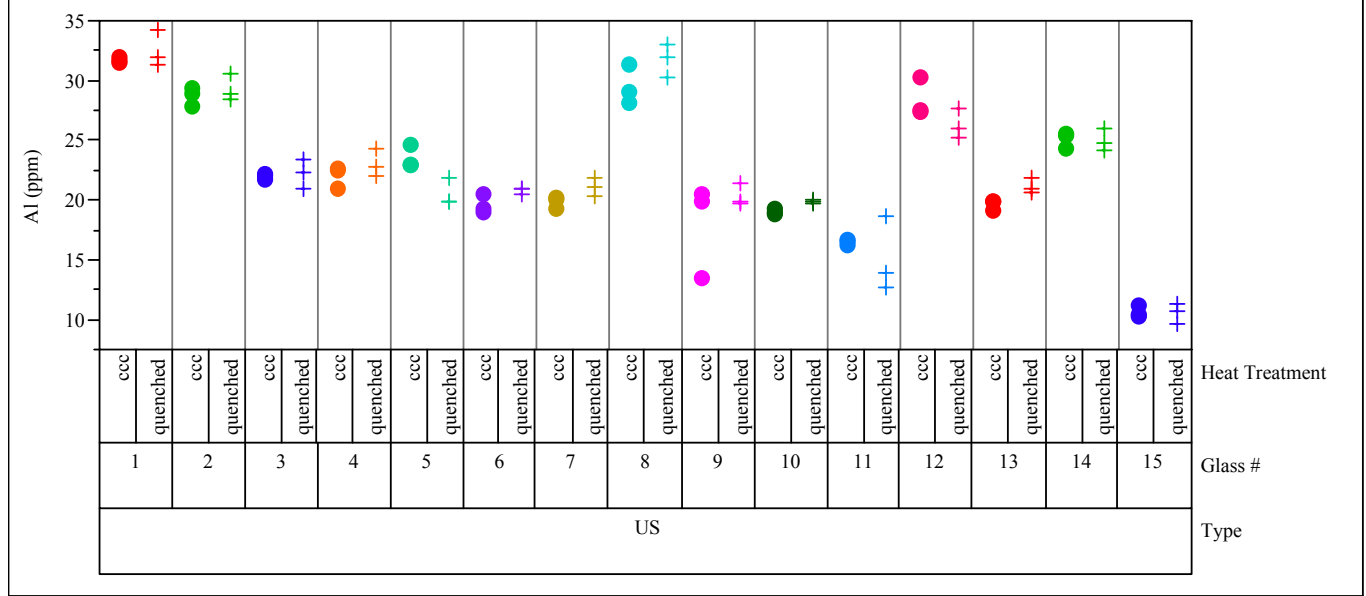

Set $=1$ Variability Chart for B (ppm)

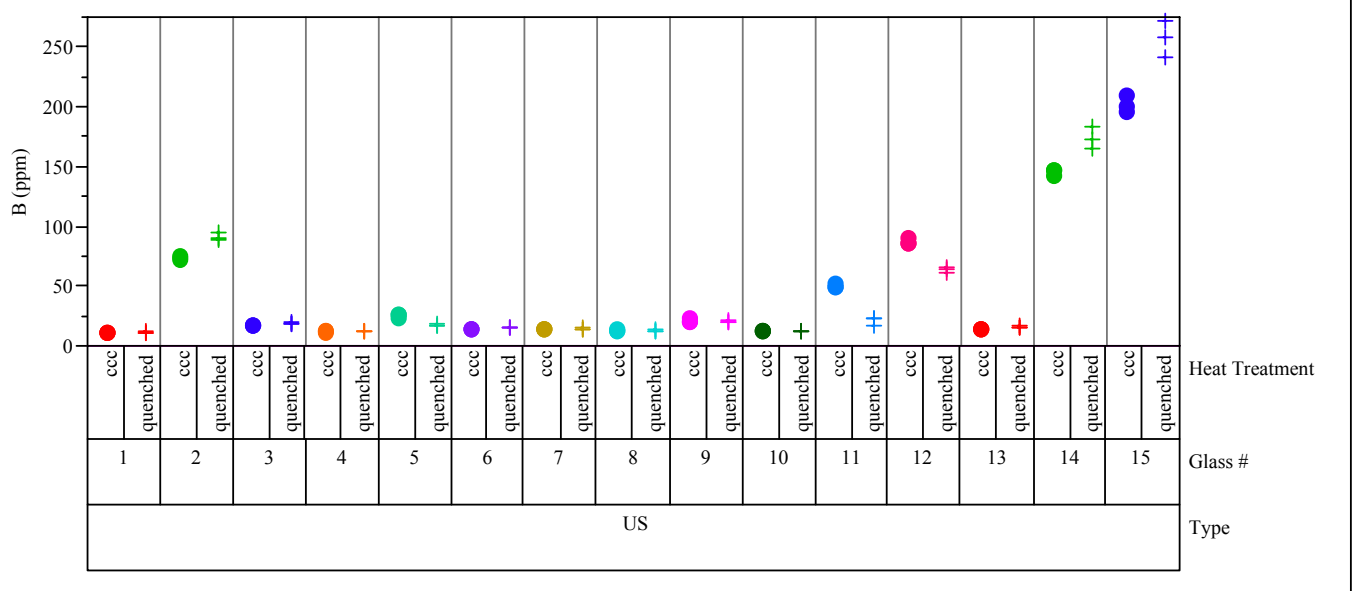

Set=1 Variability Chart for Fe (ppm)

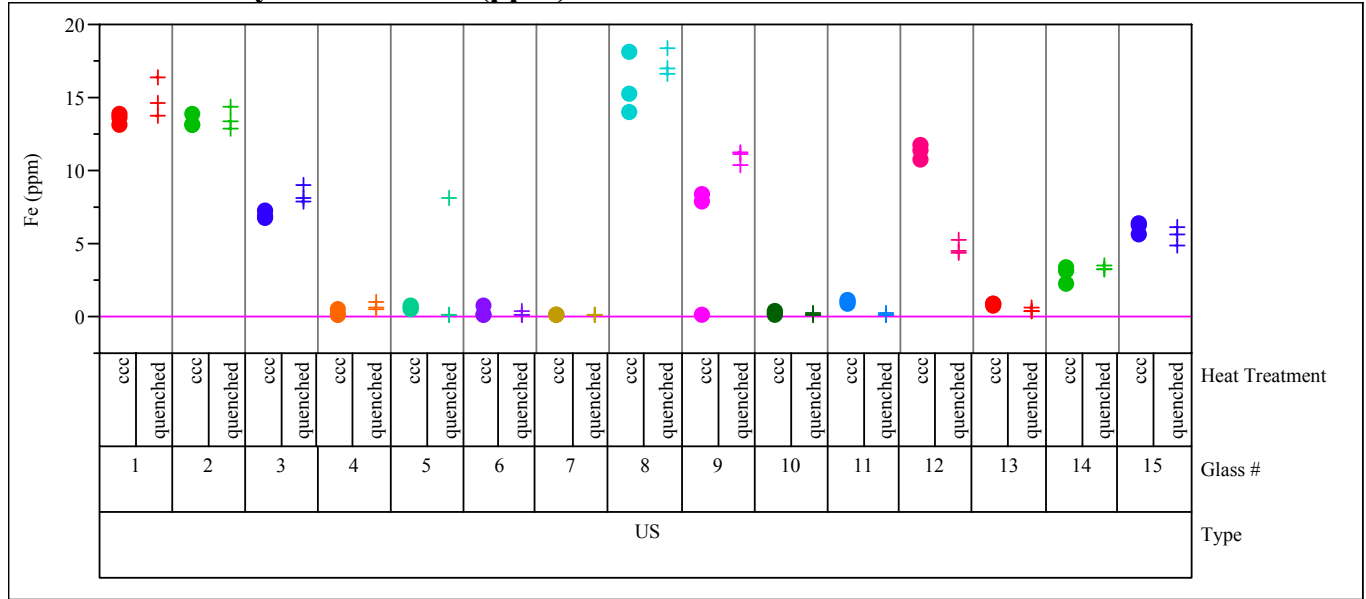


Exhibit B5. Laboratory PCT Measurements by Glass Number for Study Glasses

Set $=1$ Variability Chart for Li (ppm)

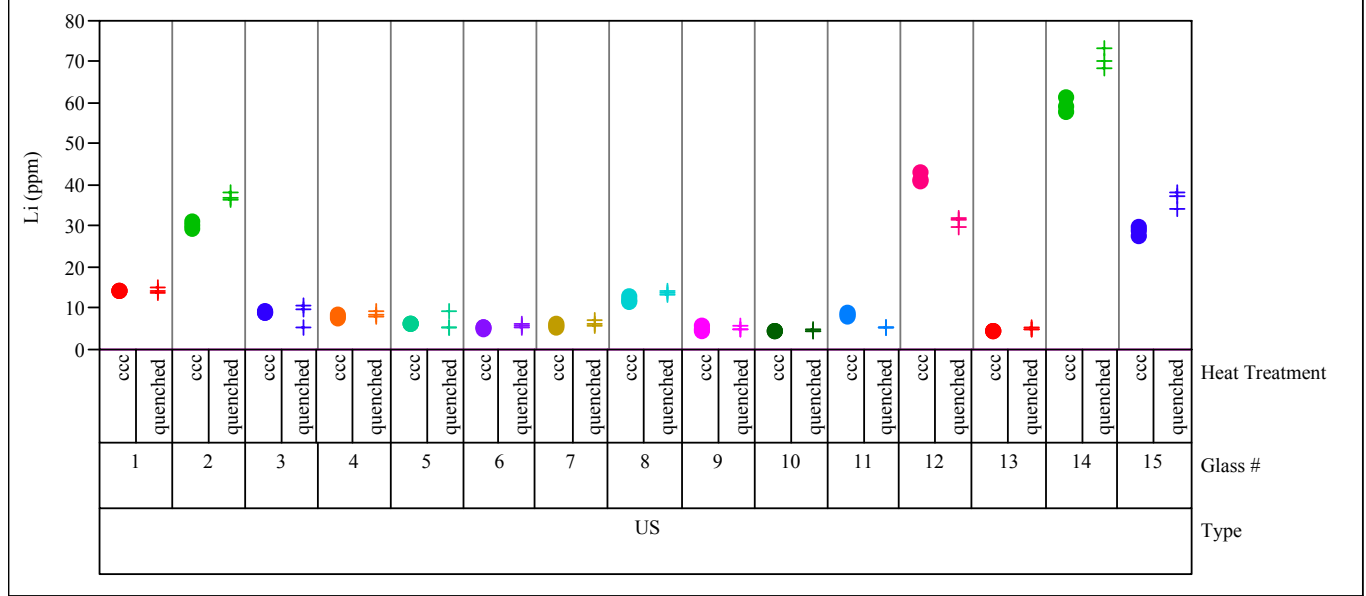

Set $=1$ Variability Chart for Na (ppm)

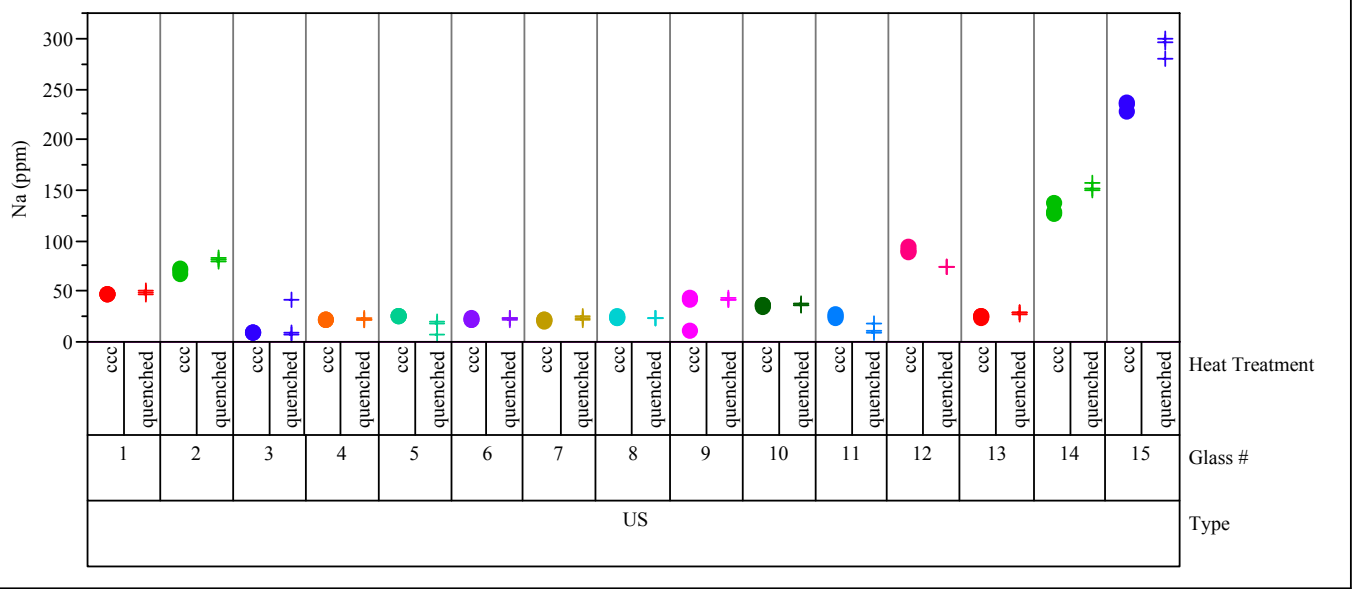

Set=1 Variability Chart for Si (ppm)

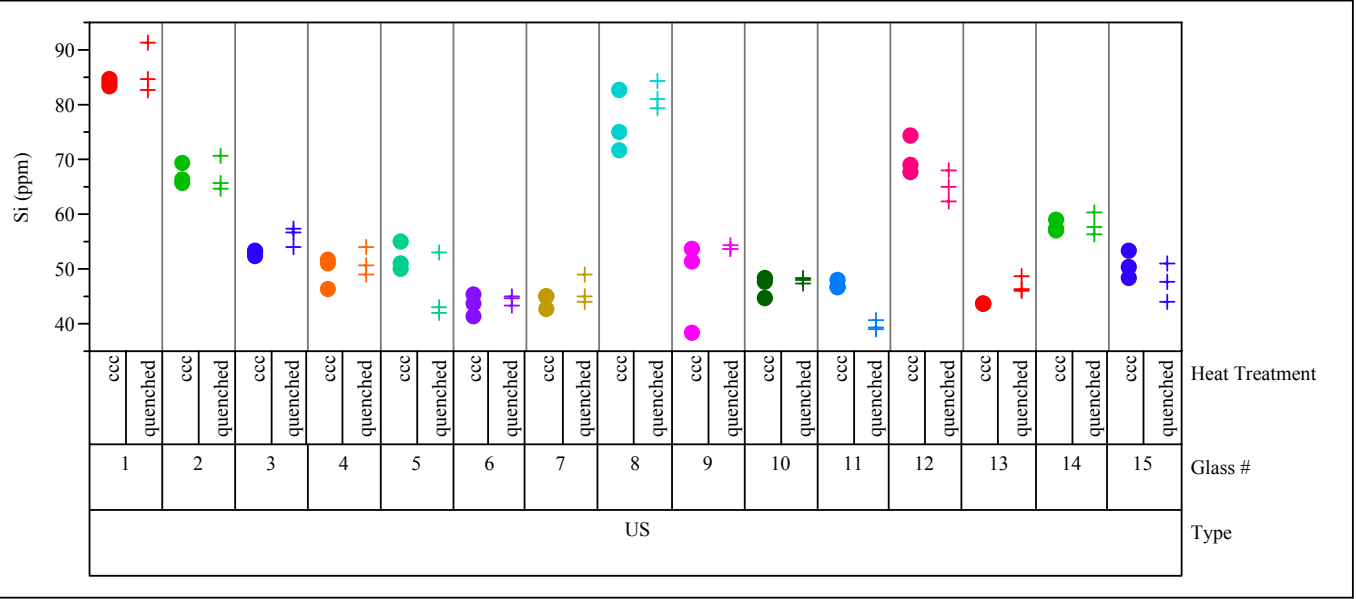


Exhibit B5. Laboratory PCT Measurements by Glass Number for Study Glasses

Set $=2$ Variability Chart for Al (ppm)

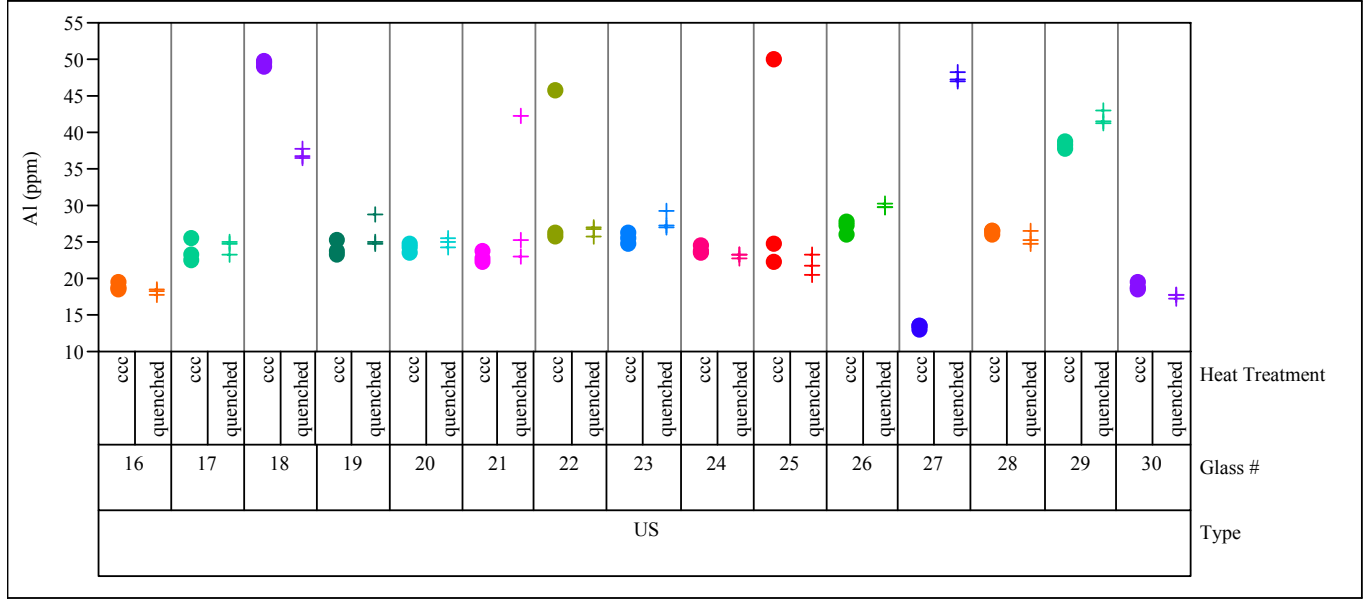

Set $=2$ Variability Chart for B (ppm)

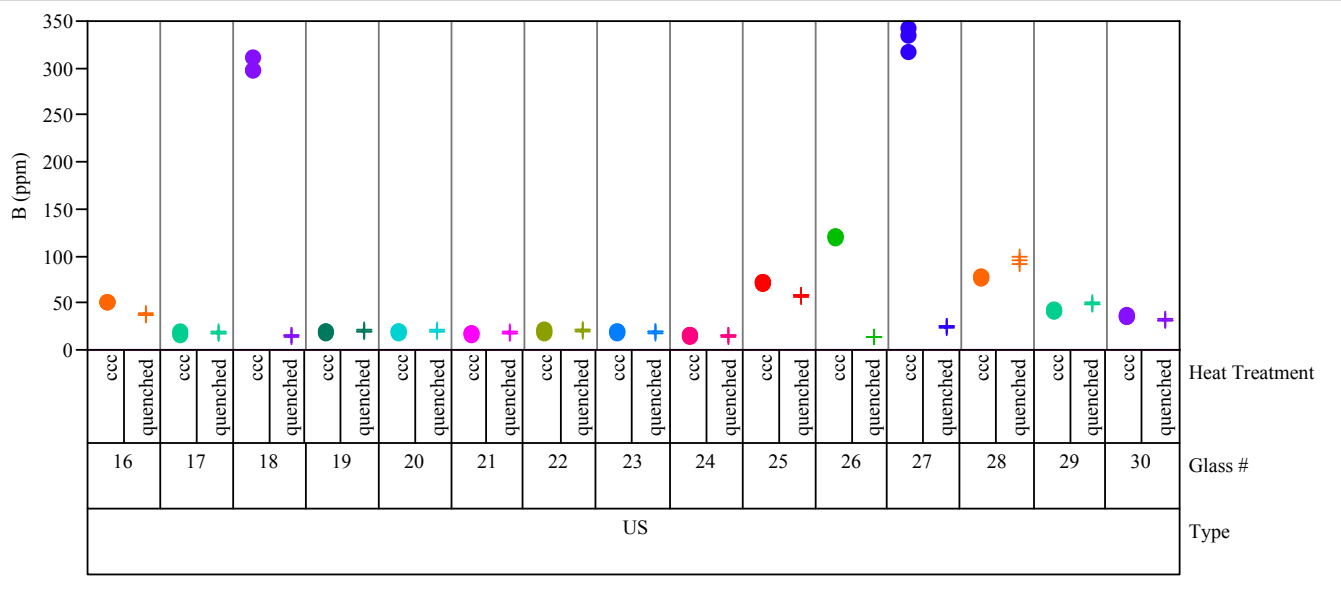

Set=2 Variability Chart for Fe (ppm)

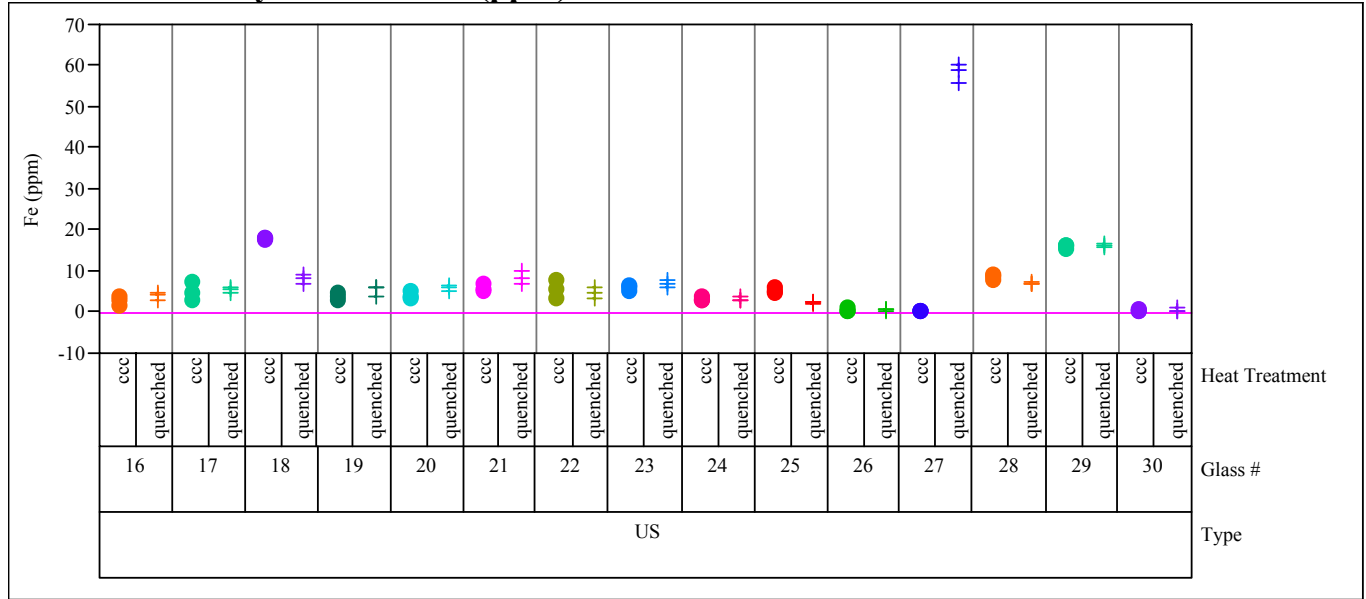


Exhibit B5. Laboratory PCT Measurements by Glass Number for Study Glasses

Set=2 Variability Chart for Li (ppm)

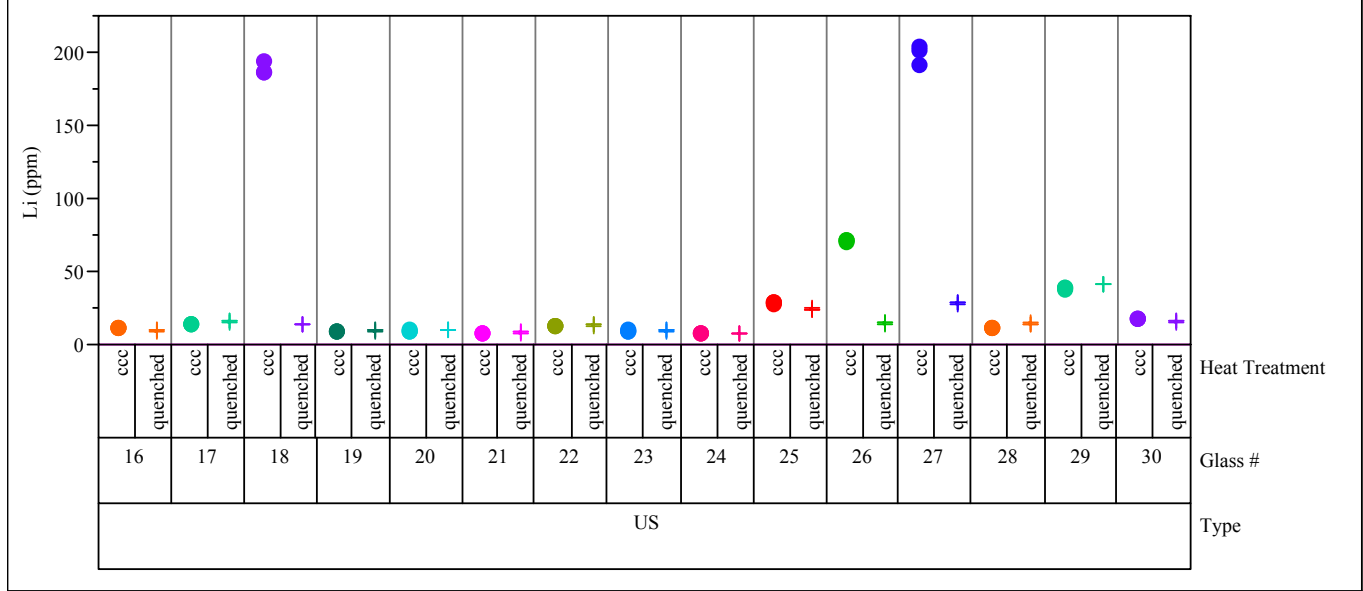

Set=2 Variability Chart for Na (ppm)

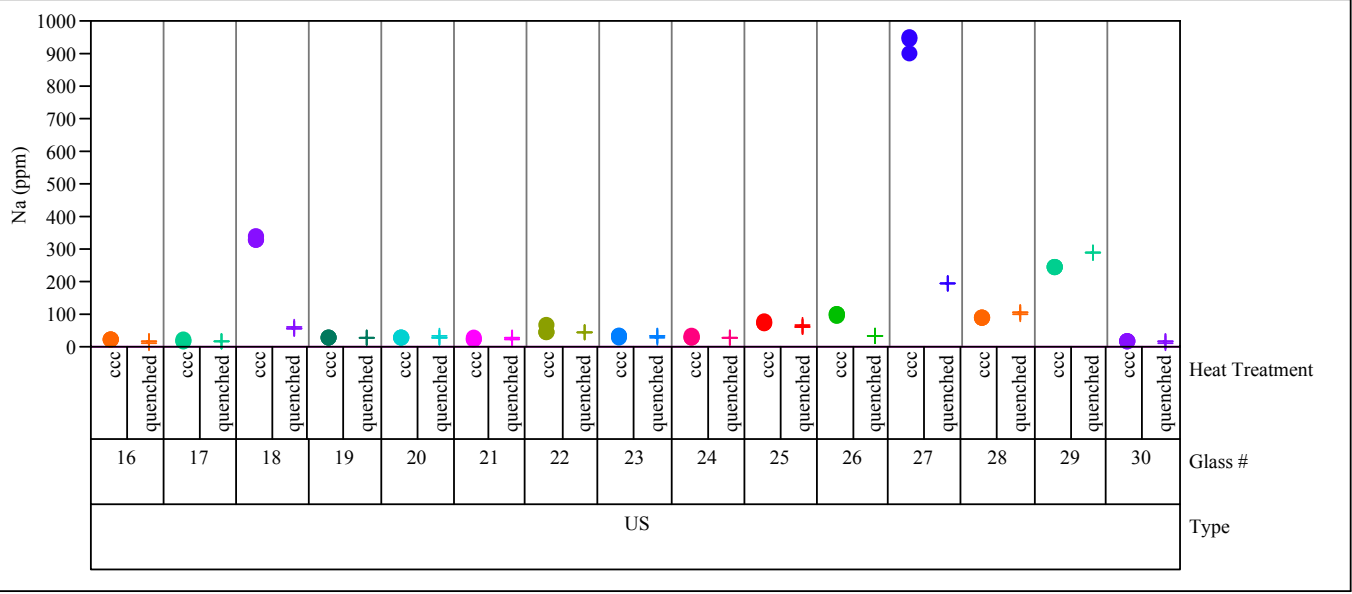

Set=2 Variability Chart for Si (ppm)

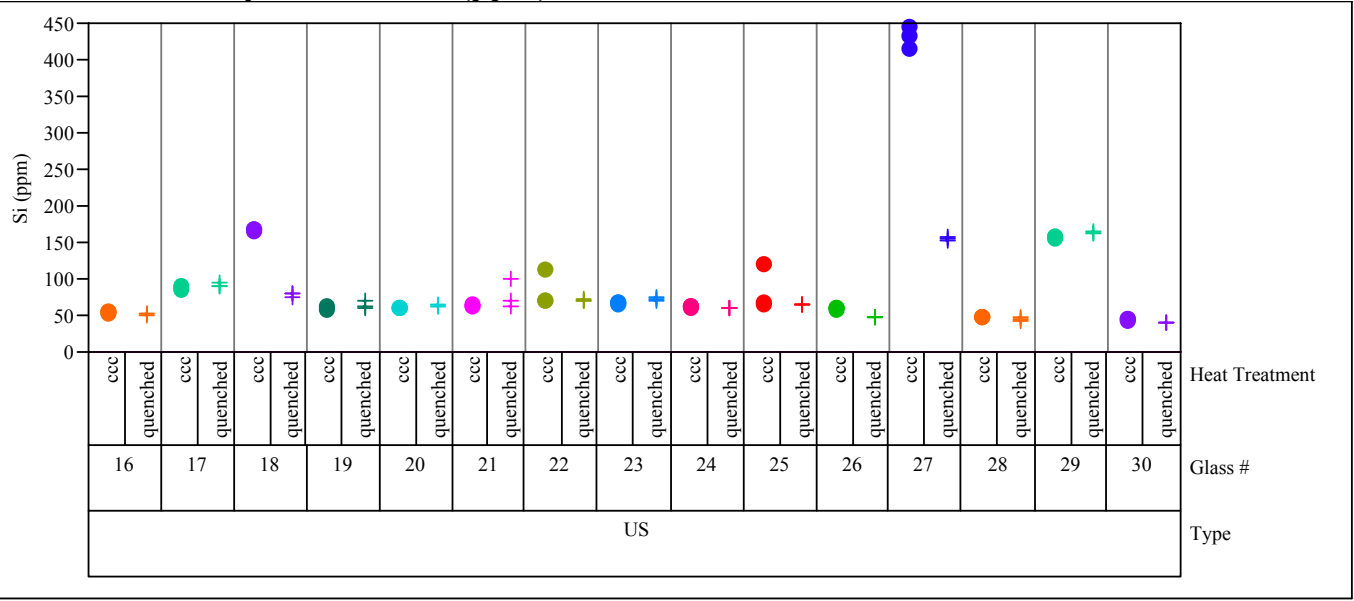


Exhibit B5. Laboratory PCT Measurements by Glass Number for Study Glasses

Set $=3$ Variability Chart for Al (ppm)

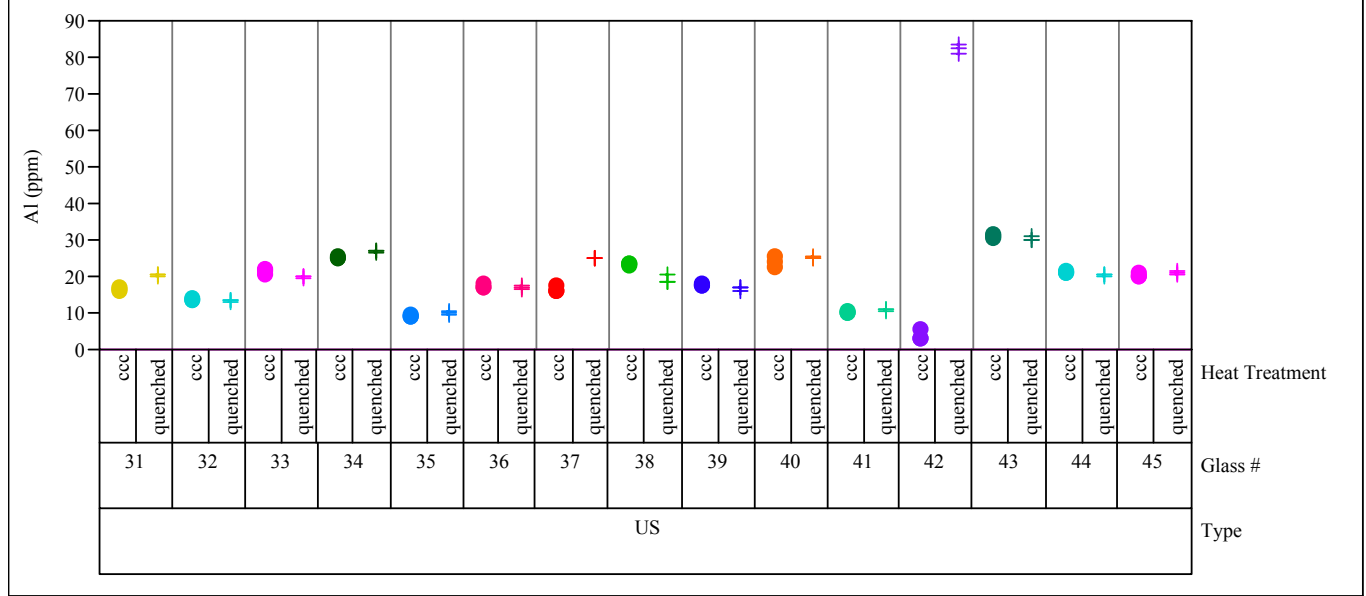

Set $=3$ Variability Chart for $\mathbf{B}(\mathrm{ppm})$

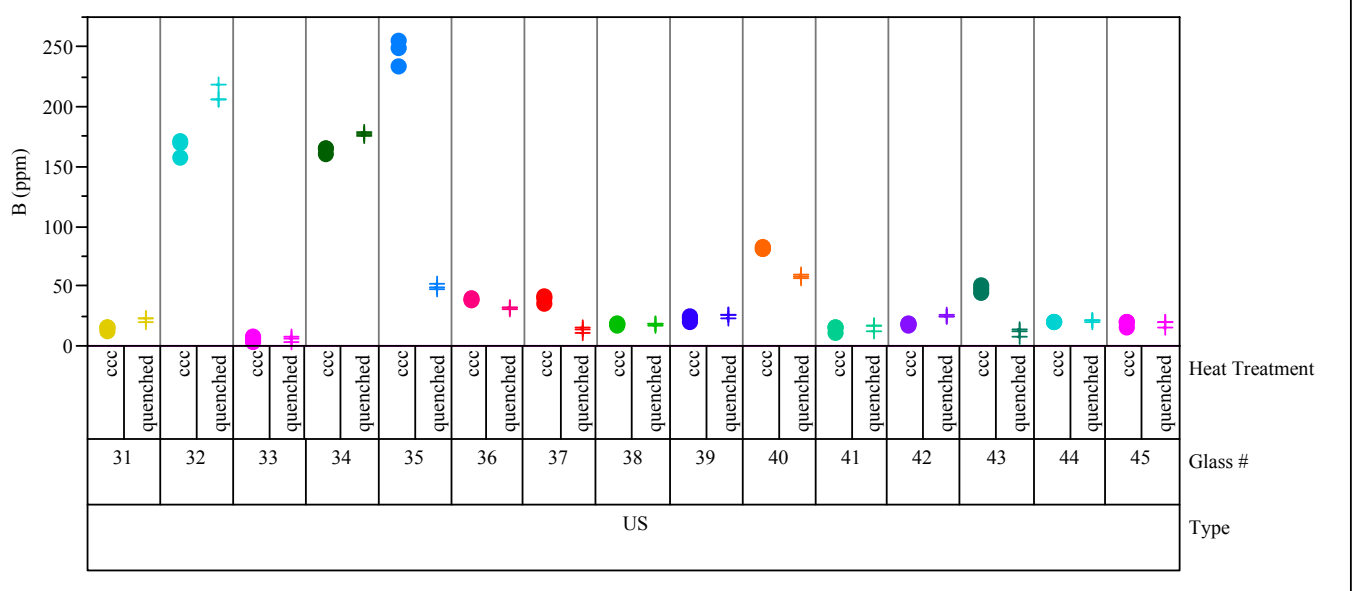

Set $=3$ Variability Chart for Fe (ppm)

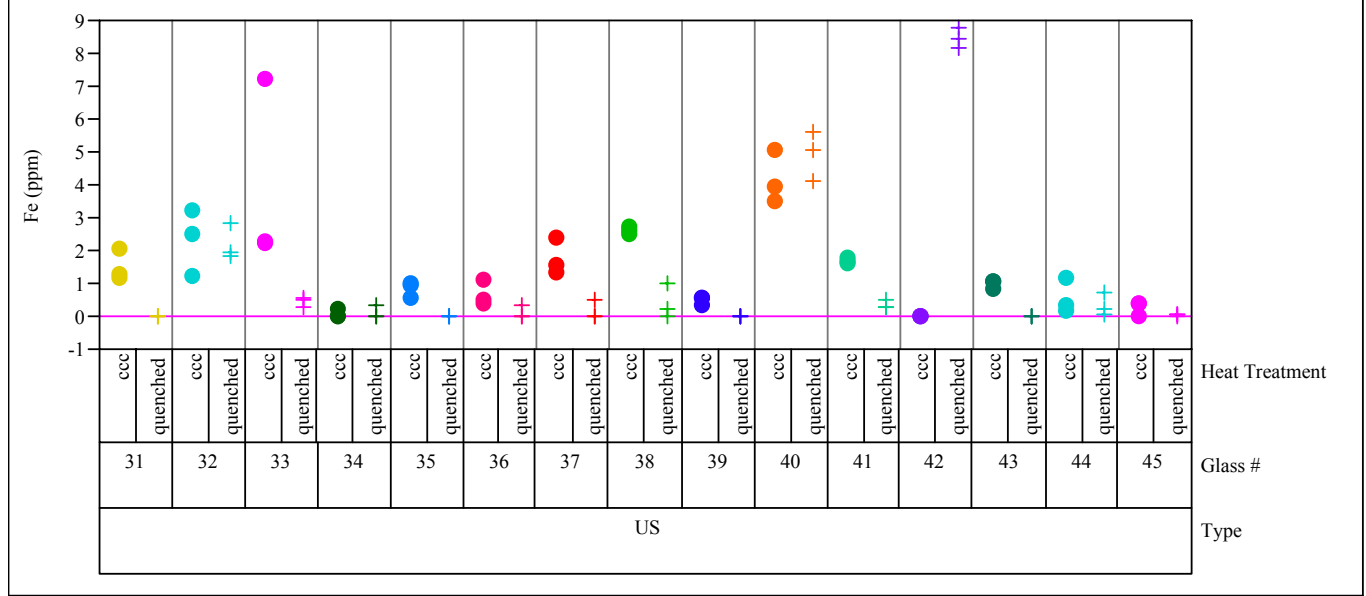


Exhibit B5. Laboratory PCT Measurements by Glass Number for Study Glasses

Set $=3$ Variability Chart for Li (ppm)

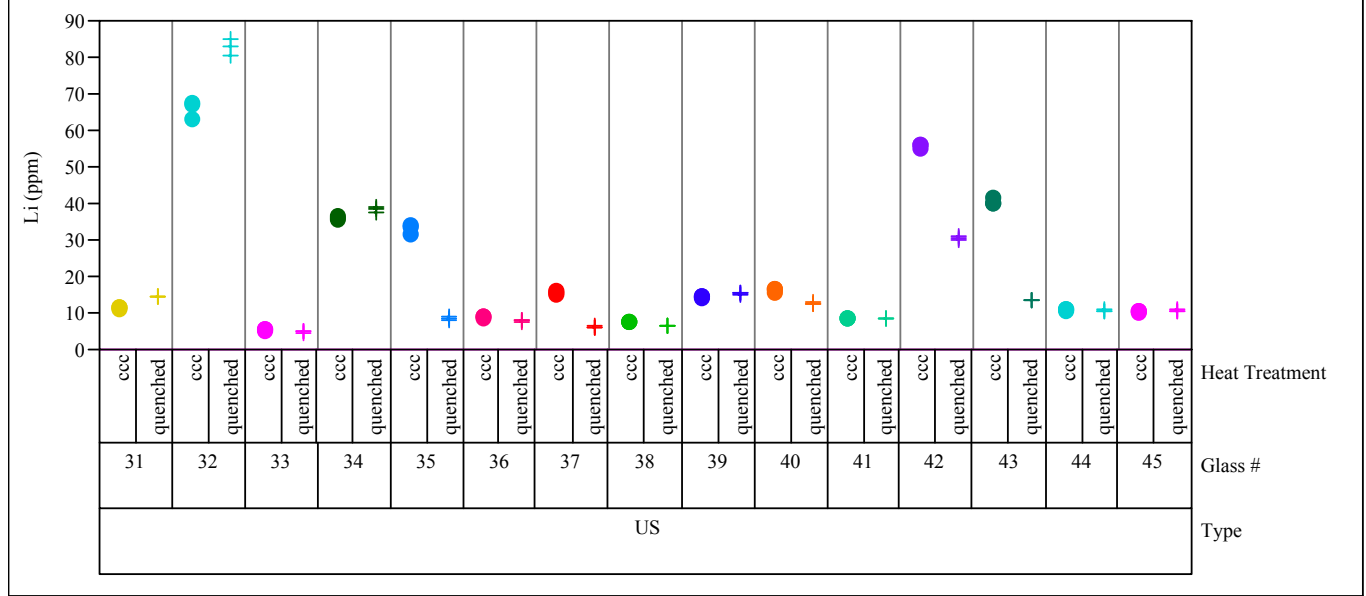

Set $=3$ Variability Chart for Na (ppm)

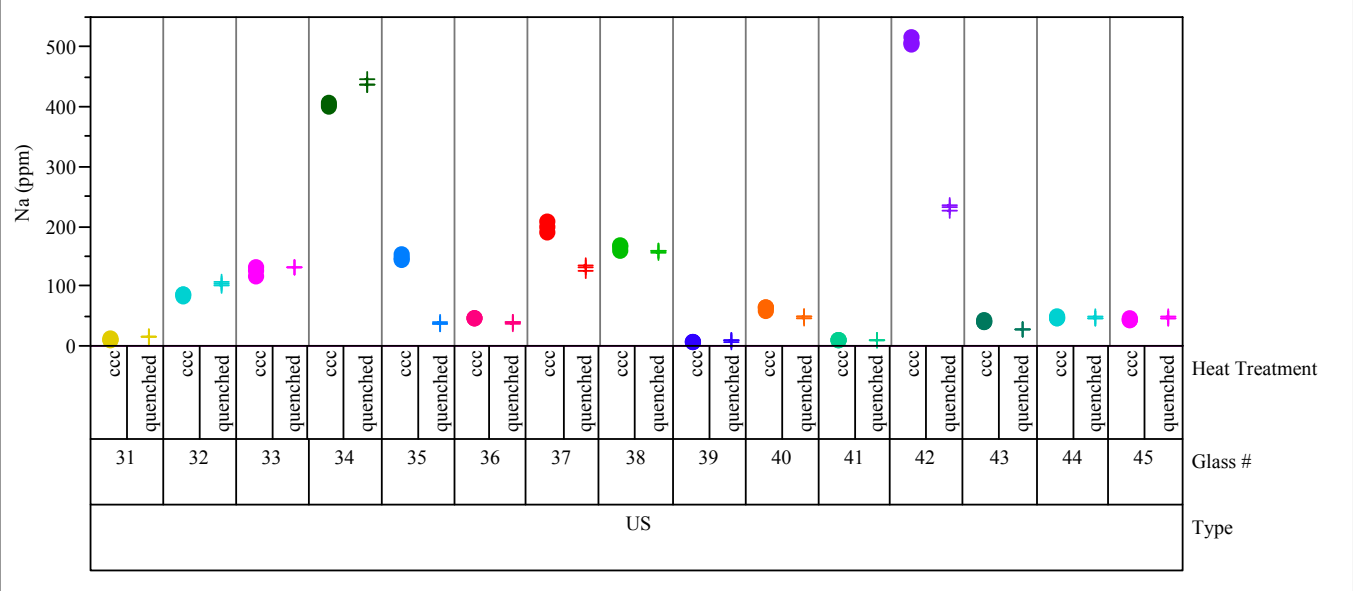

Set $=3$ Variability Chart for $\mathrm{Si}(\mathrm{ppm})$

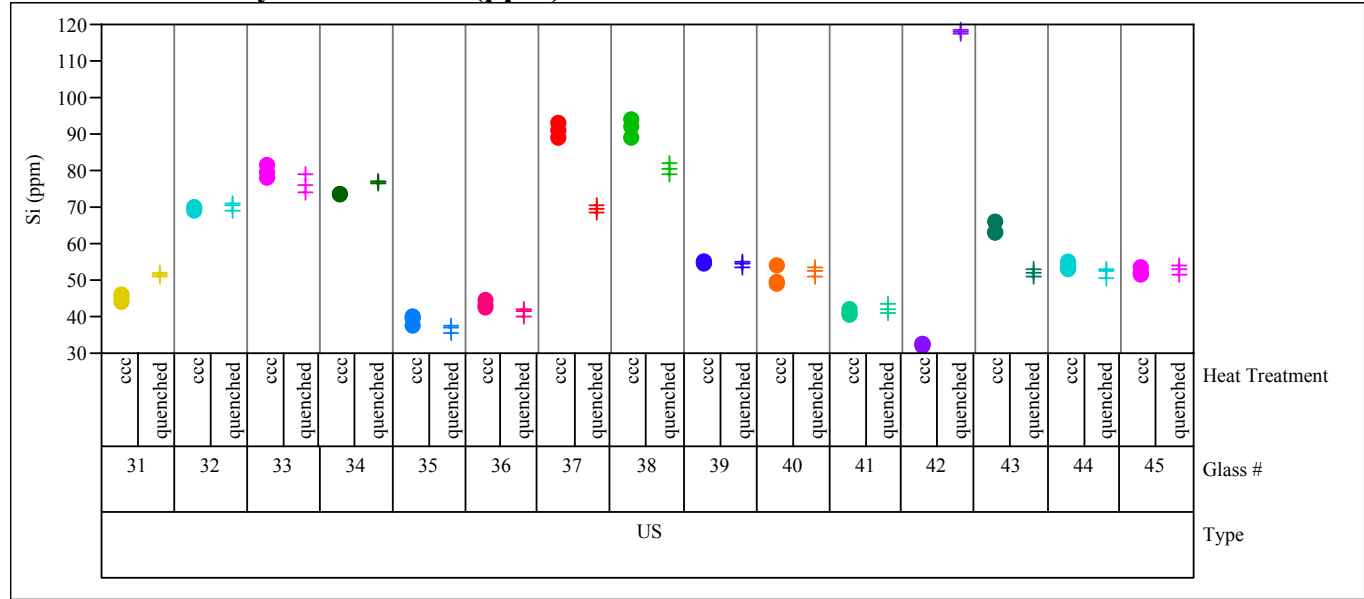


Exhibit B6. Correlations and Scatter Plots of Normalized PCTs by Set Over All Compositional Views and Heat Treatments

Multivariate Set $=1$

\section{Correlations}

$\log \mathrm{NL}[\mathrm{B}(\mathrm{g} / \mathrm{L})]$

$\log \mathrm{NL}[\mathrm{Li}(\mathrm{g} / \mathrm{L})]$

$\log \mathrm{NL}[\mathrm{Na}(\mathrm{g} / \mathrm{L})]$

$\log \mathrm{NL}[\mathrm{Si}(\mathrm{g} / \mathrm{L})]$

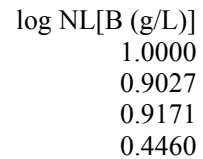

1.0000

0.9027

0.9171

0.4460
$\log \mathrm{NL}[\operatorname{Li}(\mathrm{g} / \mathrm{L})]$
0.9027
1.0000
0.7939
0.3794
$\log \mathrm{NL}[\mathrm{Na}(\mathrm{g} / \mathrm{L})]$
0.9171
0.7939
1.0000
$\log \mathrm{NL}[\mathrm{Si}(\mathrm{g} / \mathrm{L})]$
0.4460
0.3794
0.5105

Scatterplot Matrix

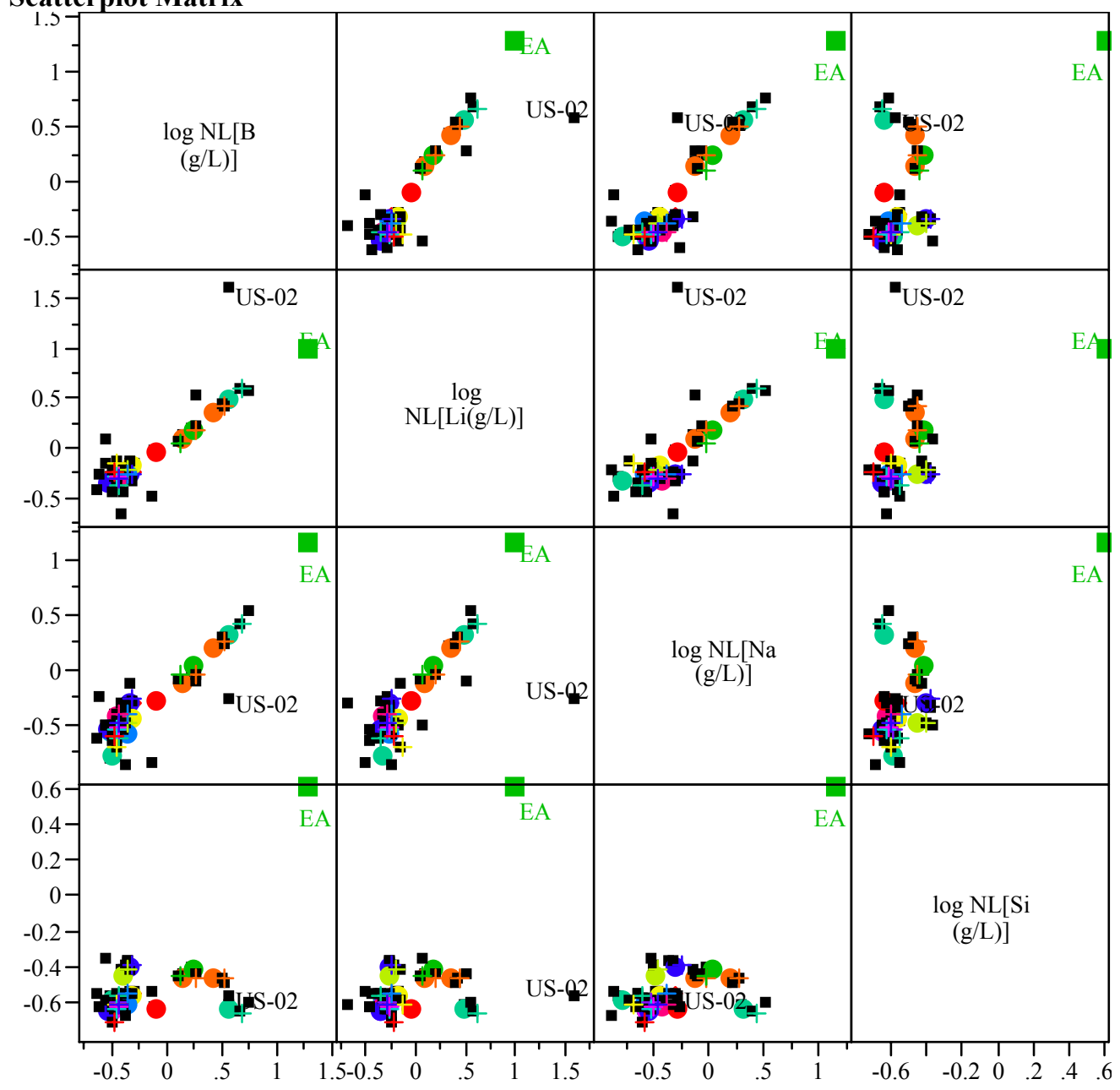


Exhibit B6. Correlations and Scatter Plots of Normalized PCTs by Set Over All Compositional Views and Heat Treatments

Multivariate Set $=2$

\section{Correlations}

$\log \mathrm{NL}[\mathrm{B}(\mathrm{g} / \mathrm{L})]$

$\log \mathrm{NL}[\mathrm{Li}(\mathrm{g} / \mathrm{L})]$

$\log \mathrm{NL}[\mathrm{Na}(\mathrm{g} / \mathrm{L})]$

$\log \mathrm{NL}[\mathrm{Si}(\mathrm{g} / \mathrm{L})]$

$$
\begin{array}{r}
\log \mathrm{NL}[\mathrm{B}(\mathrm{g} / \mathrm{L})] \\
1.0000 \\
0.9328 \\
0.8440 \\
0.7530
\end{array}
$$
$\log \mathrm{NL}[\operatorname{Li}(\mathrm{g} / \mathrm{L})]$
0.9328
1.0000
0.8049
0.7382
$\log \mathrm{NL}[\mathrm{Na}(\mathrm{g} / \mathrm{L})]$
0.8440
0.8049
1.0000
$\log \mathrm{NL}[\mathrm{Si}(\mathrm{g} / \mathrm{L})]$
0.7530
0.7382
0.8616

4 rows not used due to missing or excluded values or frequency or weight variables missing, negative or less than one.

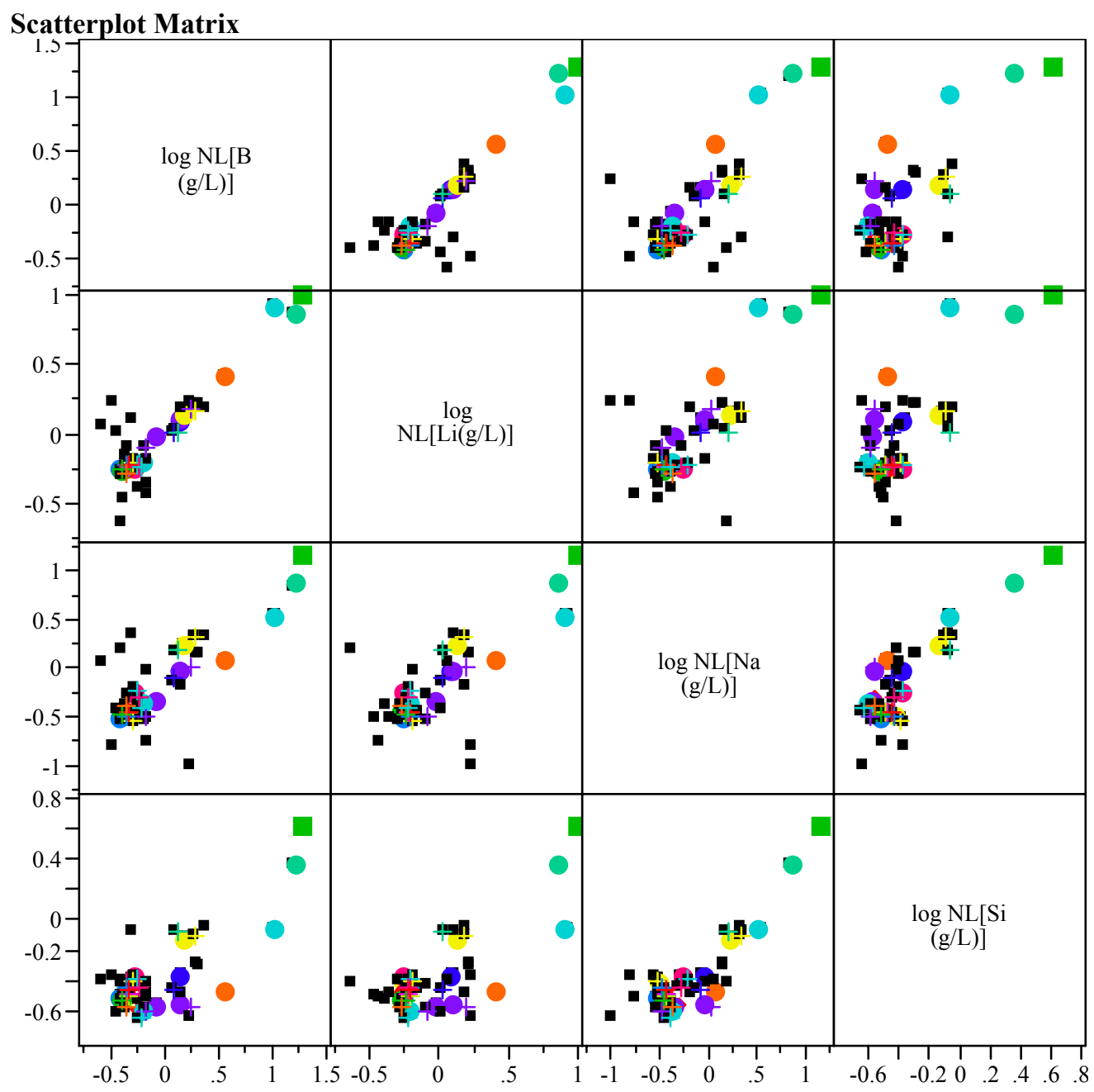


Exhibit B6. Correlations and Scatter Plots of Normalized PCTs by Set Over All Compositional Views and Heat Treatments

Multivariate Set $=3$

\section{Correlations}

$\log \mathrm{NL}[\mathrm{B}(\mathrm{g} / \mathrm{L})]$

$\log \mathrm{NL}[\mathrm{Li}(\mathrm{g} / \mathrm{L})]$

$\log \mathrm{NL}[\mathrm{Na}(\mathrm{g} / \mathrm{L})]$

$\log \mathrm{NL}[\mathrm{Si}(\mathrm{g} / \mathrm{L})]$

$$
\begin{array}{r}
\log \mathrm{NL}[\mathrm{B}(\mathrm{g} / \mathrm{L})] \\
1.0000 \\
0.8918 \\
0.6438 \\
0.3747
\end{array}
$$
$\log \mathrm{NL}[\mathrm{Li}(\mathrm{g} / \mathrm{L})]$
0.8918
1.0000
0.7083
0.3800
$\log \mathrm{NL}[\mathrm{Na}(\mathrm{g} / \mathrm{L})]$
0.6438
0.7083
1.0000
0.5243
$\log \mathrm{NL}[\mathrm{Si}(\mathrm{g} / \mathrm{L})]$
0.3747
0.3800
0.5243

4 rows not used due to missing or excluded values or frequency or weight variables missing, negative or less than one.

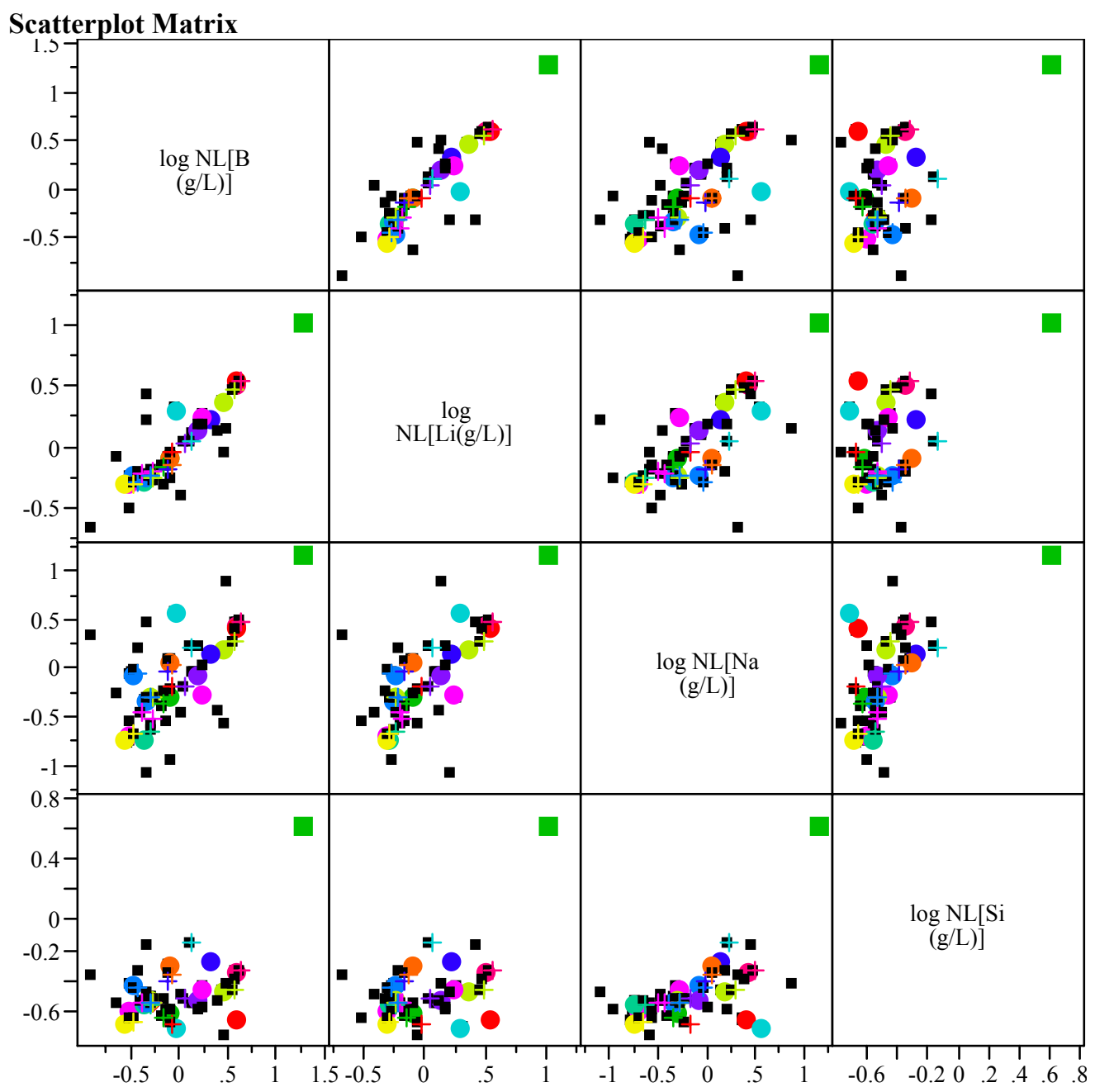




\section{Exhibit B7. Effects of Heat Treatment on PCT $\log (\mathrm{ppm})$-Response of Study Glasses}

Matched Pairs Glass \#=1

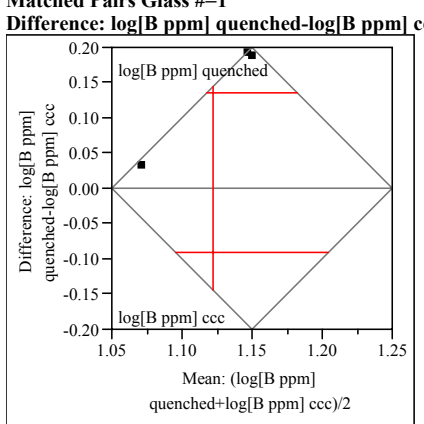

$\log [\mathrm{B}$ ppm] quenched 1.19082 t-Ratio 2.580203 $\begin{array}{lllr}\log [\mathrm{B} \text { ppm] ccc } & 1.05436 & \text { DF } & 2 \\ \text { Mean Difference } & 0.13646 & \text { Prob }>|t| & 0.1231\end{array}$

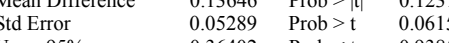
Upper95\% $\quad 0.36402 \quad$ Prob $<$ t 0.9385 Lower $95 \%$

Correlatio 3
-0.3979

Difference: $\log [\mathrm{Li}$ ppm] quenched-log[Li ppm] ccc

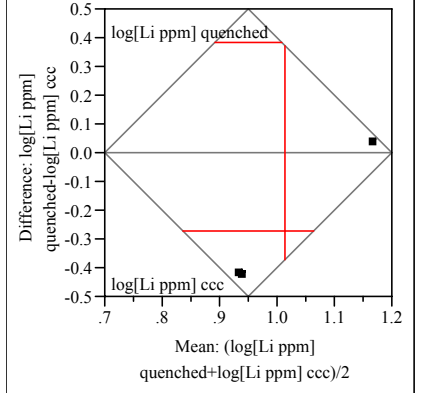

$\log [\mathrm{Li}$ ppm] quenched $0.87789 \quad$ t-Ratio $\quad-1.78678$ \begin{tabular}{llll}
$\log [\mathrm{Li} \mathrm{ppm}] \mathrm{ccc}$ & 1.15007 & $\mathrm{DF}$ & 2 \\
& -0.2722 & $\mathrm{PF}$ & -1.78678 \\
\hline
\end{tabular} $\begin{array}{llll}\text { Mean Diffrchco } & -0.2722 & \text { Prob }>|t| & 0.2159 \\ \text { Std Error } & 0.15233 & \text { Prob }>t & 0.8921\end{array}$ $\begin{array}{llll}\text { Upper95\% } & 0.38324 & \text { Prob }<\text { t } & 0.8979\end{array}$ Lower95\% $-0.9276$

Correlatio

0.30559

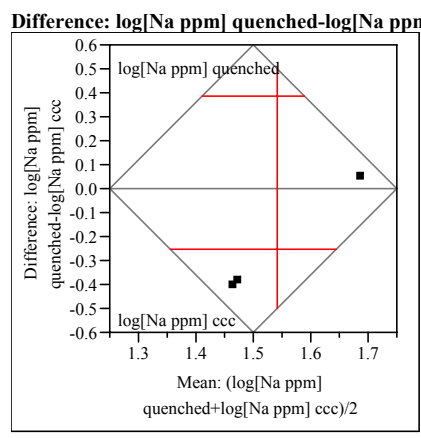

$\log [$ Na ppm] quenched $1.41568 \quad \mathrm{t}$-Ratio $\quad-1.69674$ $\begin{array}{llll}\log [\mathrm{Na} p \mathrm{ppm}] \mathrm{ccc} & 1.66694 & \mathrm{DF} & 2\end{array}$ Std Error $\begin{array}{llll}\text { Upper95\% } & 0.148589 & \text { Prob }<\mathrm{t} & 0.884 \\ & -.81159\end{array}$ Lower $95 \%$ $-0.888$

Correlation $\quad-0.957$

Difference: $\log [\mathrm{Si}$ ppm] quenched-log[Si ppm] cce

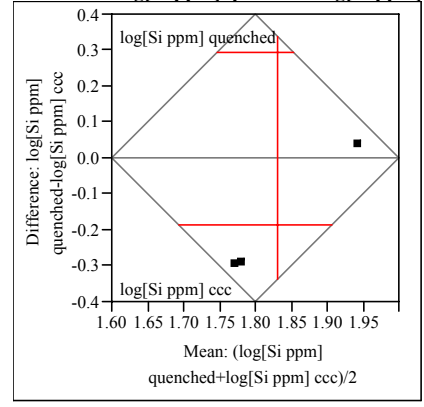

$\log [$ Si ppm] quenched $1.73828 \quad$ t-Ratio $\quad-1.67156$ $\begin{array}{llll}\log [\mathrm{Si} \mathrm{ppm}] \mathrm{ccc} & 1.9237 & \mathrm{DF} & 2\end{array}$ $\begin{array}{llll}\text { Mean Difference } & -0.1854 & \text { Prob }>|t| & 0.2366 \\ \text { Std Error } & 0.11092 & \text { Prob }>t & 0.817\end{array}$ $\begin{array}{llll}\text { Std Error } & 0.11092 & \text { Prob }>\mathrm{t} & 0.8817 \\ \text { Upper95\% } & 0.29185 & \text { Prob }<\mathrm{t} & 0.1183\end{array}$ Upper $95 \%$ $-0.6627$ $\begin{array}{lr}\mathrm{N} & 3 \\ \text { Correlation } & -0.0304\end{array}$
Matched Pairs Glass $\#=2$ Differen

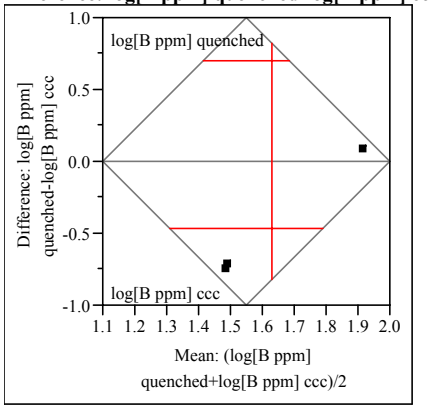

$\log [\mathrm{B}$ ppm] quenched $\quad 1.39388 \quad \mathrm{t}$-Ratio $\quad-1.72444$ $\begin{array}{llll}\log [\mathrm{B} \text { ppm }] \mathrm{ccc} & 1.86421 & \mathrm{DF} & \\ & & 0.27274 & \mathrm{DP}\end{array}$ $\begin{array}{llll} & -0.4703 & \text { Prob }>|t| & 0.2268 \\ \text { Mean Difference } & 0.27274 & \text { Pob }>t & 0.896\end{array}$ $\begin{array}{llll}\text { Std Error } & 0.27274 & \text { Prob }>t & 0.8866 \\ \text { Upper95\% } & 0.70319 & \text { Prob }<\mathrm{t} & 0.113\end{array}$ (1) $\begin{array}{rll}0.70319 & \text { Prob }<\mathrm{t} \quad 0.1134 \\ -1.6438 & & \end{array}$

Correlation 3

Difference: $\log [\mathbf{L i}$ ppm] quenched-log $[\mathbf{L i}$ ppm] ccc

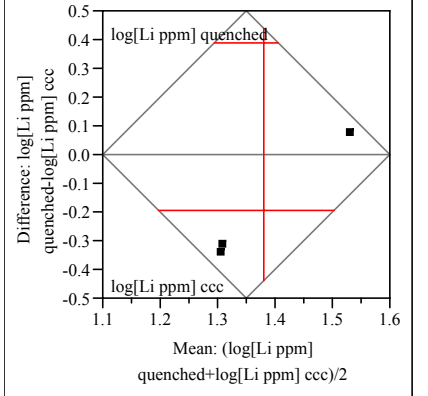

$\log [\mathrm{Li}$ ppm] quenched $\quad 1.284 \quad \mathrm{t}$-Ratio $\quad-1.44458$ $\begin{array}{lrlr}\log [\mathrm{Li} \text { ppm] } \mathrm{ccc} & 1.47938 & \mathrm{DF} & 2 \\ \text { Mean Difference } & -0.1954 & \text { Prob }>|t| & 0.2854\end{array}$ $\begin{array}{llll}\text { Mean Difference } & -0.1954 & \text { Prob }>|t| & 0.2854 \\ \text { Std Error } & 0.13525 & \text { Prob }>t & 0.8573\end{array}$ $\begin{array}{llll}\text { Upper } 95 \% & 0.38654 & \text { Prob }<\mathrm{t} & 0.1427\end{array}$ Lower95\% -0.7773 Prob $<1 \quad 0.14$ Correlation

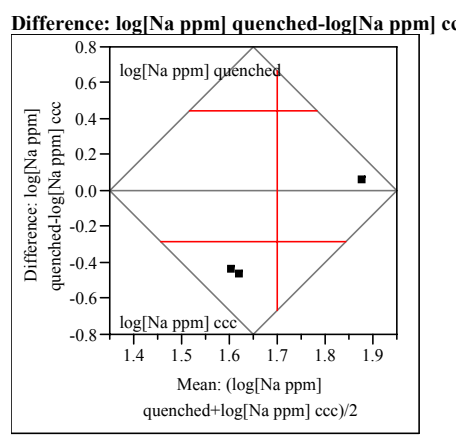

$\log [\mathrm{Na} p \mathrm{pm}]$ quenched $1.55682 \quad \mathrm{t}$-Ratio $\quad-1.68182$ $\begin{array}{llll}\log [\mathrm{Nappm}] \mathrm{ccc} & 1.84319 & \mathrm{DF} & 2\end{array}$ $\begin{array}{llll}\text { Upper95\% } & 0.44626 & \text { Prob }<\mathrm{t} & 0.1173\end{array}$ $-1.019$

$\mathrm{N}$

Difference: $\log [\mathrm{Si}$ ppm] quenched-log[Si ppm] cce

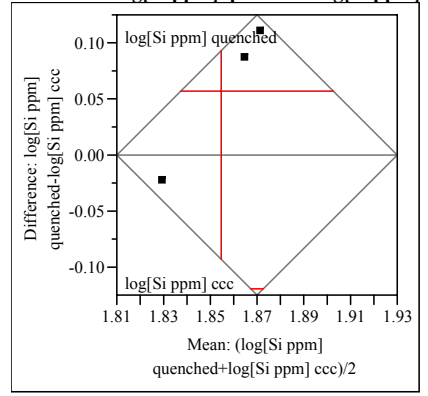

$\log [$ Si ppm] quenched 1.88365 t-Ratio 1.39674

$\begin{array}{llll}\log [\mathrm{Si} \text { ppm }] \text { ccc } & 1.82631 & \mathrm{DF} & 2 \\ \text { Mean Difference } & 0.05734 & \text { Prob }>|t| & 0.2973\end{array}$ $\begin{array}{llll}\text { Std Error } & 0.04105 & \text { Prob } & \\ \text { Upper95\% } & 0.23397 & \text { Prob }<t & 0.1486\end{array}$ Lower95\% $\quad-0.1193$

Correlation $\quad-0.998$ 


\section{Exhibit B7. Effects of Heat Treatment on PCT $\log (\mathrm{ppm})$-Response of Study Glasses}

Matched Pairs Glass \#=3

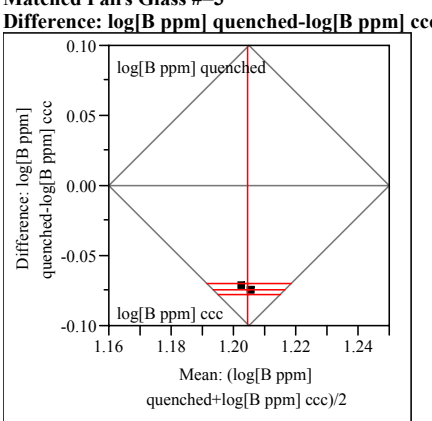

$\log [\mathrm{B}$ ppm] quenched 1.16733 t-Ratio $\quad-83.2763$ $\begin{array}{lllr}\log [\mathrm{B} \text { ppm] ccc } & 1.24166 & \text { DF } & 2 \\ \text { Mean Difference } & -0.0743 & \text { Prob }>|t| & 0.0001\end{array}$ $\begin{array}{llll}\text { Mean Difference } & -0.0743 & \text { Prob }>|t| & 0.0001 \\ \text { Std Error } & 0.00089 & \text { Prob }>t & 0.9999\end{array}$ $\begin{array}{llll}\text { Std Error } & 0.00089 & \text { Prob }>\mathrm{t} & 0.9999 \\ \text { Upper95\% } & -0.0705 & \text { Prob }<\mathrm{t} & <.0001 \\ \text { Lower95\% } & -0.0782 & & \end{array}$ $-0.0782$

Correlation

Difference: $\log [$ Li ppm] quenched-log[Li ppm] cce

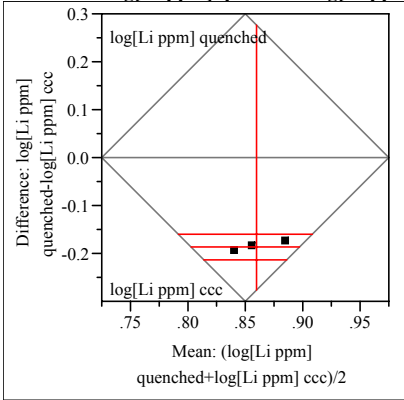

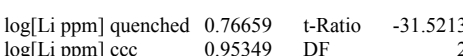
$\begin{array}{llll}\log [\mathrm{Li} p \mathrm{ppm}] \mathrm{ccc} & 0.95349 & \mathrm{DF} & \\ \text { Mean Difference } & -0.1869 & \text { Prob }>|\mathrm{t}| & 0.0010 \\ & 0.0593 & \text { Pro } & 0.995\end{array}$ $\begin{array}{llll}\text { Std Error } & -0.1869 & \text { Prob }>|t| & 0.0010 \\ \text { Stence } & 0.00593 & \text { Prob }>t & 0.9995 \\ \text { U } & -0.124 & & \end{array}$ $\begin{array}{llll}\text { Upper95\% } & -0.1614 & \text { Prob }<\mathrm{t} & 0.0005 \\ \text { Lower95\% } & -0.2124 & & \end{array}$ $\mathrm{N}$ $-0.2124$ 0.99772

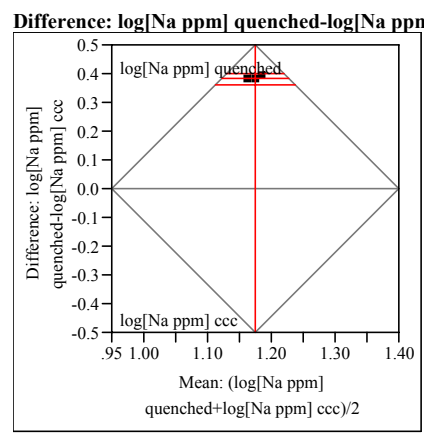

$\log [\mathrm{Na}$ ppm] quenched $1.36576 \quad \mathrm{t}$-Ratio $\quad 87.87286$ $\begin{array}{llll}\log [\mathrm{Na} p \mathrm{ppm}] \mathrm{ccc} & 0.98398 & \mathrm{DF} & 87.87286\end{array}$ \begin{tabular}{llll}
0.38178 & Prob $>|t|$ & 0.000 \\
\hline & 0.003 &
\end{tabular} $\begin{array}{llll}\text { Std Error } & 0.00434 & \text { Prob }>t & <.000 \\ \text { Upper95\% } & 0.40047 & \text { Prob }<\mathrm{t} & 0.9999\end{array}$ Uppergs\% 0.36308

$\mathrm{N}$ 0.90427

Difference: $\log [\mathrm{Si}$ ppm] quenched-log[Si ppm] cce

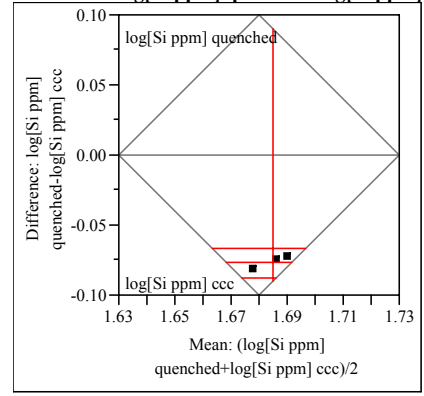

$\log [$ Si ppm] quenched $1.64614 \quad \mathrm{t}$-Ratio $\quad-31.5579$

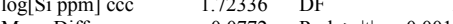

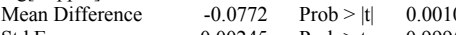
Upper95\% $\quad-0.0667 \quad$ Pob $<1 \quad 0.0005$ $\begin{array}{llll}\text { Upper95\% } & -0.0667 & \text { Prob }<\mathrm{t} & 0.0005 \\ \text { Lower95\% } & -0.0877 & & \end{array}$

Correlation $\quad 0.99925$
Matched Pairs Glass \#=4 Differed

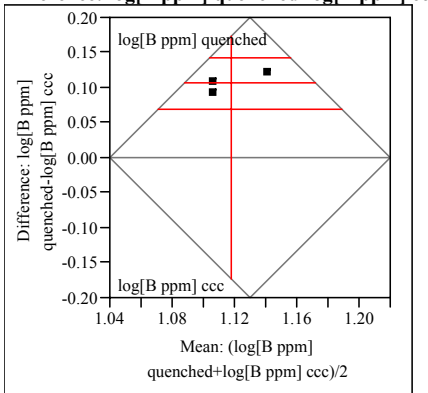

$\log [\mathrm{B} \mathrm{ppm}]$ quenched $\quad 1.17053 \quad \mathrm{t}$-Ratio $\quad 12.44794$ $\begin{array}{lrlr}\log [\mathrm{B} \text { ppm] } \mathrm{ccc} & 1.06472 & \mathrm{DF} & 2 \\ \mathrm{Mean} \text { Difference } & 0.10582 & \text { Prob }>\text { |t } & 0.0064\end{array}$ $\begin{array}{lrll}\text { Mean Difference } & 0.10582 & \text { Prob }>|t| & 0.0064 \\ \text { Std Error } & 0.0085 & \text { Prob }>t & 0.0032\end{array}$ $\begin{array}{lrll}\text { Std Error } & 0.0085 & \text { Prob }>\mathrm{t} & 0.0032 \\ \text { Upper95\% } & 0.14239 & \text { Prob }<\mathrm{t} & 0.9968\end{array}$ Lower95\%

Correlation .90581 Difference: $\log [\mathrm{Li}$ ppm] quenched-log[Li ppm] cce

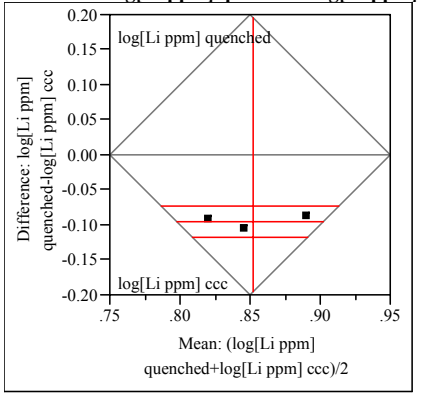

$\log [\mathrm{Li} p p m]$ quenched $\quad 0.8036 \quad$ t-Ratio $\quad-18.889$ $\begin{array}{llll}\log [\mathrm{Li} \text { ppm }] \text { ccc } & 0.89978 & \mathrm{DF} & 2\end{array}$ $\begin{array}{llll}\text { Mean Difference } & -0.0962 & \text { Prob }>|t| & 0.0028 \\ \text { Std Error } & 0.00509 & \text { Prob }> & 0.9986\end{array}$ $\begin{array}{llll}\text { Std Error } & 0.00509 & \text { Prob }>t & 0.9986 \\ \text { Upper95\% } & -0.0743 & \text { Prob }<\mathrm{t} & 0.001\end{array}$ Lower95\% $\begin{array}{ccc}-0.0743 & \text { Prob }<\mathrm{t} & 0.0014 \\ -0.1181 & & \end{array}$ Correlation

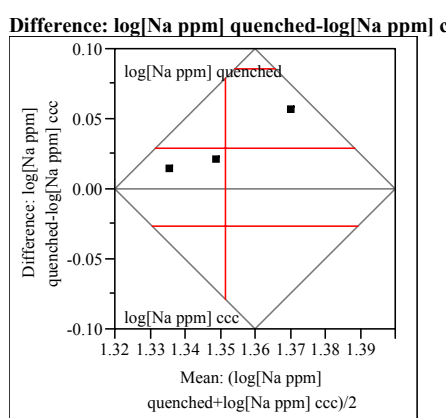

$\log [\mathrm{Na}$ ppm] quenched $1.36632 \quad$ t-Ratio 2.238247

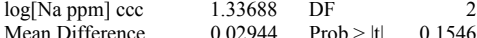
$\begin{array}{llll}\text { Mean Difference } & 0.02944 & \text { Prob }>|t| & 0.1546 \\ \text { Std Error } & 0.01315 & \text { Prob }>t & 0.0773\end{array}$ $\begin{array}{llll}\text { Std Error } & 0.01315 & \text { Prob }>t & 0.0773 \\ \text { Upper95\% } & 0.08604 & \text { Prob }<\mathrm{t} & 0.9227\end{array}$ Upper $95 \%$ 0.08604 -0.027
3
0.86729 Difference: $\log [\mathrm{Si}$ ppm] quenched-log[Si ppm] cce

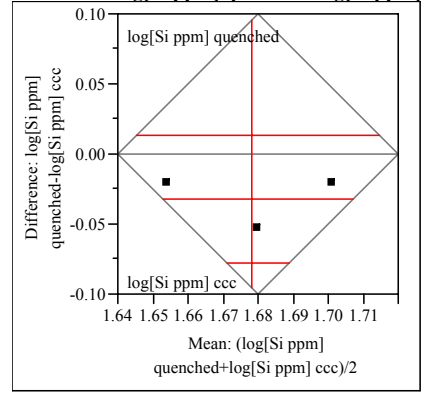

$\log [$ Si ppm] quenched $\quad 1.6623 \quad \mathrm{t}$-Ratio $\quad-3.01029$ $\begin{array}{llll}\log [\mathrm{Si} \text { ppm }] \mathrm{ccc} & 1.6941 & \text { DF } & -3.01029 \\ & -0.0318 & \text { PF } & \end{array}$ $\begin{array}{llll}\text { Mean Difference } & -0.0318 & \text { Prob }>|t| & 0.0949 \\ \text { Std } & 0.0057 & \text { Prob }>t & 0.945\end{array}$ $\begin{array}{llll}\text { Std Error } & 0.01057 & \text { Prob }>t & 0.9525 \\ \text { Upper95\% } & 0.01366 & \text { Prob }<\mathrm{t} & 0.0475\end{array}$ Loweres $9 \%$ $\mathrm{N}$ Correlation 


\section{Exhibit B7. Effects of Heat Treatment on PCT $\log (\mathrm{ppm})$-Response of Study Glasses}

Matched Pairs Glass \#=5 Difference: $\log [\mathbf{B}$ ppm] quenched-log $[B$ ppm] cce

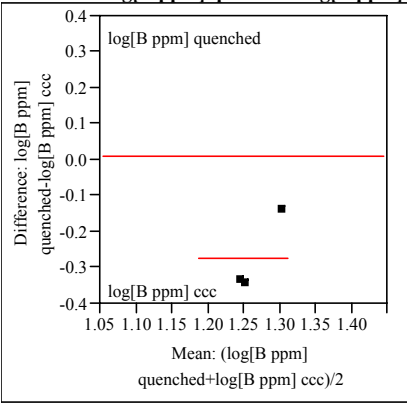

$\log [\mathrm{B}$ ppm] quenched $\quad 1.1288 \quad$ t-Ratio $\quad-4.16666$

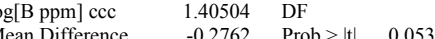
$\begin{array}{llll} & 1.0 .2762 & \text { Prob }>|t| & 0.053 \mid \\ \text { Std Error } & 0.0663 & \text { Prob }>t & 0.9735\end{array}$ $\begin{array}{llll}\text { Upper95\% } & 0.00602 & \text { Prob }>t & 0.9735 \\ & 0.00615 & & \end{array}$

$\mathrm{N}$ Correlation $-0.9736$

Difference: $\log [$ Li ppm] quenched-log $[\mathrm{Li}$ ppm] $\mathrm{ccc}$

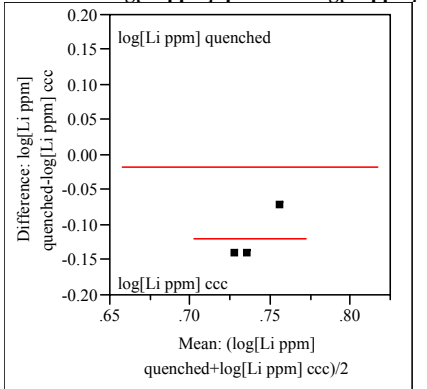

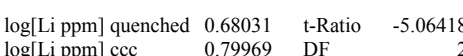

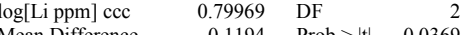
$\begin{array}{llll}\text { Std Error } & -0.1194 & \text { Prob }>|t| & 0.0369 \\ \text { Unt } & 0.02357 & \text { Prob }>t & 0.9816\end{array}$ $\begin{array}{llll}\text { Upper95\% } & -0.018 & \text { Prob }<\text { t } \quad 0.0184\end{array}$ Lower95\% $\mathrm{N}$ $-0.2208$ $-0.7794$

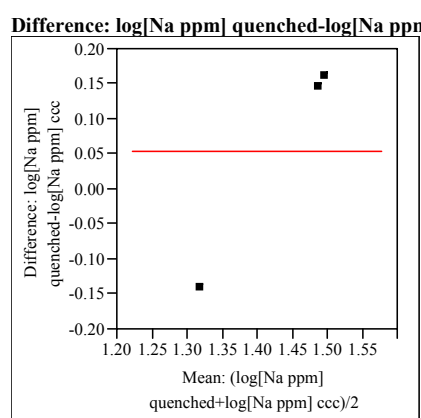

$\log [\mathrm{Na}$ ppm] quenched $1.46051 \quad \mathrm{t}$-Ratio $\quad 0.55147$

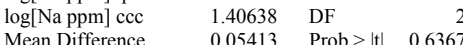
$\begin{array}{llll}\text { Mean Difference } & 0.05413 & \text { Prob }>|t| & 0.6367 \\ \text { Std Error } & 0.09815 & \text { Prob }>t & 0.3183\end{array}$ $\begin{array}{llll}\text { Std Error } & 0.09815 & \text { Prob }>\text { t } & 0.3183 \\ \text { Upper95\% } & 0.47643 & \text { Prob }<\text { t } & 0.6817\end{array}$ Upper95\% $\begin{array}{rrr}0.47643 & \text { Prob }<\mathrm{t} & 0.6817 \\ -0.3682 & & \end{array}$

$\begin{array}{ll}\text { Correlation } & 0.99931 \\ \text { Difference: } \log [\mathbf{S i} \text { ppm] quenched-log [Si ppm] ccc }\end{array}$

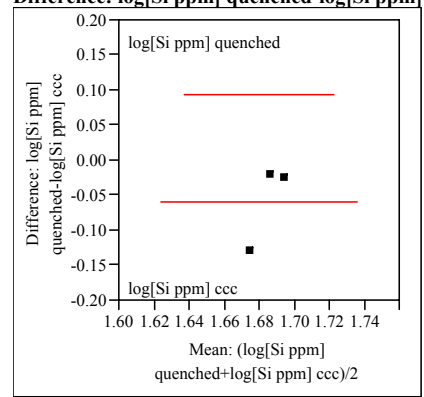

$\log [$ Si ppm] quenched $1.65522 \quad$ t-Ratio $\quad-1.68297$ $\begin{array}{lllll}\log [\mathrm{Si} \text { ppm] } \mathrm{ccc} & 1.71516 & \mathrm{DF} & 2\end{array}$ $\begin{array}{lllll} & \text { Std Error } & 0.03561 & \text { Prob }>\text { t } & 0.2382\end{array}$ $\begin{array}{lllll}\text { Upper95\% } & 0.09329 & \text { Prob }<\mathrm{t} & 0.1172\end{array}$ $-0.2132$ $-0.9546$
Matched Pairs Glass \#=6 Difference $\log [\mathbf{B}$ ppm] quenched-log $[$ B ppm] cec

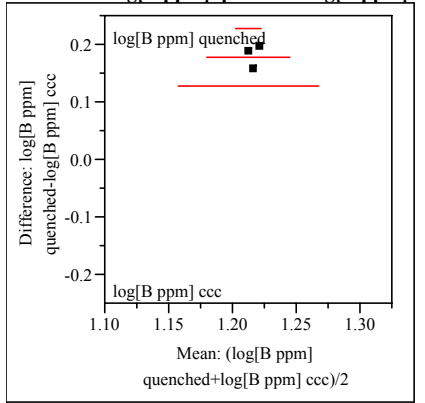

$\log [\mathrm{B} \mathrm{ppm}]$ quenched $1.30572 \quad \mathrm{t}$-Ratio $\quad 15.26768$ $\begin{array}{lllr}\log [\mathrm{B} \text { ppm }] \text { quenched } & 1.30572 & \text { t-Ratio } & 15.26768 \\ \log [\mathrm{B} \mathrm{ppm}] \mathrm{ccc} & 1.12704 & \text { DF } & 2 \\ & 0.17867 & & \end{array}$ $\begin{array}{lrll}\text { Mean Difference } & 0.17867 & \text { Prob }>|t| & 0.0043 \\ \text { Std } & 0.0217 & \text { Prob }>t & 0.0021\end{array}$ $\begin{array}{lrll}\text { Std Error } & 0.0117 & \text { Prob }>\mathrm{t} & 0.0021 \\ \text { Upper95\% } & 0.22903 & \text { Prob }<\mathrm{t} & 0.9979\end{array}$ Lpwer95\%

$\mathrm{N}$ 3
-0.6758

Difference: $\log [$ Li ppm] quenched-log $[\mathrm{Li}$ ppm $] \mathrm{ccc}$

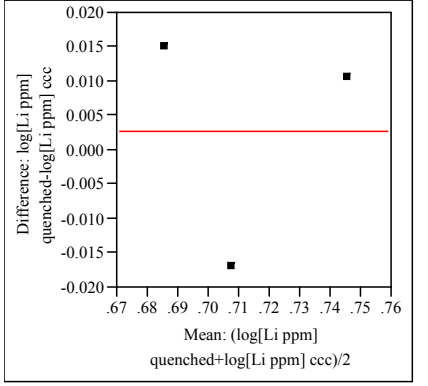

$\log [\mathrm{Li} p p m]$ quenched $\quad 0.7143 \quad$ t-Ratio $\quad 0.276921$ $\begin{array}{lrlr}\log [\mathrm{Li} \text { ppm] ccc } & 0.71154 & \text { DF } & 2 \\ \text { Mean Difference } & 0.00277 & \text { Prob }>|t| & 0.8078\end{array}$ $\begin{array}{llll}\text { Std Error } & 0.00998 & \text { Prob }>t & 0.4039 \\ \text { Upper95\% } & 0.04573 & \text { Prob }<\mathrm{t} & 0.5961\end{array}$ Upper95\% Lower95\%

Correlation

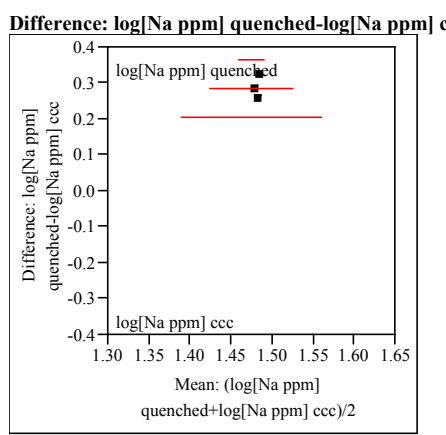

$\log [\mathrm{Na}$ ppm] quenched $1.62417 \quad$ t-Ratio $\quad 15.32363$ $\begin{array}{llll}\log [\mathrm{Na} \text { ppm] } \mathrm{ccc} & 1.34006 & \mathrm{DF} & \end{array}$ $\begin{array}{lll}0.28411 & \text { Prob }>|t| & 0.0042\end{array}$ $\begin{array}{llll}\text { Upper95\% } & 0.01854 & \text { Prob }>t & 0.0021 \\ & 0.36388 & \text { Prob }<\mathrm{t} & 0.9979\end{array}$ Lower95\%

Difference: $\log [\mathrm{Si}$ ppm] quenched-log[Si ppm] ccc

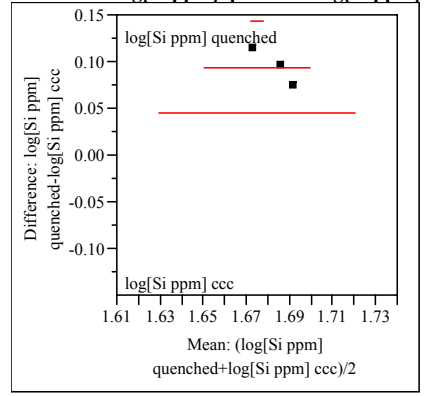

$\log [\mathrm{Si} \mathrm{ppm}]$ quenched $1.73061 \quad$ t-Ratio $\quad 8.162041$ $\begin{array}{llll}\log [\mathrm{Si} \text { ppm] ccc } & 1.63654 & \mathrm{DF} & 2 \\ \text { Mean Difference } & 0.09406 & \text { Prob }>|\mathrm{t}| & 0.0147\end{array}$ $\begin{array}{llll}\text { Upper } 95 \% & 0.14365 & \text { Prob }<\mathrm{t} & 0.9927\end{array}$ Lower95\% $\quad 0.0444$

Correlation $\quad-0.1556$ 


\section{Exhibit B7. Effects of Heat Treatment on PCT $\log (\mathrm{ppm})$-Response of Study Glasses}

Matched Pairs Glass \#=7

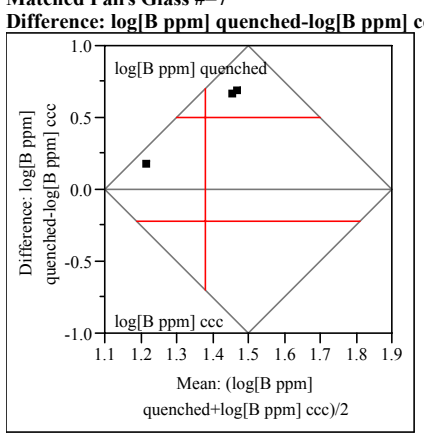

$\log [\mathrm{B}$ ppm] quenched $\quad 1.62964 \quad \mathrm{t}$-Ratio $\quad 2.998126$ $\begin{array}{llll}\log [\mathrm{B} \mathrm{ppm}] \mathrm{ccc} & 1.1298 & \mathrm{DF} & 2\end{array}$ $\begin{array}{llll}\text { Mean Difference } & 0.49984 & \text { Prob }>|t| & 0.0956\end{array}$ $\begin{array}{llll}\text { Std Error } & 0.16672 & \text { Prob }>t & 0.0478 \\ \text { Upperg5\% } & 1.21718 & \text { Prob }<\mathrm{t} & 0.9522 \\ \text { Lower95\% } & -0.2175 & & \end{array}$ Correlation 3
-2939

Difference: $\log [$ Li ppm] quenched-log $[$ Li ppm $]$ ccc

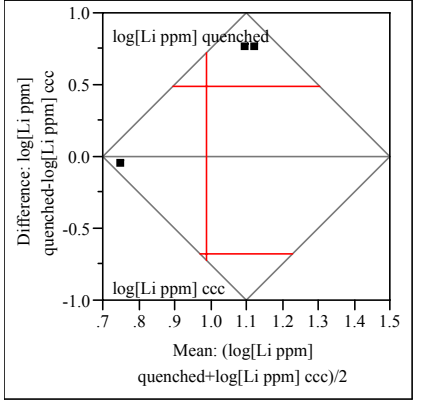

$\log [\mathrm{Li}$ ppm] quenched $1.23107 \quad \mathrm{t}$-Ratio 1.786155 $\begin{array}{lll}0.74659 & \mathrm{DF} & 2\end{array}$ $\begin{array}{llll}\text { Mean Difference } & 0.48447 & \text { Prob }>|t| & 0.2160 \\ \text { Std Error } & 0.27124 & \text { Prob }>t & 0.1080\end{array}$ $\begin{array}{llll}\text { Std Error } & 0.27124 & \text { Prob }>\mathrm{t} & 0.1080 \\ \text { Upper95\% } & 1.65152 & \text { Prob }<\mathrm{t} & 0.8920\end{array}$ Upper95\% $\mathrm{N}$ $\begin{array}{ll}1.65152 & \text { Prob }<\mathrm{t} \quad 0.8920 \\ -0.6826 & \end{array}$ Correlation

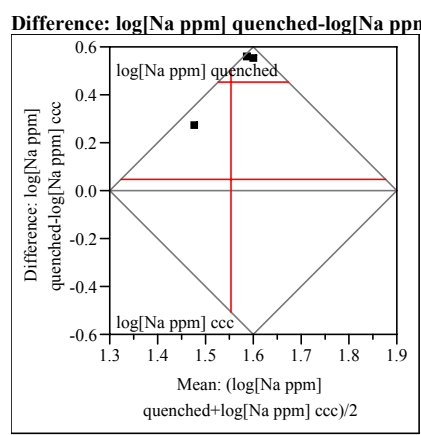

$\log [\mathrm{Na}$ ppm] quenched $1.78223 \quad \mathrm{t}$-Ratio 4.759205

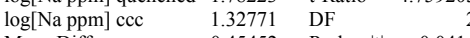
$\begin{array}{llll}\text { Mean Difference } & 0.45452 & \text { Prob }>|t| & 0.0414\end{array}$ $\begin{array}{llll}\text { Std Error } & 0.0955 & \text { Prob }>t & 0.0207 \\ \text { Upper95\% } & 0.0655 & \text { Prob }<\text { t } & 0.9793\end{array}$ $\begin{array}{lll}0.86545 & \text { Prob }<\mathrm{t} & 0.9793\end{array}$

Corelation 0.0436

Difference: $\log [\mathrm{Si}$ ppm] quenched-log[Si ppm] cce

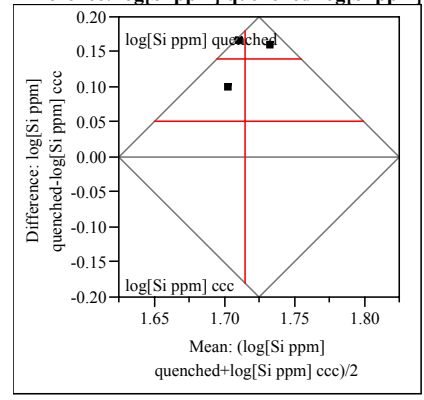

$\log [$ Si ppm] quenched $1.78581 \quad$ t-Ratio 6.67614 $\log [\mathrm{Si} \mathrm{ppm}] \mathrm{ccc} \quad 1.64495$ DF $\quad 0.676$

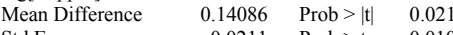
$\begin{array}{lrll}\text { Std Error } & 0.0211 & \text { Prob }>t & 0.0109 \\ \text { Upper95\% } & 0.23165 & \text { Prob }<\text { t } & 0.9891\end{array}$ Lower95\% 0.05008

Correlation 3
-0.2194
Matched Pairs Glass \#=8 Differes $\log [\mathrm{B} \mathrm{ppm}]$ quenched-log $[\mathrm{B}$ ppm] ccc

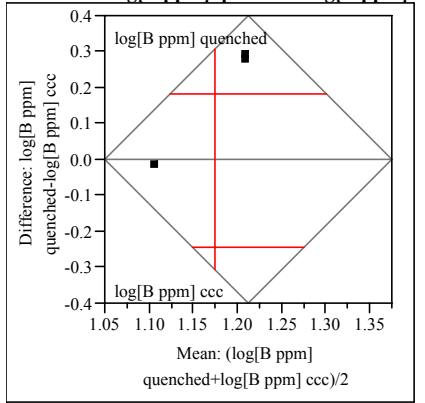

$\log [\mathrm{B}$ ppm] quenched $1.26552 \quad \mathrm{t}$-Ratio $\quad 1.838664$

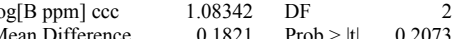

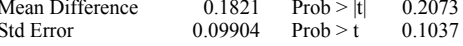
$\begin{array}{llll}\text { Upper95\% } & 0.60822 & \text { Prob }<\mathrm{t} & 0.8963\end{array}$ $-0.2$

$\mathrm{N}$ $\begin{array}{r}3 \\ -9.9967 \\ \hline\end{array}$

Difference: $\log [$ Li ppm] quenched-log $[$ Li ppm] cce

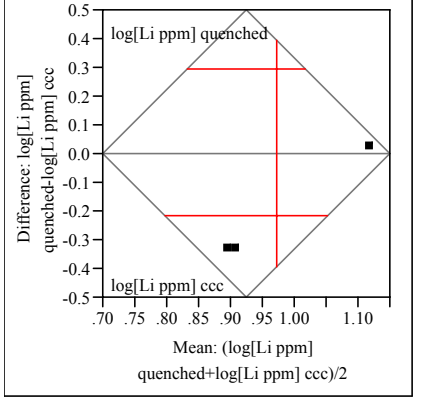

$\log [$ Li ppm] quenched $0.86475 \quad$ t-Ratio $\quad-1.83197$

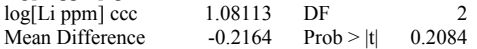
$\begin{array}{llll}\text { Mean Difference } & -0.2164 & \text { Prob }>|t| & 0.2084 \\ \text { Std Error } & 0.11811 & \text { Prob }>t & 0.8958\end{array}$ $\begin{array}{llll}\text { Std Error } & 0.11811 & \text { Prob }>t & 0.8958 \\ \text { Upper95\% } & 0.29182 & \text { Prob }<\mathrm{t} & 0.1042\end{array}$ Lower $95 \%$ $\mathrm{N}$ Correlation 0.96764

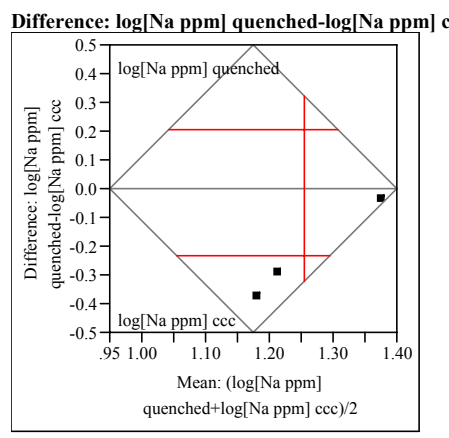

$\log [\mathrm{Na} p \mathrm{pm}]$ quenched $1.13679 \quad \mathrm{t}$-Ratio $\quad-2.3022$ $\begin{array}{llll}\log [\mathrm{Na} p \mathrm{ppm}] \mathrm{ccc} & 1.37289 & \mathrm{DF} & 2\end{array}$ $\begin{array}{llll}\text { Upper95\% } & 0.20516 & \text { Prob }<\mathrm{t} & 0.0740\end{array}$ Lower95\%

Correlation $\quad 0.89235$

Difference: $\log [\mathrm{Si}$ ppm] quenched-log[Si ppm] cce

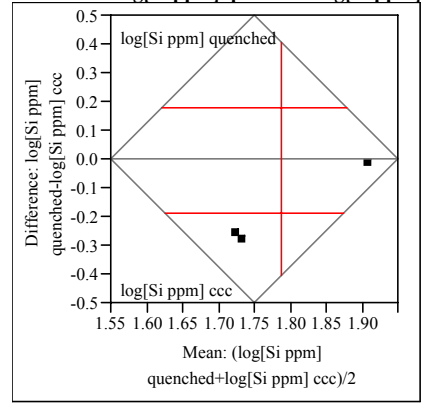

$\log [\mathrm{Si} \mathrm{ppm}]$ quenched $1.69418 \quad \mathrm{t}$-Ratio $\quad-2.2044$ $\begin{array}{llll}\log [\mathrm{Si} \mathrm{ppm}] \mathrm{ccc} & 1.88195 & \text { DF } & 2 \\ & 0.0878 & \text { PF } & 0.1583\end{array}$ $\begin{array}{llll}\text { Mean Difference } & -0.1878 & \text { Prob }>|t| & 0.1583 \\ \text { Std Error } & 0.08518 & \text { Prob }>t & 0.920\end{array}$ $\begin{array}{llll}\text { Std Error } & 0.08518 & \text { Prob }>t & 0.9208 \\ \text { Upper95\% } & 0.17872 & \text { Prob }<\mathrm{t} & 0.0792\end{array}$ Correlation $\quad 0.94295$ 


\section{Exhibit B7. Effects of Heat Treatment on PCT $\log (\mathrm{ppm})$-Response of Study Glasses}

Matched Pairs Glass \#=9

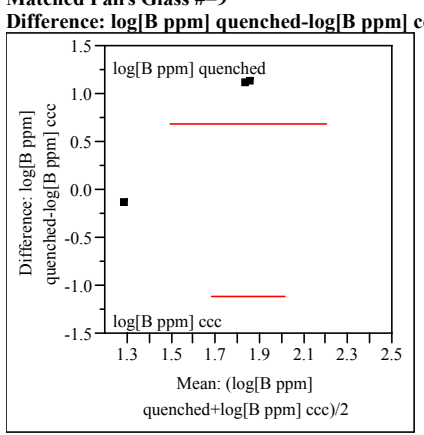

$\log [\mathrm{B}$ ppm] quenched $2.00342 \quad \mathrm{t}$-Ratio $\quad 1.648221$ $\begin{array}{lllll}\log [\mathrm{B} \mathrm{ppm}] \mathrm{ccc} & 1.31511 & \mathrm{DF} & 2\end{array}$ $\begin{array}{llll} & & & \\ \text { Std Error } & 0.68831 & \text { Prob }>|t| & 0.2411 \\ \text { Un } & 0.41761 & \text { Prob }>t & 0.1205\end{array}$$$
\begin{array}{r}
-1.1085 \\
3
\end{array}
$$

$\mathrm{N}$ Correlation $-0.9698$

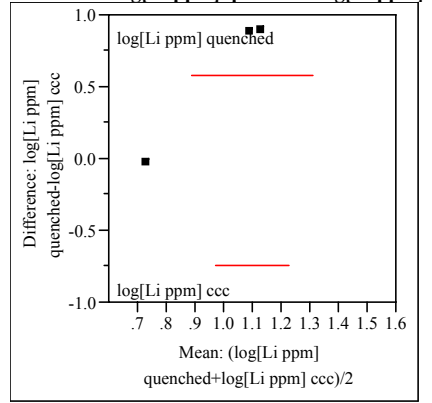

$\log [$ Li ppm] quenched $1.27116 \quad \mathrm{t}$-Ratio 1.885421 $\begin{array}{lrlr}\text { log[Li ppm] ccc } & 0.69372 & \text { DF } & 2 \\ \text { Mean Difference } & 0.57744 & \text { Prob }>|t| & 0.2000\end{array}$ $\begin{array}{llll}\text { Std Error } & 0.30627 & \text { Prob }>\mathrm{t} & 0.1000 \\ \text { Upper95\% } & 1.89519 & \text { Prob }<\mathrm{t} & 0.9000\end{array}$ $\mathrm{N}$ -0.7403
3
-0.9226 $\begin{array}{llll}\text { Upper95\% } & 2.48514 & \text { Prob }<\mathrm{t} & 0.8795\end{array}$

Difference: $\log [$ Li ppm] quenched-log $[$ Li ppm] cec $\begin{array}{llll}\text { Mean Difference } & 0.57744 & \text { Prob }>|t| & 0.2000 \\ \text { Std Error } & 0.30627 & \text { Prob }>t & 0.1000\end{array}$

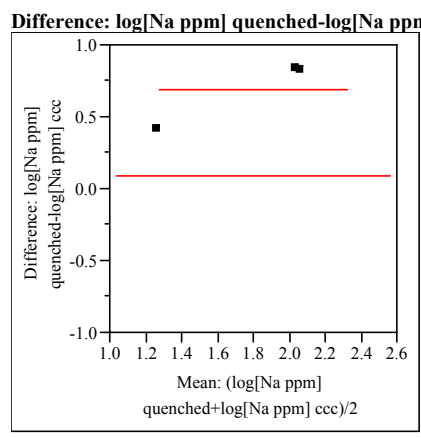

$\log [\mathrm{Na}$ ppm] quenched $2.12894 \quad \mathrm{t}$-Ratio $\quad 4.922628$ $\begin{array}{llll}\log [\mathrm{Na} p \mathrm{ppm}] \mathrm{ccc} & 1.43768 & \mathrm{DF} & \\ \mathrm{M} & 0.14042 & \mathrm{P} & \end{array}$ $\begin{array}{lll}0.69126 & \text { Prob }>|t| & 0.038 \\ 0.14042 & \text { P }>\text { c } & 0.094\end{array}$ $\begin{array}{llll}\text { Upper95\% } & 0.14042 & \text { Prob }>\mathrm{t} & 0.0194 \\ & 1.29546 & \text { Prob }<\mathrm{t} & 0.9806\end{array}$ 0.08706

$\mathrm{N}$

Difference: $\log [\mathrm{Si}$ ppm] quenched-log[Si ppm] cce

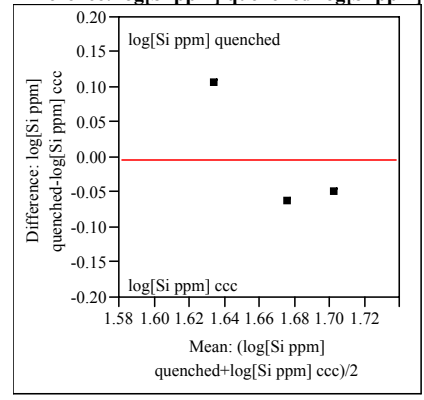

$\log [$ Si ppm] quenched 1.66913 t-Ratio $\quad-0.07077$ $\log [\mathrm{Si} \mathrm{ppm}] \mathrm{ccc} \quad 1.67302 \quad \mathrm{DF} \quad-0.07077$ $\begin{array}{lll}1.67302 & \text { DF } & 0.0500\end{array}$

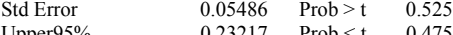
Lower95\% $-0.2399$

Correlation

3
-0.5927
Matched Pairs Glass \#=10 Difference: $\log [\mathbf{B}$ ppm] quenched-log $[\mathbf{B}$ ppm] cec

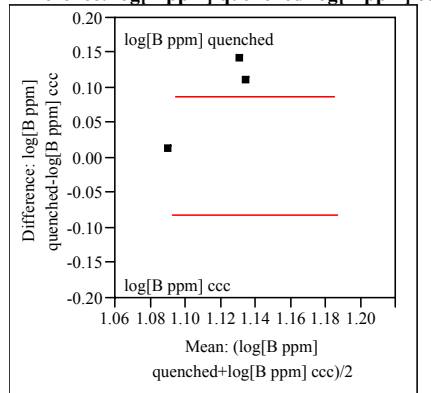

$\begin{array}{llll}\log [\mathrm{B} \text { ppm] quenched } & 1.16219 & \text { t-Ratio } & 2.217548 \\ \log [\mathrm{B} p \text { pm }] \text { ccc } & 1.07564 & \mathrm{DF} & 2\end{array}$ $\begin{array}{lllr}\log [\mathrm{B} \text { ppm] ccc } & 1.07564 & \mathrm{DF} & 2 \\ \text { Mean Difference } & 0.08655 & \text { Prob }>|t| & 0.1569\end{array}$ $\begin{array}{llll}\text { Mean Difference } & 0.08655 & \text { Prob }>|t| & 0.1569 \\ \text { Std Error } & 0.03903 & \text { Prob }>t & 0.0784\end{array}$ $\begin{array}{llll}\text { Std Error } & 0.03903 & \text { Prob }>\mathrm{t} & 0.0784 \\ \text { Upper } 95 \% & 0.25449 & \text { Prob }<\mathrm{t} & 0.9216\end{array}$ (1)

$\mathrm{N}$ $-0.0814$

Correlation $-0.73$ Difference: $\log [\mathrm{Li}$ ppm] quenched-log[Li ppm] cce

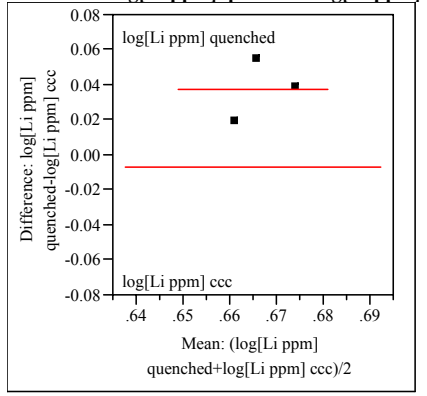

$\log [$ Li ppm] quenched $\quad 0.68562 \quad \mathrm{t}$-Ratio $\quad 3.616171$ $\begin{array}{llll}\log [\mathrm{Li} \text { ppm] ccc } & 0.64831 & \mathrm{DF} & 2 \\ \text { Mean Differec } & 0.03731 & \mathrm{PF} & 0.0687\end{array}$ $\begin{array}{llll} & 0.03731 & \text { Prob }>|t| & 0.0687 \\ \text { Std Error } & 0.01032 & \text { Prob }>t & 0.0343\end{array}$ $\begin{array}{lrll}\text { Std Error } & 0.01032 & \text { Prob }>\text { t } & 0.0343 \\ \text { Upper95\% } & 0.0817 & \text { Prob }<\text { t } & 0.9657 \\ \text { Lower95\% } & -0.0071 & & \end{array}$ Correlation

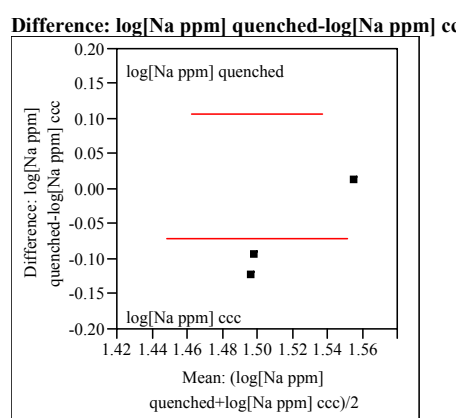

$\log [\mathrm{Na}$ ppm] quenched $1.48137 \quad$ t-Ratio $\quad-1.7148$ $\begin{array}{llll}\log [\mathrm{Na} p \mathrm{pm}] \mathrm{ccc} & 1.55156 & \mathrm{DF} & 2 \\ \text { Men Difference } & 0.0702 & \text { Prob }>|\mathrm{t}| & 0.2285\end{array}$ $\begin{array}{llll}\text { Mean Difference } & -0.0702 & \text { Prob }>|\uparrow| & 0.2285 \\ \text { Std Error } & 0.04094 & \text { Prob }>t & 0.8857\end{array}$ $\begin{array}{llll}\text { Upper95\% } & 0.10594 & \text { Prob }<\mathrm{t} & 0.1143 \\ & -0.2463 & & \end{array}$ $-0.2463$ Correlation $\quad-0.296$ Difference: $\log [\mathrm{Si}$ ppm] quenched-log[Si ppm] ccc

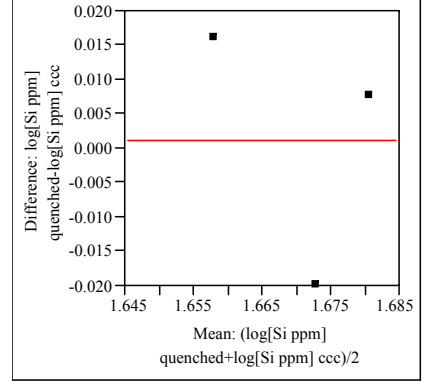

$\log [$ Si ppm] quenched $1.67097 \quad$ t-Ratio $\quad 0.106787$ $\begin{array}{lrlr}\log [\mathrm{Si} \text { ppm] ccc } & 1.66982 & \text { DF } & 2 \\ \text { Mean Difference } & 0.00116 & \text { Prob }>|t| & 0.9247\end{array}$ $\begin{array}{llll}\text { Mean Difference } & 0.00116 & \text { Prob }>|t| & 0.9247 \\ \text { Std Error } & 0.01085 & \text { Prob }>t & 0.4624\end{array}$ $\begin{array}{llll}\text { Std Error } & 0.01085 & \text { Prob }>\mathrm{t} & 0.4624 \\ \text { Upper95\% } & 0.04782 & \text { Prob }<\mathrm{t} & 0.5376 \\ & & & \end{array}$ Lower95\% Correlation $\quad 0.3$ 


\section{Exhibit B7. Effects of Heat Treatment on PCT $\log (\mathrm{ppm})$-Response of Study Glasses}

Matched Pairs Glass \#=11

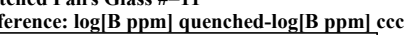

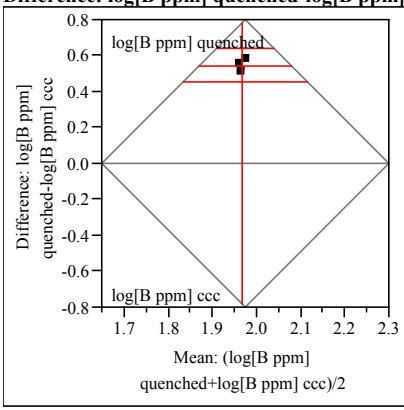

$\log [\mathrm{B}$ ppm] quenched $2.23973 \quad \mathrm{t}$-Ratio $\quad 25.68979$ $\begin{array}{llll}\log [\mathrm{B} \mathrm{ppm}] \mathrm{ccc} & 1.69444 & \mathrm{DF} & 2\end{array}$ $\begin{array}{llll}\text { Mean Difference } & 0.54529 & \text { Prob }>|t| & 0.0015 \\ 0 & 0.02123 & \text { Prob }>\text { t } & 0.008\end{array}$ $\begin{array}{llll}\text { Std Error } & 0.02123 & \text { Prob }>\mathrm{t} & 0.0008 \\ \text { Upper95\% } & 0.63662 & \text { Prob }<\mathrm{t} & 0.9992\end{array}$ (1) 0.45396

Correlation $-0.8498$

Difference: $\log [$ Li ppm] quenched-log $[\mathbf{L i}$ ppm] ccc

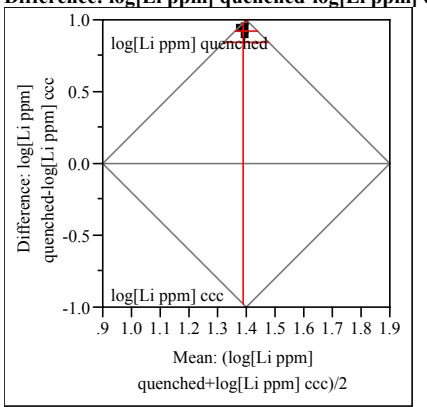

$\log [$ Li ppm] quenched $1.84903 \quad$ t-Ratio $\quad 55.34711$ $\begin{array}{lrlr}\log [\mathrm{Li} \text { ppm] } \mathrm{ccc} & 0.92863 & \mathrm{DF} & 2 \\ \text { Mean Difference } & 0.9204 & \text { Prob }>\text { t } & 0.0003\end{array}$ $\begin{array}{lrll}\text { Mean Difference } & 0.9204 & \text { Prob }>|t| & 0.0003 \\ \text { Std Error } & 0.01663 & \text { Prob }>t & 0.0002\end{array}$ $\begin{array}{llll}\text { Std Error } & 0.01663 & \text { Prob }>\text { t } & 0.0002 \\ \text { Upper95\% } & 0.99195 & \text { Prob }<\mathrm{t} & 0.9998\end{array}$ $\mathrm{N}$ $\begin{array}{lll}0.99195 & \text { Prob }<\mathrm{t} \quad 0.9998 \\ 0.84885 & & \end{array}$ Correlation

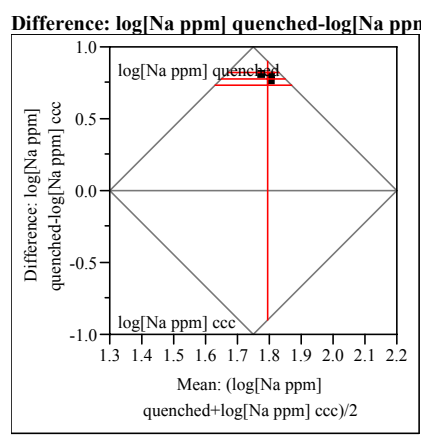

$\log [\mathrm{Na}$ ppm] quenched $2.18428 \quad \mathrm{t}$-Ratio $\quad 71.8358$ $\begin{array}{lllr}\text { log[Na ppm] ccc } & 1.40421 & \text { DF } & 2 \\ \text { Mean Difference } & 0.78007 & \text { Prob }>|t| & 0.0002\end{array}$ $\begin{array}{lllll}\text { Std Error } & 0.01086 & \text { Prob }>t & <.0001 \\ \text { Upper95\% } & 0.82679 & \text { Prob }<\text { t } & 0.9999\end{array}$ Lower $95 \%$

$\mathrm{N}$ Correlation 3.6409

Difference: $\log [\mathrm{Si}$ ppm] quenched-log[Si ppm] ccc

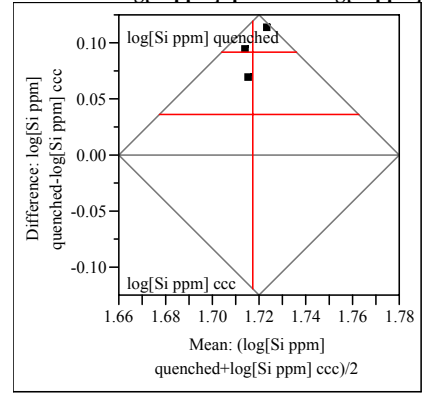

$\log [\mathrm{Si}$ ppm] quenched 1.76326 t-Ratio 7.20635 $\begin{array}{lrll}\log [\mathrm{Si} p \mathrm{ppm}] \mathrm{ccc} & 1.67206 & \mathrm{DF} & 2 \\ \text { Mean Difference } & 0.0912 & \text { Prob }>|t| & 0.0187\end{array}$ $\begin{array}{lrll}\text { Mean Difference } & 0.0912 & \text { Prob }>|t| & 0.0187 \\ \text { Std Error } & 0.01266 & \text { Prob }>t & 0.0094\end{array}$ $\begin{array}{llll}\text { Std Error } & 0.01266 & \text { Prob }>\text { t } & 0.0094 \\ \text { Upper95\% } & 0.14566 & \text { Prob }<\text { t } & 0.9906 \\ \text { Lower95\% } & 0.03675 & & \end{array}$ 0.03675

Correlation atched Pairs Glass \#=12 政

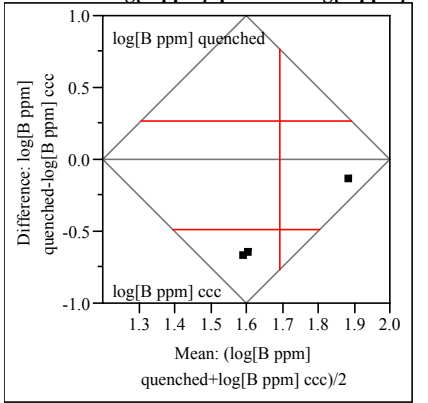

$\log [\mathrm{B}$ ppm] quenched $1.44973 \quad \mathrm{t}$-Ratio $\quad-2.8037$

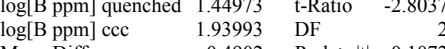
$\begin{array}{llll}\text { Mean Difference } & -0.4902 & \text { Prob }>|t| & 0.1072\end{array}$ $\begin{array}{llll}\text { Std Error } & 0.17484 & \text { Prob }>t & 0.9464\end{array}$ $\begin{array}{llll}\text { Upper95\% } & 0.26208 & \text { Prob }<\mathrm{t} & 0.0536 \\ \text { Lower95\% } & -1.2425 & & \end{array}$

$\mathrm{N}$ 3
0.99638

Difference: $\log [\mathbf{L i ~ p p m ]}$ quenched-log $[\mathrm{Li}$ ppm] ccc

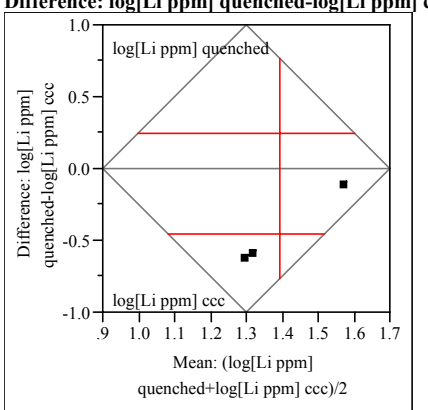

$\log [\mathrm{Li}$ ppm] quenched $1.16929 \quad \mathrm{t}$-Ratio $\quad-2.77204$ $\begin{array}{lrlr}\log [\mathrm{Li} \text { ppm] } \mathrm{ccc} & 1.61968 & \mathrm{DF} & 2 \\ \text { Mean Difference } & -0.4504 & \text { Prob }>|t| & 0.1092\end{array}$ $\begin{array}{llll}\text { Mean Difference } & -0.4504 & \text { Prob }>|t| & 0.1092 \\ \text { Std Error } & 0.16248 & \text { Prob }>t & 0.9454\end{array}$ $\begin{array}{llll}\text { Std Error } & 0.16248 & \text { Prob }>t & 0.9454 \\ \text { Upper95\% } & 0.24869 & \text { Prob }<\mathrm{t} & 0.0546\end{array}$ Lower $95 \%$ $\mathrm{N}$
Correlation -1.1495 Prob $<1 \quad 0.0546$

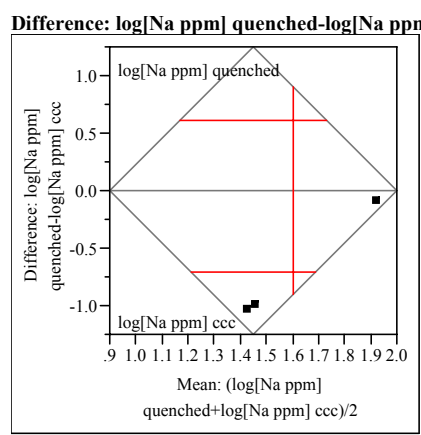

$\log [\mathrm{Na} p \mathrm{pm}]$ quenched $1.24508 \quad \mathrm{t}$-Ratio $\quad-2.3166$ $\begin{array}{llll}\log [\mathrm{Nappm}] \mathrm{ccc} & 1.95737 & \mathrm{DF} & 2\end{array}$ Std Error $\begin{array}{lllll}\text { Upper95\% } & 0.61064 & \text { Prob }<\text { t } & 0.0732\end{array}$ Lower95\%

Correlation 0.99996

Difference: $\log [\mathrm{Si}$ ppm] quenched-log $[\mathrm{Si}$ ppm] ccc

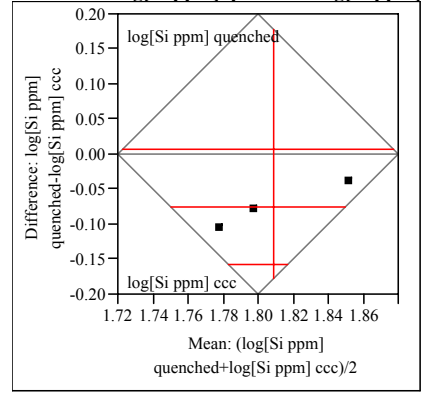

$\log [$ Si ppm] quenched $1.77096 \quad$ t-Ratio $\quad-3.906$ $\log [\mathrm{Si}$ ppm] $\mathrm{ccc} \quad 1.84646$ $\begin{array}{lll}1.84675 & \text { Prob }>|t| & 0.0597\end{array}$ $\begin{array}{llll}\text { Std Error } & 0.01933 & \text { Prob }>t & 0.9701 \\ \text { Upper } 95 \% & 0.00767 & \text { Prob }<\mathrm{t} & 0.0299\end{array}$ Lower95\% $\quad-0.1587$ Correlation $\quad 0.99125$ 


\section{Exhibit B7. Effects of Heat Treatment on PCT $\log (\mathrm{ppm})$-Response of Study Glasses}

Matched Pairs Glass \#=13

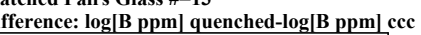

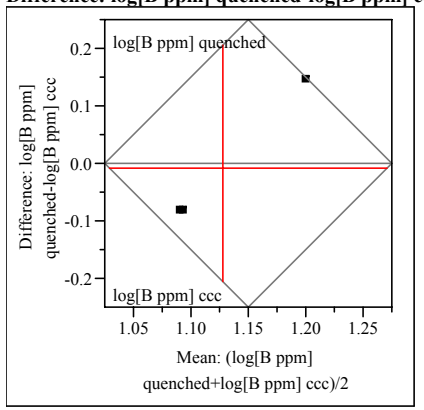

$\log [\mathrm{B}$ ppm] quenched $1.12361 \quad \mathrm{t}$-Ratio $\quad-0.10088$ $\begin{array}{llll}\log [\mathrm{B} \mathrm{ppm}] \mathrm{ccc} & 1.13122 & \mathrm{DF} & \\ & -0.0752 & & \end{array}$ $\begin{array}{llll}\text { Mean Difference } & -0.0076 & \text { Prob }>|t| & 0.9288 \\ & 0.07552 & \text { Prob }>t & 0.5356\end{array}$ $\begin{array}{llll}\text { Std Error } & 0.07552 & \text { Prob }>t & 0.5356 \\ \text { Upper95\% } & 0.31733 & \text { Prob }<\mathrm{t} & 0.4644\end{array}$ (1)

$\mathrm{N}$ 3
-0.8176

Difference: $\log [$ Li ppm] quenched-log $[\mathbf{L i}$ ppm] ccc

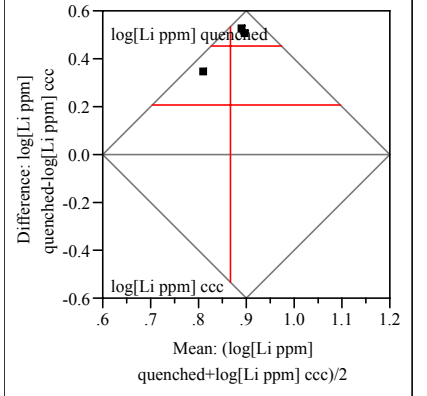

$\log [$ Li ppm] quenched $1.09221 \quad$ t-Ratio 7.939569 $\begin{array}{lll}0.63789 & \mathrm{DF} & 2\end{array}$ $\begin{array}{llll}\text { Mean Difference } & 0.45433 & \text { Prob }>|t| & 0.0155 \\ \text { Std Error } & 0.05722 & \text { Prob }>t & 0.0077\end{array}$ $\begin{array}{llll}\text { Std Error } & 0.05722 & \text { Prob }>\text { t } & 0.0077 \\ \text { Upper95\% } & 0.70054 & \text { Prob }<\text { t } & 0.9923\end{array}$ $\mathrm{N}$ $\begin{array}{lll}0.70054 & \text { Prob }<\mathrm{t} \quad 0.9923 \\ 0.20812 & & \end{array}$ Correlation

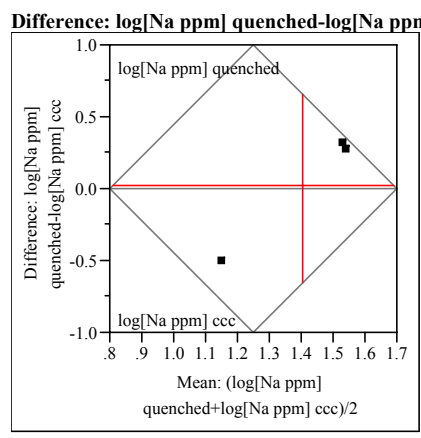

$\log [\mathrm{Na}$ ppm] quenched $1.41799 \quad \mathrm{t}$-Ratio $\quad 0.079734$ $\begin{array}{lllr}\text { log[Na ppm] ccc } & 1.39676 & \text { DF } & 2 \\ \text { Mean Difference } & 0.02123 & \text { Prob }>|t| & 0.9437\end{array}$ $\begin{array}{llll}\text { Std Error } & 0.26624 & \text { Prob }>\mathrm{t} & 0.4719 \\ \text { Upper95\% } & 1.16675 & \text { Prob }<\mathrm{t} & 0.5281\end{array}$ $\begin{array}{rll}1.16675 & \text { Prob }<\mathrm{t} & 0.5281\end{array}$ Lower $95 \%$

$\mathrm{N}$ Correlation $-1.1243$

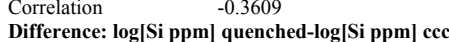

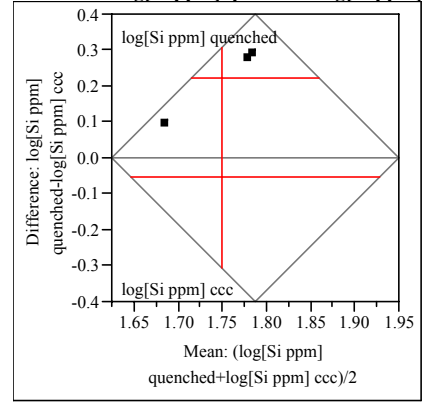

$\log [$ Si ppm] quenched $1.85915 \quad$ t-Ratio $\quad 3.48292$ $\begin{array}{llll}\log [\mathrm{Si} \mathrm{ppm}] \mathrm{ccc} & 1.63905 & \mathrm{DF} & 2\end{array}$ $\begin{array}{llll}\text { Mean Difference } & 0.2201 & \text { Prob }>|t| & 0.073\end{array}$ $\begin{array}{llll}\text { Std Error } & 0.06319 & \text { Prob }>t & 0.0367 \\ \text { Upper95\% } & 0.49201 & \text { Prob }<\mathrm{t} & 0.9633\end{array}$ $-0.0518$ Correlation
Matched Pairs Glass \#=14

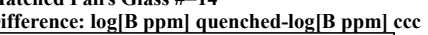

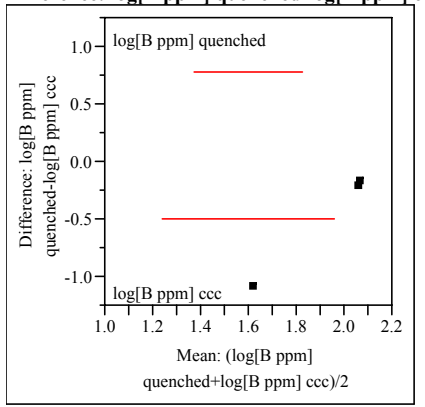
$\begin{array}{lllr}\log [\mathrm{B} \mathrm{ppm}] \text { quenched } & 1.66613 & \text { t-Ratio } & -1.67249 \\ \log [\mathrm{B} \mathrm{ppm}] \mathrm{ccc} & 2.16299 & \text { DF } & 2\end{array}$ $\begin{array}{llll}\text { Mean Difference } & -0.4969 & \text { Prob }>|t| & 0.2364\end{array}$ $\begin{array}{llll}\text { Std Error } & 0.29708 & \text { Prob }>\text { t } & 0.8818 \\ \text { Upper95\% } & 0.78137 & \text { Prob }<\text { t } & 0.1182\end{array}$

Lower95\% $\begin{array}{rll}0.78137 & \text { Prob }<\mathrm{t} \quad 0.1182 \\ -1.7751 & & \end{array}$

$\mathrm{N}$ Correlation 3
-0.5233

Difference: $\log [\mathbf{L i ~ p p m ]}]$ quenched-log $[\mathbf{L i}$ ppm] ccc

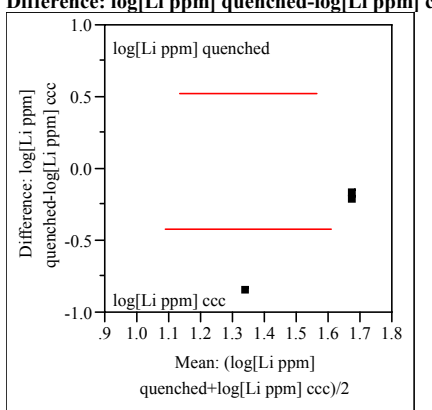

$\log [$ Li ppm] quenched 1.35205 t-Ratio -1.9165 $\begin{array}{lll}1.77358 & \mathrm{DF} & 2\end{array}$ $\begin{array}{llll}\text { Mean Difference } & -0.4215 & \text { Prob }>|t| & 0.1953\end{array}$ $\begin{array}{llll}\text { Std Error } & 0.21994 & \text { Prob }>\mathrm{t} & 0.9023 \\ \text { Upper95\% } & 0.52481 & \text { Prob }<\mathrm{t} & 0.0977\end{array}$ (1) $\mathrm{N}$ $\begin{array}{rrr}0.52481 & \text { Prob }<\mathrm{t} & 0.0977 \\ -1.3679 & \end{array}$ Correlation

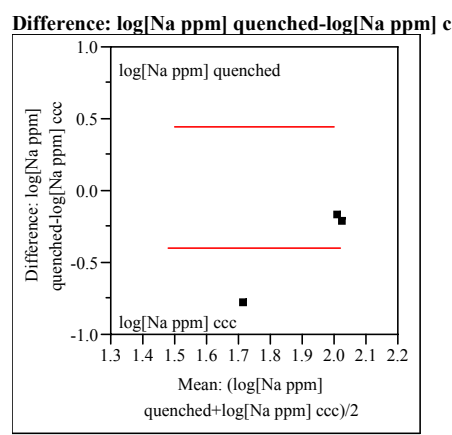

$\log [\mathrm{Na} p \mathrm{ppm}]$ quenched $1.71895 \quad \mathrm{t}$-Ratio $\quad-2.0244$ $\begin{array}{llll}\log [\mathrm{Na} \mathrm{ppm}] \mathrm{ccc} & 2.11572 & \mathrm{DF} & 2\end{array}$ Std Error $\begin{array}{lrll}\text { Upper95\% } & 0.4465 & \text { Prob }<\mathrm{t} & 0.090\end{array}$ Lower95\%

Difference: $\log [\mathrm{Si}$ ppm] quenched-log[Si ppm] ccc

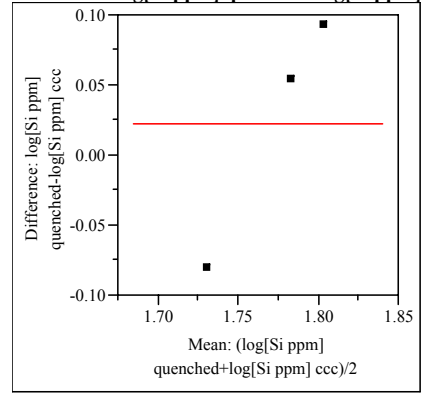

$\log [$ Si ppm] quenched $\quad 1.783 \quad$ t-Ratio 0.41441

$\begin{array}{lrllr}\log [\mathrm{Si} \text { ppm] ccc } & 1.7613 & \mathrm{DF} & 2 \\ \text { Mean Difference } & 0.0217 & \text { Prob }>|t| & 0.7188\end{array}$

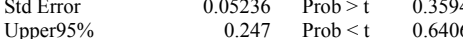
$-0.2036$ 3 


\section{Exhibit B7. Effects of Heat Treatment on PCT $\log (\mathrm{ppm})$-Response of Study Glasses}

Matched Pairs Glass \#=15

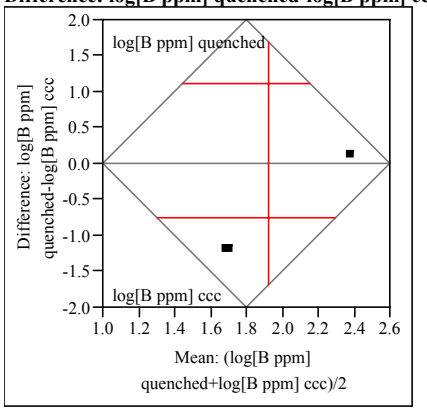

$\log [\mathrm{B}$ ppm] quenched $1.54018 \quad \mathrm{t}$-Ratio $\quad-1.74465$ $\begin{array}{llll}\log [\mathrm{B} \mathrm{ppm}] \mathrm{ccc} & 2.30444 & \mathrm{DF} & 2\end{array}$ $\begin{array}{llll}\text { Mean Difference } & -0.7643 & \text { Prob }>|t| & 0.2232 \\ & 0.43805 & \text { Prob }>t & 0.8884\end{array}$ $\begin{array}{llll}\text { Std Error } & 0.43806 & \text { Prob }>t & 0.8884 \\ \text { Upper95\% } & 1.12056 & \text { Prob }<\mathrm{t} & 0.1116 \\ \text { Lower95\% } & -2.6491 & & \end{array}$ Upper $95 \%$

$\mathrm{N}$ Correlation 3

Difference: $\log [$ Li ppm] quenched-log $[$ Li ppm] cce

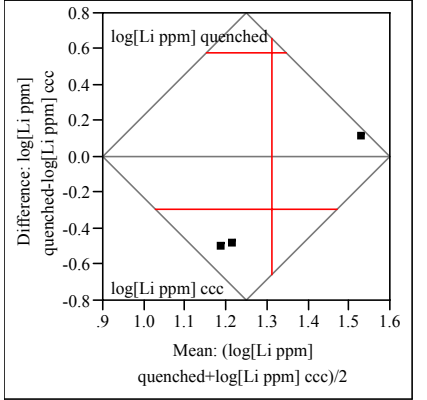

$\log [$ Li ppm] quenched $\quad 1.16318 \quad \mathrm{t}$-Ratio $\quad-1.46101$ $\begin{array}{lrlr}\log [\mathrm{Li} \text { ppm] } \mathrm{ccc} & 1.45886 & \text { DF } & 2 \\ \text { Mean Difference } & -0.2957 & \text { Prob }>|t| & 0.2815\end{array}$ $\begin{array}{llll}\text { Std Error } & 0.20238 & \text { Prob }>t & 0.8593\end{array}$ $\begin{array}{llll}\text { Upper95\% } & 0.57509 & \text { Prob }<\text { t } & 0.1407\end{array}$

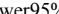
Correlation

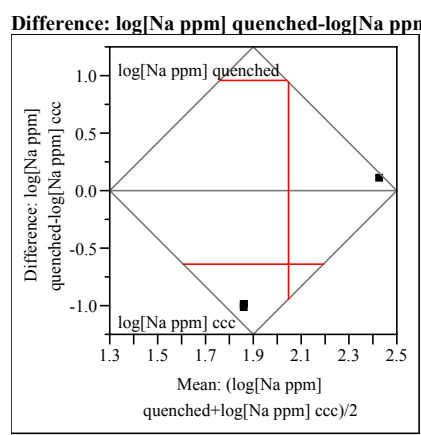

$\log$ [Na ppm] quenched $1.72605 \quad \mathrm{t}$-Ratio $\quad-1.72306$ $\begin{array}{lrlr}\log [\mathrm{Na} p \mathrm{ppm}] \mathrm{ccc} & 2.36793 & \text { DF } & -1.72306 \\ \log [\mathrm{Na} \text { pan Difference } & -0.6419 & \text { Prob }>|\mathrm{t}| & 0.2270 \\ \text { Mean } & 0.37259 & \end{array}$ $\begin{array}{llll}\text { Std Error } & 0.37253 & \text { Prob }>\mathrm{t} & 0.8865 \\ \text { Upper95\% } & 0.96097 & \text { Prob }<\mathrm{t} & 0.1135\end{array}$

Lower $95 \%$ $\begin{array}{rrr}0.96097 & \text { Prob }<\mathrm{t} \quad 0.1135\end{array}$

$\mathrm{N}$ (1)

Difference: $\log [\mathrm{Si}$ ppm] quenched-log[Si ppm] ccc

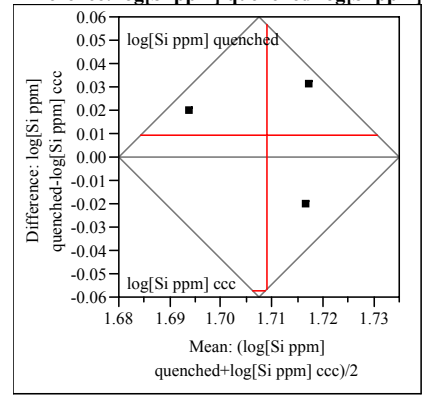

$\log [$ Si ppm] quenched 1.71395 t-Ratio 0.61507 $\begin{array}{llll}\log [\mathrm{Si} \text { ppm }] \mathrm{ccc} & 1.70438 & \mathrm{DF} & 2 \\ \text { Mean Difference } & 0.00958 & \text { Prob }>|t| & 0.6012 \\ \text { Sed } & 0.01557 & \text { Pob }>1 & 0.3006\end{array}$ $\begin{array}{llll}\text { Std Error } & 0.01557 & \text { Prob }>t & 0.3006 \\ \text { Upper95\% } & 0.07658 & \text { Prob }<\text { t } & 0.6994 \\ \text { Lower95\% } & -0.0574 & & \end{array}$ $-0.0574$

Correlation

$-0.0122$
Matched Pairs Glass \#=16 Difference: $\log [\mathbf{B}$ ppm] quenched-log $[\mathbf{B}$ ppm] cec

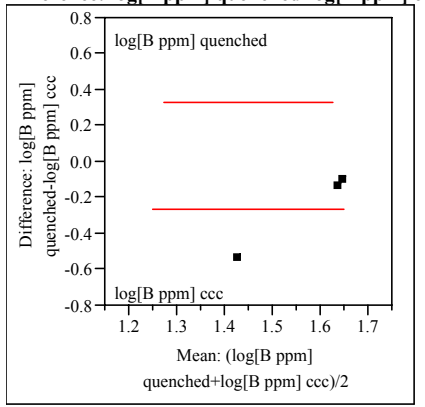
$\begin{array}{lllr}\log [\mathrm{B} \mathrm{ppm}] \text { quenched } & 1.43673 & \text { t-Ratio } & -1.91472 \\ \log [\mathrm{B} \mathrm{ppm}] \mathrm{ccc} & 1.70327 & \mathrm{DF} & 2 \\ \text { D } & -0.2665 & & \end{array}$ $\begin{array}{llll}\text { Mean Difference } & -0.2665 & \text { Prob }>|t| & 0.1956\end{array}$ $\begin{array}{llll}\text { Std Error } & 0.13921 & \text { Prob }>\text { t } & 0.9022 \\ \text { Upper95\% } & 0.33241 & \text { Prob }<\text { t } & 0.0978\end{array}$ $\begin{array}{rll}0.33241 & \text { Prob }<\mathrm{t} \quad 0.0978 \\ -0.8655 & & \end{array}$ 55232

$\mathrm{N}$ Correlation $\quad 0.55232$

Difference: $\log [\mathbf{L i ~ p p m ]}]$ quenched-log $[\mathrm{Li} \mathrm{ppm}] \mathrm{ccc}$

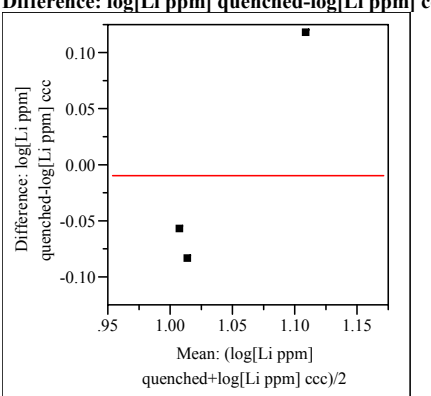

$\log [$ Li ppm] quenched $\quad 1.03872 \quad$ t-Ratio $\quad-0.1448$ $\begin{array}{lllll}\log [\mathrm{Li} \mathrm{ppm}] \mathrm{ccc} & 1.04786 & \mathrm{DF} & 2\end{array}$ $\begin{array}{llll}\text { Std Error } & 0.06318 & \text { Prob }>|t| & 0.8981 \\ & 0.0626 & \text { Prob }>t & 0.5509\end{array}$ $\begin{array}{llll}\text { Upper95\% } & 0.26267 & \text { Prob }<\mathrm{t} & 0.4491\end{array}$ Lower95\% $-0.28$

Correlation

0.21102

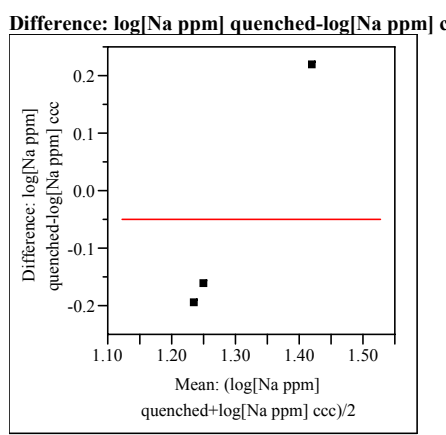

$\log [\mathrm{Na}$ ppm] quenched $1.27656 \quad \mathrm{t}$-Ratio $\quad-0.3696$ $\begin{array}{lllll}\log [\mathrm{Na} \text { ppm] } \mathrm{ccc} & 1.32555 & \mathrm{DF} & & \end{array}$ $\begin{array}{llll}\text { Mean Difference } & -0.049 & \text { Prob }>|t| & 0.747\end{array}$ $\begin{array}{llll}\text { Std Error } & 0.13255 & \text { Prob }>t & 0.6264\end{array}$ $\begin{array}{lll}0.52131 & \text { Prob }<\mathrm{t} \quad 0.3736\end{array}$ Lower $95 \%$

Correlation -0.9973

Difference: $\log [\mathrm{Si}$ ppm] quenched-log $[\mathrm{Si}$ ppm] cce

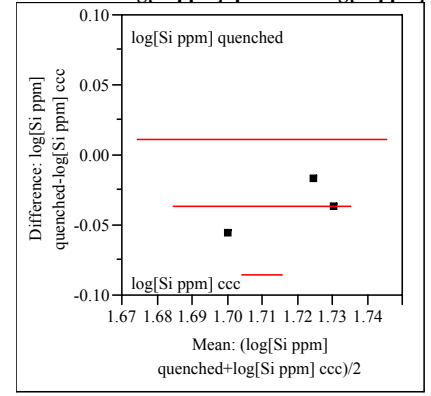

$\log [$ Si ppm] quenched $1.69998 \quad \mathrm{t}$-Ratio $\quad-3.34576$

$\begin{array}{lrll}\log [\mathrm{Si} \text { ppm }] \text { ccc } & 1.7372 & \text { DF } & 2 \\ \text { Mean Difference } & -0.0372 & \text { Prob }>|t| & 0.0789\end{array}$ Upper95\% $\quad 0.01064$ Prob $<$ t $\quad 0.0395$ Lower95\% $\quad-0.085$

$\begin{array}{lr}\mathrm{N} \\ \text { Correlation } & 0.63218\end{array}$ 


\section{Exhibit B7. Effects of Heat Treatment on PCT $\log (\mathrm{ppm})$-Response of Study Glasses}

Matched Pairs Glass \#=17

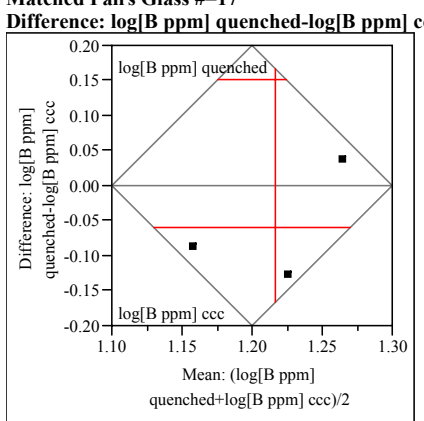

$\log [\mathrm{B}$ ppm] quenched $\quad 1.18596 \quad \mathrm{t}$-Ratio $\quad-1.2246$ $\begin{array}{lrlr}\log [\mathrm{B} \text { ppm] ccc } & 1.24636 & \mathrm{DF} & 2 \\ \text { Mean Difference } & -0.0604 & \text { Prob }>|\mathrm{t}| & 0.3454 \\ & 0.04932 & & \end{array}$ $\begin{array}{llll}\text { Mean Difference } & -0.0604 & \text { Prob }>|t| & 0.3454 \\ \text { Std Error } & 0.04932 & \text { Prob }>t & 0.8273\end{array}$ $\begin{array}{lrll}\text { Std Error } & 0.04932 & \text { Prob }>\text { t } & 0.8273 \\ \text { Upper95\% } & 0.1518 & \text { Prob }<\mathrm{t} & 0.1727\end{array}$ Upper95\%

Correlation $-0.2726$

Difference: $\log [$ Li ppm] quenched-log $[$ Li ppm] ccc

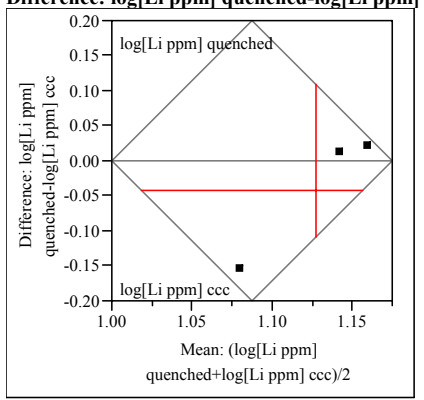

$\log [\mathrm{Li} \mathrm{ppm}]$ quenched $1.10638 \quad \mathrm{t}$-Ratio $\quad-0.72256$ $\begin{array}{lrlr}\text { log[Li ppm] ccc } & 1.1476 & \text { DF } & 2 \\ \text { Mean Difference } & -0.0412 & \text { Prob }>|t| & 0.5450\end{array}$ $\begin{array}{llll}\text { Std Error } & 0.05705 & \text { Prob }>t & 0.7275\end{array}$ \begin{tabular}{llll} 
Upper95\% & 0.20425 & Prob $<\mathrm{t}$ & 0.2725 \\
\hline & & &
\end{tabular} Lower95\%

Correlation

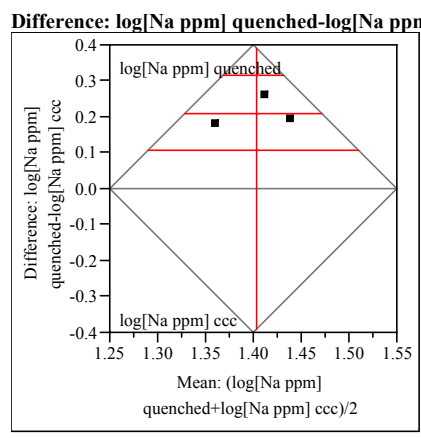

$\log [\mathrm{Na}$ ppm] quenched $1.50846 \quad \mathrm{t}$-Ratio $\quad 8.680455$ $\begin{array}{lrlr}\log [\mathrm{Na} \text { ppm] } \mathrm{ccc} & 1.29869 & \mathrm{DF} & 2 \\ \text { Mean Difference } & 0.20978 & \text { Prob }>|t| & 0.0130\end{array}$ $\begin{array}{llll}\text { Std Error } & 0.02417 & \text { Prob }>t & 0.0065 \\ \text { Upper95\% } & 0.31376 & \text { Prob }<\mathrm{t} & 0.9935\end{array}$

Lower95\%

$\mathrm{N}$ 0.59135

Difference: $\log [\mathrm{Si}$ ppm] quenched-log $[\mathrm{Si}$ ppm] ccc

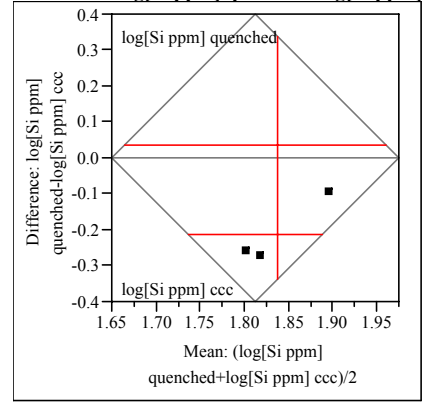

$\log [\mathrm{Si}$ ppm] quenched $1.73249 \quad$ t-Ratio $\quad-3.6916$ $\begin{array}{llll}\log [\mathrm{Si} \text { ppm }] \mathrm{ccc} & 1.9444 & \text { DF } & 2\end{array}$ $\begin{array}{lrlll}\text { Mean Difference } & -0.2119 & \text { Prob }>|t| & 0.0662 \\ \text { Std } & 0.0574 & \text { Prob }>t & 0.9669\end{array}$ $\begin{array}{lrll}\text { Std Error } & 0.0574 & \text { Prob }>t & 0.9669 \\ \text { Upper95\% } & 0.03508 & \text { Prob }<\text { t } & 0.0331\end{array}$ Upper95\% $-0.458$

Correlation $\quad 0.05324$
Matched Pairs Glass \#=18 Differes $\log [\mathbf{B}$ ppm] quenched-log $[B$ ppm $]$ ccc

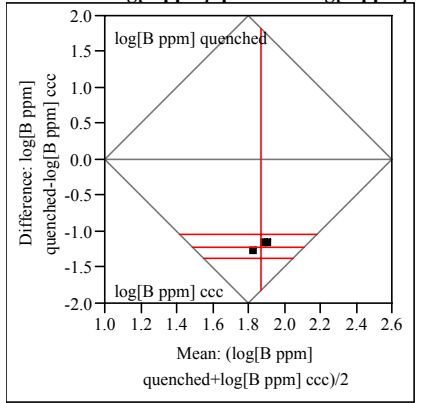

$\log [\mathrm{B}$ ppm] quenched $\quad 1.2643 \quad$ t-Ratio $\quad-30.4693$ $\begin{array}{llll}\log [\mathrm{B} \mathrm{ppm}] \mathrm{ccc} & 2.48023 & \mathrm{DF} & \end{array}$ $\begin{array}{llll}\text { Mean Difference } & -1.2159 & \text { Prob }>|t| & 0.0011 \\ & 0.03991 & \text { Pob }>< & 0.0995\end{array}$ $\begin{array}{llll}\text { Std Error } & 0.03991 & \text { Prob }>t & 0.9995 \\ \text { Upper95\% } & -1.0442 & \text { Prob }<t & 0.0005\end{array}$ (pper95\% $\begin{array}{rll}-1.0442 & \text { Prob }<\mathrm{t} \quad 0.0005 \\ -1.3876 & & \end{array}$

Correlation 3
0.66011

Difference: $\log [\mathrm{Li}$ ppm] quenched-log $[\mathrm{Li}$ ppm] cce

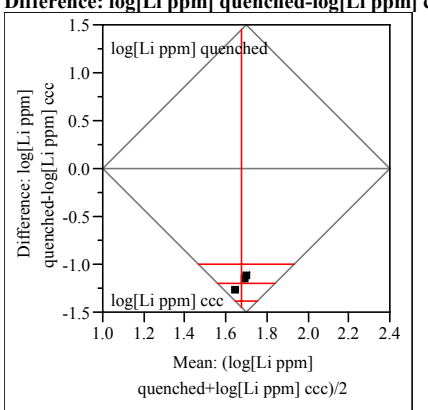

$\log [$ Li ppm] quenched $1.08224 \quad$ t-Ratio $\quad-26.0543$ $\begin{array}{lrlr}\log [\mathrm{Li} \text { ppm] } \mathrm{ccc} & 2.27616 & \text { DF } & 2 \\ \text { Mean Difference } & -1.1939 & \text { Prob }>|t| & 0.0015\end{array}$ $\begin{array}{llll}\text { Mean Difference } & -1.1939 & \text { Prob }>|t| & 0.0015 \\ \text { Std Error } & 0.04582 & \text { Prob }>t & 0.9993\end{array}$ $\begin{array}{llll}\text { Std Error } & 0.04582 & \text { Prob }>t & 0.9993 \\ \text { Upper } 95 \% & -0.9967 & \text { Prob }<\mathrm{t} & 0.0007\end{array}$ Upper95\% $\mathrm{N}$
Correlation $-1.3911 \quad$ Prob $<1 \quad 0.0007$

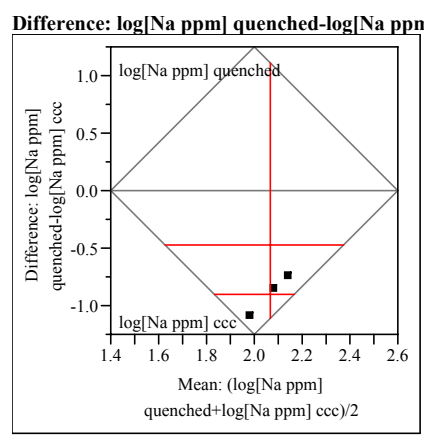

$\log [$ Na ppm] quenched $1.61671 \quad$ t-Ratio $\quad-9.0312$ $\begin{array}{llll}\log [\mathrm{Na} \text { ppm }] \mathrm{ccc} & 2.51993 & \mathrm{DF} & 2\end{array}$ $\begin{array}{llll}\text { Mean Difference } & -0.9032 & \text { Prob }>|t| & 0.0120\end{array}$ $\begin{array}{llll}\text { Std Error } & 0.10001 & \text { Prob }>t & 0.9940 \\ \text { Upper95\% } & -0.4729 & \text { Prob }<t & 0.0060\end{array}$ Upporgs\% $\begin{array}{rll}-0.4729 & \text { Prob }<\mathrm{t} & 0.0060 \\ -1.3335 & & \end{array}$ $\mathrm{N}$

Difference: $\log [\mathrm{Si}$ ppm] quenched-log [Si ppm] $\mathrm{ccc}$

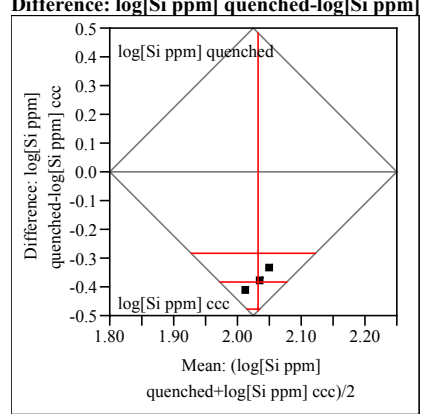

$\log [$ Si ppm] quenched $1.84189 \quad \mathrm{t}$-Ratio $\quad-16.815$ $\begin{array}{llll}\log [\mathrm{Si} \mathrm{ppm}] \mathrm{ccc} & 2.22286 & \mathrm{DF} & 2\end{array}$ $\begin{array}{llll}\text { Mean Difference } & -0.381 & \text { Prob }>|t| & 0.0035 \\ \text { Std Error } & 0.02266 & \text { Prob }>t & 0.9982\end{array}$ $\begin{array}{llll}\text { Std Error } & 0.02266 & \text { Prob }>t & 0.9982 \\ \text { Upper95\% } & -0.2835 & \text { Prob }<\mathrm{t} & 0.0018\end{array}$ Lower95\% $\quad-0.4784$

Correlation $\quad-0.1965$ 


\section{Exhibit B7. Effects of Heat Treatment on PCT $\log (\mathrm{ppm})$-Response of Study Glasses}

Matched Pairs Glass \#=19

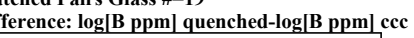

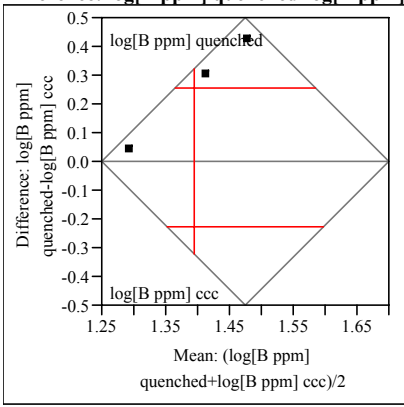

$\log [\mathrm{B}$ ppm] quenched $\quad 1.52142 \quad \mathrm{t}$-Ratio $\quad 2.257608$ $\begin{array}{llll}\log [\mathrm{B} \text { ppm }] \mathrm{ccc} & 1.26717 & \mathrm{DF} & 2\end{array}$ $\begin{array}{llll}\text { Mean Difference } & 0.25425 & \text { Prob }>|t| & 0.1525 \\ & 0.11262 & \text { Prob }>\text { t } & 0.0763\end{array}$ $\begin{array}{llll}\text { Std Error } & 0.11262 & \text { Prob }>\mathrm{t} & 0.0763 \\ \text { Upper95\% } & 0.73882 & \text { Prob }<\mathrm{t} & 0.9237\end{array}$ (1) $-0.230$

$\mathrm{N}$ Correlation $\begin{array}{r}3 \\ -0.6488 \\ \hline\end{array}$

Difference: $\log [\mathbf{L i}$ ppm] quenched-log $[\mathrm{Li}$ ppm] ccc

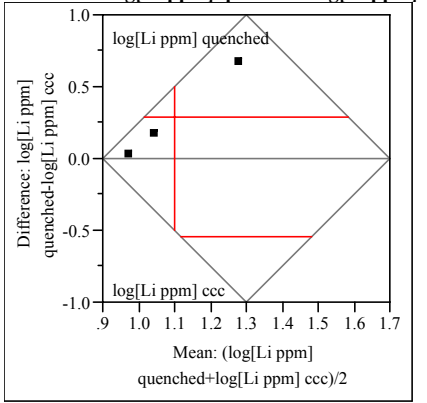

$\log [$ Li ppm] quenched $1.24168 \quad \mathrm{t}$-Ratio 1.485197 $\begin{array}{lrlr}\log [\mathrm{Li} p \mathrm{ppm}] \mathrm{ccc} & 0.95529 & \mathrm{DF} & 2 \\ \text { Mean Difference } & 0.28639 & \text { Prob }>|t| & 0.2758\end{array}$ $\begin{array}{llll}\text { Mean Difference } & 0.28639 & \text { Prob }>|t| & 0.2758 \\ \text { Std Error } & 0.19283 & \text { Prob }>t & 0.1379\end{array}$ $\begin{array}{llll}\text { Std Error } & 0.19283 & \text { Prob }>\text { t } & 0.1379 \\ \text { Upper95\% } & 1.11607 & \text { Prob }<\mathrm{t} & 0.8621\end{array}$

$\mathrm{N}$

Correlation

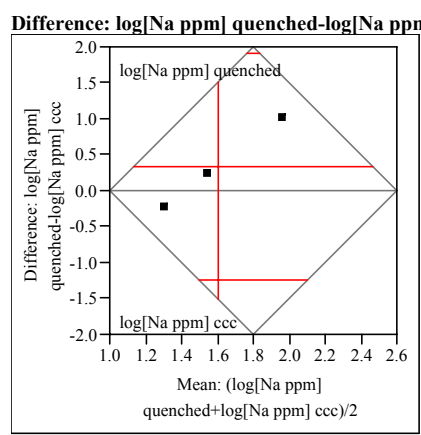

$\log [\mathrm{Na}$ ppm] quenched $1.76636 \quad \mathrm{t}$-Ratio $\quad 0.8980$ $\begin{array}{lllll}\log [\mathrm{Na} \mathrm{ppm}] \mathrm{ccc} & 1.4374 & \mathrm{DF} & 2\end{array}$ $\begin{array}{llll}\text { Mean Difference } & 0.32896 & \text { Prob }>|t| & 0.4640\end{array}$ $\begin{array}{llll}\text { Std Error } & 0.36632 & \text { Prob }>t & 0.2320 \\ \text { Upper95\% } & 1.90512 & \text { Prob }<\mathrm{t} & 0.7680\end{array}$

Lower95\%

Correlation 1.85237

Difference: $\log [\mathrm{Si}$ ppm] quenched-log[Si ppm] ccc

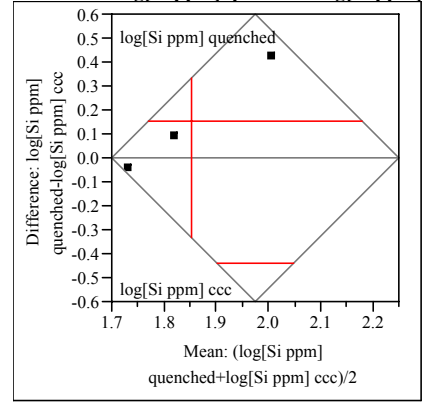

$\log [\mathrm{Si}$ ppm] quenched $1.92935 \quad \mathrm{t}$-Ratio $\quad 1.112651$ $\begin{array}{llll}\log [\mathrm{Si} \mathrm{ppm}] \mathrm{ccc} & 1.77618 & \mathrm{DF} & 2\end{array}$ $\begin{array}{llll}\text { Mean Difference } & 0.15317 & \text { Prob }>|t| & 0.3817 \\ \text { Std } & 0.13766 & \text { Prob }>t & 0.1908\end{array}$

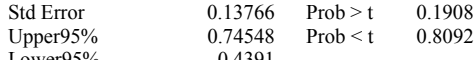

$\mathrm{N}$ Correlation $-0.4391$ 3
0.9792
Tatched Pairs Glass \#=20

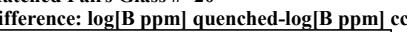

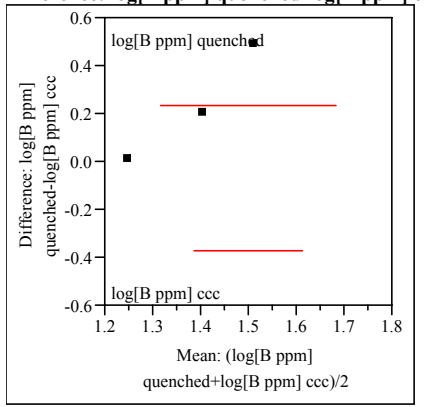

$\log [\mathrm{B} \mathrm{ppm}]$ quenched $1.50198 \quad \mathrm{t}$-Ratio $\quad 1.635977$ $\begin{array}{llll}\log [\mathrm{B} \mathrm{ppm}] \mathrm{ccc} & 1.27162 & \mathrm{DF} & 2\end{array}$ $\begin{array}{llll} & & & \\ \text { Mean Difference } & 0.23036 & \text { Prob }>|t| & 0.2435 \\ \text { Std Error } & 0.14081 & \text { Prob }>t & 0.1217\end{array}$ $\begin{array}{llll}\text { Utd Error } & 0.14081 & \text { Prob }>\mathrm{t} & 0.1217 \\ \text { Upper95\% } & 0.8362 & \text { Prob }<\mathrm{t} & 0.8783\end{array}$ $-0.3755$

Correlation $\quad 0.39164$

Difference: $\log [\mathbf{L i}$ ppm] quenched-log $[\mathbf{L i}$ ppm] cec

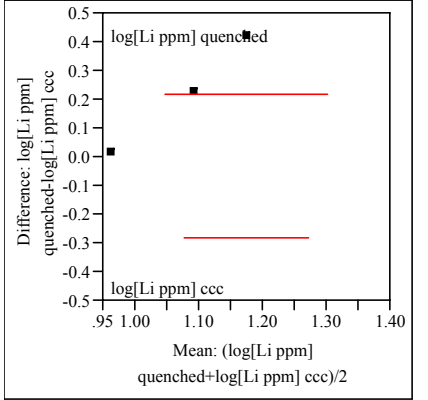

$\log [\mathrm{Li}$ ppm] quenched $1.18554 \quad \mathrm{t}$-Ratio 1.857828 $\begin{array}{llll}\log [\mathrm{Li} \text { ppm] } \mathrm{ccc} & 0.96813 & \text { DF } & 2 \\ \text { Mean Difference } & 0.21742 & \text { Prob }>|t| & 0.2043\end{array}$ $\begin{array}{llll}\text { Mean Difference } & 0.21742 & \text { Prob }>|t| & 0.2043 \\ \text { Std Error } & 0.11703 & \text { Prob }>t & 0.1022\end{array}$ $\begin{array}{llll}\text { Std Error } & 0.11703 & \text { Prob }>\text { t } & 0.1022 \\ \text { Upper95\% } & 0.72094 & \text { Prob }<\mathrm{t} & 0.8978\end{array}$ (1) $\mathrm{N}$ $-0.2861 \quad$ Prob $<1 \quad 0.828$ Correlation $\quad 0.43993$

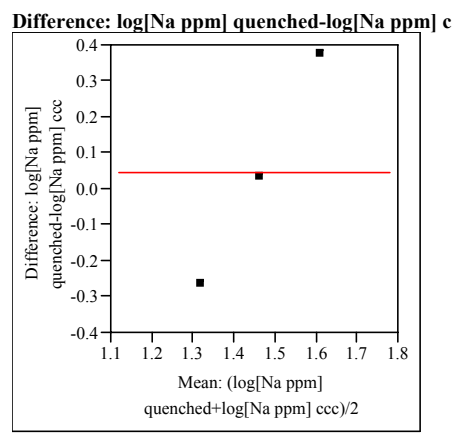

$\log [\mathrm{Na}$ ppm] quenched $1.48599 \quad$ t-Ratio $\quad 0.244706$ $\begin{array}{llll}\log [\mathrm{Na} \text { ppm] ccc } & 1.44091 & \text { DF } & 2 \\ \text { Mean Difference } & 0.04508 & \text { Prob }>|t| & 0.8295\end{array}$ Std Error $\begin{array}{llll}\text { Upper95\% } & 0.83777 & \text { Prob }<\mathrm{t} & 0.5852 \\ & & & \end{array}$ $-0.7476$

Correlation $\quad-0.9454$

Difference: $\log [\mathrm{Si}$ ppm] quenched-log[Si ppm] ccc

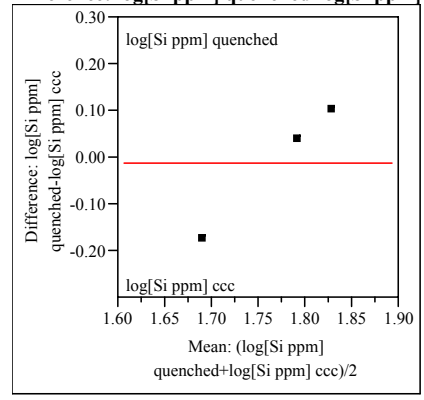

$\log [$ Si ppm] quenched $\quad 1.7632 \quad \mathrm{t}$-Ratio $\quad-0.16937$

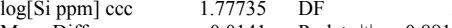

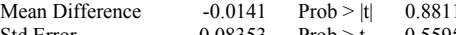

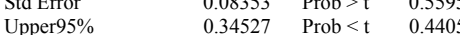
$\begin{array}{ll}\text { Upper95\% } & 0.3455 \\ \text { Lower95\% } & -0.3736\end{array}$

Correlation $\quad-0.279$ 


\section{Exhibit B7. Effects of Heat Treatment on PCT $\log (\mathrm{ppm})$-Response of Study Glasses}

Matched Pairs Glass \#=21 Difference $\log [\mathrm{B} \mathrm{ppm]}$ quenched-log $[\mathrm{B}$ ppm] cec

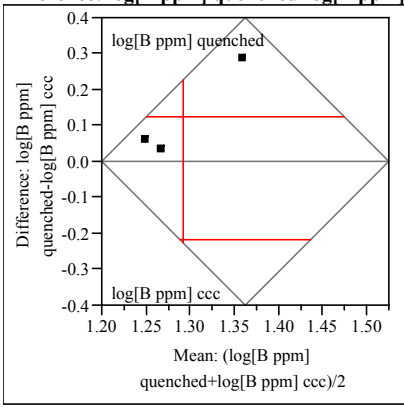

$\log [\mathrm{B}$ ppm $]$ quenched $1.35398 \quad \mathrm{t}$-Ratio $\quad 1.56386$ $\begin{array}{llll}\log [\mathrm{B} \mathrm{ppm}] \mathrm{ccc} & 1.22859 & \mathrm{DF} & 2\end{array}$ $\begin{array}{llll}\text { Mean Difference } & 0.12538 & \text { Prob }>|t| & 0.2583 \\ & 0.08017 & \text { Prob }>t & 0.129\end{array}$ $\begin{array}{lllll}\text { Std Error } & 0.08017 & \text { Prob }>t & 0.1291 \\ \text { Upper95\% } & 0.47035 & \text { Prob }<\text { t } & 0.8709\end{array}$ $\begin{array}{llll}\text { Upper95\% } & 0.47035 & \text { Prob }<\mathrm{t} & 0.870 \\ \text { Lower95\% } & -0.2196 & \end{array}$

$\mathrm{N}$ Correlation 3

Difference: $\log [\mathbf{L i}$ ppm] quenched-log $[\mathbf{L i}$ ppm $] \mathrm{ccc}$

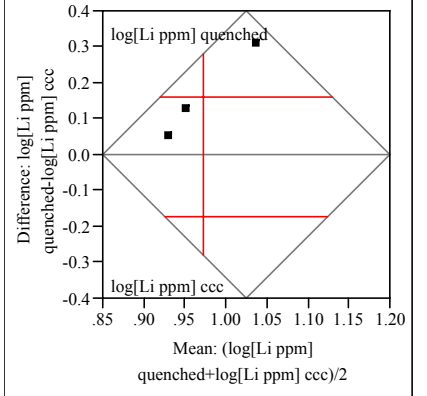

$\log [$ Li ppm] quenched $1.05202 \quad$ t-Ratio 2.064312 $\begin{array}{lll}1.05202 & \mathrm{DF} & 2 \\ 0\end{array}$ $\begin{array}{llll}\text { Mean Difference } & 0.15939 & \text { Prob }>|t| & 0.1750\end{array}$ $\begin{array}{llll}\text { Std Error } & 0.07721 & \text { Prob }>\text { t } & 0.0875 \\ \text { Upper } & 0.95 \% & & \end{array}$ $\begin{array}{llll}\text { Upper95\% } & 0.49161 & \text { Prob }<\mathrm{t} & 0.9125 \\ \text { Lower95\% } & -0.1728 & & \end{array}$ $\mathrm{N}$ 3
-0.8546

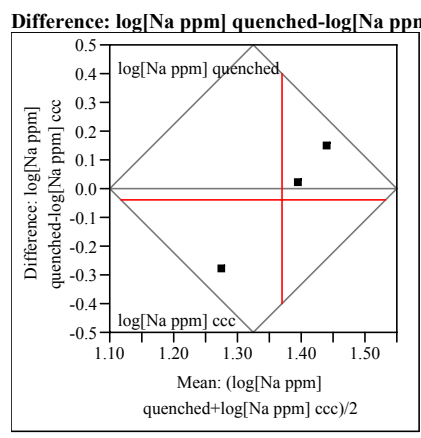

$\log [$ Na ppm] quenched $1.34915 \quad \mathrm{t}$-Ratio $\quad-0.32866$ $\begin{array}{llll}\log [\mathrm{Na} \text { ppm] ccc } & 1.39065 & \mathrm{DF} & 2\end{array}$ $\begin{array}{llll}\text { Upper95\% } & 0.12628 & \text { Prob }>\mathrm{t} & 0.6132 \\ & 0.50184 & \text { Prob }<\mathrm{t} & 0.3868\end{array}$ $-0.5849$

$\mathrm{N}$ $-0.9957$

Difference: $\log [\mathrm{Si}$ ppm] quenched-log[Si ppm] cce

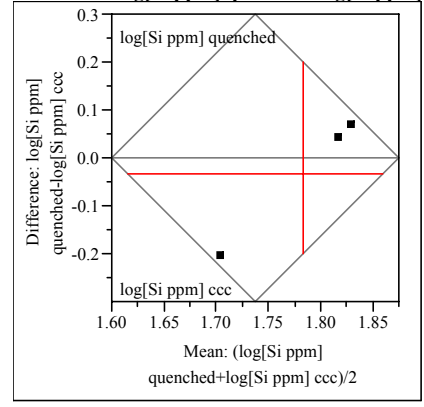

$\log [\mathrm{Si}$ ppm $]$ quenched $\quad 1.7668 \quad$ t-Ratio $\quad-0.38706$ $\begin{array}{llll}\log [\mathrm{Si} \mathrm{ppm}] \mathrm{ccc} & 1.80047 & \mathrm{DF} & 2\end{array}$ $\begin{array}{llll}\text { Mean Difference } & -0.0337 & \text { Prob }>|t| & 0.7360 \\ \text { StI } & 0.08699 & \text { Prob }>t & 0.6320\end{array}$ $\begin{array}{lrll}\text { Std Error } & 0.08699 & \text { Prob }>t & 0.6320 \\ \text { Upper95\% } & 0.3406 & \text { Prob }<\text { t } & 0.3680\end{array}$ Upper $95 \%$

$-0.407$

$\mathrm{N}$ Correlation
Matched Pairs Glass \#=2

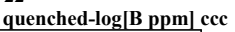

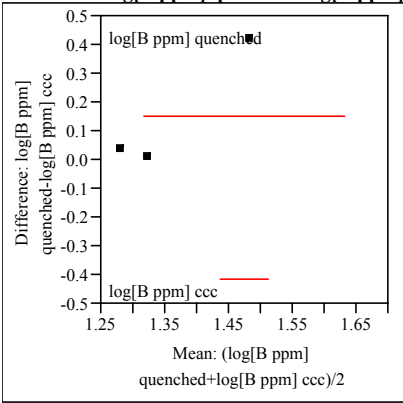

$\log [\mathrm{B}$ ppm] quenched $1.43825 \quad \mathrm{t}$-Ratio $\quad 1.153353$ $\begin{array}{lllll}\log [\mathrm{B} \text { ppm }] \mathrm{ccc} & 1.28565 & \mathrm{DF} & 2\end{array}$ $\begin{array}{lrlr} & 0.1526 & \text { Prob }>|t| & 0.3680 \\ \text { Mean Difference } & 0.13231 & \text { Prob }>t & 0.1840\end{array}$ $\begin{array}{llll}\text { Std Error } & 0.13231 & \text { Prob }>\text { t } & 0.1840 \\ \text { Upper95\% } & 0.72187 & \text { Prob }<\mathrm{t} & 0.8160\end{array}$ Lower $95 \%$

Correlation 3
-0.2555

Difference: $\log [$ Li ppm] quenched-log $[\mathbf{L i}$ ppm $]$ cec

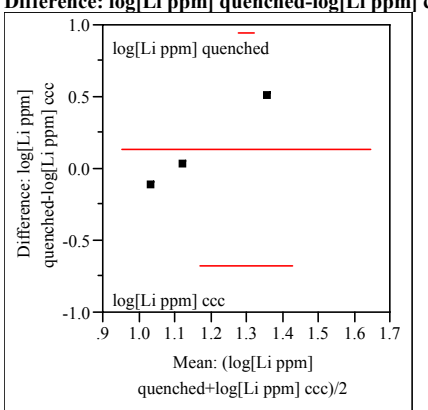

$\log [$ Li ppm] quenched $1.23767 \quad$ t-Ratio $\quad 0.706067$ $\begin{array}{lrlr}\log [\mathrm{Li} \text { ppm] } \mathrm{ccc} & 1.1043 & \text { DF } & 2 \\ \text { Mean Difference } & 0.13337 & \text { Prob }>|t| & 0.5533\end{array}$ $\begin{array}{lrll}\text { Mean Difference } & 0.13337 & \text { Prob }>|t| & 0.5533 \\ \text { Std Error } & 0.1889 & \text { Prob }>t & 0.2767\end{array}$ $\begin{array}{lrll}\text { Std Error } & 0.1889 & \text { Prob }>t & 0.2767 \\ \text { Upper95\% } & 0.94614 & \text { Prob }<t & 0.7233\end{array}$ Lower95\% $\mathrm{N}$ Correlation

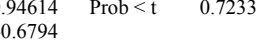
3
0.59323

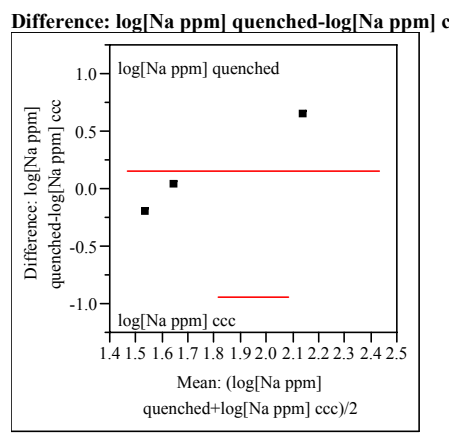

$\log [\mathrm{Na}$ ppm] quenched $1.84954 \quad \mathrm{t}$-Ratio $\quad 0.593834$ $\begin{array}{lllll}\log [\mathrm{Na} \text { ppm] } \mathrm{ccc} & 1.6979 & \mathrm{DF} & 0.593834\end{array}$ $\begin{array}{llll}\text { Mean Difference } & 0.15163 & \text { Prob }>|t| & 0.6128\end{array}$ $\begin{array}{llll}\text { Std Error } & 0.25535 & \text { Prob }>t & 0.3064 \\ \text { Upper95\% } & 1.2503 & \text { Prob }<\mathrm{t} & 0.693\end{array}$ Lower95\%

Correlation $\quad 0.9655$

Difference: $\log [\mathrm{Si}$ ppm] quenched-log[Si ppm] ccc

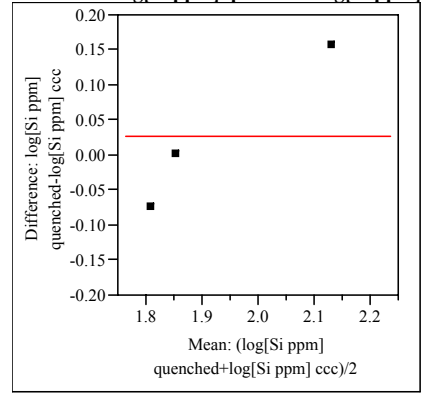

$\log [\mathrm{Si}$ ppm] quenched $1.94378 \quad$ t-Ratio 0.39964 $\log [\mathrm{Si} \mathrm{ppm]}] \mathrm{ccc} \quad 1.91662$

$\begin{array}{llll}1.93662 & \text { Prob }>|t| & 0.7281\end{array}$ $\begin{array}{llll}\text { Std Error } & 0.06796 & \text { Prob }>t & 0.3640 \\ \text { Upper95\% } & 0.31955 & \text { Prob } & 0.6360\end{array}$ Lower95\% $\quad-0.265$

Correlation $\quad 0.9881$ 


\section{Exhibit B7. Effects of Heat Treatment on PCT $\log (\mathrm{ppm})$-Response of Study Glasses}

Matched Pairs Glass \#=23

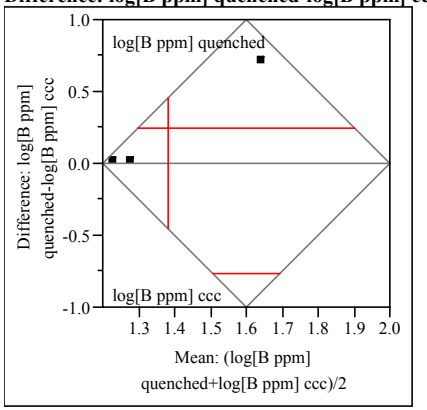

$\log [\mathrm{B}$ ppm $]$ quenched $\quad 1.5024 \quad \mathrm{t}$-Ratio $\quad 1.042925$ $\begin{array}{llll}\log [\mathrm{B} \mathrm{ppm}] \mathrm{ccc} & 1.2585 & \mathrm{DF} & 2\end{array}$ $\begin{array}{llll}\quad 0.2439 & \text { Prob }>|t| & 0.4065 \\ \text { Mean Difference } & 0.2386 & \text { Prob }>\text { r } & 0.2732\end{array}$ $\begin{array}{llll}\text { Std Error } & 0.23386 & \text { Prob }>\mathrm{t} & 0.2032 \\ \text { Upper95\% } & 1.25013 & \text { Prob }<\mathrm{t} & 0.7968\end{array}$ $-0.7623$

$\mathrm{N}$ Correlation 0.69156

Difference: $\log [$ Li ppm] quenched-log $[$ Li ppm] ccc

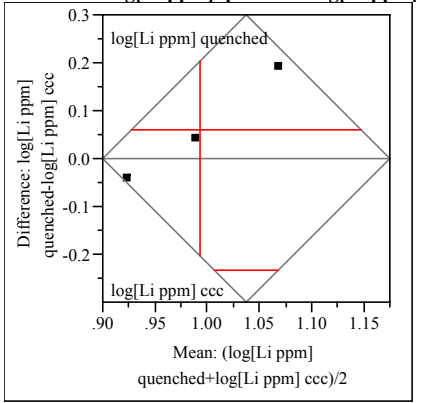

$\log [\mathrm{Li}$ ppm] quenched $1.02388 \quad \mathrm{t}$-Ratio 0.895058 $\begin{array}{lllr}\text { log[Li ppm] ccc } & 0.96231 & \text { DF } & 2 \\ \text { Mean Difference } & 0.06157 & \text { Prob }>|t| & 0.4652\end{array}$ $\begin{array}{llll}\text { Mean Difference } & 0.06157 & \text { Prob }>|t| & 0.4652 \\ \text { Std Error } & 0.06879 & \text { Prob }>t & 0.2326\end{array}$ $\begin{array}{llll}\text { Upper95\% } & 0.35753 & \text { Prob }<t & 0.7674\end{array}$ ower95\%

Correlation

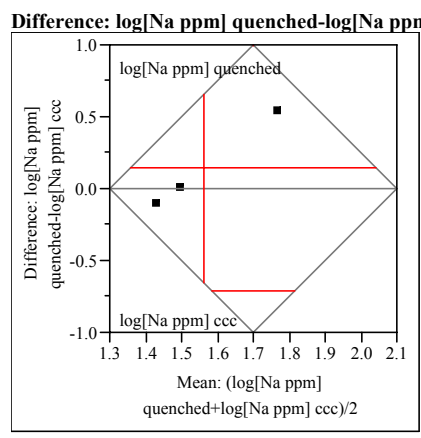

$\log$ [Na ppm] quenched $1.63431 \quad \mathrm{t}$-Ratio 0.726046 $\begin{array}{llll}\log [\mathrm{Na} \text { ppm] } \mathrm{ccc} & 1.49054 & \mathrm{DF} & 0.72604\end{array}$ $\begin{array}{llll} & 0.14377 & \text { Prob }>|t| & 0.5433\end{array}$ $\begin{array}{llll}\text { Std Error } & 0.19802 & \text { Prob }>t & 0.2716 \\ \text { Upper95\% } & 0.9956 & \text { Prob }<\mathrm{t} & 0.7284\end{array}$ Uppergs\% $\begin{array}{rll}0.99576 & \text { Prob }<\mathrm{t} \quad 0.7284\end{array}$

Difference: $\log [\mathrm{Si}$ ppm] quenched-log $[\mathrm{Si}$ ppm] ccc

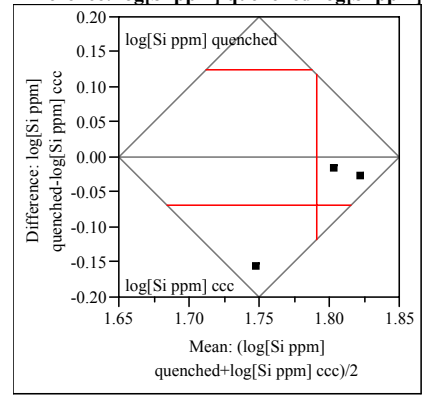

$\log [$ Si ppm] quenched 1.75702 t-Ratio -1.53207

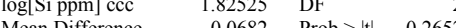
$\begin{array}{llll}1 & 0.0482 & \text { Prob }>|\mathrm{t}| & 0.2652\end{array}$ Upter Error wer $95 \%$ $-0.2599$

Correlation 0.02215
Matched Pairs Glass $\#=24$ Difference log [B ppm] quenched-log [B ppm] cce

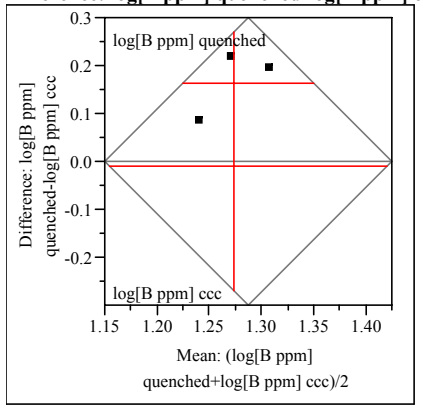

$\log [\mathrm{B}$ ppm $]$ quenched $1.35549 \quad \mathrm{t}$-Ratio $\quad 4.048568$ $\begin{array}{llll}\log [\mathrm{B} \mathrm{ppm}] \mathrm{ccc} & 1.19062 & \mathrm{DF} & 2\end{array}$ $\begin{array}{llll}\text { Mean Difference } & 0.16487 & \text { Prob }>|t| & 0.0559 \\ \text { Std } & 0.04072 & \text { Prob }>t & 0.0280\end{array}$ $\begin{array}{llll}\text { Std Error } & 0.04072 & \text { Prob }>t & 0.0280 \\ \text { Upper95\% } & 0.34008 & \text { Prob }<t & 0.9720\end{array}$ Upper $95 \%$

$\mathrm{N}$ 3

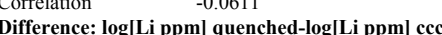

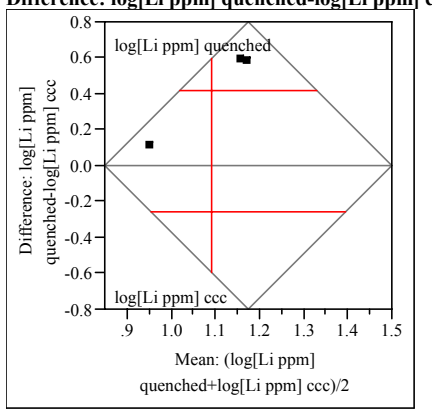

$\log [$ Li ppm] quenched $\quad 1.3038 \quad$ t-Ratio $\quad 2.68432$ $\begin{array}{llll}\text { Mean Difference } & 0.42193 & \text { Prob }>|t| & 0.1153 \\ \text { Std Error } & 0.15718 & \text { Prob }>t & 0.0576\end{array}$ $\begin{array}{llll}\text { Std Error } & 0.15718 & \text { Prob }>\text { t } & 0.0576 \\ \text { Upper95\% } & 1.09824 & \text { Prob }<\text { t } & 0.9424\end{array}$ (1) $\mathrm{N}$ $\begin{array}{llll}-0.2544 & \text { Prob }<\mathrm{t} & 0.942 \\ - & \end{array}$ Correlation

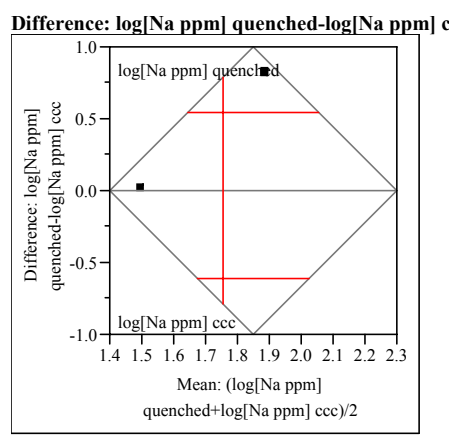

$\log [\mathrm{Na}$ ppm] quenched $2.02612 \quad$ t-Ratio $\quad 2.02569$ $\begin{array}{llll}\log [\mathrm{Na} \text { ppm }] \mathrm{ccc} & 1.48187 & \mathrm{DF} & 2\end{array}$ $\begin{array}{lll}0.54425 & \text { Prob }>|t| & 0.180\end{array}$ $\begin{array}{llll}\text { Std Error } & 0.26868 & \text { Prob }>t & 0.0900 \\ \text { Upper95\% } & 1.70027 & \text { Prob }<\text { t } & 0.9100\end{array}$ Upper $95 \%$ $\begin{array}{lll}.70027 & \text { Prob }<\mathrm{t} \quad 0.9100\end{array}$

$\mathrm{N}$

Difference: $\log [\mathbf{S i}$ ppm] quenched-log[Si ppm] cce

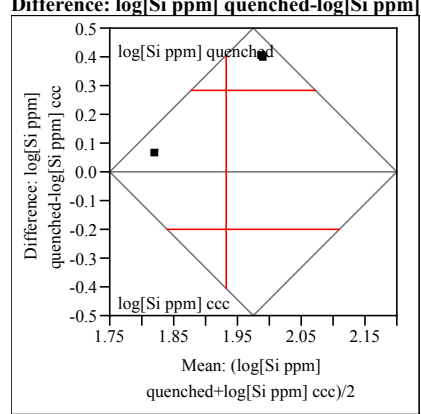

$\log [$ Si ppm] quenched $2.07513 \quad$ t-Ratio 2.531956 $\begin{array}{llll}\log [\mathrm{Si} \mathrm{ppm}] \mathrm{ccc} & 1.78966 & \mathrm{DF} & 2\end{array}$ $\begin{array}{llll}\text { Mean Difference } & 0.28547 & \text { Prob }>|t| & 0.1270 \\ \text { Std Error } & 0.11275 & \text { Prob }>t & 0.0635\end{array}$ $\begin{array}{llll}\text { Std Error } & 0.11275 & \text { Prob }>t & 0.0635 \\ \text { Upper95\% } & 0.77058 & \text { Prob }<\mathrm{t} & 0.9365 \\ & & & \end{array}$ $-0.1996$ Correlation $\quad-0.3648$ 


\section{Exhibit B7. Effects of Heat Treatment on PCT $\log (\mathrm{ppm})$-Response of Study Glasses}

Matched Pairs Glass \#=25

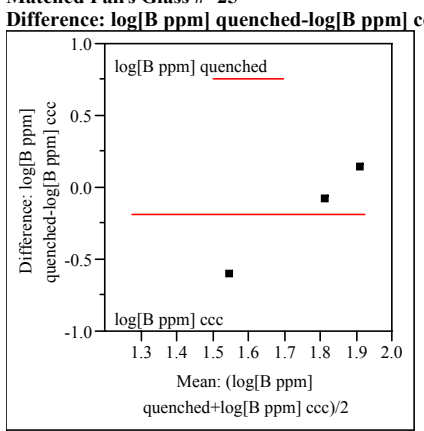

$\log [\mathrm{B}$ ppm $]$ quenched $1.66183 \quad$ t-Ratio $\quad-0.86688$ $\begin{array}{llll}\log [\mathrm{B} \mathrm{ppm}] \mathrm{ccc} & 1.85293 & \mathrm{DF} & 2\end{array}$ $\begin{array}{llll}\text { Mean Difference } & -0.1911 & \text { Prob }>|t| & 0.4774 \\ & 0.2045 & \text { Prob } & 0.756\end{array}$ $\begin{array}{llll}\text { Std Error } & 0.22045 & \text { Prob }>\mathrm{t} & 0.7613 \\ \text { Upper95\% } & 0.75742 & \text { Prob }<\mathrm{t} & 0.2387 \\ \text { Lower95\% } & -1.1396 & & \end{array}$

$\mathrm{N}$ Correlatio

3

Difference: $\log [$ Li ppm] quenched-log $[$ Li ppm $]$ cce

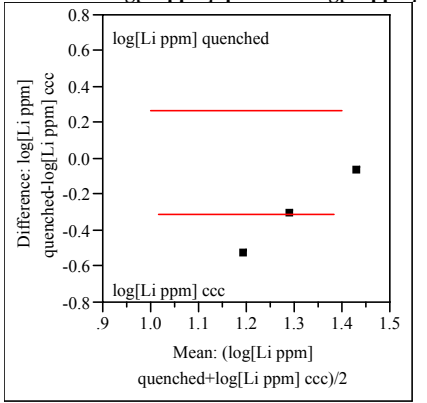

$\log [$ Li ppm] quenched $1.15091 \quad$ t-Ratio $\quad-2.29522$ $\begin{array}{llll}\text { Mean Difference } & -0.3072 & \text { Prob }>|t| & 0.1486 \\ \text { Std Error } & 0.13386 & \text { Prob }>t & 0.9257\end{array}$ $\begin{array}{llll}\text { Std Error } & 0.13386 & \text { Prob }>\text { t } & 0.9257 \\ \text { Upper95\% } & 0.26872 & \text { Prob }<\mathrm{t} & 0.0743\end{array}$ Upper95\% $\mathrm{N}$ $\begin{array}{lll}0.26872 & \text { Prob }<\mathrm{t} & 0.0743 \\ -0.8832 & & \end{array}$ Correlation

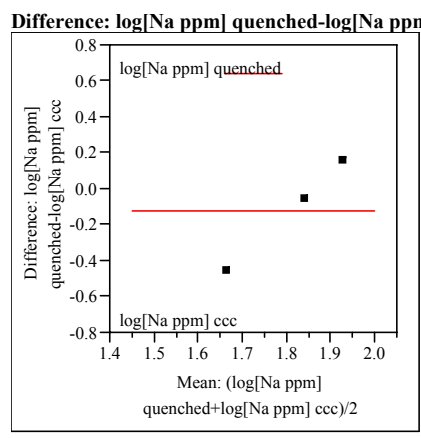

$\log [\mathrm{Na}$ ppm] quenched $1.74833 \quad \mathrm{t}$-Ratio $\quad-0.69641$ \begin{tabular}{lllr}
$\log [\mathrm{Na} p \mathrm{ppm}] \mathrm{ccc}$ & 1.87251 & DF & -0.6964 \\
$\log$ Mean Difference & -0.1242 & Prob $>|\mathrm{t}|$ & 0.5582 \\
\hline & 0.17832 &
\end{tabular} $\begin{array}{llll}\text { Std Error } & 0.17832 & \text { Prob }>t & 0.7209\end{array}$ $0.64306 \quad$ Prob $<\mathrm{t} \quad 0.279$ Lower $95 \%$ $-0.8914$

Correlation

Difference: $\log [\mathrm{Si}$ ppm] quenched-log[Si ppm] ccc

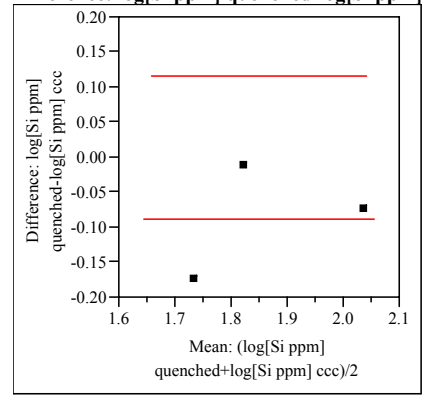

$\log [$ Si ppm] quenched $1.82009 \quad \mathrm{t}$-Ratio $\quad-1.85$ $\log [\mathrm{Si} \mathrm{ppm}] \mathrm{ccc} \quad 1.90794$

Prob $>|t| 0.204$ $\begin{array}{llll}\text { Std Error } & 0.04734 & \text { Prob }>\text { t } & 0.8977 \\ \text { Upper95\% } & 0.11582 & \text { Prob }>t & 0.1023\end{array}$ $-0.2915$

Correlation 3
0.89009
Matched Pairs Glass $\#=26$ Difference $\log [\mathbf{B}$ ppm] quenched-log $[$ p ppm] cece

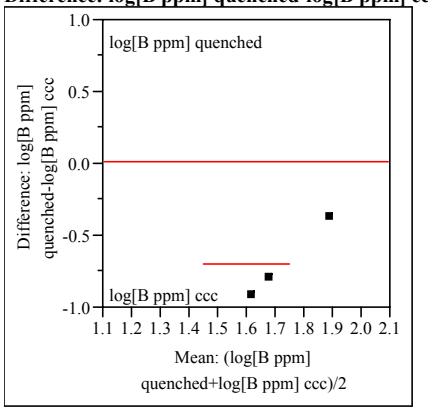

$\log [\mathrm{B}$ ppm] quenched $1.38048 \quad \mathrm{t}$-Ratio $\quad-4.2334$ $\begin{array}{llll}\log [\mathrm{B} \mathrm{ppm}] \mathrm{ccc} & 2.07758 & \mathrm{DF} & \end{array}$ $\begin{array}{llll} & -0.6971 & \text { Prob }>|t| & 0.051 \\ \text { Std Error } & 0.6967 & \text { Prob }><t & 0.9742\end{array}$ $\begin{array}{lrlll}\text { Std Error } & 0.16467 & \text { Prob }>t & 0.9742 \\ \text { Upper95\% } & 0.0114 & \text { Prob }<\mathrm{t} & 0.025\end{array}$ Upper95\% $\begin{array}{rll}0.0114 & \text { Prob }<\mathrm{t} \quad 0.0258\end{array}$ $\mathrm{N}$ Correlation $\begin{array}{r}3 \\ 0.52715 \\ \hline\end{array}$

Difference: $\log [\mathbf{L i ~ p p m ]}]$ quenched-log $[\mathrm{Li}$ ppm $] \mathrm{ccc}$

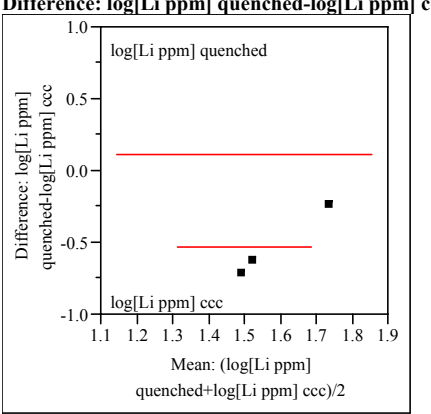

$\log [$ Li ppm] quenched 1.31613 t-Ratio $\quad-3.5710$ $\begin{array}{lrlr}\log [\mathrm{Li} \text { ppm] } \mathrm{ccc} & 1.85088 & \mathrm{DF} & 2 \\ \text { Mean Difference } & -0.5347 & \text { Prob }>|t| & 0.0703\end{array}$ $\begin{array}{llll}\text { Mean Difference } & -0.5347 & \text { Prob }>|t| & 0.0703 \\ \text { Std Error } & 0.14975 & \text { Prob }>t & 0.9649\end{array}$ Upper95\% $\quad 0.10956 \quad$ Prob $<\mathrm{t} \quad 0.0351$ Lower95\% $-1.1791$ Correlation

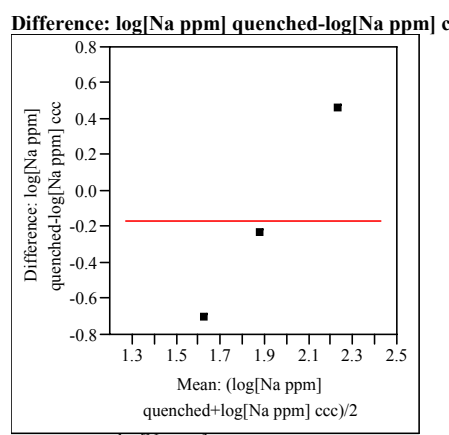

$\log [$ Na ppm] quenched $\quad 1.8298 \quad$ t-Ratio $\quad-0.4909$ $\begin{array}{llll}\log [\mathrm{Na} \text { ppm }] \mathrm{ccc} & 1.99601 & \mathrm{DF} & 2\end{array}$ $\begin{array}{llll} & -0.1662 & \text { Prob }>|t| & 0.6721\end{array}$ $\begin{array}{llll}\text { Std Error } & 0.33858 & \text { Prob }>t & 0.6640 \\ \text { Up } & 1.29059 & \text { Pro }>t & 0.3360\end{array}$ Upper $95 \%$ $\begin{array}{lll}.29059 & \text { Prob }<t \quad 0.3360\end{array}$

Difference: $\log [\mathrm{Si}$ ppm] quenched-log[Si ppm] ccc

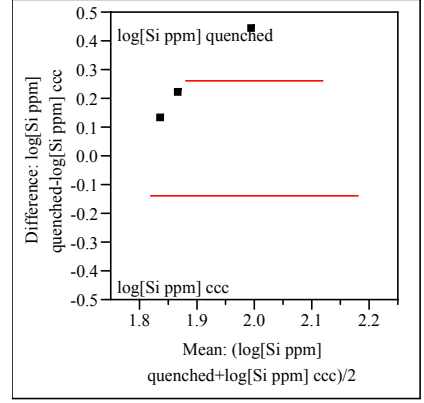

$\log [$ Si ppm] quenched $2.02927 \quad$ t-Ratio 2.793854 $\begin{array}{llll}\log [\mathrm{Si} \mathrm{ppm}] \mathrm{ccc} & 1.76876 & \mathrm{DF} & 2\end{array}$ $\begin{array}{llll}\text { Mean Difference } & 0.26051 & \text { Prob }>|t| & 0.1078 \\ \text { Std Error } & 0.09324 & \text { Prob }>t & 0.0539\end{array}$ $\begin{array}{lrlll}\text { Std Error } & 0.09324 & \text { Prob }>t & 0.0539 \\ \text { Upper95\% } & 0.6617 & \text { Prob }<\mathrm{t} & 0.9461\end{array}$ Lower $95 \% \quad-0.1407$

Correlation $\quad 0.42284$ 


\section{Exhibit B7. Effects of Heat Treatment on PCT $\log (\mathrm{ppm})$-Response of Study Glasses}

Matched Pairs Glass \#=27

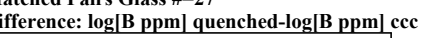

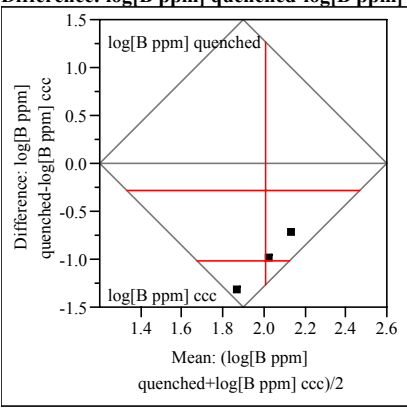

$\log [\mathrm{B}$ ppm] quenched $1.49885 \quad \mathrm{t}$-Ratio $\quad-5.97686$

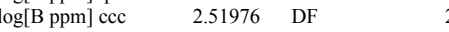
$\begin{array}{lll}\text { Mean Difference } & -1.0209 & \text { Prob }>|t| \\ & 0.17081 & 0.0269\end{array}$ $\begin{array}{lrll}\text { Std Error } & 0.17081 & \text { Prob }>t & 0.9866 \\ \text { Upper95\% } & -0.286 & \text { Prob }<\mathrm{t} & 0.0134\end{array}$ (1) -0.286
-1.758 $\quad$ Prob $<\mathrm{t} \quad 0.0134$

$\mathrm{N}$ 93

Difference: $\log [\mathbf{L i}$ ppm] quenched-log $[\mathbf{L i}$ ppm $]$ ccc

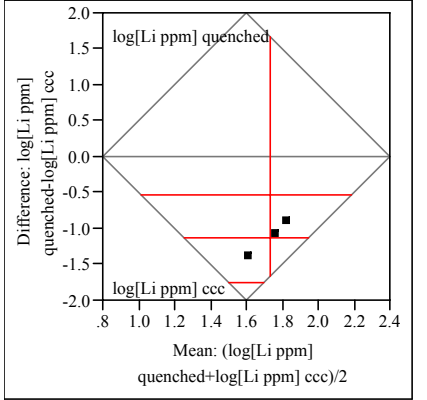

$\log [\mathrm{Li}$ ppm $]$ quenched $1.15976 \quad$ t-Ratio $\quad-7.9894$ $\begin{array}{llll}\log [\mathrm{Li} \mathrm{ppm}] \mathrm{ccc} & 2.29847 & \mathrm{DF} & -7.984 \\ & -1.1387 & \mathrm{P} & \end{array}$ $\begin{array}{llll}\text { Mean Difference } & -1.1387 & \text { Prob }>|t| & 0.0153 \\ & 0.14253 & \text { Pro } & 0\end{array}$ $\begin{array}{llll}\text { Std Error } & 0.14253 & \text { Prob }>t & 0.9923 \\ \text { Upper95\% } & -0.5255 & \text { Prob }<\mathrm{t} & 0.0077\end{array}$ Upper95\% $\mathrm{N}$ Correlation

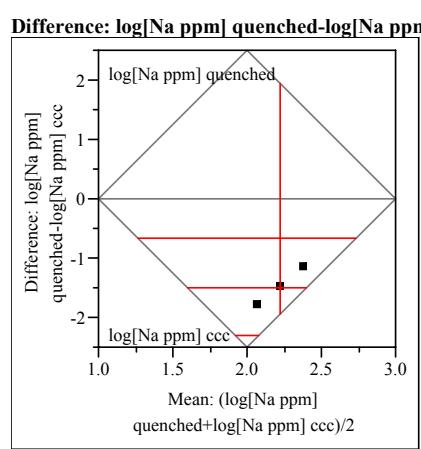

$\log [\mathrm{Na}$ ppm] quenched $1.47389 \quad \mathrm{t}$-Ratio $\quad-7.86426$ $\log [\mathrm{Na} \mathrm{ppm}] \mathrm{ccc} \quad 2.96992$ DF $\quad-7.86426$ $\begin{array}{lll}\text { Mean Difference } & -1.496 & \text { Prob }>|t| \\ 0.0158\end{array}$ $\begin{array}{llll}\text { Std Error } & -1.4023 & \text { Prob }>t & 0.992 \\ \text { Uppera5\% } & -0.6775 & \text { Pob }<1 & 0.007\end{array}$ Upper95\% $-2.3145$

Correlation 3
-0.9169 Difference: $\log [\mathrm{Si}$ ppm] quenched-log[Si ppm] cec

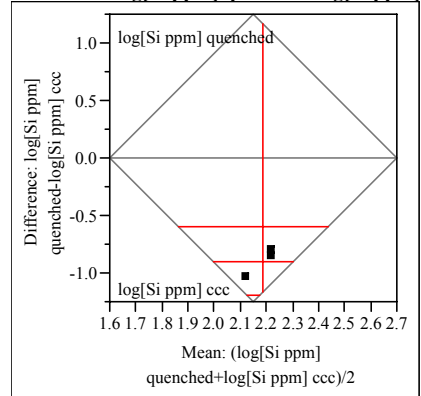

$\log [$ Si ppm] quenched $\quad 1.73492 \quad \mathrm{t}$-Ratio $\quad-12.8529$ $\begin{array}{llll}\log [\mathrm{Si} \text { ppm] } \mathrm{ccc} & 2.63442 & \mathrm{DF} & 2\end{array}$ $\begin{array}{llll}\text { Mean Difference } & -0.8995 & \text { Prob }>|t| & 0.0060 \\ \text { Std Error } & 0.06998 & \text { Prob }>t & 0.9970\end{array}$ Upper95\% $\quad-0.5984 \quad$ Prob $<$ t $\quad 0.0030$ Lower95\%

Correlation
Matched Pairs Glass \#=28 m] quenched-log $[\mathbf{B}$ ppm $]$

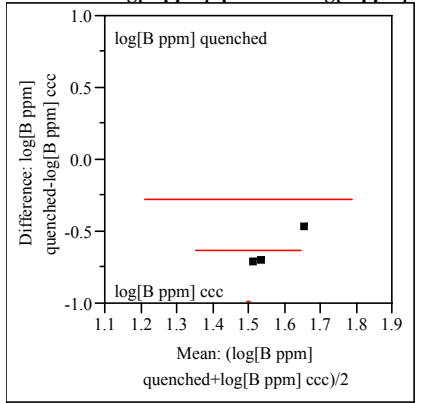

$\log [\mathrm{B}$ ppm] quenched $1.24866 \quad \mathrm{t}$-Ratio $\quad-7.74715$

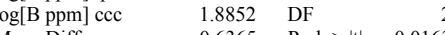
$\begin{array}{llll}\text { Mean Difference } & -0.6365 & \text { Prob }>|t| & 0.0163 \\ & 0.08216 & \text { Prob }>t & 0.9919\end{array}$ $\begin{array}{llll}\text { Std Error } & 0.08216 & \text { Prob }>t & 0.9919 \\ \text { Upper95\% } & -0.283 & \text { Prob }<\text { t } & 0.008\end{array}$ Upper95\% -0.283
-0.9901 $\quad$ Prob $<\mathrm{t} \quad 0.0081$ $\mathrm{N}$ Correlation 3
0.63157

Difference: $\log [\mathrm{Li}$ ppm] quenched-log $[\mathrm{Li}$ ppm] $\mathrm{ccc}$

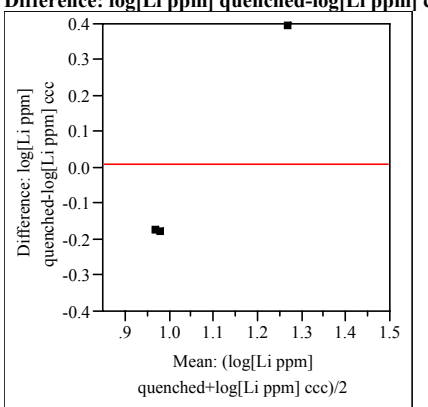

$\log [$ Li ppm] quenched $1.07739 \quad$ t-Ratio $\quad 0.052782$ $\begin{array}{lrlr}\log [\mathrm{Li} \text { ppm] ccc } & 1.06731 & \text { DF } & 2 \\ \text { Mean Difference } & 0.01007 & \text { Prob }>|t| & 0.9627\end{array}$ $\begin{array}{llll}\text { Mean Difference } & 0.01007 & \text { Prob }>|t| & 0.9627 \\ \text { Std Error } & 0.19085 & \text { Prob }>t & 0.4814 \\ \text { U } & 0.53125 & \text { Pro }<1 & 0.86\end{array}$ $\begin{array}{llll}\text { Std Error } & 0.19085 & \text { Prob }>t & 0.4814 \\ \text { Upper95\% } & 0.83125 & \text { Prob }<\mathrm{t} & 0.5186\end{array}$ (1) $\mathrm{N}$ $-0.8111 \quad$ Prob $<1 \quad 0.5186$ Correlation

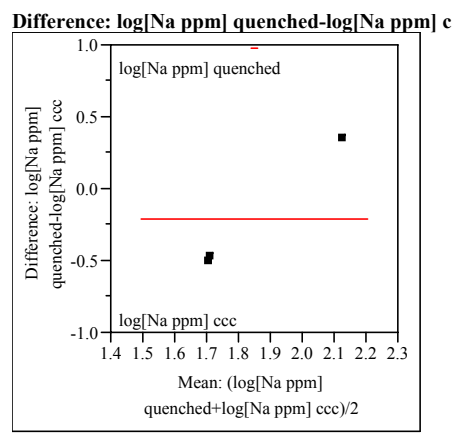

$\log [\mathrm{Na} p \mathrm{pm}]$ quenched $1.73859 \quad \mathrm{t}$-Ratio $\quad-0.7736$ $\begin{array}{llll}\log [\mathrm{Na} \text { ppm] ccc } & 1.95396 & \mathrm{DF} & 2 \\ \mathrm{Mean} \text { Difffence } & -0.2154 & \mathrm{PF}>\mathrm{lt} & 0.5201\end{array}$ Std Error $\quad 0.2784$ Prob $>t \quad 0.7400$ $\begin{array}{lllll}\text { Upper95\% } & 0.98248 & \text { Prob }<\text { t } & 0.2600\end{array}$ $-1.4132$

$\mathrm{N}$ $-0.2368$

Difference: $\log [\mathrm{Si}$ ppm] quenched-log $[\mathrm{Si}$ ppm] cce

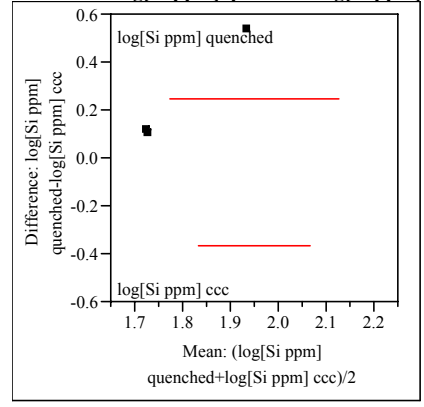

$\log [$ Si ppm] quenched $1.91878 \quad$ t-Ratio 1.736279 $\begin{array}{lllll}\log [\mathrm{Si} \mathrm{ppm}] \mathrm{ccc} & 1.67054 & \mathrm{DF} & \\ & & 0.14298 & \text { Prob }>|t| & 0.2246\end{array}$ $\begin{array}{llll}\text { Mean Difference } & 0.24824 & \text { Prob }>|t| & 0.224 \\ \text { Std Error } & 0.14298 & \text { Prob }>t & 0.1123\end{array}$ $\begin{array}{llll}\text { Std Error } & 0.14298 & \text { Prob }>\text { t } & 0.1123 \\ \text { Upper95\% } & 0.86342 & \text { Prob }<\text { t } & 0.8877\end{array}$ Lower $95 \% \quad-0.3669$

Correlation $\quad-0.7259$ 


\section{Exhibit B7. Effects of Heat Treatment on PCT $\log (\mathrm{ppm})$-Response of Study Glasses}

Matched Pairs Glass \#=29

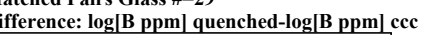

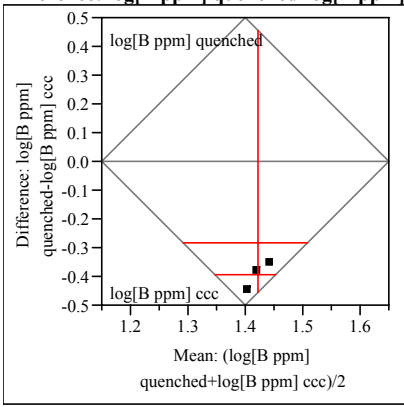

$\log [\mathrm{B}$ ppm] quenched $1.22296 \quad \mathrm{t}$-Ratio $\quad-14.7915$ $\begin{array}{llll}\log [\mathrm{B} \mathrm{ppm}] \mathrm{ccc} & 1.61917 & \mathrm{DF} & 2\end{array}$ \begin{tabular}{llll} 
Mean Difference & -0.3962 & Prob $>|t|$ & 0.0045 \\
\hline & 0.02679 & Prob $>t$ & 0.0977
\end{tabular} $\begin{array}{lrll}\text { Std Error } & 0.02679 & \text { Prob }>t & 0.9977 \\ \text { Upper95\% } & -0.281 & \text { Prob }<\mathrm{t} & 0.0023 \\ \text { Lower95\% } & -0.5115 & & \end{array}$ (1)

$\mathrm{N}$ 3
-0.4976

Difference: $\log [\mathbf{L i}$ ppm] quenched-log $[\mathbf{L i}$ ppm] ccc

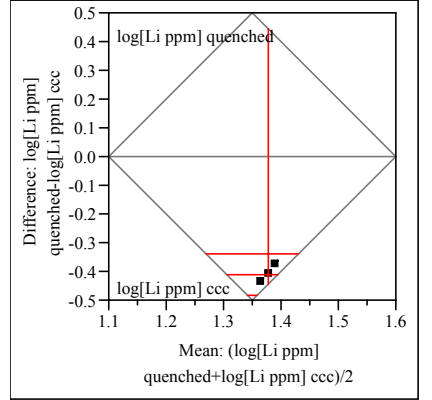

$\log [$ Li ppm] quenched $1.17244 \quad$ t-Ratio $\quad-23.92$

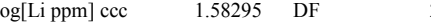
$\begin{array}{lll}\text { Mean Difference } & -0.4105 & \text { Prob }>|t| \\ 0.0017\end{array}$ $\begin{array}{llll}\text { Std Error } & 0.01716 & \text { Prob }>t & 0.999 \\ \text { Ut } & -0.3367 & \text { Prob } & \end{array}$ Upper $95 \%$ 0.4843 Correlation

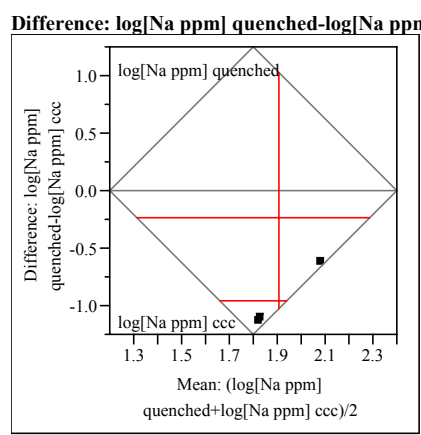

$\log [\mathrm{Na}$ ppm] quenched $1.42853 \quad \mathrm{t}$-Ratio $\quad-5.6756$ $\begin{array}{lllll}\log [\mathrm{Na} \text { ppm] } \mathrm{ccc} & 2.38422 & \mathrm{DF} & \end{array}$ $\begin{array}{llll} & -0.9557 & \text { Prob }>|t| & 0.0297\end{array}$ $\begin{array}{llll}\text { Std Error } & 0.16839 & \text { Prob }>t & 0.9852 \\ \text { Upper95\% } & -.2312 & \text { Pob } & 0.0148\end{array}$ $\begin{array}{rll}-0.2312 & \text { Prob }<\mathrm{t} & 0.0148\end{array}$ Lower95\%

Conction

Difference: $\log [\mathrm{Si}$ ppm] quenched-log[Si ppm] $\mathrm{ccc}$

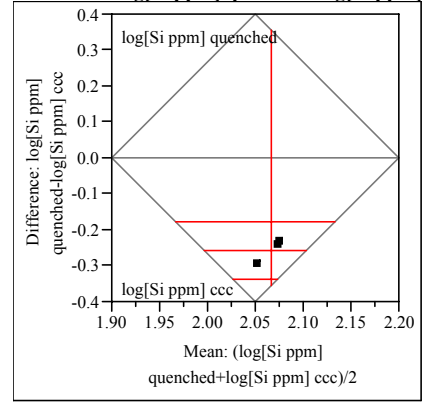

$\log [$ Si ppm] quenched $1.93692 \quad \mathrm{t}$-Ratio $\quad-13.8824$ $\begin{array}{llll}\log [\mathrm{Si} \mathrm{ppm}] \mathrm{ccc} & 2.19559 & \mathrm{DF} & 2\end{array}$ $\begin{array}{llll}\text { Mean Difference } & -0.2587 & \text { Prob }>|t| & 0.0051 \\ \text { Std } & 0.0163 & \text { Prob }>t & 0.9974\end{array}$ $\begin{array}{llll}\text { Std Error } & 0.01863 & \text { Prob }>t & 0.9974 \\ \text { Upper95\% } & -0.1785 & \text { Prob }<\mathrm{t} & 0.0026\end{array}$ Upper95\% $\mathrm{N}$ $-0.1785$ Correlation
Matched Pairs Glass \#=30

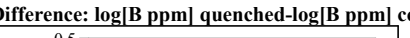

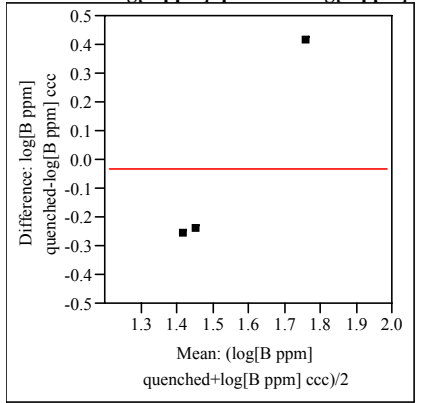

$\log [\mathrm{B}$ ppm] quenched $1.52666 \quad \mathrm{t}$-Ratio $\quad-0.14259$ $\begin{array}{llll}\log [\mathrm{B} \mathrm{ppm}] \mathrm{ccc} & 1.55816 & \mathrm{DF} & 2\end{array}$ $\begin{array}{llll}\text { Mean Difference } & -0.0315 & \text { Prob }>|t| & 0.8997\end{array}$ $\begin{array}{llll}\text { Std Error } & 0.22094 & \text { Prob }>\text { t } & 0.5502 \\ \text { Upper95\% } & 0.91911 & \text { Prob }<\text { t } & 0.4498\end{array}$

Lower95\%

$\mathrm{N}$ Correlation 0.982

Difference: $\log [\mathbf{L i} p p m]$ quenched-log $[\mathbf{L i}$ ppm] cce

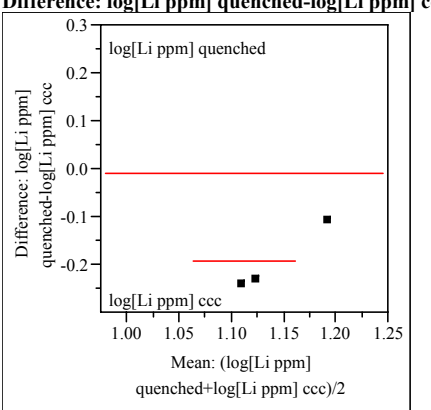

$\log [$ Li ppm] quenched 1.04417 t-Ratio $\quad-4.5516$ $\begin{array}{lrlr}\log [\mathrm{Li} \text { ppm] } \mathrm{ccc} & 1.23884 & \text { DF } & 2 \\ \text { Mean Difference } & -0.1947 & \text { Prob }>|t| & 0.0450\end{array}$ $\begin{array}{llll}\text { Mean Difference } & -0.1947 & \text { Prob }>|t| & 0.0450 \\ \text { Std Error } & 0.04277 & \text { Prob }>t & 0.9775\end{array}$ $\begin{array}{llll}\text { Std Error } & 0.04277 & \text { Prob }>\text { t } & 0.9775 \\ \text { Upper95\% } & -0.0106 & \text { Prob }<\mathrm{t} & 0.0225\end{array}$ Upper95\% $\mathrm{N}$ $-0.3787 \quad$ Prob $<1 \quad 0.0225$ Correlation

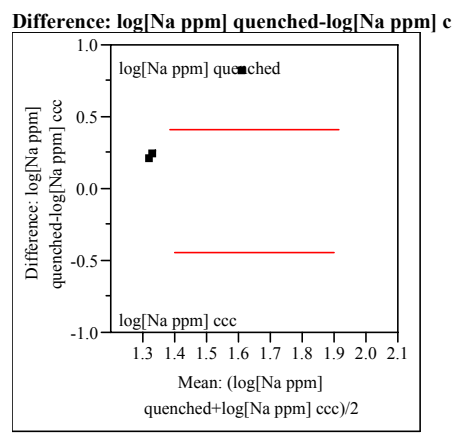

$\log [\mathrm{Na} p \mathrm{ppm}]$ quenched $1.62662 \quad \mathrm{t}$-Ratio $\quad 2.08003$ $\begin{array}{lllll}\log [\mathrm{Na} \text { ppm] ccc } & 1.21481 & \text { DF } & \end{array}$ Std Error $\begin{array}{llll}\text { Std Error } & 0.19798 & \text { Prob }>t & 0.0865 \\ \text { Upper5\% } & 1.26366 & \text { Prob }<\mathrm{t} & 0.9135\end{array}$ Lower95\%

Correlation -0.8892

Difference: $\log [\mathrm{Si}$ ppm] quenched-log[Si ppm] ccc

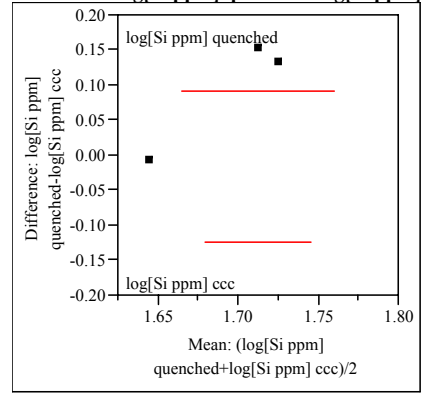

$\log [\mathrm{Si} \mathrm{ppm}]$ quenched 1.74009 t-Ratio 1.82322 $\log [\mathrm{Si} \mathrm{ppm}] \mathrm{ccc} \quad 1.64827 \quad \mathrm{DF}$

$\begin{array}{llll}1.64827 & \text { Drob }>|t| & 0.2098\end{array}$ $\begin{array}{llll}\text { Std Error } & 0.05036 & \text { Prob } & 0.1049 \\ \text { Upper95\% } & 0.30851 & \text { Prob }<t & 0.8951\end{array}$ Lower95\% $\quad-0.1249$

Correlation $\quad 0.00599$ 


\section{Exhibit B7. Effects of Heat Treatment on PCT $\log (\mathrm{ppm})$-Response of Study Glasses}

Matched Pairs Glass \#=31 Difference $\log [B$ ppm] quenched-log $[B$ ppm $]$ ccc

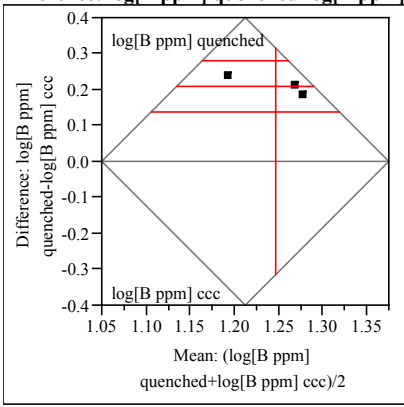

$\log [\mathrm{B}$ ppm] quenched $\quad 1.3513 \quad \mathrm{t}$-Ratio $\quad 12.85101$ $\begin{array}{llll}\log [\mathrm{B} \mathrm{ppm}] \mathrm{ccc} & 1.14159 & \mathrm{DF} & 2\end{array}$ $\begin{array}{llll}\text { Mean Difference } & 0.20971 & \text { Prob }>|t| & 0.0060\end{array}$ $\begin{array}{llll}\text { Std Error } & 0.01632 & \text { Prob }>\mathrm{t} & 0.0030 \\ \text { Upper95\% } & 0.27992 & \text { Prob }<\mathrm{t} & 0.9970\end{array}$ (1) 0.1395

Correlation $\quad 0.95814$

Difference: $\log [$ Li ppm] quenched-log $[\mathrm{Li} \mathrm{ppm]} \mathrm{ccc}$

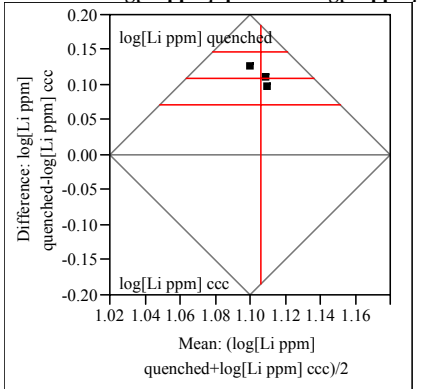

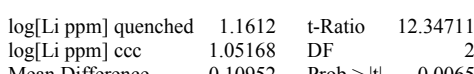
$\begin{array}{llll}\text { Std Error } & 0.00887 & \text { Prob }>|t| & 0.0065 \\ \text { Unt } & 0.1003 & 0.0032\end{array}$ $\begin{array}{llll}\text { Upper95\% } & 0.14769 & \text { Prob }<\text { t } & 0.9968\end{array}$ Lower95\% $\mathrm{N}$ Correlation 0.07136 3
-0.6797

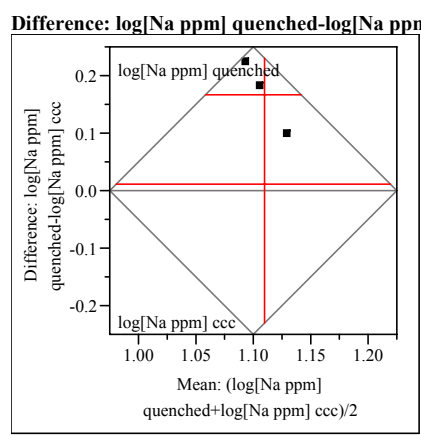

$\log [\mathrm{Na}$ ppm] quenched $1.19285 \quad \mathrm{t}$-Ratio $\quad 4.571295$ $\begin{array}{lllr}\log [\mathrm{Na} p \text { pm] ccc } & 1.02539 & \text { DF } & 2 \\ \text { Mean Difference } & 0.16746 & \text { Prob }>|t| & 0.0447\end{array}$ $\begin{array}{llll}\text { Std Error } & 0.03663 & \text { Prob }>\mathrm{t} & 0.0223 \\ \text { Upper95\% } & 0.32507 & \text { Prob }<\mathrm{t} & 0.9777\end{array}$ Lower $95 \%$ 0.00984

Correlation

Difference: $\log [\mathrm{Si}$ ppm] quenched-log[Si ppm] cce

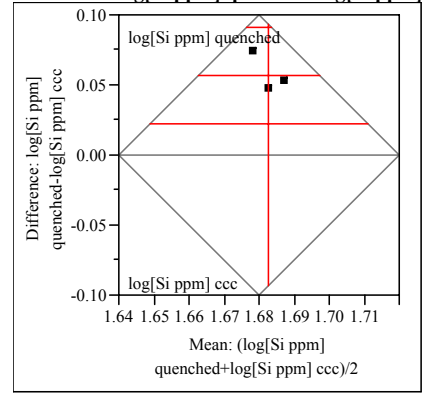

$\log [\mathrm{Si} \mathrm{ppm}]$ quenched 1.71133 t-Ratio 7.14406 $\log [\mathrm{Si} \mathrm{ppm}] \mathrm{ccc} \quad 1.6542$

$\begin{array}{lll}1.0572 & \text { Prob }>|t| & 0.0190\end{array}$ $\begin{array}{lrll}\text { Upper95\% } & 0.008 & \text { Prob }>t & 0.0095 \\ & 0.09153 & \text { Prob }<\text { t } & 0.9905\end{array}$ Lower95\% 0.02272

Correlation

$-0.5686$
Matched Pairs Glass \#=32 ifference: $\log [B$ ppm] quenched-log $[B$ ppm $]$ c

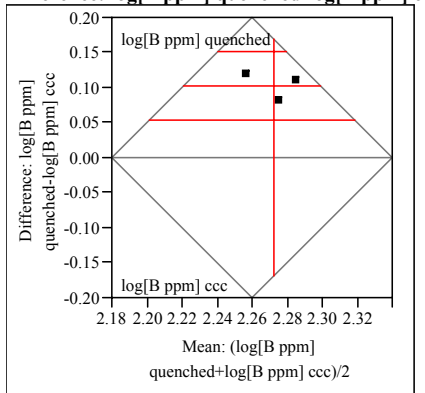

$\log [\mathrm{B}$ ppm] quenched $2.32323 \quad$ t-Ratio $\quad 9.182655$ $\begin{array}{lrlr}\log [\mathrm{B} \mathrm{ppm}] \mathrm{ccc} & 2.22097 & \mathrm{DF} & 2 \\ \text { Mean Difference } & 0.10226 & \text { Prob }>\text { tt } & 0.0117\end{array}$ $\begin{array}{llll}\text { Mean Difference } & 0.10226 & \text { Prob }>|t| & 0.0117 \\ \text { Std Error } & 0.01114 & \text { Prob }>t & 0.0058\end{array}$ $\begin{array}{llll}\text { Std Error } & 0.01114 & \text { Prob }>\mathrm{t} & 0.0058 \\ \text { Upper95\% } & 0.15018 & \text { Prob }<\mathrm{t} & 0.9942\end{array}$ Upper95\%

Correlation 0.40651 Difference: $\log [\mathrm{Li}$ ppm] quenched-log[Li ppm] cce

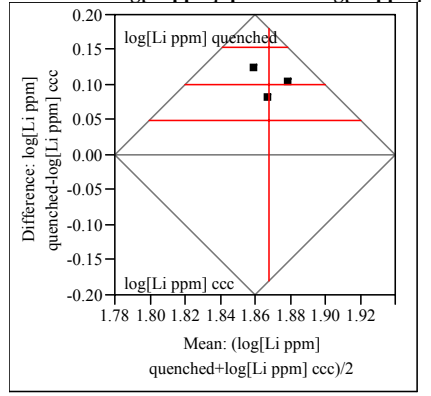

$\log [$ Li ppm] quenched $\quad 1.9187 \quad$ t-Ratio $\quad 8.272258$ $\begin{array}{llll}\log [\mathrm{Li} \text { ppm] ccc } & 1.81787 & \mathrm{DF} & 2\end{array}$ $\begin{array}{llll}0.10083 & \text { Prob }>|t| & 0.0143 \\ & 0.01219 & \text { Prob }>>t & 0.072\end{array}$ $\begin{array}{llll}\text { Std Error } & 0.01219 & \text { Prob }>\mathrm{t} & 0.0072 \\ \text { Upper95\% } & 0.15327 & \text { Prob }<\mathrm{t} & 0.9928 \\ \text { Lower95\% } & 0.04838 & & \end{array}$ Correlation

$-0.0643$

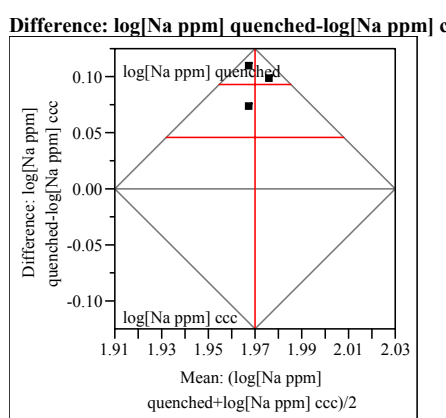

$\log [\mathrm{Na}$ ppm] quenched $2.01624 \quad$ t-Ratio 8.536903

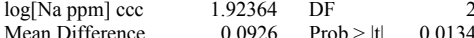
$\begin{array}{lrll}\text { Mean Difference } & 0.0926 & \text { Prob }>|t| & 0.0134 \\ \text { Std Error } & 0.01085 & \text { Prob }>t & 0.0067\end{array}$ $\begin{array}{llll}\text { Std Error } & 0.01085 & \text { Prob }>t & 0.0067 \\ \text { Upper95\% } & 0.13927 & \text { Prob }<\text { t } & 0.9933\end{array}$ Upper95\%

0.13927

Correlation $\quad-0.5664$

Difference: $\log [\mathrm{Si}$ ppm] quenched-log[Si ppm] $\mathrm{ccc}$

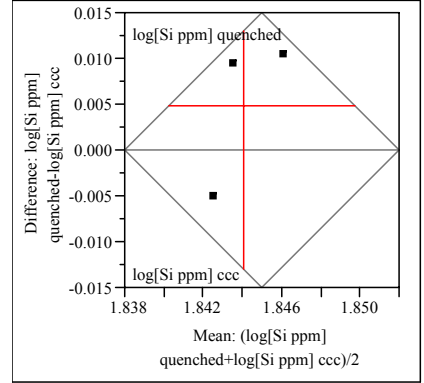

$\log [$ Si ppm] quenched $1.84646 \quad$ t-Ratio $\quad 0.96027$ $\begin{array}{lllll}\log [\mathrm{Si} \text { ppm] } \mathrm{ccc} & 1.84164 & \mathrm{DF} & 2\end{array}$ $\begin{array}{llll}\text { Mean Difference } & 0.00482 & \text { Prob }>|t| & 0.4382 \\ \text { Std Error } & 0.00502 & \text { Prob }>t & 0.2191\end{array}$ $\begin{array}{llll}\text { Std Error } & 0.00502 & \text { Prob }>\text { t } & 0.2191 \\ \text { Upper95\% } & 0.02641 & \text { Prob }<\text { t } & 0.7809\end{array}$ Upper $95 \%$ .0264 Correlation - -0.8257 


\section{Exhibit B7. Effects of Heat Treatment on PCT $\log (\mathrm{ppm})$-Response of Study Glasses}

Matched Pairs Glass \#=33

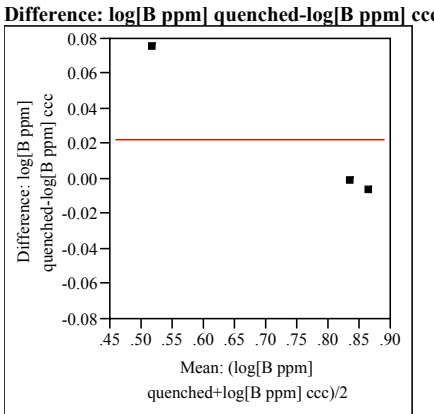

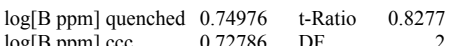
$\begin{array}{lrll}\log [\mathrm{B} p \mathrm{ppm}] \mathrm{ccc} & 0.72786 & \mathrm{DF} & 2 \\ \text { Mean Difference } & 0.0219 & \text { Prob }>|t| & 0.4949 \\ & 0.025 & \end{array}$ $\begin{array}{lrlll}\text { Mean Difference } & 0.0219 & \text { Prob }>|t| & 0.4949 \\ \text { Std Error } & 0.02646 & \text { Prob }>t & 0.2474\end{array}$ $\begin{array}{llll}\text { Std Error } & 0.02646 & \text { Prob }>\mathrm{t} & 0.2474 \\ \text { Upper95\% } & 0.13577 & \text { Prob }<\mathrm{t} & 0.7526\end{array}$ Upper95\%

0.13577

$\mathrm{N}$ Correlation .99999 Difference: $\log [$ Li ppm] quenched-log[Li ppm] cce

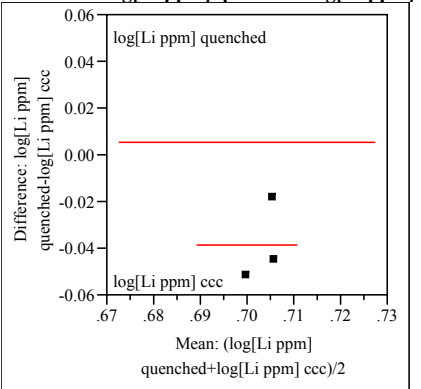

$\log [$ Li ppm] quenched $0.68416 \quad \mathrm{t}$-Ratio $\quad-3.77563$ $\begin{array}{llll}\text { log[Li ppm] ccc } & 0.72288 & \text { DF } & 2 \\ \text { Mean Difference } & -0.0387 & \text { Prob }>|t| & 0.0635\end{array}$ $\begin{array}{llll}\text { Std Error } & -0.0387 & \text { Prob }>|t| & 0.0635 \\ \text { Prob } & 0.01025 & \text { Prob }>t & 0.9682\end{array}$ $\begin{array}{llll}\text { Upper95\% } & 0.0054 & \text { Prob }<\text { t } & 0.0318\end{array}$ Lower $95 \%$ $-0.0828$

Correlation

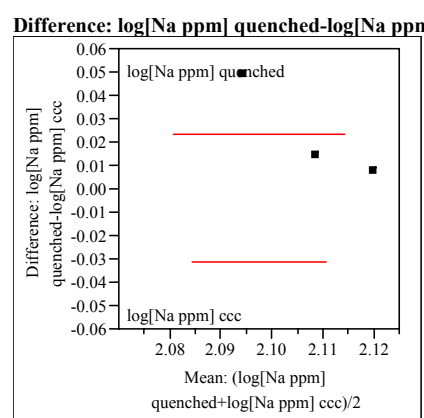

$\log [\mathrm{Na}$ ppm] quenched $2.11911 \quad \mathrm{t}$-Ratio $\quad 1.821401$ $\begin{array}{lllr}\log [\mathrm{Na} \text { ppm] ccc } & 2.09592 & \text { DF } & 2 \\ \text { Mean Difference } & 0.02319 & \text { Prob }>|t| & 0.2101\end{array}$ $\begin{array}{llll}\text { Mean Difference } & 0.02319 & \text { Prob }>|t| & 0.2101 \\ \text { Std Error } & 0.01273 & \text { Prob }>t & 0.1051\end{array}$ $\begin{array}{llll}\text { Std Error } & 0.01273 & \text { Prob }>t & 0.1051 \\ \text { Upper95\% } & 0.07797 & \text { Prob }<\text { t } & 0.8949\end{array}$ Upper $95 \%$ Lower95\% $-0.031$

Correlation 0.45924 Difference: $\log [\mathrm{Si}$ ppm] quenched-log[Si ppm] cce

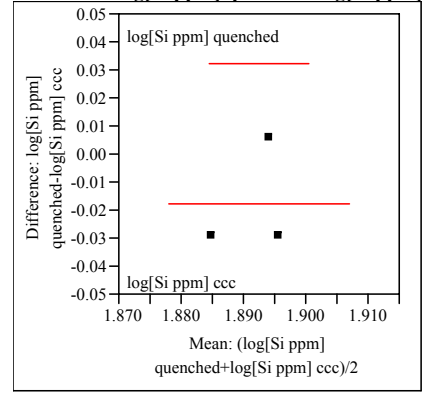

$\log [$ Si ppm] quenched $1.88259 \quad \mathrm{t}$-Ratio $\quad-1.52333$ $\begin{array}{llll}\log [\mathrm{Si} \text { ppm] ccc } & 1.90031 & \mathrm{DF} & 2 \\ \text { Mean Difference } & 0.0177 & \text { Prob }>|t| & 0.2671\end{array}$ Mean Differer
Std Error $\begin{array}{llll}\text { Upper95\% } & 0.03233 & \text { Prob }<\mathrm{t} & 0.1336\end{array}$ Lower95\% Correlation
Matched Pairs Glass \#=34

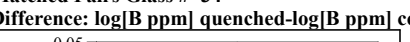

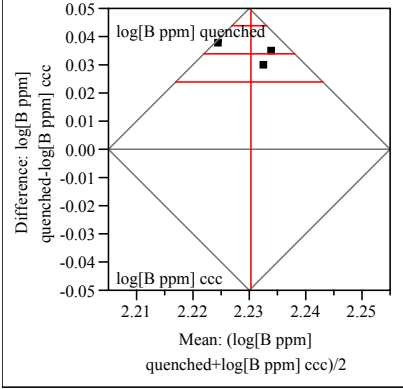

$\log [\mathrm{B}$ ppm $]$ quenched 2.24715 t-Ratio 14.47072 $\begin{array}{lllr}\log [\mathrm{B} \text { ppm] } \mathrm{ccc} & 2.21349 & \mathrm{DF} & 2 \\ \text { Mean Difference } & 0.03366 & \text { Prob }>\text { |t } & 0.0047\end{array}$ $\begin{array}{llll}\text { Mean Difference } & 0.03366 & \text { Prob }>|t| & 0.0047 \\ \text { Std Error } & 0.00233 & \text { Prob }>t & 0.0024\end{array}$ $\begin{array}{llll}\text { Std Error } & 0.00233 & \text { Prob }>\text { t } & 0.0024 \\ \text { Upper95\% } & 0.04366 & \text { Prob }<\text { t } & 0.9976\end{array}$

Correlation 0.81323 Difference: $\log [\mathrm{Li}$ ppm] quenched-log[Li ppm] cce

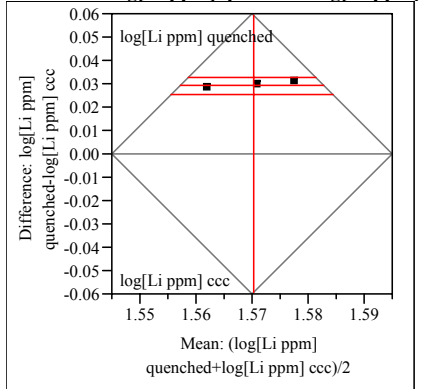

$\log [\mathrm{Li}$ ppm $]$ quenched $\quad 1.58479 \quad$ t-Ratio $\quad 35.45425$ $\begin{array}{llll}\log [\mathrm{Li} \mathrm{ppm}] \mathrm{ccc} & 1.5556 & \mathrm{DF} & 2\end{array}$ $\begin{array}{llll} & 0.02918 & \text { Prob }>|t| & 0.0008 \\ \text { Std Error } & 0.00082 & \text { Prob }>t & 0.0004\end{array}$ $\begin{array}{lrll}\text { Upper95\% } & 0.0008273 & \text { Prob }>\mathrm{t} & 0.0004 \\ \text { Urob }<\mathrm{t} & 0.9996\end{array}$ $\mathrm{N}$ 0.02564 3
0.99985

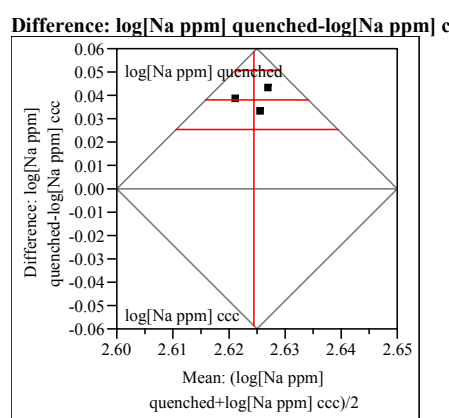

$\log [\mathrm{Na}$ ppm] quenched $2.64345 \quad \mathrm{t}$-Ratio $\quad 12.90315$ $\begin{array}{llll}\log [\mathrm{Na} p \mathrm{pm}] \mathrm{ccc} & 2.60566 & \mathrm{DF} & 2 \\ \text { Men Difference } & 0.03778 & \text { Prob }>|\mathrm{t}| & 0.0060\end{array}$ $\begin{array}{llll}\text { Mean Difference } & 0.03778 & \text { Prob }>|t| & 0.0060 \\ \text { Std Error } & 0.00293 & \text { Prob }>t & 0.0030\end{array}$ $\begin{array}{llll}\text { Std Error } & 0.00293 & \text { Prob }>\text { t } & 0.0030 \\ \text { Upper95\% } & 0.05038 & \text { Prob }<\text { t } & 0.9970\end{array}$ Upper $95 \%$ $\begin{array}{ll}\mathrm{N} & 3 \\ \mathrm{~N} & \\ \mathrm{Correlation} & 0.19286\end{array}$ Difference: $\log [\mathrm{Si}$ ppm] quenched-log[Si ppm] ccc

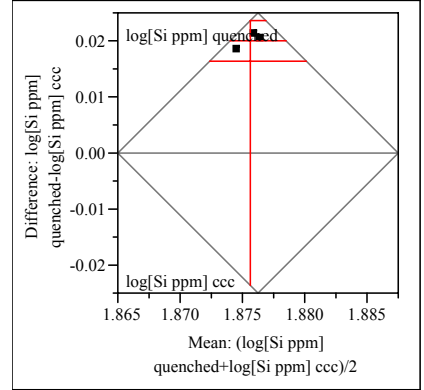

$\log [$ Si ppm] quenched $1.88556 \quad \mathrm{t}$-Ratio $\quad 24.02154$ $\begin{array}{llll}\log [\mathrm{Si} \mathrm{ppm]} \operatorname{ccc} & 1.86564 & \mathrm{DF} & 24 \\ \text { Mean Differec } & 0.01992 & \text { Prob }>|t| & 0.0017\end{array}$

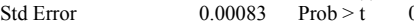
$\begin{array}{llll}\text { Upper95\% } & 0.02349 & \text { Prob }<\mathrm{t} & 0.999\end{array}$ 0.01635 


\section{Exhibit B7. Effects of Heat Treatment on PCT $\log (\mathrm{ppm})$-Response of Study Glasses}

Matched Pairs Glass \#=35

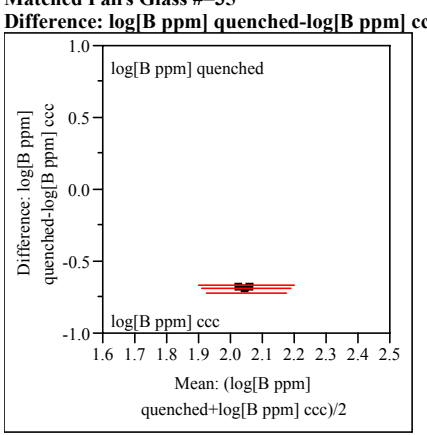

$\log [\mathrm{B}$ ppm] quenched $\quad 1.69629 \quad \mathrm{t}$-Ratio $\quad-122.207$ $\begin{array}{llll}\log [\mathrm{B} p \mathrm{ppm}] \mathrm{ccc} & 2.38986 & \mathrm{DF} & \\ \text { Mean Difference } & 0.0968 & \mathrm{PF} & \end{array}$ \begin{tabular}{llll} 
Std Error & -0.6936 & Prob $>|t|$ & $<.000$ \\
\hline & 0.00568 & Prob $>t$ & 1.000
\end{tabular} $\begin{array}{llll}\text { Upper95\% } & -0.6692 & \text { Prob }<\mathrm{t} & <.000\end{array}$

Correlation

$$
\begin{array}{r}
3 \\
0.87164
\end{array}
$$

Difference: $\log [$ Li ppm] quenched-log $[$ Li ppm] cec

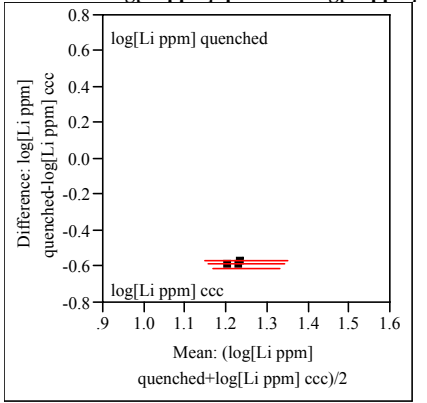

$\log [$ Li ppm] quenched $0.92718 \quad$ t-Ratio $\quad-100.502$ $\begin{array}{lrlr}\log [\mathrm{Li} \text { ppm] ccc } & 1.51762 & \text { DF } & 2 \\ \text { Mean Difference } & -0.5904 & \text { Prob }>|t| & <.0001\end{array}$ $\begin{array}{llll}\text { Std Error } & 0.00587 & \text { Prob }>t & 1.0000\end{array}$ $\begin{array}{llll}\text { Upper95\% } & -0.5652 & \text { Prob }<\text { t } & <.0001 \\ \text { Lower95\% } & -0.6157 & & \end{array}$ Der95\% Correlation

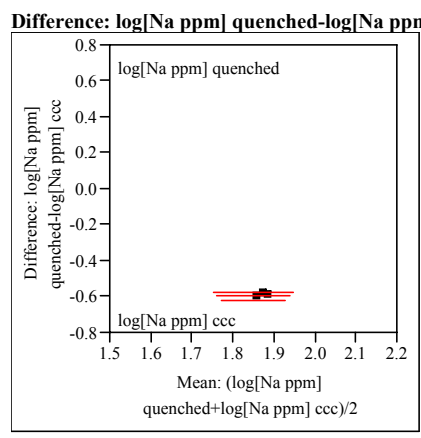

$\log [$ Na ppm] quenched $\quad 1.5725 \quad \mathrm{t}$-Ratio $\quad-108.138$

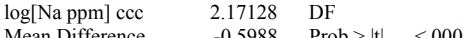
Std Error $\quad 0.00554 \quad$ Prob $>1$ $\begin{array}{ccc}-0.575 & \text { Prob }<\mathrm{t} & <.000\end{array}$

Correlation $\quad 0.86292$

Difference: $\log [\mathrm{Si}$ ppm] quenched-log[Si ppm] cce

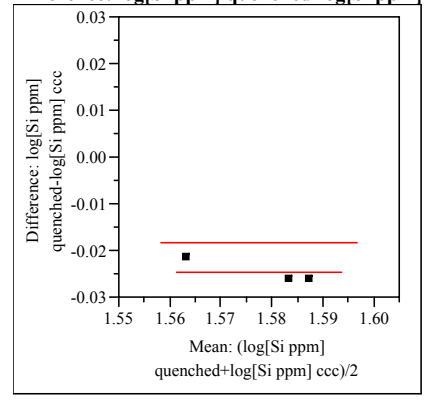

$\log [$ Si ppm] quenched 1.56549 t-Ratio -16.304 $\log [\mathrm{Si} \mathrm{ppm}] \mathrm{ccc} \quad 1.5903 \quad \mathrm{DF} \quad 1.3041$ $\begin{array}{llllll}1.5028 & \text { DF } & 0.0037\end{array}$ Upper $95 \%$ Prob $<t \quad 0.0019$ $-0.0314$

Correlation 0.99924
Tatched Pairs Glass \#=36 Difference: $\log \left[\mathbf{B}\right.$ ppm] quenched-log $\left[\mathbf{B}\right.$ ppm] $\mathrm{cec}^{-}$

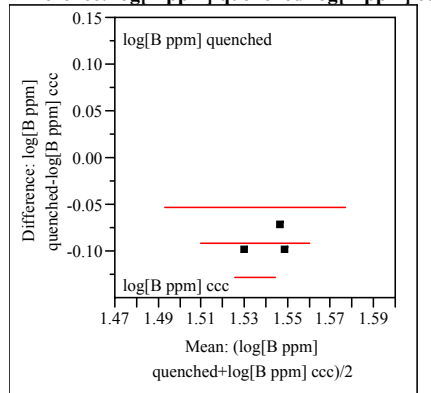

$\log [\mathrm{B}$ ppm] quenched $1.49584 \quad \mathrm{t}$-Ratio $\quad-10.4455$

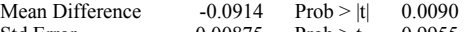
$\begin{array}{llll}\text { Std Error } & 0.00875 & \text { Prob }>t & 0.9955 \\ \text { Upper95\% } & -0.0538 & \text { Prob } & 0.0045\end{array}$ $\begin{array}{llll}\text { Upper95\% } & -0.0538 & \text { Prob }<\mathrm{t} & 0.0045 \\ \text { Lower95\% } & -0.1291 & & \end{array}$ 3
0.32915 Correlation Difference: $\log [\mathrm{Li}$ ppm] quenched-log[Li ppm] ccc

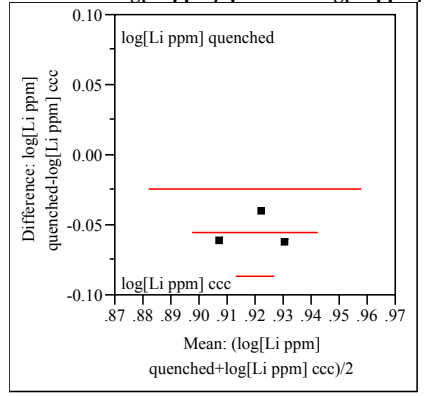

$\log [$ Li ppm] quenched $0.89196 \quad$ t-Ratio $\quad-7.76365$ $\begin{array}{lrlr}\log [\text { Li ppm] ccc } & 0.94792 & \text { DF } & 2 \\ \text { Mean Difference } & & 0.056 & \text { P }\end{array}$ $\begin{array}{lrlll} & & -0.056 & \text { Prob }>t \mid & 0.0162 \\ \text { Std Error } & 0.00721 & \text { Prob }>t & 0.9919\end{array}$ $\begin{array}{lrll}\text { Upper95\% } & -0.0249 & \text { Prob }<\mathrm{t} & 0.0081\end{array}$ Lower95\% $-0.087$ Correlation

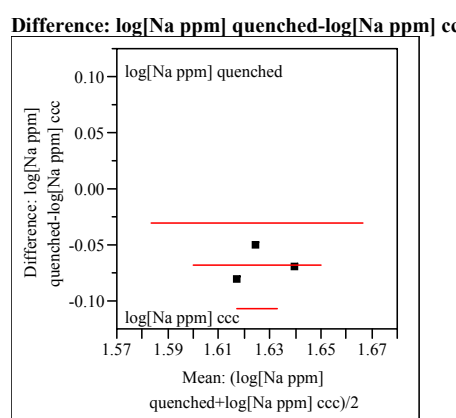

$\log [\mathrm{Na}$ ppm] quenched $1.59276 \quad \mathrm{t}$-Ratio $\quad-7.64874$ $\begin{array}{llll}\log [\mathrm{Na} p \mathrm{ppm}] \mathrm{ccc} & 1.66106 & \mathrm{DF} & -7.6487 \\ \text { Mean Difference } & -0.0683 & \text { Prob }>\text { tt } & 0.0167\end{array}$ $\begin{array}{llll}\text { Std Error } & -0.0683 & \text { Prob }>|t| & 0.0167 \\ \text { S } & 0.00893 & \text { Prob }>t & 0.9917\end{array}$ Upper95\% $\quad-0.0299 \quad$ Prob $<$ t $\quad 0.0083$ Lower95\% $-0.0299$ Correlation .38129 Difference: $\log [\mathrm{Si}$ ppm] quenched-log[Si ppm] cce

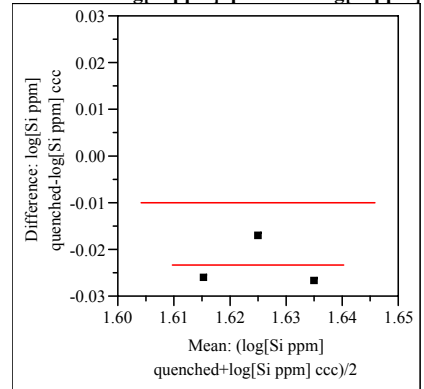

$\log [$ Si ppm] quenched $\quad 1.6133 \quad$ t-Ratio $\quad-7.4621$

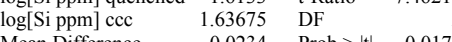
Std Error $\begin{array}{llll}\text { Upper } 95 \% & -0.0099 & \text { Prob }<\mathrm{t} \quad 0.008\end{array}$ Lower $95 \%$

0.85883 


\section{Exhibit B7. Effects of Heat Treatment on PCT $\log (\mathrm{ppm})$-Response of Study Glasses}

Matched Pairs Glass \#=37 Difference: $\log [\mathbf{B}$ ppm] quenched-log $[\mathbf{B}$ ppm] $\mathbf{c c c}$

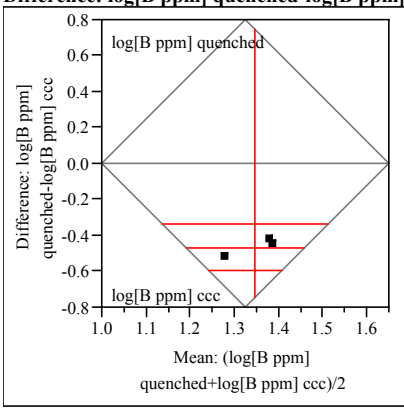

$\log [\mathrm{B}$ ppm] quenched $1.11413 \quad \mathrm{t}$-Ratio $\quad-15.3933$

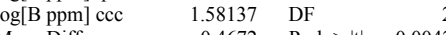
$\begin{array}{llll}\text { Mean Difference } & -0.4672 & \text { Prob }>|t| & 0.0042\end{array}$ $\begin{array}{llll}\text { Std Error } & 0.03035 & \text { Prob }>t & 0.9979 \\ \text { Upper95\% } & -0.3366 & \text { Prob }<\mathrm{t} & 0.0021 \\ \text { Lower95\% } & -0.5978 & & \end{array}$ (1)

Correlation 3

Difference: $\log [\mathbf{L i}$ ppm] quenched-log $[\mathbf{L i}$ ppm] $\mathbf{c c c}$

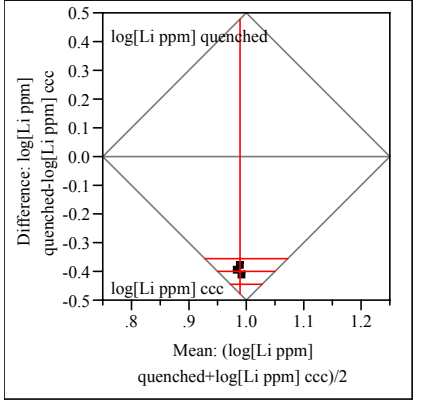

$\log [$ Li ppm] quenched $0.78805 \quad$ t-Ratio $\quad-40.0206$

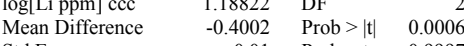
$\begin{array}{lrll}\text { Std Error } & 0.01 & \text { Prob }>t & 0.9997 \\ \text { Upper95\% } & -0.3571 & \text { Prob }<\mathrm{t} & 0.0003 \\ & & & \end{array}$ $\mathrm{N}$ $\begin{array}{ccc}-0.3571 & \text { Prob }<\mathrm{t} & 0.0003 \\ -0.4432 & & \end{array}$ Correlation

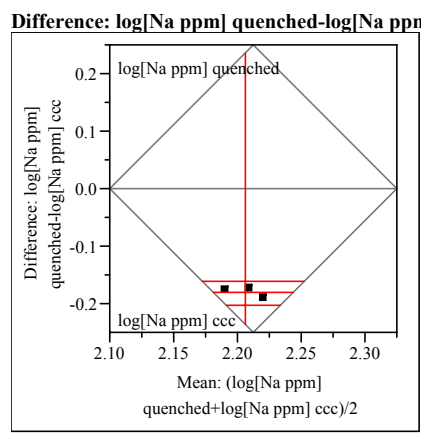

$\log [$ Na ppm] quenched $\quad 2.1157 \quad$ t-Ratio $\quad-36.4016$ $\begin{array}{llll}\log [\mathrm{Na} \text { ppm] } \mathrm{ccc} & 2.29715 & \mathrm{DF} & \end{array}$ $\begin{array}{llll}\text { Mean Difference } & -0.1815 & \text { Prob }>|t| & 0.0008\end{array}$ $\begin{array}{lrlll}\text { Std Error } & 0.00498 & \text { Prob }>t & 0.9996 \\ \text { Upper95\% } & -0.16 & \text { Prob }<\text { t } & 0.0004\end{array}$

Lower $95 \%$

Difference: $\log [\mathrm{Si}$ ppm] quenched-log[Si ppm] ccc

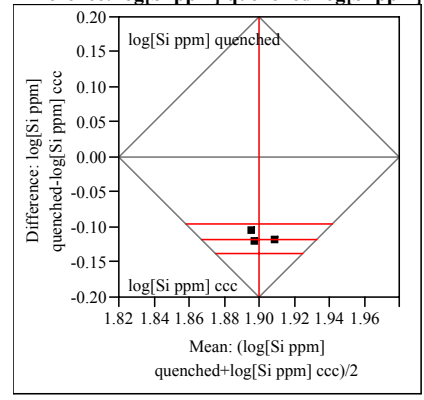

$\log [$ Si ppm] quenched $1.84196 \quad \mathrm{t}$-Ratio $\quad-22.926$ $\begin{array}{llll}\log [\mathrm{Si} \mathrm{ppm}] \mathrm{ccc} & 1.9587 & \mathrm{DF} & 2\end{array}$

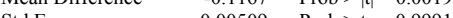
$\begin{array}{llll}\text { Std Error } & 0.00509 & \text { Prob }>\text { t } & 0.9991 \\ \text { Upper95\% } & -0.0948 & \text { Pob } & 0.1\end{array}$ Lower95\% $-0.1386$

Correlation

0.52864
Matched Pairs Glass $\#=38$ Difference: $\log [\mathrm{B}$ ppm] quenched-log $[\mathrm{B} \mathrm{ppm}] \mathrm{ccc}$

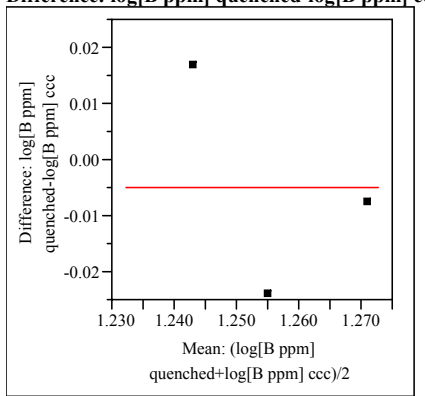

$\log [\mathrm{B} p p m]$ quenched $1.25382 \quad \mathrm{t}$-Ratio $\quad-0.43277$ $\begin{array}{llll}\log [\mathrm{B} \text { ppm] } \mathrm{ccc} & 1.25894 & \mathrm{DF} & 2 \\ \text { Mean Difference } & -0.0051 & \text { Prob }>|t| & 0.7074\end{array}$ $\begin{array}{llll}\text { Mean Difference } & -0.0051 & \text { Prob }>|t| & 0.7074 \\ \text { Std Error } & 0.01182 & \text { Prob }>t & 0.6463\end{array}$ $\begin{array}{llll}\text { Std Error } & 0.01182 & \text { Prob }>t & 0.6463 \\ \text { Upper } 95 \% & 0.04573 & \text { Prob }<\mathrm{t} & 0.3537\end{array}$ Lower95\%

$\mathrm{N}$$$
-0.056
$$

Correlation 3
0.35774 Difference: $\log [\mathrm{Li}$ ppm] quenched-log[Li ppm] ccc

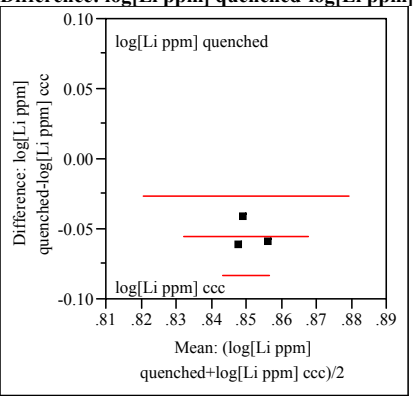

$\log [$ Li ppm] quenched $0.82352 \quad$ t-Ratio $\quad-8.39666$ $\begin{array}{lrlr}\log [\mathrm{Li} \text { ppm] ccc } & 0.87854 & \text { DF } & 2 \\ \text { Mean Difference } & -0.055 & \text { Prob }>\text { |t } & 0.0139\end{array}$ $\begin{array}{lrll} & -0.055 & \text { Prob }>|t| & 0.0139 \\ \text { Std Error } & 0.00655 & \text { Prob }>t & 0.9931\end{array}$ $\begin{array}{llll}\text { Upper95\% } & -0.0268 & \text { Prob }<\mathrm{t} & 0.0069\end{array}$ Lower95\% $-0.0832$

Correlation

$-0.2072$

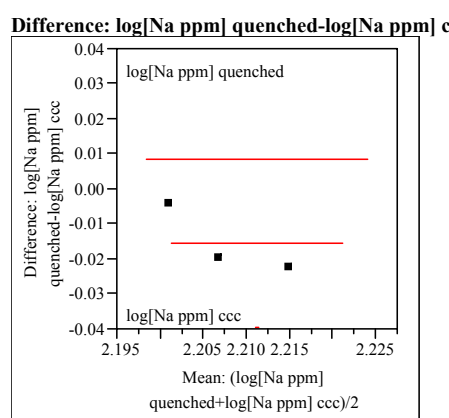

$\log [\mathrm{Na}$ ppm] quenched $2.19972 \quad \mathrm{t}$-Ratio $\quad-2.79623$ $\begin{array}{llll}\log [\mathrm{Na} \text { ppm] } \mathrm{ccc} & 2.21534 & \mathrm{DF} & 2 \\ \text { Men Difference } & -0.0156 & \text { Prob }>|t| & 0.1076\end{array}$ $\begin{array}{llll}\text { Mean Difference } & -0.0156 & \text { Prob }>|t| & 0.1076 \\ \text { Std Error } & 0.00558 & \text { Prob }>t & 0.9462\end{array}$ $\begin{array}{llll}\text { Std Error } & 0.00558 & \text { Prob }>\mathrm{t} & 0.9462 \\ \text { Upper95\% } & 0.00841 & \text { Prob }<\mathrm{t} & 0.0538\end{array}$ Upper $95 \%$ 0.00841

Correlation

0.63589

Difference: $\log [\mathrm{Si}$ ppm] quenched-log[Si ppm] ccc

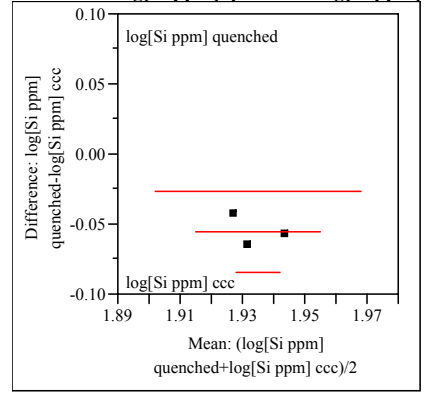

$\log [\mathrm{Si}$ ppm] quenched $\quad 1.90635 \quad$ t-Ratio $\quad-8.1613$ $\begin{array}{lrlr}\log [\mathrm{Si} \text { ppm] ccc } & 1.96184 & \mathrm{DF} & 2 \\ \text { Mean Difference } & -0.0555 & \text { Prob }>|t| & 0.0147\end{array}$ $\begin{array}{llll}\text { Std Error } & 0.0068 & \text { Prob }>t \quad 0.9927\end{array}$ $\begin{array}{llll}\text { Upper } 95 \% & -0.0262 & \text { Prob }<\mathrm{t} & 0.0073\end{array}$ -0.0847
3

0.37604 


\section{Exhibit B7. Effects of Heat Treatment on PCT $\log (\mathrm{ppm})$-Response of Study Glasses}

Matched Pairs Glass \#=39

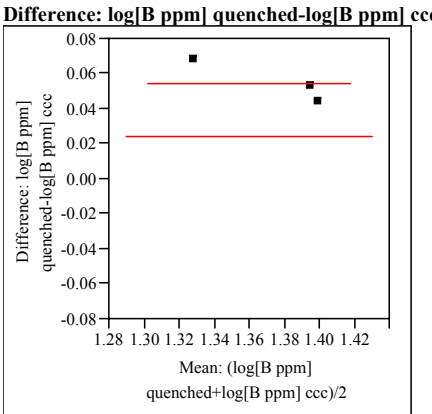

$\log [\mathrm{B}$ ppm] quenched $1.40092 \quad$ t-Ratio $\quad 7.586194$ $\begin{array}{lrlr}\log [\mathrm{B} \text { ppm] } \mathrm{ccc} & 1.34631 & \mathrm{DF} & 2 \\ \text { Mean Difference } & 0.05462 & \text { Prob }>|t| & 0.0169\end{array}$ $\begin{array}{lrll}\text { Mean Difference } & 0.05462 & \text { Prob }>|t| & 0.0169 \\ \text { Std Error } & 0.0072 & \text { Prob }>t & 0.0085 \\ \text { L } & 0.0236 & & \end{array}$ $\begin{array}{lrll}\text { Std Error } & 0.0072 & \text { Prob }>\mathrm{t} & 0.0085 \\ \text { Upper95\% } & 0.08559 & \text { Prob }<\mathrm{t} & 0.9915\end{array}$

Correlation 0.99492 Difference: $\log [\mathrm{Li}$ ppm] quenched-log[Li ppm] cce

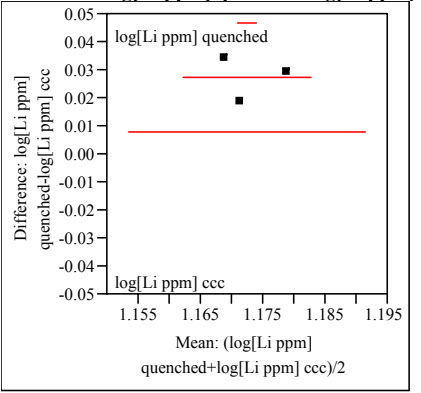

$\log [\mathrm{Li}$ ppm $]$ quenched $\quad 1.1864 \quad \mathrm{t}$-Ratio $\quad 6.009573$ $\begin{array}{llll}\log [\mathrm{Li} \text { ppm }] \mathrm{ccc} & 1.15934 & \mathrm{DF} & 2\end{array}$ $\begin{array}{llll}0.02706 & \text { Prob }>|t| & 0.0266\end{array}$ $\begin{array}{lrll}\text { Std Error } & 0.0045 & \text { Prob }>t & 0.0133 \\ \text { Upper95\% } & 0.04643 & \text { Prob }<\mathrm{t} & 0.9867\end{array}$ ( 0.00769

Correlation

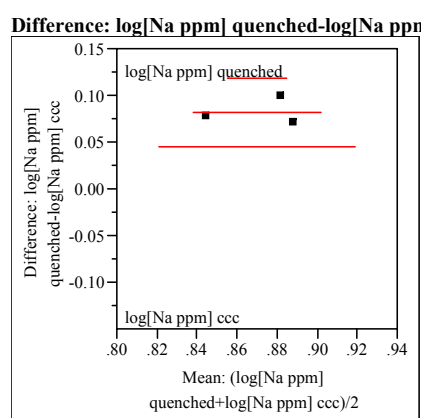

$\log [\mathrm{Na}$ ppm] quenched $0.91185 \quad \mathrm{t}$-Ratio $\quad 9.495575$ $\begin{array}{llll}\log [\mathrm{Nappm}] \mathrm{ccc} & 0.83067 & \mathrm{DF} & 2 \\ \text { Mean Difference } & 0.08118 & \text { P } & 0.0109\end{array}$ $\begin{array}{llll}\text { Mean Difference } & 0.08118 & \text { Prob }>|t| & 0.0109 \\ \text { Std Error } & 0.00855 & \text { Prob }>t & 0.0055\end{array}$ $\begin{array}{llll}\text { Std Error } & 0.00855 & \text { Prob }>t & 0.0055 \\ \text { Upper95\% } & 0.11797 & \text { Prob }<\text { t } & 0.9945\end{array}$ Upper95\% 0.11797 Prob $<t \quad 0.9945$

Correlation 3
0.82107

Difference: $\log [\mathbf{S i}$ ppm] quenched-log[Si ppm] ccc

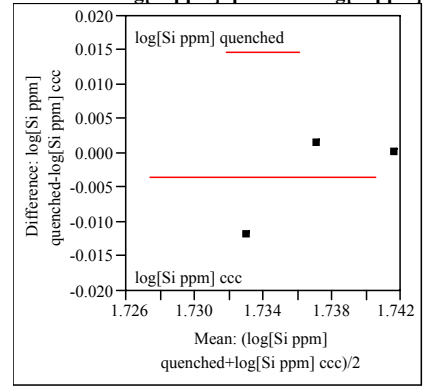

$\log [\mathrm{Si}$ ppm] quenched $1.73547 \quad \mathrm{t}$-Ratio $\quad-0.84006$

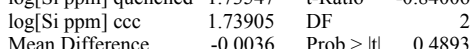
$\begin{array}{llll}\text { Mean Difference } & -0.0036 & \text { Prob }>|t| & 0.4893\end{array}$ $\begin{array}{llll}\text { Std Error } & 0.00425 & \text { Prob }>t & 0.7554 \\ \text { Upper95\% } & 0.01473 & \text { Prob }<\text { t } & 0.2446\end{array}$ Upper $95 \%$ $\mathrm{N}$ Correlation 0.25863
Matched Pairs Glass \#=40 Difference: $\log [\mathrm{B}$ ppm] quenched-log[B ppm] $\mathrm{c}$

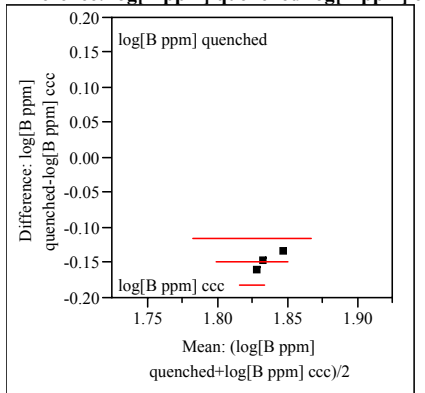

$\log [\mathrm{B} \mathrm{ppm}]$ quenched $1.76156 \quad \mathrm{t}$-Ratio $\quad-18.9705$ $\begin{array}{llll}\text { Mean Difference } & -0.1487 & \text { Prob }>|t| & 0.0028\end{array}$ $\begin{array}{lrll}\text { Std Error } & 0.00784 & \text { Prob }>\text { t } & 0.9986 \\ \text { Upper95\% } & -0.115 & \text { Prob }<\mathrm{t} & 0.0014\end{array}$ Upper95\%

$\mathrm{N}$ $\begin{array}{lll}-0.115 & \text { Prob }<\mathrm{t} & 0.0014 \\ -0.1824 & & \end{array}$ Correlation 0.76927 Difference: $\log [\mathrm{Li}$ ppm] quenched-log[Li ppm] ccc

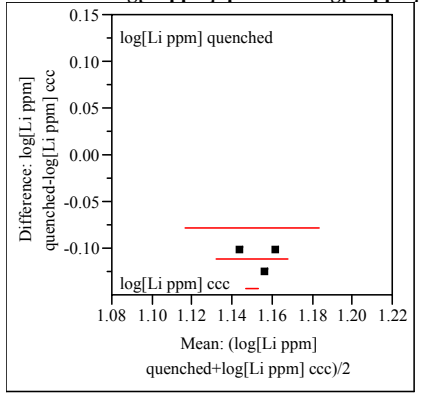

$\log [\mathrm{Li}$ ppm] quenched $1.09838 \quad \mathrm{t}$-Ratio $\quad-14.6096$ $\begin{array}{lllr}\log [\text { Li ppm] ccc } & 1.20956 & \text { DF } & 2 \\ \text { Mean Difference } & -0.1112 & \text { Prob }>\text { tt } & 0.0047\end{array}$ $\begin{array}{lll}0.00761 & \text { Prob }>t & 0.9977\end{array}$ $\begin{array}{llll}\text { Upper95\% } & -0.0784 & \text { Prob }<\mathrm{t} & 0.0023\end{array}$ Lower95\% $-0.1439$

Correlation

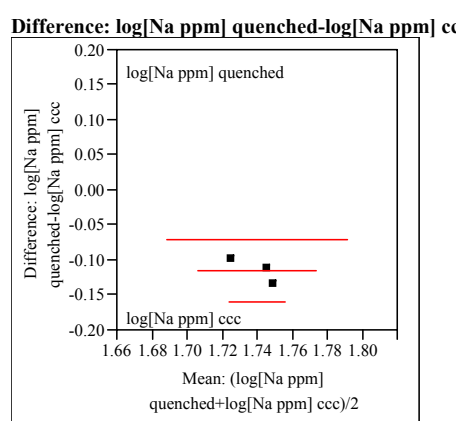

$\log [\mathrm{Na}$ ppm] quenched $1.68172 \quad \mathrm{t}$-Ratio $\quad-11.2277$ $\begin{array}{llll}\log [\mathrm{Na} p \mathrm{ppm}] \mathrm{ccc} & 1.79785 & \mathrm{DF} & 2 \\ \text { Mean Difference } & -0.1161 & \text { Prob }>\text { t } & 0.0078\end{array}$ $\begin{array}{llll}\text { Mean Difference } & -0.1161 & \text { Prob }>|t| & 0.0078 \\ \text { Std Error } & 0.01034 & \text { Prob }>t & 0.9961\end{array}$ $\begin{array}{llll}\text { Std Error } & 0.01034 & \text { Prob }>t & 0.9961 \\ \text { Upper95\% } & -0.0716 & \text { Prob }<\mathrm{t} & 0.0039\end{array}$ Upper $95 \%$ $-0.0716$ Correlation 0.61771 Difference: $\log [\mathrm{Si}$ ppm] quenched-log[Si ppm] ccc

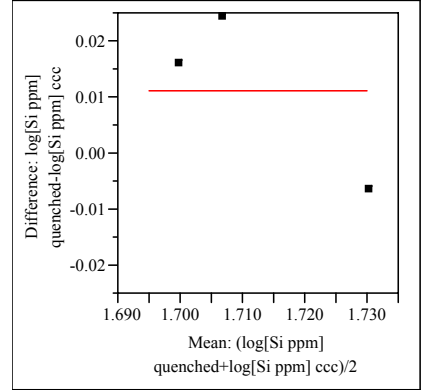

$\log [$ Si ppm] quenched $1.71779 \quad \mathrm{t}$-Ratio $\quad 1.203059$ $\begin{array}{lllll}\log [\mathrm{Si} \mathrm{ppm}] \mathrm{ccc} & 1.70668 & \mathrm{DF} & & \\ \text { Mean Difference } & 0.01111 & \text { Prob }>|t| & 0.3520\end{array}$ $\begin{array}{llll}\text { Std Error } & 0.00924 & \text { Prob }>t & 0.1760\end{array}$ $\begin{array}{llll}\text { Upper95\% } & 0.05086 & \text { Prob }<\mathrm{t} & 0.8240\end{array}$ $-0.0286$ $\begin{array}{ll}\mathrm{N} \\ \text { Correlation } & 0.85271\end{array}$ 


\section{Exhibit B7. Effects of Heat Treatment on PCT $\log (\mathrm{ppm})$-Response of Study Glasses}

Matched Pairs Glass \#=41

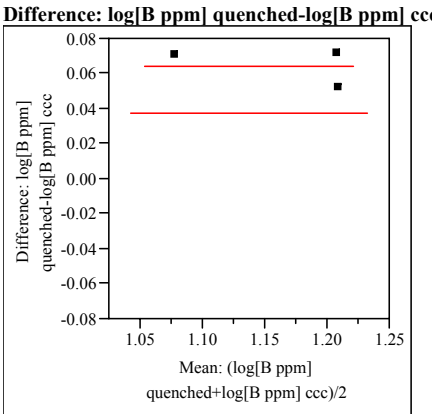

$\log [\mathrm{B} \mathrm{ppm}]$ quenched $1.19686 \quad \mathrm{t}$-Ratio $\quad 10.37207$ $\begin{array}{lrlr}\log [\mathrm{B} \text { ppm] ccc } & 1.13258 & \mathrm{DF} & 2 \\ \text { Mean Difference } & 0.06428 & \text { Prob }>|\mathrm{t}| & 0.0092\end{array}$ $\begin{array}{lrll}\text { Mean Difference } & 0.06428 & \text { Prob }>|t| & 0.0092 \\ \text { Std Error } & 0.0062 & \text { Prob }>t & 0.0046 \\ \text { L } & 0.0956 & & \end{array}$ $\begin{array}{lrll}\text { Std Error } & 0.0062 & \text { Prob > t } & 0.0046 \\ \text { Upper95\% } & 0.09094 & \text { Prob }<\text { t } & 0.9954\end{array}$

$\mathrm{N}$.

Difference: $\log [$ Li ppm] quenched-log[Li ppm] cce

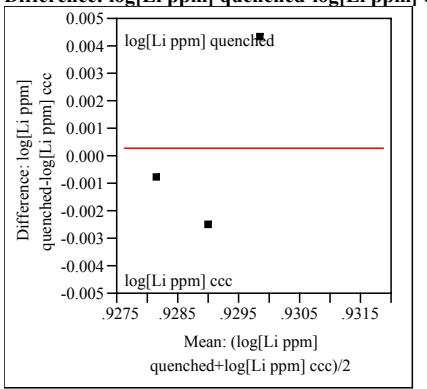

$\log [$ Li ppm] quenched $0.92914 \quad \mathrm{t}$-Ratio $\quad 0.137218$ $\log [\mathrm{Li} \mathrm{ppm}] \mathrm{ccc}-0.92886 \quad \mathrm{DF} \quad 2$ $\begin{array}{llll}\text { Mean Difference } & 0.00028 & \text { Prob }>|t| & 0.9034 \\ \text { Std Error } & 0.00205 & \text { Prob }>t & 0.4517\end{array}$ $\begin{array}{llll}\text { Std Error } & 0.00205 & \text { Prob }>\mathrm{t} & 0.4517 \\ \text { Upper95\% } & 0.00909 & \text { Prob }<\mathrm{t} & 0.5483\end{array}$ Upper95\% $-0.0085$ Correlation

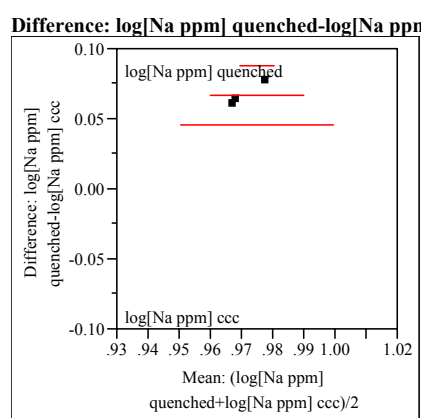

$\log [\mathrm{Na}$ ppm] quenched $1.00425 \quad \mathrm{t}$-Ratio $\quad 13.76067$ $\begin{array}{lrlr}\log [\mathrm{Na} \text { ppm] ccc } & 0.93758 & \text { DF } & 2 \\ \text { Mean Difference } & 0.06667 & \text { Prob }>|t| & 0.0052\end{array}$ $\begin{array}{llll}\text { Mean Difference } & 0.06667 & \text { Prob }>|t| & 0.0052 \\ \text { Std Error } & 0.00485 & \text { Prob }>t & 0.0026\end{array}$ $\begin{array}{llll}\text { Std Error } & 0.00485 & \text { Prob }>t & 0.0026 \\ \text { Upper95\% } & 0.08752 & \text { Prob }<\text { t } & 0.9974\end{array}$ Upper95\% 0.0458

Correlation 0.92511 Difference: $\log [$ Si ppm] quenched-log[Si ppm] cce

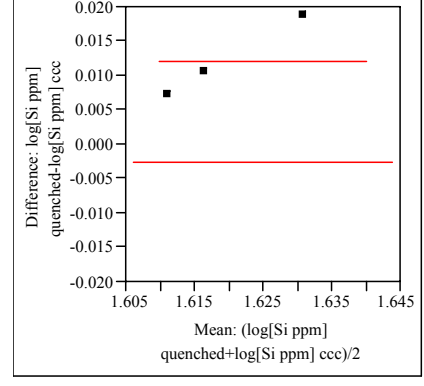

$\log [$ Si ppm] quenched $1.62541 \quad$ t-Ratio 3.529334

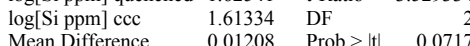
$\begin{array}{llll}\text { Mean Difference } & 0.01208 & \text { Prob }>|t| & 0.0717\end{array}$ $\begin{array}{lrll}\text { Std Error } & 0.00342 & \text { Prob }>t & 0.0359 \\ \text { Upper95\% } & 0.0268 & \text { Prob }<\text { t } & 0.9641 \\ \text { L } & & \end{array}$

Correlation
Matched Pairs Glass \#=42 ifferenged $[$ og ppm] quenched-log $[\mathrm{B}$ ppm $]$ c

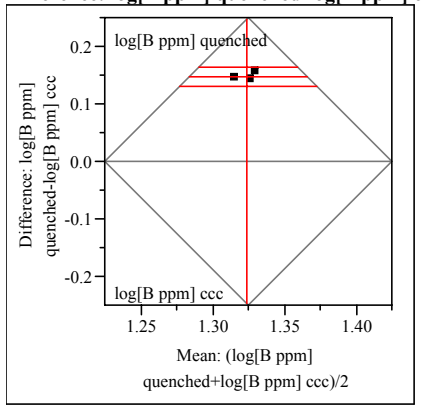

$\log [\mathrm{B}$ ppm] quenched $\quad 1.3969 \quad \mathrm{t}$-Ratio $\quad 35.57694$ $\begin{array}{llll}\operatorname{gg}[\mathrm{B} \mathrm{ppm}] \mathrm{ccc} & 1.24986 & \mathrm{DF} & 2\end{array}$

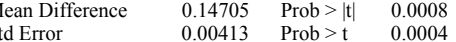
$\begin{array}{llll}\text { Std Error } & 0.00413 & \text { Prob }>t & 0.0004 \\ \text { Upper95\% } & 0.16483 & \text { Prob }<\text { t } & 0.9996\end{array}$ Lower95\%

$\mathrm{N}$ Correlation 3
0.72156

Difference: $\log [\mathrm{Li} \mathrm{ppm]}$ quenched-log $[\mathrm{Li}$ ppm] $\mathrm{ccc}$

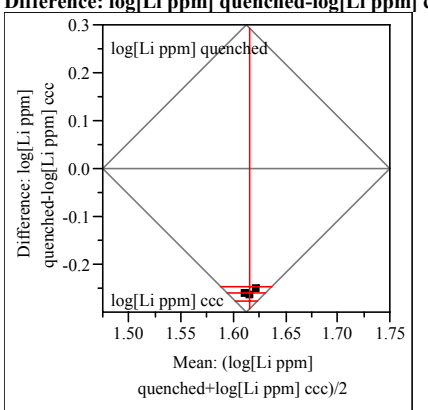

$\log [$ Li ppm] quenched $\quad 1.48505 \quad$ t-Ratio $\quad-68.9929$ $\begin{array}{lrlr}\log [\mathrm{Li} \text { ppm] } \mathrm{ccc} & 1.74646 & \text { DF } & 2 \\ \text { Mean Difference } & -0.2614 & \text { Prob }>|t| & 0.0002\end{array}$ $\begin{array}{llll}\text { Mean Difference } & -0.2614 & \text { Prob }>|t| & 0.0002 \\ \text { Std Error } & 0.00379 & \text { Prob }>t & 0.9999\end{array}$ $\begin{array}{llll}\text { Std Error } & 0.00379 & \text { Prob }>t & 0.9999 \\ \text { Upper95\% } & -0.2451 & \text { Prob }<\mathrm{t} & 0.0001\end{array}$ Upper95\% $\mathrm{N}$ Correlation

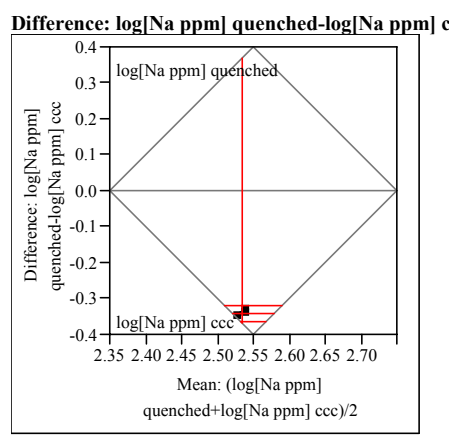

$\log [\mathrm{Na}$ ppm] quenched $2.36375 \quad$ t-Ratio $\quad-67.736$ $\begin{array}{llll}\log [\mathrm{Na} \text { ppm] } \mathrm{ccc} & 2.70662 & \mathrm{DF} & \end{array}$ $\begin{array}{ll}-0.3429 & \text { Prob }>|t| \\ 0.0 .0002 & 0.000\end{array}$

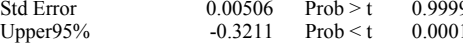
Lower $95 \%$

Correlation $-0.3646$

Difference: $\log [\mathrm{Si}$ ppm] quenched-log $[\mathrm{Si}$ ppm] ccc

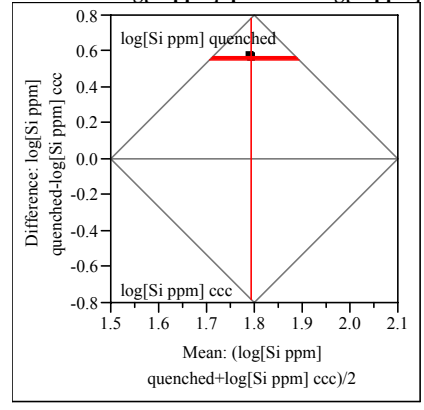

$\log [$ Si ppm] quenched $2.07209 \quad$ t-Ratio 200.441 $\begin{array}{llll}\log [\mathrm{Si} \text { ppm }] \mathrm{ccc} & 1.51188 & \mathrm{DF} & 2\end{array}$ $\begin{array}{llll}\text { Mean Difference } & 0.56021 & \text { Prob }>|t| & <.0001 \\ \text { Std Error } & 0.00279 & \text { Prob }>t & <.0001\end{array}$ $\begin{array}{llll}\text { Std Error } & 0.00279 & \text { Prob }>t & <.0001 \\ \text { Upper95\% } & 0.57224 & \text { Prob }<\mathrm{t} & 1.0000\end{array}$ Upper95\% $\quad 0.57224$ $\mathrm{N} \quad 3$ 


\section{Exhibit B7. Effects of Heat Treatment on PCT $\log (\mathrm{ppm})$-Response of Study Glasses}

Matched Pairs Glass \#=43

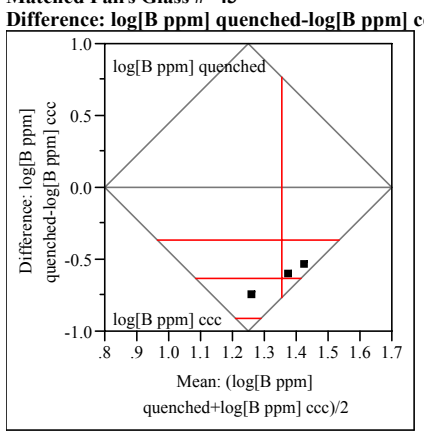

$\log [\mathrm{B}$ ppm] quenched $\quad 1.03612 \quad \mathrm{t}$-Ratio $\quad-9.99418$ $\begin{array}{llll}\log [\mathrm{B} \text { ppm }] \mathrm{ccc} & 1.67296 & \mathrm{DF} & 2\end{array}$ \begin{tabular}{llll} 
Mean Difference & -0.6368 & Prob $>|t|$ & 0.0099 \\
\hline & 0.06372 & Prob $>t$ & 0.995
\end{tabular} $\begin{array}{llll}\text { Std Error } & 0.06372 & \text { Prob }>t & 0.9951 \\ \text { Upper } 95 \% & -0.3627 & \text { Prob }<\mathrm{t} & 0.0049\end{array}$ $-0.91$

N 3
0.99931

Difference: $\log [$ Li ppm] quenched-log $[$ Li ppm] ccc

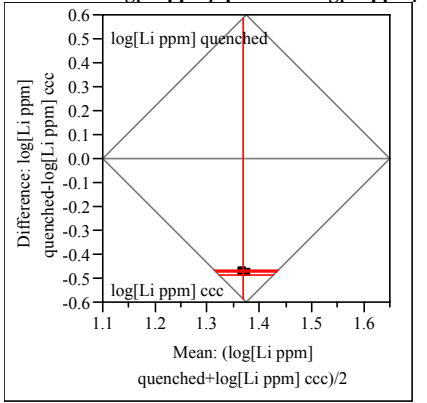

$\log [$ Li ppm] quenched $1.13175 \quad$ t-Ratio $\quad-180.592$ $\begin{array}{lrlr}\log [\mathrm{Li} \text { ppm] ccc } & 1.60742 & \text { DF } & 2 \\ \text { Mean Difference } & -0.4757 & \text { Prob }>|t| & <.0001\end{array}$ $\begin{array}{llll}\text { Mean Difference } & -0.4757 & \text { Prob }>|t| & <.0001 \\ \text { Std Error } & 0.00263 & \text { Prob }>t & 1.0000\end{array}$ $\begin{array}{llll}\text { Upper95\% } & -0.4643 & \text { Prob }<\mathrm{t} & <.0001\end{array}$ Correlation

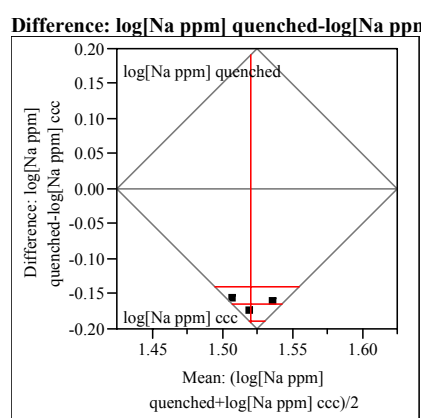

$\log [\mathrm{Na}$ ppm] quenched $\quad 1.4383 \quad \mathrm{t}$-Ratio $\quad-29.1316$ $\begin{array}{lrlr}\log [\mathrm{Na} \text { ppm] ccc } & 1.60366 & \mathrm{DF} & 2 \\ \text { Mean Difference } & -0.1654 & \mathrm{PFb}>\text { tt } & 0.0012\end{array}$ $\begin{array}{llll}\text { Mean Difference } & -0.1654 & \text { Prob }>|t| & 0.0012 \\ \text { Std Error } & 0.00568 & \text { Prob }>t & 0.9994\end{array}$ $\begin{array}{llll}\text { Std Error } & 0.00568 & \text { Prob }>t & 0.9994 \\ \text { Upper95\% } & -0.1409 & \text { Prob }<t & 0.0006\end{array}$ Upper95\% $\begin{array}{rll}-0.1409 & \text { Prob }<\mathrm{t} \quad 0.0006 \\ -0.1898 & & \end{array}$ Lower $95 \%$ .80839 Difference: $\log [\mathrm{Si}$ ppm] quenched-log[Si ppm] ccc

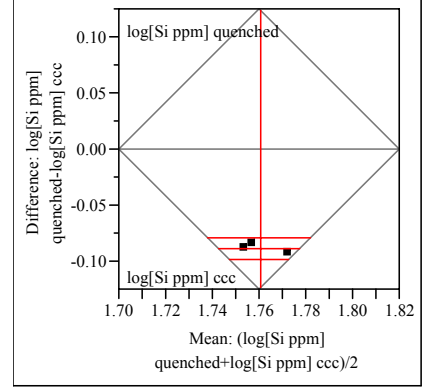

$\log [$ Si ppm] quenched $1.71642 \quad \mathrm{t}$-Ratio $\quad-38.0699$

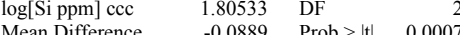
$\begin{array}{lllll}\text { Std Error } & 0.00234 & \text { Prob }>\text { t } & 0.9997\end{array}$ $\begin{array}{llll}\text { Upper95\% } & -0.0789 & \text { Prob }<\mathrm{t} & 0.0003\end{array}$ -0.0789
-0.099
0.9581 0.95891
Matched Pairs Glass \#=44 ifference: $\log [\mathbf{B}$ ppm] quenched-log[B ppm] cec

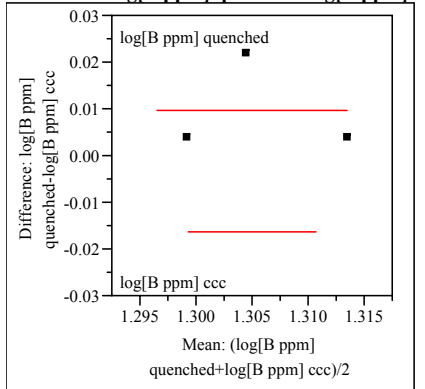

$\log [\mathrm{B} \mathrm{ppm}]$ quenched $1.31053 \quad \mathrm{t}$-Ratio $\quad 1.59706$ $\begin{array}{llll}\text { Mean Difference } & 0.00956 & \text { Prob }>|t| & 0.2513\end{array}$ $\begin{array}{llll}\text { Std Error } & 0.00599 & \text { Prob }>\mathrm{t} & 0.1257 \\ \text { Upper } 95 \% & 0.03533 & \text { Prob }<\mathrm{t} & 0.8743\end{array}$ Upper95\%

$\mathrm{N}$ $-0.0162$

Correlation 0.32406 Difference: $\log [\mathrm{Li}$ ppm] quenched-log[Li ppm] ccc

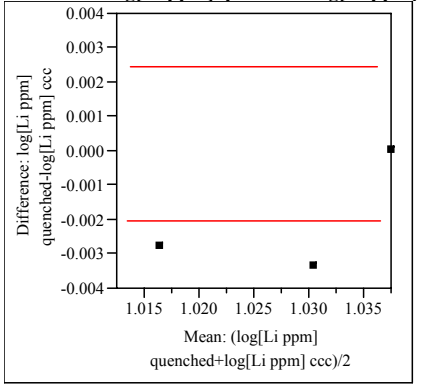

$\log [$ Li ppm] quenched $\quad 1.02703 \quad \mathrm{t}$-Ratio $\quad-1.9734$ $\begin{array}{lrlr}\log [\mathrm{Li} \text { ppm] } \mathrm{ccc} & 1.02909 & \text { DF } & 2 \\ \text { Mean Difference } & -0.0021 & \text { Prob }>|t| & 0.1872\end{array}$ $\begin{array}{llll}\text { Mean Difference } & -0.0021 & \text { Prob }>|t| & 0.1872 \\ \text { Std Error } & 0.00104 & \text { Prob }>t & 0.9064\end{array}$ $\begin{array}{llll}\text { Upper95\% } & 0.00242 & \text { Prob }<t \quad 0.0936\end{array}$ $-0.0065$ Correlation

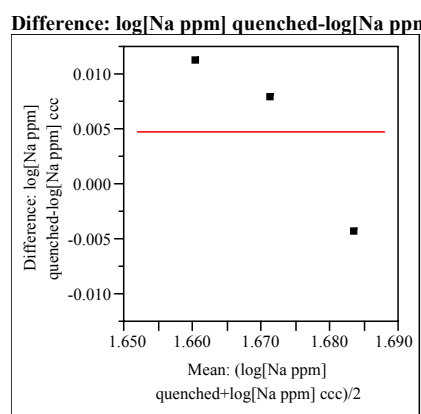

$\log [\mathrm{Na}$ ppm] quenched $1.67411 \quad \mathrm{t}$-Ratio 1.00649 $\log [\mathrm{Na} \mathrm{ppm}] \mathrm{ccc} \quad 1.66935$ DF 1.0062

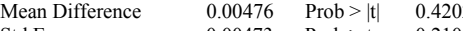
$\begin{array}{llll}\text { Std Error } & 0.0073 & \text { Prob }>\mathrm{t} & 0.2101 \\ \text { Upper95\% } & 0.02512 & \text { Prob }<\mathrm{t} & 0.7899\end{array}$ Lower $95 \%$

Correlation $\quad 0.9748$ Difference: $\log [\mathrm{Si}$ ppm] quenched-log[Si ppm] cce

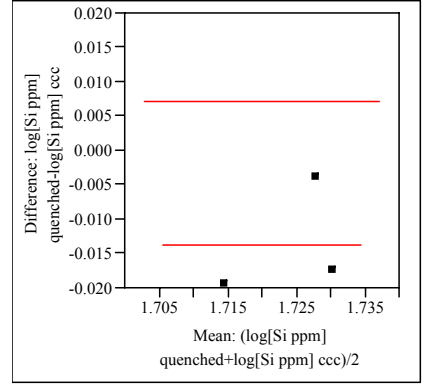

$\log [$ Si ppm] quenched $\quad 1.71731 \quad \mathrm{t}$-Ratio $\quad-2.8219$ $\log [\mathrm{Si}$ ppm] $\mathrm{ccc}-1.73102 \quad \mathrm{DF} \quad-2.82199$

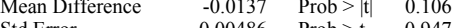
$\begin{array}{llll}\text { Std Error } & 0.00736 & \text { Prob }>\text { t } & 0.9470\end{array}$ Lower95\% 3
0.65128 
Exhibit B7. Effects of Heat Treatment on PCT $\log (\mathrm{ppm})$-Response of Study Glasses

Matched Pairs Glass \#=45

ifference: $\log [\mathbf{B}$ ppm] quenched-log$[B$ ppm] cec

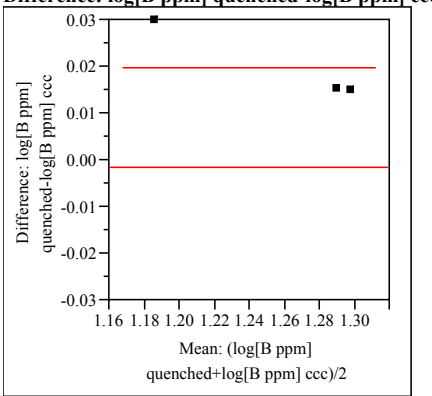

$\log [\mathrm{B}$ ppm] quenched 1.26751 t-Ratio 3.94232 $\begin{array}{llll}\log [\mathrm{B} \mathrm{ppm}] \mathrm{ccc} & 1.24779 & \mathrm{DF} & 2 \\ \text { Mean Difference } & 0.01973 & \text { Prob }>|t| & 0.0587\end{array}$ $\begin{array}{lrll}\text { Std Error } & 0.005 & \text { Prob }>t & 0.0294\end{array}$ Upper95\% $\quad 0.04126 \quad$ Prob $<$ t $\quad 0.9706$

$\mathrm{N}$ $-0.00$

Correlation $\quad 0.99998$ Difference: $\log [\mathrm{Li}$ ppm] quenched-log[Li ppm] $\mathrm{ccc}$

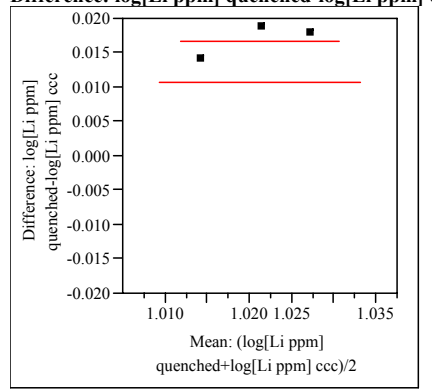

$\log [$ Li ppm] quenched 1.02935 t-Ratio 11.96197 $\begin{array}{llll}\text { Mean Difference } & 0.01676 & \text { Prob }>|t| & 0.0069\end{array}$ $\begin{array}{llll}\text { Std Error } & 0.0014 & \text { Prob }>t & 0.0035 \\ \text { Upper } 95 \% & 0.02279 & \text { Pob } & 0.965\end{array}$ Upper95\%

Correlation

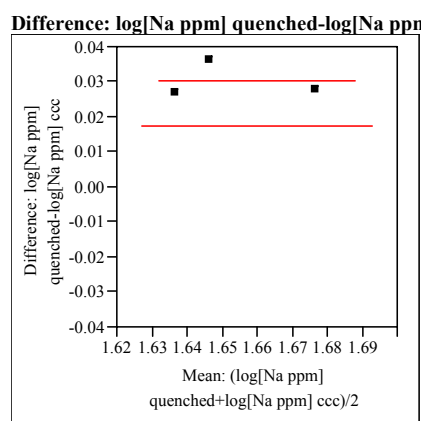

$\log [\mathrm{Na}$ ppm] quenched $1.66818 \quad \mathrm{t}$-Ratio $\quad 10.15427$ $\begin{array}{lrlr}\log [\mathrm{Na} p \mathrm{ppm}] \mathrm{ccc} & 1.63814 & \mathrm{DF} & 2 \\ \text { Mean Difference } & 0.03004 & \text { Prob }>|\mathrm{t}| & 0.0096\end{array}$ $\begin{array}{llll}\text { Std Error } & 0.00296 & \text { Prob }>t & 0.0048\end{array}$ $\begin{array}{llll}\text { Upper95\% } & 0.04277 & \text { Prob }<1 \quad 0.9952\end{array}$$$
\begin{array}{r}
3 \\
0.9714
\end{array}
$$

Difference: $\log [\mathrm{Si}$ ppm] quenched-log[Si ppm] ccc

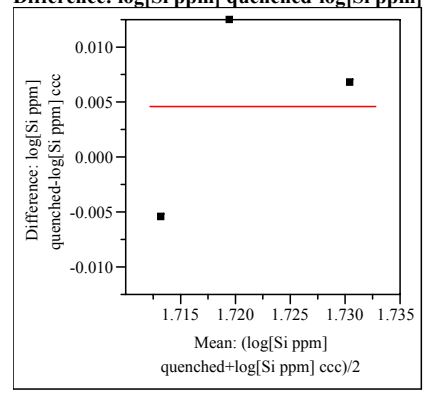

$\log [$ Si ppm] quenched $1.72327 \quad$ t-Ratio $\quad 0.849206$ $\begin{array}{lllll}\log [\mathrm{Si} \text { ppm] } \mathrm{ccc} & 1.71875 & \mathrm{DF} & 2 \\ \mathrm{M} & 0.0452 & \mathrm{PF}>\mid \mathrm{r} & 0.4852\end{array}$ $\begin{array}{llll}\text { Mean Difference } & 0.00452 & \text { Prob }>|t| & 0.4852 \\ \text { Std Error } & 0.00532 & \text { Prob }>t & 0.2426\end{array}$ $\begin{array}{llll}\text { Std Error } & 0.00532 & \text { Prob }>\mathrm{t} & 0.2426 \\ \text { Upper95\% } & 0.02742 & \text { Prob }<\mathrm{t} & 0.7574\end{array}$

\begin{tabular}{ll} 
Correlation $\quad 0.62832$ \\
\hline
\end{tabular} 
Exhibit B8. Normalized PCT Responses for Both Heat Treatments of the Study Glasses by Compositional View

Variability Gage Comp View $=$ measured

Variability Chart for $\log \mathrm{NL}[\mathrm{B}(\mathrm{g} / \mathrm{L})]$

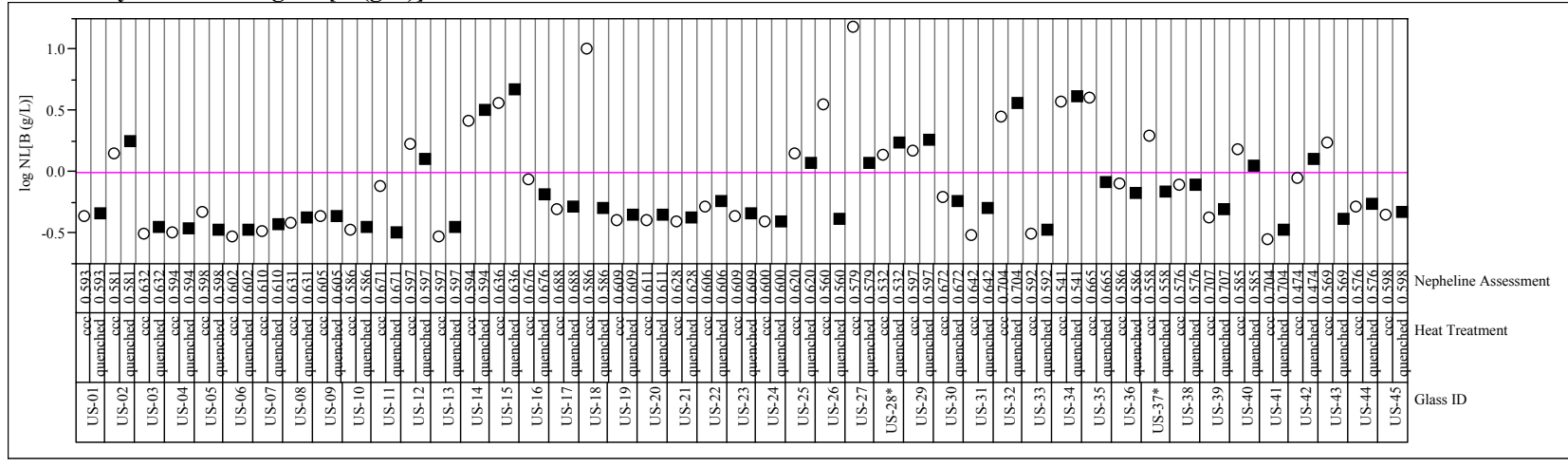

Variability Gage Comp View=measured

Variability Chart for log NL[Li(g/L)]

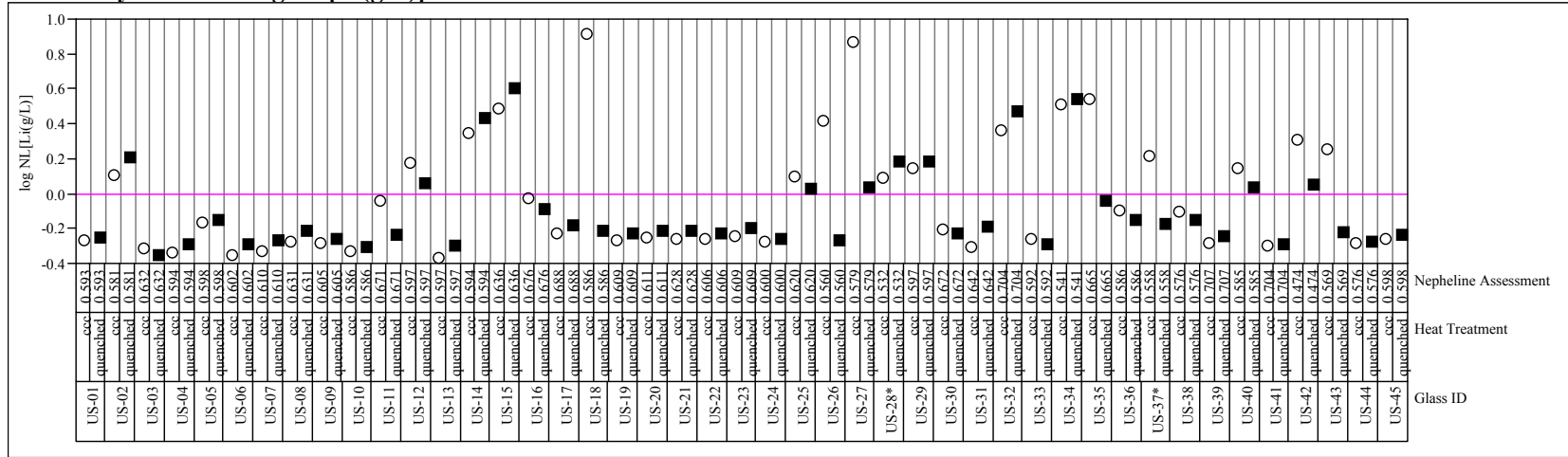

Variability Gage Comp View=measured

Variability Chart for $\log \mathrm{NL}[\mathrm{Na}(\mathrm{g} / \mathrm{L})]$

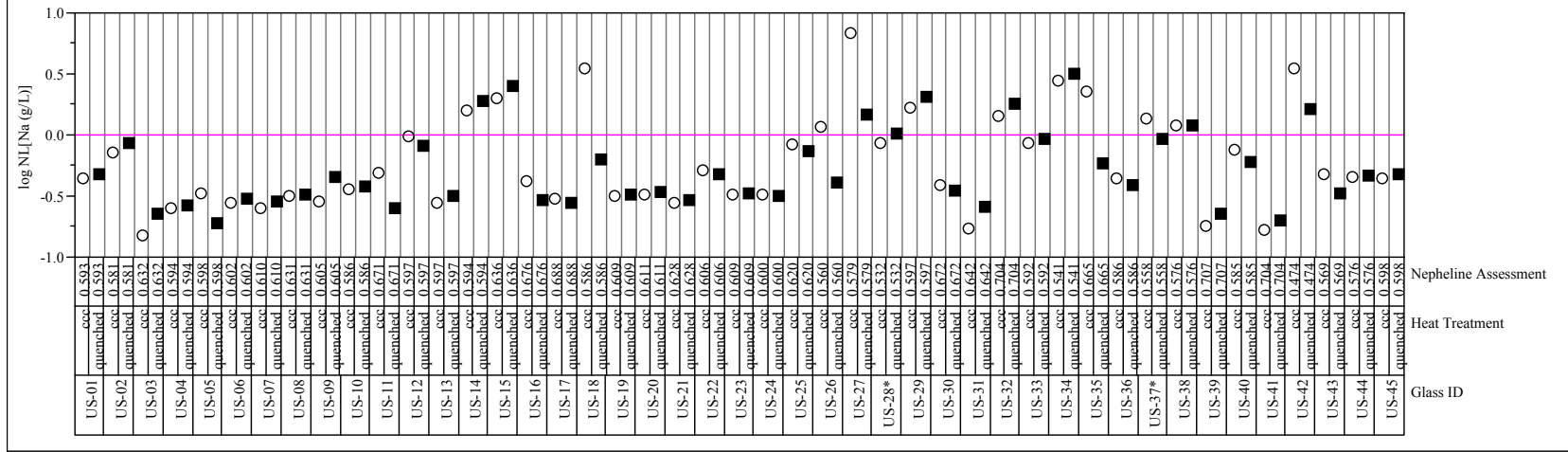


Exhibit B8. Normalized PCT Responses for Both Heat Treatments of the Study Glasses by Compositional View

Variability Gage Comp View=measured

Variability Chart for $\log \mathrm{NL}[\mathrm{Si}(\mathrm{g} / \mathrm{L})]$

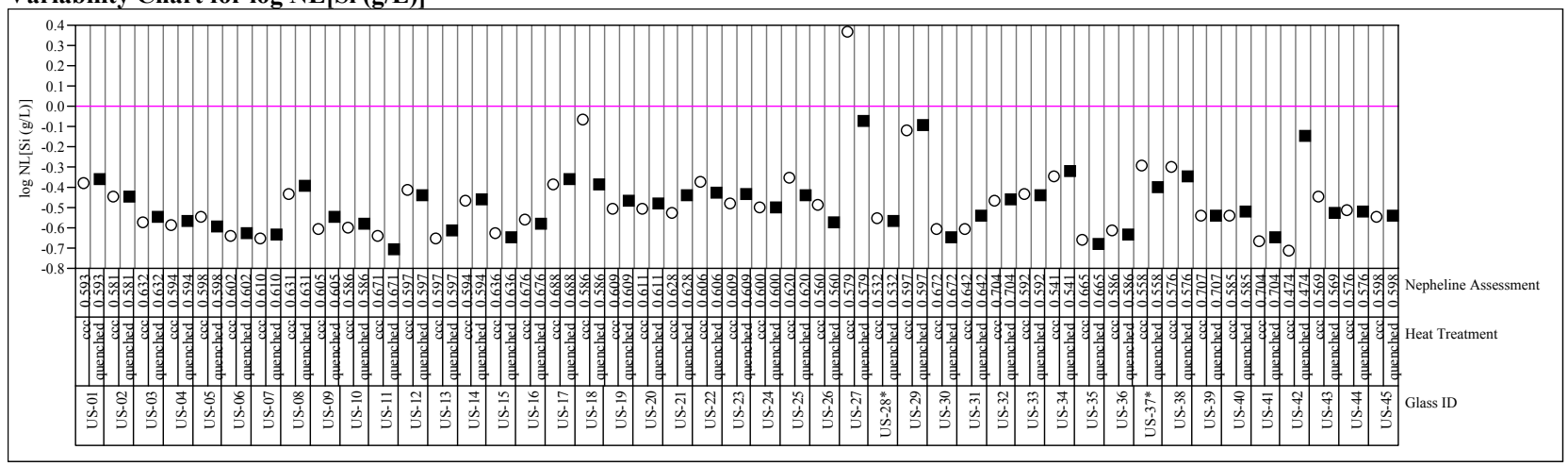

Variability Gage Comp View $=$ measured bc

Variability Chart for $\log$ NL[B (g/L)]

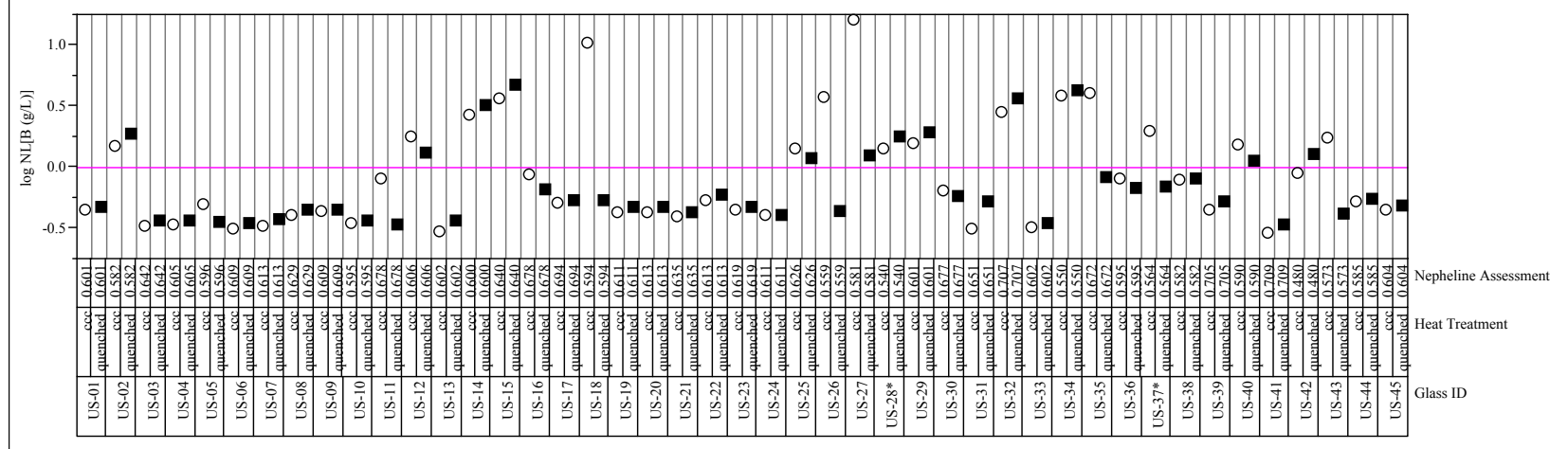

Variability Gage Comp View $=$ measured bc

Variability Chart for $\log \mathrm{NL}[\mathrm{Li}(\mathrm{g} / \mathrm{L})]$

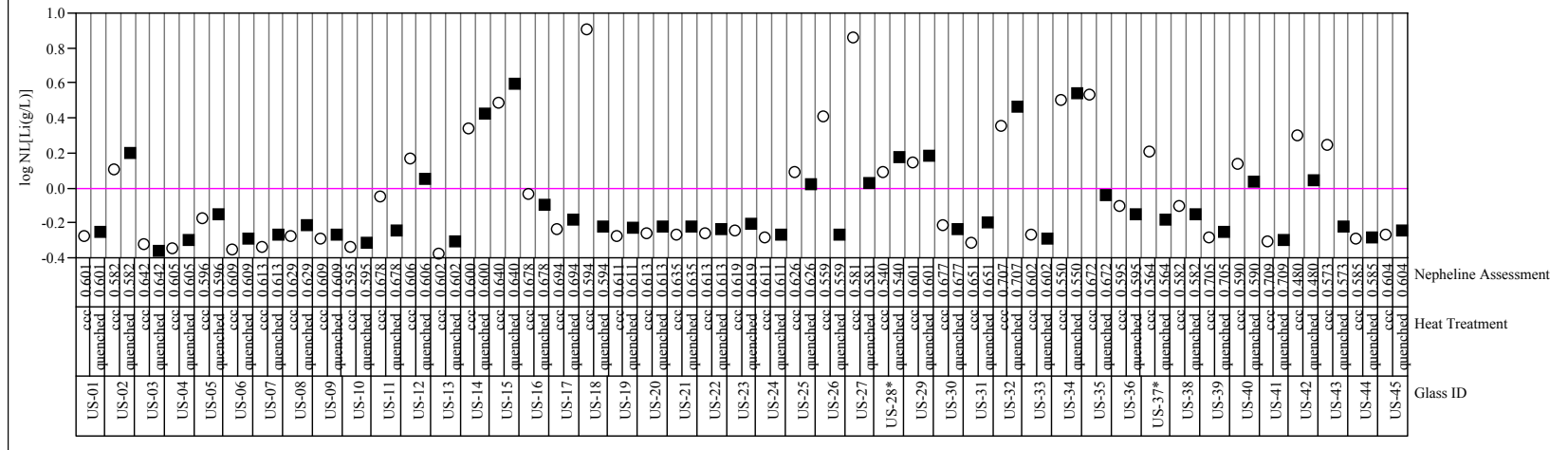


Exhibit B8. Normalized PCT Responses for Both Heat Treatments of the Study Glasses by Compositional View

Variability Gage Comp View $=$ measured bc

Variability Chart for $\log \mathrm{NL}[\mathrm{Na}(\mathrm{g} / \mathrm{L})]$

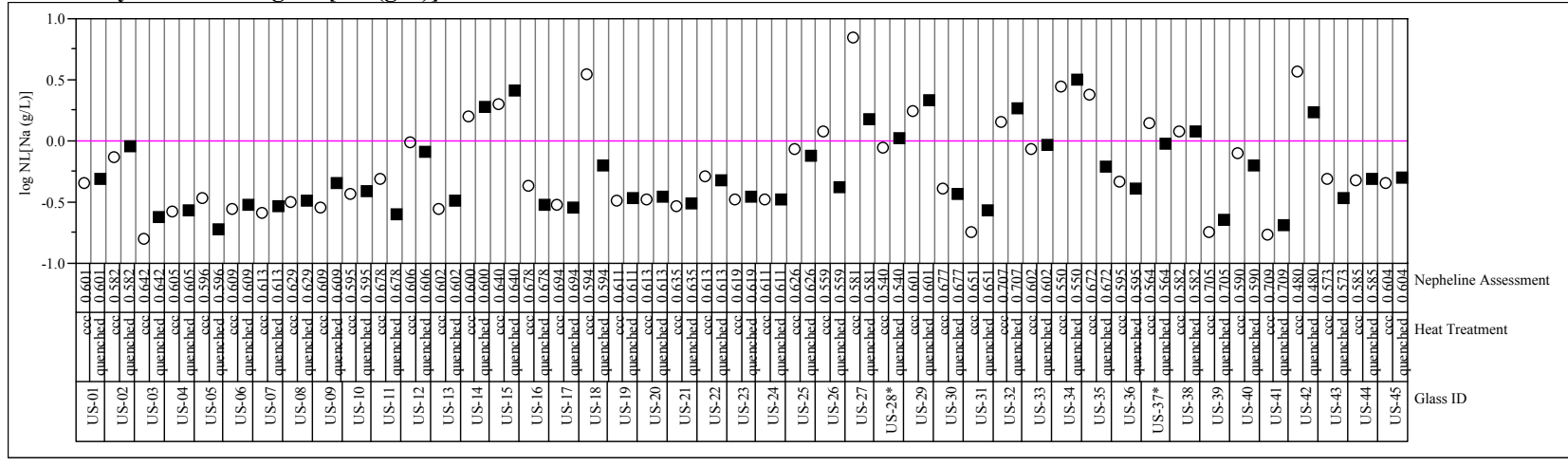

Variability Gage Comp View $=$ measured bc

Variability Chart for log NL[Si (g/L)]

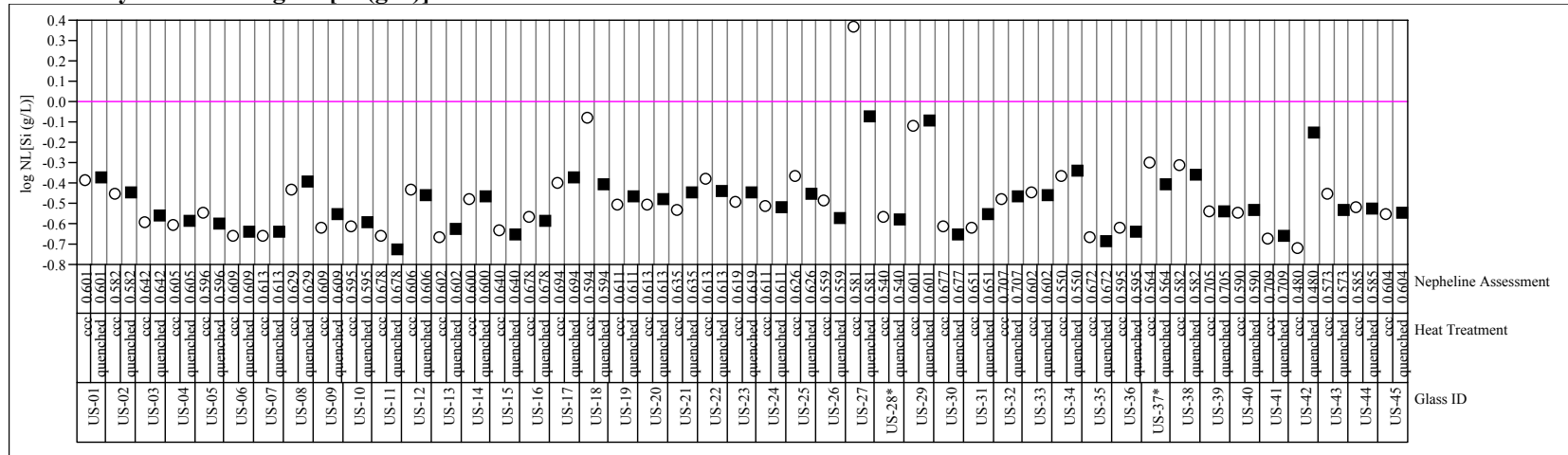

Variability Gage Comp View $=$ target

Variability Chart for $\log \mathrm{NL}[\mathrm{B}(\mathrm{g} / \mathrm{L})]$

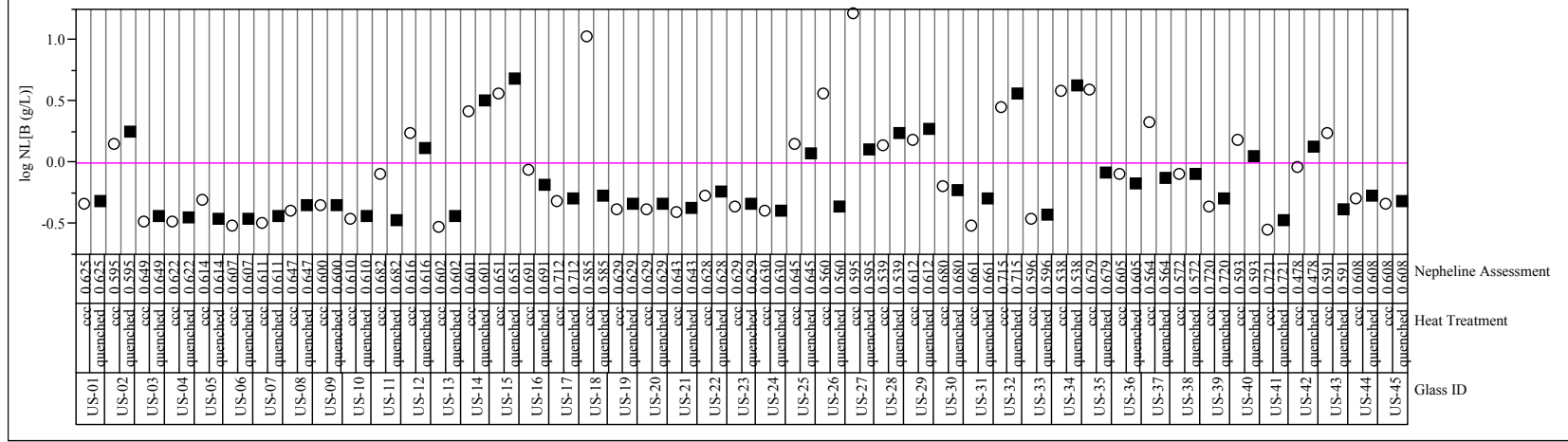


Exhibit B8. Normalized PCT Responses for Both Heat Treatments of the Study Glasses by Compositional View

Variability Gage Comp View=target

Variability Chart for $\log \mathrm{NL}[\mathrm{Li}(\mathrm{g} / \mathrm{L})]$

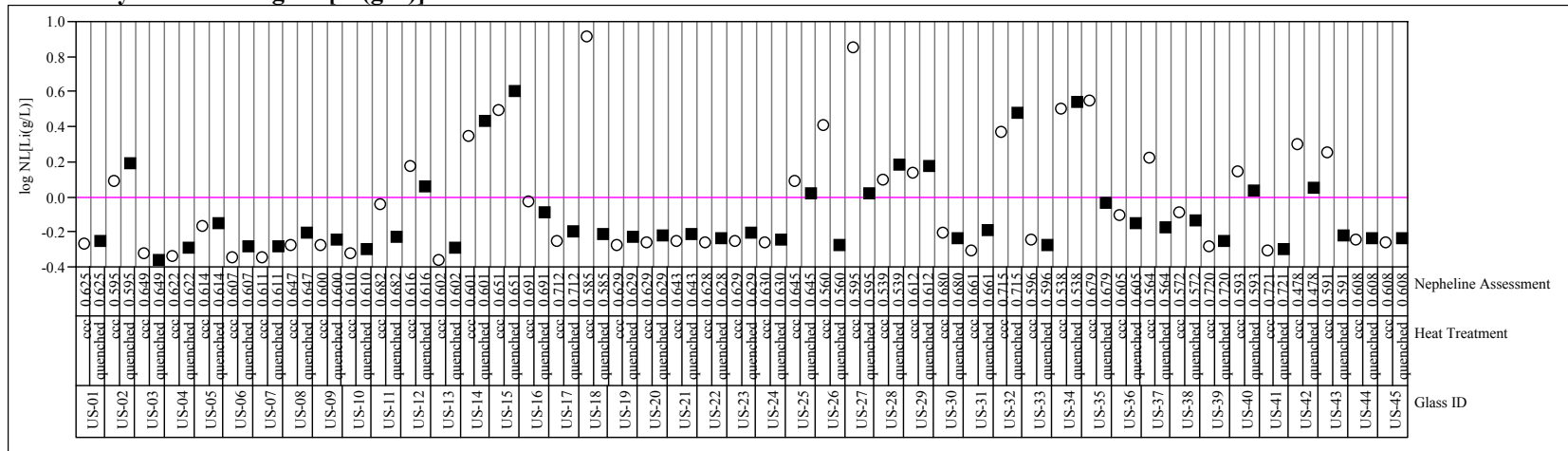

Variability Gage Comp View $=$ target

Variability Chart for $\log \mathrm{NL}[\mathrm{Na}(\mathrm{g} / \mathrm{L})]$

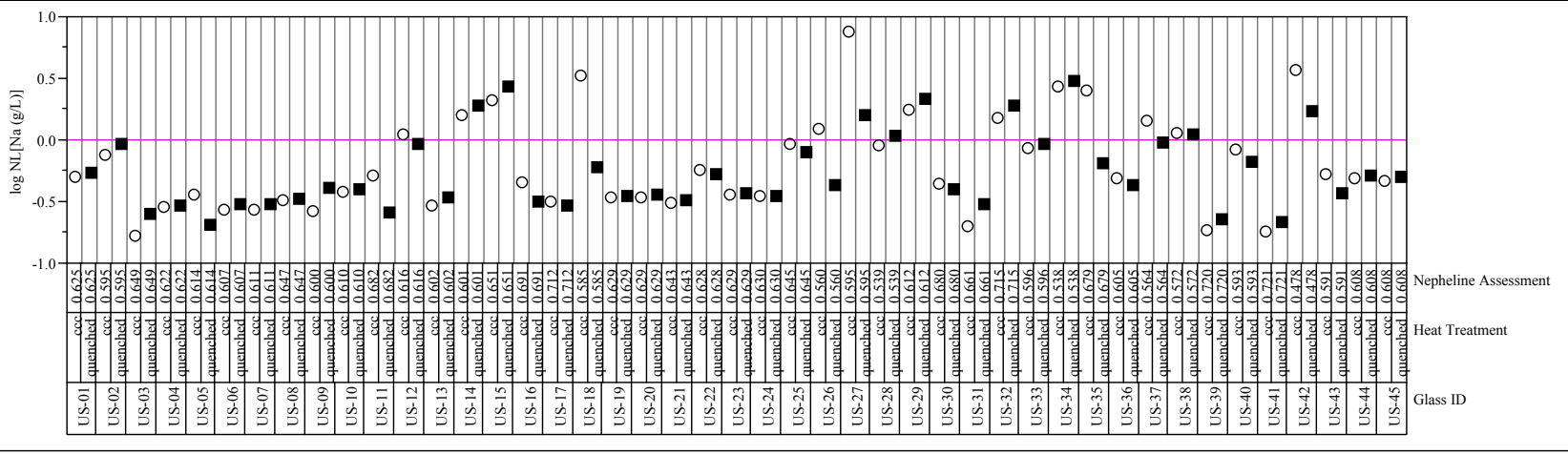

Variability Gage Comp View $=$ target

Variability Chart for $\log \mathrm{NL}[\mathrm{Si}(\mathrm{g} / \mathrm{L})]$

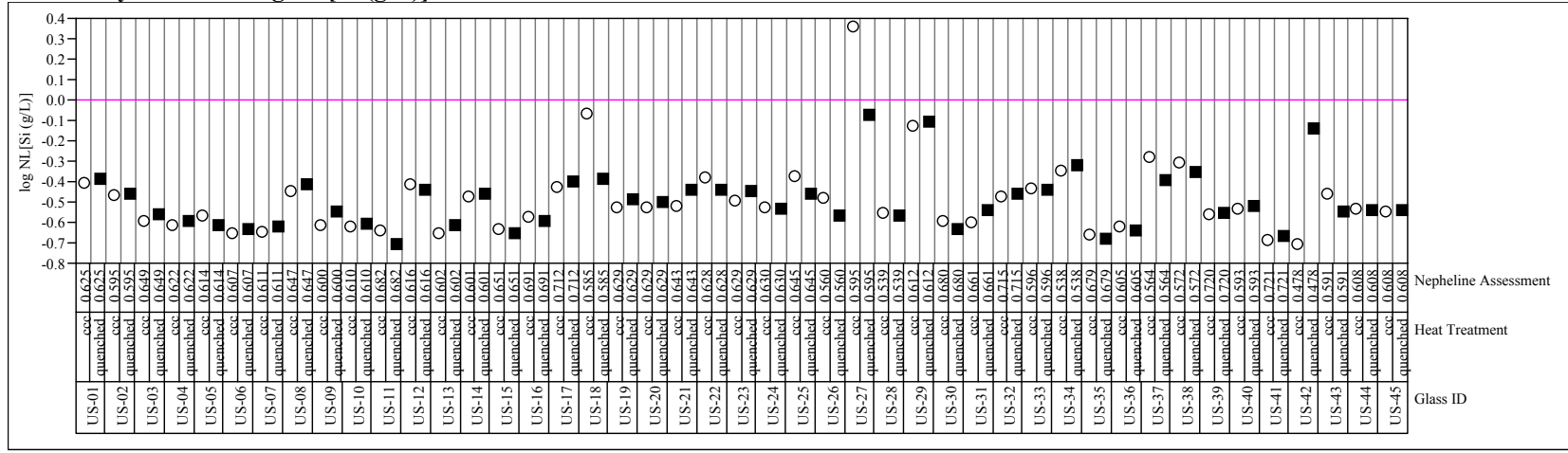

